Florida International University FIU Digital Commons

$3-17-2014$

\title{
Conformational Dynamics Associated with Ligand Binding to Vertebrate Hexa-coordinate Hemoglobins
}

Luisana Astudillo

lastu001@fiu.edu

DOI: $10.25148 /$ etd.FI14040865

Follow this and additional works at: https:// digitalcommons.fiu.edu/etd

Part of the Biochemistry Commons, Biophysics Commons, and the Chemistry Commons

\section{Recommended Citation}

Astudillo, Luisana, "Conformational Dynamics Associated with Ligand Binding to Vertebrate Hexa-coordinate Hemoglobins" (2014). FIU Electronic Theses and Dissertations. 1177.

https://digitalcommons.fiu.edu/etd/1177 


\section{FLORIDA INTERNATIONAL UNIVERSITY}

Miami, Florida

\section{CONFORMATIONAL DYNAMICS ASSOCIATED WITH LIGAND BINDING TO VERTEBRATE HEXA-COORDINATE HEMOGLOBINS}

A dissertation submitted in partial fulfillment of the requirements for the degree of DOCTOR OF PHILOSOPHY

in

\section{CHEMISTRY}

by

Luisana Astudillo 
To: Dean Kenneth G. Furton

College of Arts and Sciences

This dissertation, written by Luisana Astudillo, and entitled Conformational Dynamics Associated with Ligand Binding to Vertebrate Hexa-coordinate Hemoglobins, having been approved in respect to style and intellectual content, is referred to you for judgment.

We have read this dissertation and recommend that it be approved.

$\begin{array}{r}\hline \text { Bernard Gerstman } \\ \hline \text { Jeffrey Joens } \\ \hline \text { Andrew Terentis } \\ \hline \text { Xiaotang Wang } \\ \hline \text { Jaroslava Miksovska, Major Professor }\end{array}$

Date of Defense: March 17, 2014

The dissertation of Luisana Astudillo is approved.

$\begin{array}{r}\begin{array}{r}\text { Dean Kenneth G. Furton } \\ \text { College of Arts and Sciences }\end{array} \\ \hline \begin{array}{r}\text { Dean Lakshmi N. Reddi } \\ \text { University Graduate School }\end{array}\end{array}$

Florida International University, 2014 
(C) Copyright 2014 by Luisana Astudillo

All rights reserved. 


\section{DEDICATION}

I dedicate this work to my daughter Diana and my husband Cesar. Without their patience, support, and unconditional love, the completion of this work would not have been possible. 


\section{ACKNOWLEDGMENTS}

I would like to express my gratitude to my major Professor, Dr. Jaroslava Miksovska, for giving me the opportunity to work on this project and offer her time and dedication to teach me and train me in all the techniques available in the laboratory. I greatly appreciate all her mentoring and encouragement to publish the results of this work and present at several conferences, which I believe has helped me to become a better scientist and made my time in FIU very productive. I would also like to thank my committee members Dr. Xiaotang Wang, Dr. Andrew Terentis, Dr. Bernard Gerstman and Dr. Jeffrey Joens, for taking the time to provide me with helpful comments and ideas to improve my research.

I would like to thank our collaborators from the University of Paris-Sud IX (Orsay, France) Dr. Sophie Bernad, Valerie Derrien and Dr. Pierre Sebban, as well as Purushottam Tiwari and Dr. Yesim Darici from the Physics Department at FIU for such a productive collaboration and their helpful insights during my research.

I also thank former and present members of Dr. Miksovska's research group. Simona Horsa and Gangadhar Duhlipala thank you for your help and training during my first years in the program and for offering me your friendship through all these years. Thanks to Khoa Pham, Walter Gonzalez, David Butcher, Vanessa Capera, Antonija Tangar, and Ruben Fernandez for helpful discussions and good times in the lab.

I especially thank my family for all their support and encouragement during all these years. I am indebted to my parents for all their love, dedication and sacrifice to give me the best education possible, which has led me to become the person I am today. I thank my sister for all her support and help in times when I needed it the most. 
I also give special thanks to my husband Cesar. Without his help, love and encouragement to keep going forward, I am certain I would not have completed this dissertation. I also appreciate all the patience of my daughter Diana, because at her short age she seems to understand that mom has to complete her homework.

I also would like to thank the Department of Chemistry and Biochemistry at FIU for accepting me into their graduate program, and for their financial support as Teaching and Research Assistant. In addition, I would like to acknowledge the financial support provided by the MBRS-RISE Biomedical Research Initiative (BRI) through the 2012 BRI Student Summer Research award (NIH/NIGMSR25 GM061347). The content presented here is solely the responsibility of the author and does not necessarily represent the official views of the National Institutes of Health. 


\title{
ABSTRACT OF THE DISSERTATION \\ CONFORMATIONAL DYNAMICS ASSOCIATED WITH LIGAND BINDING TO \\ VERTEBRATE HEXA-COORDINATE HEMOGLOBINS
}

\author{
by \\ Luisana Astudillo \\ Florida International University, 2014 \\ Miami, Florida \\ Professor Jaroslava Miksovska, Major Advisor
}

Neuroglobin $(\mathrm{Ngb})$ and cytoglobin $(\mathrm{Cygb})$ are two new additions to the globin family, exhibiting heme iron hexa-coordination, a disulfide bond and large internal cavities. These proteins are implicated in cytoprotection under hypoxic-ischemic conditions, but the molecular basis of their cytoprotective function is unclear.

Herein, a photothermal and spectroscopic study of the interactions of diatomic ligands with Ngb, Cygb, myoglobin and hemoglobin is presented. The impact of the disulfide bond in Ngb and Cygb and role of conserved residues in Ngb His64, Val68, Cys55, Cys120 and Tyr44 on conformational dynamics associated with ligand binding/dissociation were investigated. Transient absorption and photoacoustic calorimetry studies indicate that $\mathrm{CO}$ photo-dissociation from $\mathrm{Ngb}$ leads to a volume expansion $\left(13.4 \pm 0.9 \mathrm{~mL} \mathrm{~mol}^{-1}\right)$, whereas a smaller volume change was determined for $\mathrm{Ngb}$ with reduced Cys $\left(\Delta \mathrm{V}=4.6 \pm 0.3 \mathrm{~mL} \mathrm{~mol}^{-1}\right)$. Furthermore, Val68 side chain regulates ligand migration between the distal pocket and internal hydrophobic cavities since Val68Phe geminate quantum yield is $~ 2.7$ times larger than that of WT Ngb. His64Gln and Tyr44Phe mutations alter the thermodynamic parameters associated with $\mathrm{CO}$ photo- 
release indicating that electrostatic/hydrogen binding network that includes heme propionate groups, Lys 67, His64, and Tyr 44 in Ngb modulates the energetics of CO photo-dissociation. In Cygb, $\mathrm{CO}$ escape from the protein matrix is fast $(<40 \mathrm{~ns})$ with a $\Delta \mathrm{H}$ of $18 \pm 2 \mathrm{kcal} \mathrm{mol}^{-1}$ in $\mathrm{Cygb}^{\mathrm{red}}$, whereas disulfide bridge formation promotes a biphasic ligand escape associated with an overall enthalpy change of $9 \pm 4 \mathrm{kcal} \mathrm{mol}^{-1}$. Therefore, the disulfide bond modulates conformational dynamics in Ngb and Cygb. I propose that in Cygb with reduced Cys the photo-dissociated ligand escapes through the hydrophobic tunnel as occurs in $\mathrm{Ngb}$, whereas the $\mathrm{CO}$ preferentially migrates through the His64 gate in Cygb ${ }^{\text {ox }}$.

To characterize Cygb surface 1,8-ANS interactions with Cygb were investigated employing fluorescence spectroscopy, ITC and docking simulations. Two 1,8-ANS binding sites were identified. One binding site is located close to the extended $\mathrm{N}$-terminus of Cygb and was also identified as a binding site for oleate. Furthermore, guanidinium hydrochloride-induced unfolding studies of Cygb reveal that the disulfide bond does not impact Cygb stability, whereas binding of cyanide slightly increases the protein stability. 


\section{TABLE OF CONTENTS}

CHAPTER

PAGE

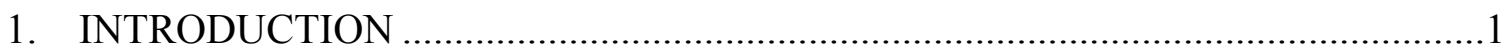

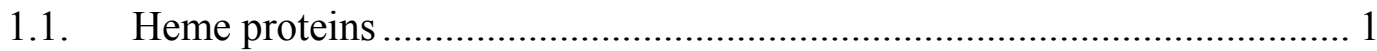

1.2. Electronic properties and absorption spectroscopy of heme proteins....... 19

1.3. Heme protein interactions with diatomic ligands ..................................... 21

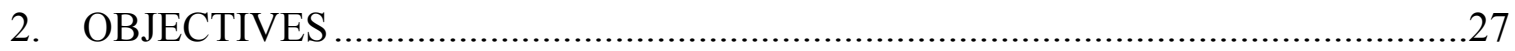

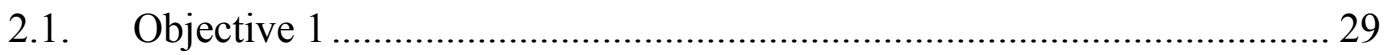

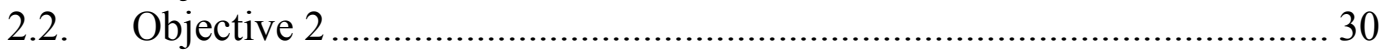

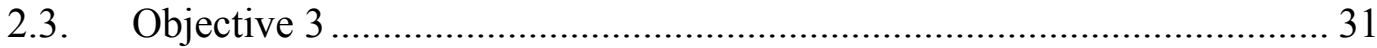

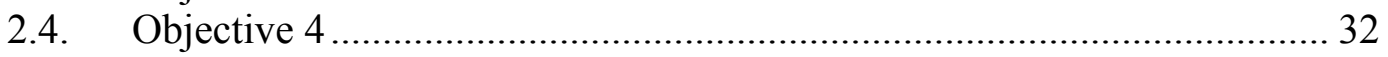

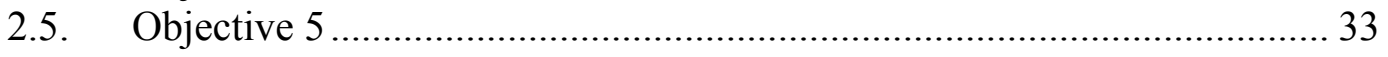

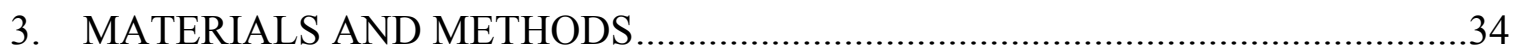

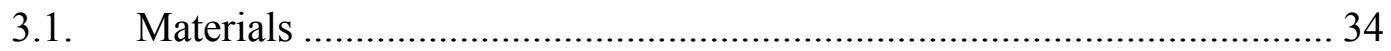

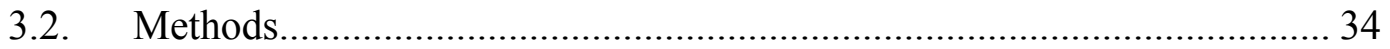

4. CONFORMATIONAL DYNAMICS IN HUMAN NEUROGLOBIN: EFFECT OF HIS 64, VAL 68 AND CYS 120 ON LIGAND MIGRATION ............72

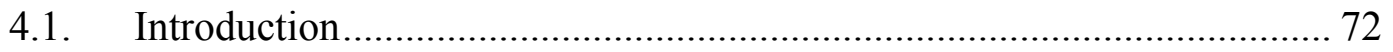

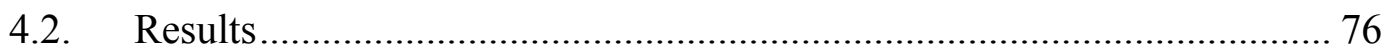

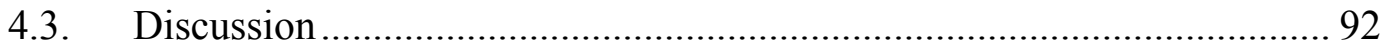

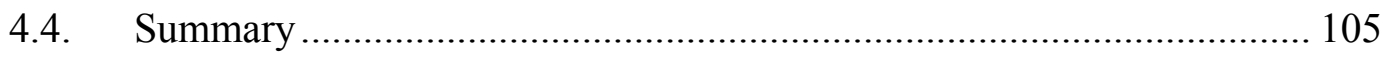

5. THE ROLE OF THE DISULFIDE BOND AND TYROSINE 44 RESIDUE IN TRANSMISSION OF STRUCTURAL INFORMATION FROM THE CD LOOP TO THE HEME BINDING POCKET IN NEUROGLOBIN .................107

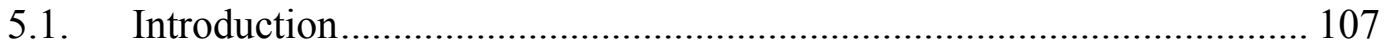

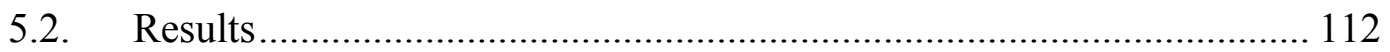

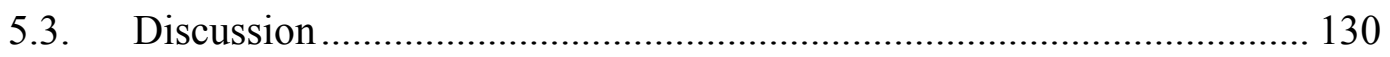

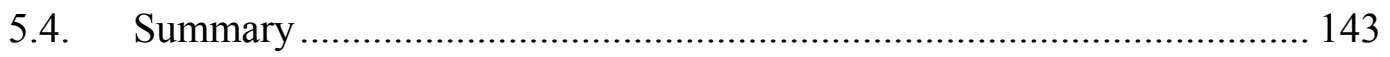

6. REDUCTION OF THE INTERNAL DISULFIDE BOND BETWEEN CYS 38 AND 83 SWITCHES THE LIGAND MIGRATION PATHWAY IN

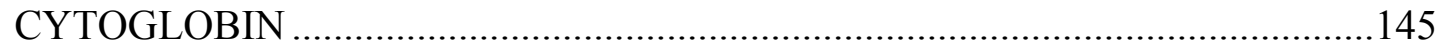

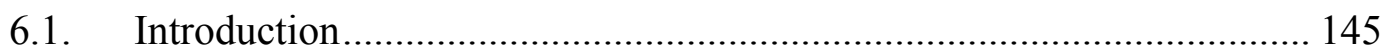

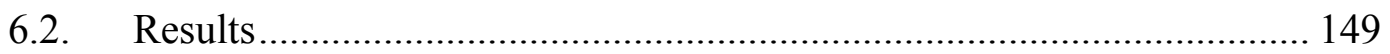

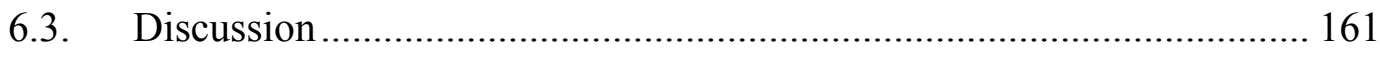

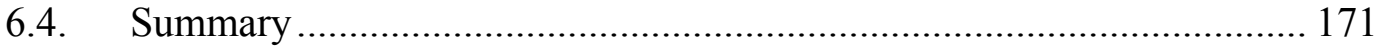

7. TIME-RESOLVED THERMODYNAMICS ASSOCIATED WITH EXOGENOUS LIGAND BINDING TO VERTEBRATE HEMOGLOBINS .........173

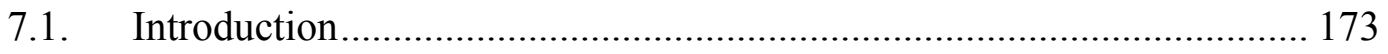




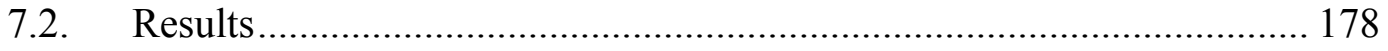

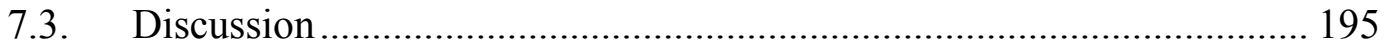

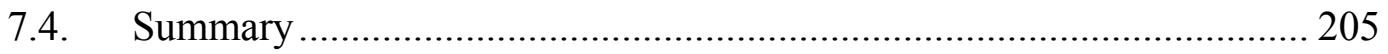

8. STUDY OF CYTOGLOBIN INTERACTIONS WITH FLUORESCENT PROBE 1-ANILINONAPHTHALENE-8-SULFONATE .......................................207

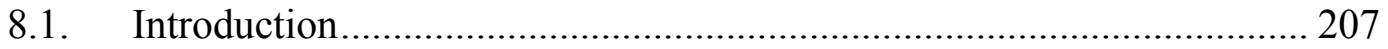

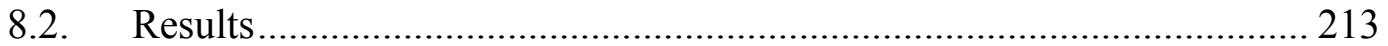

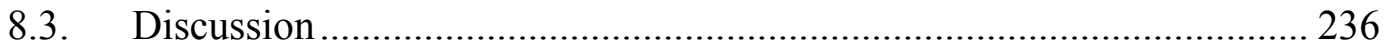

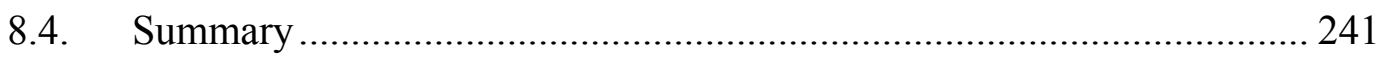

9. GUANIDINIUM HYDROCHLORIDE INDUCED UNFOLDING STUDY OF HUMAN CYTOGLOBIN AND NEUROGLOBIN ……………….................243

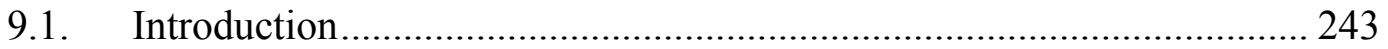

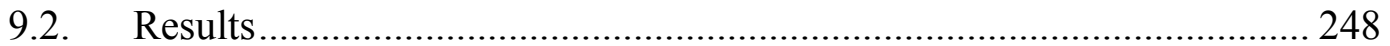

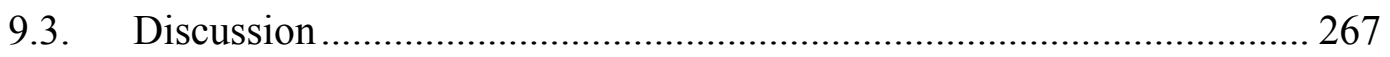

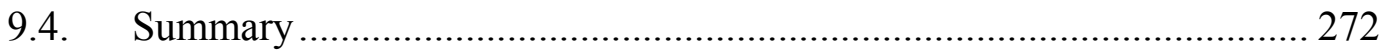

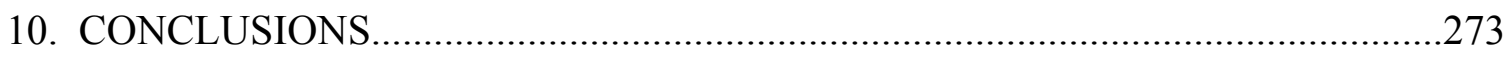

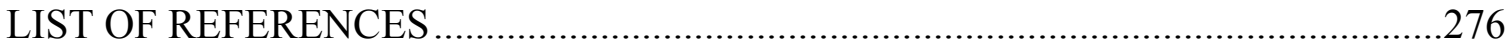

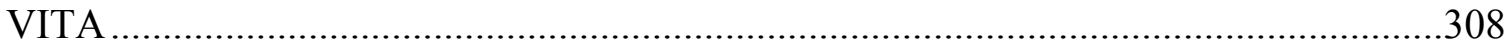




\section{LIST OF TABLES}

TABLE

PAGE

Table 3.1 Composition of running buffer for electrophoresis

Table 3.2 Composition of sample treatment buffer.

Table 3.3 Composition of Coomassie blue staining solution. .38

Table 3.4 Composition of de-staining solution .38

Table 4.1 Kinetic parameters associated with CO rebinding to wild type hNgb and mutants. Rate constants $\left(\mathrm{k}_{\mathrm{gem}}\right.$ and $\mathrm{k}_{\text {fast }}$ ) for ligand binding to fivecoordinate $\mathrm{Ngb}$ were determined at $20^{\circ} \mathrm{C}$ and $1 \mathrm{mM} \mathrm{CO}$, whereas the slow phase was measured at $35^{\circ} \mathrm{C}$ and $0.1 \mathrm{mM} \mathrm{CO}$ to increase the yield of bishistidyl $\mathrm{Ngb}$

Table 4.2 Thermodynamic parameters associated with CO photo-dissociation from Ngbs

Table 5.1 Absorption maxima of the Soret and Q bands of ferric, ferrous deoxy and $\mathrm{CO}$ adducts of wild type $\mathrm{hNgb}$, wild type rNgb and mutants.

Table 5.2 Kinetic parameters associated with $\mathrm{CO}$ geminate and bimolecular rebinding to five-coordinate wild type $\mathrm{hNgb}$, His64Gln $\mathrm{hNgb}$, and Tyr44Phe $\mathrm{hNgb}$ in the presence and absence of the internal disulfide bond.

Table 5.3 Kinetic parameters associated with $\mathrm{CO}$ bimolecular rebinding to wild type $\mathrm{hNgb}$, rNgb and mutants hNgb Cys55Ser and rNgb Gly46Cys.

Table 5.4 Thermodynamic parameters associated with CO escape from wild type $\mathrm{Ngb}$ and mutants

Table 5.5 Rate constants for $\mathrm{CO}$ association and dissociation to Ngbs

Table 5.6 Dissociation constants, $\Delta \mathrm{G}$ and $\Delta \mathrm{S}$ for $\mathrm{CO}$ dissociation from Ngbs

Table 6.1 Reaction $(\Delta \mathrm{H}$ and $\Delta \mathrm{V})$ and activation $\left(\Delta \mathrm{H}^{\#}\right.$ and $\left.\Delta \mathrm{S}^{\#}\right)$ thermodynamic parameters associated with the photo-dissociation of $\mathrm{Fe}-\mathrm{CO}$ bond and subsequent ligand escape from the protein matrix in $\mathrm{Cygb}^{\mathrm{ox}}$ and $\mathrm{Mb}$.

Table 6.2 Total volume and enthalpy changes associated with the CO photorelease from Cygb, $\mathrm{Ngb}$ and horse heart $\mathrm{Mb}$.

Table 6.3 Rate constants for $\mathrm{CO}$ binding to $\mathrm{Cygb}^{\mathrm{ox}}$ and $\mathrm{Cygb}^{\text {red }}$ obtained using MEM analysis. 
Table 6.4 Rate constants for $\mathrm{CO}$ binding to $\mathrm{Cygb}^{\mathrm{ox}}$ and $\mathrm{Cygb}^{\text {red }}$ and quantum yield for bimolecular $\mathrm{CO}$ binding determined using a four exponential decay model.

Table 6.5 Microscopic rate constants associated with $\mathrm{CO}$ migration in $\mathrm{Cygb}^{\mathrm{ox}}$ at 20 ${ }^{\circ} \mathrm{C}$.

Table 6.6 Microscopic rate constants associated with $\mathrm{CO}$ migration in $\mathrm{Cygb}^{\text {red }}$ at $20{ }^{\circ} \mathrm{C}$

Table 7.1 Volume and enthalpy changes associated with $\mathrm{O}_{2}$ dissociation from $\mathrm{Mb}$ in the temperature range of $6^{\circ} \mathrm{C}$ to $10^{\circ} \mathrm{C}$

Table 7.2 Thermodynamic parameters associated with $\mathrm{O}_{2}$ photo-release from $\mathrm{hNgb}$ and $\mathrm{hNgb}$ witn $500 \mathrm{mM} \mathrm{NaCl}$.

Table 7.3 Volume and enthalpy changes associated with $\mathrm{CO}$ photo-dissociation from $\mathrm{Hb}$.

Table 8.1 Dissociation constants and emission maximum for 1,8-ANS association to $\mathrm{Cygb}$ in its different forms determined using steady-state fluorescence spectroscopy.

Table 8.2 Fluorescence decay parameters associated with 1,8-ANS in complex with Cygb, Cygb ${ }^{\text {red }}$ and CNCygb.

Table 8.3 Thermodynamic parameters determined for Cygb-ANS complexes using ITC.

Table 8.4 Inhibitor constants determined for displacement of 1,8-ANS with sodium oleate in Cygb-ANS complexes.

Table 9.1 Free energy of unfolding $\left(\Delta G^{o} \mathrm{H}_{2} \mathrm{O}\right)$ and degree of hydrophobic exposure upon $\mathrm{GuHCl}$-induced unfolding (m) determined for $\mathrm{Cygb}, \mathrm{Cygb}^{\text {red }}$ and CNCygb employing UV-visible absorption spectroscopy.

Table 9.2 Free energy of unfolding $\left(\Delta G^{o} \mathrm{H}_{2} \mathrm{O}\right)$ and degree of hydrophobic exposure upon $\mathrm{GuHCl}$-induced unfolding (m) determined for $\mathrm{Cygb}, \mathrm{Cygb}^{\text {red }}$ and CNCygb employing CD spectroscopy.

Table 9.3 Free energy of unfolding $\left(\Delta G^{o}{ }_{H_{2} O}\right.$ ) and degree of hydrophobic exposure upon GuHCl-induced unfolding $(\mathrm{m})$ determined for wild type hNgb and Trp mutants employing UV-visible absorption spectroscopy.

Table 9.4 Fluorescence lifetime of hNgb and Trp mutants as a function of CNbinding. 


\section{LIST OF FIGURES}

FIGURE

PAGE

Figure 1.1 Top panel: Structure of iron protoporphyrin IX (heme b). Bottom panel: Ribbon representation of three dimensional structure of myoglobin showing nomenclature of $\alpha$ helices adopted for globins. The heme prosthetic group and the distal and proximal His residues are depicted as sticks.

Figure 1.2. Representation of phylogenetic relationships in vertebrate globins.

Figure 1.3 Sequence alignment of vertebrate globins $\mathrm{Hb}, \mathrm{Mb}, \mathrm{Ngb}, \mathrm{Cygb}$ and Adgb. Conserved residues are represented by a star below the sequence, whereas residues that strongly share similar properties are represented by two dots and those exhibiting weak similarity are represented by one dot below the sequence.

Figure 1.4 Ribbon representation of the three dimensional structure of horse heart $\mathrm{Hb}$ (PDB entry: 2HHB) showing $\alpha$ subunits in orange and $\beta$ subunits in blue...

Figure 1.5 Ribbon representation of the heme binding site in vertebrate globins $\mathrm{Hb}$ (PDB entry 1FDH), Mb (PDB entry 1WLA), Ngb (PDB entry 1OJ6), and Cygb (PDB entry 1V5H) exhibiting penta-coordination and hexa-coordination of the heme iron. The distal and proximal histidine residues are shown as sticks

Figure 1.6 Ribbon representation of the crystal structure of human Ngb (PDB entry: 1JO6). The heme prosthetic group is shown as red sticks, the distal His64 and proximal His96 are depicted in blue, and the Cys residues in purple.

Figure 1.7 Ribbon representation of the crystal structure of human Cygb (PDB entry: $1 \mathrm{~V} 5 \mathrm{H})$. The heme prosthetic group is shown as red sticks, the distal His81 and proximal His113 are depicted in blue, and the Cys residues in purple.

Figure 1.8 Oxidation of halogenated phenols by dehalperoxidase (DHP) in the presence of $\mathrm{H}_{2} \mathrm{O}_{2}$. The $\mathrm{X}$ represents halogen atoms $(\mathrm{X}=\mathrm{I}, \mathrm{Br}, \mathrm{Cl}, \mathrm{F})$.

Figure 1.9 Energy level diagrams showing the d-orbital electron occupancies of high and low-spin iron(II) and iron (III) complexes in an octahedral ligand field (Halcrow, 2008)......

Figure 1.10 Schematic representation of ligand rebinding to heme proteins according to the sequential three state model. 
Figure 3.1 Schematic representation of home-built TA instrumental set-up.

Figure 3.2 Contour plots of the entropy $S$ and the statistic parameter $\chi 2$ for a two dimensional $f(\lambda)$. The maximum entropy solution corresponds to the point where the gradient of $S$ is parallel to the gradient of $\chi^{2}$, where $\chi^{2}$ is close to 1. Modified from Steinbach et al. (Steinbach et al., 1992).

Figure 3.3 Schematic representation of the pressure change resulting from the heat release in photothermal methods. Modified from Gensch and Viappiani (Gensch \& Viappiani, 2003).

Figure 3.4 Schematic representation of home-built PAC instrumental set-up.

Figure 3.5 Schematic representation of wave propagation from the sample to the detector in PAC measurements.

Figure 3.6 Representative PAC acoustic traces for sample and reference compound......

Figure 3.7 Representative PAC acoustic traces for sample and reference compound in which the sample acoustic trace is shifted in phase with respect to the reference trace.....

Figure 3.8 Light modulation in the frequency domain approach. The solid line represents the modulated excitation light, whereas the dotted line represents the emitted light with a phase shift with respect to the excitation light. AC and DC denotes the amplitudes of the sinusoidal wave, and the subscripts $\mathrm{E}$ and F refer to the excited and emitted light, respectively. (From Ross \& Jameson, 2008).

Figure 3.9 Far-UV Circular dichroism spectra associated with various types of secondary structure. The solid line represents $\alpha$ helices, the dashed line represents anti-parallel $\beta$ sheets, the dotted line and short dashes represent random coils and irregular structures. (Kelly and Price, 1997).

Figure 4.1 Top: Sequence alignment of human Ngb and human Mb. Conserved residues are represented by a star below the sequence, whereas residues that strongly share similar properties are represented by two dots and those exhibiting weak similarity are represented by one dot below the sequence. Bottom: Ribbon representation of ferric hNgb (PDB entry 1JO6, molecule A) depicting the position of the heme group, proximal His 96, distal His 64, Val 68, Ser 120, and Ser 55.

Figure 4.2 CD spectra of wild type hNgb and mutants His64Gln, Val68Phe, His64Gln/Val68Phe, Cys120Ser, and Cys55Ser/Cys120Ser in the farUV region. The concentration of the protein was $40 \mu \mathrm{M}$ in $10 \mathrm{mM}$ Tris buffer ( $\mathrm{pH}$ 7.0). Spectra were corrected for the baseline of the buffer. 
Figure 4.3 Absorption spectra of ferric (black line), ferrous (red line) and CO bound (blue line) hNgb wild type in $50 \mathrm{mM}$ Tris buffer ( $\mathrm{pH} 7.0$ ).

Figure 4.4 Absorption spectra of ferric (black line), ferrous (red line) and CO bound (blue line) hNgb Cys120Ser in $50 \mathrm{mM}$ Tris buffer (pH 7.0).

Figure 4.5 Absorption spectra of ferric (black line), ferrous (red line) and CO bound (blue line) $\mathrm{hNgb}$ Cys55Ser/Cys120Ser in $50 \mathrm{mM}$ Tris buffer ( $\mathrm{pH} 7.0)$.

Figure 4.6 Absorption spectra of ferric (black line), ferrous (red line) and CO bound (blue line) $\mathrm{hNgb}$ Val68Phe in $50 \mathrm{mM}$ Tris buffer ( $\mathrm{pH} 7.0)$.

Figure 4.7 Absorption spectra of ferric (black line), ferrous (red line) and CO bound (blue line) His64Gln hNgb in $50 \mathrm{mM}$ Tris buffer ( $\mathrm{pH} 7.0$ ).

Figure 4.8 Absorption spectra of ferric (black line), ferrous (red line) and CO bound (blue line) His64Gln/Val68Phe hNgb in $50 \mathrm{mM}$ Tris buffer ( $\mathrm{pH}$ $7.0)$

Figure 4.9 Transient absorption traces measured for $\mathrm{CO}$ geminate recombination and bimolecular rebinding to Ngbs. The initial amplitudes following the photo-dissociation of $\mathrm{CO}$ were normalized to 1 . The solid lines correspond to a fit of the experimental data using a four exponential decay model. Conditions: $20 \mu \mathrm{M}$ protein in $50 \mathrm{mM}$ Tris buffer ( $\mathrm{pH}$ $7.0)$ at $20^{\circ} \mathrm{C}$ and $1 \mathrm{mM} \mathrm{CO}$.

Figure 4.10 Transient absorption traces measured for $\mathrm{CO}$ bimolecular rebinding to Ngbs. Traces were normalized to 1 . The solid lines correspond to a fit of the experimental data using a single exponential decay model. Conditions: $20 \mu \mathrm{M}$ protein in $50 \mathrm{mM}$ Tris buffer $(\mathrm{pH} 7.0)$ at $35^{\circ} \mathrm{C}$ and $0.1 \mathrm{mM} \mathrm{CO}$ to increase the yield of bishistidyl coordination.

Figure 4.11 Photoacoustic traces for wild type $\mathrm{hNgb}$ together with the reference compound 4SP. Conditions: $20 \mu \mathrm{M}$ protein in $50 \mathrm{mM}$ Tris buffer $(\mathrm{pH}$ 7.0). The absorbance of the sample matched that of the reference compound at $532 \mathrm{~nm}\left(\mathrm{~A}_{532 \mathrm{~nm}}=0.22\right)$.

Figure 4.12 Photoacoustic traces for hNgb H64Q together with the reference compound 4SP. Conditions: $20 \mu \mathrm{M}$ protein in $50 \mathrm{mM}$ Tris buffer $(\mathrm{pH}$ 7.0). The absorbance of the sample matched that of the reference compound at $532 \mathrm{~nm}\left(\mathrm{~A}_{532 \mathrm{~nm}}=0.23\right)$.

Figure 4.13 Plot of $\phi \mathrm{E}_{\mathrm{hv}}$ as a function of $\mathrm{C}_{\mathrm{p}} \rho / \beta$ for $\mathrm{CO}$ photo-release from wild type Ngb (solid squares), His64Gln (solid circles), Val68Phe (open triangles), Cys120Ser (open circles), Cys55Ser/Cys120Ser (open squares), and Cys55Ser (solid triangles). The corresponding volume and enthalpy changes were determined from the slope and intercept of 
the linear fits, respectively. The data for human $\mathrm{Ngb}$ and the Cys55Ser mutant were previously reported by Astudillo et al. (2010).

Figure 4.14 Ribbon representation of ferric mNgb (left, PDB entry 3GK9) and sperm whale $\mathrm{Mb}$ (right, PDB entry 1J52) depicting the position of the heme group, proximal His96, and distal His64, Val68, Ser120, and Ser55 in Ngb and analogous residues in Mb. Xenon cavities Xe1, Xe2, $\mathrm{Xe} 3$ and $\mathrm{Xe} 4$ are depicted as green spheres.

Figure 4.15 Overlay of modeled structure of $\mathrm{CO}$ bound $\mathrm{Fe}^{2+} \mathrm{mNgb}$ Val68Phe (blue) and $\mathrm{Fe}^{2+} \mathrm{mNgb}$ Val68Phe depicting the position of the heme group, distal His 64 and Phe 68 residue. Internal hydrophobic cavities are shown in light blue for $\mathrm{CO}$ bound $\mathrm{Fe}^{2+} \mathrm{mNgb}$ Val68Phe and in light orange for $\mathrm{Fe}^{2+} \mathrm{mNgb}$ Val68Phe. The modeled structures were obtained by minimization of $\mathrm{CO}$ bound $\mathrm{Fe}^{2+} \mathrm{mNgb}$ (PDB entry $1 \mathrm{~W} 92$ ) and $\mathrm{Fe}^{2+} \mathrm{mNgb}$ (PDB entry 2VRY) in which Ser 55 and Ser 120 were replaced by Cys employing Amber03 force field in YASARA software. Internal cavities were analyzed using a $1.4 \AA$ probe radius and the numerical algorithm in YASARA software and are shown as contour surfaces.

Figure 4.16 Overlay of crystal structure of $\mathrm{CO}$ bound $\mathrm{mNgb}$ (shown in purple, PDB entry 1W92) and $\mathrm{Fe}_{2}+\mathrm{mNgb}$ (shown in yellow, PDB entry 2VRY) showing the position of Ser 120, His 23, Glu 22 and Ser 19. The H-bond network connecting the helix $\mathrm{G}$ and the loop between the $\mathrm{A}$ and $\mathrm{B}$ helices in the $\mathrm{Fe}^{2+} \mathrm{mNgb}$ form (shown as black lines) is missing in the structure of $\mathrm{CO}$ bound $\mathrm{mNgb}$.

Figure 5.1 Sequence alignment of human, rat and mouse Ngb. The stars below the sequences represent identical amino acids shared by the three proteins, the two dots below the sequence indicates amino acid residues with significantly similar properties and the single dot indicates weak similarity between amino acid residues. The red arrows indicate the position of Cys residues in hNgb sequence.

Figure 5.2 Three dimensional structure of molecule A (left) and molecule B (right) of hNgb (PDB entry 1OJ6) showing the heterogeneity of Tyr 44 in $\mathrm{hNgb}$. Position of residues 46 and 55 are shown in purple.

Figure 5.3 Three dimensional structure of human Ngb (PDB entry 1OJ6, molecule B) depicting the electrostatic network including His 64, Tyr 44, Lys 67 and heme propionate groups.

Figure 5.4 Absorption spectra of ferric (black line), ferrous (red line) and CO bound (blue line) hNgb Tyr44Phe. Conditions: $20 \mu \mathrm{M}$ protein in 50 $\mathrm{mM}$ Tris buffer ( $\mathrm{pH} 7.0)$. 
Figure 5.5 Transient absorption traces measured for $\mathrm{CO}$ rebinding to $\mathrm{hNgb}$, $\mathrm{hNgb}^{\text {red }}$, hNgbTyr44Phe, hNgbTyr44Phe ${ }^{\text {red }}$, hNgbHis64Gln and hNgbHis64Gln ${ }^{\text {red }}$ mutated proteins. Conditions: $20 \mu \mathrm{M}$ protein in 50

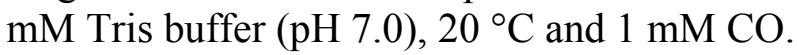

Figure 5.6 Transient absorption traces measured for $\mathrm{CO}$ rebinding to pentacoordinate hNgb, rNgb, hNgb Cys55Ser, and rNgb Gly46Ser mutated proteins. Conditions: $20 \mu \mathrm{M}$ protein in $50 \mathrm{mM}$ Tris buffer ( $\mathrm{pH}$ 7.0), 20 ${ }^{\circ} \mathrm{C}$ and $1 \mathrm{mM} \mathrm{CO}$.

Figure 5.7 Transient absorption traces measured for $\mathrm{CO}$ rebinding to bis-histidyl $\mathrm{hNgb}, \mathrm{rNgb}, \mathrm{hNgb}$ Cys55Ser, and rNgb Gly46Ser mutated proteins.

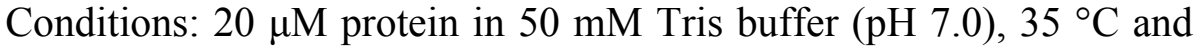
$0.1 \mathrm{mM}$ CO.

Figure 5.8 Photoacoustic traces for $\mathrm{hNgb}$ Tyr44Phe and the reference compound 4SP. Conditions: $20 \mu \mathrm{M}$ protein in $50 \mathrm{mM}$ Tris buffer $(\mathrm{pH} \mathrm{7})$. The absorbance of the sample matched that of the reference compound at $532 \mathrm{~nm}$.

Figure 5.9 Plot of $\left[E_{h v}(\phi-1)\right] / \Phi$ as a function of $C_{p} \rho / \beta$ for $C O$ photo-release from wild type $\mathrm{hNgb}, \mathrm{rNgb}$ and mutated proteins in the presence and absence DTT.

Figure 5.10 Observed rate constants associated with $\mathrm{CO}$ rebinding to $\mathrm{hNgb}$ (closed squares), $\mathrm{hNgb}^{\text {red }}$ (open squares), hNgbY44F (closed circles) and $\mathrm{hNgbY} 44 \mathrm{~F}^{\mathrm{red}}$ (open circles) as a function of concentration of $\mathrm{CO}$. Conditions: $20 \mu \mathrm{M}$ protein in $50 \mathrm{mM}$ Tris buffer $\left(\mathrm{pH} \mathrm{7.0)}, 20{ }^{\circ} \mathrm{C}\right.$ and CO concentrations from $10 \mu \mathrm{M}$ to $300 \mu \mathrm{M}$.

Figure 5.11 Overlay of minimized structures of ferrous mNgbTyr44Phe (left, shown in green) and $\mathrm{CO}$ bound mNgbTyr44Phe (right, shown in light blue) depicting residues in the vicinity of the heme binding pocket of $\mathrm{Ngb}$. The dashed lines represent hydrogen bonds and the numbers the distance in $\AA$ between $\mathrm{H}$-donor and $\mathrm{H}$-acceptor groups.

Figure 5.12 Overlay of minimized structures of ferrous mNgbTyr44Phe ${ }^{\text {red }}$ (left, shown in green) and $\mathrm{CO}$ bound mNgbTyr44Phe ${ }^{\text {red }}$ (right, shown in light blue) depicting residues in the vicinity of the heme binding pocket of $\mathrm{Ngb}$. The dashed lines represent hydrogen bonds and the numbers the distance in $\AA$ between $\mathrm{H}$-donor and $\mathrm{H}$-acceptor groups.

Figure 5.13 Modeled structures of His64Gln hNgb mutant showing the various conformations sampled by the side chain of Gln 64 (shown in gray). Residues in the vicinity of the heme binding pocket (Tyr 44, Lys 67 and Glu 60) are depicted as sticks. The population of each conformation is $20.8 \%$ (A), $14.6 \%$ (B), $11.8 \%$ (C) and $5.2 \%$ (D). 
Figure 5.14 Thermodynamic cycle determined for $\mathrm{CO}$ escape from $\mathrm{hNgb}$ Tyr44Phe mutated protein...

Figure 5.15 Thermodynamic cycle determined for $\mathrm{CO}$ escape from $\mathrm{hNgb}$ His64Gln mutated protein.

Figure 6.1 Sequence alignment of human Cygb with human Ngb and human $\mathrm{Mb}$. Amino acid residues occupying identical positions in more than one sequence are highlighted in red and rectangular boxes represent $\alpha$ helices $\mathrm{A}$ to $\mathrm{H}$. The stars correspond to the positions of the distal and proximal His residues.

Figure 6.2 Three dimensional structure of human Cygb (PDB entry: 1V5H) displaying the heme group, distal His (His 81), proximal His (His 113), Cys 38 and Cys 83 as sticks.

Figure 6.3 Absorption spectra of ferric (black line), ferrous (red line) and CO bound (blue line) $\mathrm{Cygb}^{\mathrm{ox}}$. Conditions: $20 \mu \mathrm{M}$ protein in $50 \mathrm{mM}$ Tris buffer ( $\mathrm{pH} 7.0)$.

Figure 6.4 Overlay of photoacoustic traces for $\mathrm{Cygb}^{\text {ox }}(\mathrm{A})$ and $\mathrm{Cygb}^{\text {red }}(\mathrm{C})$ with the reference compound 4SP. Normalized traces are shown on the right side for $\mathrm{Cygb}^{\mathrm{ox}}$ (B) and Cygbred (D) to better demonstrate the shift between PAC traces for the sample and reference in $\mathrm{Cygb}^{\mathrm{ox}}$. Conditions: $20 \mu \mathrm{M}$ protein in $50 \mathrm{mM}$ Tris buffer $(\mathrm{pH} \mathrm{7.0)}$. The absorbance of the sample matched that of the reference compound at $532 \mathrm{~nm}\left(\mathrm{~A}_{532} \mathrm{~nm}=0.25\right)$.

Figure 6.5 Acoustic traces for $\mathrm{CO}$ photodissociation from $\mathrm{Cygb}^{\mathrm{ox}}$ (blue line) and the reference trace (Fe(III)4SP) (black line). The PAC trace for the $\mathrm{CO}$ photo-dissociation from $\mathrm{Cygb}^{\text {ox }}$ was deconvoluted as described in Materials and Methods section (Chapter 3). Note that the calculated sample acoustic trace (red line) nearly overlays with the measured sample acoustic wave. The corresponding residuals are shown in the bottom panel.

Figure 6.6 Eyring plot for $\mathrm{CO}$ photo-release from $\mathrm{Cygb}^{\mathrm{ox}}$ obtained from the temperature dependence of $\tau_{2}$ values determined from the deconvolution of PAC traces.

Figure 6.7 Plot of $\left[\left(\phi_{1}-1\right) \mathrm{E}_{\mathrm{hv}} / \Phi\right]$ as a function of the $\mathrm{C}_{\mathrm{p}} \rho / \beta$ term for the prompt phase (solid squares) and and plot of $\left[\phi_{2} \mathrm{E}_{\mathrm{hv}} / \Phi\right]$ versus $\mathrm{C}_{\mathrm{p}} \rho / \beta$ for the slow phase (open squares) for $\mathrm{CO}$ photo-dissociation from $\mathrm{Cygb}^{\mathrm{ox}}$ and the prompt phase for ligand dissociation from $\mathrm{Cygb}^{\text {red }}$ (circles).

Figure 6.8 Transient absorption spectra of the $\mathrm{CO}$ adduct of $\mathrm{Cygb}^{\mathrm{ox}}$ at $3 \mathrm{~ns}$ (black line), $10 \mathrm{~ns}$ (gray line), and $30 \mathrm{~ns}$ (black dashed line). The excitation wavelength was $400 \mathrm{~nm}$...... 
Figure 6.9 Top: Transient absorption traces for $\mathrm{CO}$ rebinding to $\mathrm{Cygb}^{\mathrm{ox}}$ and Cygb $^{\text {red }}$. Conditions: $20 \mu \mathrm{M}$ protein in $50 \mathrm{mM}$ Tris buffer, $\mathrm{pH} 7.0$ and $1 \mathrm{mM} \mathrm{CO}$, at $20^{\circ} \mathrm{C}$. Bottom: Lifetime distribution associated with the $\mathrm{CO}$ rebinding to $\mathrm{Cygb}^{\text {ox }}$ (red line) and $\mathrm{Cygb}^{\text {red }}$ (blue line) as determined using MEM approach. The transient absorption traces were normalized to unity at the maximum of the TA curve.

Figure 6.10 Three dimensional structure of ferric human Cygb in its ligand free form (PDB entry: 1VH5) displaying the major and minor conformations of Arg 84 side-chain.

Figure 6.11 Representation of the internal cavities identified in the model structures of $\mathrm{Cygb}^{\text {red }}$ (left) and $\mathrm{Cygb}^{\text {ox }}$ (right)....

Figure 6.12 Overlay of minimized structure of ferric Cygb (PDB file: 1V5H, shown in blue) using YASARA structure molecular software as described in materials and methods section (section 2.4) and ferric Cygb double mutant (Cys38Ser and Cys83Ser) crystalized in the presence of Xe (PDB file: 1UX9, shown in orange). Internal cavities were analyzed using a $1.4 \AA$ probe radius and the numerical algorithm in YASARA software and are shown as mesh surfaces. Cys residues are shown as blue sticks and Xe atoms are depicted as yellow spheres.

Figure 6.13 Schematic representation of the impact of the disulfide bond in Cygb.

Figure 7.1 Ribbon representation of the tetrameric human Hb structure (A, PBD entry $1 \mathrm{FDH})$, horse heart $\mathrm{Mb}$ structure (B, PDB entry 1WLA) and human $\mathrm{Ngb}$ structure (C, PDB entry 1JO6). The heme prosthetic groups are shown as sticks. In the case of $\mathrm{Mb}$ and $\mathrm{Ngb}$, the distal and proximal histidines are visualized.

Figure 7.2 Structure of bezafibrate (left) and inositol hexakisphosphate (right).

Figure 7.3 Absorption spectra of ferric (black line), ferrous (red line) and $\mathrm{O}_{2}$ bound (blue line) hNgb wild type in $50 \mathrm{mM}$ Tris buffer ( $\mathrm{pH} 7.0$ ).

Figure 7.4 Schematic representation of ligand migration observed in heme proteins.

Figure 7.5 Quantum yield for bimolecular $\mathrm{O}_{2}$ rebinding to $\mathrm{Mb}$ as a function of temperature. The solid line demonstrates the trend.

Figure 7.6 PAC traces for $\mathrm{O}_{2}$ photo-dissociation from $\mathrm{O}_{2}-\mathrm{Mb}$ at $9{ }^{\circ} \mathrm{C}$. Conditions: $40 \mu \mathrm{M} \mathrm{Mb}$ dissolved in $50 \mathrm{mM}$ Hepes buffer $(\mathrm{pH}$ 7.0). The absorbance of the reference compound, $\mathrm{Fe}(\mathrm{III}) 4 \mathrm{SP}$, at the excitation wavelength of $532 \mathrm{~nm}$ was identical to that of $\mathrm{O}_{2}-\mathrm{Mb}$. 
Figure 7.7 Plot of the ratio of the acoustic amplitude for $\mathrm{O}_{2}$ photo-release from $\mathrm{Mb}$ and the reference compound as a function of the $\mathrm{C}_{\mathrm{p}} \rho / \beta$ term. The values obtained for $\phi_{1}$ that correspond to the prompt phase are shown as solid circles and the $\phi_{2}$ values corresponding to the slow phase are shown as open triangles.

Figure 7.8 Quantum yield for bimolecular $\mathrm{O}_{2}$ rebinding to human $\mathrm{Ngb}$ as a function of temperature. The solid line demonstrates the trend.

Figure 7.9 Photoacoustic traces for wild type $\mathrm{hNgb}$ and the reference compound 4SP. Conditions: $20 \mu \mathrm{M}$ protein in $50 \mathrm{mM}$ Tris buffer $(\mathrm{pH} 7)$. The absorbance of the sample matched that of the reference compound at $532 \mathrm{~nm}$.

Figure 7.10 Photoacoustic traces for $\mathrm{hNgb}$ with $500 \mathrm{mM} \mathrm{NaCl}$ and the reference compound 4SP. Conditions: $20 \mu \mathrm{M}$ protein in $50 \mathrm{mM}$ Tris buffer, 500 $\mathrm{mM} \mathrm{NaCl}(\mathrm{pH} \mathrm{7)}$. The absorbance of the sample matched that of the reference compound at $532 \mathrm{~nm}$

Figure 7.11 Plot of $\left[\mathrm{E}_{\mathrm{hv}}(\phi-1)\right] / \Phi$ as a function of $\mathrm{C}_{\mathrm{p}} \rho / \beta$ for $\mathrm{O}_{2}$ photo-release from $\mathrm{hNgb}$ (closed squares) and hNgb with $500 \mathrm{mM} \mathrm{NaCl}$ (open circles).

Figure 7.12 Quantum yield for bimolecular $\mathrm{CO}$ rebinding to $\mathrm{Hb}$ and $\mathrm{Hb}$ with effectors as a function of temperature. The error of quantum yield is \pm 0.05 . The solid line demonstrates the trend.

Figure 7.13 Photoacoustic traces for $\mathrm{CO}$ photo-dissociation from $\mathrm{CO}-\mathrm{Hb}$ complex and the reference compound $\mathrm{Fe}(\mathrm{III}) 4 \mathrm{SP}$. Conditions: $40 \mu \mathrm{M} \mathrm{Hb}$ in 100 $\mathrm{mM}$ HEPES buffer $\mathrm{pH} 7.0$ and $20{ }^{\circ} \mathrm{C}$. The absorbance of the reference compound matched the absorbance of the sample at $532 \mathrm{~nm}$.

Figure 7.14 Plot of the ratio of the acoustic amplitude for the $\mathrm{CO}$ photodissociation from the $\mathrm{CO}-\mathrm{Hb}$ complex and the reference compound as a function of the temperature dependent factor $(\mathrm{Cp} \rho / \beta)$ term.

Figure 7.15 The thermodynamic profile for $\mathrm{CO}$ photo-dissociation from $\mathrm{Hb}$ in the absence of effector and in the presence of BZF and IHP. The arrows indicate the respective scale.

Figure 8.1 Three dimensional structure of Cygb (PDB entry: 2DC3, molecule A in orange and molecule B in light blue) homodimer. The heme prosthetic groups are shown in red and Cys residues in blue. Distal His 81and proximal His 113 residues are shown as orange sticks in molecule A and as blue sticks in molecule B. Extended $\mathrm{C}$ - and $\mathrm{N}$-termini are highlighted in green.

Figure 8.2 Structure of 1-anilino-8-naphthalene sulfonate (1,8-ANS) (left) and sodium oleate (right). 
Figure 8.3 Fluorescence emission of $40 \mu \mathrm{M}$ of 1,8-ANS in buffer (black line) and in the presence of $20 \mu \mathrm{M}$ ferric Cygb (blue line). The buffer used was $50 \mathrm{mM}$ Tris, $\mathrm{pH}$ 7.0. The excitation wavelength was $350 \mathrm{~nm}$.

Figure 8.4 Fluorescence emission of different concentrations of 1,8-ANS in the presence of Cygb (A), $\mathrm{Cygb}^{\text {red }}(\mathrm{B}), \mathrm{CO}-\mathrm{Fe}^{2+} \mathrm{Cygb}(\mathrm{C})$, and $\mathrm{CNCygb}^{\text {red }}$ (D). Conditions: $20 \mu \mathrm{M}$ protein in $50 \mathrm{mM}$ Tris $(\mathrm{pH}$ 7.0) for Cygb and $\mathrm{CO}-\mathrm{Fe}^{2+} \mathrm{Cygb}$, containing $10 \mathrm{mM}$ DTT for $\mathrm{Cygb}^{\text {red }}$, and containing 10 $\mathrm{mM}$ DTT and $10 \mathrm{mM} \mathrm{KCN}$ for CNCygb ${ }^{\text {red }}$. The excitation wavelength was $350 \mathrm{~nm}$.

Figure 8.5 Titration curves of 1,8-ANS binding to $\mathrm{Cygb} \mathrm{Cygb}^{\text {red }}, \mathrm{CNCygb}$, $\mathrm{CNCygb}^{\text {red }}, \mathrm{Fe}^{2+} \mathrm{Cygb}$ and $\mathrm{CO}-\mathrm{Fe}^{2+} \mathrm{Cygb}$. Conditions: $20 \mu \mathrm{M}$ protein in $50 \mathrm{mM}$ Tris, $\mathrm{pH} 7.0$.

Figure 8.6 Fluorescence emission of $40 \mu \mathrm{M}$ of 1,8-ANS in buffer (black line) and in the presence of $20 \mu \mathrm{M} \mathrm{Ngb}$. The buffer used was $50 \mathrm{mM}$ Tris, $\mathrm{pH}$ 7.0. The excitation wavelength was $350 \mathrm{~nm}$. The sharp peak with a maximum at $485 \mathrm{~nm}$ corresponds to light scattering.

Figure 8.7 Fluorescence emission of $40 \mu \mathrm{M}$ of 1,8-ANS in buffer (black line), in the presence of $20 \mu \mathrm{M}$ ferric Cygb (blue line) and $20 \mu \mathrm{M}$ ferric $\Delta \mathrm{N} \Delta \mathrm{C}$ Cygb (red line). Conditions: $50 \mathrm{mM}$ Tris, $\mathrm{pH}$ 7.0. The excitation wavelength was $350 \mathrm{~nm}$.

Figure 8.8 Time-resolved fluorescence in the frequency domain data determined for ANS in 1,8-ANS:Cygb (black squares), 1,8-ANS:Cygb ${ }^{\text {red }}$ (blue circles) and 1,8-ANS:CNCygb (red triangles) complexes. Phase delay data are shown as closed symbol and modulation ratio data are displayed as open symbols. Solid lines represent the fit to the data using a three exponential decay model. The data were analyzed using Globals software. Conditions: $20 \mu \mathrm{M}$ protein, $40 \mu \mathrm{M}$ 1,8-ANS in 50 $\mathrm{mM}$ Tris ( $\mathrm{pH} 7.0$ ), containing $10 \mathrm{mM}$ DTT for $\mathrm{Cygb}^{\text {red }}$, and $10 \mathrm{mM}$ $\mathrm{KCN}$ for CNCygb. The reference compound used was POPOP solubilized in ethanol ( $\tau=1.35 \mathrm{~ns})$ (Lakowicz, 2006)

Figure 8.9 Isothermal titration calorimetry of ANS binding to Cygb (A), CNCygb (B), $\mathrm{Cygb}^{\text {red }}$ (C) and $\mathrm{CNCygb}^{\text {red }}$ (D). Top: Raw data for the titration of the protein $(20 \mu \mathrm{M})$ with $2 \mathrm{mM}$ ANS in $50 \mathrm{mM}$ Tris buffer $(\mathrm{pH} 7)$ at $25^{\circ} \mathrm{C}$. Bottom: Integrated heats of binding obtained from fitting the raw data shown in the top panel after subtracting the heat of dilution. The solid line represents the best fit to the experimental data using a two sites binding model. The buffer used for $\mathrm{CN}^{-}$bound Cygb samples contained $10 \mathrm{mM} \mathrm{KCN}$ and the buffer for $\mathrm{Cygb}^{\text {red }}$ samples contained a final concentration of DTT of $1 \mathrm{mM}$. 
Figure 8.10 Fluorescence emission of 1,8-ANS in complex with Cygb (1:1) in the absence and presence of increasing concentration of sodium oleate. Conditions: $20 \mu \mathrm{M}$ Cygb, $20 \mu \mathrm{M}$ 1,8-ANS in $1 \mathrm{mM}$ Hepes, $\mathrm{pH}$ 7.0.

Figure 8.11 Fluorescence emission of 1,8-ANS in complex with $\mathrm{Cygb}^{\text {red }}(1: 1)$ in the absence and presence of increasing concentration of sodium oleate. Conditions: $20 \mu \mathrm{M} \mathrm{Cygb}^{\text {red }}, 20 \mu \mathrm{M}$ 1,8-ANS in $1 \mathrm{mM}$ Hepes, $\mathrm{pH} 7.0$, containing $1 \mathrm{mM}$ DTT.

Figure 8.12 Fluorescence emission of 1,8-ANS in complex with CNCygb (1:1) in the absence and presence of increasing concentration of sodium oleate. Conditions: $20 \mu \mathrm{M}$ CNCygb, $20 \mu \mathrm{M}$ 1,8-ANS in $1 \mathrm{mM}$ Hepes, $\mathrm{pH}$ 7.0, containing $10 \mathrm{mM} \mathrm{KCN}$....

Figure 8.13 Titration curves for addition of sodium oleate to Cygb-ANS, $\mathrm{Cygb}^{\text {red }}$ ANS, and CNCybg-ANS complexes. The concentration of the protein and 1,8-ANS were $20 \mu \mathrm{M}$. The buffer used was $1 \mathrm{mM}$ Hepes for Cygb-ANS, $1 \mathrm{mM}$ Hepes containing $10 \mathrm{mM}$ DTT for $\mathrm{Cygb}^{\text {red }}$-ANS, and $1 \mathrm{mM}$ Hepes containing $10 \mathrm{mM} \mathrm{KCN}$ for CNCygb-ANS.

Figure 8.14 Representation of binding site with highest affinity determined from the docking of 1,8-ANS to Cygb (PDB file: 2DC3, molecule B)

Figure 8.15 Docking of oleate to Cygb (PDB file: 2DC3, molecule B). The solvent accessible surface of Cygb structure is shown in light blue color, whereas oleate structure is shown as sticks.

Figure 8.16 Docking of 1,8-ANS to Cygb (PDB file: 2DC3, molecule B). The solvent accessible surface of Cygb structure is shown in light blue color, whereas 1,8-ANS structure is shown as sticks.

Figure 8.17 Representation of solvent accessible surface area of residues involved in the interaction of Cygb (PDB file: 2DC3, molecule B) with 1,8ANS

Figure 8.18 Representation of solvent accessible surface area of residues involved in the interaction of Cygb (PDB file: 2DC3, molecule B) with oleate that are also involved in the interaction of Cygb with 1,8 -ANS.

Figure 9.1 Structure of guanidinium hydrochloride $(\mathrm{GuHCl})$.

Figure 9.2 Top: Three dimensional structure of hNgb (PDB entry 1OJ6, molecule A) depicting the heme prosthetic group, distal His 64, proximal His 96, and residues Trp 13, Trp 133 and Trp 148 as sticks. Botton: Distance between the heme iron and Trp residues in $\mathrm{hNgb}$ 
Figure 9.3 UV-visible absorption spectra of Cygb in the presence of increasing concentrations of $\mathrm{GuHCl}$ from 0 to $6 \mathrm{M}$. The concentration of the proteins was approximately $10 \mu \mathrm{M}$ in $10 \mathrm{mM}$ Tris buffer $(\mathrm{pH} 7.0)$. .

Figure 9.4 UV-visible absorption spectra of $\mathrm{Cygb}^{\text {red }}$ in the presence of increasing concentrations of $\mathrm{GuHCl}$ from 0 to $6 \mathrm{M}$. The concentration of the proteins was approximately $10 \mu \mathrm{M}$. The buffer used was $10 \mathrm{mM}$ Tris buffer ( $\mathrm{pH}$ 7.0) containing $10 \mathrm{mM}$ DTT.

Figure 9.5 UV-visible absorption spectra of CNCygb in the presence of increasing concentrations of $\mathrm{GuHCl}$ from 0 to $6 \mathrm{M}$. The concentration of the proteins was approximately $10 \mu \mathrm{M}$. The buffer used for Cygb was 10 $\mathrm{mM}$ Tris buffer ( $\mathrm{pH} 7.0)$ containing $10 \mathrm{mM} \mathrm{KCN}$.

Figure 9.6 Unfolding curves of Cygb, $\mathrm{Cygb}^{\text {red }}$ and $\mathrm{CNCygb}$ monitored using UVvisible absorption spectroscopy as a function of $\mathrm{GuHCl}$ concentration. The concentration of the protein was $\sim 10 \mu \mathrm{M}$ in $10 \mathrm{mM}$ Tris buffer, $\mathrm{pH}$ 7.0. The buffer used for $\mathrm{Cygb}^{\text {red }}$ samples contained $10 \mathrm{mM}$ DTT and for CNCygb samples contained $10 \mathrm{mM} \mathrm{KCN}$.

Figure 9.7 Far-UV Circular dichroism spectra of Cygb in the presence of increasing concentrations of $\mathrm{GuHCl}$. The concentration of the proteins was $40 \mu \mathrm{M}$ in $10 \mathrm{mM}$ Tris buffer ( $\mathrm{pH} 7.0)$.

Figure 9.8 Far-UV Circular dichroism spectra of $\mathrm{Cygb}^{\text {red }}$ in the presence of increasing concentrations of $\mathrm{GuHCl}$. The concentration of the proteins was $40 \mu \mathrm{M}$. The buffer used was $10 \mathrm{mM}$ Tris buffer $(\mathrm{pH}$ 7.0) containing $10 \mathrm{mM}$ DTT.

Figure 9.9 Far-UV Circular dichroism spectra of CNCygb in the presence of increasing concentrations of $\mathrm{GuHCl}$. The concentration of the proteins was $40 \mu \mathrm{M}$. The buffer used for Cygb was $10 \mathrm{mM}$ Tris buffer ( $\mathrm{pH}$ 7.0) containing $10 \mathrm{mM} \mathrm{KCN}$......

Figure 9.10 Unfolding curves of Cygb, $\mathrm{Cygb}^{\text {red }}$ and CNCygb monitored using CD spectroscopy as a function of $\mathrm{GuHCl}$ concentration. The concentration of the protein was $40 \mu \mathrm{M}$ in $10 \mathrm{mM}$ Tris buffer, $\mathrm{pH}$ 7.0. The buffer used for $\mathrm{Cygb}^{\text {red }}$ samples contained $10 \mathrm{mM}$ DTT and for CNCygb samples contained $10 \mathrm{mM} \mathrm{KCN}$.

Figure 9.11 Overlay of unfolding curves of Cygb, $\mathrm{Cygb}^{\text {red }}$ and CNCygb determined using UV-visible spectroscopy (open symbols) and CD spectroscopy (closed symbols) as a function of $\mathrm{GuHCl}$ concentration.

Figure 9.12 Overlay of UV-visible absorption spectra of wild type hNgb and Trp mutants W13Y/W133Y, W13Y/W148Y, and W133Y/W148Y. The concentration of the protein was $10 \mu \mathrm{M}$ in $50 \mathrm{mM}$ Tris buffer $(\mathrm{pH} 7.0) \ldots . . .258$ 
Figure 9.13 UV-visible absorption spectra of wild type hNgb in the presence of increasing concentrations of $\mathrm{GuHCl}$ from 0 to $6 \mathrm{M}$. The concentration of the proteins was approximately $10 \mu \mathrm{M}$ in $10 \mathrm{mM}$ Tris buffer (pH $7.0)$

Figure $9.14 \mathrm{UV}$-visible absorption spectra of $\mathrm{hNgb} \mathrm{W} 13 \mathrm{Y} / \mathrm{W} 133 \mathrm{Y}$ in the presence of increasing concentrations of $\mathrm{GuHCl}$ from 0 to $6 \mathrm{M}$. The concentration of the proteins was approximately $10 \mu \mathrm{M}$ in $10 \mathrm{mM}$ Tris buffer ( $\mathrm{pH} 7.0)$.

Figure 9.15 UV-visible absorption spectra of $\mathrm{hNgb} \mathrm{W} 13 \mathrm{Y} / \mathrm{W} 148 \mathrm{Y}$ in the presence of increasing concentrations of $\mathrm{GuHCl}$ from 0 to $6 \mathrm{M}$. The concentration of the proteins was approximately $10 \mu \mathrm{M}$ in $10 \mathrm{mM}$ Tris buffer ( $\mathrm{pH} 7.0)$.

Figure 9.16 UV-visible absorption spectra of $\mathrm{hNgb}$ W133Y/W148Y in the presence of increasing concentrations of $\mathrm{GuHCl}$ from 0 to $6 \mathrm{M}$. The concentration of the proteins was approximately $10 \mu \mathrm{M}$ in $10 \mathrm{mM}$ Tris buffer ( $\mathrm{pH} 7.0)$.

Figure 9.17 Unfolding curves of wild type hNgb and Trp mutants monitored using UV-visible absorption spectroscopy as a function of $\mathrm{GuHCl}$ concentration. The concentration of the protein was $\sim 10 \mu \mathrm{M}$ in 10 $\mathrm{mM}$ Tris buffer, $\mathrm{pH}$ 7.0.

Figure 9.18 Far-UV CD spectra of wild type hNgb and mutants W13Y/W133Y, $\mathrm{W} 13 \mathrm{Y} / \mathrm{W} 148 \mathrm{Y}$ and $\mathrm{W} 133 \mathrm{Y} / \mathrm{W} 148 \mathrm{Y}$. The concentration of the protein was $40 \mu \mathrm{M}$ in $10 \mathrm{mM}$ Tris buffer ( $\mathrm{pH} 7.0)$. Spectra were corrected for the baseline of the buffer

Figure 9.19 Time-resolved fluorescence in the frequency domain data determined for wild type $\mathrm{hNgb}$ (black squares) and double Trp mutants $\mathrm{W} 13 \mathrm{Y} / \mathrm{W} 133 \mathrm{Y}$ (blue triangles), W13Y/W148Y (red circles) and W133Y/W148Y (green diamonds). Phase delay data are shown as open symbol and modulation ratio data are displayed as closed symbols. Solid lines represent the fit to the data using a sum of three exponential decay model retrieved using Vinci software. The concentration of the protein was $7 \mu \mathrm{M}$ in $50 \mathrm{mM}$ Tris ( $\mathrm{pH} 7$ ).

Figure 9.20 Time-resolved fluorescence in the frequency domain data determined for $\mathrm{CN}$ - adducts of wild type hNgb (black squares) and double Trp mutants W13Y/W133Y (blue triangles), W13Y/W148Y (red circles) and $\mathrm{W} 133 \mathrm{Y} / \mathrm{W} 148 \mathrm{Y}$ (green diamonds). Phase delay data are shown as open symbol and modulation ratio data are displayed as closed symbols. Solid lines represent the fit to the data using a sum of three exponential decay model retrieved using Globals software. The 
concentration of the protein was $7 \mu \mathrm{M}$ in $50 \mathrm{mM}$ Tris $(\mathrm{pH}$ 7)

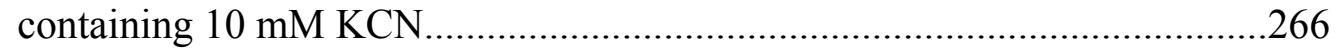

Figure 9.21 Ribbon representation of the dimensional structure of human Ngb (PDB entry 1OJ6), Cygb (PDB entry 2DC3) and Mb (PDB entry 1WLA) depicting hydrophobic residues in helices $\mathrm{A}, \mathrm{G}$ and $\mathrm{H}$ as sticks. 


\section{LIST OF ABREVIATIONS AND ACRONYMS}

\begin{tabular}{|c|c|}
\hline ABBREVIATION & FULL NAME \\
\hline$\Phi$ & Quantum yield \\
\hline $1,8-\mathrm{ANS}$ & 1-anilinonaphthalene- 8 -sulfonic acid \\
\hline 2,6-ANS & 2-anilinonaphthalene-6-sulfonic acid \\
\hline Adgb & Androglobin \\
\hline Ar & Argon \\
\hline $\mathrm{BZF}$ & Bezafibrate \\
\hline $\mathrm{CD}$ & Circular dichroism \\
\hline Cygb & Cytoglobin \\
\hline $\mathrm{Cygb}^{\mathrm{ox}}$ & Cytoglobin oxidized \\
\hline $\mathrm{Cygb}^{\text {red }}$ & Cytoglobin reduced \\
\hline $\mathrm{CN}^{-}$ & Cyanide ligand \\
\hline $\mathrm{CO}$ & Carbon monoxide \\
\hline Cyt c & Cytochrome c \\
\hline DTT & Dithiothreitol \\
\hline DOSS & Dioctyl sulfosuccinate \\
\hline $\mathrm{Fe}(\mathrm{III}) 4 \mathrm{SP}$ & $\mathrm{Fe}^{3+}$ tetrakis(4-sulfonatophenyl)phorphine \\
\hline FePPIX & Iron protoporphyrin IX \\
\hline $\mathrm{GuHCl}$ & Guanidine hydrochloride \\
\hline $\mathrm{Hb}$ & Hemoglobin \\
\hline HEPES & 4-(2-hydroxyethyl)-1-piperazineethanesulfonic \\
\hline HOMO & Highest occupied molecular orbital \\
\hline
\end{tabular}




\begin{tabular}{|c|c|}
\hline IHP & Inositol hexakisphosphate \\
\hline $\mathrm{K}_{\mathrm{a}}$ & Association constant \\
\hline $\mathrm{KCN}$ & Potassium cyanide \\
\hline $\mathrm{K}_{\mathrm{d}}$ & Dissociation constant \\
\hline $\mathrm{K}_{\mathrm{i}}$ & Inhibition constant \\
\hline LUMO & Lowest unoccupied molecular orbital \\
\hline MEM & Maximum entropy method \\
\hline Ngb & Neuroglobin \\
\hline $\mathrm{hNgb}$ & Human neuroglobin \\
\hline $\mathrm{hNgb}^{\text {red }}$ & Human neuroglobin reduced \\
\hline $\mathrm{mNgb}$ & Mouse neuroglobin \\
\hline NO & Nitrogen oxide \\
\hline $\mathrm{rNgb}$ & Rat neuroglobin \\
\hline $\mathrm{O}_{2}$ & Oxygen \\
\hline $\mathrm{O}_{2}-\mathrm{Mb}$ & Oxymyoglobin \\
\hline PAC & Photoacoustic calorimetry \\
\hline SDS & Sodium dodecyl sulfate \\
\hline TA & Transient absorption spectroscopy \\
\hline$\Delta \mathrm{H}$ & Enthalpy change \\
\hline$\Delta \mathrm{H}^{\#}$ & Activation enthalpy change \\
\hline$\Delta \mathrm{V}$ & Volume change \\
\hline RNS & Reactive nitrogen species \\
\hline ROS & Reactive oxygen species \\
\hline
\end{tabular}




\section{INTRODUCTION}

\subsection{Heme proteins}

Heme proteins constitute one of the largest classes of metalloproteins found in nature and are characterized by the presence of a heme moiety as the prosthetic group that may be covalently or non-covalently bound to the apoprotein (Everse, 2004). These metalloproteins are remarkably versatile and perform a wide variety of functions, including respiratory functions such as oxygen storage and transport (myoglobin and hemoglobin), catalysis (catalases), electron transfer (cytochromes), substrate oxidation by peroxides (peroxidases), and oxygen sensors (FixL) (Everse, 2004; Larsen and Miksovska, 2007; Rodgers, 1999).

The versatility displayed by heme proteins have been attributed to the type of heme prosthetic group and the amino acid residues surrounding the heme pocket (Anderson and Chapman, 2005). The heme group consists of a porphyrin ring bound to an iron ion via coordination bonds with the four pyrrole nitrogens from the porphyrin. Axial ligation to the iron, from one or two amino acid side chains, maintains the heme in position within the protein (Anderson and Chapman, 2005). Amino acid residues that bind to the heme iron typically contain lone pairs of electrons that allow them to form coordination bonds with the heme iron, such as histidine, methionine, tyrosine or cysteine (Larsen and Miksovska, 2007). In the case of histidine coordination, other types of interactions may be also involved including hydrogen bonding with other amino acid residues. In addition, heme orientation is influenced by interactions between propionate and vinyl side chains and the protein backbone (Anderson and Chapman, 2005). 
Amino acid residues occupying the fifth axial position in heme proteins are called proximal ligands, which could be a histidine residue in globin, peroxidases and nitrophorins (Anderson and Chapman, 2005; Dunford, 1999; Lecomte et al., 2005), or a cysteine residue in cytochrome P450, nitric oxide synthase, and chloroperoxidase (Anderson and Chapman, 2005; Dunford, 1999). The sixth axial position, situated above the heme plane, can be either vacant or occupied by an amino acid residue. Those residues coordinating to the sixth axial position are called distal ligands and could be a methionine (i.e., cytochrome c) (Anderson and Chapman, 2005) or a histidine residue (i.e. cytochrome b, neuroglobin, cytoglobin and androglobin) (Al-Attar and de Vries, 2013; Dewilde et al., 2001; Hoogewijs et al., 2012; Pesce et al., 2002).

One of the most extensively studied families of heme proteins belongs to the globin family. Globins have been identified in many taxa, including bacteria, plants and animals (Hardison, 1998). According to Vinogradov et al. (2013) globins in all kingdoms of life are classified into three types: M (myoglobin-like), S (sensor), and T (truncated) globins. In this context, the bacterial globin family comprises three sub-families that have been further classified into two structural classes: the 3/3 (3-over-3) and 2/2 (2-over-2) fold globins. The $3 / 3$ globin fold bacterial globins encompases the $M$ family, comprising the flavohemoglobins (FHbs) and related single domain globins (SDgbs), the S family consisting of globin coupled sensors (GCS), protoglobins (Pgb) and sensor single domain globins (SSDgb). The third family ( $\mathrm{T}$ family) consists of truncated globins (TrHbs) in which the $3 / 3$ fold is reduced to a $2 / 2$ fold resulting from a shorter or absent helix A and conversion of the $\mathrm{F}$ helix into a loop. The $\mathrm{T}$ family is further classified into three structurally distinct families, TrHb1 (T1), TrHb2 (T2) and TrHb3 (T3) (Vinogradov et 
al., 2013). In plants, two structurally different types of hemoglobins have been identified: the $3 / 3$ myoglobin-like hemoglobins and the truncated hemoglobins exhibiting the $2 / 2$ fold (Vazquez-Limon et al., 2012; Vinogradov et al., 2011). The 3/3 plant hemogloins comprise symbiotic hemoglobins (sHbs) and leghemoglobins (Lbs), which are synthesized in nodules of nitrogen-fixing plants, and non-symbiotic hemoglobins (nsHbs), which have been found in diverse organs of bryophytes and angiosperms (Vazquez-Limon et al., 2012). The nsHbs are further classified into two classes, namely nsHbs-1 and nsHbs-2, on the basis of their oxygen affinity and sequence homology (Vazquez-Limon et al., 2012). Some of the bacterial globin sub-families are also present in eukaryotes. For example, TrHb1 and TrHb2 are present in ciliates and algae, $\operatorname{TrHb} 2$ in plants, and FHHbs and SSDgbs are found in fungi (Vazquez-Limon et al., 2012).

Structurally, members of the globin family are small proteins arranged into six to eight $\alpha$-helices, named $\mathrm{A}$ to $\mathrm{H}$ on the basis of myoglobin $(\mathrm{Mb})$ nomenclature (Figure 1.1) (Everse, 2004; Lecomte et al., 2005), connected by short coils. In case of $3 / 3$ globins these $\alpha$-helices display a characteristic 3-over-3- $\alpha$-helical sandwich structure or the socalled "globin fold" that contains a non-covalently bound iron protoporphyrin IX (heme b) as the prosthetic group that is axially ligated by the side chain of a conserved histidine residue at the $8^{\text {th }}$ position of helix F (Lecomte et al., 2005). Iron protoporphyrin IX (FePPIX) (Figure 1.1) is a macrocycle constituted by four pyrrole rings that are linked by four methine bridges $(\alpha, \beta, \gamma, \delta)$ and form coordination bonds with an iron ion in its ferrous or ferric state. In addition to the canonical $3 / 3$ globin fold, a subfamily of proteins that display a characteristic fold denoted as $2 / 2$ hemoglobin $(\mathrm{Hb})$ fold conform the truncated hemoglobins (trHb) family (Lecomte et al., 2005). These proteins are 20-30 
amino acid residues shorter than $\mathrm{Mb}$ and the $2 / 2 \mathrm{Hb}$ fold only involves $\mathrm{BC} / \mathrm{E}$ and $\mathrm{G} / \mathrm{H}$ helices. In terms of physiological function, TrHbs have been proposed to act as small molecule sensors, oxygen carriers and pseudo-enzymes (Lecomte et al., 2005).
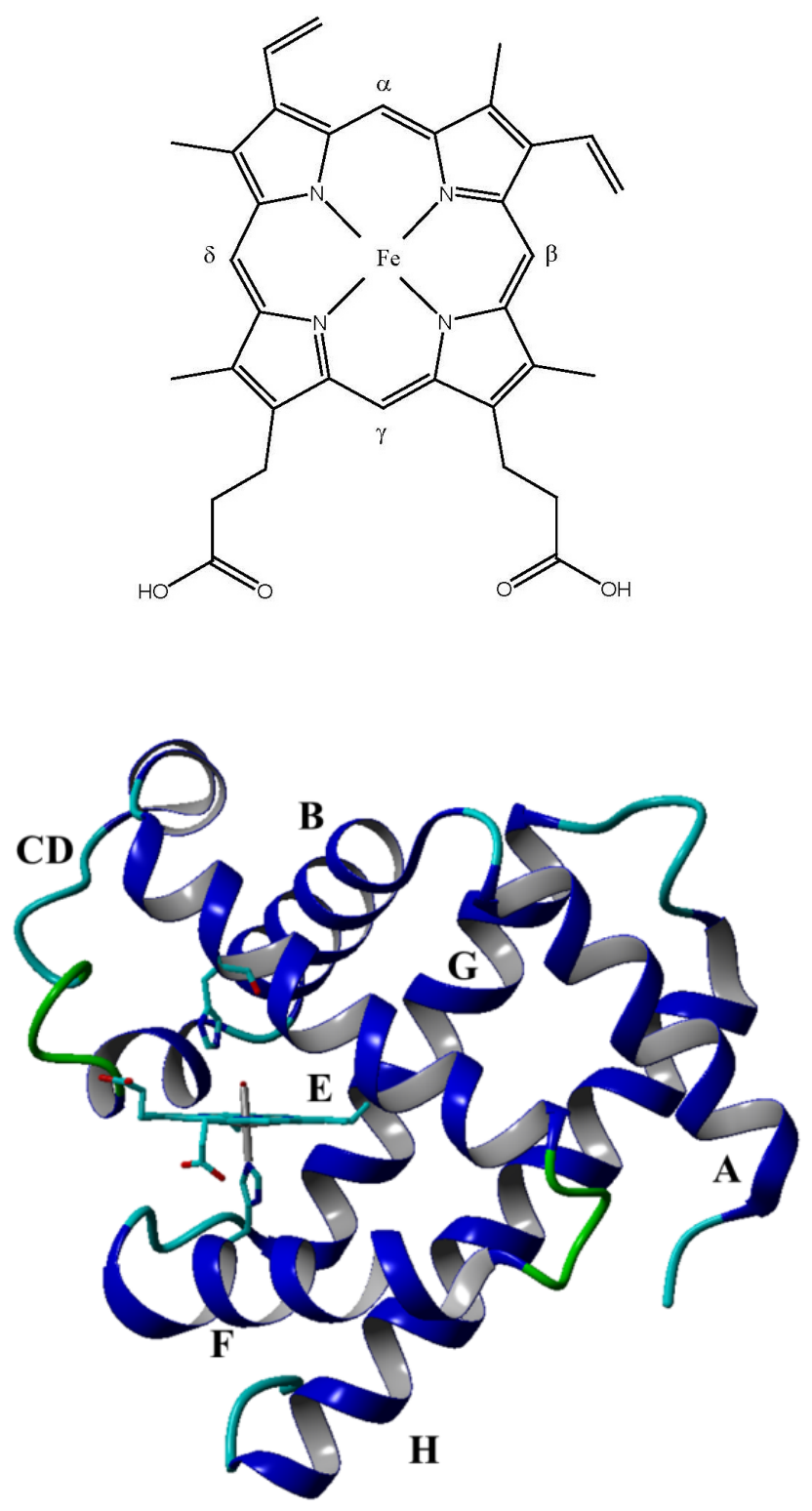

Figure 1.1 Top panel: Structure of iron protoporphyrin IX (heme b). Bottom panel: Ribbon representation of three dimensional structure of myoglobin showing nomenclature of $\alpha$ helices adopted for globins. The heme prosthetic group and the distal and proximal His residues are depicted as sticks. 
Until a decade ago, only two globins were thought to be expressed in vertebrates: myoglobin and hemoglobin. Burmester et al. (Burmester et al., 2002; Burmester et al., 2000) discovered two new heme proteins through an extensive DNA database search, namely neuroglobin (Ngb) and cytoglobin (Cygb) in 2000 and 2002, respectively. The DNA database search then led to the discovery of additional globins in vertebrates including globin $\mathrm{E}(\mathrm{GbE})$, globin $\mathrm{X}(\mathrm{GbX})$, globin $\mathrm{Y}(\mathrm{GbY})$ and the more recently androglobin (Adgb) was discovered by Hoogewijs et al. (Brunori and Vallone, 2007; Hoogewijs et al., 2012). At present, only five globins have been discovered in humans: $\mathrm{Hb}, \mathrm{Mb}, \mathrm{Ngb}$, Cygb, and Adgb. Interestingly, these globins have been shown to differ in structure and tissue distribution, suggesting different physiological functions.

A representation of the phylogenetic distribution of globins in vertebrates is shown in Figure 1.2. Although $\mathrm{Ngb}$ and $\mathrm{GbX}$ have been recently discovered, phylogenetic and genetic analysis of vertebrate globins indicates that $\mathrm{Ngb}$ and $\mathrm{GbX}$ belong to a branch that diverged before the split of Protostomia and Deuterostomia (700 million years ago) (Roesner et al., 2005). Molecular evolutionary analysis has also revealed that Adgb is phylogenetically more ancient than Ngb (Hoogewijs et al., 2012). On the other hand, $\mathrm{Cygb}, \mathrm{Mb}$ and $\mathrm{Hb}$ branches separated at the time of protostomiandeuterostomian divergence, indicating that these proteins diverged at a later stage (Roesner et al., 2005). The sequence alignment of vertebrate globins $\mathrm{Hb}$ ( $\alpha$ and $\beta$ chains), $\mathrm{Mb}, \mathrm{Ngb}$, Cygb and the globin domain of Adgb (Figure 1.3) shows the low sequence homology among these proteins. Interestingly, Cygb and Adgb contain a larger number of amino acid residues than $\mathrm{Hb}, \mathrm{Mb}$ and $\mathrm{Ngb}$. Consequently, Cygb exhibits extended $\mathrm{N}$ and C-termini, whereas Adgb only displays an extended N-terminus. The role of these 
extended termini in Cygb is not clear, but it has been proposed that they may be involved in association with lipids and/or protein-protein interactions (Oleksiewicz et al., 2011; Reeder et al., 2011).

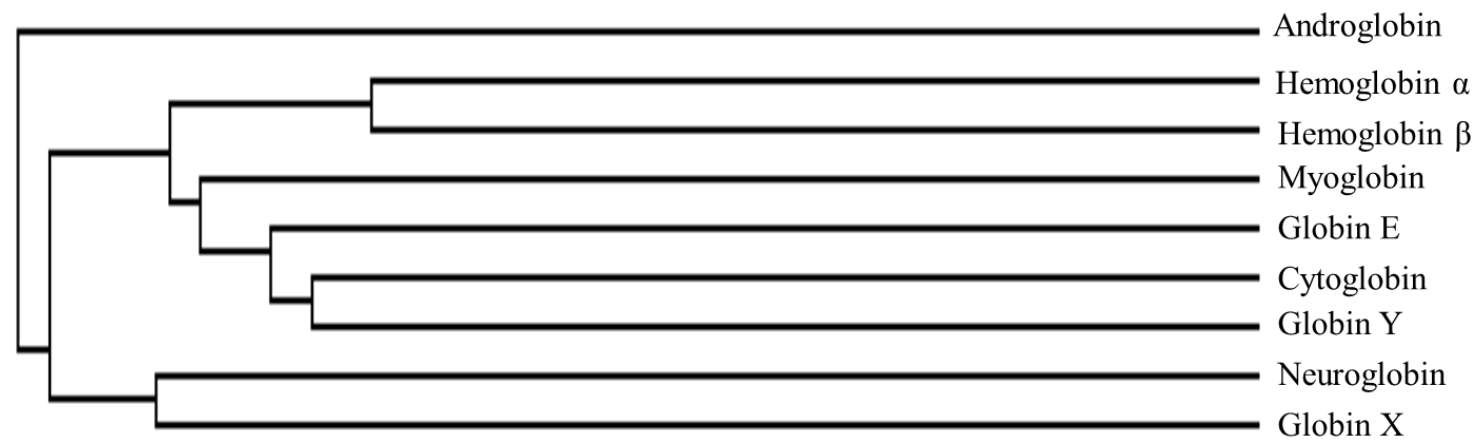

Figure 1.2. Representation of phylogenetic relationships in vertebrate globins.

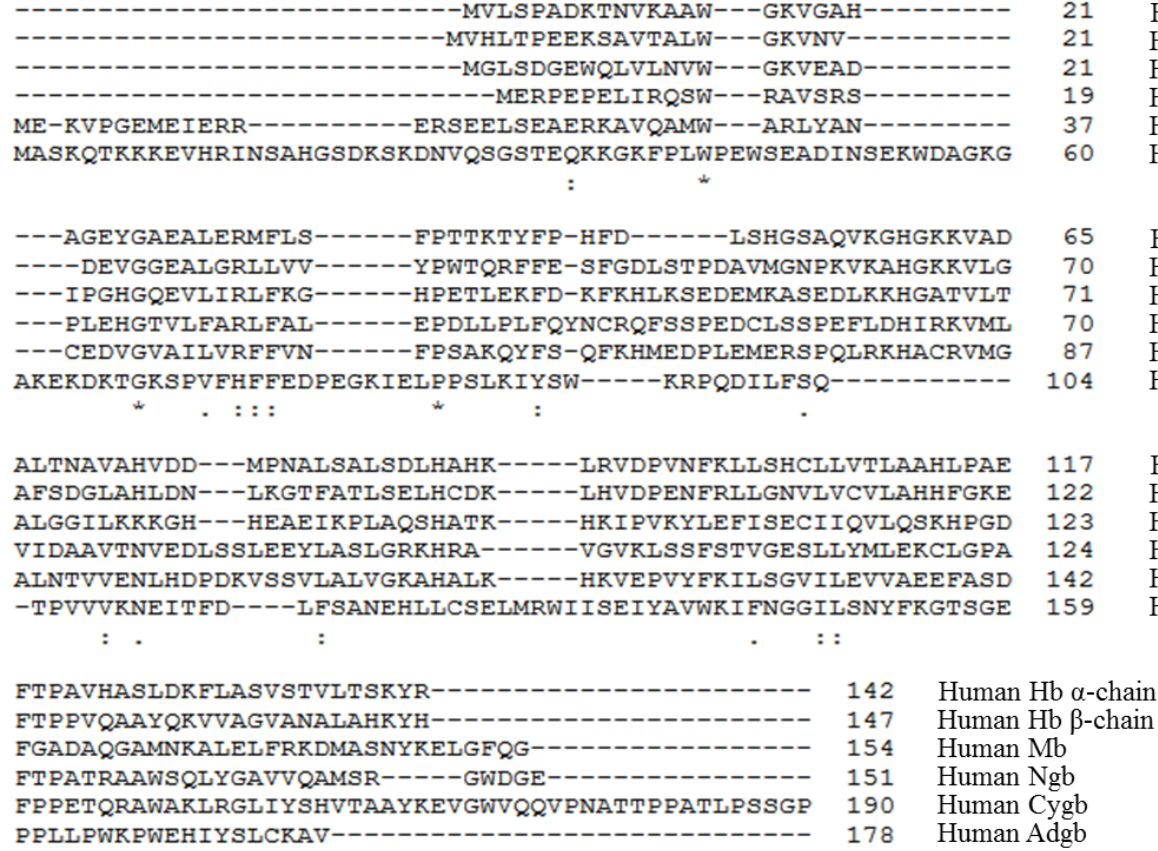

Figure 1.3 Sequence alignment of vertebrate globins $\mathrm{Hb}, \mathrm{Mb}, \mathrm{Ngb}, \mathrm{Cygb}$ and Adgb. Conserved residues are represented by a star below the sequence, whereas residues that strongly share similar properties are represented by two dots and those exhibiting weak similarity are represented by one dot below the sequence. 


\subsubsection{Myoglobin and hemoglobin}

Among vertebrate globins, $\mathrm{Mb}$ and $\mathrm{Hb}$ have been the most widely studied members of this family and have been used as models to understand structure-function relationship in proteins. Myoglobin is a monomer mainly found in cardiac and striated muscle acting as an oxygen storage protein that facilitates oxygen diffusion to mitochondria (Brunori, 2000). Hemoglobin is found in red blood cells and serves to transport oxygen in the circulatory system (Hardison, 1998). The Hb protein is a heterotetramer composed of two $\alpha$-chain subunits of 141 amino acid residues and two $\beta$ chain subunits comprising 146 amino acid residues with each of the subunits containing a heme group (Figure 1.4). The interaction between $\alpha$ and $\beta$ subunits results in cooperativity of oxygen binding, which is physiologically relevant because it allows fast and efficient oxygen release over a narrow range of blood oxygen tensions (Perutz et al., 1998; Safo et al., 2011). In addition to cooperativity, the oxygen affinity of $\mathrm{Hb}$ is modulated by the binding of allosteric ligands at sites that are distant from the heme iron, including protons, chloride ions, and inorganic phosphates (Safo et al., 2011; Yonetani and Laberge, 2008). These ions preferentially bind and stabilize the low affinity deoxy conformation of $\mathrm{Hb}$. In addition, heterotropic effectors including 2,3-diphosphoglycerate (2,3-BPG), inositol hexaphosphate (IHP), and bezafibrate (BZF) have been shown to bind to $\mathrm{R}$ (relaxed) and $\mathrm{T}$ (tense) states of $\mathrm{Hb}$ and induce global conformational changes influencing the stability of the tetramer (Eaton et al., 2007; Schay et al., 2006). 


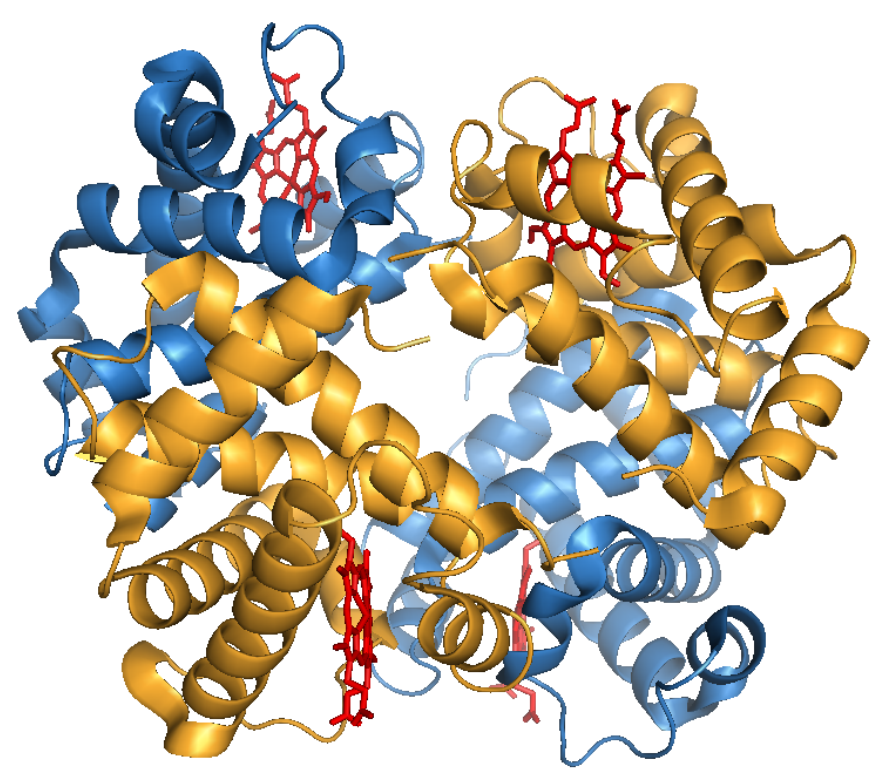

Figure 1.4 Ribbon representation of the three dimensional structure of horse heart $\mathrm{Hb}$ (PDB entry: $2 \mathrm{HHB}$ ) showing $\alpha$ subunits in orange and $\beta$ subunits in blue.

Both $\mathrm{Mb}$ and $\mathrm{Hb}$ contain a penta-coordinate heme iron that provides an open ligand binding site to accommodate exogenous ligands such as $\mathrm{O}_{2}, \mathrm{CO}$ and $\mathrm{NO}$ (Lecomte et al., 2005). For this reason, heme iron penta-coordination has been regarded as the common characteristic of vertebrate heme proteins. However, the discovery of Ngb displaying iron hexa-coordination led researchers to classify vertebrate globins into two classes: penta-coordinate hemoglobins $(\mathrm{Mb}$ and $\mathrm{Hb})$ and hexa-coordinate hemoglobins (Ngb, Cygb, and Adgb). A representation of the heme binding site in vertebrate globins is shown in Figure 1.5. 

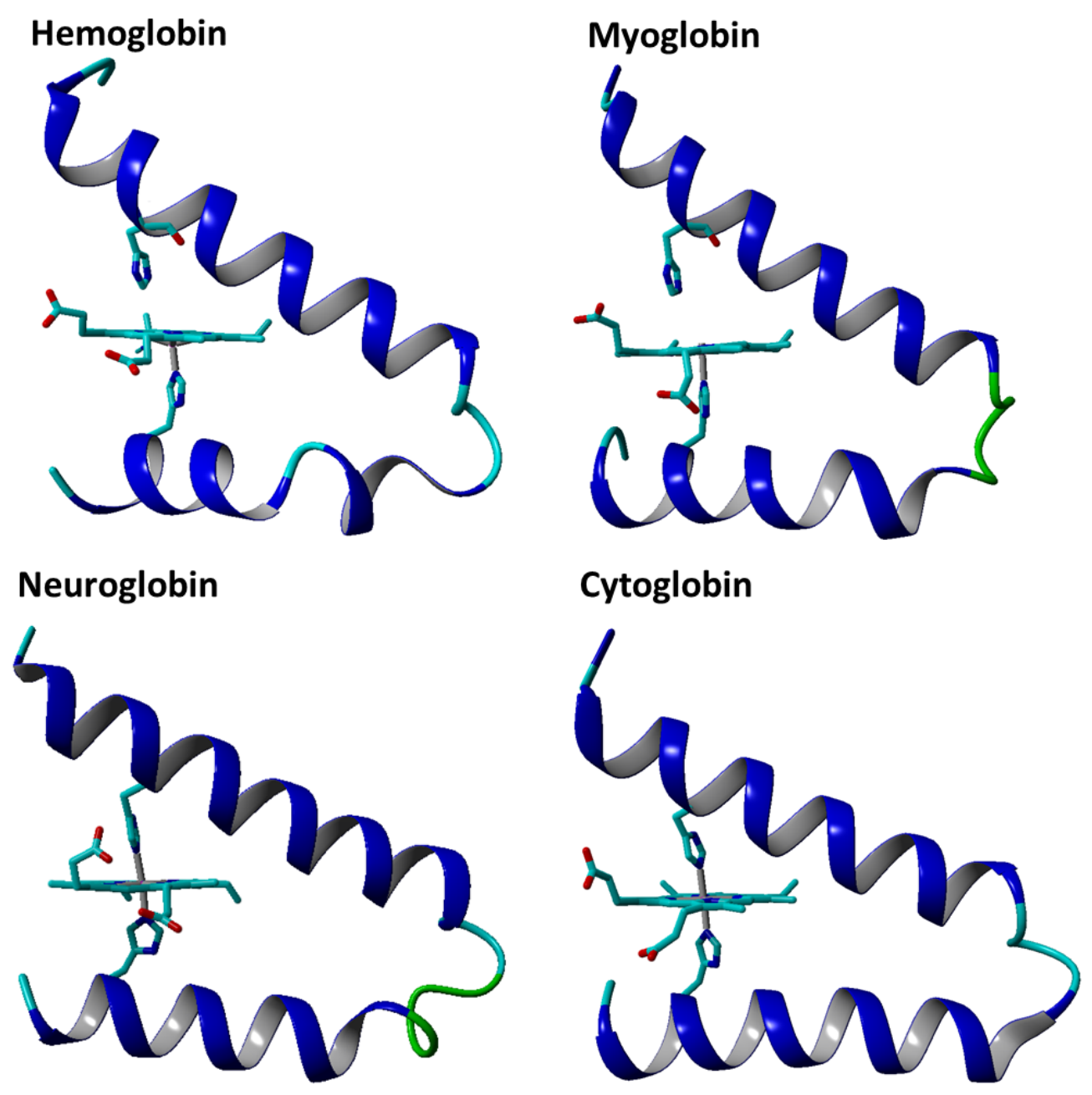

Figure 1.5 Ribbon representation of the heme binding site in vertebrate globins $\mathrm{Hb}$ (PDB entry $1 \mathrm{FDH}), \mathrm{Mb}$ (PDB entry 1WLA), Ngb (PDB entry 1OJ6), and Cygb (PDB entry $1 \mathrm{~V} 5 \mathrm{H})$ exhibiting penta-coordination and hexa-coordination of the heme iron. The distal and proximal histidine residues are shown as sticks.

Although the discovery of hexa-coordinate globins in vertebrates was surprising, heme hexa-coordination has been previously observed in globins from plants and bacteria (Hargrove, 2000). For example, class 1 non-symbiotic hemoglobins (nsHbs) are sixcoordinated and exhibit low affinity for oxygen (Smagghe et al., 2009). It has been suggested that class $1 \mathrm{nsHbs}$ are responsible for maintaining the redox and energy status 
of plant cells during fermentative metabolism, which occurs during hypoxia (Igamberdiev et al., 2006). Class $1 \mathrm{nsHbs}$ are characterized by their bishistidine hexacoordination in both the reduced and ferrous states. In addition, class $1 \mathrm{nsHbs}$ form dimers consisting of two identical subunits of $\sim 18 \mathrm{kDa}$ and contain one or two Cys residues per molecule. In case of barley hemoglobin (class $1 \mathrm{nsHb}$ ), it was demonstrated that Cys 79 forms intermolecular disulfide bonds, which contribute to stabilize the quaternary structure of the protein (Bykova et al., 2006). The dimer interface of class 1 non-symbiotic $\mathrm{Hbs}$ is formed by close contacts between the $\mathrm{G}$ helix and the region formed by the B and $\mathrm{C}$ helices of the partner subunit (Hargrove et al., 2000). Although many functions have been proposed for hexa-coordinate hemoglobins from diverse organisms, no clear role has been identified yet for these proteins (Kakar et al., 2010).

\subsubsection{Neuroglobin}

Neuroglobin $(\mathrm{Ngb})$ is a small protein comprising 151 amino acid residues $(\sim 17$ $\mathrm{kDa}$ ) that is mainly expressed in neurons of the peripheral and central nervous system, retina and some endocrine tissues (Brunori and Vallone, 2007; Burmester et al., 2000). Both in vitro and in vivo studies have suggested that $\mathrm{Ngb}$ over-expression protects the brain against hypoxic and ischemic insults (Khan et al., 2007; Sun et al., 2001). In particular, Ngb over-expression in human neuroblastoma cell lines (SH-SY5Y) increased cell survival under anoxia condition as well as in the case of glucose deprivation (Fordel et al., 2006). In addition, increased levels of $\mathrm{Ngb}$ in transgenic mice significantly protected the brain tissues from hypoxic insult, whilst decreased levels of Ngb resulted in increased tissue damage (Khan et al., 2007). The molecular basis of Ngb neuro-protection is still not well understood. However, several plausible mechanisms have been proposed, 
including: i) oxygen storage and/or transport, ii) scavenger of reactive oxygen species (ROS) or reactive nitrogen species (RNS), iii) detoxification of harmful NO excess by conversion of NO to nitrate (dioxygenase activity), and iv) oxygen/redox sensor (Brunori and Vallone, 2007; Burmester and Hankeln, 2009; Hankeln et al., 2005). The current focus of identification of $\mathrm{Ngb}$ physiological function involves characterization of structural changes associated with $\mathrm{Ngb}$ interactions with diatomic ligands as well as a search for Ngb intracellular partners.

Wakasugi et al. (2003) reported that ferric Ngb binds exclusively to the GDP bound form of the $\alpha$-subunit of heterotrimeric $G\left(G_{\alpha i}\right)$ protein acting as a guanine nucleotide dissociation inhibitor (GDI) (i.e., inhibiting the rate of exchange of GDP to GTP). In addition, since Ngb in its ferrous ligand bound form did not exhibit GDI activity, human Ngb would function as a oxidative stress-responsive sensor for signal transduction in the brain (Wakasugi et al., 2003). Heterotrimeric G proteins belong to a superfamily of regulatory GTP hydrolyses that have crucial roles as molecular switches in the regulation of downstream effector molecules, which are involved in the signaling pathways of several G protein coupled receptor (Sprang, 1997). Heterotrimeric G proteins consist of an $\alpha$ subunit that contains a guanine nucleotide binding pocket and intrinsic GTPase activity, a $\beta$ - and a $\gamma$-subunit that form a complex often refered to as the $\beta \gamma$ subunit. Upon ligand binding to a G protein coupled receptor (GPCR) on the cell surface, the $\alpha$-subunit of G protein $(\mathrm{G} \alpha)$ exchanges GTP for GDP and detaches from the $\mathrm{G}_{\beta \gamma}$ complex. These subunits become active and interact with diverse downstream effectors, including adenylate cyclase, $\mathrm{G}$ protein regulated kinases, ion channels and phospholipase C to initiate specific cellular responses (Hepler and Gilman, 1992; Katz et 
al., 1992; Sprang, 1997). The activation/deactivation of G proteins can be regulated by three classes of protein modulators: guanine nucleotide exchange factors (GEFs) that stimulate GDP dissociation and GTP association, guanine nucleotide dissociation inhibitors (GDIs) that inhibit GDP dissociation, and GTPase-activating proteins (GAPs) that enhance GTP hydrolysis (Sprang, 1997).

Site-directed mutagenesis in combination with mass spectrometry and measurement of GDI activity for $\mathrm{G}_{\alpha}$ suggested that residues Glu 53 and Glu 60, located within the CD-D region in human Ngb, are crucial for the GDI activity in human Ngb (Kitatsuji et al., 2007; Takahashi et al., 2013; Wakasugi et al., 2005; Wakasugi and Morishima, 2005). At the molecular level, it was proposed that Glu 60 of Ngb interacts with Ser 206 of $\mathrm{G}_{\alpha \mathrm{i}}$ (located in the region that contacts $\mathrm{G} \beta \gamma$ ), and Glu 53 in Ngb wih Ser 44 of $\mathrm{G}_{\alpha \mathrm{i}}$ (adjacent to the GDP-binding site), thereby binding of $\mathrm{Ngb}$ would facilitate the release of $\mathrm{G}_{\beta \gamma}$ from $\mathrm{G}_{\alpha}$ and also inhibit the exchange of GDP for GTP (Kitatsuji et al., 2007). Therefore, residues located within the CD loop in human Ngb are crucial for the binding of $\mathrm{Ngb}$ to $\mathrm{G}_{\mathrm{ai}}$ protein emphasizing the importance of the CD-D region for protein-protein interactions (Wakasugi et al., 2003; Watanabe et al., 2012). The encounter of human Ngb with $G_{\alpha i}$ was proposed to be facilitated by flotillin-1, a lipid raft microdomain-associated protein that was identified as an interacting partner of $\mathrm{Ngb}$ (Wakasugi et al., 2004). Under oxidative stress conditions, flotillin-1 recruits human Ngb to lipid rafts, where human $\mathrm{Ngb}$ then binds to $\mathrm{G}_{\alpha \mathrm{i}}$ (also present in lipid rafts) and acts as a GDI for $G_{a i}$ though prevention of decrease in cAMP concentration, thus leading to protection against cell death (Watanabe et al., 2012). Noteworthy, the interaction between $\mathrm{Ngb}$ and the heterotrimeric G protein appears to be species specific since zebrafish Ngb, 
which shares $\sim 50 \%$ sequence homology with human Ngb, does not exhibit GDI activity for $\mathrm{G}_{\alpha}$ (Wakasugi and Morishima, 2005). The difference in GDI activity between human Ngb and zebrafish Ngb was proposed to be related to the presence of a Glu residue at position 53 in mammalian Ngbs that is occupied by a Pro residue in fish Ngbs, however the physiological relevance of the specificity observed in mammalian Ngbs toward GDI activity is unclear (Kitatsuji et al., 2007).

On the other hand, Brittain et al. and others (Brittain et al., 2010b; Fago et al., 2008; Raychaudhuri et al., 2010) showed that Ngb inhibits apoptosis in vivo through the binding to cytochrome c, thereby preventing pro-caspase 9 activation. Surface plasmon resonance studies combined with docking simulations indicate that $\mathrm{Ngb}$ binds to Cyt $\mathrm{c}$ with moderate affinity with a $\mathrm{K}_{\mathrm{d}}=45 \mu \mathrm{M}$ and the authors suggested that the interactions are mostly electrostatic because of the dependence of $K_{d}$ on the solution ionic strength (Bonding et al., 2008). The authors showed that formation of the complex between Ngb and Cyt $\mathrm{c}$ is entropy driven, with a positive $\Delta \mathrm{H}=1.9 \mathrm{kcal} \mathrm{mol}^{-1}$ and $\Delta \mathrm{S}=14.8 \mathrm{cal} \mathrm{deg}^{-1}$ $\mathrm{mol}^{-1}$ at $37{ }^{\circ} \mathrm{C}$. The docking simulations suggest that Cyt $\mathrm{c}$ binds to $\mathrm{Ngb}$ at a site covering the area of the exposed heme so that the two heme groups are in close proximity, and the residues Lys 25 and Lys 72 were identified as crucial residues in the interaction between Ngb and Cyt c (Bonding et al., 2008; Brittain et al., 2010b). These amino acid residues have been previously identified as key residues in the interaction of Cyt c with Apaf-1, the major cytosolic protein involved in apoptosome assembly, suggesting competition of Ngb with Apaf-1 for Cyt c binding site (Brittain et al., 2010b). 
An intramolecular disulfide bond is present in human $\mathrm{Ngb}(\mathrm{hNgb})$ that connects Cys 46 within the CD loop with Cys 55 from the E helix (Hamdane et al., 2003). There is another Cys residue (Cys 120) in the sequence of Ngb that does not participate in intramolecular disulfide bond formation since it is located too far from the other two Cys. Despite the high sequence homology between $\mathrm{hNgb}$ and rodent $\mathrm{Ngbs}(\sim 94 \%)$, the internal disulfide bond is absent in rodent Ngbs (rat, mouse) because a Gly residue is found at position 46. Reduction of the internal disulfide bond decreases the rate of His64 dissociation from heme iron by a factor of approximately 10 times resulting in decreased hNgb affinity for $\mathrm{O}_{2}$ (Hamdane et al., 2003). Therefore, Hamdane et al. (2003) proposed that the interaction between $\mathrm{hNgb}$ and $\mathrm{O}_{2}$ may be coupled to the intracellular redox state of the cell through the rupture and/or formation of the disulfide bond.

The crystal structures of the CCC $\rightarrow$ GSS mutated protein of ferric human Ngb (Figure 1.6), as well as the $\mathrm{CC} \rightarrow \mathrm{SS}$ mutated protein of ferric, ferrous and $\mathrm{CO}$ bound adduct of mouse Ngb have been elucidated (Pesce et al., 2003, 2004b; Vallone et al., 2004a; Vallone et al., 2004b). Superposition of the crystal structure of the ligand free and ligand bound forms of Ngb indicates that ligand binding leads to large structural changes, including a heme sliding mechanism that has been proposed to be species specific, and reorganization of internal cavities (Vallone et al., 2004b). 


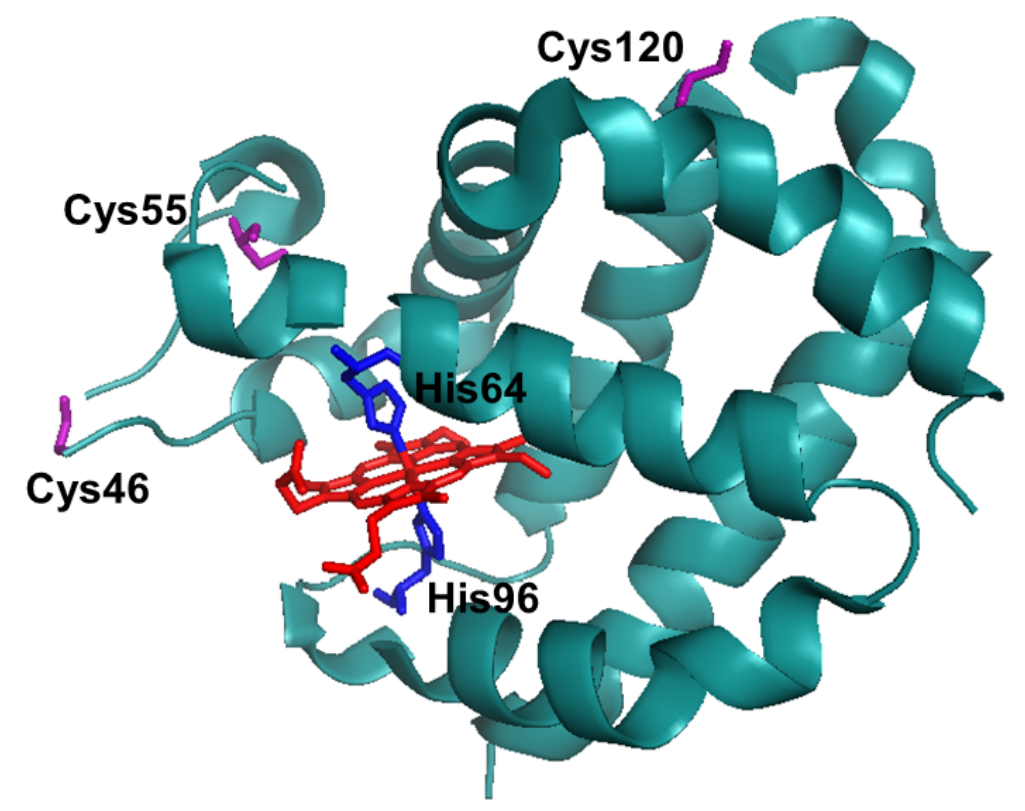

Figure 1.6 Ribbon representation of the crystal structure of human Ngb (PDB entry: 1JO6). The heme prosthetic group is shown as red sticks, the distal His64 and proximal His96 are depicted in blue, and the Cys residues in purple.

\subsubsection{Cytoglobin}

Cytoglobin comprises 190 amino acid residues $(\sim 21 \mathrm{kDa})$ with extended $\mathrm{N}$ - and C-termini that is localized in the nucleus and cytoplasm of several tissues, specifically in fibroblasts and related cell types (Burmester et al., 2002; Makino et al., 2006; Nakatani et al., 2004). Cytoglobin is overexpressed in fibrosis and neurodegenerative disorders and down-regulated in some types of cancers, such as head and neck cancer (Fordel et al., 2004; He et al., 2011; Xu et al., 2006). Moreover, Cygb has been shown to inhibit cancer cell growth in vitro, which indicates a tumor suppressor role (Kawada and Le, 2011; Shivapurkar et al., 2008). Recently, Reeder et al. (2011) reported that binding of lipids, such as oleate and cardiolipin, to Cygb enforces the protein's transition from hexa- to penta-coordinate state, suggesting a role for $\mathrm{Cygb}$ in lipid transport. However, the 
molecular mechanism of Cygb cytoprotection remains under investigation. Some studies point out diverse roles of Cygb, including oxygen storage, oxygen sensing, as decomposition of reactive oxygen species (ROS), nitric dioxygenase activity, and lipid transport (Fago et al., 2004c; Halligan et al., 2007; Mammen et al., 2004; Oleksiewicz et al., 2011; Pesce et al., 2002; Reeder et al., 2011).

An interesting structural feature of Cygb, also found in human $\mathrm{Ngb}$, is the presence of an internal disulfide bond. There are two cysteine residues in Cygb, Cys 38 and Cys 83, forming a disulfide bond (Hamdane et al., 2003). The three-dimensional structure of ferric Cygb is shown in Figure 1.7. Two histidine residues are positioned in the fifth and sixth axial positions of the iron atom, His 113 as the proximal ligand and His 81 as the distal ligand. Reduction of the disulfide bond in Cygb decreases the rate of distal His81 dissociation from heme iron, but to a lesser extent than observed for Ngb, by a factor of approximately two times indicating a weaker coupling between the disulfide bond and ligand binding in this protein (Hamdane et al., 2003).

The crystal structure for both ferric and $\mathrm{CO}$ adduct of Cygb have been elucidated (de Sanctis et al., 2004a; Makino et al., 2011; Makino et al., 2006). Superposition of the $\mathrm{CO}$ bound structure of Cygb with its ferric form shows ligand induced conformational changes in CD-D corner and the E-helix of Cygb (Makino et al., 2011). Molecular dynamics simulations of deoxy and $\mathrm{CO}$ bound human Cygb indicate that ligand-induced structural changes are mainly modulated by rearrangement of loops and cavities instead of the heme sliding mechanism proposed for Ngb (Zhang et al., 2011a). The structural differences between $\mathrm{Ngb}$ and Cygb suggest different physiological functions and mechanism of interaction with ligands (Zhang et al., 2011a). 


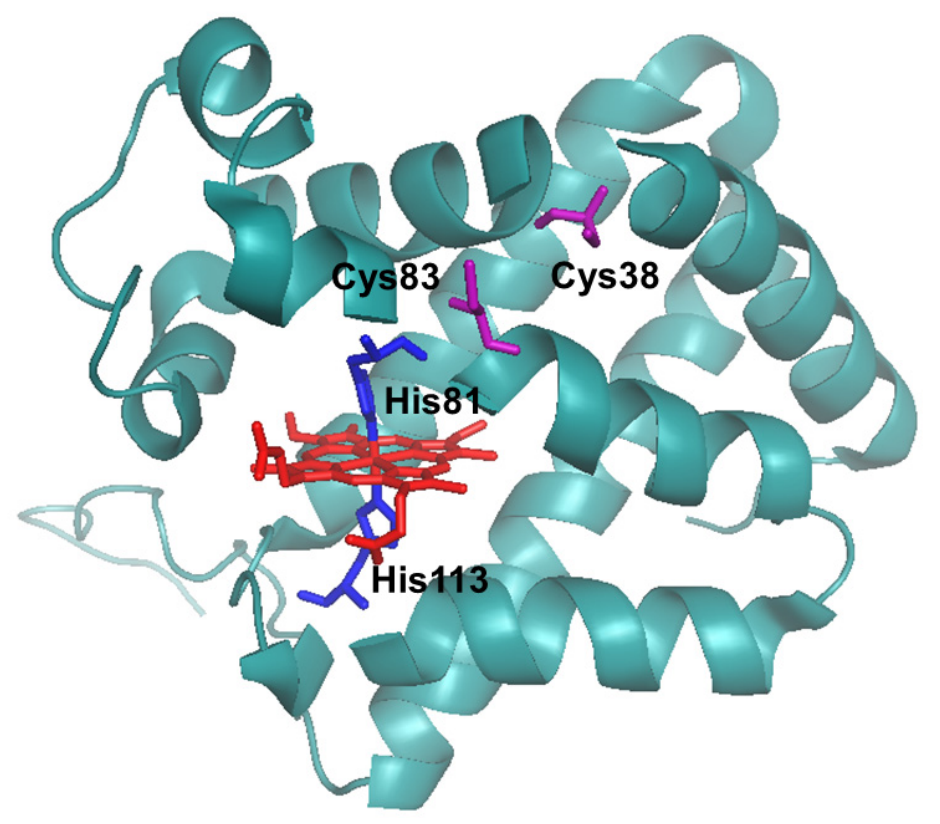

Figure 1.7 Ribbon representation of the crystal structure of human Cygb (PDB entry: $1 \mathrm{~V} 5 \mathrm{H})$. The heme prosthetic group is shown as red sticks, the distal His81 and proximal His 113 are depicted in blue, and the Cys residues in purple.

\subsubsection{Androglobin}

The recently discovered androglobin (Adgb) is a multidomain protein containing an $\mathrm{N}$-terminal calpain-like domain, an internal globin domain that has undergone internal shuffling of a-helices, and an IQ calmodulin binding motif (Hoogewijs et al., 2012). In mammals, Adgb is preferentially expressed in testis. The physiological role of Adgb is still not known, but it has been proposed to be involved in redox-signaling or oxygen sensing (Hoogewijs et al., 2012). The three dimensional structure of Adgb has not been elucidated yet.

In addition to vertebrate Ngbs and Cygb that show structural similarities with $\mathrm{Mb}$, other globins such as dehaloperoxidase (DHP) and the globin coupled sensor HemAT exhibit high structural similarities to $\mathrm{Mb}$, but distinct functions. Dehaloperoxidase is non- 
vertebrate hemoglobin that is expressed by the terebellid polychaete Amphitrite ornate. Secretion of DHP by Amphitrite ornata enables this organism to live in coastal marine environments contaminated with haloaromatic compounds originated from human related activities or secreted by other organisms (Chen et al., 1996). Although DHP has different structural properties than other peroxidases, including horseradish peroxidase, it still has a peroxidase function that enables it to oxidize halogenated phenols into quinones in the presence of $\mathrm{H}_{2} \mathrm{O}_{2}$ (Figure 1.8). The active site of DHP consists of a protoporphyrin IX that is covalently attached to the apo-protein through the proximal His 89 . In addition, the proximal His in DHP forms a hydrogen bond with Leu 83 that is weaker than that observed in other peroxidases and the distal pair of hydrophobic residues Arg-His, which is crucial for the enzymatic activity in other peroxidases, is missing in DHP (Franzen et al., 2006; LaCount et al., 2000; Osborne et al., 2004).

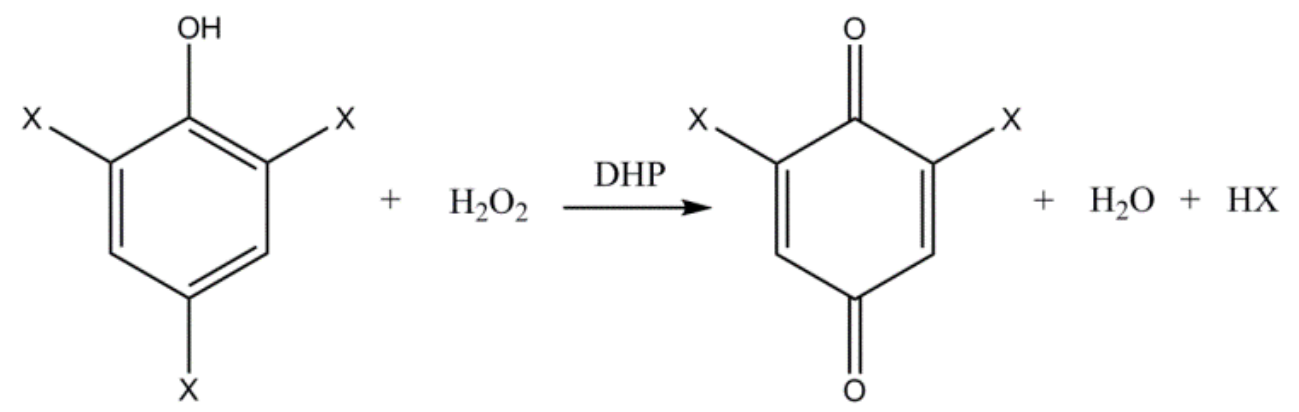

Figure 1.8 Oxidation of halogenated phenols by dehalperoxidase (DHP) in the presence of $\mathrm{H}_{2} \mathrm{O}_{2}$. The $\mathrm{X}$ represents halogen atoms $(\mathrm{X}=\mathrm{I}, \mathrm{Br}, \mathrm{Cl}, \mathrm{F})$.

The HemAT proteins belong to the globin coupled sensors family, which represent a unique class of oxygen sensing heme proteins found in both Archaea and Bacteria. The first two members of the globin coupled sensors were HemAT-Hs from the archeae Halobacterium salinarum and HemAT-Bs from the gram positive prokaryote 
Bacillus subtilis (Hou et al., 2000). These proteins contain an N-terminal heme sensor myoglobin-like domain and a C-terminal domain homologous to the cytoplasmic signaling domain of Tsr, a methyl-accepting chemotaxis protein from Escherichia coli ( 30\% sequence similarity) (Hou et al., 2000). The myoglobin-like domain of HemAT proteins share conserved proximal residues His (F8), Pro (C2), and Phe (CD1) with Mb corresponding to amino acid residues Pro55, His123, Phe61 for HemAT-Hs and Pro56, His123, Phe69 for HemAT-Hs (Mokdad et al., 2007; Zhang and Phillips, 2003a, b). It has been proposed that HemAT function is related to the aerotaxic response exhibited by microorganisms (migratory response towards or away from oxygen), especifically HemAT-Hs is involved in an aerophilic response, whereas HemAT-Bs function is related to an aerophobic response (Hou et al., 2001; Hou et al., 2000; Yu et al., 2002).

\subsection{Electronic properties and absorption spectroscopy of heme proteins}

\subsubsection{Electronic properties}

The presence of the heme prosthetic group in globins provides an excellent opportunity to study functional and structural properties of this class of proteins using steady state and time resolved spectroscopic techniques. The electronic configuration of iron atom is $[\mathrm{Ar}] 3 \mathrm{~d}^{6} 4 \mathrm{~s}^{2}$. Ions $\mathrm{Fe}^{2+}$ and $\mathrm{Fe}^{3+}$ tend to form octahedral complexes in which the splitting of the energy levels results in three degenerate $e_{g}$ orbitals $\left(d_{x y}, d_{x z}, d_{y z}\right)$ and two degenerate $\mathrm{t}_{2 \mathrm{~g}}$ orbitals $\left(\mathrm{d}_{\mathrm{z}}^{2}, \mathrm{~d}_{\mathrm{x}-\mathrm{y}}^{2}{ }^{2}\right)$ (Figure 1.9) (Lippard and Berg, 1994). The energy levels corresponding to the electrons from the d-orbitals in heme proteins are altered from those found in the free metal ion as a consequence of a phenomenon called ligand-field splitting. Energy-level diagrams are used to show the position of the energy levels, which depend on the strength of the ligand field (Lippard and Berg, 1994). These diagrams have 
been useful to correlate some of the physical properties of metal centers found in proteins, including their absorption spectra and magnetic properties, with their structures or reactivity (Lippard and Berg, 1994; Perry et al., 2002). The strength of the ligand field at the metal center is determined by the atoms from the ligand that coordinate to the metal center. In fact, the ability of ligands to split the d-orbitals varies according to the spectrochemical series (Lippard and Berg, 1994).

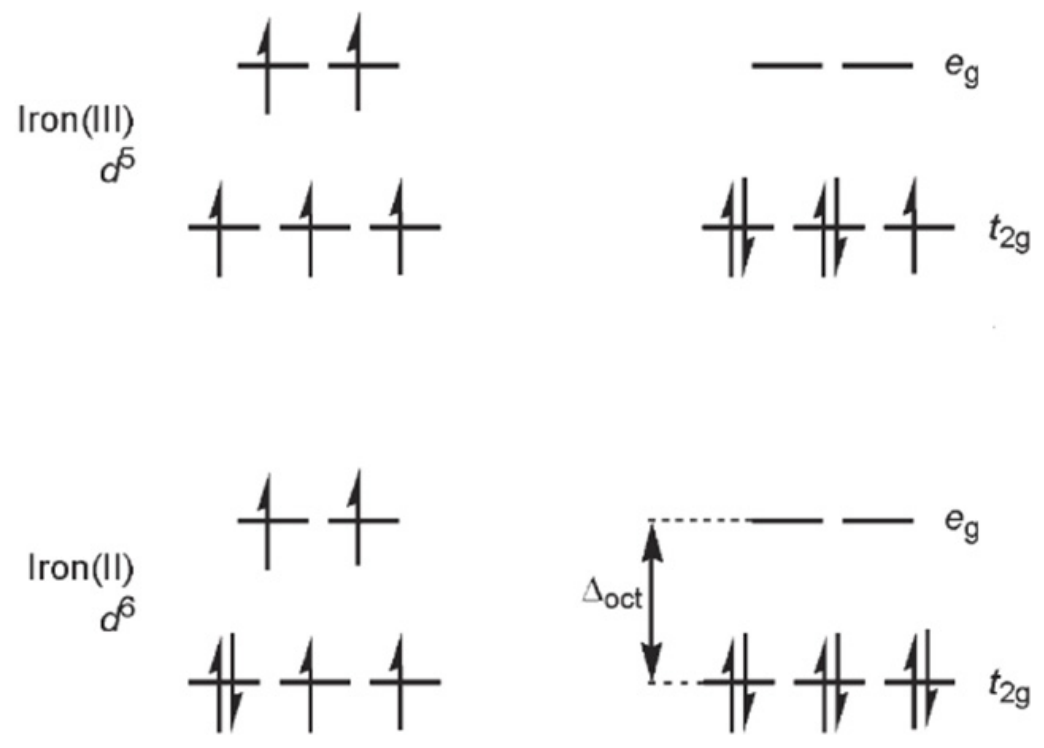

High spin

Low spin

Figure 1.9 Energy level diagrams showing the d-orbital electron occupancies of high and low-spin iron(II) and iron (III) complexes in an octahedral ligand field (Halcrow, 2008).

\subsubsection{Absorption spectroscopy}

There are three main sources of electronic spectra in metal complexes, namely internal ligand bands such as those found in porphyrins, transitions associated purely with metal orbitals such as d-d transitions, and charge transfer bands between metal and 
ligand. These spectral properties have been used to assign oxidation state of the metal, follow reactions, and identify chemical species (Lippard and Berg, 1994).

Heme proteins exhibit characteristic absorption spectra with absorption bands that result from the transition between two HOMO orbitals $\left(\mathrm{a}_{1 \mathrm{u}}\right.$ and $\mathrm{a}_{2 u}$ orbital) and two degenerate LUMO orbitals (e $\mathrm{e}_{\mathrm{g}}$ orbitals) (Larsen and Miksovska, 2007). The Soret band is a high intensity band (also referred to as B band) that results from the $\mathrm{a}_{1 \mathrm{u}}$ to $\mathrm{e}_{\mathrm{g}}$ transition and appears in the near ultraviolet region of the spectrum at approximately $400 \mathrm{~nm}$. In addition, there is a number of low intensity bands referred to as $\mathrm{Q}$ bands that appear in the visible region of the spectrum, between 450 and $700 \mathrm{~nm}$, arising from the $\mathrm{a}_{2 \mathrm{u}}$ to $\mathrm{e}_{\mathrm{g}}$ transitions. Soret and Q bands maxima are influenced by the oxidation state of the metal center, interactions between the metal and the porphyrin ring, as well as the type of ligands coordinating to the metal (Perry et al., 2002).

High spin complexes of $\mathrm{Fe}^{3+}$ porphyrins are characterized by a Soret band maximum located at approximately $400 \mathrm{~nm}$, whereas low spin complexes increase the strength of the axial ligand field resulting in a red shifted Soret band at approximately $410 \mathrm{~nm}$ (Perry et al., 2002). Reduction of the heme iron $\left(\mathrm{Fe}^{3+}\right.$ to $\left.\mathrm{Fe}^{2+}\right)$ leads to a bathochromic shift of the Soret band and upon addition of diatomic ligands, including $\mathrm{CO}, \mathrm{NO}$ and $\mathrm{O}_{2}$, subsequent formation of the adducts results in hypsochromic shift of the Soret band maximum with respect to the ferrous form (Nienhaus and Nienhaus, 2005b).

\subsection{Heme protein interactions with diatomic ligands}

The presence of the heme group allows heme proteins to bind reversibly to diatomic ligands, including $\mathrm{O}_{2}, \mathrm{CO}$ and $\mathrm{NO}$ at the distal coordination site (Anderson and Chapman, 2005). Ligand binding to heme proteins is strongly influenced by residues 
within the distal heme pocket in terms of orientation of the ligand and ligand-Fe bond lability through non-covalent interactions including hydrogen bonding and hydrophobicity (Anderson and Chapman, 2005). Moreover, distal pocket residues also play a role in substrate binding and recognition serving as substrate binding sites for proteins like plant peroxidases and cytochromes $\mathrm{P} 450$, since they are designed to place the substrate in a specific orientation and at a specific distance from the heme active site (Larsen and Miksovska, 2007).

The photo-cleavable nature of the Fe-ligand bond is advantageous for ligand migration studies in heme proteins, since it allows monitoring of transient deoxy intermediates using spectroscopic techniques, including time-resolved absorption spectroscopy (Carver et al., 1990; Esquerra et al., 2010; Gibson et al., 1986; Goldbeck et al., 2006; Rohlfs et al., 1990) and time-resolved X-ray crystallography (Schmidt et al., 2005; Srajer et al., 1996). In particular, $\mathrm{Mb}$ and $\mathrm{Hb}$ have been extensively studied using these techniques as model systems to understand the mechanism of ligand migration in heme proteins, as well as ligand affinity and specificity (Brunori, 2000).

Ligand migration in heme proteins was described by Henry et al. (1983) using the sequential three state model (Figure 1.10). Photo-dissociation of the Fe-ligand bond results in two global kinetic steps. The cleaved ligand can be temporarily trapped within the protein matrix and from there rebind back to the heme iron in the so called "geminate rebinding" or it can diffuse from the protein matrix into the surrounding solvent in the so called "bimolecular rebinding" (Henry et al., 1983). Geminate rebinding does not depend on the concentration of the ligand (e.g., $\mathrm{NO}, \mathrm{O}_{2}$ and $\mathrm{CO}$ ) and occurrs in the nanosecond time scale. On the other hand, bimolecular rebinding occurs on significantly longer time 
scales in the range of microseconds to milliseconds (Henry et al., 1983; Jongeward et al., 1988; Schaad et al., 1993).

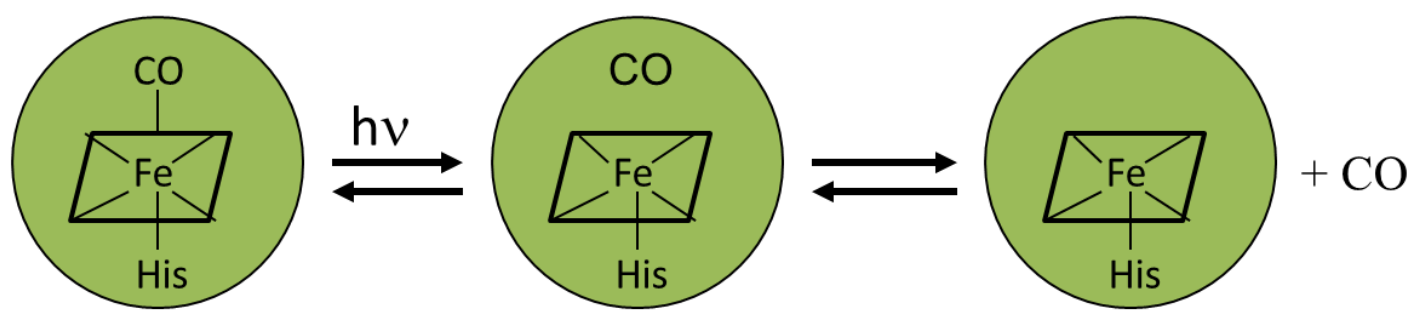

Figure 1.10 Schematic representation of ligand rebinding to heme proteins according to the sequential three state model.

The microscopic rate constants associated with ligand migration in heme proteins can be analyzed using the simple three-state model according to the following equation:

$$
L-H b \underset{k_{21}}{\stackrel{k_{12}}{\rightleftarrows}} H b_{5 c}: L \underset{k_{32}}{\stackrel{k_{23}}{\rightleftarrows}} H b_{5 c}+L
$$

Where state 1 corresponds to the ligand bound protein, state 2 represents the penta-coordinate hemoglobin $\left(\mathrm{Hb}_{5 \mathrm{c}}\right)$ with the ligand within the protein matrix in the socalled geminate pair, and state 3 is the free $\mathrm{Hb}_{5 \mathrm{c}}$ with the ligand into the surrounding solvent. Since the concentration of the ligand used for determination of ligand geminate and bimolecular rebinding to $\mathrm{Hb}_{5 \mathrm{c}}$ is usually much larger than the concentration of the protein and in case of $\mathrm{CO}$ the concentration of the ligand is $\sim 1 \mathrm{mM}$, the following approximations can be used: $[\mathrm{L}]=10^{-3} \mathrm{M}, \mathrm{k}_{21}>>\mathrm{k}_{12}$, and $\mathrm{k}_{23}>>\mathrm{k}_{32}[\mathrm{~L}]$ (Ciaccio et al., 2004).

$$
k_{\text {gem }}=k_{21}+k_{23}
$$




$$
\begin{aligned}
& \frac{A_{\text {gem }}}{A_{\text {total }}}=\frac{k_{21}}{k_{23}+k_{21}} \\
& k_{\text {bim }}=\frac{k_{32} k_{21}}{k_{23}+k_{21}}
\end{aligned}
$$

Where $\mathrm{k}_{\mathrm{gem}}$ is the rate constant for ligand geminate recombination, $\mathrm{A}_{\text {total }}$ is the total absorbance change for ligand rebinding to $\mathrm{Hb}_{5 \mathrm{c}}$ (geminate recombination and bimolecular rebinding), $\mathrm{A}_{\mathrm{gem}}$ is the absorbance change upon ligand geminate recombination, and $\mathrm{k}_{\mathrm{bim}}$ corresponds to the rate constant for ligand bimolecular rebinding.

By combining equations 1.2 to 1.4 , the individual kinetic rate constants associated to ligand dissociation and rebinding to $\mathrm{Hb}_{5 \mathrm{c}}$ presented in equation 1.1 can be calculated using the following equations:

$$
\begin{aligned}
& k_{32}=\frac{k_{\text {bim }} A_{\text {total }}}{A_{\text {gem }}} \\
& k_{21}=\frac{k_{\text {gem }} A_{\text {gem }}}{A_{\text {total }}} \\
& k_{23}=k_{\text {gem }}-k_{21}
\end{aligned}
$$

The quantum yield for each process, defined as the ratio between the amplitude of the kinetic step and the total amplitude, strongly depends on the character of the ligand and the protein (Henry et al., 1983). The NO molecule rebinds predominantly through geminate rebinding (Ye et al., 2002) with a quantum yield close to unity in most proteins. In $\mathrm{Mb}, \mathrm{CO}$ rebinds predominantly through bimolecular rebinding with a quantum yield close to one $\left(\Phi_{\text {bim }}=0.96\right)$ (Henry et al., 1983), whereas the quantum yield for 
bimolecular $\mathrm{O}_{2}$ rebinding to heme proteins is significantly lower (Carver et al., 1990; Walda et al., 1994).

The mechanism of oxygen entrance to $\mathrm{Mb}$ and some $\mathrm{Hbs}$ was proposed to occur though a so-called "histidine gate" by Perutz (Perutz, 1989) upon elucidation of the crystal structure of $\mathrm{Mb}$, in which the distal His swings out of the heme pocket allowing the ligand to escape from the protein matrix. Kinetic studies demonstrated that the observed rate of entrance of the ligand $\left(k_{\text {on }}\right)$ increased when the distal His64 in Mb was replaced with small hydrophobic residues such as Ala or Gly, whereas it decreased when His64 was replaced by bulky amino acid residues such as Trp (Olson et al., 2007; Rohlfs et al., 1990). Moreover, the crystal structure of $\mathrm{Mb}$ at low $\mathrm{pH}$ showed that the distal His is displaced towards the solvent in what was called the "open conformation" (Yang and Phillips, 1996), which was supported by kinetic results that provided evidence of increased $k_{\text {on }}$ at lower $\mathrm{pH}$ (Tian et al., 1993).

The crystal structures of $\mathrm{Mb}$ also revealed another interesting feature of globins related to the presence of hydrophobic cavities within the protein matrix. These cavities are also denoted as xenon binding sites because they can bind Xe atoms (Brunori and Gibson, 2001). Therefore, the presence of these cavities in Mb suggested possible alternative routes for ligand entry and escape (Brunori and Gibson, 2001; Savino et al., 2009).

Although, the histidine gate mechanism has been considered as the main pathway for $\mathrm{O}_{2}$ entry to $\mathrm{Hb}$ and $\mathrm{Mb}$, several other plausible pathways have been also proposed as a result of thermal fluctuations in proteins, which suggest that most likely the histidine gate mechanism is one of the many pathways in ligand migration from globins (Salter et al., 
2012). Accesibility of the ligand to the active site of the protein may also occur through ligand channels that provide entry/exit pathways from the solvent to the distal heme pocket (Lecomte et al., 2005). These channels and/or pathways are modulated by protein conformational changes as a result of "gated" ligand access and protein motions that are regulated by protein dynamics.

The different pathways observed in globins may have important physiological roles that are still not well understood. For instance, these pathways may have enzymatic functions as it has been proposed in the catalysis of $\mathrm{NO}$ to $\mathrm{NO}_{3}{ }^{-}$reaction by some oxyHbs and oxyMbs, or serve as transient docking sites for ligand storage (Anderson and Chapman, 2005). Such pathways could also provide various routes for the ligand to enter and/or escape the protein, which would also increase the available surface for the capture and release rates of gas molecules (Savino et al., 2009).

Overall, the affinity of heme proteins for ligands is affected by several factors, including the reactivity of the ligand for the heme which can be modulated by the type of residues occupying the proximal and distal axial positions. The proximal residues largely impact the heme electronic structure, whilst the distal residues may interact directly with the bound ligand through non-covalent interactions (Anderson and Chapman, 2005). In addition, the reactivity of the ligand also depends on the ligand accessibility to the distal site of the protein that is modulated by the presence of i) blocking residues coordinating to the heme iron, ii) tunnels connecting the distal site with the surrounding solvent, and iii) gates that affect proteins dynamics thus controlling ligand access and escape routes (Lecomte et al., 2005; Vetromile et al., 2011). 


\section{OBJECTIVES}

Conformational dynamics in proteins play a crucial role in their function, activity and association to interacting partners. Chemical changes and atomic motions in biological systems typically occur on time scales ranging from femtoseconds to seconds. Therefore, kinetics and thermodynamics associated with relevant protein dynamics, including local conformational changes, binding to ligands, and protein folding, have been usually investigated through the use of time-resolved spectroscopic methods (Braslavsky and Heibel, 1992; Brunori, 2000; Chekmarev et al., 2005; Chen et al., 1997; Gensch and Viappiani, 2003; Vetromile et al., 2011).

Traditional techniques such as UV-vis spectroscopy have been extensively used to monitor time-resolved structural changes in the vicinity of the chromophore. The photolability of the ligand-iron bond in combination with the sensitivity of the absorption spectra of heme proteins to changes in the vicinity of the heme iron have proved to be advantageous to probe time-resolved local dynamics that provide critical information related to local chromophore/protein dynamics using transient absorption spectroscopy (Nienhaus and Nienhaus, 2005b). However, optical methods are limited in their ability to characterize the thermodynamic parameters, in terms of molar volume and enthalpy changes, associated with the ligand binding event on fast time scales. Photothermal methods, such as photoacoustic calorimetry, provide an opportunity to monitor dynamics and energetics of conformational changes in proteins that are otherwise "silent" with other time-resolved spectroscopic techniques, including time-resolved absorption spectroscopy and time-resolved fluorescence. The use of photoacoustic calorimetry allows characterization of volume and enthalpy changes associated with ligand binding to 
proteins occurring on physiologically relevant time scales (ns to $\mu \mathrm{s}$ ) (Larsen and Miksovska, 2007; Vetromile et al., 2011). The overall goal of these studies is to gain a deeper understanding of the relationship between dynamics and function in proteins. Besides being a subject of basic scientific interest, this information will be useful in the fields of protein science and medicine (Anderson and Chapman, 2005).

In this work, conformational dynamics associated with ligand binding to four vertebrate globins, namely $\mathrm{Hb}, \mathrm{Mb}, \mathrm{Ngb}$, and Cygb, were investigated using steady-state and time-resolved spectroscopic methods. The role of individual amino acid residues on conformational dynamics associated with CO binding to Ngb as well as the impact of the disulfide bond in Ngb and Cygb was probed by time-resolved photothermal and spectroscopic methods. In addition to understanding the interactions of vertebrate globins with $\mathrm{CO}$, a part of this study was devoted to characterizing how distinct ligands, $\mathrm{CO}$ and $\mathrm{O}_{2}$, affect the thermodynamic parameters associated with ligand photodissociation in vertebrate globins. The overall goal of this study is to provide molecular insight into the mechanism of ligand interactions with vertebrate hemoglobins. In particular, a thorough study of the kinetics and thermodynamics of ligand migration in hexacoordinate globins as well as their equilibrium dynamics is crucial to have a better understanding of their physiological functions in vertebrates. Since Ngb and Cygb have been proposed to protect cells under hypoxia/ischemia and oxidative stress conditions, the information obtained from this study will in the long term provide molecular bases for development of novel targets for therapeutic agents against hypoxia, neurodegenerative diseases, and/or cancer. 


\subsection{Objective 1}

Determine the role of His64, Val68 and Cys120 residues on the dynamics and energetics of structural changes associated with $\mathrm{CO}$ binding to $\mathrm{Ngb}$ using transient absorption spectroscopy and photoacoustic calorimetry

Protein function is related to protein flexibility and the interaction between a protein and an exogenous molecule requires the protein to be able to change its conformation. Although, this variation in conformation may be small, solely involving the rearrangement of a few amino acid residues or side chains, it can also be large and involve long-range interactions within the protein. Dynamics and energetics associated with ligand migration have been extensively studies in model proteins such as $\mathrm{Mb}$. Although $\mathrm{O}_{2}$ is considered the physiological ligand of globins, carbon monoxide has been widely used as a probe for ligand binding studies in heme proteins because of the high yield of $\mathrm{CO}$ photodissociation, which is close to unity in $\mathrm{Mb}$, the stability of the $\mathrm{CO}$ adducts of these proteins, and the comparable size between $\mathrm{CO}$ and $\mathrm{O}_{2}$. The fact that most studies of ligand migration have used $\mathrm{CO}$ as a probe is helpful in comparing the results of this study to previously reported results.

In this study, I took advantage of the sensitivity of photothermal methods to monitor the role of two conserved residues in globins located in the distal pocket, His64 andVal68, as well as conserved Cys residue among Ngbs (Cys 55 and Cys120), on the thermodynamic profiles of exogenous binding to Ngb and ligand migration pathways. This study provided the first time-resolved thermodynamic profiles for ligand migration in $\mathrm{Ngb}$ with the concomitant conformational transitions, which contributes to the 
understanding of the mechanism of ligand migration in this protein. The results of this objective are shown in chapter 4 and have been published in (Astudillo et al., 2012).

\subsection{Objective 2}

Determine the impact of the disulfide bond on the dynamics and energetics of structural changes associated with $\mathrm{CO}$ binding to Ngb and Cygb using transient absorption spectroscopy and photoacoustic calorimetry.

Disulfide bonds in proteins have often been associated with enhacement of protein stability (Wedemeyer et al., 2000). Recently, it was shown that disulfide bonds in proteins can have additional functions including regulation of enzymatic activity and sensing, and acting as molecular switches in cells that regulate several processes such as transcription (Nagahara, 2011; Wouters et al., 2010). Ngb and Cygb are the only two heme proteins in vertebrates that carry a disulfide bond, although the precise contribution of the disulfide bond to Ngb and Cygb remains unclear.

In this work, time-resolved absorption spectroscopy and photoacoustic calorimetry were used to monitor the impact of the disulfide bond on conformational dynamics associated with $\mathrm{CO}$ binding to $\mathrm{Ngb}$ and Cygb. The impact of the internal disulfide bond present in human Ngb and Cygb on the structural changes between the ligand free and the ligand bound form of the protein was evaluated by comparing the thermodynamic profiles for the wild type protein in the presence and absence of the disulfide bond. The disulfide bond was reduced by incubation of the protein with dithiothreitol or through replacement of Cys residues with Gly or Ser residues. In addition, since the internal disulfide bond is missing in wild type rat $\mathrm{Ngb}$, a rat $\mathrm{Ngb}$ mutated protein with an engineered disulfide bond was also characterized. Moreover, the 
role of the distal Tyr residue in transmitting structural information between the heme pocket and the disulfide bond in human Ngb was assessed by characterization of a Tyr44 $\rightarrow$ Phe mutated protein. The main hypothesis was that the presence of the disulfide bond affects the kinetic and thermodynamic profiles of hexa-coordinate hemoglobins in vertebrates. Characterization of global structural changes associated with ligand binding in these heme proteins is crucial for a detailed understanding of of role of the disulfide bond in hexa-coordinate hemoglobins. The results of this objective are described in chapters 5 and 6 and have been published in (Astudillo et al., 2013; Astudillo et al., 2010).

\subsection{Objective 3}

Determination of thermodynamic parameters associated with $\mathrm{O}_{2}$ photodissociation from $\mathrm{Mb}$ and $\mathrm{Ngb}$ and $\mathrm{CO}$ photo-dissociation from $\mathrm{Hb}$ in the presence of heterotrophic effectors

Photoacoustic calorimetry was utilized to characterize thermodynamic parameters associated with oxygen photodissociation from $\mathrm{Mb}$ and human $\mathrm{Ngb}$ to obtain a better description of how these proteins discriminate between different exogenous ligands, such as $\mathrm{CO}$ and $\mathrm{O}_{2}$. Although $\mathrm{O}_{2}$ is considered the physiological ligand for these proteins, $\mathrm{O}_{2}$

photodissociation from $\mathrm{Mb}$ and $\mathrm{Ngb}$ has not been previously investigated. In addition, thermodynamic profiles of $\mathrm{CO}$ photodissociation from $\mathrm{Hb}$ in the presence of heterotrophic allosteric effector bezafibrate and inositol hexakishexaphosphate were constructed and compared with that of $\mathrm{Hb}$ in the absence of allosteric effectors. The results of this part of the study are described in chapter 7 and partially published in (Miksovska and Astudillo, 2011). 


\subsection{Objective 4}

Characterization of cytoglobin interactions with the fluorescent probe 1anilinonaphthalene-8-sulfonate and sodium oleate.

Crystallographic data and sequence homology indicates that cytoglobin contains extended $\mathrm{N}$ - and C-termini that may be involved in association of the protein with intracellular partners such as other proteins or small molecules. A recent study shows that Cygb associates with the lipids oleic acid and cardiolipin, which suggests a possible role of Cygb in lipid transport (Reeder et al., 2011).

To provide insight into Cygb interactions with lipids, the fluorescence probe 1anilinonaphthalene-8-sulfonate $(1,8$-ANS) was used as a spectral tool to characterize the interactions of the protein with a hydrophobic molecule. In addition, replacement studies of ANS-protein complexes have been previously used to characterize binding of fatty acids with proteins. In this study, steady-state and time-resolved fluorescence spectroscopy were employed to characterize the association of Cygb with the dye 1,8ANS, as well as the interactions of the protein with fatty acids such as oleic acid through replacement studies of ANS-protein complexes. To determine the thermodynamic parameters associated with binding of the 1,8-ANS probe to Cygb, isothermal titration calorimetry was employed to characterize the interaction of 1,8-ANS with Cygb. The impact of the disulfide bond and exogenous ligands on Cygb was probed by characterizing the protein reduced with DTT and the cyanide adduct of Cygb. In addition, molecular docking simulations were used to complement the experimental data and obtain structural information about the sites where 1,8-ANS and oleate bind to Cygb. The results of this part of the study are described in chapter 8 . 


\subsection{Objective 5}

Characterization of guanidine hydrochloride induced unfolding of hexacoordinate vertebrate globins

To obtain insight into the stability of Cygb and $\mathrm{Ngb}$, chemical unfolding of these proteins was studied using the chaotropic agent guanidine hydrochloride. The impact of the disulfide bond and the binding of exogenous ligands on the stability of the proteins were also investigated by characterizing the protein reduced with DTT and the cyanide adduct of the proteins. The spectral changes of the Soret band upon addition of $\mathrm{GuHCl}$ were monitored using steady-state absorption spectroscopy in the visible region of the spectrum, whereas the changes in the secondary structure of the proteins were monitored using far-UV circular dichorism spectroscopy. The results of this part of the study are described in chapter 9. 


\section{MATERIALS AND METHODS}

\subsection{Materials}

The $\mathrm{Fe}^{3+}$ tetrakis(4-sulfonatophenyl)phorphine [Fe(III)4SP] was purchased from Frontier Scientific Inc. Myoglobin, hemoglobin, inositol hexakisphosphate (IHP), bezafibrate (BZF), dithiothreitol (DTT), potassium cyanide $(\mathrm{KCN})$, and $5-\delta$ aminolevulenic acid were purchased from Sigma-Aldrich. All other reagents were purchased from Fisher Scientific. All reagents were used as received.

\subsection{Methods}

\subsubsection{Protein expression and purification}

Transformed cells containing plasmids for wild type human Ngb, rat Ngb, human Cygb and mutants were kindly provided by Dr. Pierre Sebban (Paris Sud University, Orsay, France). The coding sequence of Ngb and Cygb with a six-His tag at the Nterminus was cloned into a pET15b expression vector. The DNA sequence of the recombinant plasmid product was confirmed through DNA sequencing. The QuickChange site-directed mutagenesis method (Stratagene) was employed to introduce mutations into the $\mathrm{Ngb}$ and Cygb coding sequence. The expression vectors were then transformed into Escherichia coli strain BL21.

\subsubsection{Neuroglobin isolation and purification}

Transformed Ngb cells were grown in $50 \mathrm{~mL}$ of Luria-Bertani medium supplemented with $100 \mathrm{mg} \mathrm{L}{ }^{-1}$ of ampicillin for $8 \mathrm{~h}$ at $37{ }^{\circ} \mathrm{C}$. Subsequently, $10 \mathrm{~mL}$ of culture were transferred into $1 \mathrm{~L}$ of Terrific Broth medium supplemented with $100 \mathrm{mg} \mathrm{L}^{-1}$ of ampicillin and $170 \mathrm{mg} \mathrm{L}^{-1}$ of 5-aminolevulenic acid. Cells were incubated at $37^{\circ} \mathrm{C}$ and $200 \mathrm{rpm}$ until the absorbance at $600 \mathrm{~nm}\left(\mathrm{~A}_{600}\right)$ reached 1.1. The culture was induced by 
addition of isopropyl $\beta$-D-1-thiogalactopyranoside to a final concentration of $0.4 \mathrm{mM}$. Cells were then incubated overnight at $30{ }^{\circ} \mathrm{C}$, collected by centrifugation for $10 \mathrm{~min}$ at $5000 \mathrm{rpm}(2700 \mathrm{~g})$ and $4{ }^{\circ} \mathrm{C}$ (Allegra 64R, Beckman Coulter), and homogenized in 50 $\mathrm{mM}$ Tris buffer and $5 \mathrm{mM}$ DTT $(\mathrm{pH} 8.0)$. The suspension was sonicated with a sonic dismembrator (model 100, Fisher Scientific) and centrifuged at $24000 \mathrm{~g}$ to remove membrane debris. The supernatant was filtered through a $0.2 \mu \mathrm{m}$ membrane and loaded into a Ni-NTA column (Qiagen) that was previously equilibrated with $10 \mathrm{mM}$ Tris buffer (pH 8.0). The column was then washed with $10 \mathrm{mM}$ Tris buffer $(\mathrm{pH} 8.0)$ containing increasing concentrations of imidazole $(5,10,15$ and $20 \mathrm{mM})$ until the absorbance at 280 $\mathrm{nm}$ was less than 0.03 . Neuroglobin was eluted with $10 \mathrm{mM}$ Tris buffer $(\mathrm{pH} 8.0)$ containing $40 \mathrm{mM}$ imidazole. Fractions with an $\mathrm{A}_{\text {soret }} / \mathrm{A}_{280}$ ratio higher than 3.0 were collected, concentrated using Amicon Millipore concentrators, and dialyzed overnight against $50 \mathrm{mM}$ Tris buffer ( $\mathrm{pH}$ 7.0). The purity of the protein was assessed using sodium dodecyl sulfate electrophoresis.

\subsubsection{Cytoglobin isolation and purification}

Transformed human Cygb cells were grown in $50 \mathrm{~mL}$ of Luria-Bertani medium

supplemented with $100 \mathrm{mg} \mathrm{L}^{-1}$ of ampicillin for $8 \mathrm{~h}$ at $37{ }^{\circ} \mathrm{C}$. Subsequently, $5 \mathrm{~mL}$ of culture were transferred into $300 \mathrm{~mL}$ of Terrific Broth medium supplemented with 100 $\mathrm{mg} \mathrm{L}^{-1}$ of ampicillin and $170 \mathrm{mg} \mathrm{L}^{-1}$ of 5 -aminolevulenic acid. Cells were incubated at $37^{\circ} \mathrm{C}$ and $250 \mathrm{rpm}$ until the absorbance at $600 \mathrm{~nm}\left(\mathrm{~A}_{600}\right)$ reached 1.1. The culture was induced by addition of isopropyl $\beta$-D-1-thiogalactopyranoside to a final concentration of $0.4 \mathrm{mM}$. Cells were then incubated overnight at $30^{\circ} \mathrm{C}$, collected by centrifugation for 10 min at $5000 \mathrm{rpm}(2700 \mathrm{~g})$ and $4{ }^{\circ} \mathrm{C}$ (Allegra 64R, Beckman Coulter), and homogenized in 
$50 \mathrm{mM}$ Tris buffer and $5 \mathrm{mM}$ DTT $(\mathrm{pH} 8.0)$. The suspension was sonicated with a sonic dismembrator (model 100, Fisher Scientific) and centrifuged at $24000 \mathrm{~g}$ to remove membrane debris. The supernatant was filtered through a $0.2 \mu \mathrm{m}$ membrane and loaded into a Ni-NTA column (Qiagen) that was previously equilibrated with $10 \mathrm{mM}$ Tris buffer (pH 8.0). The column was then washed with $10 \mathrm{mM}$ Tris buffer $(\mathrm{pH} 8.0)$ containing increasing concentrations of imidazole $(5,10,15$ and $20 \mathrm{mM})$ until the absorbance at 280 $\mathrm{nm}$ was less than 0.03. Cytoglobin was eluted with $10 \mathrm{mM}$ Tris buffer $(\mathrm{pH}$ 8.0) containing $40 \mathrm{mM}$ imidazole. Fractions with an $\mathrm{A}_{\text {Soret }} / \mathrm{A}_{280}$ ratio higher than 2.5 were collected, concentrated using Amicon Millipore concentrators, and dialyzed overnight against $50 \mathrm{mM}$ Tris buffer ( $\mathrm{pH}$ 7.0). The purity of the protein was assessed using sodium dodecyl sulfate electrophoresis.

\subsubsection{Sodium dodecyl sulfate electrophoresis}

Sodium dodecyl sulfate (SDS) electrophoresis was performed according to the procedure described by Gallagher (Gallagher, 2001). Electrophoresis gels were purchased

from Lonza (PAGEr ${ }^{\mathrm{TM}}$ Gold Precast gels). The composition of the running buffer is shown in Table 3.1. Protein samples for electrophoresis were prepared by mixing the protein solution with $20 \mu \mathrm{L}$ of treatment buffer (Table 3.2). Subsequently, the protein samples were boiled in a water bath for 5 minutes. 
Table 3.1 Composition of running buffer for electrophoresis

\begin{tabular}{ll}
\hline Components & Amount \\
\hline Tris-HCl buffer (pH 8.3) & $0.025 \mathrm{M}$ \\
Glycine & $0.192 \mathrm{M}$ \\
SDS & $0.1 \%$ \\
\hline
\end{tabular}

Table 3.2 Composition of sample treatment buffer

\begin{tabular}{ll}
\hline Components & Amount \\
\hline Tris-HCl buffer (pH 6.8) & $0.125 \mathrm{M}$ \\
SDS & $4 \%$ \\
Glycerol & $20 \%$ \\
Bromophenol blue & $0.02 \%$ \\
DTT & $0.2 \mathrm{M}$ \\
\hline
\end{tabular}

The gel was placed into the electrophoresis chamber. Subsequently, $5 \mu \mathrm{L}$ of treatment buffer containing the sample were loaded into each lane of the electrophoresis gel. Electrophoresis was carried out using a constant voltage of $125 \mathrm{~V}$ with varying current using a power supply (Model FB300, Fisher Scientific) until the protein samples reached the bottom of the gel. The gel was removed from the glass plates and stained using a Coomassie blue staining solution overnight. The gels were then de-stained by placing the gel into a de-staining solution for approximately 2 hours. The composition of the staining and the de-staining solution are listed in Tables 3.3 and 3.4, respectively. 
Table 3.3 Composition of Coomassie blue staining solution

\begin{tabular}{lc}
\hline Components & Amount \\
\hline Coomassie blue R-350 & $0.1 \%$ \\
Acetic acid & $10 \%$ \\
Methanol & $20 \%$ \\
Water & $69 \%$ \\
\hline
\end{tabular}

Table 3.4 Composition of de-staining solution

\begin{tabular}{lc}
\hline Components & Amount \\
\hline Acetic acid & $10 \%$ \\
Methanol & $50 \%$ \\
Water & $40 \%$ \\
\hline
\end{tabular}

\subsubsection{Transient absorption spectroscopy (TA) and photoacoustic calorimetry (PAC)}

\subsubsection{Sample preparation for TA and PAC measurements}

\subsection{Myoglobin}

Oxymyoglobin samples were prepared by dissolving the protein in $50 \mathrm{mM}$ HEPES buffer $\mathrm{pH}$ 7.0. $\mathrm{CO}$ bound $\mathrm{Mb}$ samples were prepared by dissolving the protein in $50 \mathrm{mM}$ Tris or HEPES buffer $\mathrm{pH}$ 7.0. The protein samples were placed into $0.2 \mathrm{~cm} \times 1.0$ $\mathrm{cm}$ or $0.5 \mathrm{~cm}$ x $1.0 \mathrm{~cm}$ quartz cuvettes, sealed with a septum cap, and purged with Ar for $10 \mathrm{~min}$. Then, the heme iron was reduced by addition of a freshly prepared solution of 
sodium dithionite. The quality of deoxymyoglobin (deoxyMb) was verified by UVvisible spectroscopy. Oxymyoglobin was obtained by bubbling air through the deoxyMb sample, whereas $\mathrm{CO}$ bound $\mathrm{Mb}$ was obtained by purging the deoxyMb sample with $\mathrm{CO}$ for approximately $5 \mathrm{~min}$. Formation of oxyMb and $\mathrm{CO}$ bound $\mathrm{Mb}$ samples were monitored using UV-visible absorption spectroscopy (single-beam spectrophotometer Cary 50, Varian). The concentration of the samples were calculated using an extinction coefficient $\left(\varepsilon_{408 \mathrm{~nm}}\right)$ of $188 \mathrm{mM}^{-1} \mathrm{~cm}^{-1}$ at $408 \mathrm{~nm}$ for the met form of $\mathrm{Mb}$ (Antonini and Brunori, 1971).

\subsection{Hemoglobin}

The $\mathrm{CO}$ bound hemoglobin sample was prepared by dissolving $\mathrm{Hb}$ in $100 \mathrm{mM}$ HEPES buffer $\mathrm{pH} 7.0$ into a $0.2 \mathrm{~cm} \times 1.0 \mathrm{~cm}$ or $0.5 \times 1.0 \mathrm{~cm}$ quartz cuvette. The concentration of allosteric effectors was $5 \mathrm{mM}$ for bezafibrate (BZF) and $1 \mathrm{mM}$ for inositol hexaphosphate (IHP). The sample was then sealed with a septum cap and purged with Ar for 10 min, reduced with a small amount of sodium dithionite to prepare deoxyhemoglobin (deoxyHb), and subsequently purged with $\mathrm{CO}$ for approximately 1 min. Preparation of $\mathrm{CO}-\mathrm{Hb}$ adducts was checked by UV-vis spectroscopy (Cary 50, Varian). The sample concentration was calculated using an extinction coefficient $\left(\varepsilon_{405 \mathrm{~nm}}\right)$ of $179 \mathrm{mM}^{-1} \mathrm{~cm}^{-1}$ at $405 \mathrm{~nm}$ for the met form of $\mathrm{Hb}$ (Antonini and Brunori, 1971).

\subsection{Neuroglobin and cytoglobin}

Neuroglobin and cytoglobin were prepared in $50 \mathrm{mM}$ Tris buffer ( $\mathrm{pH}$ 7.0). The CO bound ferrous samples were prepared by placing the samples into $0.5 \mathrm{~cm} \times 1.0 \mathrm{~cm}$ or $0.2 \mathrm{~cm} \times 1.0 \mathrm{~cm}$ quartz cuvettes sealed with a septum cap. Samples were then purged with Ar for approximately 10 min and reduced with a few microliters of freshly prepared 
$1 \mathrm{mM}$ sodium dithionate. Subsequently, the sample was purged with $\mathrm{CO}$, and the formation of the $\mathrm{CO}$ bound protein was verified by monitoring the $\mathrm{UV}$-visible absorption spectra (single-beam spectrophotometer, Cary 50, Varian). Protein concentrations were

calculated using an extinction coefficient at $532 \mathrm{~nm}\left(\varepsilon_{532}\right)$ of $10.7 \mathrm{mM}^{-1} \mathrm{~cm}^{-1}$ for the met form (Fago et al., 2004b).

\subsubsection{Transient absorption spectroscopy (TA)}

Transient absorption spectroscopy, also known as flash photolysis and timeresolved absorption spectroscopy, is a technique that consists in using a short laser pulse to trigger photochemical reactions and the subsequent detection of transient or short-lived intermediates (Chen et al., 1997). Time-dependent changes in the absorption spectra of chromophores can provide kinetic information to obtain insight into biological mechanisms, including the number of transient intermediates involved in a photo-induced reaction and the lifetime of each species. Therefore, fast events in biological systems, occurring on the nanosecond to millisecond time scale, such as bond cleavage and formation or structural relaxations may be monitored optically by the use of suitable TA instruments (Chen et al., 1997). The TA technique has been widely used to study kinetics of photochemical reactions, including biologically relevant photochemical processes such as electron transfer reactions in metalloproteins, kinetics of electron-transfer initiated protein folding, and ligand binding to heme proteins (Rosell and Mauk, 2011).

\subsection{TA theory}

Transient absorption spectroscopy relies on the use of a probe beam to monitor the absorbance of the sample before and after excitation with a short laser pulse. The excitation light source is used to photo-trigger a reaction, including bond cleavage, 
isomerization, or protein/peptide folding (Rosell and Mauk, 2011), which leads to changes in the absorption spectrum of the biomolecule under investigation. These protoinduced changes in the absorption spectrum are monitored with the probe source, which generally consists of a continuous wave (cw) light source such as a Xe lamp (Abbruzzetti et al., 2008).

In particular, TA has been extensively employed to study the kinetics of ligand binding to heme proteins because of the high sensitivity of their absorption spectra to the environment of the heme and the photo-lability of the Fe-ligand bond (Nienhaus and Nienhaus, 2005b). Transient absorption experiments performed on heme proteins consist in cleavage of the bond between the heme iron and the ligand with a short laser pulse and monitoring of ligand rebinding to the heme iron by measuring the light intensity (I(t)) at a particular wavelength as a function of time. The resulting changes in transmitted light from the probe beam, I $(t<0)$, and after laser excitation, $I(t)$, are related to the change in absorbance $(\triangle \mathrm{A})$ according to equation 3.1.

$$
\Delta A(t)=-\log \frac{I(t)}{I\left(t<t_{0}\right)}
$$

The wavelength of interest is usually located in the near-UV part of the spectrum since the Soret band of the heme, located in this region $(400-450 \mathrm{~nm})$, is sensitive to the ligation state of the heme iron and changes in its surroundings (Nienhaus and Nienhaus, 2005b). 


\subsection{TA instrumentation}

Kinetics of ligand rebinding to heme proteins in this study $(\mathrm{Mb}, \mathrm{Hb}, \mathrm{Ngb}$, and Cygb) on a micro- to millisecond time scale were observed using a home-built transient absorption instrument described previously (Belogortseva et al., 2007). A schematic representation of the TA instrumental set up is shown in Figure 3.1.

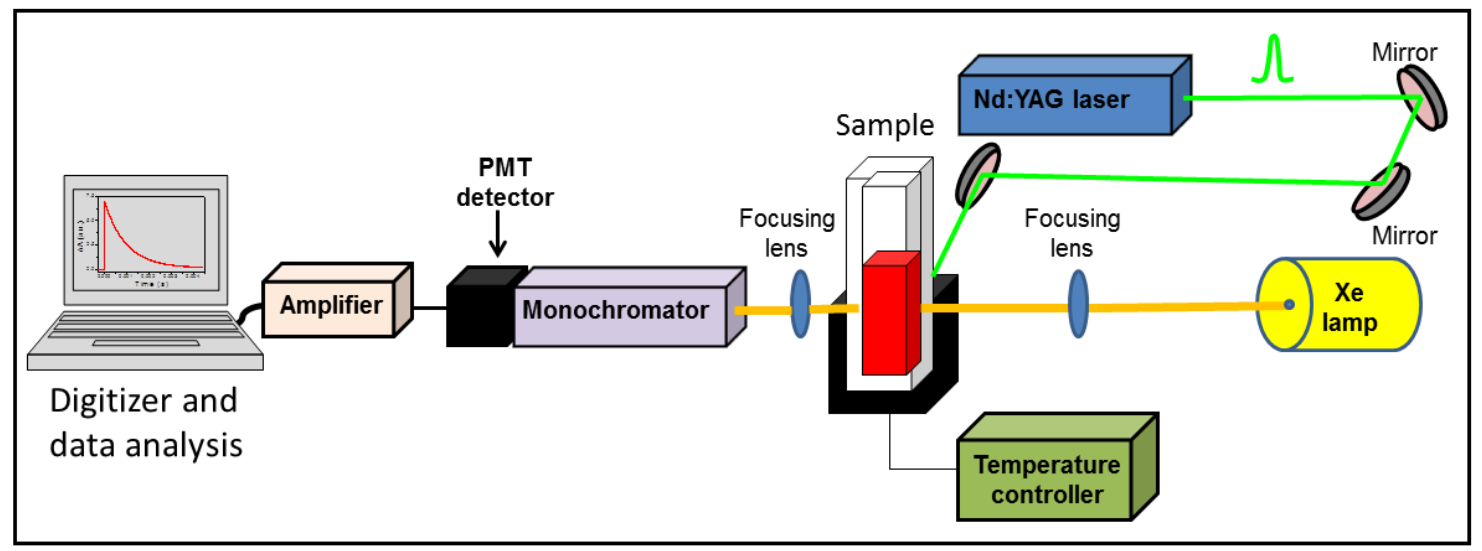

Figure 3.1 Schematic representation of home-built TA instrumental set-up.

The sample in an optical cell was placed into a temperature-controlled cell holder (Flash 300, Quantum Northwest), and the probe beam (output from a $200 \mathrm{~W}$ Xe arc lamp, Newport) was focused on the center of the cell. Ligand photo-dissociation was triggered using a $532 \mathrm{~nm}$ output from a Nd:YAG laser $(500 \mu \mathrm{J}, 7 \mathrm{~ns}$ pulse width, Minilite II, Continuum) operating at $1 \mathrm{~Hz}$ repetition rate. The emerging light from the probe beam was passed through a monochromator (Jovin Yvon), and the change in absorbance was detected at $440 \mathrm{~nm}$ for $\mathrm{Mb}$ and $436 \mathrm{~nm}$ for $\mathrm{Hb}, \mathrm{Ngb}$ and Cygb using an amplified photodiode (PDA 10A, Thornlabs) or a photodiode (model 818-BB-22, Newport) attached to an amplifier (C6438-01, Hamamatsu). The signals were subsequently stored in a $400 \mathrm{MHz}$ digitizer (Wave Surfer 42Xs, LeCroy). The energy of the laser pulse was 
kept below $30 \mu \mathrm{J}$ in the case of $\mathrm{HbCO}$ experiments to prevent photo-cleavage of more than one ligand at a time.

Submicrosecond CO rebinding kinetics was probed using a broadband pumpprobe transient absorption spectrometer (EOS, Ultrafast Systems, LLC, Sarasota, FL). These experiments were performed in Ultrafast Systems, Sarasota, FL. Photodissociation of CO was triggered using a Ti-sapphire laser ( $\sim 35$ fs pulse, Legend Elite, Coherent) with an output wavelength of $400 \mathrm{~nm}$. The change in absorbance was detected through a fiber optic cable coupled to a multichannel spectrometer with CMOS sensors $(1.5 \mathrm{~nm}$ resolution). TA traces were analyzed using Origin, version 8.0 (OriginLab Corp).

All transient absorption measurements were carried out on 20-40 $\mu \mathrm{M}$ samples in 50 or $100 \mathrm{mM}$ Tris or HEPES buffer, $\mathrm{pH} 7.0$, placed into a $2 \mathrm{~mm}$ path quartz cell.

\subsection{Quantum yield determination}

The quantum yield $(\Phi)$ for ligand binding to $\mathrm{Mb}$ and $\mathrm{Hb}$ was determined as described previously by Belogortseva et al. (2007). The quantum yield was determined by comparing the change in the sample absorbance at $440 \mathrm{~nm}$ with that of a reference of known quantum yield, according to equation 3.2. CO bound myoglobin was used as the reference since its quantum yield for bimolecular rebinding was previously reported by Henry et al. $\left(\Phi_{\text {ref }}=0.96\right)$ (Henry et al., 1983$)$.

$$
\Phi=\frac{\Delta A_{\text {sam }} \Delta \varepsilon_{\text {ref }} \Phi_{\text {ref }}}{\Delta A_{\text {ref }} \Delta \varepsilon_{\text {sam }}}
$$

where $\Delta \mathrm{A}_{\text {sam }}$ and $\Delta \mathrm{A}_{\text {ref }}$ are the absorbance change of the sample and reference at $440 \mathrm{~nm}$, respectively, and $\Delta \varepsilon_{\text {sam }}$ and $\Delta \varepsilon_{\text {ref }}$ are the change in extinction coefficient between 
the $\mathrm{CO}$ bound and reduced form of the sample and the reference, respectively, determined from the UV-visible steady-state absorption spectra.

In the case of ligand rebinding to $\mathrm{Ngb}$ and $\mathrm{Cygb}$, the quantum yield was calculated from the ratio of the amplitude of the geminate and bimolecular rebinding.

\subsection{Ligand rebinding and data analysis}

Nanosecond kinetics associated with $\mathrm{CO}$ rebinding to $\mathrm{Ngb}$ and $\mathrm{Cygb}$ were determined by fitting the change in absorbance $(\Delta \mathrm{A})$ trace at $436 \mathrm{~nm}$ using a multiexponential decays model (Equation 3.3).

$$
\Delta A=\sum_{i} A_{i} e^{\frac{t}{\tau_{i}}}
$$

where $\tau_{\mathrm{i}}$ correspond to the lifetime and and $\mathrm{A}_{\mathrm{i}}$ refers to the amplitude for each kinetic step, respectively.

The best fit was obtained using a four exponential decay model. The quality of the fit was evaluated by comparing the residuals retrieved by the fit as well as visual inspection of how well the calculated trace fitted the experimental trace for each measurement. To obtain the error associated to rate constants, kinetic traces at different wavelengths were fitted to the same model and the average and standard deviation of 5 or more values were calculated.

In addition to the exponential decay model, the maximum entropy method (MEM) was employed to fit the kinetic traces. The MEM is a mathematical inversion method that has been applied to analyze data in diverse fields, including radioastronomy, neutron scattering, fluorescence and ligand binding (Gull and Daniell, 1978; Lavalette et al., 1991; Livesey and Brochon, 1987; Steinbach, 1996). The basic idea of MEM is that a rate 
distribution $f(\lambda)$ is represented by a discrete set of data, $f\left(\lambda_{j}\right)$, and the uncertainty resulting from the specification of the function $f\left(\lambda_{j}\right)$ is measured by the Shannon-Jaynes entropy, $S$. The entropy $\mathrm{S}$ is maximized subject to constraining the statistic parameter $\chi^{2}$ to 1 (Figure 3.2). Therefore, the MEM is not based on a specific model and does not introduce any correlations into $f(\lambda)$. Consequently the rate distribution is directly retrieved from the experimental data (Steinbach et al., 1992; Steinbach et al., 2002).

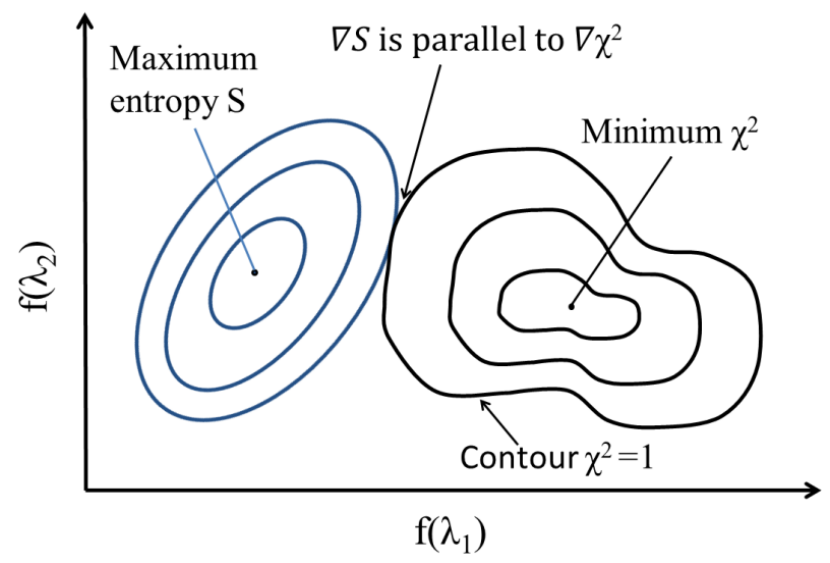

Figure 3.2 Contour plots of the entropy $\mathrm{S}$ and the statistic parameter $\chi^{2}$ for a two dimensional $f(\lambda)$. The maximum entropy solution corresponds to the point where the gradient of $\mathrm{S}$ is parallel to the gradient of $\chi^{2}$, where $\chi^{2}$ is close to 1 . Modified from Steinbach et al. (Steinbach et al., 1992).

The program MEMexp developed by Steinbach et al. (Steinbach et al., 2002) was employed to analyze the kinetics traces measured for $\mathrm{CO}$ rebinding to $\mathrm{Ngb}$ and $\mathrm{Cygb}$ with the MEM. The MemExp program uses the maximum entropy method (MEM) and either nonlinear least squares (NLS) or maximum likelihood (ML) fitting to analyze a general time-dependent signal in terms of distributed and discrete lifetimes (Steinbach, 2012; Steinbach et al., 2002). The program analyses kinetics using one or two distributions of effective $\log$-lifetimes, $\mathrm{g}(\log \tau)$ and $\mathrm{h}(\log \tau)$, according to equation 3.4. 


$$
F_{i}=D_{0} \int_{-\infty}^{+\infty} d \log \tau[g(\log \tau)-h(\log \tau)] e^{-t_{i} / \tau}+\sum_{k=0}^{3}\left(b_{k}-c_{k}\right)\left(\frac{t_{i}}{t_{\max }}\right)^{k}
$$

where $D_{0}$ corresponds to a normalization constant, the functions $g(\log \tau)$ and $\mathrm{h}(\log \tau)$ correspond to the distributions describing decaying and rising kinetics, respectively, and the polynomial function describes the experimental baseline. Coefficients of the baseline are scaled using the constant parameter $t_{\max }$ in order to maintain them comparable in magnitude. The constant $\mathrm{D}_{0}$ can be estimated from the experimental data, assuming that the temporal window of the measurements includes all kinetic processes involved.

The part of MEMexp program that uses the MEM is based on the Cornwell and Evans algorithm (Cornwell and Evans, 1985). Kinetic parameters are fitted to equation 3.4 by iteration through second order optimization of the entropy $\mathrm{S}$ (equation 3.5), in which the statistic parameter $\chi^{2}$ is constrained to unity (Steinbach et al., 2002).

$$
S(\mathbf{f}, \mathbf{F})=\sum_{j=1}^{M}\left[f_{j}-F_{j}-f_{j} \ln \frac{f_{j}}{F_{j}}\right]
$$

The $\chi^{2}$ statistic parameter measures the accuracy of the calculated data set $\left(\mathrm{F}_{\mathrm{i}}\right)$ to the experimental data set $\left(\mathrm{D}_{\mathrm{i}}\right)$, according to equation 3.6.

$$
\chi^{2}=\frac{1}{N} \sum_{i=1}^{N}\left(\frac{F_{i}-D_{i}}{\sigma_{i}}\right)^{2}
$$

Optimization of the fitting is then achieved by maximizing the function $\mathrm{Q}$ described by equation 3.7 .

$$
Q=S-\lambda \chi^{2}-\alpha I
$$


The parameters $\lambda$ and I represent two Lagrange multipliers. The iteration of the MEM calculation employs Newton-Raphson optimizations of the function Q by gradually adjusting the Lagrange multipliers $\lambda$ and I until the change in Q is very small (Steinbach et al., 2002). Upon completion of the iteration process, also referred to as the MEM convergence, the software MEMexp recommends a distributed and discrete fits as optimal descriptions of the kinetic process.

\subsection{Analysis of microscopic rate constants}

The microscopic rate constants associated with $\mathrm{CO}$ rebinding to penta-coordinate wild type $\mathrm{Cygb}\left(\mathrm{Cygb}^{\mathrm{ox}}\right)$ and $\mathrm{Cygb}$ with reduced $\mathrm{Cys}\left(\mathrm{Cybg}^{\text {red }}\right)$ were analyzed according to the two models described in chapter 6 (scheme 6.1). The differential equations corresponding to ligand migration in $\mathrm{Cygb}^{\mathrm{ox}}$ can be solved analytically as previously reported by Sottini et al. (2004) for hemoglobin and the microscopic rate constants can be calculated according to equations 3.8 to 3.13 . The overall geminate rebinding rate $\left(\mathrm{k}_{\mathrm{gem}}\right)$ can be described as:

$$
k_{\text {gem }}=k_{B A}+k_{B C}+k_{B D}
$$

Also, the geminate phase can be described in terms of the observed rates and amplitudes for each geminate step, according to equation 3.9.

$$
k_{\text {gem }}=\frac{A_{\text {gem } 1} k_{\text {gem } 1}{ }^{2}+A_{\text {gem } 2} k_{\text {gem } 2}{ }^{2}}{k_{B A}}
$$

Microscopic rate constant $\mathrm{k}_{\mathrm{BA}}, \mathrm{k}_{\mathrm{CB}}, \mathrm{k}_{\mathrm{BC}}$, and $\mathrm{k}_{\mathrm{BD}}$ were calculated according to the following equations:

$$
k_{B A}=A_{g e m 1} k_{g e m 1}+A_{g e m 2} k_{g e m 2}
$$




$$
\begin{aligned}
& k_{C B}=k_{\text {gem } 1}+k_{\text {gem } 2}-k_{\text {gem }} \\
& k_{B C}=k_{\text {gem }}-k_{B A}-k_{B D} \\
& k_{B D}=\frac{A_{\text {bim }} k_{B A}}{1-A_{\text {bim }}}
\end{aligned}
$$

where $\mathrm{A}_{\mathrm{gem} 1}$ and $\mathrm{A}_{\mathrm{gem} 2}$ correspond to the amplitude of the geminate phase 1 and 2, respectively, whereas $A_{b i m}$ represent the amplitude of the bimolecular phase with the major population in Cygb $\left(\mathrm{A}_{\text {bim1 } 1}\right)$. Parameters $\mathrm{k}_{\mathrm{gem} 1}$ and $\mathrm{k}_{\mathrm{gem} 2}$ are the observed rate constants for geminate phase 1 and 2 , respectively, and $\mathrm{k}_{\mathrm{bim}}$ is the observed overall bimolecular rate constant.

The CO rebinding rate can be determined using the steady-state approximation since the concentration of the protein in the intermediate state B can be considered constant. Then, the microscopic rate constant $\mathrm{k}_{\mathrm{DB}}$ can be calculated from equation 3.14.

$$
k_{D B}[C O]=\frac{k_{b i m} k_{C B}\left(k_{g e m}-k_{B C}\right)-k_{b i m}{ }^{2} k_{g e m}}{k_{C B} k_{B A}-\left(k_{g e m}-k_{B D}\right) k_{\text {bim }}}
$$

The concentration of $\mathrm{CO}$ under our experimental conditions was $1 \mathrm{mM}$.

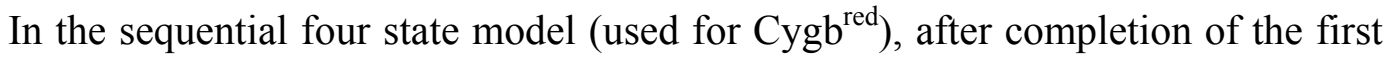
geminate phase, the fractional amount of absorbance change associated with escape of the ligand to the solvent, $\mathrm{f}_{\mathrm{gem} 2}$, can be described as shown below.

$$
f_{g 2}=\frac{1-A_{g e m 1}-A_{g e m 2}}{1-A_{g e m 1}}
$$

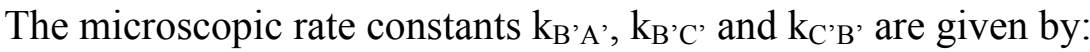

$$
k_{B^{\prime} A^{\prime}}=k_{\text {geml }}\left(1-\frac{\Phi_{\text {gem }}}{f_{\text {gem2 } 2}}\right)
$$




$$
\begin{aligned}
k_{B^{\prime} C^{\prime}} & =\frac{k_{B^{\prime} A^{\prime}} \Phi_{g e m}}{1-\Phi_{g e m}} \\
k_{C^{\prime} B^{\prime}} & =\frac{k_{g e m 2}\left(1-f_{g e m 2}\right)}{1-\frac{\Phi_{g e m}}{f_{g e m 2}}}
\end{aligned}
$$

The microscopic rate constants associated with ligand escape from state $C^{\prime}$ to state D' as well as ligand rebinding from state D' and C' were calculated for each conformation ("accessible" and "restricted") as described in the following expressions:

$$
\begin{aligned}
& k^{a c c}{ }_{C^{\prime} D^{\prime}}=k_{C^{\prime} B^{\prime}}\left(\frac{1}{A_{b i m 1}}-1\right) \\
& k^{r e s t}{ }_{C^{\prime} D^{\prime}}=k_{C^{\prime} B^{\prime}}\left(\frac{1}{A_{b i m 2}}-1\right)
\end{aligned}
$$

In this sequential model the bimolecular rate constant can be described as the product of the geminate quantum yield and the microscopic bimolecular rebinding rate (Scott et al., 2001), according to equation 3.21.

$$
k_{\text {bim }}=k_{D^{\prime} C^{\prime}} \Phi_{g e m}
$$

where the overall geminate quantum yield can be described in terms of the microscopic rate constants as:

$$
\Phi_{g e m}=\frac{k_{B^{\prime} A^{\prime}} k_{C^{\prime} D^{\prime}}}{k_{B^{\prime} A^{\prime}} k_{C^{\prime} D^{\prime}}+k_{C^{\prime} B^{\prime}} k_{B^{\prime} C^{\prime}}+k_{B^{\prime} A^{\prime}} k_{B^{\prime} C^{\prime}}}
$$

Then, the microscopic rate constant associated with ligand rebinding from state $\mathrm{D}$ ' to state C' for the "accessible conformation" ( $\left(\mathrm{k}^{\mathrm{acc}} \mathrm{D}^{\prime} \mathrm{C}\right.$ ') and "restricted conformation" $\left(\mathrm{k}^{\text {rest }}{ }^{\prime} \mathrm{C}^{\prime}\right)$ can be calculated using equations 3.23 and 3.24, respectively. 


$$
\begin{aligned}
& k_{D^{\prime} C^{\prime}}^{a c c}=\frac{k_{b i m 1}}{\Phi_{g e m}} \\
& k^{r e s t} D^{\prime} C^{\prime}=\frac{k_{b i m 2}}{\Phi_{g e m}}
\end{aligned}
$$

\subsubsection{Photoacoustic calorimetry (PAC)}

In order to fully understand the mechanism of a reaction it is not just necessary to determine the kinetics of the reaction but also the thermodynamic parameters associated with the reaction. Thermodynamic parameters reveal the energy levels of reactants and products, as well as the driving forces that are necessary for the reaction to occur.

Investigation of conformational dynamics and energetics associated with biological processes that occur on physiologically relevant time scales is still a challenge in molecular biophysics (Vetromile et al., 2011). Although the high sensitivity of timeresolved optical methods allow probing structural changes in the vicinity of chromophores, which provide information about local dynamics occurring in picosecond to millisecond time scales, these techniques have limited ability to probe directly thermodynamic events in proteins (molar volumes, enthalpy changes) occurring on these time scales (Larsen and Miksovska, 2007; Vetromile et al., 2011). Photothermal methods such as photoacoustic calorimetry (PAC) are powerful techniques that have been used to determine the magnitude of reaction volume and enthalpy changes as well as the time scale of conformational changes occurring on physiologically relevant time scales in proteins (Gensch and Viappiani, 2003; Larsen and Miksovska, 2007). PAC is used to monitor the magnitude and time profiles of overall reaction enthalpy and volume changes

associated with global conformational changes in proteins that are "optically silent" (do 
not affect directly the absorption spectrum of the chromophore) occurring between $\sim 50$ ns - $10 \mu$ s (Miksovska et al., 2008). Some examples of the use of PAC are in the study of conformational changes associated with ligand photo-release from heme proteins $(\mathrm{Mb}$, $\mathrm{Hb}$, FixL, Cyt P450, etc) and heme protein model complexes, as well as to probe the catalytic cycle of rhodopsin, bacteriorhodopsin and photosynthetic reaction centers (Braslavsky, 1986; Braslavsky and Heibel, 1992; Larsen and Miksovska, 2007; Peters et al., 1991; Vetromile et al., 2011).

\subsection{PAC theory}

The physical principle behind photothermal methods is that upon photo-excitation of a molecule there is a concomitant dissipation of excess energy through vibrational relaxation to the ground state that is accompanied by thermal heating of the surrounding solvent (Gensch and Viappiani, 2003; Larsen and Miksovska, 2007).

In the case of PAC, the rapid change in solution temperature results in changes in the solution volume of the illuminated cylinder (Gensch and Viappiani, 2003), as shown in Figure 3.3. 


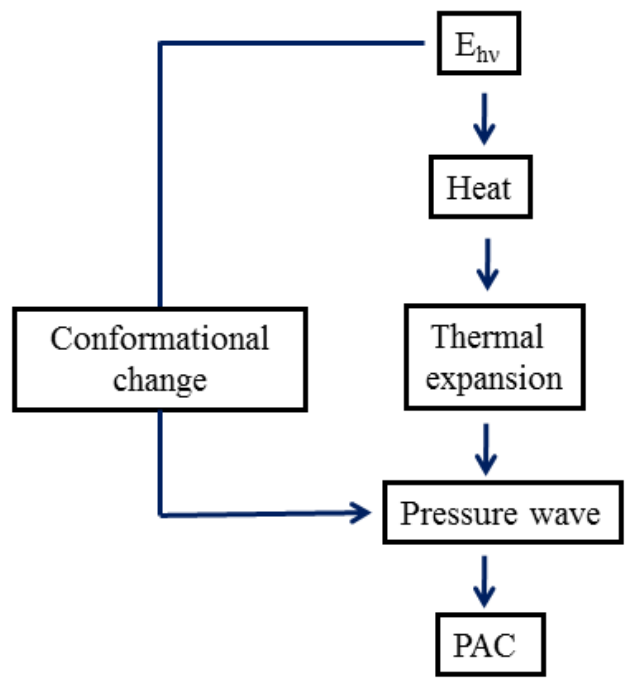

Figure 3.3 Schematic representation of the pressure change resulting from the heat release in photothermal methods. Modified from Gensch and Viappiani (Gensch \& Viappiani, 2003).

The mathematical expressions that describe the wave generation in PAC have been previously reviewed (Larsen and Miksovska, 2007). The change in pressure ( $\Delta \mathrm{P})$ at some point due to changes in solution volume can be described by equation 3.25.

$$
\Delta P=2 \pi f_{a} \nu_{a} \Delta x \rho
$$

where $f_{a}$ is the frequency of the sound wave, $v_{a}$ is the acoustic velocity, $\Delta x$ corresponds to the volume displacement in one dimension, and $\rho$ is the solvent density. The change in volume of a cylinder of radius $R$ and length $l$ that results from an adiabatic, isobaric expansion can be written as:

$$
\pi R^{2} l-\pi(R+\Delta R)^{2} l=\beta V \Delta T
$$

where $\beta$ corresponds to the volumetric expansion coefficient. The change in temperature in the illuminated area can be described as (Laman and Falvey, 1996; Larsen and Miksovska, 2007): 


$$
\Delta T=\frac{2 \alpha E_{a}}{\pi \rho C_{p} \hbar \omega}
$$

where $\alpha$ is the absorption coefficient, $E_{a}$ is the total energy per pulse, $C_{p}$ is the heat capacity of the solvent, $\hbar \omega$ is the energy per photon in the pump pulse. Then, substitution of equation 3.26 into equation 3.27 and assuming a point source of heat results in the following expressions:

$$
\begin{aligned}
& \pi R^{2} l-\pi(R+\Delta R)^{2} l=\beta V\left(\frac{2 \alpha E_{a}}{\pi \rho C_{p} \hbar \omega}\right) \\
& \pi R^{2} l-\pi\left(R^{2}+2 R \Delta R+\Delta R^{2}\right) l=\beta V\left(\frac{2 \alpha E_{a}}{\pi \rho C_{p} \hbar \omega}\right)
\end{aligned}
$$

Since $\mathrm{R}>>\Delta \mathrm{R}$ for a point source, then equation 3.12 can be reorganized to obtain $\Delta \mathrm{R}$.

$$
\Delta R=-\left(\frac{\beta}{C_{p} \rho}\right)\left(\frac{2 \alpha E_{a} R}{h \omega}\right)
$$

The $\Delta \mathrm{R}$ term in equation 3.30 is directly proportional to $\Delta x$ in equation 3.25 , and then the equation for $\Delta x$ can be described as shown below.

$$
\Delta x=B \Delta R
$$

where $B$ represents a proportionality constant between $\Delta x$ and $\Delta R$. Substitution of equations 3.30 and 3.31 into equation 3.25 results in an expression for the change in pressure $\Delta P$.

$$
P=B 2 f_{a} \nu_{a}\left(\frac{\beta}{C_{p}}\right)\left(\frac{2 \alpha E_{a} R}{h \omega}\right)
$$


For a fixed excitation system, the terms $f_{a}$ and $R$ can be included in the proportionality constant B', which results in equation 3.33 .

$$
P=B^{\prime}\left(\frac{v_{a} \beta}{C_{p}}\right)\left(\alpha E_{0}\right)
$$

where $E_{o}$ is the total energy of the excitation pulse normalized to the photon energy. The pressure change generates an acoustic wave in solution that is governed by the following wave equation:

$$
\nabla \Psi_{(r, t)}-\left(\frac{1}{v_{s}^{2}}\right) \frac{\partial^{2} \Psi_{(r, t)}}{\partial t^{2}}=-4 \pi h_{(r, t)}
$$

where $v_{\mathrm{s}}$ is the speed of sound in the medium, $\Psi(\mathrm{r}, \mathrm{t})$ is the wave amplitude at the observation coordinates $\mathrm{r}$ and $\mathrm{t}$, and $\mathrm{h}\left(\mathrm{r}^{\prime}, \mathrm{t}^{\prime}\right)$ is a heat source function. The solution to this equation is of the form:

$$
\Psi(r, t)=\frac{1}{4 \pi} \int d t^{\prime \prime} \int d r^{\prime} g\left(r, r^{\prime}, t, t^{\prime}\right) h\left(r, t^{\prime}\right)
$$

where $\mathrm{g}\left(\mathrm{r}, \mathrm{r}^{\prime}, \mathrm{t}, \mathrm{t}^{\prime}\right)$ is a Green's function that solves the wave equation for the given impulse heat function. At $r^{\prime}=0$ (point source) the impulse function can be written in terms of a heat function as:

$$
h\left(r^{\prime}, t^{\prime}\right)=\delta\left(r^{\prime}\right) f\left(t^{\prime}\right)
$$

where $\delta$ denotes the Dirac delta function and $f\left(t^{\prime}\right)$ describes the temporal behavior of the heat source. The solution to equation 3.35 gives the acoustic wave amplitude as:

$$
\Psi\left(r_{0}, t\right)=\frac{f\left(t-\frac{r_{0}}{v_{s}}\right)}{4 \pi r_{0}}
$$


where $\mathrm{r}_{0} / v_{\mathrm{s}}$ term is a propagation delay term and the $1 / \mathrm{r}_{0}$ is an energy conservation term associated with spherical emitters. For a single heat source transient with a lifetime $\tau, \mathrm{f}\left(\mathrm{t}^{\prime}\right)$ can be written as:

$$
f\left(t^{\prime}\right)=\frac{h_{0} e^{-t^{\prime} \tau}}{\tau} \vartheta\left(t^{\prime}\right)
$$

where $\vartheta\left(t^{\prime}\right)$ is a Heaviside unit step function. The wave amplitude can now be written as:

$$
\Psi\left(r_{0}, t\right)=\left(\frac{h_{0}}{4 \pi r_{0}}\right)\left(\frac{e^{-t^{\prime \prime} / \tau}}{\tau}\right) \vartheta\left(t^{\prime}\right)
$$

The acoustic wave described by $\Psi\left(\mathrm{r}_{0}, \mathrm{t}\right)$ represents the wave amplitude observed at the transducer. The transducer response to the wave amplitude is that of an under-damped oscillator with an impulse response given by:

$$
G\left(t, t^{\prime \prime}\right)=A \sin \left(v\left(t-t^{\prime}\right)\right) e^{-\left(t-t^{\prime \prime}\right) / \tau_{0}}
$$

with an amplitude A, a characteristic oscillation frequency $v$ and a relaxation time $\tau_{0}$. The transducer response (piezoelectric crystal) is found by the convolution of the impulse response G(t,t'), with the acoustic amplitude, $\Psi\left(\mathrm{r}_{0}, \mathrm{t}\right.$ ') giving

$$
V(t)=\int_{-\infty}^{t} G\left(t, t^{\prime \prime}\right) \Psi\left(r_{0}, t^{\prime \prime}\right) d t^{\prime \prime}
$$

Evaluation of equation 3.41 gives equation 3.42 for the transducer response.

$$
V(t)=\left(\frac{h_{0} A}{4 \pi r_{0}}\right)\left(\frac{v \tau}{1+v^{2} \tau^{2}}\right)\left\{e^{-t / \tau}-e^{-t / \tau_{0}}\left[\cos (v t)-\frac{1}{v \tau} \sin (v t)\right]\right\}
$$

where $\tau$ is the lifetime of the process in which the heat is evolved. Equation 3.42 can be used to model the relationship between the lifetime of the heat evolving process 
and the amplitude at the transducer. The maximum transducer amplitude can be determined for cases in which $\tau<<1 / \nu$ and for cases in which $\tau>>1 / \nu$.

$$
\begin{array}{ll}
V(t)=\left(\frac{h_{0} A}{4 \pi r_{0}}\right) e^{-t / \tau_{0}} & \text { For } \tau \nu<<1 \\
V(t)=\left(\frac{h_{0} A}{4 \pi r_{0}}\right)\left(\frac{1}{v \tau}\right) e^{-t / \tau}+e^{-t / \tau_{0}} & \text { For } \tau \nu>>1
\end{array}
$$

\subsection{PAC instrumentation}

The PAC instrumental setup has been described previously (Miksovska et al., 2008). A schematic representation of the PAC instrumental setup is shown in Figure 3.4. Samples were placed into a temperature controlled cuvette holder (Quantum Northwest). The $532 \mathrm{~nm}$ output from a Nd:YAG laser $(50 \mu \mathrm{J}, 7 \mathrm{~ns}$ pulse width, Minilite II, Continuum) was passed through a narrow slit $(100 \mu \mathrm{m})$ placed in front of the optical cell. A $1 \mathrm{MHz}$ acoustic detector (model RV103, Panametrics) that consists of a piezoelectric transducer was attached to the side of the cell using a thin layer of honey to ensure a tight connection between the cell and the transducer. A schematic representation of the wave propagation in PAC is shown in Figure 3.5. The acoustic signal was amplified using an ultrasonic amplifier (model 5662, Parametrics) and stored in a $400 \mathrm{MHz}$ digitizer (Wave Surfer $42 \mathrm{Xs}$, LeCroy). In order to improve the signal-to-noise ratio, 25-50 traces were averaged. Photoacoustic traces were then analyzed using Origin, version 8.0 (OriginLab Corp.). 


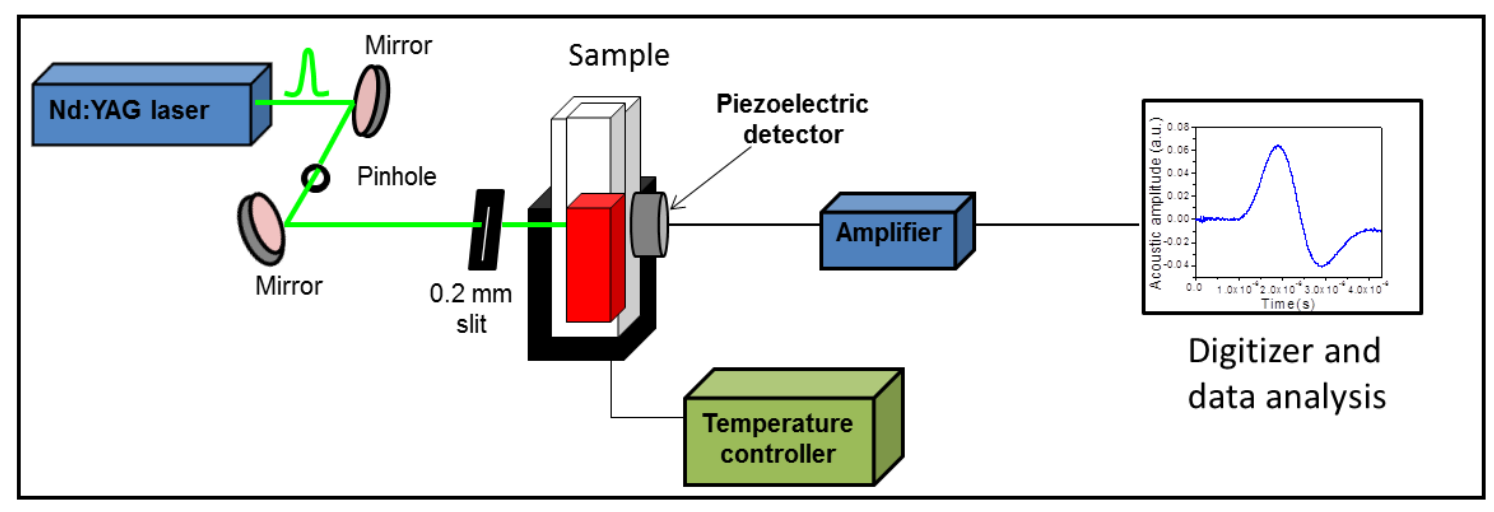

Figure 3.4 Schematic representation of home-built PAC instrumental set-up.

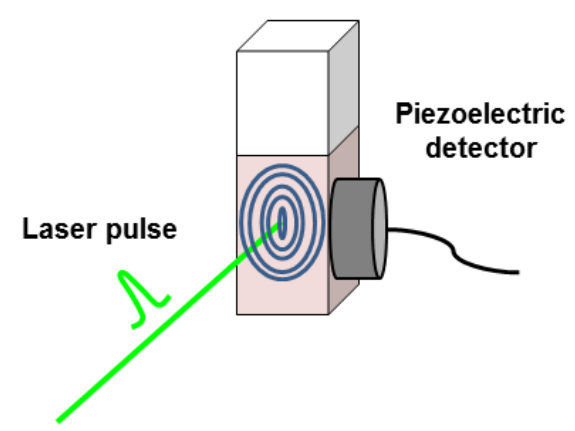

Figure 3.5 Schematic representation of wave propagation from the sample to the detector in PAC measurements.

\subsection{PAC data analysis}

The data analysis used for PAC has been previously reviewed (Gensch and Viappiani, 2003; Larsen and Miksovska, 2007). The amplitude of the acoustic signals for the sample (S) and reference compound (R) correspond to the difference between the first maximum and minimum of the acoustic wave in each case, as shown in Figure 3.6. 


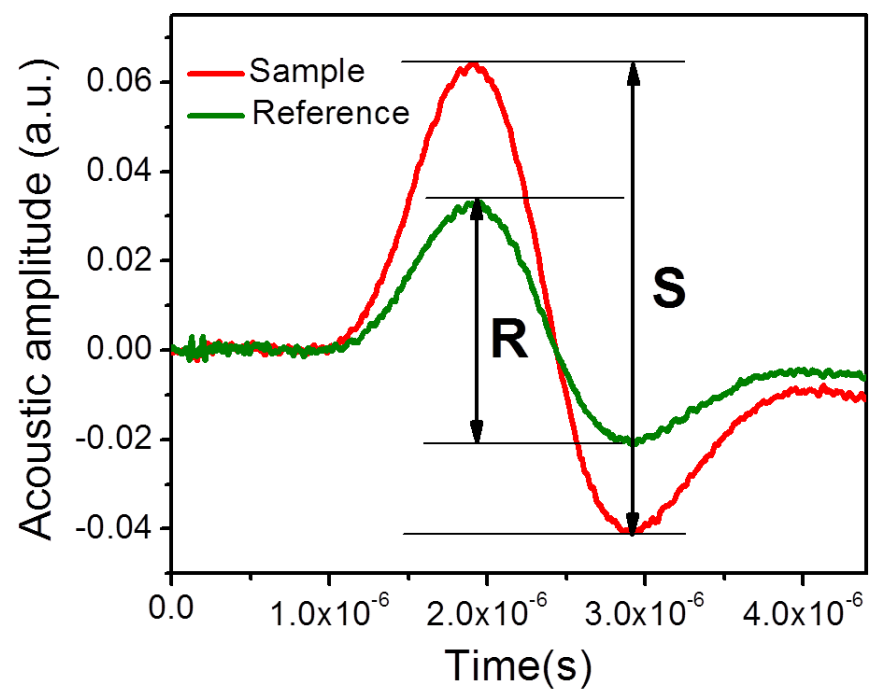

Figure 3.6 Representative PAC acoustic traces for sample and reference compound.

The amplitude of the sample acoustic signal can be described according to the following equation:

$$
S=K E_{a}\left(Q \frac{\beta}{C_{p} \rho}+\Delta V_{\text {nonthermal }}\right)
$$

where the $Q\left[\beta /\left(C_{p} \rho\right)\right]$ term corresponds to the thermal volume change ( $\left.\Delta V_{\text {thermal }}\right)$ and $\Delta V_{\text {nonthermal }}$ represents the volume change resulting from the photochemical processes such as cleavage of the Fe-CO bond, structural changes, solvation, and/or electrostriction. The thermal expansion coefficient, $\beta$, of water is temperature dependent and has a value of zero at $3.9^{\circ} \mathrm{C}$ (Peters et al., 1991). The terms $E_{a}$ and $K$ correspond to the number of Einsteins absorbed and the instrument response parameter, respectively. In order to calibrate the instrument and eliminate the instrument response parameter, the signal of a calorimetric reference was measured under identical conditions to those used for the sample. The reference compound, $\mathrm{Fe}(\mathrm{III}) 4 \mathrm{SP}$, does not undergo any photochemistry and 
releases absorbed energy into the surrounding solvent with a yield of unity (Abbruzzetti et al., 1999). The amplitude of the reference acoustic signal, $R$, can be then expressed as shown below.

$$
R=K E_{a} E_{h v}\left(\frac{\beta}{C_{p} \rho}\right)
$$

where $\mathrm{E}_{\mathrm{hv}}$ is the energy of the photon at the excitation wavelength.

In order to separate the contributions from the thermal and non-thermal volume changes to the acoustic signal, the acoustic traces were measured as a function of temperature (usually in the temperature range from $5{ }^{\circ} \mathrm{C}$ to $35^{\circ} \mathrm{C}$ ). By taking the ratio of the amplitude of the sample and reference acoustic waves and plotting them as a function of the temperature dependent term $\left[\left(C_{p} \rho\right) / \beta\right]$, according to equation 3.47 , the amount of heat released to the solution can be extrapolated from the intercept and the non-thermal volume change can be determined from the slope of the linear plot.

$$
\phi E_{h v}=\left(\frac{S}{R}\right) E_{h v}=Q+\left(\frac{C_{p} \rho}{\beta}\right) \Delta V_{\text {nonthermal }}
$$

For processes with quantum yield, $\Phi$, of less than 1, the reaction enthalpy $(\Delta H)$ and volume change $(\Delta V)$ are determined according to equations 3.48 and 3.49 , respectively.

$$
\begin{aligned}
& \Delta H=\frac{E_{h v}-Q}{\Phi} \\
& \Delta V=\frac{\Delta V_{\text {nonthermal }}}{\Phi}
\end{aligned}
$$

Since the piezoelectric transducer is not only sensitive to the amplitude of the acoustic wave but also to the temporal profile of the acoustic wave, reactions occurring 
within the time resolution of the PAC instrumentation (typically from approximately 50 ns to $10 \mu \mathrm{s})$ can be resolved. In this case, the acoustic trace is shifted in phase with respect to the reference traces as shown in Figure 3.7.

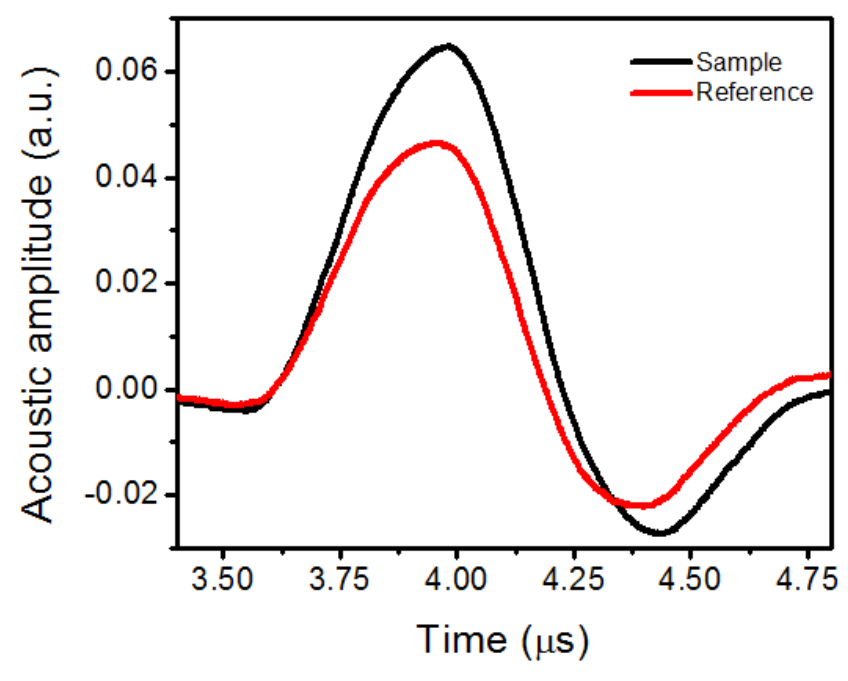

Figure 3.7 Representative PAC acoustic traces for sample and reference compound in which the sample acoustic trace is shifted in phase with respect to the reference trace.

The time-dependent voltage produced by the transducer, $\mathrm{E}(\mathrm{t})$, is the result of the convolution of a time-dependent heat source, $\mathrm{H}(\mathrm{t})$, with the instrument response function $\mathrm{T}(\mathrm{t})$

$$
E(t)=H(t) \otimes T(t)
$$

For a kinetic process that involves an intermediate with sequential decays $\mathrm{k}_{1}$ and $\mathrm{k}_{2}$, as shown in equation 3.51, the time dependence of the concentration for A and B can be described by equations 3.52 and 3.53 , respectively.

$$
A \stackrel{k_{1}}{\longrightarrow} B \stackrel{k_{2}}{\longrightarrow} C
$$




$$
\begin{aligned}
& {[A]=A_{0} e^{-\frac{t}{\tau_{1}}}} \\
& {[B]=\frac{A_{0} k_{1}}{k_{2}-k_{1}}\left(e^{-\frac{t}{\tau_{1}}}-e^{-\frac{t}{\tau_{2}}}\right)}
\end{aligned}
$$

Then, the time-dependent heat source can be expressed as described by equation 3.54 (Schaberle et al., 2010).

$$
H(t)=\phi_{1} e^{-\frac{t}{\tau_{1}}}+\frac{\phi_{2} k_{1}}{k_{2}-k_{1}}\left(e^{-\frac{t}{\tau_{1}}}-e^{-\frac{t}{\tau_{2}}}\right)
$$

where $\mathrm{k}=1 / \tau$. The values of $\phi_{1}$ and $\phi_{2}$, which relate to the enthalpy changes for kinetic processes 1 and 2 , and the associated relaxation times $\tau_{1}$ and $\tau_{2}$ are obtained by a deconvolution procedure. The program SoundAnalysis (Quantum Northwest) was employed to fit the PAC traces. The $\tau_{1}$ parameter was fixed at $1 \mathrm{~ns}$, indicating that the first kinetic process occurs within the laser pulse, while the remaining three parameters ( $\tau_{2}, \phi_{1}$ and $\left.\phi_{2}\right)$ were varied. This set of values is used to calculate $\mathrm{H}(\mathrm{t})$, which is then convoluted with the instrument response function $\mathrm{T}(\mathrm{t})$, obtained from a calibration compound, to generate a calculated experimental wave, $E_{\exp }(t)$. The accuracy of the fit is evaluated by comparing the calculated wave to the experimental wave and visual inspection of the residuals.

\subsubsection{Fluorescence spectroscopy}

Fluorescence spectroscopy is a widely used technique to study the structure and dynamics of proteins since the high sensitivity of this technique and the response of fluorescent probes to their microenvironment provide information about local fluctuations in the vicinity of the fluorophore (Lakowicz, 2006). In general, the 
characteristics of fluorescence, namely spectrum, quantum yield, and lifetime, are affected by any excited-state process involving interactions of the excited molecule with its close environment (Valeur, 2001).

Fluorescence studies of proteins usually rely on either intrinsic or extrinsic fluorescence. Intrinsic fluorescence of proteins results from fluorescent amino acids in the protein sequence (i.e., phenylalanine, tyrosine and tryptophan residues), whereas the extrinsic fluorescence relies on the use of fluorophores that are non-covalently associated with the protein as probes, such as 8-anilino-1-naphthalenesulphonic acid (1,8-ANS), porphyrins, nicotinamide adenine dinucleotide (NADH) or flavin adenine dinucleotide (FAD) (Ross and Jameson, 2008).

\subsubsection{Steady-state fluorescence spectroscopy}

Steady-state fluorescence emission spectra and polarization data were recorded using a PC1 fluorometer (ISS, Illinois). The excitation wavelength used for fluorescence studies of intrinsic Trp residues in Ngb wild type and single Trp mutants was $295 \mathrm{~nm}$. The Ngb samples were prepared by dissolving the protein stock solution in $50 \mathrm{mM}$ Tris buffer, $\mathrm{pH}$ 7.0, to a final concentration of $7 \mu \mathrm{M}$. Cyanide bound Ngb samples were

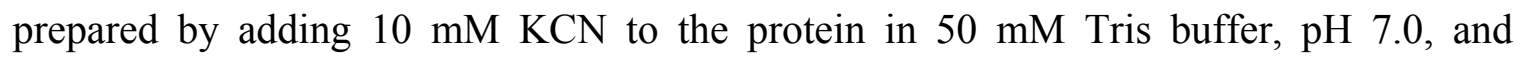
formation of the cyanide complexes was monitored using UV-visible spectroscopy.

In the study of binding of the extrinsic fluorescent probe 1,8-ANS to Cygb, the emission spectra was recorded using $20 \mu \mathrm{M}$ protein dissolved in $50 \mathrm{mM}$ Tris buffer, $\mathrm{pH}$ 7.0 , that was incubated with $40 \mu \mathrm{M}$ probe $(1,8-\mathrm{ANS})$ for 30 minutes at room temperature before the measurement. The wavelength used for excitation of 1,8-ANS was $350 \mathrm{~nm}$ and the emission was monitored in the range of $400 \mathrm{~nm}$ and $600 \mathrm{~nm}$. The concentration of 
1,8-ANS was determined using an extinction coefficient at $350 \mathrm{~nm}$ of $4950 \mathrm{mM}^{-1} \mathrm{~cm}^{-1}$ (Weber and Joung, 1964). Replacement studies of the hydrophobic probe 1,8-ANS with sodium oleate were performed by titration of Cygb-ANS complexes dissolved in 1 or 5 mM HEPES buffer, $\mathrm{pH} 7.0$, with $1 \mathrm{mM}$ sodium oleate solution. The Cygb-ANS complexes were incubated for 30 minutes at room temperature before titration with the sodium oleate solution.

\subsubsection{Time-resolved fluorescence spectroscopy}

\subsection{Theory}

The fluorescence decay time or fluorescence lifetime is one of the most important characteristics of a fluorescent molecule because it defines the time window at which dynamic phenomena can be observed (Valeur, 2001). Fluorescence lifetime measurements can therefore provide information about structural fluctuations as well as conformational heterogeneity in proteins since changes in fluorescence lifetimes can result from structural perturbations in the vicinity of intrinsic or extrinsic fluorescent probes (Lakowicz, 2006; Valeur, 2001).

In practice, fluorescence lifetimes are usually in the range of picoseconds to a few nanoseconds and can be measured using either the impulse or harmonic response method, also referred to as the time domain and frequency domain approach, respectively (Ross and Jameson, 2008). In the time domain approach the sample excited with a short laser pulse and the emission intensity is recorded as a function of time. In the frequency domain approach, a continuous light source is typically used to excite molecules, such as a CW laser or Xe lamp, and the intensity of this light is modulated sinusoidally at high frequencies $(\omega)$. As a consequence of the persistence of the excited state, fluorophores 
that are subjected to the excitation light produce a modulated emission that is shifted in phase $(\phi)$ with respect to the excited light and the amplitude of the emitted light is modulated as shown in Figure 3.8 (Lakowicz, 2006; Ross and Jameson, 2008).

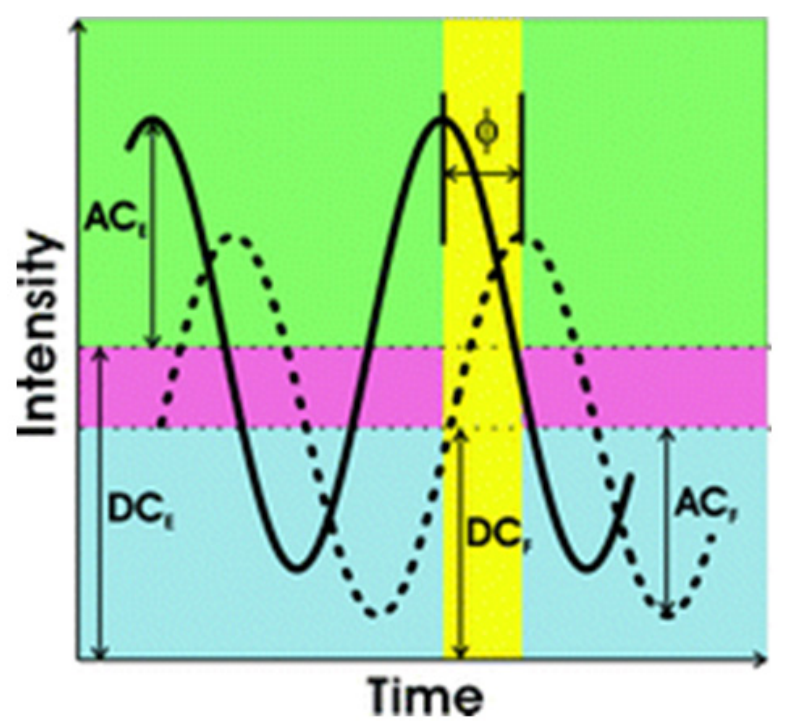

Figure 3.8 Light modulation in the frequency domain approach. The solid line represents the modulated excitation light, whereas the dotted line represents the emitted light with a phase shift with respect to the excitation light. AC and DC denotes the amplitudes of the sinusoidal wave, and the subscripts $\mathrm{E}$ and $\mathrm{F}$ refer to the excited and emitted light, respectively. (From Ross \& Jameson, 2008).

The excitation intensity and the fluorescence intensity are described by equations 3.55 and 3.56, respectively (Ross and Jameson, 2008).

$$
\begin{aligned}
& E(t)=E_{0}\left[1+M_{E} \sin (\omega t)\right] \\
& F(t)=F_{0}\left[1+M_{F} \sin (\omega t+\phi]\right.
\end{aligned}
$$

where $\mathrm{E}(\mathrm{t})$ and $\mathrm{E}_{0}$ are the excitation intensities, $\mathrm{F}(\mathrm{t})$ and $\mathrm{F}_{0}$ are the fluorescence intensities at time $\mathrm{t}$ and time zero, respectively, $\phi$ is the phase delay between the excitation and emission, $\mathrm{M}_{\mathrm{E}}$ is the modulation of the excitation, and $\mathrm{M}_{\mathrm{F}}$ is the modulation 
of the fluorescence. The angular modulation frequency, $\omega$, is proportional to the linear modulation frequency as described by equation 3.57 .

$$
\omega=2 \pi f
$$

In the case of fluorophores with a single lifetime, the lifetime can be directly calculated from the phase delay, $\phi$, according to equation 3.58 .

$$
\tau=\frac{\tan \phi}{\omega}
$$

Alternatively, the lifetime can also be calculated from the relative values of the emission modulation $\left(\mathrm{M}_{\mathrm{F}}\right)$ and excitation modulation $\left(\mathrm{M}_{\mathrm{E}}\right)$ that depend on the $\mathrm{AC}$ to $\mathrm{DC}$ components of the excitation and the emission as shown in equations 3.59 to 3.62 .

$$
\begin{aligned}
& M=\frac{M_{F}}{M_{E}} \\
& M_{E}=\left(\frac{A C_{E}}{D C_{E}}\right) \\
& M_{F}=\left(\frac{A C_{F}}{D C_{F}}\right) \\
& M=\frac{1}{\sqrt{1+(\omega \tau)^{2}}}
\end{aligned}
$$

The wave forms corresponding to the excitation and emission can also be described by the transforms of the impulse response function according to equations 3.63 and 3.64.

$$
N=\frac{\int_{0}^{\infty} I(t) \sin \omega t d t}{\int_{0}^{\infty} I(t) d t}
$$




$$
D=\frac{\int_{0}^{\infty} I(t) \cos \omega t d t}{\int_{0}^{\infty} I(t) d t}
$$

where $I(t)$ is the impulse response function at any time $t, N$ and $D$ correspond to the sine and cosine transforms of the impulse response function, respectively, and $\omega$ is the frequency.

In the case of a system with multiple lifetimes, the excited light has to be modulated over a wide range of frequencies and the corresponding phase shifts and modulation ratios have to be measured in order to characterize the multi-exponential decay. The sine and cosine transforms of the impulse response function for a multiexponential decay are described by equations 3.65 and 3.66 (Lakowicz et al., 1984).

$$
\begin{aligned}
& N . J=\sum_{i=1}^{n} \frac{\alpha_{i} \omega \tau_{i}{ }^{2}}{\left(1+\omega^{2} \tau_{i}^{2}\right)} \\
& D . J=\sum_{i}^{n} \frac{\alpha_{i} \tau_{i}}{\left(1+\omega^{2} \tau_{i}^{2}\right)}
\end{aligned}
$$

where $\alpha_{\mathrm{i}}$ corresponds to the pre-exponential factors, $\tau_{\mathrm{i}}$ represents the individual lifetimes and $\mathrm{J}$ is described as $J=\sum_{i} \alpha_{i} \tau_{i}$. The values for the phase delay and modulation ratios are calculated using equations 3.67 and 3.68 , respectively.

$$
\begin{aligned}
& \phi=\tan ^{-1}(N / D) \\
& M=\sqrt{N^{2}+D^{2}}
\end{aligned}
$$

The experimental data can be fitted using a suitable model, such as a sum of discrete exponential decays or Gaussian model, to determine the lifetimes and the corresponding pre-exponential factors. The parameters $\alpha_{\mathrm{i}}$ and $\tau_{\mathrm{i}}$ are varied to obtain the 
best fit between the experimental data and the calculated values, and the quality of the fit is then judged by the reduced chi-squared value $\left(\chi^{2}\right)$, which is given by:

$$
\chi^{2}=\sum \frac{\left[\left(P_{c}-P_{m}\right) / \sigma_{P}\right]^{2}+\left[\left(M_{c}-M_{m}\right) / \sigma_{M}\right]^{2}}{2 n-f-1}
$$

where $P$ and $M$ correspond to the phase and modulation data, respectively, the subscripts $\mathrm{c}$ and $\mathrm{m}$ refer to the calculated and measured values, and $\sigma_{\mathrm{P}}$ and $\sigma_{\mathrm{M}}$ are the standard deviations of each phase and modulation measurement, respectively. The denominator in equation 3.69 describes the degrees of freedom, where $n$ is the number of modulation frequencies and $f$ corresponds to the number of free parameters. The best fit between the calculated values and the experimental values are typically characterized by $\chi^{2}$ values close to 1 (Lakowicz et al., 1984).

In the frequency domain approach, the fluorescence data is typically described in terms of the contributions of various components to the emission or fractional intensities, $f_{i}$, which correspond to the contribution of the $\mathrm{i}^{\text {th }}$ component to the photocurrent. On the other hand, the pre-exponential factors are related to the actual number of fluorescing species being observed. The relationship between the pre-exponential terms, $\alpha_{\mathrm{i}}$, and the fractional intensities is given by the expression shown in equation 3.70 (Lakowicz et al., 1984; Ross and Jameson, 2008).

$$
f_{i}=\frac{\alpha_{i} \tau_{i}}{\sum_{j} \alpha_{j} \tau_{j}}
$$

\subsection{Sample preparation and measurements}

Frequency domain fluorescence measurements were performed using a Chronos FD fluorometer (ISS, Illinois). A $300 \mathrm{~nm}$ LED was used as the excitation light source for 
Trp excitation, whereas a $378 \mathrm{~nm}$ LED was used for 1,8-ANS measurements. The light source was modulated in the frequency range of 20 to $200 \mathrm{MHz}$, which allows resolution of lifetimes up to tens of picoseconds. P-terphenyl and 2,5-diphenyl oxazole compounds were used as standards to calibrate the instrument. The lifetime of P-terphenyl and 2,5diphenyl oxazole in ethanol have been reported previously to be 1.05 and $1.45 \mathrm{~ns}$, respectively (Boens et al., 2007). The concentration of the sample and the reference were adjusted to obtain identical integrated emission intensity at the detection wavelengths. Data analysis was performed using the Vinci software (ISS, Illinois), which allows to use single or multi-exponential decay models as well as lifetime distribution models (Gaussian, Lorentzian, etc.) to obtain appropriate fits. The quality of the fit was judged on the basis of the chi squared values and visual inspection of the fitting curve and corresponding residuals. Samples for lifetime measurements were prepared as previously described for the steady-state fluorescence measurements.

\subsubsection{Isothermal Titration Calorimetry (ITC)}

Dissociation constants and thermodynamic parameters associated with 1,8-ANS binding to Cygb were determined using ITC. Isothermal titration calorimetry measurements were performed by Mr. Khoa Pham (Chemistry and Biochemistry Department, Florida International University) on a VP-ITC titration calorimeter (MicroCal Inc., Northampton, MA). The stock Cygb solution used to prepare samples for ITC experiments was dialyzed against $50 \mathrm{mM}$ Tris buffer, $\mathrm{pH} 7.0$, overnight at $4{ }^{\circ} \mathrm{C}$. Cygb and 1,8-ANS samples were prepared using the same batch of dialysis buffer to minimize artifacts resulting from minor differences in buffer composition. Injections were started after stability of the baseline was achieved. The sample cell $(1.4 \mathrm{~mL})$ was 
loaded with $20 \mu \mathrm{M} \mathrm{Cygb}$ solution and the concentration of 1,8-ANS in the syringe (287 $\mu \mathrm{L}$ ) was $2 \mathrm{mM}$. Titration experiments consisted of 28 injections of $10 \mu \mathrm{L}$ aliquots of $1,8-$ ANS with 2 min intervals between injections. The temperature was kept constant at 25 ${ }^{\circ} \mathrm{C}$. The data were subsequently corrected by subtracting a small heat of diffusion determined in control experiments from the binding heat. The isotherm curves were analyzed using Origin software, version 7.0 (OriginLab Corp.) employing a sequential binding model.

\subsubsection{Stability studies}

Denaturation curves of $\mathrm{Ngb}$ and Cygb with guanidine hydrochloride were determined by monitoring the change in absorbance of the Soret band or the change in the ellipticity at $222 \mathrm{~nm}$ in the far-UV region of the CD spectra of the proteins in the presence of increasing concentrations of $\mathrm{GuHCl}$ from $0 \mathrm{M}$ to $6 \mathrm{M}$. Protein samples in the presence and absence of $\mathrm{GuHCl}$ were slowly mixed for $1 \mathrm{~h}$ at room temperature before measurements.

\subsubsection{UV-VIS Absorption spectroscopy}

Protein samples $(10 \mu \mathrm{M})$ were prepared by dissolving the protein in $50 \mathrm{mM}$ Tris buffer (pH 7.0). Samples were placed into $2 \mathrm{~mm}$ optical path length quartz cuvettes and the absorption spectra were measured with a single beam UV-vis spectrometer (Cary 50, Varian) in the $200 \mathrm{~nm}$ to $800 \mathrm{~nm}$ range. All spectra were corrected for the buffer baseline used in each experiment.

\subsubsection{Circular dichroism spectroscopy}

Circular dichroism (CD) measures the difference in absorption of left and right circularly polarized light by an optically active sample. The $\mathrm{CD}$ technique is a useful tool 
to examine the secondary structure of peptides and proteins because different types of polypeptide secondary structure have distinct type of CD spectra in the ultraviolet region corresponding to absorption by the peptide carbonyl groups: (i) far-UV CD spectra of random coils show a positive band at $212 \mathrm{~nm}$ that corresponds to the $\mathrm{n} \rightarrow \pi^{*}$ transition, and a negative band at $195 \mathrm{~nm}$ that is related to the $\pi \rightarrow \pi^{*}$ transition; (ii) far-UV CD spectra of $\beta$ sheets shows a characteristic negative band at $218 \mathrm{~nm}$ that corresponds to the $\pi \rightarrow \pi^{*}$ transition; and (iii) far-UV CD spectra of $\alpha$ helices display two bands at $208 \mathrm{~nm}$ and $222 \mathrm{~nm}$ that correspond to the $\pi \rightarrow \pi^{*}$ and $\mathrm{n} \rightarrow \pi^{*}$ transitions, respectively, as shown in Figure 3.8 (Ranjbar and Gill, 2009). Therefore, the amount of alpha helix, beta sheet, and other structures can be estimated from the CD spectrum of a protein, which is advantageous to monitor folding to unfolding transitions in proteins (Kelly and Price, 1997).

Circular dichroism spectra were measured with a J15 CD spectrometer (JASCO, USA). Protein samples $(40 \mu \mathrm{M})$ were prepared in $10 \mathrm{mM}$ Tris buffer $(\mathrm{pH} 7.0)$ and placed into $2 \mathrm{~mm}$ optical path length quartz cuvettes. The far-ultraviolet spectra were measured in the range of $190 \mathrm{~nm}$ to $300 \mathrm{~nm}$ and represent the average of at least 3 accumulations. Spectra were acquired at a scan speed of $100 \mathrm{~nm} \mathrm{~min}^{-1}$ with a response time of $1 \mathrm{~s}$ and the resolution of $1 \mathrm{~nm}$. All spectra were corrected for the buffer baseline used in each experiment. 


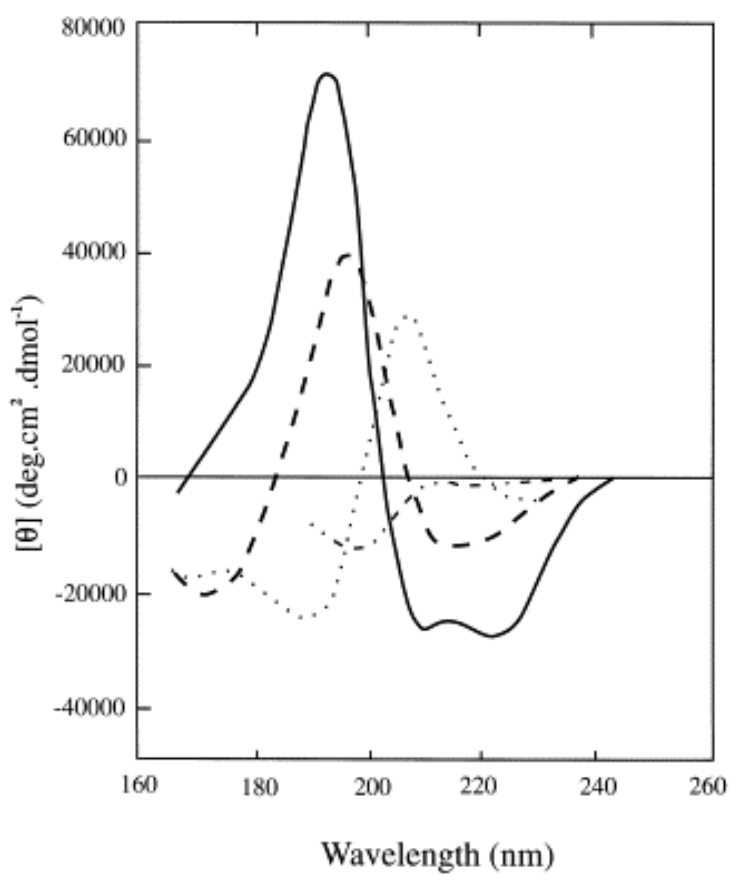

Figure 3.9 Far-UV Circular dichroism spectra associated with various types of secondary structure. The solid line represents $\alpha$ helices, the dashed line represents anti-parallel $\beta$ sheets, the dotted line and short dashes represent random coils and irregular structures. (Kelly and Price, 1997). 


\section{CONFORMATIONAL DYNAMICS IN HUMAN NEUROGLOBIN: EFFECT OF HIS 64, VAL 68 AND CYS 120 ON LIGAND MIGRATION}

(The work described in this chapter was published in Astudillo,L., Bernad, S., Derrien, V., Sebban, P., Miksovska, J. (2012) Biochemistry, 51, 9984-9994)

\subsection{Introduction}

Neuroglobin $(\mathrm{Ngb})$ is a small heme-containing globin, composed of 151 amino acid residues, that is mainly expressed in the brain and retina of vertebrates (Burmester et al., 2000). Over-expression of $\mathrm{Ngb}$ was observed in rat neurons exposed to hypoxia and focal ischemia conditions (Sun et al., 2001). An increased level of Ngb expression also promotes neuron survival after ischemic insults (Sun et al., 2001; Sun et al., 2003). The molecular basis of the neuroprotective function of $\mathrm{Ngb}$ remains unclear, but several plausible mechanisms have been proposed, including oxygen storage (Dewilde et al., 2001; Sun et al., 2001), oxygen/redox sensing (Wakasugi et al., 2003), decomposition of reactive oxygen species (Herold et al., 2004; Van Doorslaer et al., 2003), and inhibition of apoptosis (Brittain et al., 2010a; Fago et al., 2006). In addition, Wakasugi et al. (2003) reported that ferric $\mathrm{Ngb}$ binds to the GDP-bound form of the $\alpha$-subunit of the heterotrimeric $\mathrm{G}$ protein acting as a guanine nucleotide dissociation inhibitor and the interactions between $\mathrm{hNgb}$ and the G protein may promote cell survival (Wakasugi et al., 2011). Alternatively, Brittain et al. (2010a; 2010b) showed that in vivo Ngb inhibits apoptosis through the binding to cytochrome $\mathrm{c}$ and preventing pro-caspase 9 activation.

The three dimensional structure of Ngb can be nearly superimposed with that of myoglobin $(\mathrm{Mb})$, despite the low degree of sequence homology ( 30\%) (Figure 4.1$)$ (Arcovito et al., 2008; Pesce et al., 2003; Vallone et al., 2004a; Vallone et al., 2004b). 
Neuroglobin displays the classical 3-over-3 $\alpha$-helical globin fold with the heme iron being hexa-coordinated in both ferric and ferrous forms with residues His96 and His64 serving as endogenous ligands. The distal His64 can be readily replaced by diatomic ligands, including $\mathrm{O}_{2}, \mathrm{CO}$ and $\mathrm{NO}$ that reversibly bind to the heme iron (Dewilde et al., 2001; Trent et al., 2001; Van Doorslaer et al., 2003). The competition between the distal histidine and exogenous ligand leads to biphasic ligand binding kinetics, with His64 dissociation being the rate-limiting step (Dewilde et al., 2001).

In addition to hexa-coordination of the heme iron, other structural factors alter interactions of $\mathrm{Ngb}$ with diatomic ligands. An overlay of the crystal structure of the ligand free and CO-bound mouse $\mathrm{Ngb}(\mathrm{mNgb})$ reveals that the association of the ligand with the heme iron triggers reorganization of the heme distal pocket that is unique among vertebrate globins, including sliding of the heme group deeper into the distal cavity, reorganization of helix F, and alteration of C-D and E-F loop mobility (Vallone et al., 2004b). However, such heme displacement may be species-dependent as shown in computational studies (Bocahut et al., 2009; Nadra et al., 2008). 

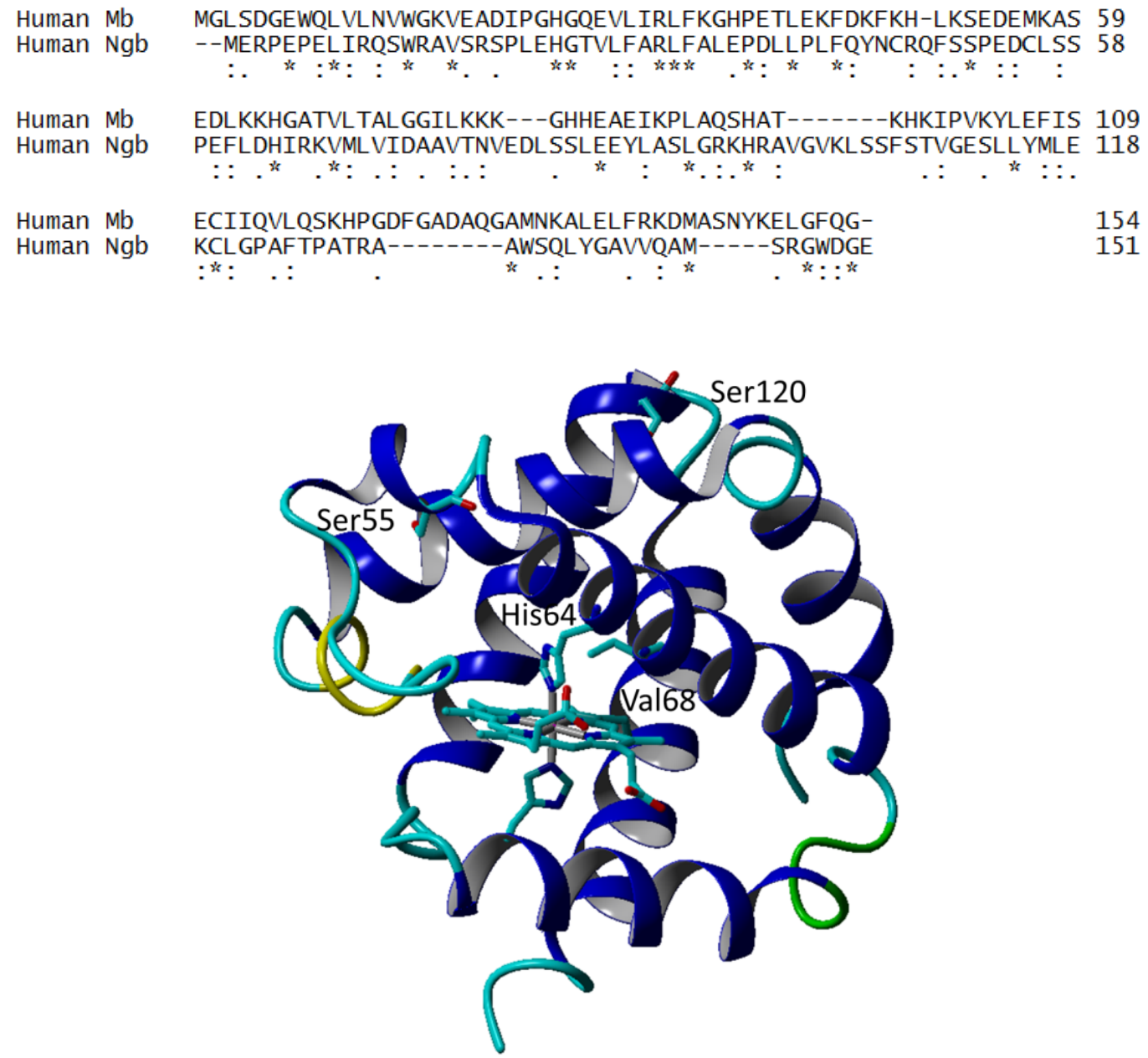

Figure 4.1 Top: Sequence alignment of human Ngb and human Mb. Conserved residues are represented by a star below the sequence, whereas residues that strongly share similar properties are represented by two dots and those exhibiting weak similarity are represented by one dot below the sequence. Bottom: Ribbon representation of ferric $\mathrm{hNgb}$ (PDB entry 1JO6, molecule A) depicting the position of the heme group, proximal His 96, distal His 64, Val 68, Ser 120, and Ser 55.

An intriguing structural feature of human $\mathrm{Ngb}(\mathrm{hNgb})$ is the presence of an internal disulfide bond between Cys46 and Cys55 that is missing in rat Ngb (rNgb) and mouse Ngb (mNgb). Hamdane et al. (2003) have demonstrated that the reduction of the internal disulfide bond or replacement of one of the Cys residues decreases the rate of dissociation of His64 from heme iron by a factor of 10, resulting in a decrease of the 
affinity of $\mathrm{hNgb}$ for $\mathrm{O}_{2}$. On the basis of these results, it was proposed that the interaction between $\mathrm{Ngb}$ and $\mathrm{O}_{2}$ may be coupled to the intracellular redox state through the rupture and/or formation of the internal disulfide bond (Hamdane et al., 2003). It was recently shown that the structural volume change associated with photo-dissociation of $\mathrm{CO}$ from $\mathrm{rNgb}$ or $\mathrm{hNgb}$ lacking the disulfide bond is approximately 3 times larger than that measured for wild-type hNgb, suggesting that the internal disulfide bond also impacts the structural dynamics associated with binding of $\mathrm{CO}$ to the five-coordinate $\mathrm{Fe}^{2+} \mathrm{Ngb}$ (see chapter 5) (Astudillo et al., 2010). The molecular mechanism of how structural changes in the C-D loop are relayed to the heme distal pocket remains unclear, although Phe28 was recently identified as a key residue in the communication between the C-D loop and the heme pocket (Ezhevskaya et al., 2011).

The crystal structures of $\mathrm{Fe}^{2+} \mathrm{mNgb}$ and $\mathrm{COmNgb}$ also show an extensive network of hydrophobic cavities that partially overlay with the Xe cavities identified in $\mathrm{Mb}$ (Moschetti et al., 2009). The volume and the spatial organization of this hydrophobic network depend on the heme iron coordination. Furthermore, the transition from the sixcoordinate CO-bound $\mathrm{Fe}^{2+} \mathrm{mNgb}$ to six-coordinate bishistidyl $\mathrm{Fe}^{2+} \mathrm{mNgb}$ is associated with an increase in the cavity volume and opening of a direct pathway between the heme binding pocket and surrounding solvent (Vallone et al., 2004b).

The physiological role of the hydrophobic cavities remains unclear; however, they may provide ligand migration pathways and/or temporary storage for diatomic gases. Indeed, experimental and theoretical studies have shown that ligand exchange between the heme binding pocket and surrounding solvent is significantly faster in Ngb than in Mb (Abbruzzetti et al., 2009; Anselmi et al., 2011; Astudillo et al., 2010). Anselmi et al. 
(2011) have proposed that the photo-released ligand escapes from the distal pocket through two hydrophobic cavities that are analogous to the $\mathrm{Xe}_{2}$ and $\mathrm{Xe}_{4}$ sites in $\mathrm{Mb}$ and with an estimated time for the escape of $\mathrm{CO}$ from the protein matrix of $25 \mathrm{~ns}$. These results are consistent with the results of previous transient absorption studies that report a rate constant for the release of the ligand from the protein matrix of $1.4 \times 10^{8} \mathrm{~s}^{-1}$ (Abbruzzetti et al., 2009), which is approximately 20 times faster than in $\mathrm{Fe}^{2+} \mathrm{Mb}(\mathrm{k}=5.3$ x $10^{6} \mathrm{~s}^{-1}$ ), and with results of photoacoustic calorimetry (PAC) studies indicating that the $\mathrm{CO}$ escapes into the surrounding solvent within $50 \mathrm{~ns}$ subsequent to photo-dissociation of the Fe-CO bond (Astudillo et al., 2010).

To provide a detailed insight into the mechanism of interaction of diatomic ligands with Ngb and the role of conserved distal pocket residues His64 and Val68 in the binding of the ligand to $\mathrm{hNgb}, \mathrm{PAC}$ and transient absorption (TA) spectroscopy were used to characterize the time-resolved thermodynamics and kinetics of $\mathrm{CO}$ photo-release and thermal binding to Ngb. Moreover, the impact of the two conserved cysteine residues, Cys 120 and Cys55, on ligand binding properties is examined. The Cys120 residue is located at the interface of helix G and the loop between helices A and B, whereas Cys55 is found within the flexible C-D loop and forms an internal disulfide bond with Cys46 in hNgb.

\subsection{Results}

\subsubsection{Circular dichroism spectroscopy}

The far-UV CD spectra of wild type hNgb and mutants His64Gln, Val68Phe, His64Gln/Val68Phe, Cys120Ser, and Cys55Ser/Cys120Ser hNgb in $10 \mathrm{mM}$ Tris buffer (pH 7.0) are shown in Figure 4.2. The CD spectrum of wild type hNgb exhibits two 
negative peaks at $208 \mathrm{~nm}$ and $222 \mathrm{~nm}$ that are characteristic of $\alpha$-helical structure, as previously reported for hNgb (Hamdane et al., 2005; Zhao et al., 2006). The $\alpha$-helical content $\left(f_{H}\right)$ was calculated using equation 4.1, according to Chen et al. (1972).

$$
f_{H}=\frac{\left.-[\theta]_{222 n m}+2340\right)}{30300}
$$

Where $[\theta]_{222 \mathrm{~nm}}$ represents the mean molar ellipticity at $222 \mathrm{~nm}$.

Analysis of the secondary structure of $\mathrm{Ngb}$ resulted in $\sim 76 \% \alpha$-helical content and matches well previously reported values of $78 \%$ of $\alpha$-helical content and $22 \%$ of other forms, which is comparable to those of other globins such as Mb (76\%) (Hamdane et al., 2005). The data in the $190 \mathrm{~nm}$ to $240 \mathrm{~nm}$ range were also uploaded into the K2D3 server (Louis-Jeune et al., 2012) to determine the secondary structure content and the following results were retrieved for the wild type protein: $\sim 66 \%$ of $\alpha$-helix and $\sim 3 \%$ of $\beta$-sheet. The CD spectra of Val68Phe, Cys120Ser, and Cys55Ser/Cys120Ser hNgb are similar to the $\mathrm{CD}$ spectrum of wild type $\mathrm{hNgb}$, hence these mutations do not affect $\mathrm{t}$ a large extent the secondary structure of the protein. On the other hand, a lower CD signal at $222 \mathrm{~nm}$ was observed for mutated proteins in which the distal His was replaced by Gln, hNgb His64Gln and hNgb His64Gln/Val68Phe, than the one observed for wild type $\mathrm{hNgb}$. The calculated $\alpha$-helical content of these mutated proteins was $\sim 72 \%$ and $\sim 74 \%$ for hNgb His64Gln and hNgb His64Gln/Val68Phe, respectively. These results indicate a small decrease of $\alpha$-helical content in these mutated proteins, likely as a result of destabilization of $\mathrm{hNgb}$ secondary structure upon substitution of the distal His. 


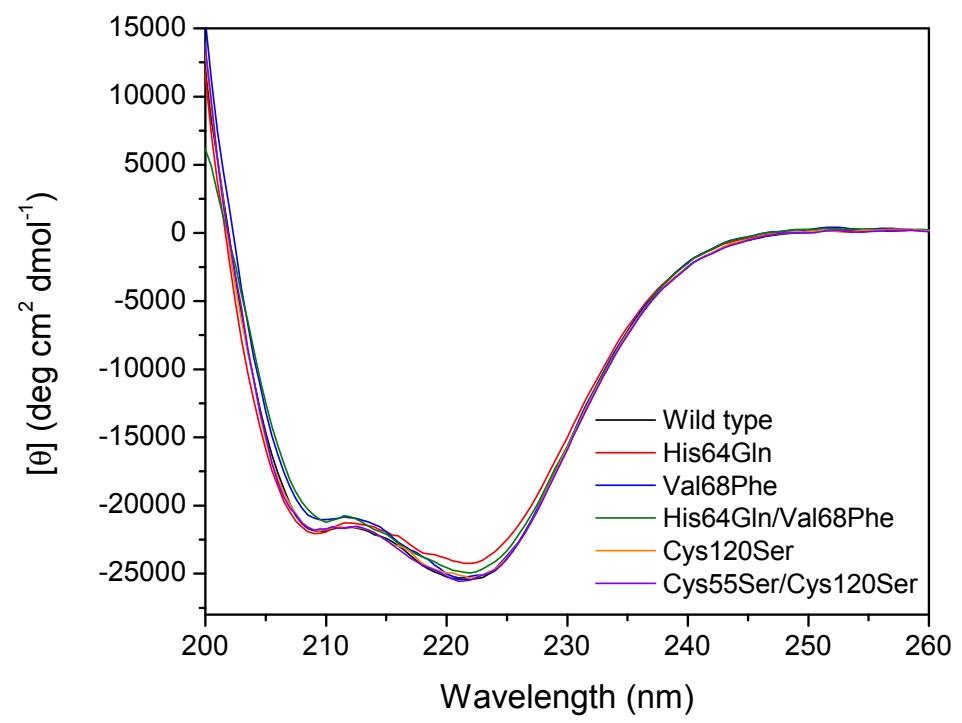

Figure 4.2 CD spectra of wild type $\mathrm{hNgb}$ and mutants His64Gln, Val68Phe, His64Gln/Val68Phe, Cys120Ser, and Cys55Ser/Cys120Ser in the far-UV region. The concentration of the protein was $40 \mu \mathrm{M}$ in $10 \mathrm{mM}$ Tris buffer (pH 7.0). Spectra were corrected for the baseline of the buffer.

\subsubsection{UV-visible spectroscopy}

The steady-state absorption spectra of ferric, deoxy, and CO bound wild type $\mathrm{hNgb}$ at $\mathrm{pH} 7.0$ are displayed in Figure 4.3. The ferric form of $\mathrm{hNgb}$ exhibits a Soret band at $413 \mathrm{~nm}$ and two visible bands at $535 \mathrm{~nm}$ and $561 \mathrm{~nm}$. Reduction of the heme iron results in a bathochromic shift of the Soret band to $424 \mathrm{~nm}$ and the two Q bands in the visible part of the spectrum shift to shorter wavelengths (529 and $559 \mathrm{~nm})$. The CO adduct of $\mathrm{hNgb}$ exhibits a Soret band at $417 \mathrm{~nm}$ and $\alpha$ and $\beta$ bands located at 560 and 537 $\mathrm{nm}$, respectively, consistent with the formation of low-spin six-coordinate heme iron. The absorbance maxima observed for $\mathrm{hNgb}$ are identical to those previously reported by Dewilde et al. (Dewilde et al., 2001) for the ferric, ferrous, and CO adduct of $\mathrm{hNgb}$, and 
these spectral features are characteristic of hexacoordinate hemoglobins, including cytochromes, indicating that $\mathrm{Ngb}$ is a hexa-coordinated globin in which the distal His 64 is the endogenous ligand (Dewilde et al. 2001).

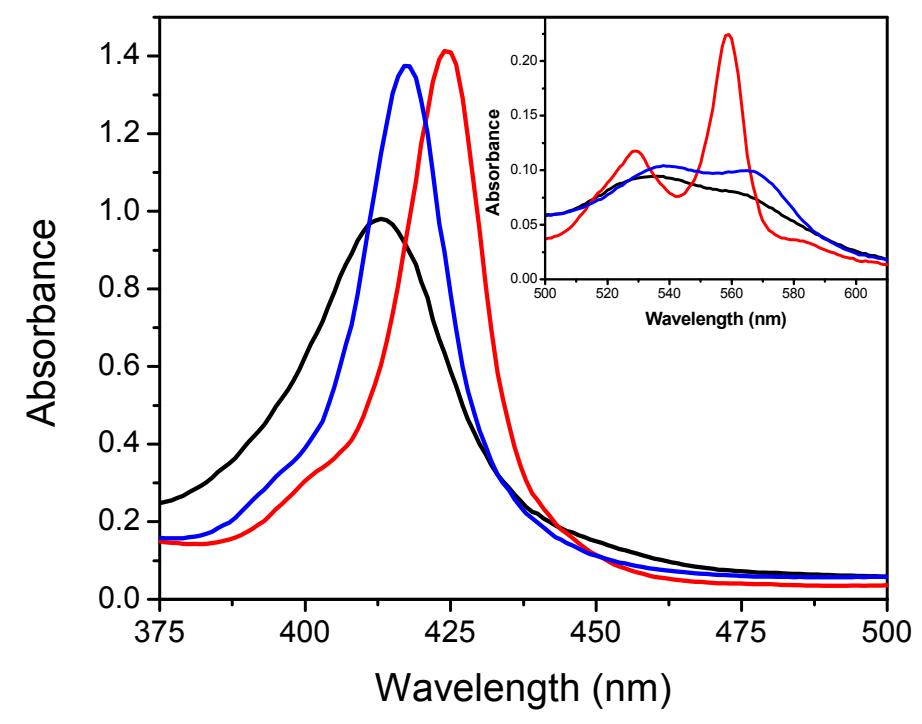

Figure 4.3 Absorption spectra of ferric (black line), ferrous (red line) and $\mathrm{CO}$ bound (blue line) $\mathrm{hNgb}$ wild type in $50 \mathrm{mM}$ Tris buffer ( $\mathrm{pH} 7.0$ ).

The absorption spectra of ferric, ferrous and $\mathrm{CO}$ adducts of hNgb strains Cys120Ser (Figure 4.4), Cys55Ser/Cys120Ser (Figure 4.5) and Val68Phe (Figure 4.6), are comparable to the spectra of wild type $\mathrm{hNgb}$, whereas the absorption spectra of $\mathrm{hNgb}$ strains His64Gln and His64Gln/Val68Phe are distinct from that of hNgb wild type.

The steady state absorption spectra of ferric, ferrous deoxy and $\mathrm{CO}$ bound His64Gln $\mathrm{hNgb}$ at $\mathrm{pH} 7$ are displayed in Figure 4.7. The $\mathrm{Fe}^{3+} \mathrm{His} 64 \mathrm{Gln}$ mutated protein exhibits a Soret band at $408 \mathrm{~nm}$, a pronounced visible band near $539 \mathrm{~nm}$, and two additional bands at 575 and $630 \mathrm{~nm}$. These bands positions are identical to those observed for other Ngb His64 mutants (Nienhaus et al., 2004; Van Doorslaer et al., 2003) 
and similar to those observed in the spectra of other globins with a hexa-coordinate highspin $\mathrm{Fe}^{3+}$ heme iron having a weakly bound water molecule in the position of the axial ligand (Quillin et al., 1993). Analogous absorption spectra were reported for African elephant Mb with naturally occurring Gln64 (Tada et al., 1998). Reduction of the heme iron produces a bathochromic shift of the Soret band to $420 \mathrm{~nm}$ with a shoulder near 432 $\mathrm{nm}$ and an additional absorption band situated at $556 \mathrm{~nm}$. This spectrum is similar to that reported for the His64Leu (Dewilde et al., 2001; Nienhaus et al., 2010) and His64Val (Tiso et al., 2011) mutants of Ngb.

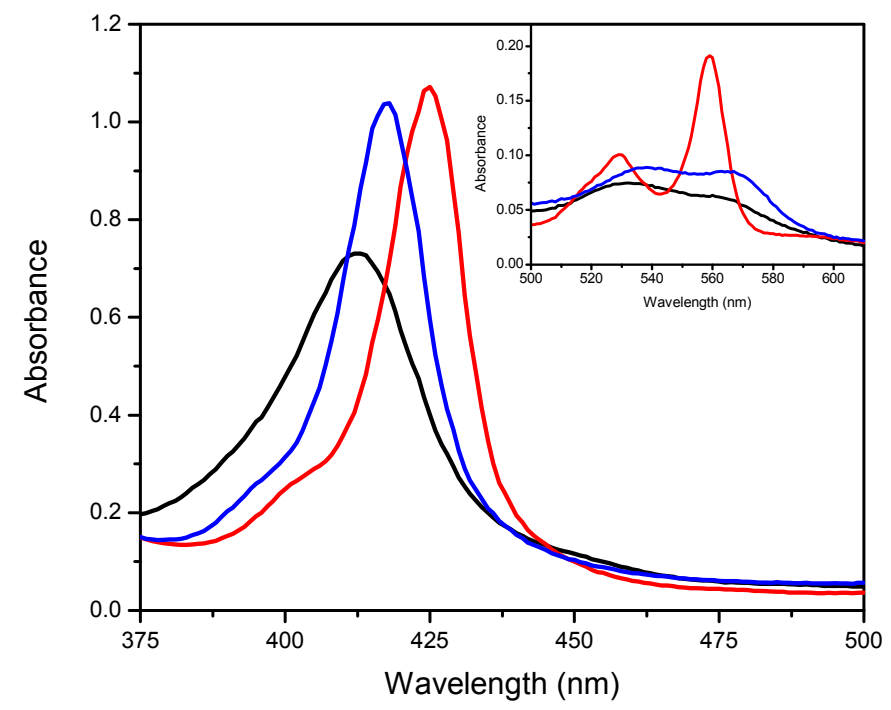

Figure 4.4 Absorption spectra of ferric (black line), ferrous (red line) and CO bound (blue line) $\mathrm{hNgb}$ Cys120Ser in $50 \mathrm{mM}$ Tris buffer (pH 7.0). 


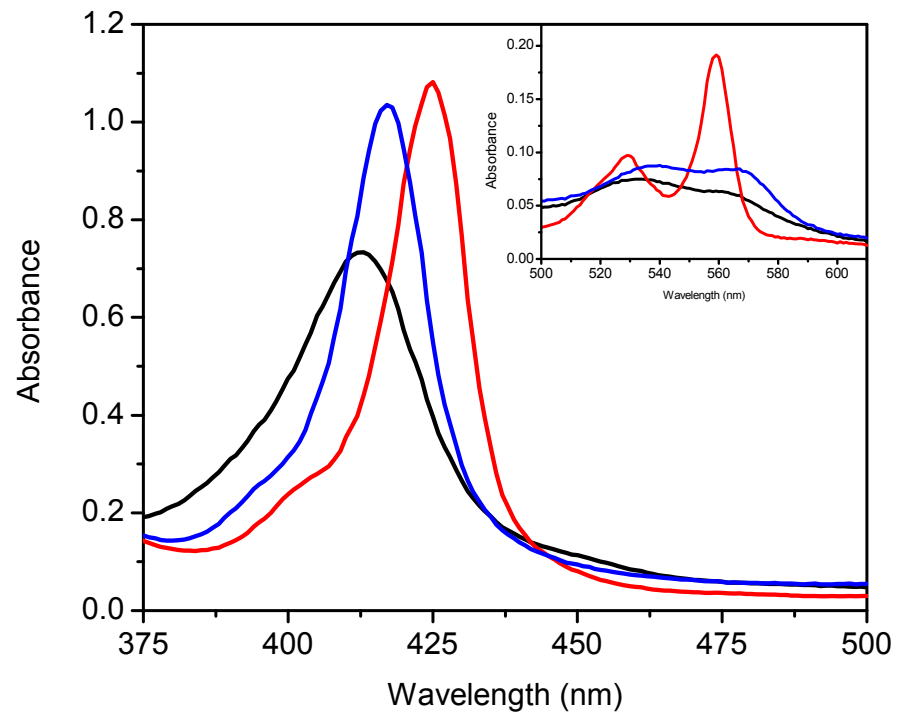

Figure 4.5 Absorption spectra of ferric (black line), ferrous (red line) and CO bound (blue line) $\mathrm{hNgb}$ Cys55Ser/Cys120Ser in $50 \mathrm{mM}$ Tris buffer (pH 7.0).

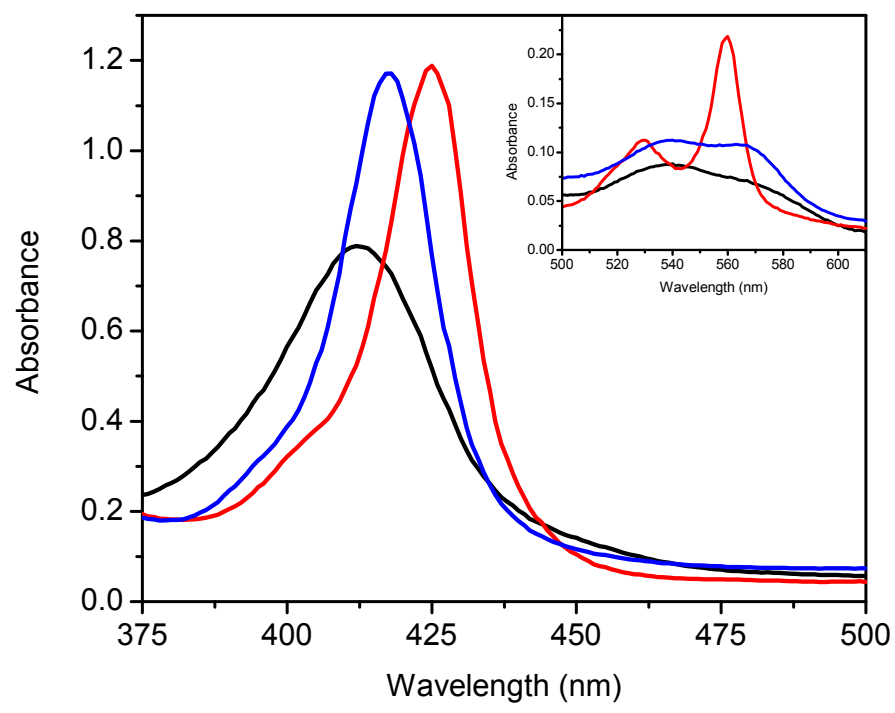

Figure 4.6 Absorption spectra of ferric (black line), ferrous (red line) and $\mathrm{CO}$ bound (blue line) $\mathrm{hNgb}$ Val68Phe in $50 \mathrm{mM}$ Tris buffer (pH 7.0). 
The shoulder at $432 \mathrm{~nm}$ and the visible band at $556 \mathrm{~nm}$ observed in the spectrum of $\mathrm{Fe}^{2+} \mathrm{His} 64 \mathrm{Gln}$ mutated protein are consistent with a penta-coordinate high-spin heme iron, whereas the Soret band maximum at $420 \mathrm{~nm}$ indicates the presence of an $\mathrm{Ngb}$ fraction with a hexa-coordinate low-spin heme iron. Nienhaus et al. (2010) have proposed that a water molecule or $\mathrm{OH}^{-}$group may serve as a sixth axial ligand in the distal site of the His64Leu mutant in $\mathrm{Fe}^{2+} \mathrm{mNgb}$, whereas Uno et al. (2004) have identified Lys67 as a possible sixth axial ligand at alkali $\mathrm{pH}$. The $\mathrm{CO}$ bound form of the His64Gln mutant exhibits a Soret band at $417 \mathrm{~nm}$ and $\alpha$ and $\beta$ bands located at 567 and $539 \mathrm{~nm}$, respectively, that are consistent with the formation of low-spin six-coordinate heme iron.

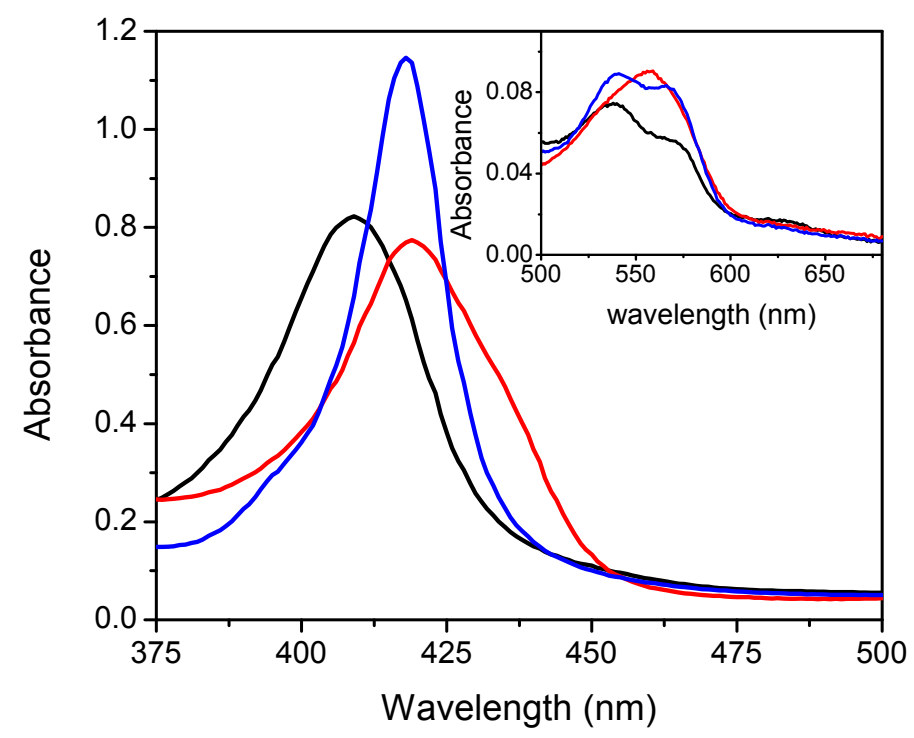

Figure 4.7 Absorption spectra of ferric (black line), ferrous (red line) and CO bound (blue line) His64Gln hNgb in $50 \mathrm{mM}$ Tris buffer (pH 7.0).

The UV-visible absorption spectra recorded for the His64Gln/Val68Phe double mutant (Figure 4.8) is similar to those for His64Gln mutant. However, the shoulder at 
$432 \mathrm{~nm}$ and the visible band at $556 \mathrm{~nm}$ are more pronounced in the spectrum of $\mathrm{Fe}^{2+} \mathrm{His} 64 \mathrm{Gln} / \mathrm{Val} 68 \mathrm{Phe}$ mutated protein than in the spectrum of $\mathrm{Fe}^{2+} \mathrm{His} 64 \mathrm{Gln}$, indicating a higher fraction of penta-coordinate high-spin heme iron in the double mutant than in the single mutant.

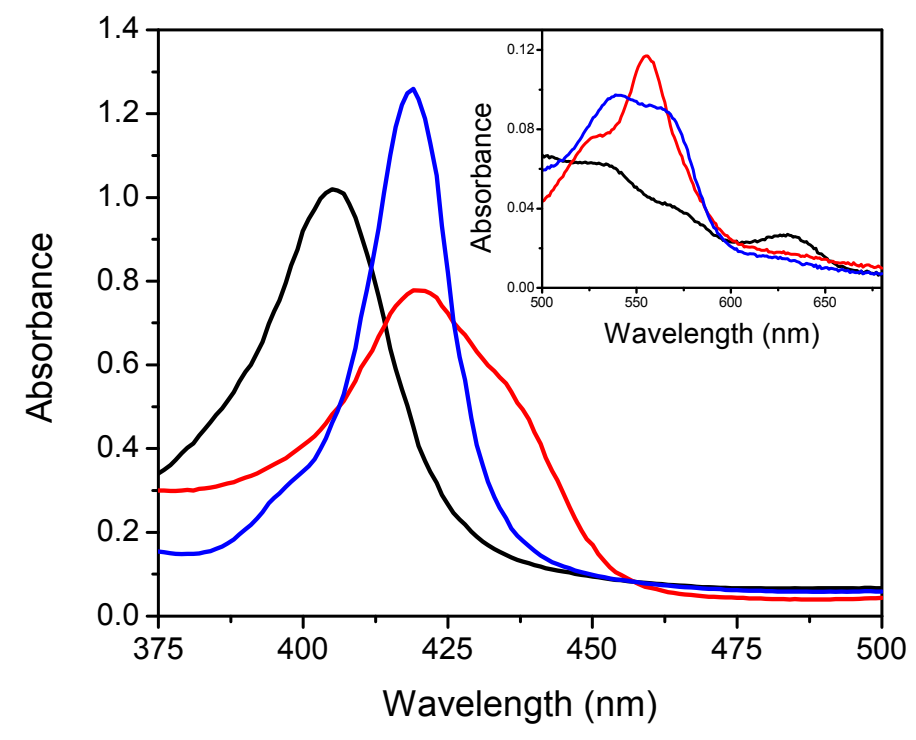

Figure 4.8 Absorption spectra of ferric (black line), ferrous (red line) and $\mathrm{CO}$ bound (blue line) His64Gln/Val68Phe hNgb in $50 \mathrm{mM}$ Tris buffer (pH 7.0).

\subsubsection{Transient absorption spectroscopy}

The kinetics for rebinding of the ligand to $\mathrm{Ngb}$ are more complex than those of binding of the diatomic ligand to penta-coordinate globins because of the hexacoordinate nature of $\mathrm{Ngb}$ and the heterogeneity of the heme group (Kiger et al., 2004). Previous studies have shown that $\mathrm{CO}$ rebinding is multiphasic, with geminate $\mathrm{CO}$ rebinding taking place on a nanosecond time scale and bimolecular rebinding of $\mathrm{CO}$ to the five-coordinate heme on the microsecond time scale (Abbruzzetti et al., 2009; Kriegl et al., 2002). Subsequent rebinding of $\mathrm{CO}$ to the six-coordinate bis-histidine heme is observed on the 
millisecond to second time scale, with the distal His dissociation representing the ratelimiting step (Kiger et al., 2004). Because the penta-coordinate and hexa-coordinate Ngb species are in equilibrium, the ratio of the fast to slow phase of bimolecular $\mathrm{CO}$ rebinding can be modified by varying the ligand concentration and temperature. Under ligand saturation conditions and at ambient temperature, the fraction of the bis-histidine heme iron is negligible, and thus, only single phase bimolecular rebinding of $\mathrm{CO}$ to fivecoordinate heme iron is observed (Dewilde et al., 2001; Kriegl et al., 2002).

Figure 4.9 shows TA traces on the nano- to microsecond time scale for rebinding of $\mathrm{CO}$ to five-coordinate $\mathrm{Ngb}$ wild type and mutated proteins studied at $20^{\circ} \mathrm{C}$ under $\mathrm{CO}$ saturating conditions $(\sim 1 \mathrm{mM} \mathrm{CO})$. The geminate quantum yield $\left(\Phi_{\text {gem }}\right)$ was determined by taking the ratio of the amplitude of the absorbance change corresponding to the geminate process $\left(\mathrm{A}_{\text {gem }}\right)$ with respect to the total absorbance change $\left(\mathrm{A}_{\text {total }}\right)$ (Table 4.1). The quantum yield for $\mathrm{CO}$ geminate rebinding is not affected by the His64Gln, Cys120Ser and Cys55Ser/Cys120Ser mutations $\left(\Phi_{\mathrm{gem}}=0.32\right)$. A large increase in $\Phi_{\mathrm{gem}}$ was observed for Val68Phe $\operatorname{Ngb}\left(\Phi_{\mathrm{gem}}=0.85\right)$ and for the double mutant His64Gln/Val68Phe $\left(\Phi_{\text {gem }}=0.96\right)$.

The TA traces were analyzed using a multi-exponential decay model:

$$
\Delta A=\sum_{i} A_{i} e\left(\frac{t}{\tau_{i}}\right)
$$

Where $\tau_{\mathrm{i}}$ and $\mathrm{A}_{\mathrm{i}}$ corresponds to the lifetime and amplitude for each kinetic step, respectively. 


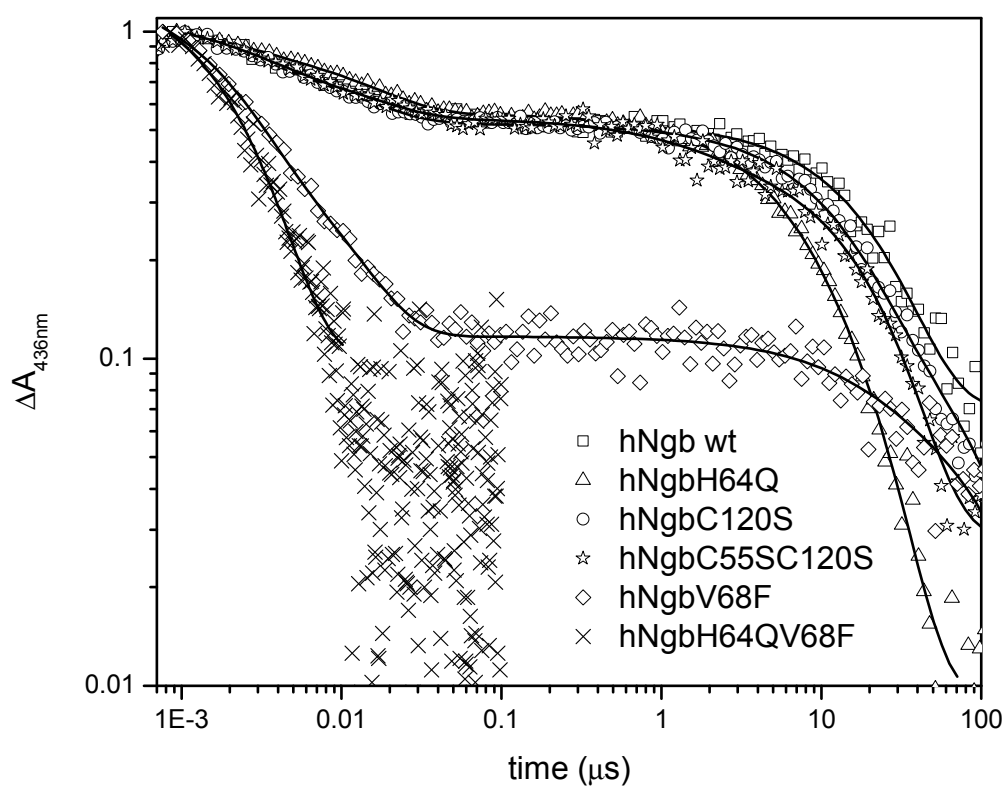

Figure 4.9 Transient absorption traces measured for $\mathrm{CO}$ geminate recombination and bimolecular rebinding to Ngbs. The initial amplitudes following the photo-dissociation of $\mathrm{CO}$ were normalized to 1 . The solid lines correspond to a fit of the experimental data using a four exponential decay model. Conditions: $20 \mu \mathrm{M}$ protein in $50 \mathrm{mM}$ Tris buffer $(\mathrm{pH} 7.0)$ at $20^{\circ} \mathrm{C}$ and $1 \mathrm{mM} \mathrm{CO}$.

Table 4.1 Kinetic parameters associated with $\mathrm{CO}$ rebinding to wild type $\mathrm{hNgb}$ and mutants. Rate constants $\left(\mathrm{k}_{\mathrm{gem}}\right.$ and $\mathrm{k}_{\text {fast }}$ ) for ligand binding to five-coordinate $\mathrm{Ngb}$ were determined at $20^{\circ} \mathrm{C}$ and $1 \mathrm{mM} \mathrm{CO}$, whereas the slow phase was measured at $35^{\circ} \mathrm{C}$ and $0.1 \mathrm{mM} \mathrm{CO}$ to increase the yield of bishistidyl $\mathrm{Ngb}$

\begin{tabular}{|c|c|c|c|c|c|c|c|c|c|c|}
\hline Sample & $\begin{array}{c}\mathrm{k}_{\mathrm{gem} 1} \\
\left(10^{8} \mathrm{~s}^{-1}\right)\end{array}$ & $\begin{array}{l}A_{\text {gem } 1} \\
(\%)\end{array}$ & $\begin{array}{c}\mathrm{k}_{\mathrm{gem} 2} \\
\left(10^{7} \mathrm{~s}^{-1}\right)\end{array}$ & $\begin{array}{l}A_{\text {gem } 2} \\
(\%)\end{array}$ & $\begin{array}{c}\mathrm{k}_{\text {fast } 1} \\
\left(\mu \mathrm{M}^{-1} \mathrm{~s}^{-1}\right)\end{array}$ & $\begin{array}{l}A_{\text {fast1 }} \\
(\%)\end{array}$ & $\begin{array}{c}\mathrm{k}_{\text {fast2 }} \\
\left(\mu \mathrm{M}^{-1} \mathrm{~s}^{-1}\right)\end{array}$ & $\begin{array}{l}A_{\text {fast2 }} \\
(\%)\end{array}$ & $\begin{array}{c}\mathrm{k}_{\text {slow }} \\
\left(\mu \mathrm{M}^{-1} \mathrm{~s}^{-1}\right)\end{array}$ & $\Phi_{\mathrm{gem}}$ \\
\hline WT & $2.3 \pm 0.8$ & 32.0 & $3.4 \pm 0.5$ & 15.4 & $61 \pm 7$ & 35.0 & $9 \pm 2$ & 17.6 & $0.10 \pm 0.01$ & 0.32 \\
\hline H64Q & $3.8 \pm 0.6$ & 13.3 & $7.2 \pm 0.9$ & 29.2 & $312 \pm 24$ & 27.5 & $95 \pm 3$ & 30.0 & - & 0.32 \\
\hline C120S & $5.0 \pm 2.0$ & 22.7 & $8.0 \pm 3.0$ & 26.7 & $90 \pm 16$ & 33.6 & $19 \pm 7$ & 17.0 & $0.09 \pm 0.01$ & 0.32 \\
\hline $\mathrm{C} 55 \mathrm{~S} / \mathrm{C} 120 \mathrm{~S}$ & $3.2 \pm 0.5$ & 37.6 & $4.7 \pm 0.5$ & 13.8 & $153 \pm 31$ & 12.3 & $29 \pm 4$ & 36.2 & $0.08 \pm 0.01$ & 0.32 \\
\hline V68F & $5.6 \pm 0.4$ & 66.5 & $10.9 \pm 0.4$ & 30.0 & $77 \pm 36$ & 3.6 & $9 \pm 3$ & 9.9 & $0.08 \pm 0.01$ & 0.85 \\
\hline H64Q/V68F & $4.8 \pm 0.3$ & & - & & - & & & & - & 0.96 \\
\hline
\end{tabular}


CO geminate rebinding is found to be biphasic with the following rate constants for the fast and slow phases in the wild type protein: $k_{g e m l}=(2.3 \pm 0.8) \times 10^{8} \mathrm{~s}^{-1}$ and $k_{\text {gem } 2}$ $=(3.4 \pm 0.5) \times 10^{7} \mathrm{~s}^{-1}$, respectively (Table 4.1). The observed rate constant for the slower geminate rebinding, $k_{\text {gem2}}$, matches well with that reported by Abbruzzetti et al. (2009). Similar rate constants for $\mathrm{CO}$ geminate rebinding were resolved for other mutated proteins studies here except the His64Gln/Val68Phe double mutant for which only CO geminate rebinding with a single rate constant of $(4.8 \pm 0.3) \times 10^{7} \mathrm{~s}^{-1}$ was assessed. The observed heterogeneity reflects the fast ligand rebinding from the primary and secondary ligand docking sites that were identified by time-resolved IR spectroscopy (Nienhaus and Nienhaus, 2005a). The multiphasic CO geminate rebinding from alternative docking sites was also reported for $\mathrm{CO}$ geminate rebinding in $\mathrm{hNgb}$ encapsulated in silica gels (Abbruzzetti et al., 2009), although the fast rate constant reported here was not resolved.

The bimolecular rebinding of $\mathrm{CO}$ to five-coordinate $\mathrm{Ngb}$ is also multiphasic for wild type $\mathrm{Ngb}$, with the rate constants for the fast and slow phases being $61 \pm 7 \mu \mathrm{M}^{-1} \mathrm{~s}^{-1}$ $(\sim 65 \%)$ and $9 \pm 2 \mu \mathrm{M}^{-1} \mathrm{~s}^{-1}(\sim 35 \%)$, respectively. The biphasic bimolecular rebinding of $\mathrm{CO}$ to five-coordinate $\mathrm{Ngb}$ was observed previously and associated with the heterogeneous orientation of the heme group in Ngb (Kiger et al., 2004). The presence of two heme isomers was confirmed in an NMR study showing that the isomers are rotated by $180^{\circ}$ and populated with a ratio of 2:1 (Du et al., 2003), which matches the ratio of amplitudes for the fast and slow phases reported here.

The replacement of Cys120 with Ser or Val68 with Phe does not alter the rate of $\mathrm{CO}$ bimolecular rebinding. On the other hand, significantly faster $\mathrm{CO}$ bimolecular rebinding was observed for the His64Gln mutant with rate constants of $312 \pm 24$ and $95 \pm$ 
$3 \mu \mathrm{M}^{-1} \mathrm{~s}^{-1}$ exhibiting equal amplitudes. Similar acceleration for the CO bimolecular rebinding was observed in the Cys120Ser/Cys55Ser double mutant with rate constants of $153 \pm 31(\sim 24 \%)$ and $29 \pm 4 \mu \mathrm{M}^{-1} \mathrm{~s}^{-1}(\sim 76 \%)$ for the fast and slow phases of bimolecular association, respectively.

The pseudo-first order rate constants for rebinding of $\mathrm{CO}$ to hexacoordinate bishistidyl Ngb $\left(\mathrm{k}_{\text {slow }}\right)$ were determined under conditions that increase the population of bishistidyl $\mathrm{hNgb}\left(35^{\circ} \mathrm{C}\right.$ and $\sim 0.1 \mathrm{mM} \mathrm{CO}$ ) (Figure 4.10). TA traces were fitted using a single exponential decay and the rate constants are listed in table 4.1. Substitution of Val68 with Phe or Cys120 with Ser does not alter the rate constants for CO rebinding relative to that of the wild type protein. The rate constant for rebinding of $\mathrm{CO}$ to bishistidyl hNgb in the Cys55Ser/Cys120Ser double mutant $\left(\mathrm{k}_{\text {slow }}=0.08 \pm 0.01 \mu \mathrm{M}^{-1} \mathrm{~s}^{-1}\right)$ is somewhat larger than the rate constant observed for the Cys55Ser single mutant $\left(\mathrm{k}_{\text {slow }}=\right.$ $0.052 \mu \mathrm{M}^{-1} \mathrm{~s}^{-1}$ ) (Astudillo et al., 2010), and described in chapter 5. Such an impact of the Cys120 mutation in the double mutant on the rate constant for rebinding of $\mathrm{CO}$ to bishistidyl $\mathrm{Fe}^{2+} \mathrm{hNgb}$ is surprising considering that this residue is located close to the protein surface, approximately $18 \AA$ from the heme binding pocket. Because the rate-limiting step for rebinding of $\mathrm{CO}$ to bis-histidyl $\mathrm{Fe}^{2+} \mathrm{hNgb}$ is distal histidine dissociation, these data suggest that substitution of Cys120 with Ser leads to long-range structural changes that impact heme iron coordination. 


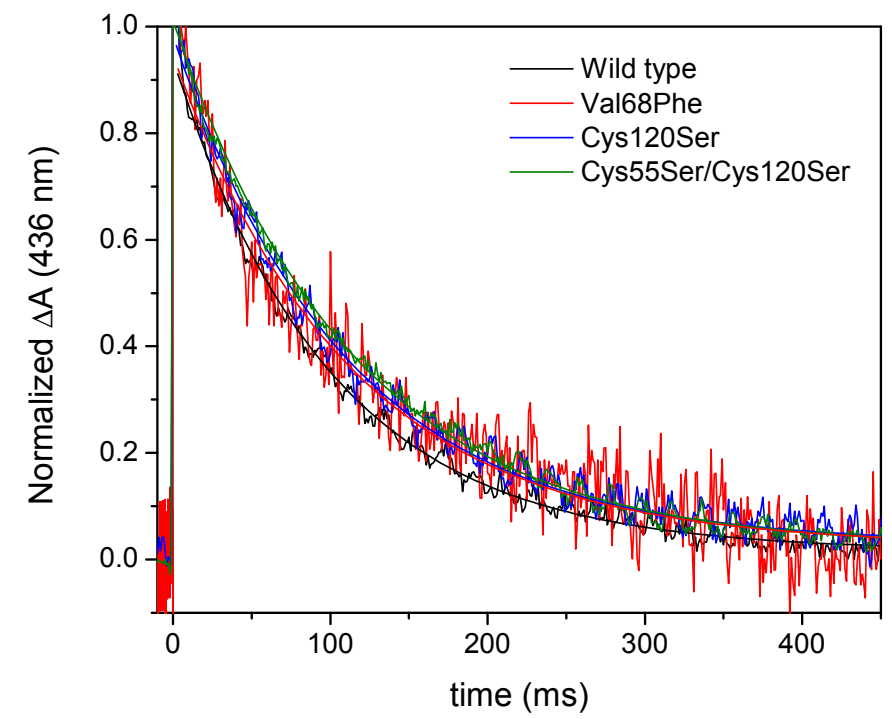

Figure 4.10 Transient absorption traces measured for $\mathrm{CO}$ bimolecular rebinding to Ngbs. Traces were normalized to 1 . The solid lines correspond to a fit of the experimental data using a single exponential decay model. Conditions: $20 \mu \mathrm{M}$ protein in $50 \mathrm{mM}$ Tris buffer $(\mathrm{pH} 7.0)$ at $35^{\circ} \mathrm{C}$ and $0.1 \mathrm{mM} \mathrm{CO}$ to increase the yield of bishistidyl coordination.

\subsubsection{Photoacoustic calorimetry results}

An overlay of photoacoustic traces for photo-dissociation of $\mathrm{CO}$ from wild type $\mathrm{hNgb}$ and His64Gln hNgb mutated protein together with the reference compound, 4SP, are shown in Figures 4.11 and 4.11, respectively. The sample acoustic traces overlay in phase with the reference traces, indicating the absence of volume and/or enthalpy changes on the time scale between $\sim 20 \mathrm{~ns}$ and $\sim 5 \mu \mathrm{s}$. Similar photoacoustic time profiles have been observed for the other mutated proteins investigated. 


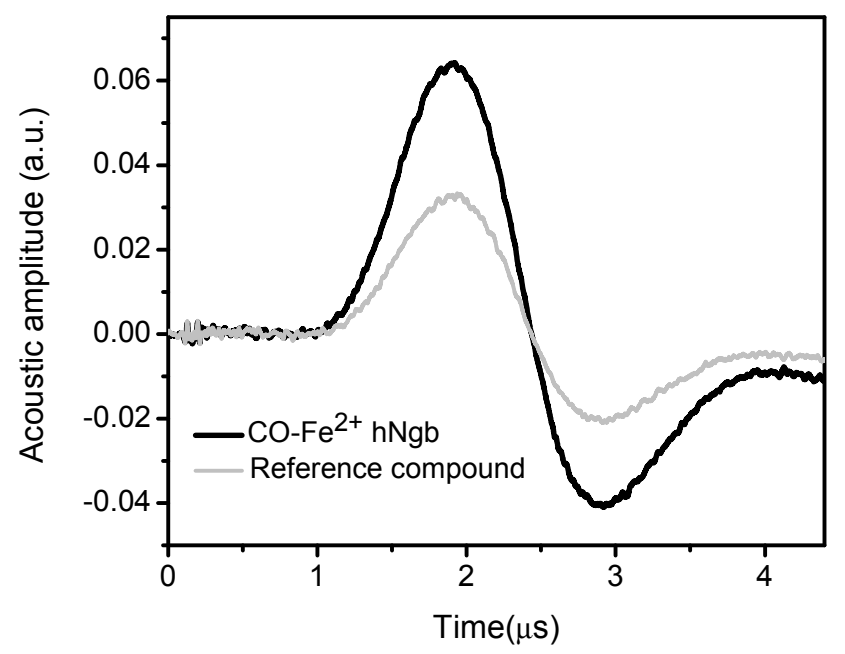

Figure 4.11 Photoacoustic traces for wild type hNgb together with the reference compound 4SP. Conditions: $20 \mu \mathrm{M}$ protein in $50 \mathrm{mM}$ Tris buffer $(\mathrm{pH}$ 7.0). The absorbance of the sample matched that of the reference compound at $532 \mathrm{~nm}$ $\left(\mathrm{A}_{532 \mathrm{~nm}}=0.22\right)$.

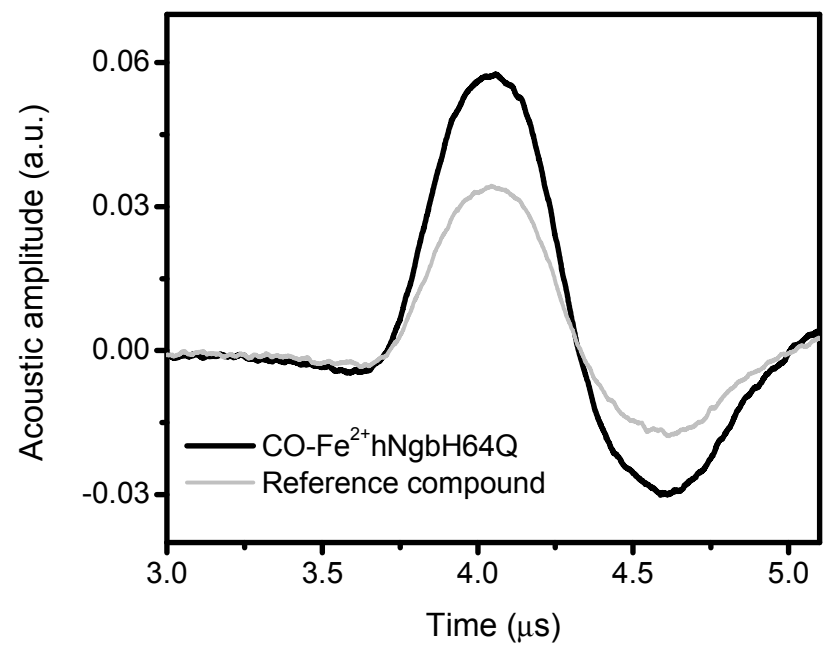

Figure 4.12 Photoacoustic traces for $\mathrm{hNgb}$ H64Q together with the reference compound 4SP. Conditions: $20 \mu \mathrm{M}$ protein in $50 \mathrm{mM}$ Tris buffer ( $\mathrm{pH}$ 7.0). The absorbance of the sample matched that of the reference compound at $532 \mathrm{~nm}\left(\mathrm{~A}_{532 \mathrm{~nm}}=0.23\right)$. 
Plots of the PAC amplitude ratios as a function of the thermal coefficient $\left[\left(C_{p} \rho\right) / \beta\right]$ over the temperature range of $16-35^{\circ} \mathrm{C}$ are displayed in Figure 4.13. The reaction volume and enthalpy changes were determined from the slope and intercept of the linear plot, respectively, as described in the Materials and Methods section, and are listed in Table 4.2. From the reaction volume change, the structural volume change, $\Delta \mathrm{V}_{\text {str }}$, that describes the difference between the partial molar volume of the fivecoordinate $\mathrm{Ngb}$ and $\mathrm{CO}-$ bound $\mathrm{Ngb}$ can be determined according to the equation 4.3.

$$
\Delta V=V_{C O}+V_{N g b}-V_{C O N g b}
$$

Where $\mathrm{V}_{\mathrm{CO}}$ is the partial molar volume of $\mathrm{CO}\left(35 \mathrm{~mL} \mathrm{~mol}^{-1}\right)$ (Hara et al., 1996) and $\mathrm{V}_{\mathrm{Ngb}}$ is the partial molar volume of $\mathrm{Ngb}$.

The photo-dissociation of $\mathrm{CO}$ from WT $\mathrm{hNgb}$ is associated with a $\Delta \mathrm{V}_{\text {str }}$ of $-21.6 \pm$ $0.9 \mathrm{~mL} \mathrm{~mol}^{-1}$, and slightly more negative structural volume changes were observed for His64Gln and Cys120Ser proteins. Replacement of Val68 with Phe has a significant impact on the structural volume change $\left(\Delta \mathrm{V}_{\text {str }}=-37 \pm 2 \mathrm{~mL} \mathrm{~mol}^{-1}\right)$ likely because of a larger structural reorganization of the distal pocket in this mutant. The overall enthalpy change for the photo-dissociation of CO from WT hNgb is $20 \pm 4 \mathrm{kcal} \mathrm{mol}^{-1}$ and largely reflects the enthalpy of the Fe-CO bond energy (Astudillo et al., 2010). Interestingly, all mutated proteins studied here exhibit negative enthalpy changes for $\mathrm{CO}$ photolysis with a $\Delta \mathrm{H}$ of $-3 \pm 4 \mathrm{kcal} \mathrm{mol}^{-1}$ for the Cys120Ser mutant and $-48 \pm 7 \mathrm{kcal} \mathrm{mol}^{-1}$ for the Val68Phe mutant. We did not characterize volume and enthalpy changes associated with photo-dissociation of $\mathrm{CO}$ from the double mutant His64Gln/Val68Phe, because the quantum yield for geminate recombination is close to unity. 


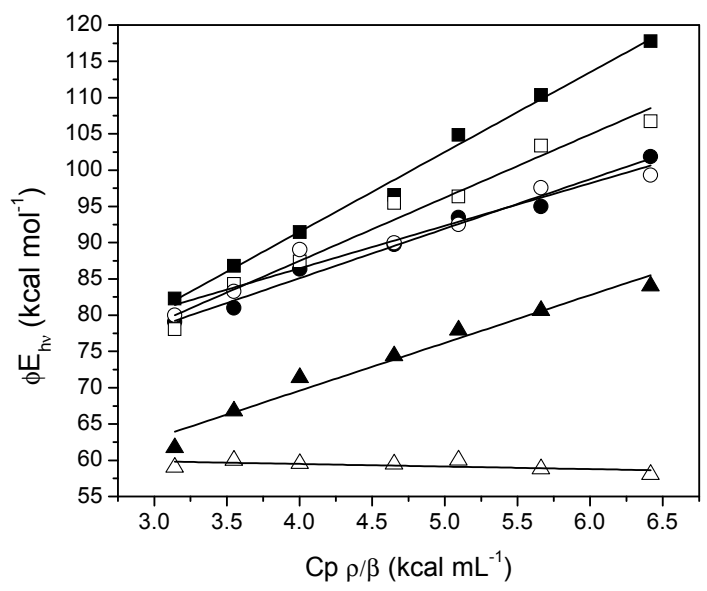

Figure 4.13 Plot of $\phi \mathrm{E}_{\mathrm{hv}}$ as a function of $\mathrm{C}_{\mathrm{p}} \rho / \beta$ for $\mathrm{CO}$ photo-release from wild type $\mathrm{Ngb}$ (solid squares), His64Gln (solid circles), Val68Phe (open triangles), Cys120Ser (open circles), Cys55Ser/Cys120Ser (open squares), and Cys55Ser (solid triangles). The corresponding volume and enthalpy changes were determined from the slope and intercept of the linear fits, respectively. The data for human $\mathrm{Ngb}$ and the Cys55Ser mutant were previously reported by Astudillo et al. (2010).

Table 4.2 Thermodynamic parameters associated with $\mathrm{CO}$ photo-dissociation from Ngbs.

\begin{tabular}{lccc}
\hline & $\Delta \mathrm{H}\left(\mathrm{kcal} \mathrm{mol}^{-1}\right)$ & $\Delta \mathrm{V}\left(\mathrm{mL} \mathrm{mol}^{-1}\right)$ & $\Delta \mathrm{V}_{\text {str }}\left(\mathrm{mL} \mathrm{mol}^{-1}\right)^{b}$ \\
\hline Wild type $^{\mathrm{a}}$ & $20 \pm 4$ & $13.4 \pm 0.9$ & $-21.6 \pm 0.9$ \\
His64Gln & $-6 \pm 3$ & $10.0 \pm 0.5$ & $-25.0 \pm 0.5$ \\
Val68Phe & $-48 \pm 7$ & $-2.4 \pm 1.5$ & $-37.4 \pm 1.5$ \\
Cys120Ser & $-3 \pm 4$ & $7.5 \pm 0.6$ & $-27.5 \pm 0.6$ \\
Cys55Ser/Cys120Ser & $-6 \pm 4$ & $12.2 \pm 0.9$ & $-22.5 \pm 0.9$ \\
\end{tabular}

${ }^{a}$ From Astudillo et al. (2010).

${ }^{\mathrm{b}}$ The structural volume change was determined using the equation $\Delta V_{s t r}=\Delta V-V_{m}^{\circ}$, where $V_{m}{ }^{\circ}$ is the partial molar volume of CO $\left(35 \mathrm{~mL} \mathrm{~mol}^{-1}\right)$ (Hara et al., 1996). 


\subsection{Discussion}

\subsubsection{Role of distal residues His64 and Val68 in controlling ligand migration in hNgb}

Conserved distal pocket residues His64 and Val68 play a crucial role in regulating the affinity for diatomic ligands in vertebrate globins. In Ngb, His64 modulates the affinity for $\mathrm{CO}$ and $\mathrm{O}_{2}$ (Dewilde et al., 2001; Pesce et al., 2003), it is responsible for the pH dependence of $\mathrm{O}_{2}$ binding (Fago et al., 2004b), and protects against formation of tyrosine-phenoxyl radicals in the presence of hydrogen peroxide (Lardinois et al., 2008).

In this work, it is shown that the replacement of His64 with a polar Gln residue of a comparable size moderately alters the rate for bimolecular rebinding of $\mathrm{CO}$ to the fivecoordinate $\mathrm{hNgb}$ with the rate constant being approximately 5 and 10 times larger than the rate constant for the fast and slow bimolecular rebinding in WT, respectively. A similar increase in the rate constant for the fast phase of bimolecular rebinding was reported previously for the His64Gln substitution by Hamdane et al. (2003) $\left(\mathrm{k}_{\text {fast }}=290\right.$ $\left.\mu \mathrm{M}^{-1} \mathrm{~s}^{-1}\right)$ and by others for $\mathrm{hNgb}$ mutants with a Leu $\left(\mathrm{k}_{\mathrm{fast}}=237 \mu \mathrm{M}^{-1} \mathrm{~s}^{-1}\right)$ (Nienhaus et al., 2004) or Val $\left(\mathrm{k}_{\text {fast }}=230 \mu \mathrm{M}^{-1} \mathrm{~s}^{-1}\right)$ (Dewilde et al., 2001) residue in position 64. In those studies, the heterogeneous rebinding of $\mathrm{CO}$ to the five-coordinate heme iron was also detected; however, the rate constant for the slow phase was not reported.

The biphasic rebinding of $\mathrm{CO}$ to the His64Val mutant was observed at $\mathrm{pH} 9.5$ with rate constants similar to those reported here $\left(\mathrm{k}_{\text {fast } 1}=350 \mu \mathrm{M}^{-1} \mathrm{~s}^{-1}\right.$, and $\mathrm{k}_{\text {fast2 }}=45 \mu \mathrm{M}^{-}$ ${ }^{1} \mathrm{~s}^{-1}$ ); however, the authors assigned the slower process to the partial coordination of Lys67 (Uno et al., 2004). On the basis of the $\mathrm{mNgb}$ crystal structure, it was proposed that binding of $\mathrm{CO}$ to the heme iron requires the distal histidine side chain swinging out of the 
distal pocket and a concomitant breakage of the electrostatic/hydrogen bonding network among Lys67, heme 7-propionate, and Tyr44. Substitution of distal His with Gln opens the access to the distal pocket, resulting in a lowering of the activation barrier for exogenous ligand binding.

The relatively moderate impact of the His64 side chain on the ligand migration within the protein matrix is evident from the quantum yield value for the $\mathrm{CO}$ geminate rebinding that is identical to that measured for $\mathrm{WT} h \mathrm{hgb}\left(\Phi_{\mathrm{gem}}=0.32\right)$. In comparison, an analogous replacement in swMb results in a substantial increase in the amplitude for CO geminate rebinding $\left(\Phi_{\text {gem }} \sim 0.04\right.$ in $\mathrm{WT} \mathrm{Mb}$, and $\Phi_{\text {gem }}=0.67$ in the His64Gln $\mathrm{Mb}$ mutant) (Lambright et al., 1994). These results show that in Ngb the residue occupying position 64 does not influence the migration of the ligand between the heme distal pocket and adjacent hydrophobic cavities and/or escape to the surrounding solvent.

The replacement of Val68 with Phe, on the other hand, significantly increases the amplitude for geminate CO rebinding $\left(\Phi_{\text {gem }}=0.85\right)$, indicating that the presence of the bulky benzyl side chain at position 68 introduces a steric block for $\mathrm{CO}$ migration between the distal pocket and the internal hydrophobic cavities. This hypothesis is further supported by the study of the His64Gln/Val68Phe double mutant in which the escape of the photo-dissociated ligand into the surrounding solvent is nearly completely hindered, which is evident from the geminate quantum yield approaching unity. Interestingly, the impact of the His64Gln and Val68Phe mutation on the $\mathrm{CO}$ geminate process is not additive, suggesting that the simultaneous mutation of both distal residues leads to a reorganization of the volume and/or dynamics of the heme distal pocket and/or nearby internal cavities. 
In Ngb, Val68 and the other conserved hydrophobic residues, Leu27, Val109, and Leu113, surround the $\mathrm{Xe}_{4}$ cavity that is located in a position analogous to that in $\mathrm{Mb}$ (Moschetti et al., 2009) (Figure 4.14). Yin et al. (2008) have probed the structure of the heme binding site in the His64Gln/Val68Phe $\mathrm{hNgb}$ double mutant using solution ${ }^{1} \mathrm{H}$ NMR. They reported that the Phe side chain adopts a spatial orientation similar to that found in the structure of the Mb triple mutant (His64Gln/Leu29Phe/Val68Phe) (Nguyen et al., 1998), with the side chain blocking the $\mathrm{Xe}_{4}$ cavity. The reduction of the volume in $\mathrm{Xe}_{4}$ cavity thus results in an efficient blockage of migration of $\mathrm{CO}$ between the distal pocket and adjacent hydrophobic cavities, confirming that the residue at position 68 exhibits an analogous functional role in both Ngb and penta-coordinate vertebrate globins (Dantsker et al., 2005). To obtain further insight into the factors affecting ligand migration in Ngb upon replacement of Val68 by Phe, the Val68 residue was replaced by Phe in the structures of $\mathrm{CO}$ bound $\mathrm{Fe}^{2+} \mathrm{mNgb}$ and $\mathrm{Fe}^{2+} \mathrm{mNgb}$, then these structures were minimized using Amber03 force field in YASARA structure software. In addition, internal hydrophobic cavities were calculated employing a $1.4 \AA$ probe radius and the numerical algorithm. The modeled structures of $\mathrm{CO}$ bound $\mathrm{Fe}^{2+} \mathrm{mNgb}$ Val68Phe and $\mathrm{Fe}^{2+} \mathrm{mNgb}$ Val68Phe (Figure 4.15) show repositioning of the side chain of Phe 68 residue and a different organization of the internal hydrophobic cavities in the ligand bound form and ligand free form of the mutated protein. $\mathrm{In} \mathrm{Fe}^{2+} \mathrm{mNgb}$ Val68Phe, the side chain is blocking the hydrophobic tunnel previously reported for $\mathrm{Fe}^{2+} \mathrm{mNgb}$ that includes $\mathrm{Xe}_{4}$ cavity. 

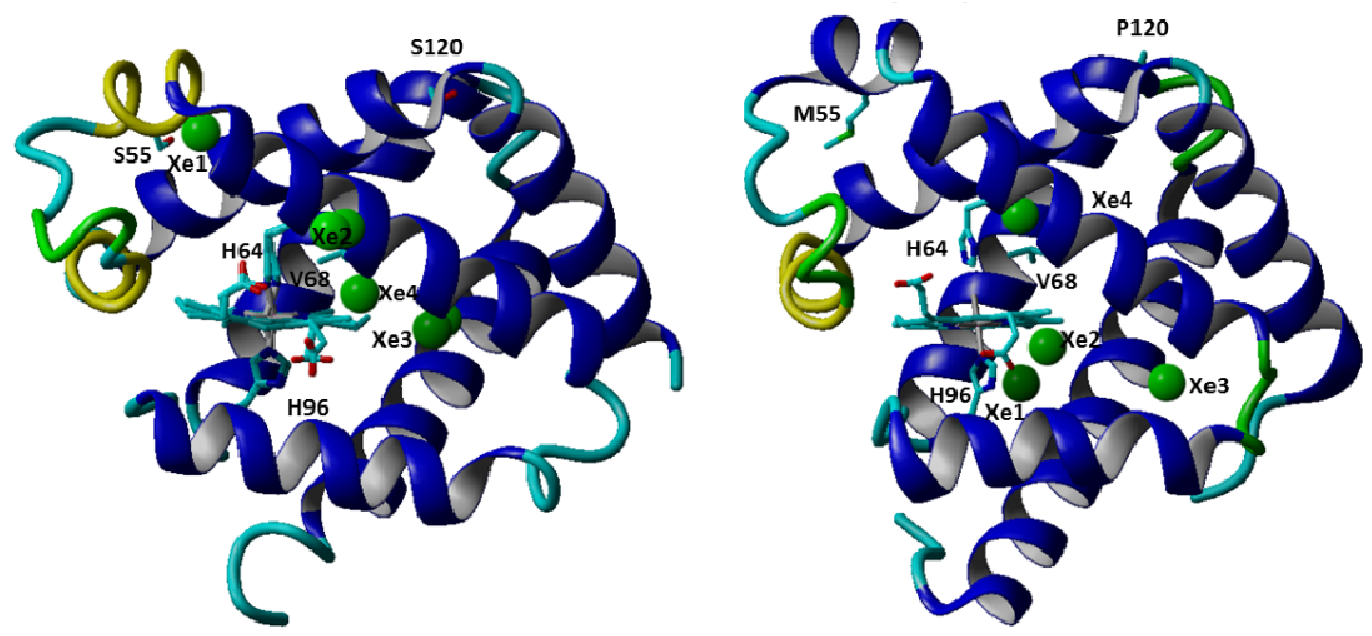

Figure 4.14 Ribbon representation of ferric mNgb (left, PDB entry 3GK9) and sperm whale $\mathrm{Mb}$ (right, PDB entry 1J52) depicting the position of the heme group, proximal His96, and distal His64, Val68, Ser120, and Ser55 in Ngb and analogous residues in Mb. Xenon cavities Xe1, Xe2, Xe3 and Xe4 are depicted as green spheres.

The mutation of distal residue His64 or Val68 significantly alters the thermodynamics of structural changes associated with ligand photo-release. The photorelease of CO from WT hNgb is endothermic $\left(\Delta \mathrm{H}=20 \pm 4 \mathrm{kcal} \mathrm{mol}^{-1}\right)$ and associated with a negative structural volume change $\left(\Delta \mathrm{V}_{\text {str }}=-21.6 \pm 0.9 \mathrm{~mL} \mathrm{~mol}^{-1}\right)$ (Astudillo et al., 2010). In the structure of $\mathrm{WT} \mathrm{Fe} \mathrm{e}^{2+} \mathrm{mNgb}$, a water molecule was resolved in the vicinity of the distal histidine that is missing in the structure of $\mathrm{CO}$ bound $\mathrm{Fe}^{2+} \mathrm{mNgb}$. The internal water molecule (Wat9 in PDB entry 1Q1F) is surrounded by the side chains of three Phe residues (Phe28, Phe42, and Phe61) and stabilized within the protein matrix through a hydrogen bond with the His64 side chain, the carbonyl oxygen of Phe61, and weak hydrogen bonds to the hydroxyl group of Tyr44 and the carbonyl oxygen of Glu60. 


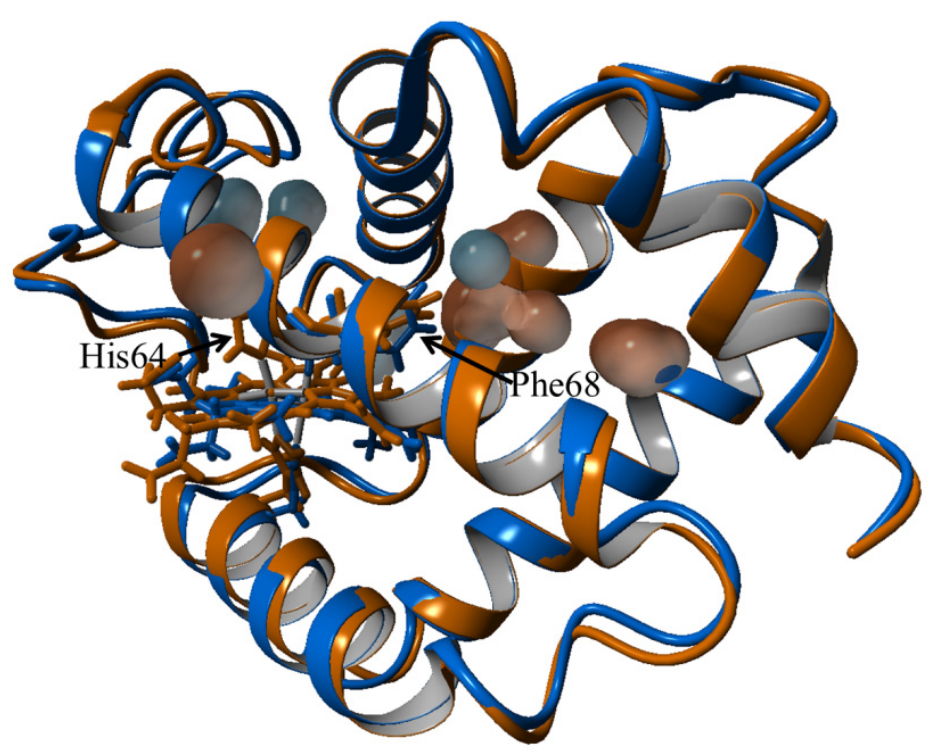

Figure 4.15 Overlay of modeled structure of $\mathrm{CO}$ bound $\mathrm{Fe}^{2+} \mathrm{mNgb}$ Val68Phe (blue) and $\mathrm{Fe}^{2+} \mathrm{mNgb}$ Val68Phe depicting the position of the heme group, distal His 64 and Phe 68 residue. Internal hydrophobic cavities are shown in light blue for $\mathrm{CO}$ bound $\mathrm{Fe}^{2+} \mathrm{mNgb}$ Val68Phe and in light orange for $\mathrm{Fe}^{2+} \mathrm{mNgb}$ Val68Phe. The modeled structures were obtained by minimization of $\mathrm{CO}$ bound $\mathrm{Fe}^{2+} \mathrm{mNgb}$ (PDB entry $1 \mathrm{~W} 92$ ) and $\mathrm{Fe}^{2+} \mathrm{mNgb}$ (PDB entry 2VRY) in which Ser 55 and Ser 120 were replaced by Cys employing Amber03 force field in YASARA software. Internal cavities were analyzed using a $1.4 \AA$ probe radius and the numerical algorithm in YASARA software and are shown as contour surfaces.

The negative structural volume change determined for photo-dissociation of $\mathrm{CO}$ from WT $\mathrm{hNgb}$ is consistent with the fast uptake of a water molecule that is concomitant with the escape of $\mathrm{CO}$ from the protein matrix. This hypothesis is further supported by the absolute magnitude of the structural volume change that is similar to the partial molar volume of water $\left(\mathrm{V}_{\mathrm{m}}{ }^{\circ}=18 \mathrm{~mL} \mathrm{~mol}{ }^{-1}\right)$. Because the water molecule forms hydrogen bonds with nearby amino acid residues, the uptake into the protein matrix does not result in a significant enthalpy change, and the observed enthalpy of $20 \mathrm{kcal} \mathrm{mol}^{-1}$ corresponds to the photodissociation of the Fe-CO bond. Xu et al. (2011) have proposed that an electrostatic/hydrogen bonding network formed by Lys67, Tyr44, His64, and heme 
7-propionate stabilizes the heme group within the heme binding pocket and $\mathrm{CO}$ association leads to the weakening of electrostatic interactions, including the breakage of the salt bridge between Lys67 and heme propionate. The enthalpy change associated with the destabilization of such polar network was estimated to be relatively small, $\sim 1.7 \mathrm{kcal} \mathrm{mol}^{-1}$ (Horovitz et al., 1990; Takano et al., 2000), and does not significantly contribute to the observed enthalpy change (Vallone et al., 2004a). In addition to $\mathrm{Fe}^{2+} \mathrm{hNgb}$ hydration, other factors such as sliding of the heme group and reshaping of the internal hydrophobic cavities may contribute to the observed volume and/or enthalpy change. However, the impact of these perturbations on the structural volume change is likely to be small as these changes are localized within the protein matrix and thus do not impact the overall protein structure and/or charge distribution on the protein surface.

The photodissociation of $\mathrm{CO}$ from the His64Gln mutant is exothermic $\left(\Delta \mathrm{H}=-6 \pm 3 \mathrm{kcal} \mathrm{mol}^{-1}\right)$ and accompanied by a negative structural volume change of $-25.0 \pm 0.5 \mathrm{~mL} \mathrm{~mol}^{-1}$. More negative volume and enthalpy changes are observed for photo-release of $\mathrm{CO}$ from the Val68Phe mutant $\left(\Delta \mathrm{H}=-48 \pm 7 \mathrm{kcal} \mathrm{mol}^{-1}\right.$, and $\left.\Delta \mathrm{V}_{\text {str }}=-37.4 \pm 1.5 \mathrm{~mL} \mathrm{~mol}^{-1}\right)$. The negative enthalpy change for photo-release of CO from $\mathrm{hNgb}$ mutants indicates that the corresponding rebinding of $\mathrm{CO}$ to five-coordinate $\mathrm{hNgb}$ is endothermic and entropically driven. A comparable positive enthalpy for binding of $\mathrm{O}_{2}$ to $\mathrm{hNgb}$ was observed by Fago et al. (2004b). These authors reported that the $\mathrm{hNgb}$ oxygenation is endothermic $\left(\Delta \mathrm{H}=12.7 \mathrm{kcal} \mathrm{mol}^{-1}\right)$ below $18^{\circ} \mathrm{C}$ and becomes exothermic at higher temperatures $\left(\Delta \mathrm{H}=-15.7 \mathrm{kcal} \mathrm{mol}^{-1}\right)$. They attributed the exothermic ligand binding to the weakening of the electrostatic/hydrogen bonding interactions among 
His64, Lys67, and heme 7-propionate at high temperatures. At low temperatures, stronger polar interactions contribute to the observed positive enthalpy for oxygen binding.

The negative reaction enthalpy observed for photo-release of CO from His64Gln $\mathrm{Fe}^{2+} \mathrm{hNgb}$ suggests that substitution of His64 promotes a reorganization of the electrostatic/hydrogen bonding network in the vicinity of the heme 7-propionate group in a similar manner to that observed for binding of oxygen to $\mathrm{hNgb}$ below $18{ }^{\circ} \mathrm{C}$. Indeed, this network of polar residues that also includes Tyr44 and a water molecule is not rigid, as evident from an overlay of $\mathrm{mNgb}$ and $\mathrm{hNgb}$ structures. For example, Lys67 forms a hydrogen bond with the heme 7-propionate in the structure of $\mathrm{Fe}^{3+} \mathrm{mNgb}$ (PDB entry 1Q1F), whereas in the structure of $\mathrm{CO}$ bound $\mathrm{mNgb}$ and $\mathrm{Fe}^{2+} \mathrm{mNgb}$ (PDB entries $1 \mathrm{~W} 92$ and 2VRY, respectively), the Lys67 side chain is positioned within hydrogen bonding distance of both heme 6- and 7-propionate group. Tyr44 is located in the highly flexible CD region (residues $40-60$ ), and its side chain was not resolved in two molecules of $\mathrm{Fe}^{3+} \mathrm{hNgb}$ (PDB entry 1OJ6), pointing toward an increased flexibility of this residue in $\mathrm{hNgb}$. In addition, computational studies have revealed that the solvent accessible area of Tyr44 increases in the CO bound form of the His64Val mutant relative to that of the WT protein, confirming that the distal His mutation alters this electrostatic network (Xu et al., 2011). These results suggest that the electrostatic/hydrogen bond network can be relatively easily reorganized either by changes in the temperature or by a single residue substitution. Xu et al. (2011) have proposed that the electrostatic/hydrogen bonding network between the heme propionate groups and the apo-protein stabilizes the position of the prosthetic group and modulates the heme sliding mechanism in Ngb. Therefore, the impact of the His64Gln substitution on the energetics of association of $\mathrm{CO}$ with five- 
coordinate hNgb may be the result of the altered heme sliding mechanism in this mutated protein.

Other factors may also contribute to the observed negative enthalpy change for CO photo-release. The UV-vis spectra revealed a small fraction of six-coordinate heme iron in $\mathrm{Fe}^{2+} \mathrm{Ngb}$ mutants lacking His64 likely because of the presence of a water molecule in the sixth axial position (Kriegl et al., 2002). Although a water molecule associated with the reduced heme iron is unusual in globins, the ferrous aquo-Mb complex was detected only below $150 \mathrm{~K}$ (Lamb et al., 1998). Strickland and Harvey (2007) have reported that binding of a water molecule to the heme model complexes of $\mathrm{Fe}^{2+}$ heme involves a low energy barrier, and it is energetically favorable by $\sim 10 \mathrm{kcal} \mathrm{mol}^{-1}$.

In the His64Gln mutant, the association of a water molecule with the ferrous heme may be facilitated by the presence of a highly reactive heme iron, which is evident from the faster kinetics for rebinding of $\mathrm{CO}$ to the five-coordinate heme iron compared to that of WT hNgb and possibly by the increased accessibility to the sixth axial position of the heme ferrous iron because of the replacement of the His64 imidazole ring with Gln. More negative enthalpy and structural volume changes were determined for photodissociation of $\mathrm{CO}$ from the Val68Phe mutant relative to the His64Gln mutant, which point toward a significant reorganization of the heme distal pocket and surrounding hydrophobic cavities and/or altered mechanism of interactions of diatomic ligands with heme iron in the Val68Phe mutant. In the absence of a crystal structure for the Val68Phe mutant, it is difficult to pinpoint structural factors that contribute to the observed thermodynamic parameters. The large negative structural volume change $\left(\Delta \mathrm{V}_{\mathrm{str}}=-37.4\right.$ $\left.\pm 1.5 \mathrm{~mL} \mathrm{~mol}^{-1}\right)$ and the exothermic enthalpy change $\left(\Delta \mathrm{H}=-48 \pm 7 \mathrm{kcal} \mathrm{mol}^{-1}\right)$ observed 
for photo-release of $\mathrm{CO}$ from the Val68Phe mutant are consistent with a reorganization of charges, including salt bridges associated with the transition between the $\mathrm{CO}$ bound $\mathrm{hNgb}$ and $\mathrm{Fe}^{2+} \mathrm{hNgb}$ that is not present in the WT protein.

\subsubsection{Role of the Cys residues in controlling ligand migration in $\mathrm{hNgb}$}

The two cysteine residues examined here, Cys55 and Cys120, are well conserved among mammalian Ngbs. Replacement of Cys120 with Ser does not alter the kinetics of geminate rebinding or bimolecular rebinding of $\mathrm{CO}$ to penta-coordinate $\mathrm{Fe}^{2+} \mathrm{hNgb}$, consistent with the position of Cys120 being near the protein surface and $\sim 19 \AA$ from the heme iron. On the other hand, bimolecular rebinding of $\mathrm{CO}$ to the five-coordinate iron in the Cys55Ser/Cys120Ser hNgb double mutant is faster than in the WT protein and displays acceleration similar to that observed for the His64Gln mutant. This result is somehow surprising because no acceleration for $\mathrm{CO}$ bimolecular rebinding was observed for the Cys55Ser hNgb single mutant, as described in chapter 5 (Astudillo et al., 2010), suggesting that simultaneous replacement of Cys120 and Cys55 leads to global structural changes that impact the rate of $\mathrm{CO}$ bimolecular rebinding.

The reaction enthalpy changes determined for photo-dissociation of $\mathrm{CO}$ from the Cys120Ser mutant and Cys55Ser/Cys120Ser double mutant are comparable and close to $0 \mathrm{kcal} \mathrm{mol}^{-1}$. On the other hand, the structural volume change measured for the Cys120Ser mutant is $\sim 6 \mathrm{~mL} \mathrm{~mol}^{-1}$ more negative than that measured for the WT protein, whereas a similar structural volume change was observed for the Cys55Ser/Cys120Ser double mutant. Previously, we reported that the single Cys55Ser mutation in hNgb does not alter the enthalpy change associated with photo-release of $\mathrm{CO}$ from $\mathrm{Ngb}$ (Astudillo et 
al., 2010), pointing out that the replacement of Cys 120 with Ser leads to a decrease in the reaction enthalpy change of $\sim 20 \mathrm{kcal} \mathrm{mol}^{-1}$.

In the crystal structure of $\mathrm{CO}$ bound $\mathrm{mNgb}$ with both Cys55 and Cys120 replaced with Ser, the hydroxyl group of Ser120 is located at the end of helix G and forms a hydrogen bond with the carbonyl oxygen of Met116, whereas in the $\mathrm{Fe}^{2+} \mathrm{mNgb}$ structure, the Ser120 side chain populates two conformations. One conformation corresponds to that found in the $\mathrm{CO}$ bound $\mathrm{Fe}^{2+} \mathrm{Ngb}$ structure, whereas the second conformation shows the Ser120 side chain rotated by $124^{\circ}$, forming a hydrogen bond with His 23 from helix B. In the $\mathrm{Fe}^{2+} \mathrm{mNgb}$ structure, the side chain of His 23 is involved in a larger hydrogen bonding network that includes Glu22 and Ser19 that is part of the A-B loop, whereas the hydrogen bond between Glu22 and His23 is missing in CO bound mNgb (Figure 4.16). Thus, I propose that in the mutants carrying a Ser residue at position 120, escape of CO from the protein matrix is associated with the fast $(\tau<50 \mathrm{~ns})$ formation of a hydrogen bond network involving Ser120, His23, and Glu22 that leads to the negative enthalpy change observed for photo-dissociation of $\mathrm{CO}$ for the Cys120Ser mutant and the Cys55Ser/Cys120Ser double mutant. In WT hNgb that carries a Cys residue at position 120, this hydrogen bonding network is missing and the observed positive enthalpy change of $20 \mathrm{kcal} \mathrm{mol}^{-1}$ represents the scission of the Fe-CO bond. These data also indicate that migration of $\mathrm{CO}$ between the protein matrix and the surrounding solvent results in reorganization of the interface between helix $\mathrm{G}$ and the A-B loop, and this interface may facilitate escape of the photo-dissociated ligand from the protein matrix. 


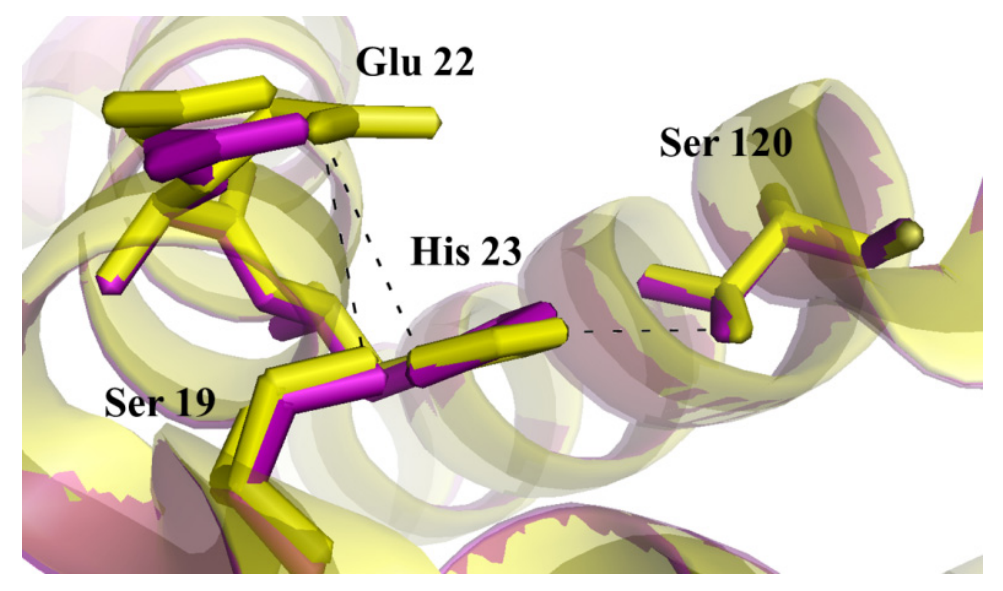

Figure 4.16 Overlay of crystal structure of CO bound mNgb (shown in purple, PDB entry 1W92) and $\mathrm{Fe}_{2}+\mathrm{mNgb}$ (shown in yellow, PDB entry 2VRY) showing the position of Ser 120, His 23, Glu 22 and Ser 19. The H-bond network connecting the helix G and the loop between the $\mathrm{A}$ and $\mathrm{B}$ helices in the $\mathrm{Fe}^{2+} \mathrm{mNgb}$ form (shown as black lines) is missing in the structure of $\mathrm{CO}$ bound $\mathrm{mNgb}$.

The analysis of the ligand migration pathways in Ngb was addressed in several computational studies. Anselmi et al. (2011) have reported that a significant fraction of the photo-dissociated $\mathrm{CO}$ molecules cross the $\mathrm{Xe}_{4}$ cavity region and escape through a phantom 1 site between helix G and the A-B loop, in excellent agreement with our experimental data. The A-B loop was also identified as a ligand transition pathway for escape of CO from Ngb, named exit 3, by Bocahut et al. (2009), although they associated this pathway with escape of $\mathrm{CO}$ from $\mathrm{hNgb}$ with the reduced disulfide bond.

The molecular mechanism of ligand migration and the role of the internal hydrophobic cavities were intensively studied in Mb using time-resolved absorption/IR spectroscopy, time-resolved X-ray crystallography, and computational studies in combination with site-directed mutagenesis (Brunori and Gibson, 2001; Olson et al., 2007; Ostermann et al., 2000; Scott et al., 2001; Srajer et al., 2001). A schematic description of the ligand migration pathways in $\mathrm{Mb}$ and $\mathrm{Ngb}$ is presented in Scheme 4.1. 

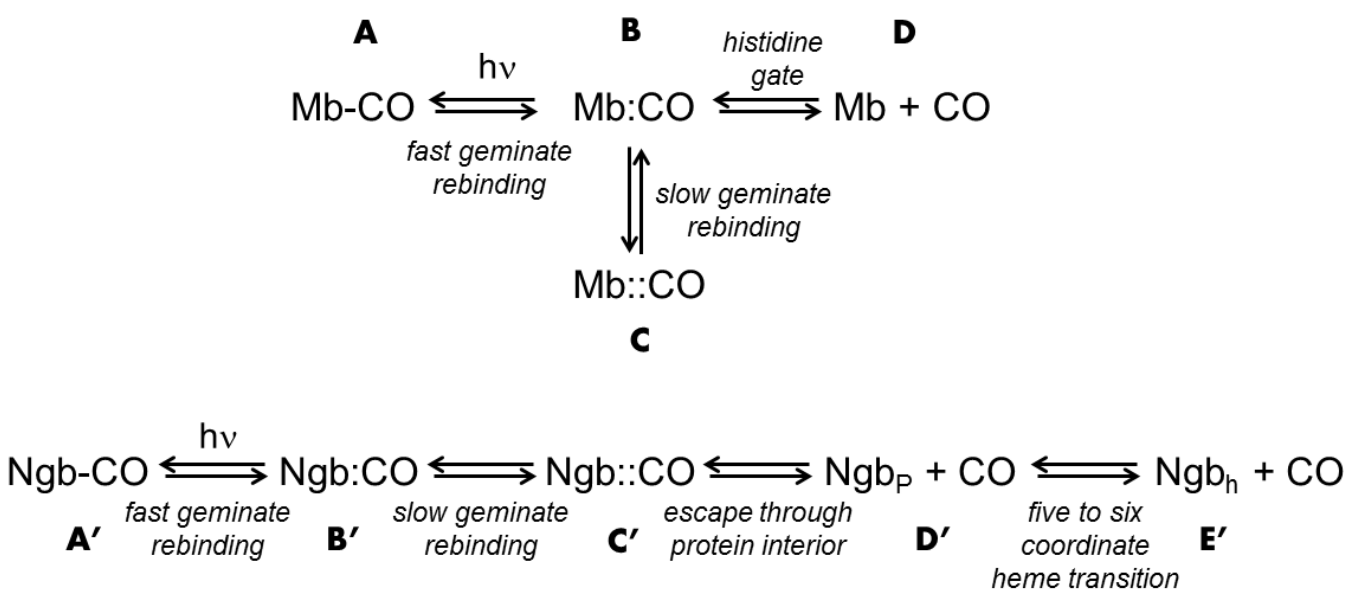

\section{Scheme 4.1}

The ligand migration in $\mathrm{Mb}$ can be described using a side path kinetic scheme (Olson et al., 2007). Upon photo-cleavage of the Fe-CO bond, the photo-released ligand rapidly translates into the primary docking site located above the heme iron plane (state B) where it remains for $<30 \mathrm{~ns}$ (Schotte et al., 2004). Subsequently, the CO molecule can either rebind to the heme iron through a geminate rebinding process, escape into the surrounding solvent (state D) through the so-called "histidine gate", or migrate into one of the nearby hydrophobic cavities, $\mathrm{Xe}_{4}$ or $\mathrm{Xe}_{1}$ site (state $\mathrm{C}$ ). To escape from the protein matrix, the photo-dissociated ligand transiently located within the hydrophobic cavities has to return to primary docking site B and then leave through the histidine gate.

The internal ligand migration in $\mathrm{Mb}$ is directed by the conformational changes of amino acid side chains located at the solvent interface and in the proximity of site B, including Ile28, Leu29, Leu32, Phe43, Phe46, His64, Val68, and Ile107 (Scott et al., 2001). For example, substitution of Leu29 with Trp blocks geminate rebinding as well as escape of the photo-dissociated ligand through the His gate and promotes ligand trapping in the $\mathrm{Xe}_{4}$ or $\mathrm{Xe}_{1}$ cavity. On the other hand, replacement of distal His64 with a nonpolar 
residue such as Leu or Val increases the rate of $\mathrm{CO}$ bimolecular rebinding as well as the geminate rebinding yield $\left(\Phi_{\text {gem }}=0.37\right.$ or 0.34 , respectively), and a significantly larger increase in the rate of geminate rebinding was observed for the His64Gln mutant $\left(\Phi_{\text {gem }}=\right.$ 0.67) (Lambright et al., 1994).

The effect of replacement of the Val68 side chain with aromatic side chains on the ligand migration pathway was probed by several groups demonstrating that the presence of a bulky side chain at position 68 results in a trapping of photo-dissociated ligands within docking site $\mathrm{B}$ and inhibition of the transition of the ligand into more remote sites, particularly the $\mathrm{Xe}_{4}$ cavity (Carver et al., 1990; Nienhaus et al., 2003; Quillin et al., 1995; Scott et al., 2001). Simultaneous alteration of both His64 and Val68 side chains effectively blocks escape of $\mathrm{CO}$ from the protein matrix, resulting in a large geminate quantum yield (Sugimoto et al., 1998).

On the basis of the kinetic and photothermal data presented here and previous spectroscopic and computational results, I propose an alternative pathway for ligand migration in Ngb. After Fe-CO bond photo-cleavage, the ligand diffuses into the primary docking site (state B') that was shown to consist of two sites: $\mathrm{B}_{1}$ ' surrounded by Phe28, Leu31, and Phe32 and site $\mathrm{B}_{2}$ ' surrounded by Phe28, His64, and Val68. The subsequent transition includes ligand migration into the $\mathrm{Xe}_{4}$ hydrophobic cavity (state C') that is facilitated by a low energy barrier, $\sim 1.1 \mathrm{~kJ} \mathrm{~mol}^{-1}$, between sites B' and C' (Nienhaus et al., 2010). As in Mb, the ligand migration between states $B^{\prime}$ and $C^{\prime}$ is gated by the side chain of Val68, which is evident from the increased rate of $\mathrm{CO}$ geminate rebinding in the Val68Phe mutant. The alteration of the His64 side chain has only a minor impact on the ligand transition between the distal pocket and site C', suggesting that in Ngb the His64 
side chain does not regulate diffusion of the ligand between internal cavities or blocks escape of the ligand from the protein matrix.

The exact pathway for escape of the ligand from state C' remains unclear. On the basis of the crystal structure of deoxy-mNgb and CO-bound $\mathrm{mNgb}$, it was suggested that the wide hydrophobic tunnel connecting the distal pocket with the surrounding solvent in the deoxy protein provides an effective pathway for ligand migration (Vallone et al., 2004b). Indeed, the $\mathrm{Xe}_{4}$ hydrophobic site is located in the vicinity of the hydrophobic tunnel entrance, indicating that the arrival of the ligand in the tunnel is regulated by the Val68 side chain. The results presented here demonstrate that the thermodynamic parameters associated with $\mathrm{CO}$ escape are altered by the hydrogen bond network connecting helix $\mathrm{G}$ and the loop between helices $\mathrm{A}$ and $\mathrm{B}$, suggesting that this region may serve as a ligand migration pathway in $\mathrm{hNgb}$ as was previously proposed by Anselmi et al. (2011). Alternatively, the rearrangement of the region between helix $\mathrm{G}$ and the A-B loop can be a part of the larger conformational transition between the ligand bound and ligand free Ngb, including the reshaping of the internal hydrophobic tunnel that provides an effective ligand escape pathway. Finally, following escape of $\mathrm{CO}$ to the solvent (state D'), the Ngb structure undergoes a considerable reorganization that involves repositioning of the heme and the re-formation of the Fe-His64 coordination bond (state E') occurring on a $100 \mathrm{~ms}$ time scale.

\subsection{Summary}

In summary, the PAC results in combination with transient absorption studies shown in this work reveal that escape of the ligand from the protein matrix in $\mathrm{hNgb}$ is 
significantly faster than in $\mathrm{Mb}$, suggesting that in $\mathrm{hNgb}$ the histidine gate mechanism does not regulate the ligand escape between the distal pocket and the surrounding solvent.

On the other hand, the highly conserved Val68 residue appears to have an analogous role in $\mathrm{hNgb}$ and in vertebrate five-coordinate globins and regulates the internal movement of the photo-dissociated ligand between the heme distal pocket and surrounding hydrophobic cavities. The thermodynamic data for photo-dissociation of the ligand from mutated proteins with Cys120 replaced with Ser indicate that the interface between helix $\mathrm{G}$ and the A-B loop may provide a pathway for the migration of $\mathrm{CO}$ from internal hydrophobic cavities to the protein exterior. The electrostatic/hydrogen bond network between the heme propionate groups and the apo-protein not only stabilizes the prosthetic group, but also influences thermodynamics of binding of the diatomic ligand to heme iron and to some extent provides a barrier for rebinding of the diatomic ligand to the five-coordinate Ngb. Altogether, these data confirm a distinct mechanism of interaction of $\mathrm{hNgb}$ with diatomic ligands compared to other vertebrate globins such as myoglobin and hemoglobin. 


\section{THE ROLE OF THE DISULFIDE BOND AND TYROSINE 44 RESIDUE IN TRANSMISSION OF STRUCTURAL INFORMATION FROM THE CD LOOP TO THE HEME BINDING POCKET IN NEUROGLOBIN}

(The work described in this chapter was partially published in Astudillo, L., Bernad, S., Derrien, V., Sebban, P., Miksovska, J. Biophys. J. 99 (2), L16-L18)

\subsection{Introduction}

Neuroglobin is a small heme protein belonging to the family of vertebrate hexacoordinated globins, predominantly found in neuronal tissues (Burmester et al., 2000), that has been proposed to have a role in neuroprotection (Greenberg et al., 2008; Sun et al., 2003). In vitro and in vivo studies have established that Ngb plays a role in the neuronal response to hypoxia and ischemia (Greenberg et al., 2008; Sun et al., 2003), although the molecular mechanism of the protective function exerted by Ngb still remains unclear. In addition, Zhang et al. (2013b) have proposed a tumor suppressor role for Ngb in hepatocellular carcinoma (HCC) since Ngb expression was down-regulated in HCC tumor tissues, whereas over-expression of Ngb suppressed HCC cell growth in vitro and tumor growth in vivo. Studies in which Ngb was knock-down in mice also support the tumor suppressor role of Ngb in HCC cells (Zhang et al., 2013b). The molecular mechanism through which $\mathrm{Ngb}$ protects cells is still not well understood, but some plausible mechanisms have been proposed including oxygen storage/transport (Burmester et al., 2000; Dewilde et al., 2001), oxygen/redox sensing (Hamdane et al., 2003), reactive oxygen or nitrogen species scavenging (Fordel et al., 2006; Zhang et al., 2013b), NO dioxygenase activity (Fago et al., 2004a; Van Doorslaer et al., 2003), and inhibition of apoptosis (Fago et al., 2008; Fago et al., 2006). 
The three dimensional structure of Ngb displays the characteristic typical 3-over3- $\alpha$ helical structure (globin fold) with several unique features, including bishistidine hexa-coordination of the heme iron in its ferric and ferrous states (Dewilde et al., 2001), an internal disulfide bond (Hamdane et al., 2003), and large internal cavities (Pesce et al., 2003). These structural features alter interactions of $\mathrm{Ngb}$ with diatomic ligands (Brunori and Vallone, 2007). For instance, the distal His residue (His 64) can be replaced by diatomic gaseous ligands $\left(\mathrm{O}_{2}, \mathrm{NO}\right.$ and $\left.\mathrm{CO}\right)$ that reversibly bind to the heme iron, hence competition between the distal histidine and exogenous ligands leads to biphasic ligand binding kinetics in which the His64 dissociation represents the rate-limiting step (Dewilde et al., 2001).

The internal disulfide bond in human Ngb (hNgb) connects the CD loop with the D helix, located between Cys46 and Cys55. Interestingly, the internal disulfide bond in Ngb is conserved among different species with exception of rodent Ngbs that carry a Gly residue at position 46, instead of a Cys residue (Figure 5.1). The internal disulfide bond in $\mathrm{Ngb}$ has been proposed to modulate interactions with other proteins (Bonding et al., 2008; Wakasugi et al., 2003). Wakasugi et al. (2003) reported that ferric Ngb binds to the $\alpha$-subunit of the heterotrimeric $G$ protein $\left(\mathrm{G}_{\alpha \mathrm{i}}\right)$ and inhibits the rate of GDP/GTP exchange, whereas the $\mathrm{CO}$ adduct does not show a significant affinity for $\mathrm{G}_{\mathrm{\alpha i}}$ suggesting for $\mathrm{Ngb}$ a role as an oxidative stress responsive sensor. Residues Glu 53 and Glu 60 were identified as possible interaction sites between $\mathrm{Ngb}$ and the $\mathrm{G}_{\alpha \mathrm{i}}$ subunit (Kitatsuji et al., 2007) and are located within a region formed by the CD loop and the D helix that undergoes a ligand induced displacement in the crystal structure of the $\mathrm{CO}$ bound protein (Vallone et al., 2004b). 


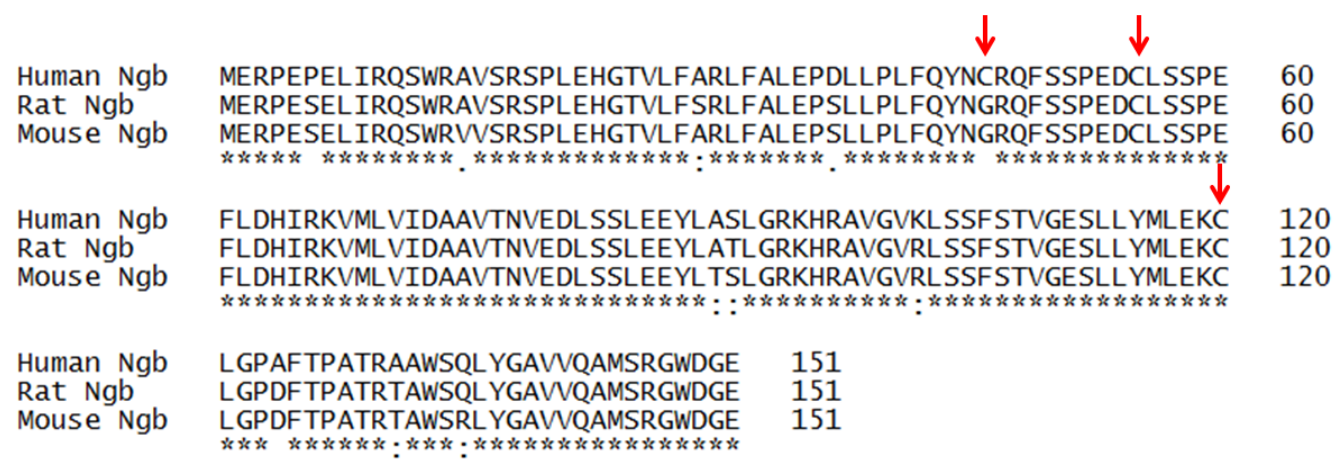

Figure 5.1 Sequence alignment of human, rat and mouse Ngb. The stars below the sequences represent identical amino acids shared by the three proteins, the two dots below the sequence indicates amino acid residues with significantly similar properties and the single dot indicates weak similarity between amino acid residues. The red arrows indicate the position of Cys residues in $\mathrm{hNgb}$ sequence.

Hamdane et al. (2003) demonstrated that reduction of the internal disulfide bond or replacement of one of the Cys residues by Ser or Gly decreases the rate of dissociation of His64 from heme iron by a factor of 10, resulting in decrease of the affinity of $\mathrm{hNgb}$ for $\mathrm{O}_{2}$. This regulation has been proposed to occur via disulfide bond induced alterations of the distal histidine dissociation rate (Hamdane et al., 2003). The molecular mechanism through which structural transitions are relayed from the CD loop to the heme distal pocket remains unclear, although Phe 28 was recently identified as a key residue in the communication between the CD loop and the heme pocket (Ezhevskaya et al., 2011). Furthermore, comparison of the crystal structures of ligand free and the $\mathrm{CO}$ adduct of mouse Ngb reveals that the association of the ligand with the heme iron triggers reorganization of the heme distal pocket that is unique among vertebrate globins, including sliding of the heme group deeper into the distal cavity, reorganization of Fhelix, and alteration of the CD and EF loop mobility (Vallone et al., 2004b). 
Inspection of the three dimensional structure of hNgb (PDB entry 1OJ6) indicates that the side chain of Tyr 44 is disordered (Figure 5.2), showing the side chain of Tyr 44 in some structures whereas the side chain of this residue is not visible in the other structures. In one of the reported structures of $\mathrm{hNgb}$, the hydroxyl group of Tyr 44 forms a hydrogen bond with the one of the heme propionate groups, which also interacts with nearby Lys 67 (Figure 5.3), suggesting that Tyr 44 may be one of the key residues that relay structural changes between the $\mathrm{CD}$ loop and the distal pocket. The interaction between Tyr 44 side chain and the heme group in $\mathrm{hNgb}$ could restrict the sliding motion that is coupled to association of exogenous ligands (Giuffre et al., 2008). Solution studies using NMR and molecular dynamic simulations indicate that inter-atomic distances increased in $\mathrm{CO}$ bound $\mathrm{Ngb}$ structure, suggesting a weaker hydrogen bond network in the structure of $\mathrm{CO}$ bound $\mathrm{Ngb}$ relative to the ligand free form (Xu et al., 2009).

Here, I present a spectroscopic investigation of the dynamics and thermodynamics of structural changes associated with the migration of exogenous ligand between the solvent and the heme active site in Ngb. To investigate the role of the internal disulfide bond on structural changes associated with ligand dissociation/binding to Ngb, I employed photoacoustic calorimetry (PAC) and transient absorption spectroscopy (TA) to determine time-resolved volume and enthalpy changes coupled to the $\mathrm{CO}$ photodissociation and the rate constants for $\mathrm{CO}$ rebinding to $\mathrm{hNgb}$ in the presence and absence of the disulfide bond. The following strains were investigated: hNgb wild type, hNgb reduced with DTT $\left(\mathrm{hNgb}^{\mathrm{red}}\right)$, Cys55Ser $\mathrm{hNgb}$ mutant, rat $\mathrm{Ngb}(\mathrm{rNgb})$, and $\mathrm{rNgb}$ Gly46Cys mutant containing an engineered disulfide bond. Indeed, in the absence of three dimensional structures for human Ngb with the disulfide bond, there is very little 
information regarding the impact of the internal disulfide bond on the conformational state of this protein. Furthermore, the role of the hydrogen bonding network within the distal pocket in Ngb and the impact of the Tyr44 amino acid side chain on transmitting structural changes in terms of disulfide formation/disruption from the CD loop to the heme iron in neuroglobin was investigated by characterizing hNgb mutants in which Tyr44 was replaced by Phe residue and the distal His 64 was substituted by Gln.
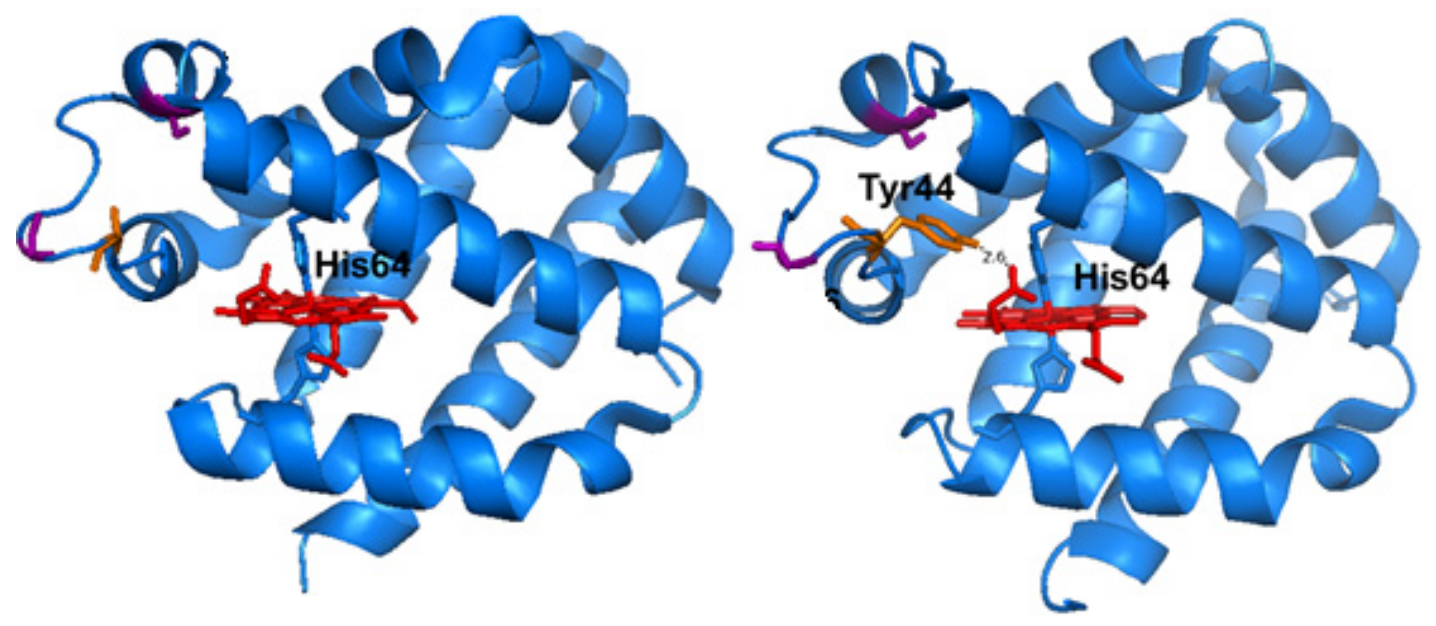

Figure 5.2 Three dimensional structure of molecule A (left) and molecule B (right) of

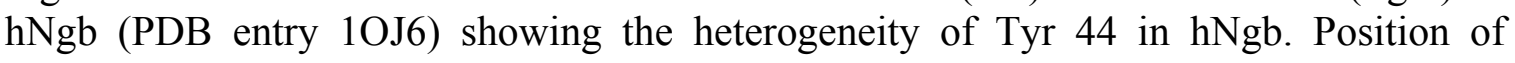
residues 46 and 55 are shown in purple. 


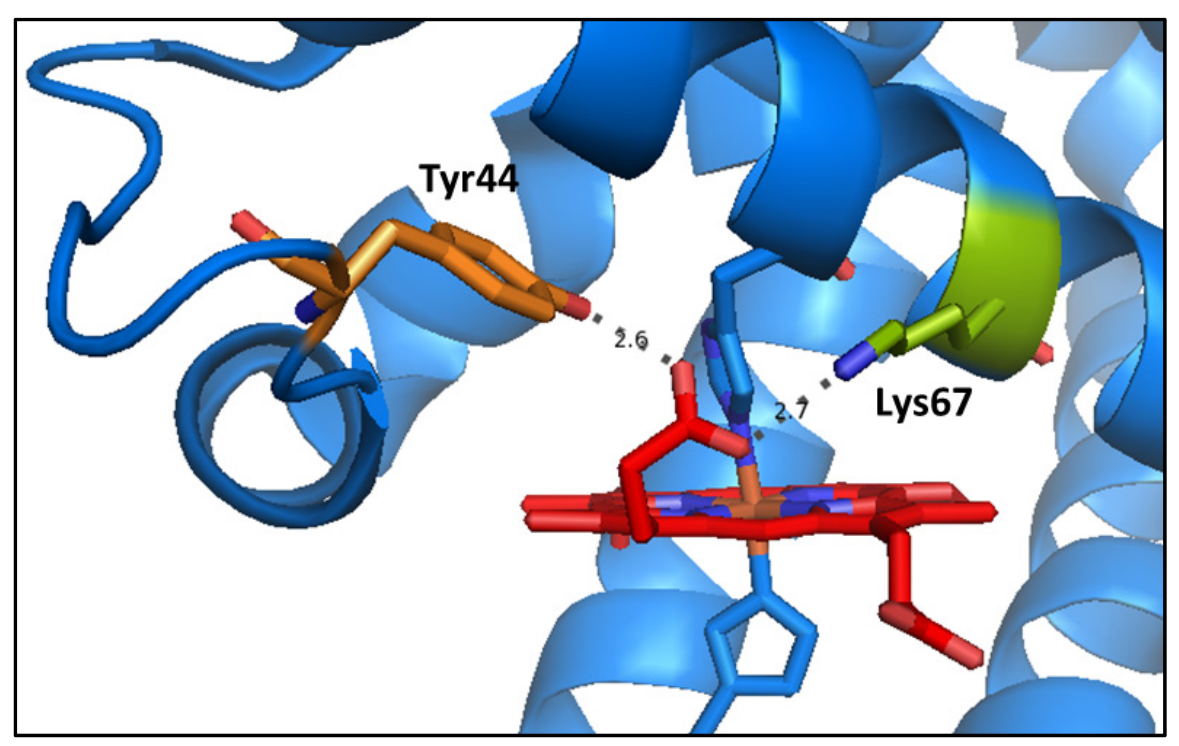

Figure 5.3 Three dimensional structure of human Ngb (PDB entry 1OJ6, molecule B) depicting the electrostatic network including His 64, Tyr 44, Lys 67 and heme propionate groups.

\subsection{Results}

\subsubsection{Steady-state absorption spectroscopy}

The steady-state absorption spectra of the ferric, ferrous and $\mathrm{CO}$ bound forms of hNgb, rNgb, rNgb Gly46Cys, and hNgb Cys55Ser mutated proteins are superimposable, indicating that replacement of residues in position 46 or 55 does not modify the electronic properties of the heme group (Table 5.1). On the other hand, replacement of the distal His 64 residue by Gln or Tyr 44 by Phe residue alters the absorption spectra of hNgb. 
Table 5.1 Absorption maxima of the Soret and Q bands of ferric, ferrous deoxy and CO adducts of wild type hNgb, wild type rNgb and mutants.

\begin{tabular}{|c|c|c|c|}
\hline Sample & & Soret band (nm) & Visible bands (nm) \\
\hline \multirow[t]{3}{*}{$\mathrm{hNgb}$} & Ferric & 413 & 535,561 \\
\hline & Ferrous & 425 & 529,559 \\
\hline & $\mathrm{CO}$ adduct & 417 & 539,565 \\
\hline \multirow[t]{3}{*}{$\mathrm{rNgb}$} & Ferric & 413 & 536,561 \\
\hline & Ferrous & 425 & 529,559 \\
\hline & $\mathrm{CO}$ adduct & 417 & 539,565 \\
\hline \multirow[t]{3}{*}{ rNgb Gly46Cys } & Ferric & 413 & 536,561 \\
\hline & Ferrous & 425 & 530,559 \\
\hline & $\mathrm{CO}$ adduct & 417 & 539,565 \\
\hline \multirow[t]{3}{*}{ hNgb Cys55Ser } & Ferric & 413 & 535,561 \\
\hline & Ferrous & 425 & 531,559 \\
\hline & $\mathrm{CO}$ adduct & 417 & 539,565 \\
\hline \multirow[t]{3}{*}{ hNgb His64Gln } & Ferric & 408 & $539,575,630$ \\
\hline & Ferrous & $420(432)^{a}$ & 556 \\
\hline & $\mathrm{CO}$ adduct & 417 & 539,567 \\
\hline \multirow[t]{3}{*}{ hNgb Tyr44Phe } & Ferric & 414 & 536,569 \\
\hline & Ferrous & $424(432)^{\mathrm{a}}$ & $529,559,583$ \\
\hline & $\mathrm{CO}$ adduct & 418 & 540,568 \\
\hline
\end{tabular}

${ }^{\mathrm{a}}$ The numbers in brackets represent the position of a shoulder. 
The steady state absorption spectra of ferric, ferrous and CO bound hNgb Tyr44Phe in $50 \mathrm{mM}$ Tris (pH 7.0) are shown in Figure 5.4. The absorption spectrum of ferric Tyr44Phe mutated protein exhibits a broad Soret band centered at $414 \mathrm{~nm}$, a pronounced visible band at $536 \mathrm{~nm}$ and a shoulder at $579 \mathrm{~nm}$. The Soret band and Q bands of $\mathrm{Fe}^{3+} \mathrm{Tyr} 44 \mathrm{Phe}$ are shifted to longer wavelengths with respect to $\mathrm{Fe}^{3+} \mathrm{hNgb}$ wild type (413 nm, $535 \mathrm{~nm}$ and $561 \mathrm{~nm})$, indicating that replacement of this residue modifies the heme pocket. Reduction of the heme iron in Tyr44Phe $\mathrm{hNgb}$ leads to a bathochromic shift of the Soret band to $424 \mathrm{~nm}$ with a shoulder at $\sim 432 \mathrm{~nm}$, and three additional bands in the visible region centered at positions $529 \mathrm{~nm}, 559 \mathrm{~nm}$, and $583 \mathrm{~nm}$. The position of the Soret band of deoxy $\mathrm{Fe}^{2+} \mathrm{Tyr} 44 \mathrm{Phe} \mathrm{hNgb}$ is comparable to that of deoxy $\mathrm{Fe}^{2+} \mathrm{hNgb}$ wild type, whereas major differences are observed in the visible region between the wild type protein and the Tyr44Phe mutated protein. The shoulder at $432 \mathrm{~nm}$ and the visible band at $556 \mathrm{~nm}$ are consistent with a fraction of penta-coordinate high-spin heme iron of approximately $23 \%$, obtained from deconvolution of the absorption spectrum, whereas the Soret band maximum at $420 \mathrm{~nm}$ indicates the presence of a fraction of Ngb with a hexa-coordinate low-spin heme iron. The absorption spectrum of the $\mathrm{CO}$ adduct of Tyr44Phe hNgb exhibits a Soret band centered at the same position as $\mathrm{CO}-\mathrm{Fe}^{2+} \mathrm{hNgb}$ $(418 \mathrm{~nm})$, and two Q band at wavelengths $540 \mathrm{~nm}$ and $568 \mathrm{~nm}$ that are consistent with the formation of low-spin hexa-coordinate heme iron.

The absorption spectrum of $\mathrm{Fe}^{2+}$ His64Gln mutant (shown in chapter 4) also indicates heterogeneity of the sample, as evident from the shoulder at $432 \mathrm{~nm}$ and the visible band at $556 \mathrm{~nm}$ that are consistent with a penta-coordinate high-spin heme iron, whereas the Soret band maximum at $420 \mathrm{~nm}$ indicates the presence of a $\mathrm{Ngb}$ fraction 
with a hexa-coordinate low-spin heme iron (Astudillo et al., 2012). Similar spectra have been previously reported for other His64 mutated proteins of hNgb, including His64Leu (Dewilde et al., 2001; Nienhaus et al., 2010) and His64Val (Tiso et al., 2011). The sixth axial ligand coordinating to the distal site that leads to the observed hexacoordinate fraction has been proposed to be a water molecule or hydroxyl group (Nienhaus et al., 2010). Moreover, residue Lys67 was identified by Uno et al. (2004) as a plausible axial ligand at basic $\mathrm{pH}$.

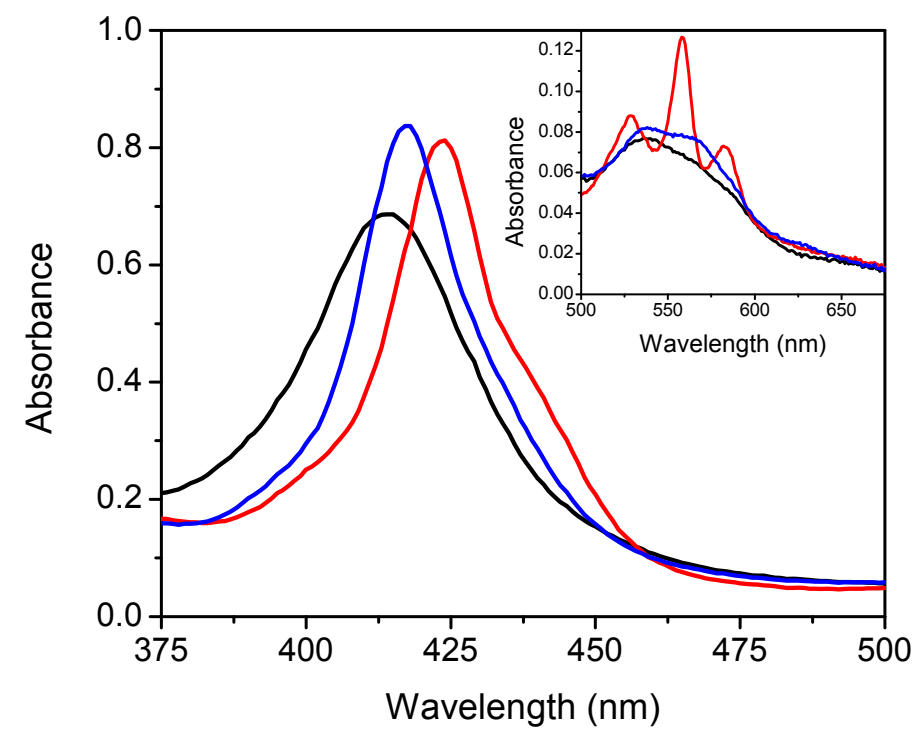

Figure 5.4 Absorption spectra of ferric (black line), ferrous (red line) and $\mathrm{CO}$ bound (blue line) $\mathrm{hNgb}$ Tyr44Phe. Conditions: $20 \mu \mathrm{M}$ protein in $50 \mathrm{mM}$ Tris buffer ( $\mathrm{pH}$ 7.0). 


\subsubsection{CO rebinding to $\mathrm{Ngb}$}

\subsubsection{The impact of the disulfide bond and distal pocket residues}

His64 and Tyr44 on CO geminate and bimolecular recombination to Ngb

Previous studies have shown that $\mathrm{CO}$ rebinding to neuroglobin is multiphasic, with geminate $\mathrm{CO}$ rebinding taking place on a nanosecond time scale and bimolecular association of $\mathrm{CO}$ to the pentacoordinate heme on the microsecond time scale (Abbruzzetti et al., 2009; Kriegl et al., 2002). Subsequent rebinding of CO to the sixcoordinate bis-histidine heme is observed on the millisecond to second time scale, with the distal His dissociation representing the rate limiting step (Kiger et al., 2004). Because the penta-coordinate and hexa-coordinate $\mathrm{Ngb}$ species are in equilibrium, the ratio of the fast to slow phase of bimolecular $\mathrm{CO}$ rebinding can be modified by varying the concentration of the ligand and the temperature. Under ligand saturation conditions and at ambient temperature, the fraction of the bis-histidine heme iron is negligible and consequently only bimolecular rebinding of $\mathrm{CO}$ to five-coordinate heme iron is observed (Dewilde et al., 2001; Kriegl et al., 2002).

Transient absorption traces on the nanosecond to microsecond time scale for $\mathrm{CO}$ rebinding to five-coordinate wild type $\mathrm{hNgb}$, Tyr44Phe and His64Gln mutated proteins studied at $20{ }^{\circ} \mathrm{C}$ under $\mathrm{CO}$ saturation conditions $(\sim 1 \mathrm{mM} \mathrm{CO})$ and in the presence and absence of the internal disulfide bond are shown in Figure 5.5. The geminate quantum yield $\left(\Phi_{\text {gem }}\right)$ was determined by taking the ratio of the amplitude of the absorbance change corresponding to the geminate process $\left(\mathrm{A}_{\mathrm{gem}}\right)$ with respect to the total absorbance change $\left(\mathrm{A}_{\text {total }}\right)$ and the calculated values are listed in Table 5.2. The quantum yield 
determined for wild type $\mathrm{hNgb}\left(\Phi_{\mathrm{gem}}=0.32\right)$ is comparable to the values determined for $\mathrm{hNgb}^{\text {red }}$ and His64Gln strains, indicating that the reduction of the disulfide bond in the wild type protein and replacement of the distal His by Gln residue does not impact the quantum yield of geminate rebinding to hNgb (Astudillo et al., 2010, 2012). An increase in the geminate quantum yield was observed for Tyr44Phe and Tyr44Phe ${ }^{\text {red }}$ mutated proteins $\left(\Phi_{\text {gem }}=0.45\right)$, indicating that disruption of the hydrogen bond between Tyr44 and one of the heme propionate groups alters the ligand migration pathways in Ngb in the presence and absence of the internal disulfide bond. A similar value for the geminate quantum yield was observed for His64Glin ${ }^{\text {red }}$ strain $\left(\Phi_{\text {gem }}=0.46\right)$. In addition, the similar effect observed on the quantum yield for geminate rebinding observed for Tyr44Phe ${ }^{\text {red }}$ and His64Gln ${ }^{\text {red }}$ suggests that in the absence of the disulfide bond, replacement of Tyr 44 by Phe and His 64 by Gln decrease the barrier for ligand recombination in Ngb.

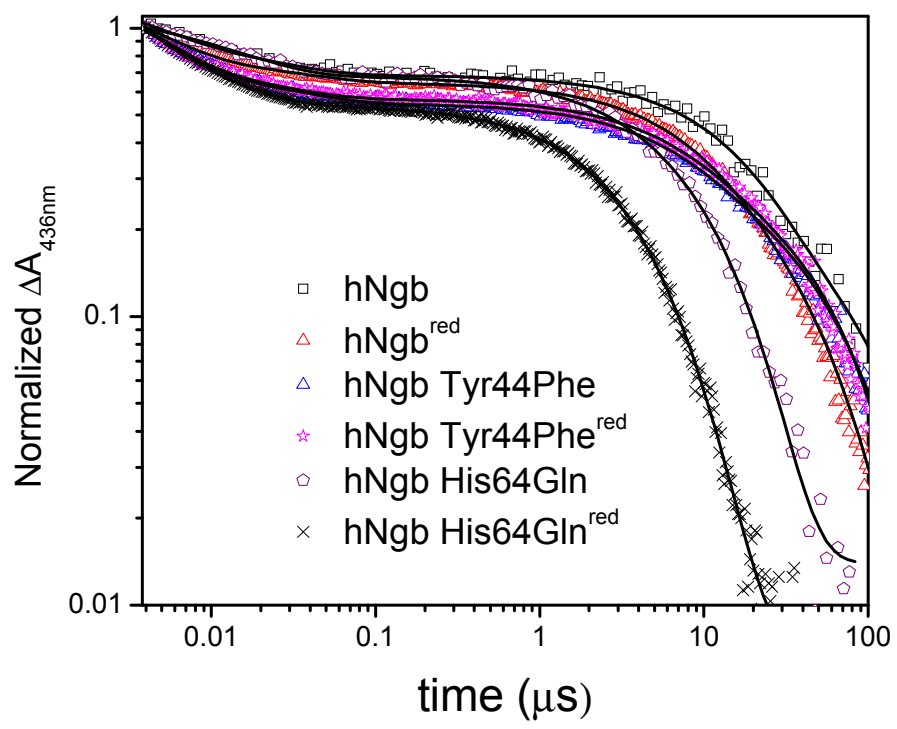

Figure 5.5 Transient absorption traces measured for $\mathrm{CO}$ rebinding to $\mathrm{hNgb}, \mathrm{hNgb}^{\text {red }}$, hNgbTyr44Phe, hNgbTyr44Phe ${ }^{\text {red }}$, hNgbHis64Gln and hNgbHis64Gln ${ }^{\text {red }}$ mutated proteins. Conditions: $20 \mu \mathrm{M}$ protein in $50 \mathrm{mM}$ Tris buffer ( $\mathrm{pH} 7.0$ ), $20^{\circ} \mathrm{C}$ and $1 \mathrm{mM} \mathrm{CO}$. 
The TA traces were analyzed using a multi-exponential decay model, according to equation 5.1.

$$
\Delta A=\sum_{i} A_{i} e^{\left(t / \tau_{i}\right)}
$$

where $\tau_{i}$ and $A_{i}$ correspond to the lifetime and amplitude for each kinetic step, respectively.

Table 5.2 Kinetic parameters associated with $\mathrm{CO}$ geminate and bimolecular rebinding to five-coordinate wild type $\mathrm{hNgb}$, His64Gln $\mathrm{hNgb}$, and Tyr44Phe $\mathrm{hNgb}$ in the presence and absence of the internal disulfide bond.

\begin{tabular}{|c|c|c|c|c|c|c|c|c|c|}
\hline Sample & $\begin{array}{c}\mathrm{k}_{\mathrm{gem} 1} \\
\left(\mathrm{x} 10^{8} \mathrm{~s}^{-1}\right)\end{array}$ & $\begin{array}{c}A_{\text {gem1 }} \\
(\%)\end{array}$ & $\begin{array}{c}\mathrm{k}_{\mathrm{gem} 2} \\
\left(\mathrm{x} 10^{7} \mathrm{~s}^{-1}\right)\end{array}$ & $\begin{array}{c}\mathrm{A}_{\text {gem2 }} \\
(\%)\end{array}$ & $\begin{array}{c}\mathrm{k}_{\mathrm{bim} 1} \\
\left(\mu \mathrm{M}^{-1} \mathrm{~s}^{-1}\right)\end{array}$ & $\begin{array}{r}A_{\text {bim1 }} \\
(\%)\end{array}$ & $\begin{array}{c}\mathrm{k}_{\mathrm{bim} 2} \\
\left(\mu \mathrm{M}^{-1} \mathrm{~s}^{-1}\right)\end{array}$ & $\begin{array}{r}A_{\text {bim2 }} \\
(\%)\end{array}$ & $\Phi_{\mathrm{gem}}$ \\
\hline $\mathrm{hNgb}$ & $2.3 \pm 0.8$ & 32.0 & $3.4 \pm 0.5$ & 15.4 & $61 \pm 7$ & 35.0 & $9 \pm 2$ & 17.6 & 0.32 \\
\hline $\mathrm{hNgb}^{\text {red }}$ & $2.5 \pm 0.9$ & 41.8 & $3.9 \pm 0.6$ & 11.7 & $226 \pm 8$ & 10.9 & $43 \pm 1$ & 35.6 & 0.32 \\
\hline His64Gln & $3.8 \pm 0.6$ & 13.3 & $7.2 \pm 0.9$ & 29.2 & $312 \pm 24$ & 27.5 & $95 \pm 3$ & 30.0 & 0.32 \\
\hline His64Gln ${ }^{\text {red }}$ & $3.0 \pm 0.6$ & 47 & $6.8 \pm 0.8$ & 16.3 & $360 \pm 16$ & 19.9 & $177 \pm 2$ & 16.4 & 0.46 \\
\hline Tyr44Phe & $2.3 \pm 0.5$ & 49.6 & $4.2 \pm 0.5$ & 12.6 & $241 \pm 6$ & 11.3 & $30 \pm 3$ & 26.5 & 0.45 \\
\hline Tyr44Phe ${ }^{\text {red }}$ & $2.4 \pm 0.6$ & 51.4 & $3.2 \pm 0.5$ & 9.4 & $238 \pm 8$ & 10.5 & $30 \pm 4$ & 28.7 & 0.45 \\
\hline
\end{tabular}

Geminate rebinding of $\mathrm{CO}$ to wild type $\mathrm{hNgb}, \mathrm{hNgb}^{\text {red }}$, His64Gln, His64Gln ${ }^{\text {red }}$, Tyr44Phe and Tyr44Phe ${ }^{\text {red }}$ mutated proteins show biphasic kinetics and the determined rate constants are listed in Table 5.2. The rate constants determined for CO geminate rebinding to wild type $\mathrm{hNgb}\left(\mathrm{k}_{\mathrm{gem} 1}=2.3 \pm 0.8 \times 10^{8} \mathrm{~s}^{-1}\right.$ and $\left.\mathrm{k}_{\mathrm{gem} 2}=3.4 \pm 0.5 \times 10^{7} \mathrm{~s}^{-1}\right)$ are comparable to the ones determined for $\mathrm{hNgb}^{\text {red }}$, His64Gln, His64Gln ${ }^{\text {red }}$, Tyr44Phe and Tyr44Phe ${ }^{\text {red }}$ mutated proteins. 
The observed heterogeneity was attributed to $\mathrm{CO}$ rebinding from primary and secondary ligand docking sites, as previously described in chapter 4 (Astudillo et al., 2012). Multiphasic kinetics has also been reported for CO geminate rebinding in Ngb and Cygb encapsulated in silica gels (Abbruzzetti et al., 2009; Gabba et al., 2013), but the fast rate constant reported here was not resolved in those studies.

The multiphasic kinetics observed for $\mathrm{CO}$ bimolecular rebinding to fivecoordinate $\mathrm{hNgb}$ have been attributed to the heterogeneous orientation of the heme group in this protein, since the ratio between the amplitude of the fast $\left(\mathrm{k}_{\mathrm{bim} 1}=61 \pm 7 \mu \mathrm{M}^{-1} \mathrm{~s}^{-1}\right.$, $\sim 65 \%$ ) and slow phase $\left(\mathrm{k}_{\mathrm{bim} 2}=9 \pm 2 \mu \mathrm{M}^{-1} \mathrm{~s}^{-1}, \sim 35 \%\right)$, matches well the ratio of $2: 1$ of the two heme isomers observed with NMR studies (Du et al., 2003). The two heme isomers differ by $180^{\circ}$ rotation around the $\alpha-\gamma$ meso axis and the ratio between these two isomers depends on the composition of the heme binding pocket (Bocahut et al., 2013; Du et al., 2003).

Reduction of the disulfide bond in hNgb leads to acceleration of $\mathrm{CO}$ bimolecular rebinding to $\mathrm{hNgb}$, as evident by the larger values determined for $\mathrm{k}_{\mathrm{bim} 1}$ and $\mathrm{k}_{\mathrm{bim} 2}$ of $226 \pm$ $8 \mathrm{M}^{-1} \mathrm{~s}^{-1}$ and $43 \pm 1 \mathrm{M}^{-1} \mathrm{~s}^{-1}$, respectively. Similar rate constants were determined for Tyr44Phe and Tyr44Phe ${ }^{\text {red }}$ proteins, indicating that reduction of the disulfide bond has a similar effect on the kinetics associated with $\mathrm{CO}$ bimolecular rebinding to $\mathrm{Ngb}$ as substitution of Tyr 44 by Phe. These results suggest that removal of the hydroxyl group in Tyr44 side chain increases accessibility of exogenous ligands to the heme iron. Interestingly, the amplitude of the fast and slow phase observed for $\mathrm{hNgb}{ }^{\text {red }}$, Tyr44Phe and Tyr44Phered are $\sim 70 \%$ to $\sim 30 \%$, which is comparable to the values determined for wild type $\mathrm{hNgb}$. 
Replacement of His 64 by Gln also accelerates both the rates for the fast and slow phase of CO bimolecular rebinding with rate constants of $312 \pm 24 \mu \mathrm{M}^{-1} \mathrm{~s}^{-1}$ and $95 \pm 3$ $\mu \mathrm{M}^{-1} \mathrm{~s}^{-1}$, respectively, and exhibit similar amplitudes (Astudillo et al., 2012). Disruption of the disulfide bond in His64Gln hNgb does not largely impact the rates associated with $\mathrm{CO}$ bimolecular rebinding to the protein.

\subsubsection{Impact of the disulfide bond on $\mathrm{CO}$ bimolecular rebinding to Ngb}

To further investigate the impact of the internal disulfide bond on the conformational dynamics of ligand binding in $\mathrm{Ngb}$, the rate constants associated with the fast and slow phases of $\mathrm{CO}$ bimolecular rebinding to the heme iron were also determined for wild type $\mathrm{hNgb}$ and $\mathrm{rNgb}$, as well as $\mathrm{hNgbCys55Ser}$ and $\mathrm{rNgbGly} 46 \mathrm{Cys}$ mutants using TA spectroscopy in the microsecond to second time scale (Figures 5.6 and 5.7). The pseudo-first order rate constants for $\mathrm{CO}$ rebinding to hexa-coordinate bis-histidine $\mathrm{Ngb}\left(\mathrm{k}_{\text {slow }}\right)$ were determined under conditions that increase the population of bis-histidyl $\mathrm{Ngb}\left(35^{\circ} \mathrm{C}\right.$ and $\left.\sim 0.1 \mathrm{mM} \mathrm{CO}\right)$. The rate constants determined for the fast and slow phases are summarized in Table 5.3.

As shown in Table 5.3, the presence of the disulfide bond moderately impacts the observed rate constants for the fast process and the reported values match well those determined previously by Hamdane et al. (2003). The rate constant for CO rebinding to six-coordinate heme is two times smaller for samples missing the internal disulfide bond (rNgb and hNgb Cys55Ser mutant) compared to hNgb and rNgb Gly46Cys, which is also in agreement with previously published data (Hamdane et al., 2003). 


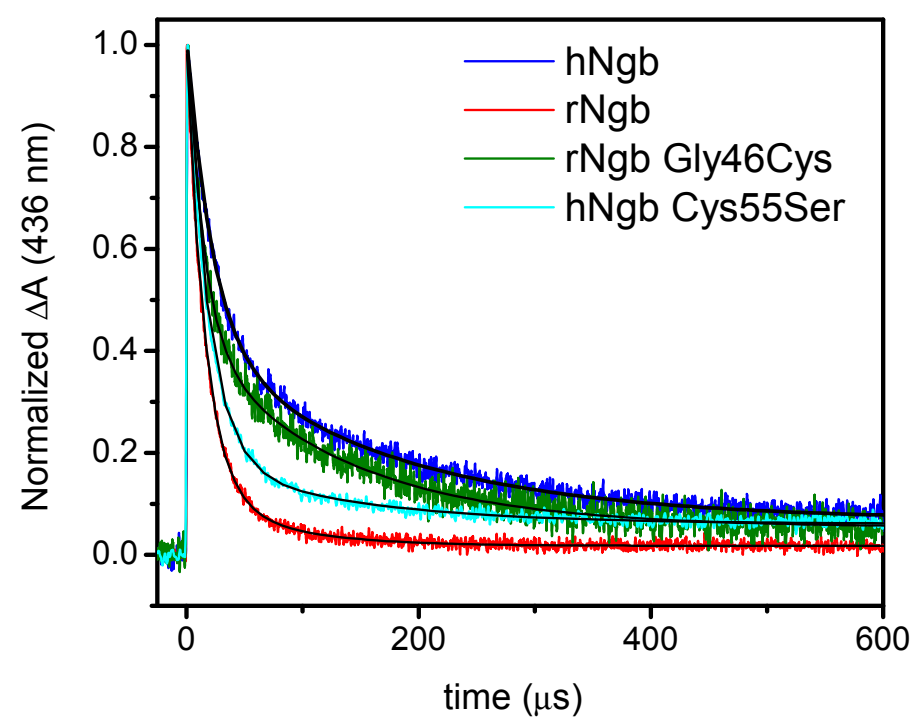

Figure 5.6 Transient absorption traces measured for $\mathrm{CO}$ rebinding to penta-coordinate $\mathrm{hNgb}$, rNgb, hNgb Cys55Ser, and rNgb Gly46Ser mutated proteins. Conditions: $20 \mu \mathrm{M}$ protein in $50 \mathrm{mM}$ Tris buffer $(\mathrm{pH} 7.0), 20^{\circ} \mathrm{C}$ and $1 \mathrm{mM} \mathrm{CO}$.

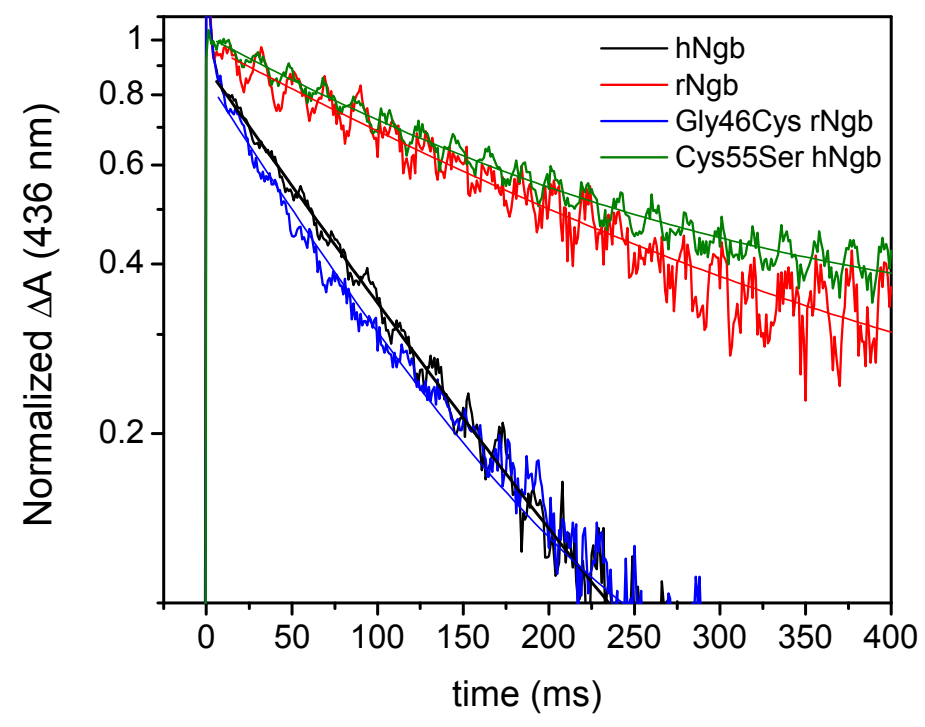

Figure 5.7 Transient absorption traces measured for $\mathrm{CO}$ rebinding to bis-histidyl hNgb, $\mathrm{rNgb}$, hNgb Cys55Ser, and rNgb Gly46Ser mutated proteins. Conditions: $20 \mu \mathrm{M}$ protein in $50 \mathrm{mM}$ Tris buffer $(\mathrm{pH} 7.0), 35^{\circ} \mathrm{C}$ and $0.1 \mathrm{mM} \mathrm{CO}$. 
Table 5.3 Kinetic parameters associated with $\mathrm{CO}$ bimolecular rebinding to wild type $\mathrm{hNgb}$, rNgb and mutants hNgb Cys55Ser and rNgb Gly46Cys.

\begin{tabular}{lccccc}
\hline \multicolumn{1}{c}{ Sample } & $\begin{array}{c}\mathrm{k}_{\text {bim1 }} \\
\left(\mu \mathrm{M}^{-1} \mathrm{~s}^{-1}\right)\end{array}$ & $\begin{array}{c}\mathrm{A}_{\text {bim1 }} \\
(\%)\end{array}$ & $\begin{array}{c}\mathrm{k}_{\text {bim2 }} \\
\left(\mu \mathrm{M}^{-1} \mathrm{~s}^{-1}\right)\end{array}$ & $\begin{array}{c}\mathrm{A}_{\text {bim2 }} \\
(\%)\end{array}$ & $\begin{array}{c}\mathrm{k}_{\text {slow }} \\
\left(\mu \mathrm{M}^{-1} \mathrm{~s}^{-1}\right)\end{array}$ \\
\hline hNgb & $62 \pm 1$ & 88 & $9 \pm 1$ & 12 & $0.10 \pm 0.01$ \\
hNgb Cys55Ser & $65 \pm 1$ & 96 & $5 \pm 1$ & 4 & $0.052 \pm 0.001$ \\
rNgb & $70 \pm 2$ & 89 & $13 \pm 2$ & 11 & $0.051 \pm 0.001$ \\
rNgb Gly46Cys & $45 \pm 1$ & 62 & $6 \pm 1$ & 38 & $0.105 \pm 0.002$ \\
\hline
\end{tabular}

\subsubsection{Photoacoustic calorimetry results}

Volume and enthalpy changes associated with $\mathrm{CO}$ photo-release from ferrous $\mathrm{Ngb}$ were determined employing PAC. In order to probe the impact of the disulfide bond in $\mathrm{hNgb}$ on volume and enthalpy changes associated with exogenous ligand photo-release, PAC measurements were performed on the proteins samples containing the disulfide bond and in proteins in which the disulfide bond was absent. The disulfide bond in $\mathrm{hNgb}$ was either chemically removed by incubation of the protein sample in $10 \mathrm{mM}$ DTT overnight to obtain $\mathrm{hNgb}^{\text {red }}$, as described in the Materials and Methods section, or removed by mutagenesis through replacement of the Cys 55 residue, which is involved in disulfide formation, by Ser residue. Since the disulfide bond is absent in rNgb, PAC measurements were also performed on rNgb wild type and a mutant of rNgb containing an engineered disulfide bond (rNgb Gly46Cys). To probe the impact of the replacement of Tyr 44 by Phe and His 64 by Gln, the following hNgb strains were also characterized using PAC: Tyr44Phe, Tyr44Phe ${ }^{\text {red }}$, His64Gln and His64Gln ${ }^{\text {red }}$. 
Representative PAC traces for CO photo-dissociation from hNgb Tyr44Phe together with the reference compound, Fe(III)tetraphenylsulfonato porphyrin (Fe4SP), are shown in Figure 5.8. The sample and reference acoustic traces overlay in phase indicating the absence of volume and enthalpy changes in $\mathrm{hNgb}$ sample on a time scale between $\sim 50 \mathrm{~ns}$ and $\sim 5 \mathrm{~ms}$, suggesting that $\mathrm{CO}$ photo-release occurs within $50 \mathrm{~ns}(\tau<$ $50 \mathrm{~ns})$. Similarly, no phase shift between the sample and the reference trace was detected for the ligand dissociation from all $\mathrm{Ngb}$ strains studied.

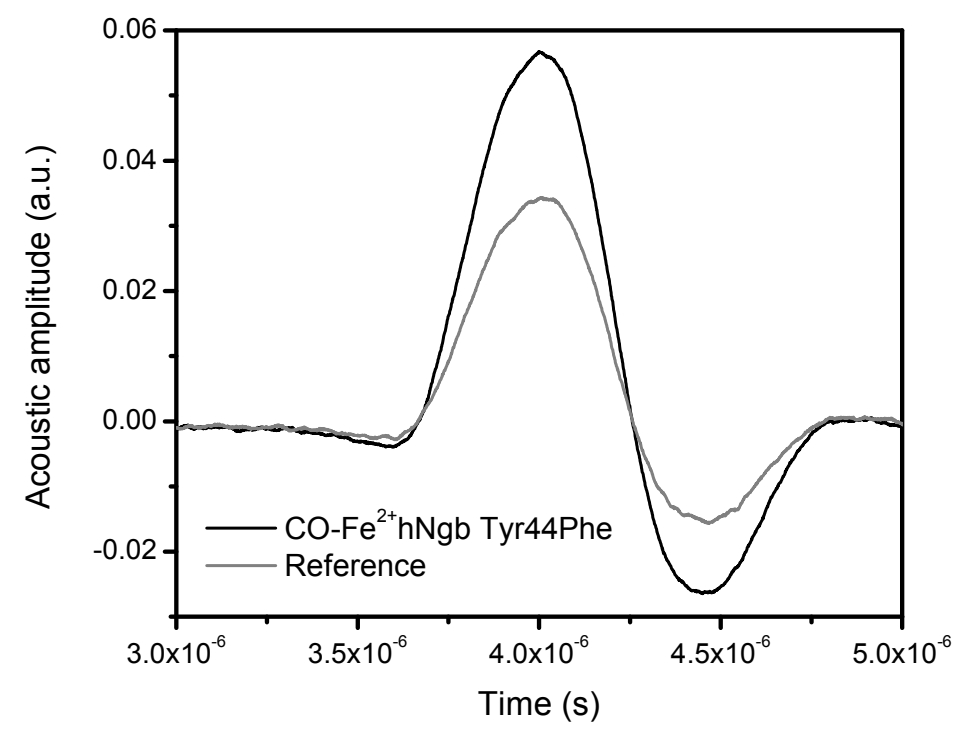

Figure 5.8 Photoacoustic traces for $\mathrm{hNgb}$ Tyr44Phe and the reference compound 4SP. Conditions: $20 \mu \mathrm{M}$ protein in $50 \mathrm{mM}$ Tris buffer ( $\mathrm{pH}$ 7). The absorbance of the sample matched that of the reference compound at $532 \mathrm{~nm}$.

The volume and enthalpy changes taking place within 50 ns upon ligand photorelease were determined from a plot of the $\left[\mathrm{E}_{\mathrm{hv}}(1-\phi)\right] / \Phi$ term as a function of the temperature dependent factor $(\mathrm{Cp} \rho / \beta)$ measured in the temperature range between $16{ }^{\circ} \mathrm{C}$ and $35^{\circ} \mathrm{C}$ (Figure 5.9), as described in the Materials and Methods section. The enthalpy 
and volume changes associated with $\mathrm{CO}$ escape from Ngbs were calculated from the intercept and slope, respectively, and are listed in table 5.4.

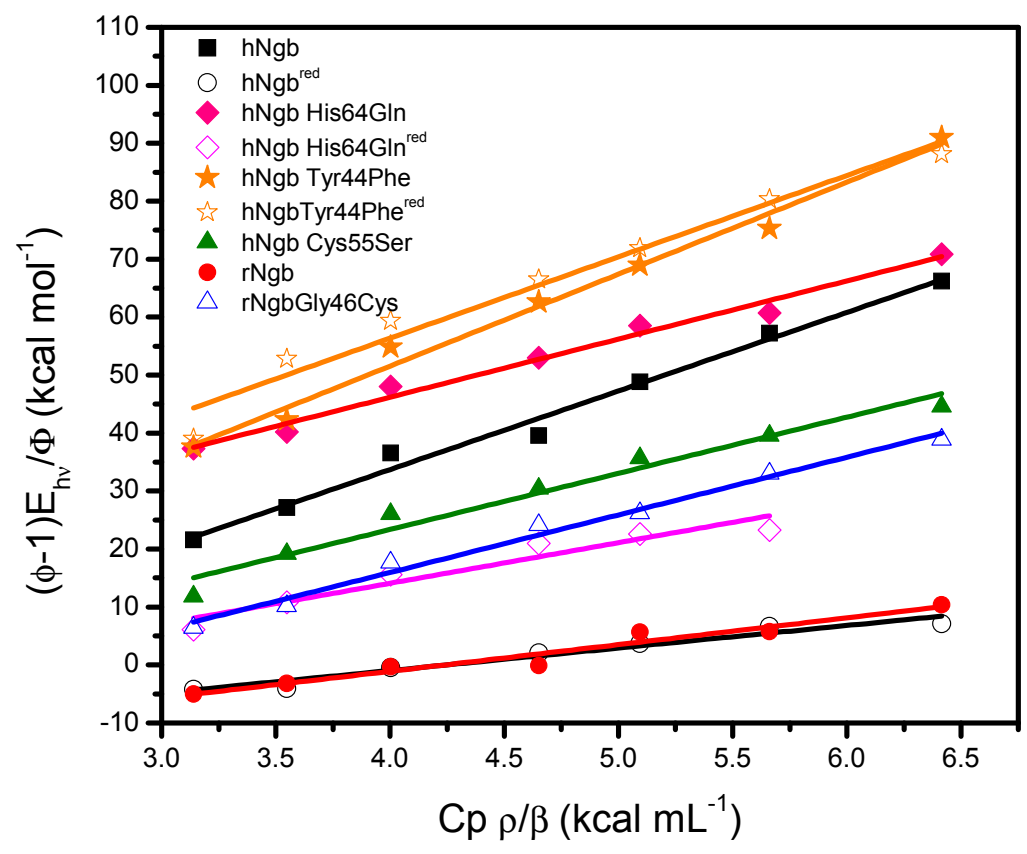

Figure 5.9 Plot of $\left[\mathrm{E}_{\mathrm{hv}}(\phi-1)\right] / \Phi$ as a function of $\mathrm{C}_{\mathrm{p}} \rho / \beta$ for $\mathrm{CO}$ photo-release from wild type $\mathrm{hNgb}, \mathrm{rNgb}$ and mutated proteins in the presence and absence DTT.

Similar values of enthalpy changes $\left(\sim 19 \mathrm{kcal} \mathrm{mol}^{-1}\right)$ were observed for $\mathrm{hNgb}$, $\mathrm{rNgb}, \mathrm{hNgb}{ }^{\text {red }}$, hNgb Cys55Ser and rNgb Gly46Cys. This value is close to the enthalpy change determined using PAC for CO photo-release from heme model complexes with imidazole as proximal ligand $\left(\Delta \mathrm{H}=17 \mathrm{kcal} \mathrm{mol}^{-1}\right)$ (Miksovska et al., 2005), suggesting that the observed enthalpy change reflects the cleavage of the $\mathrm{Fe}-\mathrm{CO}$ bond, whereas the concomitant structural changes are predominantly entropy driven. These results indicate that reduction of the internal disulfide bond in $\mathrm{hNgb}$ does not impact the enthalpy change associated with $\mathrm{CO}$ release from the protein. 
Table 5.4 Thermodynamic parameters associated with $\mathrm{CO}$ escape from wild type Ngb and mutants.

\begin{tabular}{lcc}
\hline Sample & $\Delta \mathrm{H}\left(\mathrm{kcal} \mathrm{mol}^{-1}\right)$ & $\Delta \mathrm{V}\left(\mathrm{mL} \mathrm{mol}^{-1}\right)$ \\
\hline $\mathrm{hNgb}$ & $20 \pm 4$ & $13.4 \pm 0.9$ \\
$\mathrm{hNgb}^{\text {red }}$ & $19 \pm 2$ & $4.4 \pm 0.3$ \\
$\mathrm{rNgb}$ & $19 \pm 2$ & $4.6 \pm 0.3$ \\
$\mathrm{hNgb}$ Cys55Ser & $19 \pm 2$ & $7.1 \pm 0.7$ \\
$\mathrm{rNgb}$ Gly46Cys & $19 \pm 4$ & $10.3 \pm 0.6$ \\
$\mathrm{hNgb}$ Tyr44Phe & $10 \pm 3$ & $13.3 \pm 0.6$ \\
$\mathrm{hNgb}$ Tyr44Phe & \\
$\mathrm{hNgb}$ His64Gln & $0 \pm 4$ & $11.7 \pm 0.9$ \\
$\mathrm{hNgb}$ His64Gln & $-6 \pm 3$ & $10.0 \pm 0.5$ \\
& $18 \pm 6$ & $8.8 \pm 1.2$ \\
\hline
\end{tabular}

On the other hand, the enthalpy change determined for hNgb Tyr44Phe mutated protein $\left(\Delta \mathrm{H}=10 \pm 4 \mathrm{kcal} \mathrm{mol}^{-1}\right)$ is smaller than the one determined for wild type $\mathrm{hNgb}$ and this decrease corresponds to a decrease of approximately $10 \mathrm{kcal} \mathrm{mol}^{-1}$ with respect to wild type $\mathrm{hNgb}$. This difference in enthalpy change between wild type $\mathrm{hNgb}$ and Tyr44Phe strain could be attributed to the missing hydrogen bond between the hydroxyl group of Tyr 44 side chain and one of the propionate groups from the heme group that leads to destabilization of the electrostatic network including Tyr 44, heme propionate group, His 64 an Lys 67. Reduction of the disulfide bond in hNgb Tyr44Phe leads to a larger decrease in enthalpy change associated with $\mathrm{CO}$ escape from the protein with a $\Delta \mathrm{H}$ close to zero for hNgb Tyr44Phe ${ }^{\text {red }}$. The enthalpy change associated with CO release from $\mathrm{hNgb}$ His64Gln mutated protein is exothermic $\left(\Delta \mathrm{H}=-6 \pm 3 \mathrm{kcal} \mathrm{mol}^{-1}\right)$, whereas the 
enthalpy change for $\mathrm{hNgb} H$ His $64 \mathrm{Gln}^{\text {red }}$ is endothermic with a value of $18 \pm 6 \mathrm{kcal} \mathrm{mol}^{-1}$ that is comparable to the one observed for wild type $\mathrm{hNgb}$.

The reaction volume change, $\Delta \mathrm{V}$, corresponds to differences in the partial molar volumes of the products and reactants and can be expressed as:

$$
\Delta V=V_{C O}+V_{N g b}-V_{C O N g b}
$$

where $\mathrm{V}_{\mathrm{CO}}$ corresponds to the partial molar volume of $\mathrm{CO}\left(35 \mathrm{~mL} \mathrm{~mol}^{-1}\right.$ (Hara et al., 1996)), and $\mathrm{V}_{\mathrm{Ngb}}$ and $\mathrm{V}_{\mathrm{CONgb}}$ refer to the partial molar volume of the ligand free, fivecoordinated Ngb, and CO bound Ngb, respectively. Therefore, $\mathrm{CO}$ photo-release from $\mathrm{hNgb}$ is associated with the overall structural volume change $\left(\Delta \mathrm{V}_{\mathrm{str}}=\mathrm{V}_{\mathrm{Ngb}}-\mathrm{V}_{\mathrm{CONgb}}\right)$ of $-21.6 \mathrm{~mL} \mathrm{~mol}{ }^{-1}$. A more structural volume change was detected for $\mathrm{rNgb}\left(\Delta \mathrm{V}_{\mathrm{str}}=-30.4\right.$ $\left.\mathrm{mL} \mathrm{mol}{ }^{-1}\right)$ and $\mathrm{hNgb}^{\text {red }}\left(\Delta \mathrm{V}_{\mathrm{str}}=-30.6 \mathrm{~mL} \mathrm{~mol}^{-1}\right)$. Engineering of the disulfide bond into $\mathrm{rNgb}$ structure in $\mathrm{rNgb}$ Gly46Cys mutated protein leads to a structural volume change approaching to that of $\mathrm{hNgb}\left(\Delta \mathrm{V}_{\text {str }}=-24.7 \mathrm{~mL} \mathrm{~mol}^{-1}\right)$, whereas $\mathrm{hNgb}$ Cys55Ser mutant displays a more negative volume change than $\mathrm{hNgb} \mathrm{WT}\left(\Delta \mathrm{V}_{\text {str }}=-27.9 \mathrm{~mL} \mathrm{~mol}^{-1}\right)$.

The structural volume change associated with $\mathrm{CO}$ photo-release from $\mathrm{hNgb}$ Tyr44Phe $\left(\Delta \mathrm{V}_{\text {str }}=-21.7 \mathrm{~mL} \mathrm{~mol}^{-1}\right)$ and Tyr44Phe $\mathrm{Ped}^{\text {red }}\left(\Delta \mathrm{V}_{\mathrm{str}}=-23.3 \mathrm{~mL} \mathrm{~mol}{ }^{-1}\right)$ are similar to the one determined for wild type $\mathrm{hNgb}$, indicating that replacement of Tyr44 by Phe in the presence and absence of the disulfide bond only impacts the overall reaction enthalpy change associated with $\mathrm{CO}$ photo-release from $\mathrm{Ngb}$.

Similarly, a small decrease in the overall structural volume change associated with CO photo-release was observed for hNgb His64Gln mutated protein in the presence of the disulfide bond $\left(\Delta \mathrm{V}_{\mathrm{str}}=-25.0 \mathrm{~mL} \mathrm{~mol}^{-1}\right)$ and in the absence of the disulfide bond in $\mathrm{hNgb}$ His64Gln ${ }^{\text {red }}\left(\Delta \mathrm{V}_{\mathrm{str}}=-26.2 \mathrm{~mL} \mathrm{~mol}^{-1}\right)$, compared to wild type $\mathrm{hNgb}$. 


\subsubsection{Variation of $\mathrm{CO}$ concentration}

In order to determine the thermodynamic parameters associated with $\mathrm{CO}$ rebinding five-coordinate wild type $\mathrm{Ngb}$ and mutants in terms of dissociation constant, free energy change and entropy change, $\mathrm{CO}$ rebinding to Ngbs was measured at various $\mathrm{CO}$ concentrations. In the simplest case, which is usually satisfied by penta-coordinate hemoglobins, ligand binding is described by the ratio of the on and off rates and denoted by the equilibrium coefficient $\mathrm{K}_{\mathrm{d}(\mathrm{penta})}$ as described by equation 5.3 (Uzan et al., 2004).

$$
K_{d(\text { penta })}=\frac{k_{C O, o n}}{k_{C O, o f f}}
$$

where $k_{C O, \text { on }}$ corresponds to the $\mathrm{CO}$ association rate constant and $k_{C O \text {,off }}$ refers to the $\mathrm{CO}$ dissociation rate constant.

The observed rate constants $\left(k_{\mathrm{obs}}\right)$ associated with $\mathrm{CO}$ rebinding to five-coordinate Ngbs were plotted as a function of CO concentration (Figure 5.10) and the rate of CO association $\left(k_{C O, \text { on }}\right)$ corresponds to the slope of the linear plot, whereas the rate of $\mathrm{CO}$ dissociation $\left(k_{C O \text {,off }}\right)$ corresponds to the intercept of the plot. The $\mathrm{CO}$ association and dissociation rate constants are listed in table 5.5. At very low concentrations of $\mathrm{CO}$, histidine rebinding becomes dominant and the observed rate tends to approach the on rate for histidine recombination (Uzan et al., 2004). The equilibrium dissociation constants were calculated as described in equation 5.3 and are listed in table 5.6. The change in free energy $(\Delta \mathrm{G})$ and the entropy change $(\Delta \mathrm{S})$ for $\mathrm{CO}$ dissociation from $\mathrm{hNgb}, \mathrm{hNgb}{ }^{\text {red }}$, Tyr44Phe and Tyr44Phe ${ }^{\text {red }}$ were calculated according to equations 5.4 and 5.5, respectively (Pierce et al., 1999), and the calculated values are listed in table 5.6. 


$$
\begin{aligned}
\Delta G_{d} & =-R T \ln K_{d} \\
\Delta S_{d} & =\frac{\Delta H_{d}-\Delta G_{d}}{T}
\end{aligned}
$$

where $\mathrm{R}$ is the universal gas constant, $\mathrm{T}$ is the temperature in $\mathrm{K}$, and $\Delta \mathrm{H}_{\mathrm{d}}$ is the enthalpy change determined from PAC measurements. Since $\mathrm{CO}$ bimolecular rebinding to $\mathrm{hNgb}$ and $\mathrm{hNgb}{ }^{\text {red }}$ exhibits two phases, the ligand association and dissociation rate constants as well as the $K_{d}$ were determined for the fast and slow phase as shown in tables 5.5 and 5.6.

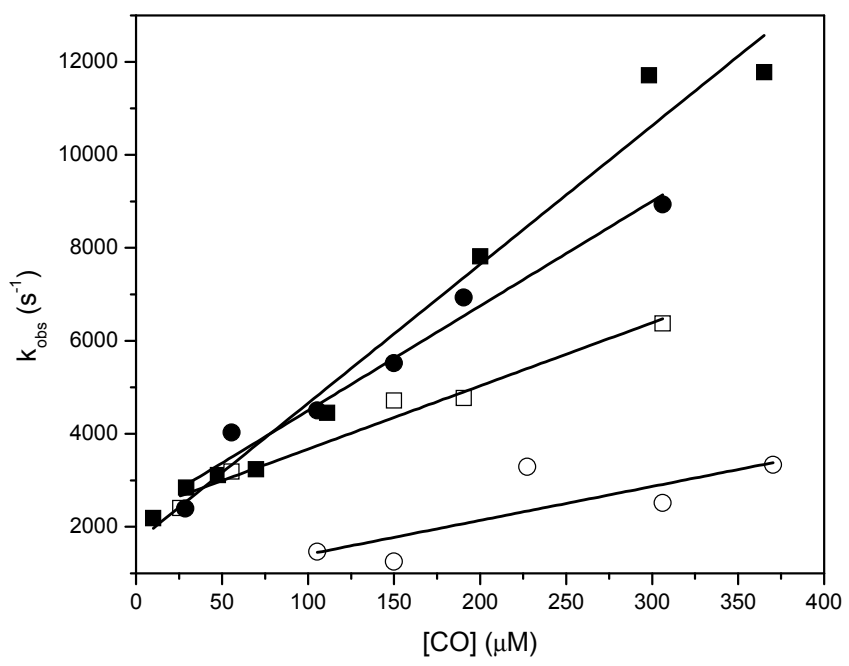

Figure 5.10 Observed rate constants associated with $\mathrm{CO}$ rebinding to $\mathrm{hNgb}$ (closed squares), $\mathrm{hNgb}^{\text {red }}$ (open squares), $\mathrm{hNgbY} 44 \mathrm{~F}$ (closed circles) and $\mathrm{hNgbY}_{44 \mathrm{~F}^{\text {red }}}$ (open circles) as a function of concentration of $\mathrm{CO}$. Conditions: $20 \mu \mathrm{M}$ protein in $50 \mathrm{mM}$ Tris buffer ( $\mathrm{pH} 7.0$ ), $20^{\circ} \mathrm{C}$ and $\mathrm{CO}$ concentrations from $10 \mu \mathrm{M}$ to $300 \mu \mathrm{M}$. 
Table 5.5 Rate constants for $\mathrm{CO}$ association and dissociation to Ngbs

\begin{tabular}{cccc}
\hline Sample & & $\mathrm{k}_{\mathrm{CO} \text {, on }}\left(\mu \mathrm{M}^{-1} \mathrm{~s}^{-1}\right)$ & $\mathrm{k}_{\mathrm{CO} \text {, ff }}\left(\mathrm{s}^{-1}\right)$ \\
\hline $\mathrm{hNgb}$ & Fast & $36 \pm 1$ & $862 \pm 48$ \\
& Slow & $4.7 \pm 0.2$ & $834 \pm 9$ \\
$\mathrm{hNgb}^{\text {red }}$ & Fast & $16.9 \pm 0.6$ & $1863 \pm 26$ \\
& Slow & $2.4 \pm 0.6$ & $656 \pm 23$ \\
Tyr44Phe & Fast+slow & $23 \pm 2$ & $2246 \pm 35$ \\
Tyr44Phe & Fast+slow & $4.9 \pm 0.9$ & $1187 \pm 40$ \\
\hline
\end{tabular}

Table 5.6 Dissociation constants, $\Delta \mathrm{G}$ and $\Delta \mathrm{S}$ for $\mathrm{CO}$ dissociation from Ngbs

\begin{tabular}{lccccc}
\hline Sample & & $\mathrm{K}_{\mathrm{d}}(\mu \mathrm{M})$ & $\Delta \mathrm{G}_{\mathrm{d}}\left(\mathrm{kcal} \mathrm{mol}^{-1}\right)$ & $\Delta \mathrm{H}_{\mathrm{d}}\left(\mathrm{kcal} \mathrm{mol}^{-1}\right)$ & $\Delta \mathrm{S}_{\mathrm{d}}\left(\mathrm{cal} \mathrm{K}^{-1} \mathrm{~mol}^{-1}\right)$ \\
\hline $\mathrm{hNgb}$ & Fast & $23.9 \pm 0.6$ & $6.2 \pm 0.2$ & $20 \pm 4$ & $47 \pm 4$ \\
& Slow & $177.5 \pm 0.9$ & $5.0 \pm 0.3$ & $20 \pm 4$ & $51 \pm 4$ \\
$\mathrm{hNgb}^{\text {red }}$ & Fast & $273.6 \pm 0.8$ & $4.8 \pm 0.2$ & $19 \pm 2$ & $49 \pm 2$ \\
& Slow & $119.4 \pm 0.7$ & $5.3 \pm 0.2$ & $19 \pm 2$ & $47 \pm 2$ \\
\multirow{2}{*}{ Tyr44Phe } & Fast+slow & $99.8 \pm 0.6$ & $5.4 \pm 0.3$ & $10 \pm 3$ & $16 \pm 4$ \\
Tyr44Phe & Fast+slow & $238.9 \pm 0.9$ & $4.9 \pm 0.4$ & $0 \pm 4$ & $-17 \pm 5$ \\
\hline
\end{tabular}

The change in free energy calculated for $\mathrm{CO}$ dissociation from $\mathrm{hNgb}\left(\Delta \mathrm{G}_{\mathrm{d}}=6.2 \pm\right.$ $\mathrm{kcal} \mathrm{mol}^{-1}$ ) is comparable to the ones determined for $\mathrm{hNgb}^{\text {red }}$, Tyr44Phe and Tyr44Phe ${ }^{\text {red }}$, indicating that reduction of the disulfide bond and disruption of the hydrogen bonding network between the hydroxyl group of Tyr 44 side chain and the propionate group of the heme does not impact the energy for $\mathrm{CO}$ dissociation from the heme group. A comparable $\Delta \mathrm{G}_{\mathrm{d}}$ of $\sim 9 \mathrm{kcal} \mathrm{mol}^{-1}$ was previously determined for wild type $\mathrm{hNgb}$ (Uno et al., 2004). On the other hand, replacement of Tyr 44 by Phe results in a lower entropy 
change and an even larger decrease in entropy was observed in Tyr44Phe ${ }^{\text {red }}$ mutant $\left(\Delta \mathrm{S}_{\mathrm{d}}=-17 \pm 5 \mathrm{cal} \mathrm{K}^{-1} \mathrm{~mol}^{-1}\right)$, which indicates a change in the degrees of freedom likely

as a result of larger overall flexibility in Tyr44Phe ${ }^{\text {red }}$. The thermodynamic parameters associated with $\mathrm{CO}$ photo-dissociation from wild type $\mathrm{Ngb}$ and mutated proteins suggest an entropy-enthalpy compensation effect. In ligand binding studies, the term entropyenthalpy compensation describes the effect observed when modification of a protein (or a ligand) results in a change in the enthalpic contribution to binding, which is partially or fully balanced by a comparable change in the entropic contribution to association, thereby resulting in no net gain in affinity (Chodera and Mobley, 2013). Several factors can contribute to observe entropy-enthalpy compensation in protein-ligand interactions, including modification of hydrogen bonding and other factors such as changes in $\mathrm{pH}$ and solvent composition (Chodera and Mobley, 2013; Meloun and Ferencikova, 2012).

\subsection{Discussion}

\subsubsection{The disulfide bond affects kinetics and energetics of $\mathrm{CO}$ migration in} Ngb

The fast ligand photo-release thermodynamics observed in PAC indicates that the major fraction of $\mathrm{CO}$ escapes from the protein through a direct pathway, either permanent or transitory, that links the Ngb heme binding pocket with the surrounding solvent. Although Abbruzzetti et al. (2009) reported that kinetics for CO migration between Ngb internal cavities occur on 100 ns time scale, those kinetics were not resolved in PAC measurements because of the very low fraction $(<10 \%)$ of "CO trapped" intermediates. The PAC results clearly indicate that the presence of the internal disulfide bond modulate overall structural changes associated with the transition from six-coordinate $\mathrm{CO}$ bound 
$\mathrm{Ngb}$ to five-coordinate deoxyNgb by approximately $8 \mathrm{~mL} \mathrm{~mol}^{-1}$. An overlay of the structure of ferrous and $\mathrm{CO}$ bound mouse $\mathrm{Ngb}$ reveals that ligand binding to the heme iron leads to the repositioning of the heme prosthetic group, reshaping of the internal cavity, and displacement of structural elements including the EF- and CD- loops (Vallone et al., 2004b). Molecular dynamics studies have suggested that the disulfide bridge restricts the flexibility of $\mathrm{Ngb}$ structure, including the $\mathrm{CD}$ loop region and $\mathrm{A}, \mathrm{C}$, and $\mathrm{E}$ helices and modulate the volume of internal cavities (Bocahut et al., 2009; Nadra et al., 2008). In addition, the heme sliding mechanism proposed in $\mathrm{rNgb}$ structure was suggested to be protein dependent and thus absent in hNgb (Anselmi et al., 2007; Bocahut et al., 2009; Nadra et al., 2008). Such restricted structural dynamics as a result of the disulfide bridge may then translate into the smaller overall structural volume changes determined for $\mathrm{hNgb}$ relative to $\mathrm{rNgb}$ or $\mathrm{hNgb}^{\text {red }}$. The Ser residue at position $55 \mathrm{in} \mathrm{hNgb}$ may form a hydrogen bond with Cys46 and stabilize the CD loop resulting in smaller structural volume change determined for the $\mathrm{hNgb}$ Cys55Ser mutated protein compared to $\mathrm{hNgb}{ }^{\text {red }}$. Considering the high sequence homology between $\mathrm{hNgb}$ and $\mathrm{rNgb}(94 \%)$, the fact that the structural volume change observed for the rNgb Gly46Cys mutant does not match that for $\mathrm{hNgb}$ is somewhat surprising. This result points out that other factors, in addition to the disulfide bond, may contribute to the difference between the overall volume changes measured for $\mathrm{hNgb}$ and $\mathrm{rNgb}$.

To further investigate the impact of the internal disulfide bond on the conformational dynamics of ligand binding in $\mathrm{Ngb}$, the rate constants for $\mathrm{CO}$ binding to the heme iron were also determined using time-resolved absorption spectroscopy. The presence of the disulfide bond only moderately impacts the observed rate constants for 
the fast process and the reported values match well those determined previously (Hamdane et al., 2003). Our data show that the rate constant for CO rebinding to sixcoordinate heme is two times smaller for samples missing the internal disulfide bond (rNgb and $\mathrm{hNgb}$ Cys55Ser mutant) compared to $\mathrm{hNgb}$ and $\mathrm{rNgb}$ Gly46Cys in agreement with the previously published data (Hamdane et al., 2003).

\subsubsection{The hydrogen bonding network that includes Tyr44 side chain affects the kinetics and thermodynamics associated with ligand migration in Ngb}

The disulfide bond in hNgb connects the CD loop with the D-helix though Cys 46 and Cys 55, respectively (Hamdane et al., 2003). As previously described, cleavage of the disulfide bond affects the kinetics of ligand rebinding to the heme iron, but the mechanism through which structural information is transmitted from the solvent exposed disulfide bond to the heme binding pocket is still not known. Inspection of the crystal structure of $\mathrm{hNgb}$ indicates that the hydroxyl group from Tyr 44 side chain, located within the CD loop, forms a hydrogen bond with one of the propionate groups of the heme. In addition, the three dimensional structure of $\mathrm{CO}$ bound $\mathrm{mNgb}$ also indicates that the interaction between the distal His 64 and Tyr 44 is involved in the displacement of the CD-D region upon CO association to the heme iron (Vallone et al., 2004b). Therefore, structural changes occurring in the CD loop might be relayed to the heme pocket though the hydrogen bonding network that involves Tyr44 and His 64 residues. In addition, further examination of the crystal structure of ferric $\mathrm{mNgb}$ suggests that this hydrogen bonding network is even larger and include nearby residues Lys 67, Glu 60, a water molecule (Astudillo et al., 2012). To probe the impact of this hydrogen bonding network 
in kinetics and energetics association with $\mathrm{CO}$ binding/dissociation from $\mathrm{hNgb}$, Tyr44Phe and His64Gln mutated proteins were characterized in the presence and absence of the internal disulfide bond employing PAC and TA.

The TA data show that replacement of Tyr 44 with a non-polar Phe residue, which has a similar size and lacks the hydroxyl group that interacts with the heme propionate group, affects the rate for $\mathrm{CO}$ bimolecular rebinding to five-coordinate $\mathrm{hNgb}$. The rate constants determined for the fast and slow phase of $\mathrm{CO}$ bimolecular rebinding to Tyr44Phe exhibit $\sim 4$ - and 3.3- fold increase, respectively, compared to the ones determined for wild type $\mathrm{hNgb}$. Acceleration of the rate constant for ligand association to hNgb upon substitution of Tyr 44 has been previously observed. Giuffre et al. (2008) reported that the rate constant for $\mathrm{O}_{2}$ association to hexa-coordinate hNgb Tyr44Asp was larger than the one determined for wild type hNgb. The faster kinetics observed for bimolecular CO association to Tyr44Phe most likely result from increased accessibility of the ligand to the distal pocket, which decreases the activation barrier for exogenous ligand binding. Since similar rate constants were determined for CO bimolecular rebinding to $\mathrm{hNgb}^{\text {red }}$ and Tyr44Phe ${ }^{\text {red }}$, the increased accessibility upon disruption of the disulfide bond and replacement of Tyr by Phe is attributed to decreased restriction of the global motions upon removal of the disulfide bridge (Ishikawa et al., 2007). Likewise, replacement of the conserved distal His 64 residue by Gln, which has a similar size and polarity as His, alters the rate for bimolecular rebinding to five-coordinate Ngb by increasing the rate constants for the fast and slow phase by $\sim 5$ and 10 times, respectively. Comparable rate constants were determined for His64Gln ${ }^{\text {red }}$. Acceleration of the rate constant associated with $\mathrm{CO}$ bimolecular rebinding to $\mathrm{hNgb}$ upon replacement of His 64 
by Gln and other residues, such as Leu and Val, has been previously observed by other groups (Dewilde et al., 2001; Hamdane et al., 2003; Nienhaus et al., 2004). Although the slow phase for $\mathrm{CO}$ bimolecular rebinding $\left(\mathrm{k}_{\mathrm{bim} 2}\right)$ was not reported in those studies, Uno et al. (2004) proposed that the slower process results from partial coordination of the hydroxyl group of nearby Lys 67 residue at $\mathrm{pH}$ 9.5.

The faster kinetics observed for $\mathrm{CO}$ bimolecular rebinding to $\mathrm{hNgb}^{\text {red }}$, Tyr44Phe, Tyr44Phe ${ }^{\text {red }}$, His64Gln and His64Gln ${ }^{\text {red }}$ might result from a transient opening of a ligand migration pathway upon disruption of the disulfide bond as well as the hydrogen bonding network connecting Tyr 44, His 64 and the heme propionate group. In a molecular dynamics simulation study of hNgb, Bocahut et al. (2009) identified Tyr 44 as part of one of the pathways of ligand migration in the protein, namely exit 8 , which also includes Phe 28, Leu 41, Phe 42, Gln 43, Phe 61, His 64 and the heme group. Interestingly, Phe 28 was proposed to be a crucial residue in relaying structural changes from the $\mathrm{CD}$ loop to the heme pocket in hNgb (Ezhevskaya et al., 2011).

Although the rate constants associated with $\mathrm{CO}$ rebinding to His64Gln and His64Gln ${ }^{\text {red }} \mathrm{hNgb}$ are comparable, the geminate quantum yield determined for His64Gln $\left(\Phi_{\mathrm{gem}}=0.32\right)$ is lower than the one determined for His64Gln ${ }^{\text {red }}$ protein $\left(\Phi_{\mathrm{gem}}=0.46\right)$. In addition, the geminate quantum yield determined for Tyr44Phe and Tyr44Phe ${ }^{\text {red }} \mathrm{hNgb}$ are identical to the quantum yield determined for His $64 \mathrm{Gln}^{\text {red }}$. These results indicate that the residue occupying position 64 in $\mathrm{hNgb}$ does not affect the migration of the ligand between the heme distal pocket and the adjacent hydrophobic cavities (Astudillo et al., 2012), whereas disruption of the hydrogen bonding network connecting Tyr 44 with the 
heme propionate group leads to a larger structural reorganization that facilitates $\mathrm{CO}$ geminate rebinding, likely by increasing the barrier for $\mathrm{CO}$ escape from the distal site.

Substitution of Tyr 44 by Phe alters the thermodynamics associated with structural changes upon $\mathrm{CO}$ photo-release in $\mathrm{hNgb}$. The photo-release of $\mathrm{CO}$ from Tyr44Phe $\mathrm{hNgb}$ is endothermic $\left(\Delta \mathrm{H}=10 \pm 3 \mathrm{kcal} \mathrm{mol}^{-1}\right)$ and associated with a negative structural volume change $\left(\Delta \mathrm{V}_{\text {struct }}=-21.7 \pm 0.6 \mathrm{~mL} \mathrm{~mol}^{-1}\right)$ that is comparable to the structural volume change determined for wild type $\mathrm{hNgb}\left(\Delta \mathrm{V}_{\text {struct }}=-21.6 \pm 0.9 \mathrm{~mL} \mathrm{~mol}^{-}\right.$ ${ }^{1}$ ). The negative structural volume change determined for $\mathrm{CO}$ photo-release from wild type $\mathrm{hNgb}$ was attributed to the fast uptake of a water molecule as the $\mathrm{CO}$ molecule escapes from the protein matrix, as described in chapter 4 . This hypothesis is further supported by the fact that the absolute magnitude of the structural volume change is similar to the partial molar volume of water of $\mathrm{V}_{\mathrm{m}}{ }^{\circ}=18 \mathrm{~mL} \mathrm{~mol}^{-1}$ (Astudillo et al., 2012).

The reaction enthalpy change determined for $\mathrm{CO}$ photo-release from $\mathrm{hNgb}$ Tyr44Phe is $10 \pm 3 \mathrm{kcal} \mathrm{mol}^{-1}$; however, the enthalpy change determined for dissociation of Fe-CO bond is $\sim 17 \mathrm{kcal} \mathrm{mol}^{-1}$ (Miksovska et al., 2005). Hence, substitution of Tyr 44 by Phe residue decreases the reaction enthalpy by $\sim 7 \mathrm{kcal} \mathrm{mol}^{-1}$. To obtain insight into the structural changes occurring upon CO photo-release from $\mathrm{hNgb}$ Tyr44Phe protein, the Tyr 44 residue in the structures of ferrous mNgb (PDB entry 2VRY) and CO bound $\mathrm{mNgb}$ (PDB entry 1W92) was replaced by Phe and both structures were minimized employing the Amber03 force field in YASARA molecular modeling software. In addition, residues Gly 46, Ser 55 and Ser 120 were replaced by Cys in both mNgb and COmNgb structures and the disulfide bridge was manually added prior minimization. 
Inspection of the minimized structures of Tyr44Phe and CO bound Tyr44Phe indicates that the side chain of Lys 67 is highly flexible and occupies different positions in the ferrous and $\mathrm{CO}$ bound structures of Ngb (Figure 5.11). In the ferrous structure of Tyr44Phe, the amino group of Lys 67 side chain forms hydrogen bonds with the carboxy group from the heme propionate group and the side chain of Asp 63. These interactions are not observed in the $\mathrm{CO}$ bound structure of Tyr44Phe mutant. The interaction between Asp 63 and Lys 67 was not observed in the wild type protein containing a disulfide bond between Cys 46 and Cys 55. Therefore, I propose that CO escape from the protein matrix in $\mathrm{hNgb}$ Tyr44Phe is associated with formation of a hydrogen bonding network (occurring within $50 \mathrm{~ns}$ ) that includes Lys 67, Asp 63 and the heme group, leading to an enthalpy change of $10 \mathrm{kcal} \mathrm{mol}^{-1}$.
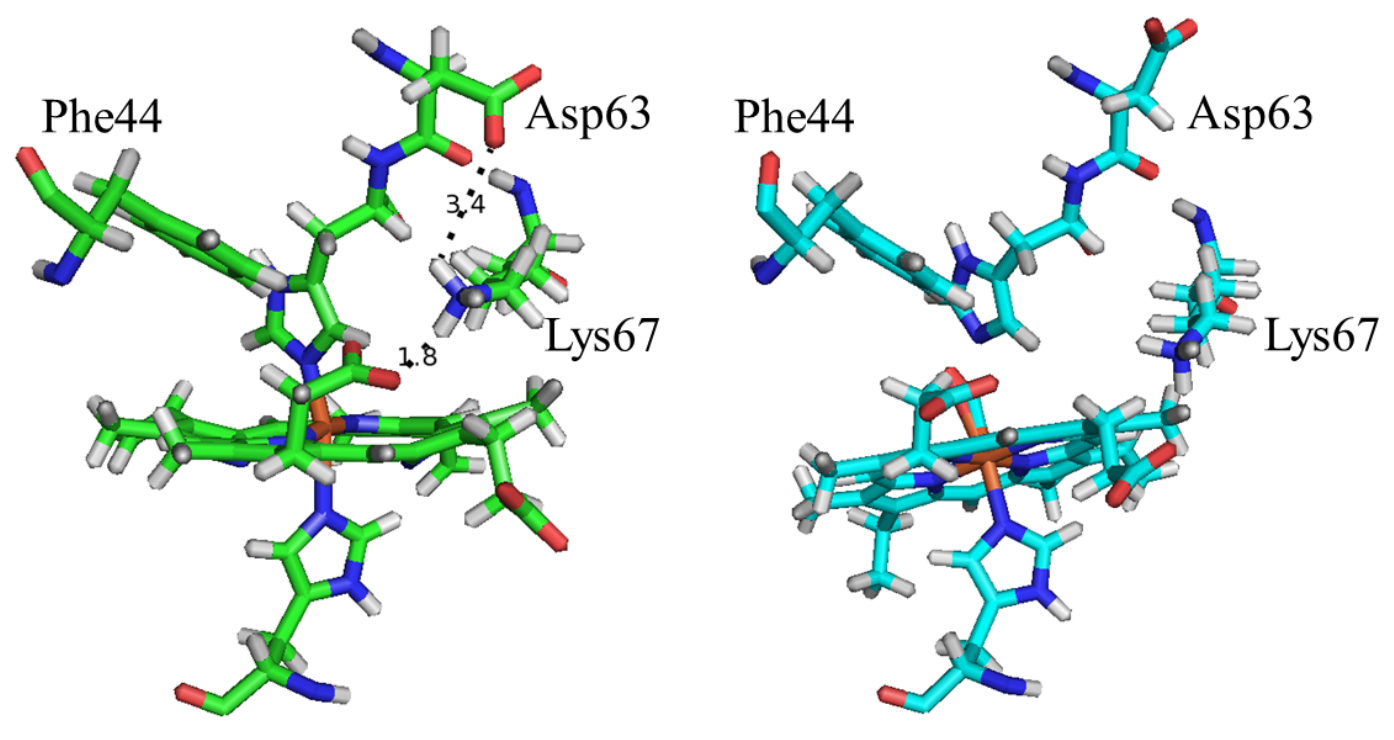

Figure 5.11 Overlay of minimized structures of ferrous mNgbTyr44Phe (left, shown in green) and $\mathrm{CO}$ bound mNgbTyr44Phe (right, shown in light blue) depicting residues in the vicinity of the heme binding pocket of Ngb. The dashed lines represent hydrogen bonds and the numbers the distance in $\AA$ between $\mathrm{H}$-donor and $\mathrm{H}$-acceptor groups. 
The reaction enthalpy change determined for $\mathrm{CO}$ photo-release from $\mathrm{hNgb}$ Tyr44Phe ${ }^{\text {red }}$ is $0 \pm 4 \mathrm{kcal} \mathrm{mol}^{-1}$, and a concomitant negative structural volume change that is comparable to the one determined for wild type hNgb. Therefore, reduction of the disulfide bond in Tyr44Phe strain leads to a decrease in the enthalpy change of $\sim 20 \mathrm{kcal}$ $\mathrm{mol}^{-1}$. This decrease in the enthalpy change is larger than the one determined for $\mathrm{hNgb}$ Tyr44, suggesting that reduction of the disulfide bond in $\mathrm{hNgb}$ Tyr44Phe may lead to disruption of a larger hydrogen bonding network in the ferrous state. In order to understand the structural changes occurring upon CO photo-release from hNgb Tyr44Phe protein leading to a larger structural enthalpy change than $\mathrm{CO}$ photo-release from wild type $\mathrm{hNgb}$, the Tyr 44 residue in the structures of ferrous $\mathrm{mNgb}$ (PDB entry 2VRY) and CO bound mNgb (PDB entry 1W92) was replaced by Phe and both structures were minimized employing the Amber03 force field in YASARA molecular modeling software. In addition, residues Gly 46, Ser 55 and Ser 120 were replaced by Cys in both $\mathrm{mNgb}$ and $\mathrm{COmNgb}$ structures prior minimization. Comparison of the minimized structures of ferrous Tyr44Phe ${ }^{\text {red }}$ and $\mathrm{CO}$ bound Tyr44Phe ${ }^{\text {red }}$ indicates that $\mathrm{CO}$ photodissociation from Tyr44Phe ${ }^{\text {red }}$ mutant leads to reorientation of the side chain of residues Lys 67 and Asp 63, which are close to the heme binding pocket, as well as residues Arg 47, Asn 45, Gln 42 that are far from the heme binding pocket but close to Cys 46 (Cys 46 forms a disulfide bond with Cys 55). As observed in the ferrous structure of Tyr44Phe, the amino group of Lys 67 side chain forms hydrogen bonds with the carboxy group from the heme propionate group and the side chain of Asp 63 in the ferrous structure of Tyr44Phe ${ }^{\text {red }}$ mutant. Additionally, the amino group from the side chain of Gln 42 forms a hydrogen bond with the carboxy group from the backbone of Cys 46, and the amino 
group from the side chain of Arg 47 forms a hydrogen bond with the carboxy group from the side chain of Asn 45 in the minimized structure of ferrous Tyr44Phe ${ }^{\text {red }}$. These interactions are not observed in the $\mathrm{CO}$ bound structure of Tyr44Phe mutant. The disruption of the disulfide bond in Tyr44Phe mutant seems to impart greater flexibility to the CD loop, where residues Arg 47, Asn 45 and Gln 42 are located, which results in a larger reorganization upon photo-cleavage of the ligand.
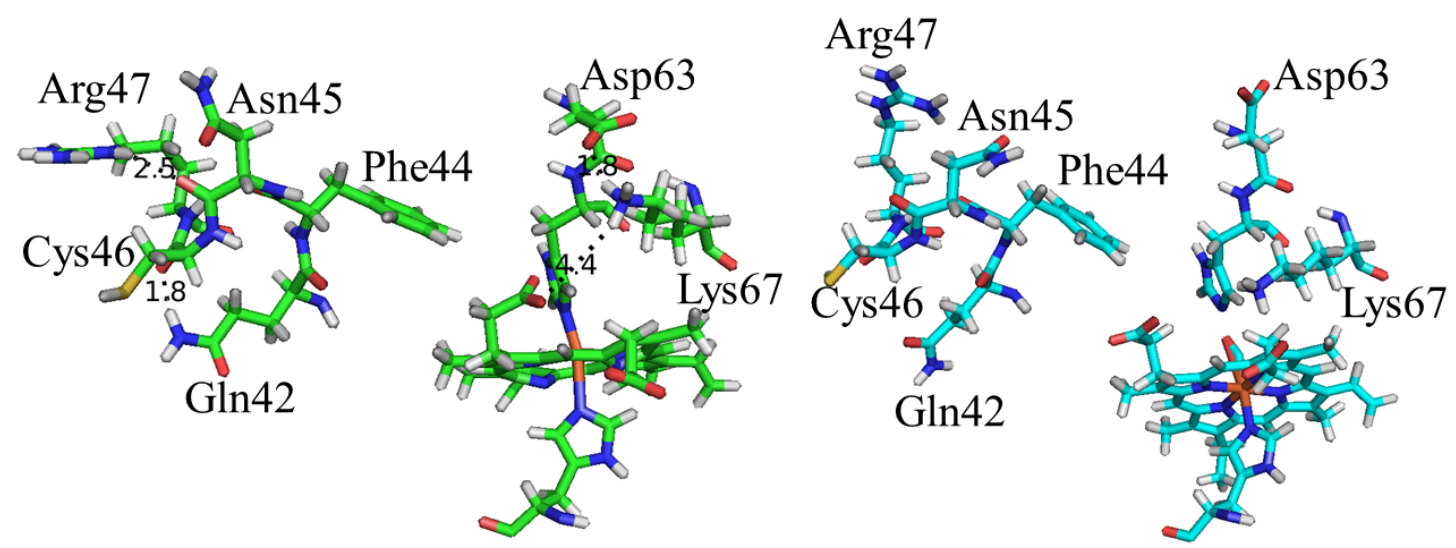

Figure 5.12 Overlay of minimized structures of ferrous mNgbTyr44Phe ${ }^{\text {red }}$ (left, shown in green) and $\mathrm{CO}$ bound $\mathrm{mNgbTyr} 44 \mathrm{Phe}^{\text {red }}$ (right, shown in light blue) depicting residues in the vicinity of the heme binding pocket of $\mathrm{Ngb}$. The dashed lines represent hydrogen bonds and the numbers the distance in $\AA$ between $\mathrm{H}$-donor and $\mathrm{H}$-acceptor groups.

Photodissociation of $\mathrm{CO}$ from His64Gln hNgb mutated protein is exothermic $(\Delta \mathrm{H}$ $=-6 \pm 3 \mathrm{kcal} \mathrm{mol}^{-1}$ ) and associated with a more negative structural volume change $\left(\Delta \mathrm{V}_{\text {struct }}=-25.0 \pm 0.5 \mathrm{~mL} \mathrm{~mol}^{-1}\right)$ than the one determined for wild type $\mathrm{hNgb}$. This exothermic enthalpy change indicates that association of $\mathrm{CO}$ to five-coordinate His64Gln mutated protein is endothermic and entropically driven. Fago et al. (2004b) reported that association of $\mathrm{O}_{2}$ to $\mathrm{hNgb}$ is endothermic $\left(\Delta \mathrm{H}=12.7 \mathrm{kcal} \mathrm{mol}^{-1}\right)$ at temperatures below $18{ }^{\circ} \mathrm{C}$, whereas oxygenation of $\mathrm{hNgb}$ becomes exothermic $\left(\Delta \mathrm{H}=-15.7 \mathrm{kcal} \mathrm{mol}^{-1}\right)$ at 
temperatures above $18{ }^{\circ} \mathrm{C}$. The exothermic ligand binding was attributed to weakening of the electrostatic/hydrogen bonding network formed among residues His 64, Lys 67 and the heme propionate group at high temperatures. Therefore, the negative enthalpy change determined for $\mathrm{CO}$ photo-release from His64Gln $\mathrm{hNgb}$ may result from reorganization of the electrostatic/hydrogen bonding network in the heme binding pocket, as previously proposed for $\mathrm{O}_{2}$ association to $\mathrm{hNgb}$. Interestingly, the reaction enthalpy change determined for $\mathrm{CO}$ photo-release from $\mathrm{hNgb} \operatorname{His} 64 \mathrm{Gln}^{\text {red }}\left(\Delta \mathrm{H}=18 \pm 6 \mathrm{kcal} \mathrm{mol}^{-1}\right)$ is positive and the structural volume change is comparable to that observed for $\mathrm{hNgb}$ His64Gln mutant. The large difference in terms of enthalpy change between His64Gln and His $64 \mathrm{Gln}^{\text {red }}$ may be related to the flexibility of the side chain of Gln at position 64 . To obtain insight into the conformational changes occurring upon replacement of His 64 by Gln in the structure of human Ngb (PDB entry 1OJ6, molecule B), the visualization program Pymol was employed to substitute His 64 by Gln and the modeled structures are shown in Figure 5.13. Inspection of the modeled structures of His64Gln indicates that the side chain of Gln 64 samples 16 conformations within the heme pocket. In the major conformation (20.8\%), the side chain of Gln 64 is solvent exposed and pointing toward the side chain of Tyr 44 and the side chain of Lys 67, whereas in the second most populate conformation (14.6\%) the side chain of Gln 64 is more buried inside the protein matrix and pointing away from residues Tyr 44 and Lys 67.

To obtain a better description of the effect of Tyr44Phe and His64Gln mutations on the thermodynamic parameters associated with $\mathrm{CO}$ photo-release from $\mathrm{hNgb}$, thermodynamic cycles for Tyr $44 \rightarrow$ Phe and His $64 \rightarrow$ Gln mutations in $\mathrm{hNgb}$ were 
constructed using the volume and enthalpy changes determined using PAC (Figures 5.14

and 5.15).
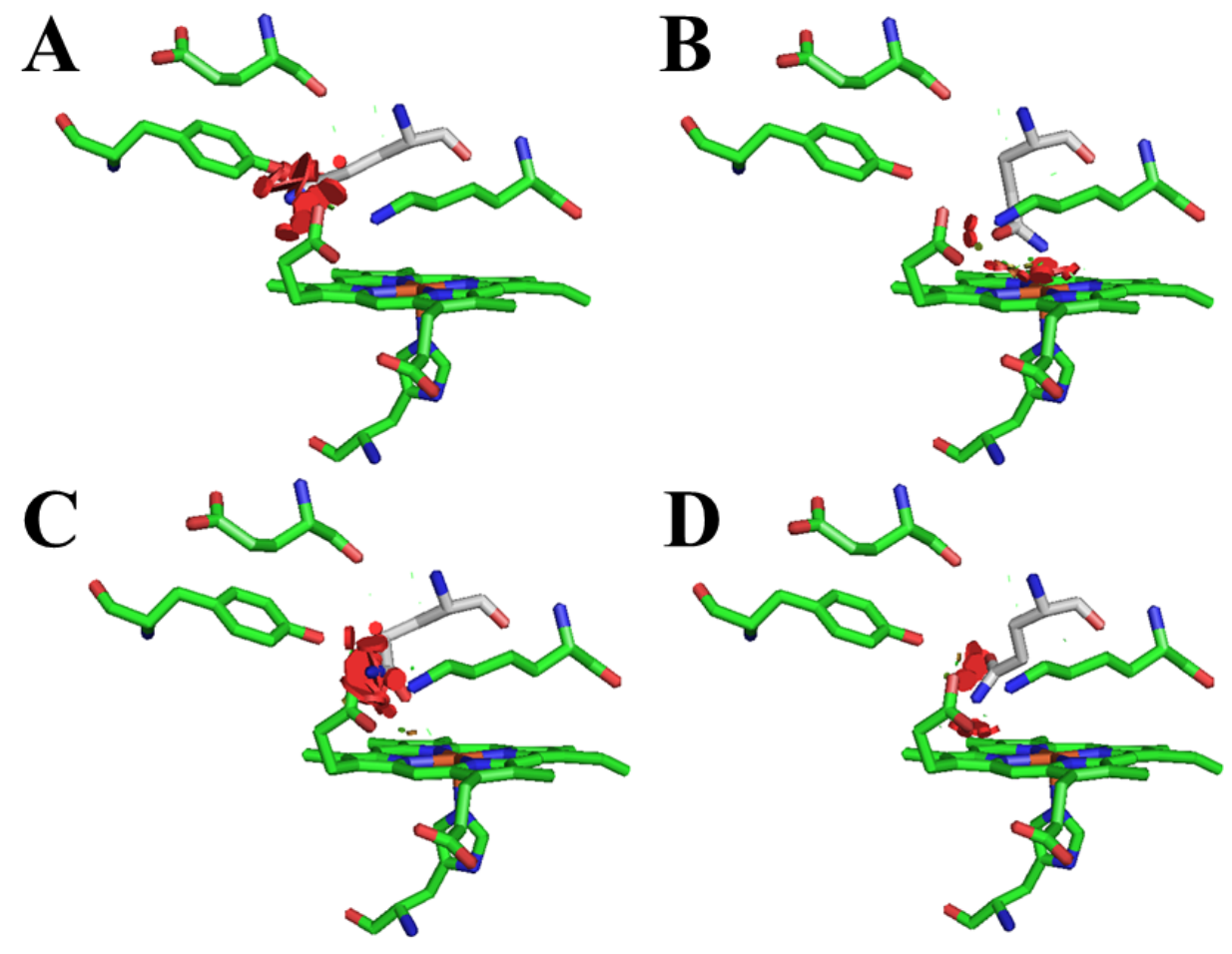

Figure 5.13 Modeled structures of His64Gln hNgb mutant showing the various conformations sampled by the side chain of Gln 64 (shown in gray). Residues in the vicinity of the heme binding pocket (Tyr 44, Lys 67 and Glu 60) are depicted as sticks. The population of each conformation is $20.8 \%(\mathrm{~A}), 14.6 \%(\mathrm{~B}), 11.8 \%(\mathrm{C})$ and $5.2 \%(\mathrm{D})$.

Substitution of Tyr44 by Phe residue in $\mathrm{hNgb}$ leads to a decrease in the enthalpy change by $10 \mathrm{kcal} \mathrm{mol}^{-1}$ and a negligible volume change, likely as a result of the disruption of hydrogen bond between Tyr 44 side chain and the heme propionate group. Reduction of the disulfide bond in $\mathrm{hNgb}$ Tyr44Phe mutated protein leads to a larger decrease of the enthalpy change by additional $10 \mathrm{kcal} \mathrm{mol}^{-1}$ as well as a small volume contraction of $1.6 \mathrm{~mL} \mathrm{~mol}^{-1}$, suggesting that breakage of the disulfide bond leads to a 
larger disruption of the hydrogen bonding network. The observed volume contraction could be attributed to the increased flexibility gained upon disruption of the disulfide bond and reorganization of internal cavities (Bocahut et al., 2009; Nadra et al., 2008). As previously described in this chapter, breakage of the disulfide bond in $\mathrm{hNgb}$ results in negligible $\Delta \Delta \mathrm{H}$ and a volume contraction of $\Delta \Delta \mathrm{V}=-9 \mathrm{~mL} \mathrm{~mol}^{-1}$. The replacement of Tyr 44 by Phe in the reduced protein results in exothermic enthalpy change $\left(\Delta \Delta \mathrm{H}=-19 \mathrm{~mL} \mathrm{~mol}^{-1}\right)$ and a concomitant volume expansion of $7.3 \mathrm{~mL} \mathrm{~mol}^{-1}$. The magnitude of the sum of $\mathrm{Ngb} \rightarrow \mathrm{Y} 44 \mathrm{~F}$ and $\mathrm{Y} 44 \mathrm{~F} \rightarrow \mathrm{Y}_{4} 4 \mathrm{~F}^{\mathrm{red}}$ is similar to the sum of $\mathrm{Ngb} \rightarrow \mathrm{Ngb}^{\text {red }}$ and Ngbred $\rightarrow \mathrm{Y}_{44} \mathrm{~F}^{\text {red }}$, suggesting a synergistic effect between the replacement of Tyr 44 by Phe and disruption of the internal disulfide bond in hNgb. The overall enthalpy change associated with the replacement of Tyr 44 by Phe and reduction of the internal disulfide bond in $\mathrm{hNgb}$ is $\Delta \mathrm{H}=-20 \mathrm{kcal} \mathrm{mol}^{-1}$ and the overall volume change is $-1.7 \mathrm{~mL} \mathrm{~mol}^{-1}$.

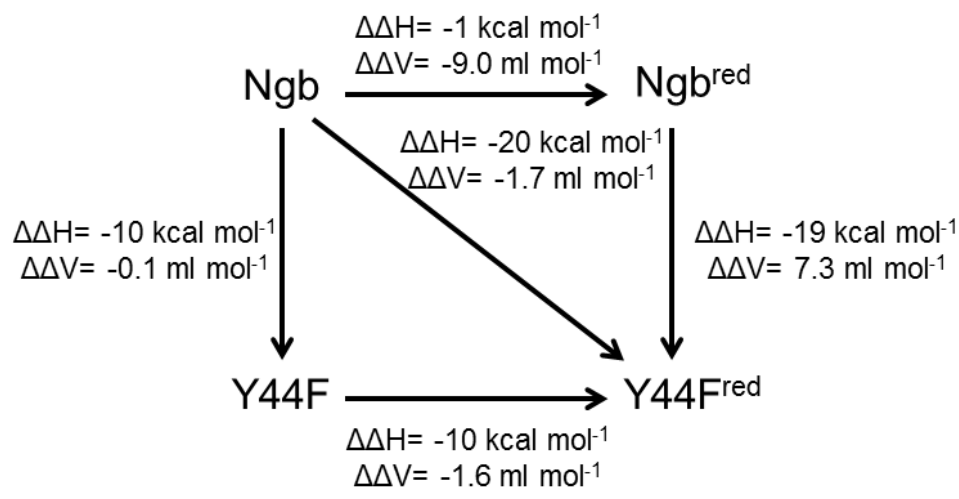

Figure 5.14 Thermodynamic cycle determined for CO escape from hNgb Tyr44Phe mutated protein. 
The displacement of the $\mathrm{CD}$ loop in $\mathrm{CO}$ bound $\mathrm{Ngb}$ has been proposed to be linked to the heme sliding mechanism through the contact of His 64 and Tyr 44 residues (Xu et al., 2011). The PAC data indicates that replacement of the distal His 64 by Gln in $\mathrm{hNgb}$ leads to a large $\Delta \Delta \mathrm{H}$ of $-26 \mathrm{kcal} \mathrm{mol}^{-1}$ and a small volume contraction of $-3.4 \mathrm{~mL}$ $\mathrm{mol}^{-1}$ (Figure 5.13). The exothermic $\Delta \Delta \mathrm{H}$ was attributed to the reorganization of the hydrogen/electrostatic interactions upon substitution of His 64 by Gln. Indeed, a molecular dynamics study of His64Val Ngb mutant shows that the solvent accessible area of Tyr 44 is larger in CO bound His64Val than in the wild type protein, hence replacement of His 64 by Val modifies the electrostatic and hydrogen bonding network in $\mathrm{Ngb}$ (Xu et al., 2011). Furthermore, it was proposed that His64Val mutation induced an upward heme sliding movement, resulting in a significant decrease in the volume of the huge internal cavity of $\mathrm{Ngb}$ (Xu et al., 2011). Disruption of the disulfide bridge in His64Gln mutated protein leads an endothermic $\Delta \Delta \mathrm{H}$ of $24 \mathrm{kcal} \mathrm{mol}^{-1}$ and a small volume contraction of $-1.2 \mathrm{~mL} \mathrm{~mol}^{-1}$. Conversely, replacement of His 64 by Gln in the $\mathrm{hNgb}^{\text {red }}$ protein results in a small $\Delta \Delta \mathrm{H}$ and a moderate volume expansion of $4.4 \mathrm{~mL} \mathrm{~mol}^{-1}$. Therefore, the overall change in enthalpy associated with substitution of His 64 by Gln and reduction of the internal disulfide bond in hNgb is $-2 \mathrm{kcal} \mathrm{mol}^{-1}$, whereas the change in volume change is $-4.6 \mathrm{~mL} \mathrm{~mol}^{-1}$. The thermodynamic data shown here demonstrates that the distal His 64 forms part of the hydrogen/electrostatic network that includes Tyr 44, Lys 67 and the 7-propionate group from the heme. 


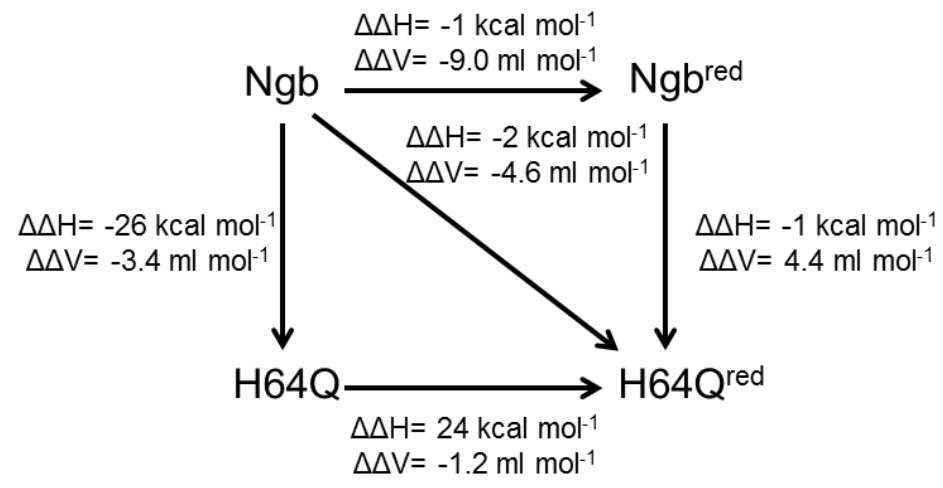

Figure 5.15 Thermodynamic cycle determined for CO escape from hNgb His64Gln mutated protein.

\subsection{Summary}

In summary, the data presented here demonstrate that the internal disulfide bond in human neuroglobin modulates structural changes associated with ligand photodissociation from the heme active site, as evident from time-resolved photothermal studies of CO photo-dissociation. The photoacoustic data reveal a $13.4 \pm 0.9 \mathrm{~mL} \mathrm{~mol}^{-1}$ volume expansion upon ligand photo-release from $\mathrm{hNgb}$, whereas the $\mathrm{CO}$ dissociation from $\mathrm{rNgb}$ leads to a significantly smaller volume change $\left(\Delta \mathrm{V}=4.6 \pm 0.3 \mathrm{~mL} \mathrm{~mol}{ }^{-1}\right)$. Reduction of the internal disulfide bond in $\mathrm{hNgb}$ leads to conformational changes nearly identical to those observed for $\mathrm{rNgb}$, as evident from the observed volume changes. In addition, the kinetic data indicate that the presence of the disulfide bond fully contributes to the increased rate constant determined for distal His 64 dissociation in $\mathrm{hNgb}$ relative to $\mathrm{rNgb}$.

In addition, the PAC data reveal that replacement of Tyr 44 by Phe and His 64 by Gln affect the thermodynamic parameters associated with $\mathrm{CO}$ photo-dissociation from $\mathrm{hNgb}$, suggesting global conformational changes upon ligand release linked to disruption 
of the large hydrogen/electrostatic network that include His 64, Tyr 44, Lys 67 and heme propionate groups. This electrostatic network was proposed to be important for transmission of structural information from the disulfide bond in the CD loop to the heme. The faster kinetics associated with bimolecular rebinding to Ngb upon replacement of Tyr44 by Phe suggest an increased accessibility of the heme iron to the ligand, likely as a result of increased flexibility of the protein upon Tyr44Phe mutation. 


\section{REDUCTION OF THE INTERNAL DISULFIDE BOND BETWEEN CYS 38 AND 83 SWITCHES THE LIGAND MIGRATION PATHWAY IN CYTOGLOBIN}

(The work described in this chapter was published in Astudillo, L., Bernad, S., Derrien, V., Sebban, P., Miksovska, J. (2013) J. Inorg. Biochem., 129, 23-29)

\subsection{Introduction}

Cytoglobin (Cygb) belongs to the family of vertebrate hexa-coordinated globins that were recently found in mammals and other vertebrates (Burmester et al., 2002; Burmester et al., 2000). Compared to other vertebrate globins, Cygb exhibits several unique structural properties that may strongly impact their intracellular function, including formation of a disulfide bond between Cys 38 and Cys 83 and extended $\mathrm{N}$ - and C-termini (Pesce et al., 2002; Trent and Hargrove, 2002). Cytoglobin is expressed in fibroblasts and fibroblast-related cell types, such as chondroblasts, osteoblasts, myofibroblasts and hepatic stellate cells, in a variety of human tissues including major organs (i.e., liver, heart, brain, retina, lungs, oesophagus and others) at low concentration ( $\mu \mathrm{M}$ level) (Oleksiewicz et al., 2013; Schmidt et al., 2004). The physiological role of this protein has not been established. In vitro and in vivo studies have shown that Cygb is over-expressed in fibrosis and under conditions of neurodegenerative disorders, whereas its expression is down-regulated in some types of cancer, including head and neck cancer (Emara et al., 2009; Emara et al., 2010). Moreover, Cygb has been shown to inhibit cancer cell growth in vitro, which indicates its tumor suppressor role (Shivapurkar et al., 2008). Numerous physiological roles including oxygen storage, oxygen sensing, nitric dioxygenase activity, protection against oxidative stress, and collagen synthesis were 
proposed (Oleksiewicz et al., 2011). Recently, it was shown that Cygb binds sodium oleate with an increased affinity $\left(\mathrm{K}_{\mathrm{d}}=0.7 \mu \mathrm{M}\right)$ and the formation of lipid-Cygb complexes promotes penta-coordinated heme iron, suggesting a possible Cygb role in lipid signaling (Reeder et al., 2011).

Human Cygb shares 25\% sequence homology with myoglobin $(\mathrm{Mb})$ and $\sim 15 \%$ homology with $\mathrm{Ngb}$ (Figure 6.1). Its core structure (residues 18 to 71) is similar to that of other globins with a typical 3-over-3- $\alpha$-helical sandwich conformation (de Sanctis et al., 2004a). The prosthetic group, protoporphyrin IX, is situated between the E and F $\alpha$ helices and bound to the polypeptide chain through the proximal and distal histidine residues, His 113 and His 81, respectively (de Sanctis et al., 2004a) (Figure 6.2). The distal histidine can be readily displaced by exogenous diatomic molecules such as $\mathrm{O}_{2}$ and $\mathrm{CO}$, that reversibly bind to the deoxy heme iron with an affinity that is comparable to that of Mb, $\mathrm{P}_{50}=0.55$ Torr and $3.4 \times 10^{-2}$ Torr, respectively (de Sanctis et al., 2004c). The Nand C-terminal regions are extended by 17 and 19 amino acid residues, respectively, and form an $\alpha$-helical and ordered loop structure (de Sanctis et al., 2004a; Makino et al., 2006). The functional role of the N- and C-terminal extensions remains unclear. Reeder et al. (2011) have proposed that these segments may be involved in the lipid binding or participate in cytoglobin association with other proteins.

Crystallographic studies indicate that Cygb forms homodimers. The structure of human Cygb shows that the dimer is stabilized by two inter-protein disulfide bridges between Cys 38 and Cys 83 and through interactions between the center of the E-helix and the $\mathrm{AB}$ corner (Sugimoto et al., 2004), whereas the dimer interface is formed by hydrophobic contacts between Phe 53 and Ile 126 in the crystal structure of a Cygb 
mutant with both Cys residues replaced by Ser (de Sanctis et al., 2004a). The presence of the inter-protein disulfide bridge in Cygb is not supported by solution studies (Hamdane et al., 2003; Lechauve et al., 2010). Lechauve et al. (2010) have shown that in the absence of a reducing agent, Cys 38 and Cys 83 form an intra-protein disulfide bridge connecting helices B and E. The intra-protein disulfide bond reduces the affinity of the distal histidine for the heme iron by approximately two-fold and consequently alters the affinity for $\mathrm{O}_{2}$ binding (Hamdane et al., 2003). Comparable modulation of the equilibrium constant for distal histidine was also observed in Ngb, even though the Cys residues involved in the formation of the disulfide bond are located in the flexible $\mathrm{CD}$ loop and a short D helix (Dewilde et al., 2001; Hamdane et al., 2003). It has been previously shown that disruption of the disulfide bond in Ngb does not have a large impact on the rate constant for $\mathrm{CO}$ escape from the protein matrix and the quantum yield for geminate ligand rebinding is not affected (Astudillo et al., 2010, 2012). Less is known about the impact of the internal disulfide bond on the structural properties of Cygb since the crystal structure of Cygb containing the internal disulfide bond has not been resolved. 


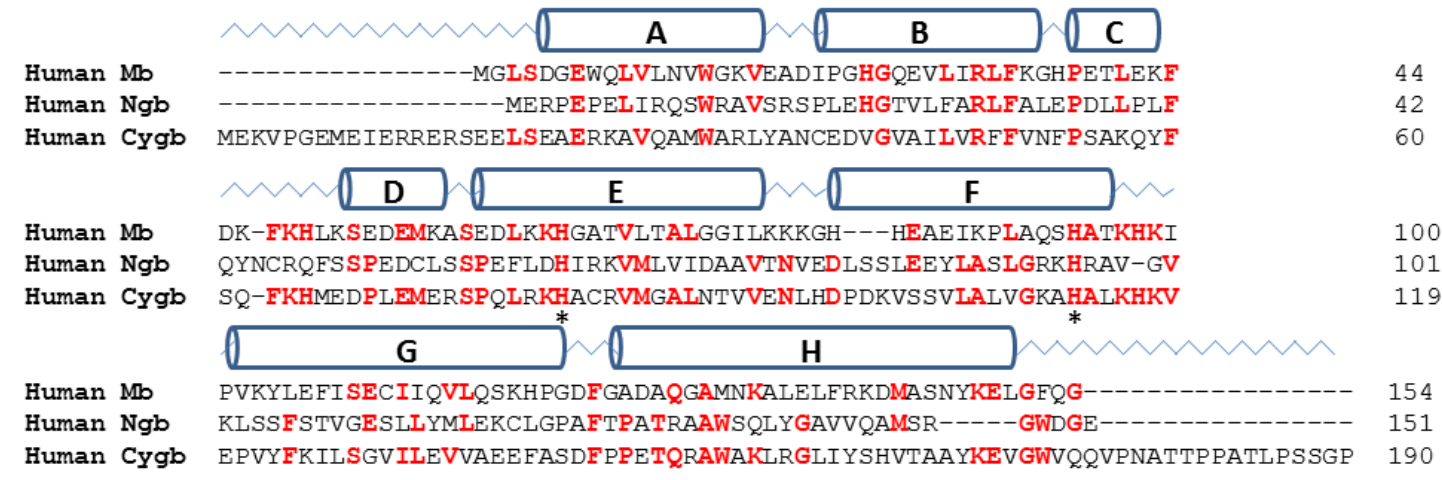

Figure 6.1 Sequence alignment of human Cygb with human Ngb and human Mb. Amino acid residues occupying identical positions in more than one sequence are highlighted in red and rectangular boxes represent $\alpha$-helices $A$ to $H$. The stars correspond to the positions of the distal and proximal His residues.

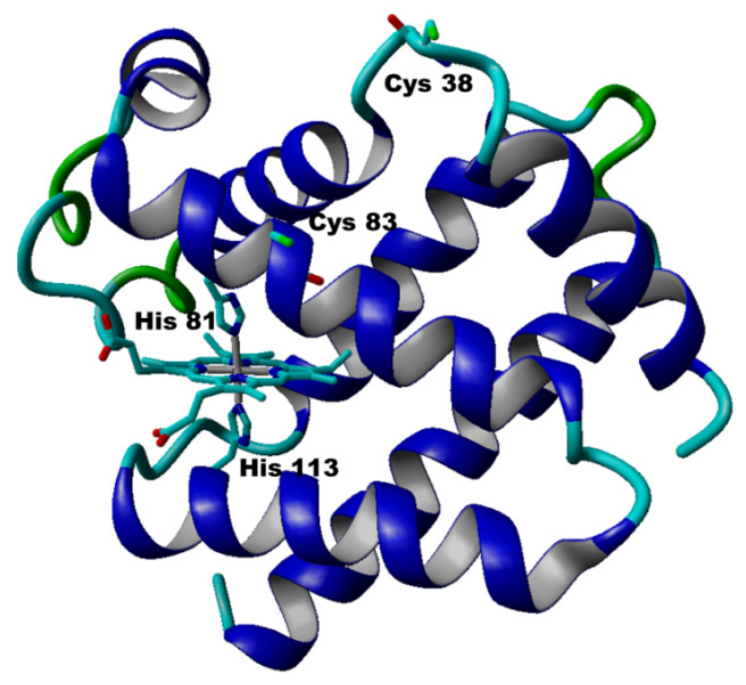

Figure 6.2 Three dimensional structure of human Cygb (PDB entry: 1V5H) displaying the heme group, distal His (His 81), proximal His (His 113), Cys 38 and Cys 83 as sticks.

Taking into consideration that in the Cygb structure Cys 38 and Cys 83 residues are located $\sim 6 \AA$ apart (Figure 6.2 ), the formation of the internal disulfide bridge requires repositioning of helix $\mathrm{B}$ and/or E. Reorientation of the $\mathrm{E}$ helix may modulate the structural properties of the distal pocket and consequently alter the ligand migration 
pathway(s) in Cygb. Here, I employed time-resolved absorption spectroscopy and timeresolved photo-acoustic calorimetry (PAC) to characterize the kinetics and thermodynamics of ligand migration in wild type Cygb $\left(\mathrm{Cygb}^{\mathrm{ox}}\right)$ and in wild type Cygb with reduced thiol groups $\left(\mathrm{Cygb}^{\text {red }}\right)$. The data reported here show that the internal disulfide bond impacts both the rate of ligand escape from the protein matrix as well as the quantum yield for the geminate rebinding to the heme iron, suggesting that the reduction of the disulfide bridge connecting helices $\mathrm{E}$ and $\mathrm{B}$ increases the energy barrier for the ligand transitions between the distal pocket, internal hydrophobic sites and surrounding solvent.

\subsection{Results}

\subsubsection{UV-visible absorption spectroscopy}

The UV-visible spectra of $\mathrm{Cygb}^{\mathrm{ox}}$ strongly resemble absorption spectra of other hexa-coordinate globins, including Ngb and plant hemoglobins with bis-histidine iron coordination. The absorption spectrum of $\mathrm{Fe}^{3+} \mathrm{Cygb}^{\mathrm{ox}}$ displays a Soret band at $416 \mathrm{~nm}$, and two Q bands located at $532 \mathrm{~nm}$ and $563 \mathrm{~nm}$, as previously reported by Sawai et al. (2003). The Soret band of $\mathrm{Fe}^{2+} \mathrm{Cygb}^{\mathrm{ox}}$ is centered at $428 \mathrm{~nm}$ and two additional Q bands are observed at $531 \mathrm{~nm}$ and $560 \mathrm{~nm}$ (Figure 6.3) (Sawai et al., 2003). Upon CO addition, the Soret band shifts to $420 \mathrm{~nm}$ and two additional bands appear at 541 and $570 \mathrm{~nm}$ that are characteristic of the low-spin hexa-coordinated heme iron with $\mathrm{CO}$ in the position of the sixth axial ligand. Reduction of the internal disulfide bond does not alter the electronic structure of the prosthetic group and the UV-visible spectra of $\mathrm{Cygb}^{\text {red }}$ are identical to those measured for $\mathrm{Cygb}^{\mathrm{ox}}$. 


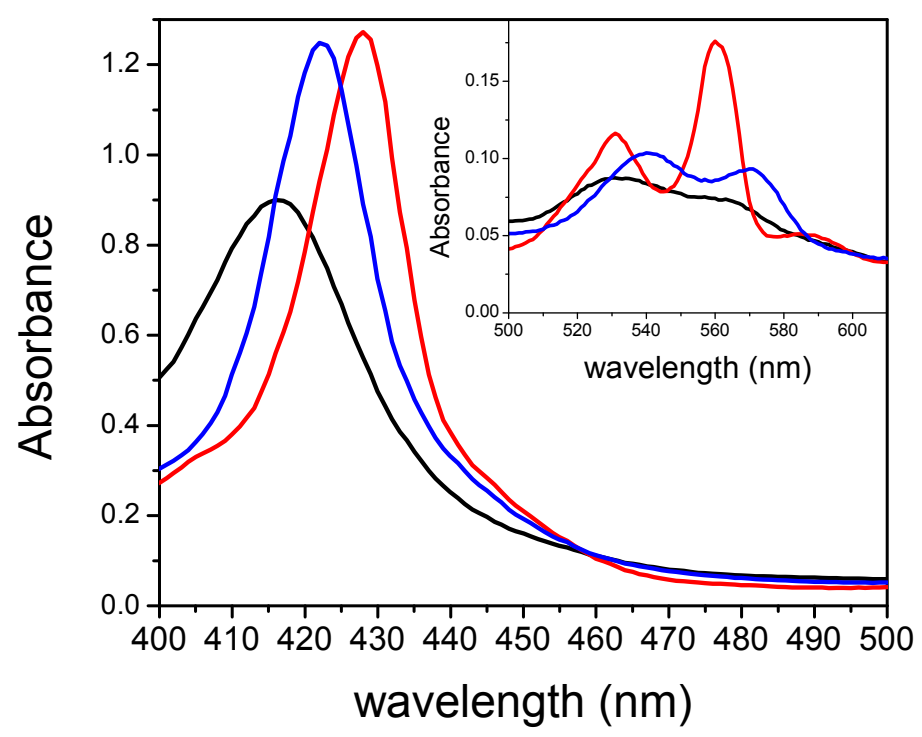

Figure 6.3 Absorption spectra of ferric (black line), ferrous (red line) and $\mathrm{CO}$ bound (blue line) $\mathrm{Cygb}^{\mathrm{ox}}$. Conditions: $20 \mu \mathrm{M}$ protein in $50 \mathrm{mM}$ Tris buffer ( $\mathrm{pH} 7.0$ ).

\subsubsection{Photoacoustic spectroscopy}

PAC traces for $\mathrm{CO}$ dissociation from $\mathrm{Cygb}^{\mathrm{ox}}$ and $\mathrm{Cygb}^{\text {red }}$ at $20{ }^{\circ} \mathrm{C}$ are shown in Fig. 6.4 together with the reference traces. The absence of a phase shift between the trace for $\mathrm{CO}$ photo-release from $\mathrm{Fe}^{2+} \mathrm{Cygb}^{\text {red }}$ and the reference trace indicates that the cleavage of the $\mathrm{Fe}-\mathrm{CO}$ bond is followed by a rapid ligand escape from the protein matrix occurring within the time resolution of our instrument ( $\sim 40 \mathrm{~ns})$. On the other hand, the acoustic trace for $\mathrm{CO}$ photo-dissociation from $\mathrm{Fe}^{2+} \mathrm{Cygb}^{\text {ox }}$ is shifted to smaller frequencies compared to the reference trace, suggesting that $\mathrm{CO}$ escape from $\mathrm{Fe}^{2+} \mathrm{Cygb}^{\mathrm{ox}}$ occurs as a multi-step process. 

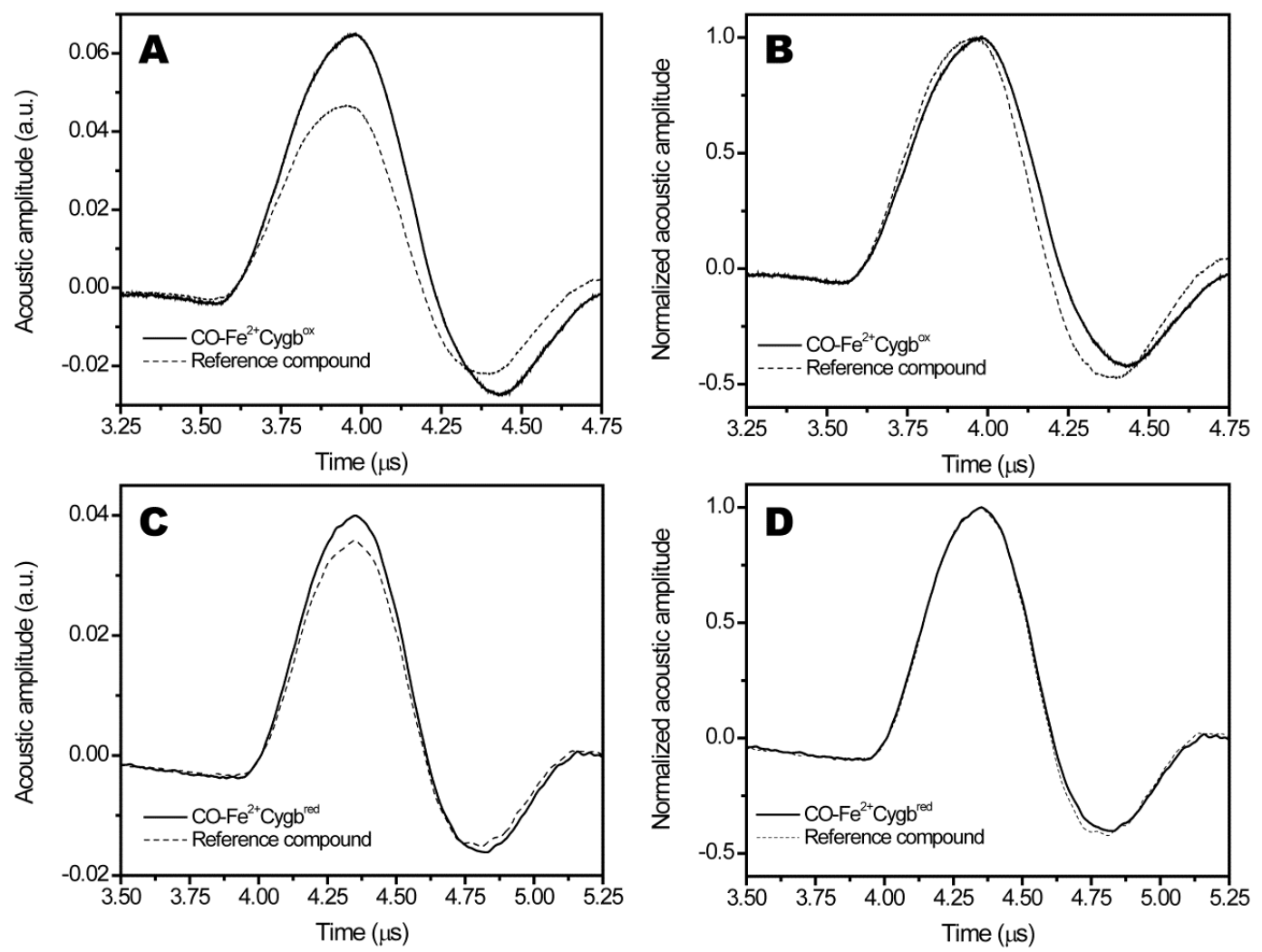

Figure 6.4 Overlay of photoacoustic traces for $\mathrm{Cygb}^{\mathrm{ox}}$ (A) and $\mathrm{Cygb}^{\text {red }}$ (C) with the reference compound 4SP. Normalized traces are shown on the right side for $\mathrm{Cygb}^{\mathrm{ox}}$ (B) and Cygbred (D) to better demonstrate the shift between PAC traces for the sample and reference in $\mathrm{Cygb}^{\mathrm{ox}}$. Conditions: $20 \mu \mathrm{M}$ protein in $50 \mathrm{mM}$ Tris buffer $(\mathrm{pH} \mathrm{7.0)}$. The absorbance of the sample matched that of the reference compound at $532 \mathrm{~nm}\left(\mathrm{~A}_{532} \mathrm{~nm}=\right.$ $0.25)$.

The SoundAnalysis software was used to analyze the PAC traces measured for $\mathrm{Cygb}^{\mathrm{ox}}$ and the fit is shown in Figure 6.5 as well as the corresponding residuals. Deconvolution of the acoustic traces reveals two distinct phases: i) a prompt phase $\left(\tau_{1<}\right.$ $40 \mathrm{~ns})$ that is associated with the photo-cleavage of the $\mathrm{CO}-\mathrm{Fe}$ bond and ligand translocation within the distal pocket and possibly its migration into distant hydrophobic cavities, and ii) a kinetic step with a lifetime $\tau_{2}=150 \mathrm{~ns}$ at $20{ }^{\circ} \mathrm{C}$ that I associate with $\mathrm{CO}$ escape into the surrounding solvent. 


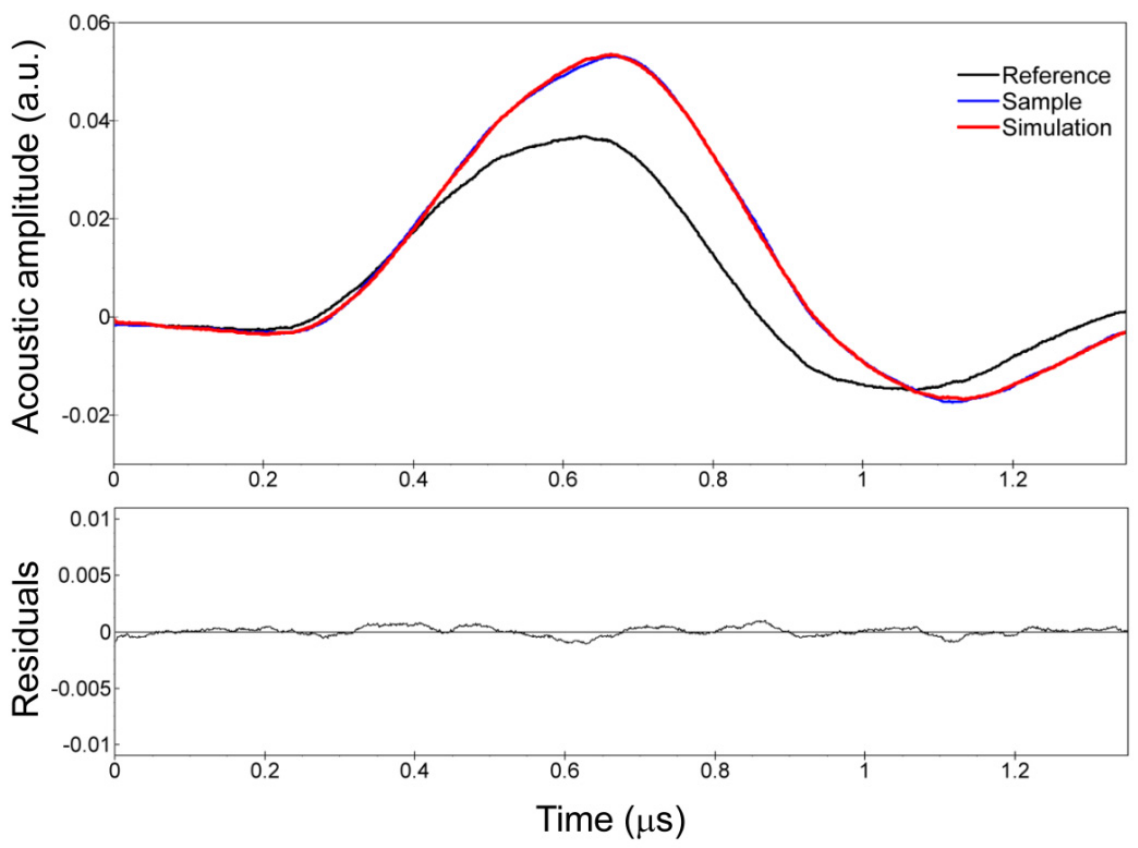

Figure 6.5 Acoustic traces for $\mathrm{CO}$ photodissociation from $\mathrm{Cygb}^{\mathrm{ox}}$ (blue line) and the reference trace (Fe(III)4SP) (black line). The PAC trace for the $\mathrm{CO}$ photo-dissociation from $\mathrm{Cygb}^{\mathrm{ox}}$ was deconvoluted as described in Materials and Methods section (Chapter 3 ). Note that the calculated sample acoustic trace (red line) nearly overlays with the measured sample acoustic wave. The corresponding residuals are shown in the bottom panel.

From the temperature dependence of $\tau_{2}$, the activation thermodynamic parameters $\left(\Delta \mathrm{H}^{\#}\right.$ and $\left.\Delta \mathrm{S}^{\#}\right)$ for $\mathrm{CO}$ release from $\mathrm{Cygb}^{\mathrm{ox}}$ were determined using Eyring plot (Fig. 6.6), according to equation 6.1 (Larsen and Miksovska, 2007). The observed values (Table 6.1) are very similar to the activation parameters for $\mathrm{CO}$ escape from horse heart $\mathrm{Mb}$, suggesting a similar mechanism of ligand escape from the protein matrix in both proteins.

$$
\ln \frac{k}{T}=-\frac{\Delta H^{\#}}{R T}+\ln \frac{k_{B}}{h}+\frac{\Delta S^{\#}}{R}
$$


where $k_{B}$ and $h$ corresponds the Boltzmann and Planck constants, respectively, $T$ is the absolute temperature, $R$ is the universal gas constant, and $\Delta \mathrm{H}^{\#}$ and $\Delta \mathrm{S}^{\#}$ are the activation enthalpy and entropy change, respectively.

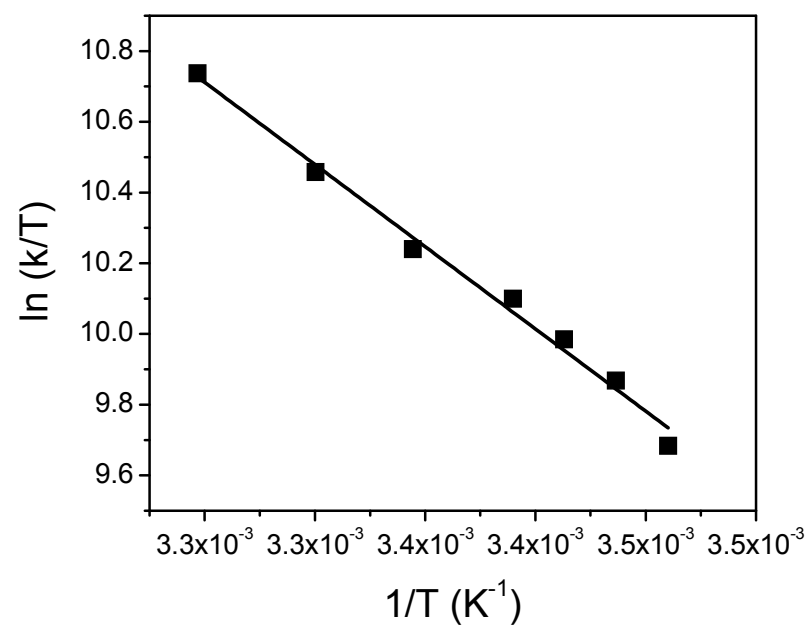

Figure 6.6 Eyring plot for $\mathrm{CO}$ photo-release from $\mathrm{Cygb}^{\mathrm{ox}}$ obtained from the temperature dependence of $\tau_{2}$ values determined from the deconvolution of PAC traces.

The thermodynamic parameters for $\mathrm{CO}$ photo-release from $\mathrm{Cygb}^{\mathrm{ox}}$ and $\mathrm{Cygb}^{\text {red }}$ were determined by plotting $\mathrm{E}_{\mathrm{hv}}\left(\phi_{1}-1\right) / \Phi$ as a function of the temperature dependent parameter $\left(C_{p} \rho / \beta\right)$ for the fast phase and and $\phi_{1} E_{h v} / \Phi$ versus $\left(C_{p} \rho / \beta\right)$ for the slow phase (Fig. 6.7), as described in the materials and methods section, and are listed in Tables 6.1 and 6.2 . 


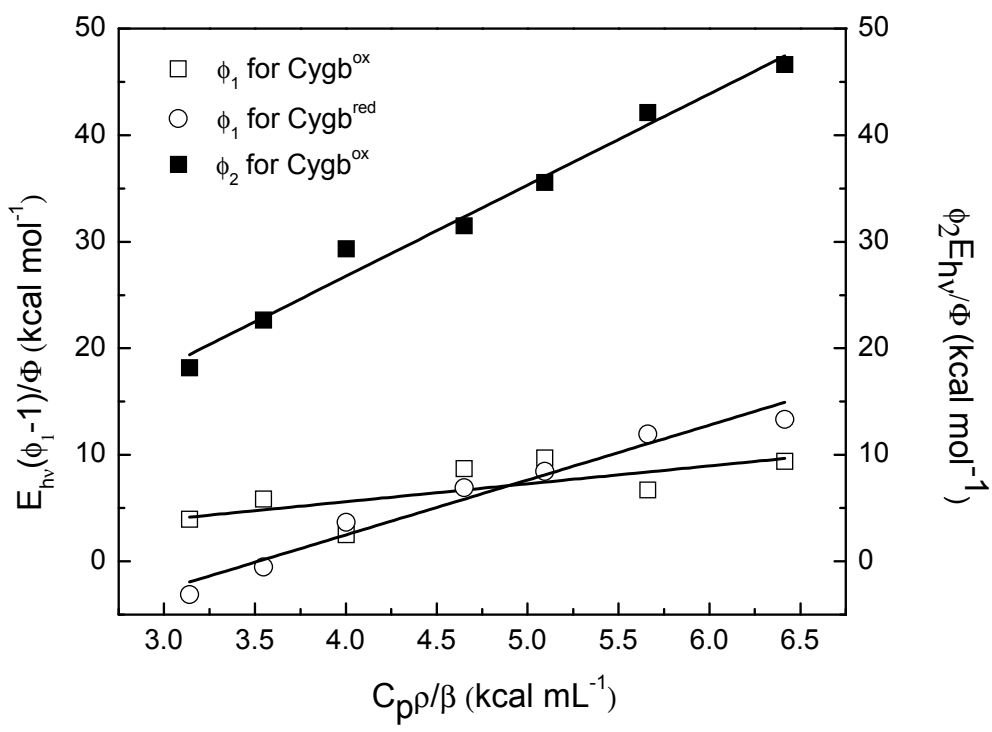

Figure 6.7 Plot of $\left[\left(\phi_{1}-1\right) \mathrm{E}_{\mathrm{hv}} / \Phi\right]$ as a function of the $\mathrm{C}_{\mathrm{p}} \rho / \beta$ term for the prompt phase (solid squares) and and plot of $\left[\phi_{2} \mathrm{E}_{\mathrm{hv}} / \Phi\right]$ versus $\mathrm{C}_{\mathrm{p}} \rho / \beta$ for the slow phase (open squares) for $\mathrm{CO}$ photo-dissociation from $\mathrm{Cygb}^{\mathrm{ox}}$ and the prompt phase for ligand dissociation from $\mathrm{Cygb}^{\text {red }}$ (circles).

Table 6.1 Reaction $(\Delta H$ and $\Delta \mathrm{V})$ and activation $\left(\Delta H^{\#}\right.$ and $\left.\Delta S^{\#}\right)$ thermodynamic parameters associated with the photo-dissociation of $\mathrm{Fe}-\mathrm{CO}$ bond and subsequent ligand escape from the protein matrix in $\mathrm{Cygb}^{\mathrm{ox}}$ and $\mathrm{Mb}$.

\begin{tabular}{lccccccc}
\hline & $\begin{array}{c}\Delta \mathrm{H}_{1} \\
\left(\mathrm{kcal} \mathrm{mol}^{-1}\right)\end{array}$ & $\begin{array}{c}\Delta \mathrm{V}_{1} \\
\left(\mathrm{~mL} \mathrm{~mol}^{-1}\right)\end{array}$ & $\begin{array}{c}\Delta \mathrm{H}_{2} \\
\left(\mathrm{kcal} \mathrm{mol}^{-1}\right)\end{array}$ & $\begin{array}{c}\Delta \mathrm{V}_{2} \\
\left(\mathrm{~mL} \mathrm{~mol}^{-1}\right)\end{array}$ & $\begin{array}{c}\tau \\
(\mathrm{ns})\end{array}$ & $\begin{array}{c}\Delta \mathrm{H}^{\#} \\
\left(\mathrm{kcal} \mathrm{mol}^{-1}\right)\end{array}$ & $\begin{array}{c}\Delta \mathrm{S}^{\#} \\
\left(\mathrm{cal} \mathrm{K}^{-1} \mathrm{~mol}^{-1}\right)\end{array}$ \\
\hline $\mathrm{Cygb}^{\mathrm{ox}^{2}}$ & $1.1 \pm 3.6$ & $1.7 \pm 0.7$ & $7.5 \pm 2.5$ & $8.6 \pm 0.5$ & 150 & $9.2 \pm 0.4$ & $4.1 \pm 1.3$ \\
$\mathrm{Mb}^{\mathrm{a}}$ & $7.4 \pm 2.0$ & $-1.7 \pm 0.5$ & $6.9 \pm 2.9$ & $12.1 \pm 0.7$ & 700 & $10.2 \pm 0.7$ & $4.0 \pm 2.2$ \\
$\mathrm{Mb}^{\mathrm{b}}$ & $-13.9 \pm 1.4$ & $2.2 \pm 0.1$ & $3.3 \pm 0.2$ & $2.1 \pm 0.1$ & 219 & $6.5 \pm 0.5$ & $5.7 \pm 0.5$ \\
$(\mathrm{pH} \mathrm{3.5)}$ & & & & & & &
\end{tabular}

${ }^{\mathrm{a}}$ Thermodynamic parameters for $\mathrm{CO}$ photo-release from horse heart $\mathrm{Mb}$ at $\mathrm{pH} 7.0$ are from Westrick and Peters (1990).

${ }^{\mathrm{b}}$ Thermodynamic parameters for $\mathrm{CO}$ photo-release from horse heart $\mathrm{Mb}$ at $\mathrm{pH} 3.5$ are from Angeloni and Feis (2003). 
Table 6.2 Total volume and enthalpy changes associated with the $\mathrm{CO}$ photo-release from Cygb, Ngb and horse heart Mb.

\begin{tabular}{lcc}
\hline & $\Delta \mathrm{H}_{\text {total }}\left(\mathrm{kcal} \mathrm{mol}^{-1}\right)$ & $\Delta \mathrm{V}_{\text {total }}\left(\mathrm{mL} \mathrm{mol}^{-1}\right)$ \\
\hline $\mathrm{Cygb}^{\text {ox }}$ & $9 \pm 4$ & $10.3 \pm 1.2$ \\
$\mathrm{Mb}^{\mathrm{a}}$ & $14 \pm 3$ & $10.4 \pm 0.7$ \\
$\mathrm{Ngb}^{\mathrm{b}}$ & $20 \pm 4$ & $13.4 \pm 0.9$ \\
$\mathrm{Cygb}^{\text {red }}$ & $18 \pm 2$ & $4.7 \pm 0.4$ \\
$\mathrm{Ngb}^{\text {red b }}$ & $19 \pm 2$ & $4.4 \pm 0.3$
\end{tabular}

${ }^{\mathrm{a}}$ Thermodynamic parameters for $\mathrm{CO}$ photo-release from $\mathrm{Mb}$ are from Westrick \& Peters (1990).

${ }^{\mathrm{b}}$ Thermodynamic parameters for $\mathrm{CO}$ photo-release from $\mathrm{Ngb}$ are from Astudillo et al. (2010).

The photo-cleavage of the Fe-CO bond and subsequent ligand release from $\mathrm{Cygb}^{\text {red }}$ are associated with an overall enthalpy change of $\Delta \mathrm{H}_{\text {total }}=18 \pm 2 \mathrm{kcal} \mathrm{mol}^{-1}$ and a small volume expansion of $\Delta \mathrm{V}_{\text {total }}=5 \pm 2 \mathrm{~mL} \mathrm{~mol}^{-1}$. In the case of $\mathrm{Fe}^{2+} \mathrm{Cygb}^{\mathrm{ox}}$, the photocleavage of the Fe-CO bond is associated with a negligible enthalpy $\left(\Delta \mathrm{H}_{1}=1.1 \pm 3.6 \mathrm{kcal}\right.$ $\left.\mathrm{mol}^{-1}\right)$ and volume change $\left(\Delta \mathrm{V}_{1}=1.7 \pm 0.7 \mathrm{~mL} \mathrm{~mol}^{-1}\right)$. The small volume change occurring within 40 ns upon Fe-CO bond cleavage is consistent with the photodissociated ligand being trapped within the protein matrix. The accompanying enthalpy change is significantly smaller than the enthalpy of the Fe-CO bond $\left(\Delta \mathrm{H}_{\mathrm{Fe}-\mathrm{CO}}=17 \mathrm{kcal}\right.$ $\mathrm{mol}^{-1}$ ) (Larsen and Miksovska, 2007) pointing out that the transition between the lowspin six-coordinate and high-spin five-coordinate heme iron triggers an exothermic structural reorganization that is confined within the protein matrix and does not contribute to the overall volume change. The subsequent ligand escape from the protein 
matrix, with a time constant of $\sim 150 \mathrm{~ns}$, is endothermic $\left(\Delta \mathrm{H}_{2}=7.5 \pm 2.5 \mathrm{kcal} \mathrm{mol}^{-1}\right)$ and leads to a small volume increase $\left(\Delta \mathrm{V}_{2}=8.6 \pm 0.5 \mathrm{~mL} \mathrm{~mol}^{-1}\right)$. The $150 \mathrm{~ns}$ time constant determined for $\mathrm{CO}$ escape from $\mathrm{Cygb}^{\mathrm{ox}}$ is similar to that reported for $\mathrm{CO}$ escape from $\mathrm{Mb}$ at acidic $\mathrm{pH}(\tau \sim 219 \mathrm{~ns}$, table 6.1) (Angeloni and Feis, 2003).

The overall volume expansion $\left(\Delta \mathrm{V}_{\text {total }}=10.3 \pm 1.2 \mathrm{~mL} \mathrm{~mol}^{-1}\right)$ and enthalpy change $\left(\Delta \mathrm{H}_{\text {total }}=9 \pm 4 \mathrm{kcal} \mathrm{mol}^{-1}\right)$ observed for ligand dissociation from $\mathrm{Fe}^{2+} \mathrm{Cygb}^{\mathrm{ox}}$ are

distinct from those determined for $\mathrm{Fe}^{2+} \mathrm{Cygb}^{\text {red }}$, pointing out that the ligand escape mechanism and concomitant structural changes are modulated by the presence of the internal disulfide bond between Cys 38 and Cys 83 .

\subsubsection{Transient absorption spectroscopy}

Transient absorption spectra of $\mathrm{CO}$ bound $\mathrm{Cygb}^{\mathrm{ox}}$ at few pump-probe delay times ( $3 \mathrm{~ns}, 10 \mathrm{~ns}$ and $30 \mathrm{~ns}$ ) are shown in Figure 6.8. The negative absorbance at $421 \mathrm{~nm}$ corresponds to the disappearance of the ground state six-coordinate ligand bound Cygb as a result of photodissociation of the ligand (Jongeward et al., 1988). The positive absorbance change at $440 \mathrm{~nm}$ corresponds to absorption by the five-coordinate ligand free Cygb. The two additional negative contributions to the spectra are observed at 539 $\mathrm{nm}$ and $575 \mathrm{~nm}$ are attributed to the $\mathrm{Q}$ bands bleaching. 


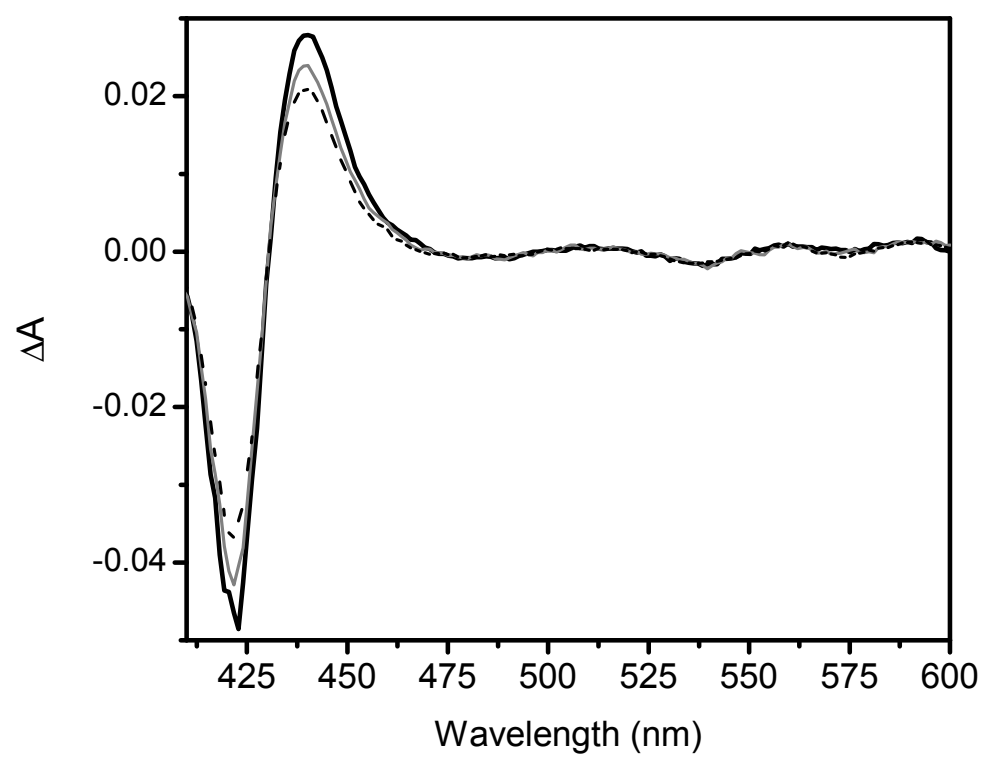

Figure 6.8 Transient absorption spectra of the $\mathrm{CO}$ adduct of $\mathrm{Cygb}^{\mathrm{ox}}$ at 3 ns (black line), $10 \mathrm{~ns}$ (gray line), and $30 \mathrm{~ns}$ (black dashed line). The excitation wavelength was $400 \mathrm{~nm}$.

The distinct ligand migration mechanism in $\mathrm{Cygb}^{\mathrm{ox}}$ and $\mathrm{Cygb}^{\text {red }}$ is also evident from the transient absorption traces for $\mathrm{CO}$ association to the five coordinate heme iron (Figure 6.9). Ligand binding to both $\mathrm{Cygb}^{\mathrm{ox}}$ and $\mathrm{Cygb}^{\mathrm{red}}$ is a multiphasic process with fast geminate rebinding taking place on the nanosecond timescale and slower bimolecular rebinding occurring on microsecond to millisecond timescales. The transient absorption traces were analyzed using either a sum of four exponential decay model or using the maximum entropy method (MEM). Both approaches revealed two phases for the geminate $\mathrm{CO}$ association as well as for the bimolecular rebinding (Table 6.3 and Table $6.4)$. 

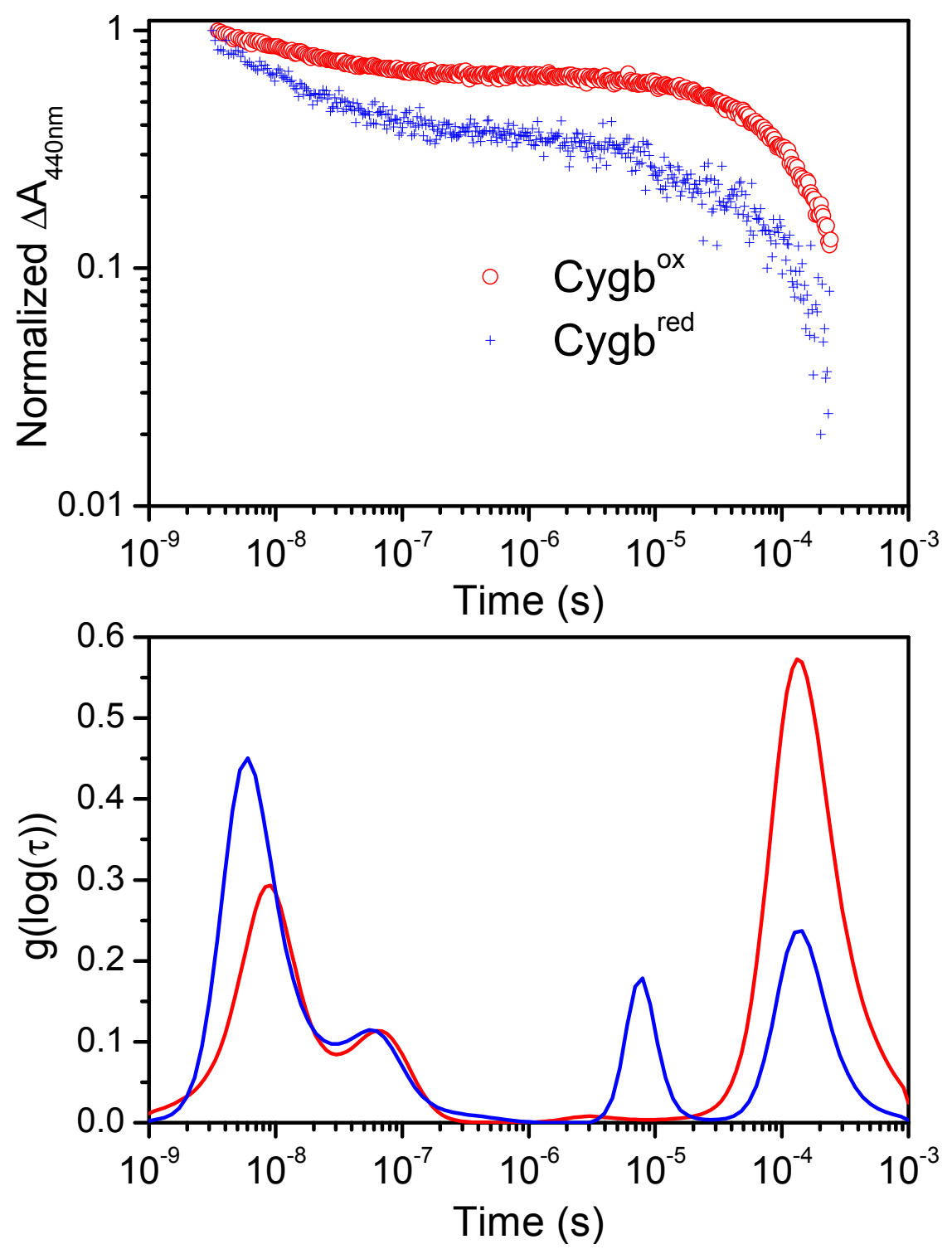

Figure 6.9 Top: Transient absorption traces for $\mathrm{CO}$ rebinding to $\mathrm{Cygb}^{\mathrm{ox}}$ and $\mathrm{Cyg}^{\mathrm{red}}$. Conditions: $20 \mu \mathrm{M}$ protein in $50 \mathrm{mM}$ Tris buffer, $\mathrm{pH} 7.0$ and $1 \mathrm{mM} \mathrm{CO}$, at $20{ }^{\circ} \mathrm{C}$. Bottom: Lifetime distribution associated with the $\mathrm{CO}$ rebinding to $\mathrm{Cygb}^{\mathrm{ox}}$ (red line) and $\mathrm{Cygb}^{\text {red }}$ (blue line) as determined using MEM approach. The transient absorption traces were normalized to unity at the maximum of the TA curve. 
Table 6.3 Rate constants for $\mathrm{CO}$ binding to $\mathrm{Cygb}^{\text {ox }}$ and $\mathrm{Cygb}^{\text {red }}$ obtained using MEM analysis

\begin{tabular}{ccccc|cccc}
\hline & \multicolumn{3}{c|}{ Geminate phase } & \multicolumn{5}{c}{ Bimolecular phase } \\
\cline { 2 - 9 } & $\mathrm{k}_{\mathrm{gem} 1}$ & $\mathrm{~A}_{\mathrm{gem} 1}$ & $\mathrm{k}_{\mathrm{gem} 2}$ & $\mathrm{~A}_{\text {gem2 }}$ & $\mathrm{k}_{\text {fast1 }}$ & $\mathrm{A}_{\text {fast1 }}$ & $\mathrm{k}_{\text {fast2 }}$ & $\mathrm{A}_{\text {fast2 }}$ \\
& $\left(\mathrm{s}^{-1}\right)$ & $(\%)$ & $\left(\mathrm{s}^{-1}\right)$ & $(\%)$ & $\left(\mu \mathrm{M}^{-1} \mathrm{~s}^{-1}\right)$ & $(\%)$ & $\left(\mu \mathrm{M}^{-1} \mathrm{~s}^{-1}\right)$ & $(\%)$ \\
\hline $\mathrm{Cygb}^{\text {ox }}$ & $1.2 \times 10^{8}$ & 29.7 & $1.5 \times 10^{7}$ & 11.3 & 325.0 & 0.8 & 7.5 & 58.2 \\
$\mathrm{Cygb}^{\text {red }}$ & $1.7 \times 10^{8}$ & 46.4 & $1.7 \times 10^{7}$ & 11.7 & 132.3 & 18.0 & 7.3 & 23.9 \\
\hline
\end{tabular}

Table 6.4 Rate constants for CO binding to $\mathrm{Cygb}^{\mathrm{ox}}$ and $\mathrm{Cygb}^{\text {red }}$ and quantum yield for bimolecular $\mathrm{CO}$ binding determined using a four exponential decay model.

\begin{tabular}{ccccc|ccccc}
\hline & \multicolumn{3}{c|}{ Geminate phase } & \multicolumn{5}{c}{ Bimolecular phase } \\
& $\mathrm{k}_{\text {gem1 }}$ & $\mathrm{A}_{\text {gem1 }}$ & $\mathrm{k}_{\text {gem2 }}$ & $\mathrm{A}_{\text {gem2 }}$ & $\mathrm{k}_{\text {fast1 }}$ & $\mathrm{A}_{\text {fast1 }}$ & $\mathrm{k}_{\text {fast2 }}$ & $\mathrm{A}_{\text {fast2 }}$ & $\Phi_{\text {gem }}$ \\
& $\left(\mathrm{s}^{-1}\right)$ & $(\%)$ & $\left(\mathrm{s}^{-1}\right)$ & $(\%)$ & $\left(\mu \mathrm{M}^{-1} \mathrm{~s}^{-1}\right)$ & $(\%)$ & $\left(\mu \mathrm{M}^{-1} \mathrm{~s}^{-1}\right)$ & $(\%)$ & \\
\hline $\mathrm{Cygb}^{\text {ox }}$ & $1.7 \times 10^{8}$ & 28.7 & $2.1 \times 10^{7}$ & 13.1 & 61.0 & 7.3 & 6.9 & 50.9 & 0.35 \\
\hline $\mathrm{Cygb}^{\text {red }}$ & $1.7 \times 10^{8}$ & 45.9 & $1.9 \times 10^{7}$ & 17.5 & 126.0 & 11.5 & 5.2 & 25.1 & 0.63 \\
\hline
\end{tabular}

$\mathrm{CO}$ geminate rebinding occurs with a rate constant of $1.7 \times 10^{8} \mathrm{~s}^{-1}$ and $\sim 2.0 \times 10^{7} \mathrm{~s}^{-1}$ in both Cygb forms, although the faster phase is associated with larger amplitude for the $\mathrm{CO}$ association to $\mathrm{Cygb}^{\text {red }}$. The faster geminate rebinding corresponds to the ligand rebinding from the primary docking site, whereas the approximately ten times slower subsequent phase reflects the ligand binding from more distant sites in the protein matrix. The rate constants for $\mathrm{CO}$ geminate rebinding to $\mathrm{Cygb}$ are significantly faster than the geminate rebinding in $\mathrm{Mb}\left(\mathrm{k}_{\mathrm{gem}}=8.3 \times 10^{6} \mathrm{~s}^{-1}\right)(\mathrm{Cao}$ et al., 2004) and are comparable with 
those determined previously for $\mathrm{CO}$ geminate rebinding to human $\mathrm{Ngb}\left(\mathrm{k}_{\mathrm{gem} 1}=2.3 \times 10^{8} \mathrm{~s}\right.$ ${ }^{1}$ and $\mathrm{k}_{\mathrm{gem} 2}=3.4 \times 10^{7} \mathrm{~s}^{-1}$ ) (Astudillo et al., 2012) and to truncated hemoglobin from Thermobifida fusca $\left(\mathrm{k}_{\mathrm{gem} 1}=5 \times 10^{8} \mathrm{~s}^{-1}\right.$ and $\left.\mathrm{k}_{\mathrm{gem} 2}=9 \times 10^{6} \mathrm{~s}^{-1}\right)$ (Marcelli et al., 2012). In addition, the increase in the amplitude of the fast geminate kinetics and overall increase in the geminate quantum yield as a consequence of the reduction of the cysteine thiol groups from 0.35 in $\mathrm{Cygb}^{\mathrm{ox}}$ to 0.63 in $\mathrm{Cygb}^{\text {red }}$ points toward a lower barrier for the geminate ligand rebinding between the distal pocket and heme iron in $\mathrm{Cyg}^{\mathrm{red}}$.

Bimolecular CO rebinding is also biphasic in both forms of Cygb. However, in the case of $\mathrm{Cygb}^{\mathrm{ox}}$, the majority of the dissociated ligand rebinds with a rate constant of $\sim 7 \mu \mathrm{M}^{-1} \mathrm{~s}^{-1}$, whereas only a minor fraction $(\sim 12 \%)$ recombines on a faster time-scale. The observed rate constant of $7 \mu \mathrm{M}^{-1} \mathrm{~s}^{-1}$ is consistent with a previously reported value of 5 $\mu \mathrm{M}^{-1} \mathrm{~s}^{-1}$ (Hamdane et al., 2003) and $5.6 \mu \mathrm{M}^{-1} \mathrm{~s}^{-1}$ (Trent and Hargrove, 2002) for $\mathrm{CO}$ binding to five coordinated $\mathrm{Cygb}^{\text {red }}$ and $\mathrm{Cygb}^{\mathrm{ox}}$, respectively. The rate constant for the faster bimolecular rebinding could not be determined unambiguously because of its small amplitude $\left(\mathrm{A}_{\text {fast }}=0.8 \%\right.$ using the four exponential decay model and $\mathrm{A}_{\text {fast }}=7.3 \%$ retrieved with the MEM approach). Multiple exponential decay analysis provided a rate constant of $61 \mu \mathrm{M}^{-1} \mathrm{~s}^{-1}$, whereas a rate constant of $325 \mu \mathrm{M}^{-1} \mathrm{~s}^{-1}$ was recovered using the MEM approach. In the absence of the disulfide bridge, CO bimolecular rebinding to $\mathrm{Cygb}^{\text {red }}$ was found to be more heterogeneous with $\sim 40 \%$ of the ligand rebinding with a rate constant of $126 \mu \mathrm{M}^{-1} \mathrm{~s}^{-1}$ and $60 \%$ rebinding with a rate constant of $5.2 \mu \mathrm{M}^{-1} \mathrm{~s}^{-1}$. Biphasic bimolecular ligand rebinding was reported for $\mathrm{Ngb}$ and attributed to the presence of two protoporphyrin IX conformations in the heme binding pocket (Abbruzzetti et al., 2009; Astudillo et al., 2012). However, the orientation of the prosthetic group in Cygb is 
significantly less heterogeneous. Bondarenko et al. (2006) have shown that the majority of the heme group in Cygb ( $90 \%)$ adopts an orientation that is analogous to that of protoporphyrin IX in $\mathrm{Mb}$ and a single heme orientation was also observed in the Cygb crystal structure (Makino et al., 2006). The access of exogenous ligands into the heme pocket was proposed to be controlled by the orientation of Arg 84 that is located in the E helix (Sawai et al., 2003).

\subsection{Discussion}

Photoacoustic calorimetry data indicates that the presence of the disulfide bond alters the thermodynamic parameters associated with $\mathrm{CO}$ photo-dissociation from Cygb, which suggests that the mechanism of ligand escape from Cygb is modulated by the presence of the internal disulfide bond. In $\mathrm{Cygb}^{\text {red }}, \mathrm{CO}$ escapes from the protein matrix to the surrounding solvent within $40 \mathrm{~ns}$ and the observed enthalpy change is consistent with cleavage of the Fe-CO bond, while the observed volume expansion is attributed to the photo-dissociated ligand being trapped within the protein matrix. On the other hand, CO photo-dissociation from $\mathrm{Cygb}^{\mathrm{ox}}$ leads to a prompt phase occurring within $40 \mathrm{~ns}$ and a subsequent slow phase with a time constant of $\sim 150 \mathrm{~ns}$ at $20^{\circ} \mathrm{C}$. The overall reaction enthalpy and volume change associated with $\mathrm{CO}$ photo-dissociation from $\mathrm{Cygb}^{\mathrm{ox}}$, as well as the activation enthalpy and entropy changes, are similar to the thermodynamic parameters previously reported for horse heart $\mathrm{Mb}$ at neutral $\mathrm{pH}$ by Westrick and Peters (1990), while the thermodynamic parameters determined for CO photo-dissociation from $\mathrm{Cygb}^{\text {red }}$ are comparable to the ones reported for human Ngb (Astudillo et al., 2012).

Interestingly, the $150 \mathrm{~ns}$ time constant determined for $\mathrm{CO}$ escape from $\mathrm{Cygb}^{\mathrm{ox}}$ is similar to that reported for $\mathrm{CO}$ escape from $\mathrm{Mb}$ at acidic $\mathrm{pH}$ ( $\tau \sim 200 \mathrm{~ns}$ ) (Angeloni and 
Feis, 2003). The fast $\mathrm{CO}$ escape at acidic $\mathrm{pH}(\mathrm{pH}=3.5)$ was associated with the stabilization of $\mathrm{Mb}$ in an open conformation with the side-chain of His 64 displaced out of the distal pocket, which pushes away Arg 45 leading to the disruption of the salt bridge between Arg 45 and the heme-6-propionate group (Yang and Phillips, 1996). The $\mathrm{pH}$ triggered structural transitions in $\mathrm{Mb}$ were investigated by several groups using spectroscopic and X-ray crystallographic techniques (Han et al., 1990; Morikis et al., 1989; Sage et al., 1991; Yang and Phillips, 1996). These studies demonstrated that the protonation of the distal histidine promotes movement of the histidine side-chain out of the distal pocket and an opening of a direct channel that connects the distal pocket with the surrounding solvent. Residue Phe46 that is located in the CD loop was identified as a key residue in controlling the histidine side-chain dynamics in Mb (Yang and Phillips, 1996). Interestingly, this residue is conserved in the $\mathrm{Mb}$ and Cygb sequence, whereas a Cys residue is found in the analogous position in human Ngb suggesting similar histidine side-chain dynamics in Cygb and $\mathrm{Mb}$. The role of the distal histidine in modulating the kinetics of ligand escape from $\mathrm{Mb}$ was also confirmed in thermal grating studies. Terazima and collaborators have characterized the $\mathrm{CO}$ photo-dissociation from several His 64 mutants of sperm whale $\mathrm{Mb}(\mathrm{swMb})$ and showed that in mutants having His 64 replaced by a non-polar amino acid residue, the $\mathrm{CO}$ escape occurs roughly twice as fast as in native $\mathrm{Mb}(\tau \sim 400 \mathrm{~ns})$ (Sakakura et al., 2002).

The transient absorption spectroscopy data also evidentiate the distinct ligand migration mechanism in $\mathrm{Cygb}^{\mathrm{ox}}$ and $\mathrm{Cygb}^{\text {red }}$ as evident from the kinetics of $\mathrm{CO}$ rebinding to five-coordinate heme iron and the different bimolecular quantum yield observed for $\mathrm{Cygb}^{\mathrm{ox}}$ and $\mathrm{Cygb}^{\mathrm{red}}$. The hetereogenety observed in the kinetics associated with $\mathrm{CO}$ 
bimolecular rebinding to $\mathrm{Cygb}^{\text {red }}$ were attributed to the heterogeneity previously reported for Arg 84 residue in Cygb structure. In the structure of CO bound Cygb, the Arg 84 sidechain adopts a single conformation that is stabilized by a hydrogen bond with the carboxyl group of Asp 40 and carbonyl oxygen of Glu 73 (Makino et al., 2011), whereas in the structure of ligand free Cygb, Arg 84 was reported to adopt two distinct orientations: a major conformation $(\sim 60 \%)$ with the side-chain oriented towards the solvent and a minor conformation $(\sim 40 \%)$ with the side-chain positioned towards the heme pocket (de Sanctis et al., 2004a) (Figure 6.10). I propose that the heterogeneity of the Arg 84 position promotes biphasic $\mathrm{CO}$ association to $\mathrm{Cygb}^{\text {red }}$ whereas the repositioning of the E-helix in $\mathrm{Cygb}^{\mathrm{ox}}$ stabilizes a single conformation of Arg 84 sidechain resulting in a nearly single phase for ligand bimolecular rebinding.

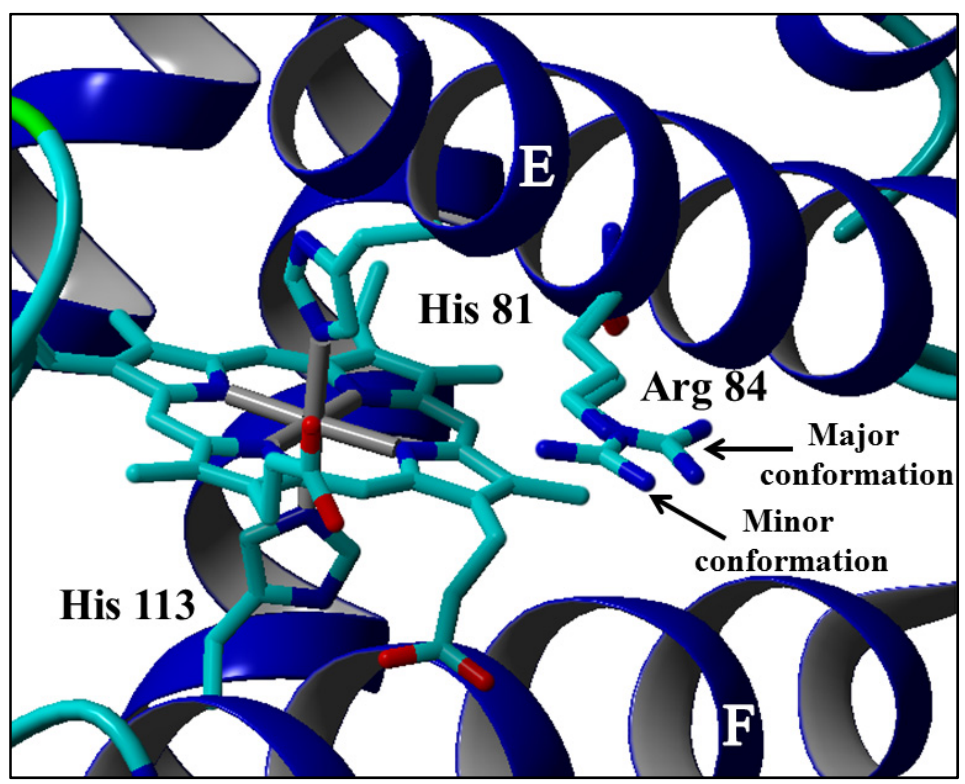

Figure 6.10 Three dimensional structure of ferric human Cygb in its ligand free form (PDB entry: 1VH5) displaying the major and minor conformations of Arg 84 side-chain. 


\subsubsection{Analysis of hydrophobic cavities in $\mathrm{Cygb}^{\circ \mathrm{x}}$ and $\mathrm{Cygb}^{\text {red }}$}

In vertebrate globins, the extensive network of hydrophobic cavities and tunnels provides access pathways for the exogenous ligand to migrate to the active center and/or allows temporary ligand trapping. Two hydrophobic cavities in $\mathrm{Mb}\left(\mathrm{Xe}_{4}\right.$ and $\left.\mathrm{Xe}_{1}\right)$ serve as temporary trapping sites for the photo-dissociated ligand, whereas in hexa-coordinated $\mathrm{Ngb}$, a long hydrophobic cavity connecting the heme binding site with the surrounding solvent may function as a ligand migration pathway (Vallone et al., 2004a). An analogous hydrophobic channel situated between $\mathrm{AB}$ and $\mathrm{GH}$ loops was found in the crystal structures of Cygb lacking the internal disulfide bridge or in the structure of Cygb with Cys 38 and Cys 83 replaced by Ser (de Sanctis et al., 2004a, b). To understand the molecular origin of the distinct ligand migration mechanism in $\mathrm{Cygb}^{\mathrm{ox}}$ and $\mathrm{Cygb}^{\mathrm{red}}$, I have employed YASARA molecular modeling software to determine the distribution of internal cavities in $\mathrm{Cygb}^{\mathrm{ox}}$. The model structures of the ligand free $\mathrm{Cygb}^{\text {red }}$ and $\mathrm{Cygb}^{\text {ox }}$ are shown in Fig. 6.11. To compare the position of cavities observed in Cygb with the

position of the Xe binding sites (de Sanctis et al., 2004b), the model structure of Cygb ${ }^{\text {red }}$ was superimposed with that of the protein in the presence of Xe gas as shown in Figure 6.12 . 

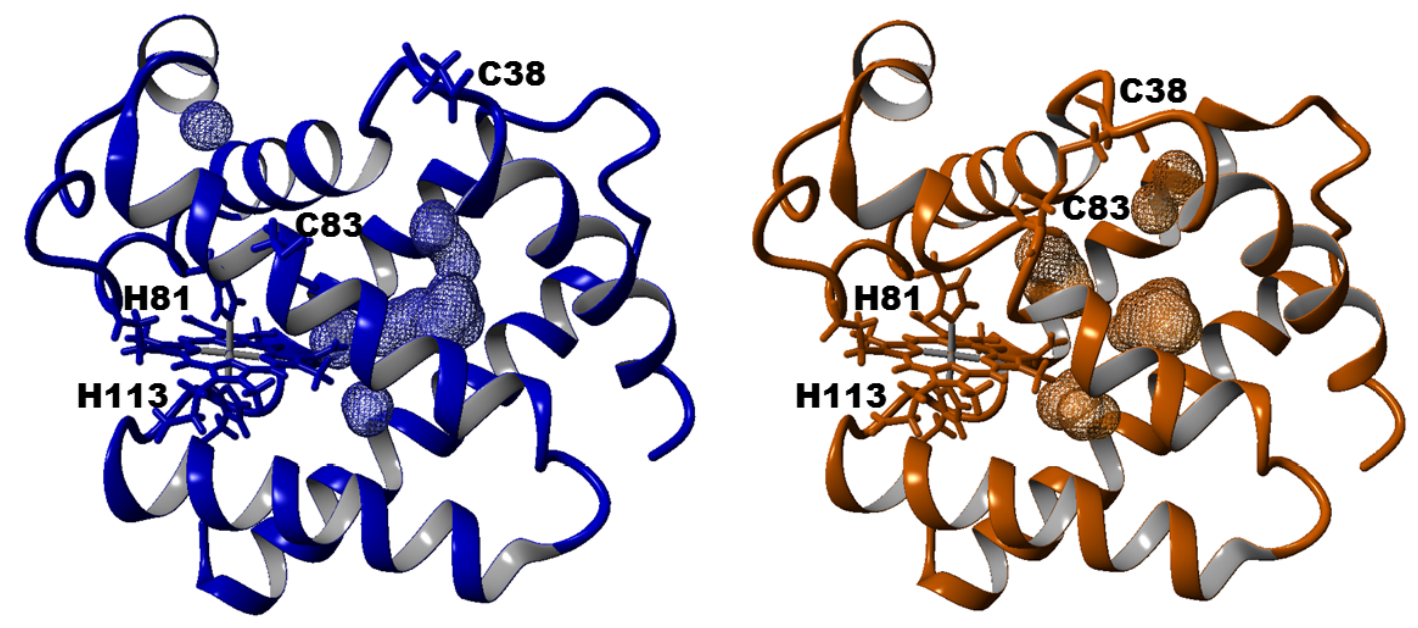

Figure 6.11 Representation of the internal cavities identified in the model structures of $\mathrm{Cygb}^{\text {red }}$ (left) and $\mathrm{Cygb}^{\text {ox }}$ (right).

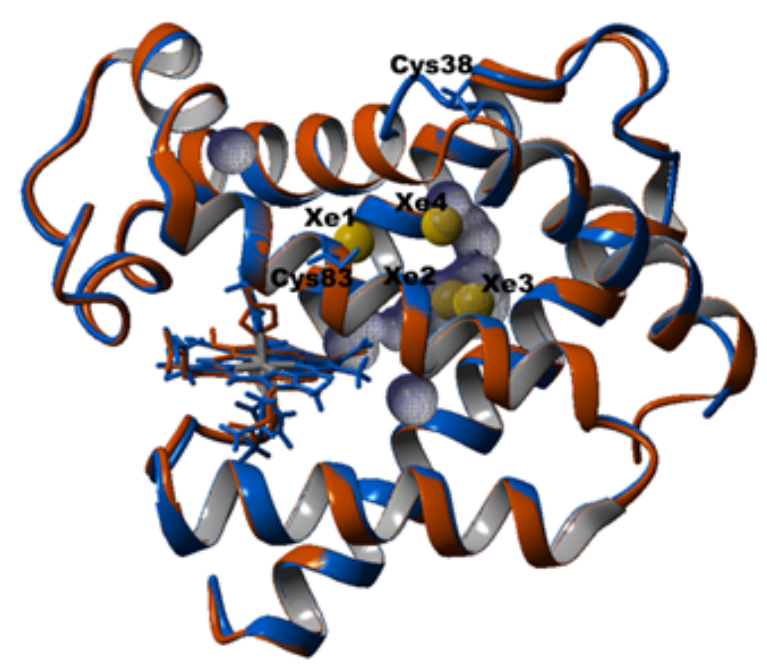

Figure 6.12 Overlay of minimized structure of ferric Cygb (PDB file: 1V5H, shown in blue) using YASARA structure molecular software as described in materials and methods section (section 2.4) and ferric Cygb double mutant (Cys38Ser and Cys83Ser) crystalized in the presence of Xe (PDB file: 1UX9, shown in orange). Internal cavities were analyzed using a $1.4 \AA$ probe radius and the numerical algorithm in YASARA software and are shown as mesh surfaces. Cys residues are shown as blue sticks and Xe atoms are depicted as yellow spheres. 
The long hydrophobic tunnel present in $\mathrm{Cygb}^{\text {red }}$ is missing in the model structure of $\mathrm{Cygb}^{\mathrm{ox}}$ and is substituted by three separated hydrophobic cavities that correspond to the three $\mathrm{Xe}$ binding sites $\left(\mathrm{Xe}_{2}, \mathrm{Xe}_{3}\right.$, and $\left.\mathrm{Xe}_{4}\right)$ previously found in the structure of Cygb crystallized in the presence of Xe gas (de Sanctis et al., 2004b).

Detailed inspection of the model structures reveals that in the structure of $\mathrm{Cygb}^{\mathrm{ox}}$, the Leu 34 and Leu 89 side-chains are repositioned in such a way that they partially block the long hydrophobic tunnel found in the $\mathrm{Cygb}^{\text {red }}$ structure. Such reorganization of the internal cavities as a result of the formation of the disulfide bridge may significantly affect the kinetics and quantum yield for the photo-dissociated ligand escape from the protein matrix by modulating the energy barriers for ligand transition between individual binding sites.

On the basis of the PAC and TA results, I propose that the presence of the long hydrophobic tunnel in the structure of $\mathrm{Cygb}^{\text {red }}$ promotes a fast escape of the photodissociated ligand from the protein matrix $(\tau<40 \mathrm{~ns})$ in a similar way as observed previously for Ngb (Anselmi et al., 2011; Astudillo et al., 2012). The enthalpy change of $18 \pm 2 \mathrm{kcal} \mathrm{mol}^{-1}$ measured for $\mathrm{CO}$ photo-release matches well with the enthalpy of the Fe-CO bond $\left(\sim 17 \mathrm{kcal} \mathrm{mol}^{-1}\right)$ (Miksovska et al., 2005) and the reaction enthalpy change measured for $\mathrm{CO}$ escape from $\mathrm{Ngb}^{\text {red }}$, suggesting a marginal enthalpy barrier for the structural transition between the $\mathrm{CO}$ bound six-coordinate and ligand free fivecoordinated heme iron. The passage of the photo-dissociated ligand through the hydrophobic channel may be facilitated by an increased flexibility of the GH loop in the free structure as observed in the computational studies (Zhang et al., 2011a). 
On the other hand, the reorganization of the internal cavities in $\mathrm{Cygb}^{\mathrm{ox}}$ increases the activation barrier for ligand passage through the internal hydrophobic cavities. On the basis of the similarity of the reaction and activation parameters associated with $\mathrm{CO}$ escape from $\mathrm{Cygb}^{\mathrm{ox}}$ and $\mathrm{Mb}$, I hypothesize that oxidation of the Cys 38 and Cys 83 thiol groups promotes ligand escape through the so-called histidine gate. Interestingly, the 150 ns time constant for $\mathrm{CO}$ release from $\mathrm{Cygb}^{\mathrm{ox}}$ is similar to the $\sim 200 \mathrm{~ns}$ time constant measured for the $\mathrm{CO}$ escape from $\mathrm{Mb}$ at low $\mathrm{pH}$ (Angeloni and Feis, 2003). These results are consistent with IR spectroscopy data on CO bound Cygb (Sawai et al., 2003) demonstrating that at neutral $\mathrm{pH}$ a significant fraction of Cygb $(\sim 40 \%)$ adopts an "open conformation" with the distal histidine side-chain oriented out of the heme pocket in a similar way as observed for the distal histidine in $\mathrm{Mb}$ at acidic $\mathrm{pH}$.

A schematic representation of the proposed mechanism of $\mathrm{CO}$ migration in $\mathrm{Cygb}^{\mathrm{ox}}$ and $\mathrm{Cygb}^{\text {red }}$ is shown in Figure 6.11.
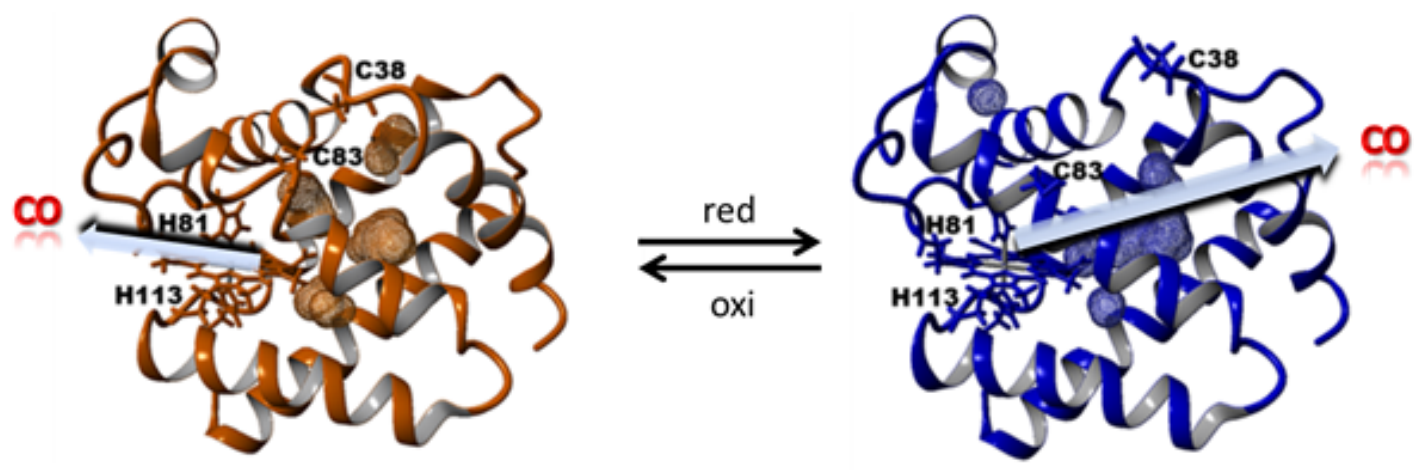

Figure 6.13 Schematic representation of the impact of the disulfide bond in Cygb.

Reduction/oxidation of the Cys38 Cys83 modifies the ligand migration in Cygb. In the absence of the internal disulfide bond, the ligand escape from the protein is fast 
and occurs through a hydrophobic channel, whereas formation of the internal disulfide bond promotes the $\mathrm{CO}$ escape through the "so-called" histidine gate.

\subsubsection{Proposed models for ligand migration in $\mathrm{Cygb}^{\mathrm{ox}}$ and $\mathrm{Cygb}^{\text {red }}$ and analysis of microscopic rate constants}

Considering the transient absorption data and thermodynamic parameters, I

propose a distinct model for the ligand migration in $\mathrm{Cygb}^{\text {ox }}$ and $\mathrm{Cygb}^{\text {red }}$ (Scheme 6.1). The presence of the multiphasic geminate kinetics requires a minimal four state sequential model to describe the ligand transitions between the individual hydrophobic cavities and solution. Since two phases are observed for the geminate $\mathrm{CO}$ rebinding to $\mathrm{Cygb}^{\mathrm{ox}}$ and the amplitude of the faster phase for the bimolecular $\mathrm{CO}$ rebinding can be neglected because of its small amplitude, a branched four state model was adopted to analyze the kinetic data as proposed previously for $\mathrm{CO}$ photo-dissociation from $\mathrm{R}$ state hemoglobin encapsulated in silica gel (Sottini et al., 2004) (Scheme 6.1, top model). According to this model, the photo-dissociated ligand initially migrates into the primary docking site (state B) and then rapidly diffuses into one of the internal hydrophobic cavities (state C). To escape from the protein matrix, the ligand has to return into the distal pocket (state B) before it migrates into the surrounding solvent (state D) through the so called "histidine gate" with the time constant of $\sim 100 \mathrm{~ns}$. 
Cygbox

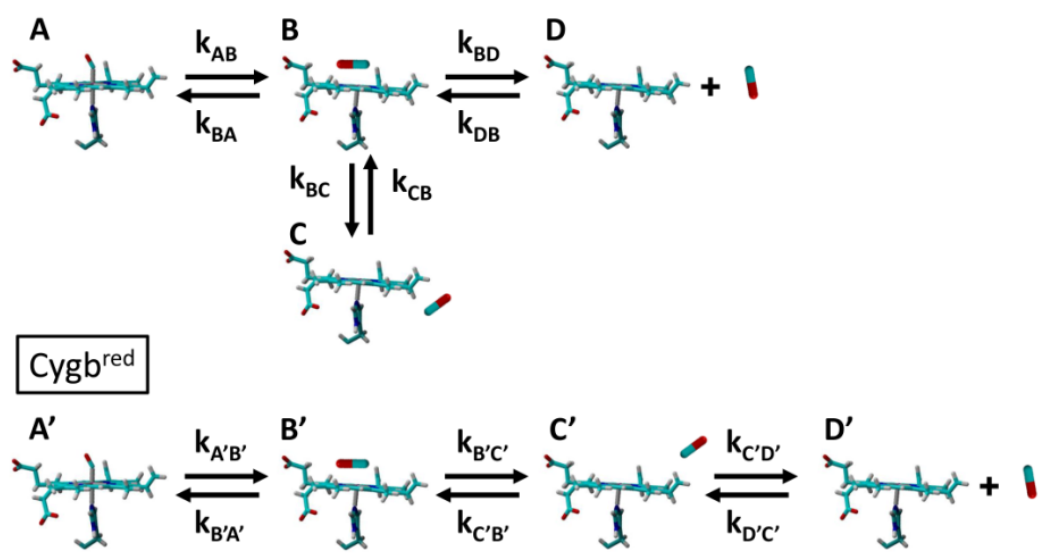

Scheme 6.1

The description of the transient absorption data analysis used to calculate the microscopic rate constants are shown in the materials and methods section (Chapter 3). Briefly, the differential equations corresponding to ligand migration in $\mathrm{Cygb}^{\mathrm{ox}}$ shown in scheme 6.1 can be solved analytically as previously reported by Sottini et al. (2004) for hemoglobin and the microscopic rate constants were determined as described in the materials and methods section (Chapter 3).

On the other hand, the presence of two kinetics of comparable amplitude for the $\mathrm{CO}$ bimolecular rebinding to $\mathrm{Cygb}^{\text {red }}$ is consistent with $\mathrm{CO}$ binding to two distinct $\mathrm{Cygb}^{\text {red }}$ populations. One population was associated with an "accessible conformation" in which Arg 84 side-chain is oriented towards the surrounding solvent facilitating a fast bimolecular $\mathrm{CO}$ rebinding $\left(\mathrm{k}=132.3 \mu \mathrm{M}^{-1} \mathrm{~s}^{-1}\right)$. The second population corresponds to $\mathrm{Cygb}^{\text {red }}$ in a "restricted conformation" in which Arg 84 side-chain is oriented toward the heme group and increases the barrier for bimolecular CO rebinding $\left(\mathrm{k}=7.3 \mu \mathrm{M}^{-1} \mathrm{~s}^{-1}\right)$. In such case, the migration of the photo-dissociated ligand in the "accessible" and 
"restricted" conformations of Cygb can be described using a four state sequential model as shown in Scheme 6.1 (bottom model). According to this model, upon photodissociation, the $\mathrm{CO}$ molecule rapidly diffuses into the primary docking site B', and from this site migrates to the nearby hydrophobic cavities (state $C^{\prime}$ ), then to the solvent. The ligand escape from the hydrophobic channel is fast, with a time constant of less than 50 ns. The individual microscopic rate constants were determined as described by Gibson et al. (1986) for n-propyl, n-butyl and tert-butyl isocyanide binding to sperm whale $\mathrm{Mb}$ (as described in the materials and methods section, chapter 3) and are listed in Table 6.6.

The microscopic rate constants for the geminate ligand association to the heme iron are fast and comparable for both $\mathrm{Cygb}^{\mathrm{ox}}$ and $\mathrm{Cygb}^{\text {red }}\left(\mathrm{k}_{\mathrm{BA}}=4 \times 10^{7} \mathrm{~s}^{-1}\right.$ and $\mathrm{k}_{\mathrm{B}^{\prime} \mathrm{A}^{\prime}}=5 \mathrm{x}$ $10^{7} \mathrm{~s}^{-1}$, respectively). Analogous geminate rebinding rates were reported previously for the $\mathrm{CO}$ association to $\mathrm{Cygb}^{\text {red }}$ (Gabba et al., 2013) and $\mathrm{Ngb}$ (Abbruzzetti et al., 2009) and were attributed to the reactive heme iron in six-coordinate globins. CO migration between the docking sites $\mathrm{B}$ and $\mathrm{C}$ and $\mathrm{B}$ ' and C' occurs on the nanosecond time scale in Cygb with the oxidized and reduced Cys residues, respectively, which is consistent with a low energy barrier separating the individual docking sites. On the other hand, the rate constant for the ligand escape from the protein matrix in $\mathrm{Cygb}^{\mathrm{ox}}$ is approximately three times smaller than that for $\mathrm{Cygb}^{\text {red }}$ suggesting that the changes in the Cygb structure due to the internal disulfide bond reduction modulate the geminate quantum yield predominantly by altering the rate of ligand escape from the protein matrix to the surrounding solvent. 
Table 6.5 Microscopic rate constants associated with $\mathrm{CO}$ migration in $\mathrm{Cygb}^{\mathrm{ox}}$ at $20^{\circ} \mathrm{C}$.

\begin{tabular}{cc}
\hline $\mathrm{k}_{\mathrm{BA}}\left(\mathrm{s}^{-1}\right)$ & $4 \times 10^{7}$ \\
\hline $\mathrm{k}_{\mathrm{BC}}\left(\mathrm{s}^{-1}\right)$ & $2 \times 10^{7}$ \\
\hline $\mathrm{k}_{\mathrm{CB}}\left(\mathrm{s}^{-1}\right)$ & $2 \times 10^{7}$ \\
\hline $\mathrm{k}_{\mathrm{BD}}\left(\mathrm{s}^{-1}\right)$ & $3 \times 10^{7}$ \\
\hline $\mathrm{k}_{\mathrm{DB}}\left(\mathrm{M}^{-1} \mathrm{~s}^{-1}\right)$ & $18 \times 10^{6}$ \\
\hline
\end{tabular}

Table 6.6 Microscopic rate constants associated with $\mathrm{CO}$ migration in $\mathrm{Cygb}^{\text {red }}$ at $20{ }^{\circ} \mathrm{C}$.

\begin{tabular}{cc}
\hline $\mathrm{k}_{\mathrm{B}^{\prime} \mathrm{A}^{\prime}}\left(\mathrm{s}^{-1}\right)$ & $5 \times 10^{7}$ \\
\hline $\mathrm{k}_{\mathrm{B}^{\prime} \mathrm{C}^{\prime}}\left(\mathrm{s}^{-1}\right)$ & $6 \times 10^{7}$ \\
\hline $\mathrm{k}_{\mathrm{C}^{\prime} \mathrm{B}^{\prime}}\left(\mathrm{s}^{-1}\right)$ & $2 \times 10^{7}$ \\
\hline $\mathrm{k}^{\mathrm{acc}} \mathrm{C}^{\prime} \mathrm{D}^{\prime}\left(\mathrm{s}^{-1}\right)$ & $9 \times 10^{7}$ \\
\hline $\mathrm{k}^{\mathrm{rest}} \mathrm{C}^{\prime} \mathrm{D}^{\prime}\left(\mathrm{s}^{-1}\right)$ & $6 \times 10^{7}$ \\
\hline $\mathrm{k}^{\mathrm{acc}} \mathrm{D}^{\prime} \mathrm{C}^{\prime}\left(\mathrm{M}^{-1} \mathrm{~s}^{-1}\right)$ & $228 \times 10^{6}$ \\
\hline $\mathrm{k}^{\mathrm{rest}} \mathrm{D}^{\prime} \mathrm{C}^{\prime}\left(\mathrm{M}^{-1} \mathrm{~s}^{-1}\right)$ & $13 \times 10^{6}$ \\
\hline
\end{tabular}

\subsection{Summary}

Despite the relatively modest impact of the internal disulfide bridge on the distal histidine affinity (Hamdane et al., 2003), the photoacoustic and transient absorption data suggest that the oxidation/reduction of the Cys thiol groups alters Cygb conformational dynamics including the flexibility of the amino acid side chains and accordingly the size, accessibility, and dynamics of internal hydrophobic cavities. These data point towards a 
more flexible distal histidine side-chain in the structure of ligand free $\mathrm{Cygb}^{\mathrm{ox}}$ that may facilitate $\mathrm{CO}$ escape from the $\mathrm{Cygb}^{\text {ox }}$ distal pocket through the so-called "histidine gate". Since the internal hydrophobic cavities found in various heme proteins are believed to have physiological roles and either facilitate ligand migration or store gaseous ligands, these results suggest that Cygb may carry out multiple physiological functions which are regulated by intracellular redox properties. 


\section{TIME-RESOLVED THERMODYNAMICS ASSOCIATED WITH EXOGENOUS LIGAND BINDING TO VERTEBRATE HEMOGLOBINS}

(The work described in this chapter was partially published in Jaroslava Miksovska and Luisana Astudillo (2011). Time-resolved thermodynamics associated with diatomic ligand dissociation from globins, Thermodynamics-Interaction StudiesSolids, Liquids and Gases, Dr. Juan Carlos Moreno Piraja (Ed.), InTech)

\subsection{Introduction}

Ligand-induced conformational transitions play an eminent role in the biological activity of proteins including recognition, signal transduction, and membrane trafficking. Conformational transitions occur over a broad time range starting from picosecond transitions that reflect reorientation of amino acid side chains to slower dynamics on the millisecond time-scale that correspond to larger domain reorganization (HenzlerWildman et al., 2007). Direct characterization of the dynamics and energetics associated with conformational changes over such a broad time range remains challenging due to limitations in experimental protocols and often due to the absence of a suitable molecular probe through which to detect structural reorganization. Photothermal methods such as photoacoustic calorimetry (PAC) and photothermal beam deflection provide a unique approach to characterize conformational transitions in terms of time resolved volume and enthalpy changes (Gensch and Viappiani, 2003; Miksovska and Larsen, 2003). Unlike traditional spectroscopic techniques that are sensitive to structural changes confined to the vicinity of a chromophore, photothermal methods monitor overall changes in volume and enthalpy allowing for the detection of structural transitions that are spectroscopically 
silent (i.e. do not lead to optical perturbations of either intrinsic or extrinsic chromophores).

Myoglobin $(\mathrm{Mb})$ and hemoglobin $(\mathrm{Hb})$ play a crucial role in the storage and transport of oxygen molecules in vertebrates and have served as model systems for understanding the mechanism through which protein dynamics regulate ligand access to the active site, ligand affinity and specificity, and, in the case of hemoglobin, oxygen binding cooperativity. Myoglobin and individual $\alpha$ - and $\beta$ - subunits of $\mathrm{Hb}$ exhibit significant structural similarities, i.e. the presence of a five coordinate heme iron with a His residue coordinated to the central iron (proximal ligand) and a characteristic "3-on-3" globin fold (Figure 1) (Park et al., 2006; Yang and Phillips, 1996). Both proteins reversibly bind small gaseous ligands such as $\mathrm{O}_{2}, \mathrm{CO}$, and NO. The photo-cleavable Feligand bond allows for the monitoring of transient deoxy intermediates using timeresolved absorption spectroscopy (Carver et al., 1990; Esquerra et al., 2010; Gibson et al., 1986) and time resolved X-ray crystallography (Milani et al., 2008; Šrajer et al., 2001).

On the basis of spectroscopic data and molecular dynamics approaches (Bossa et al., 2004; Mouawad et al., 2005), a comprehensive molecular mechanism for ligand migration in $\mathrm{Mb}$ was proposed including an initial diffusion of the photo-dissociated $\mathrm{CO}$ molecule into the internal network of hydrophobic cavities, followed by a return into the distal pocket and subsequent rebinding to heme iron or escape from the protein through a distal histidine gate. The ligand migration into internal cavities induces a structural deformation, which promotes a transient opening of a gate in the $\mathrm{CO}$ migration channel. Such transitional reorganization of the internal cavities is ultimately associated with a change in volume and/or enthalpy and thus can be probed using photothermal techniques. 
Indeed, $\mathrm{CO}$ photo-dissociation from $\mathrm{Mb}$ has been intensively investigated using PAC by several groups (Belogortseva et al., 2007; Peters et al., 1992; Vetromile et al., 2011; Westrick and Peters, 1990; Westrick et al., 1990) and these results lead to a thermodynamic description of the transient "deoxy intermediate" that is populated upon CO photo-dissociation.

The mechanism of ligand migration in $\mathrm{Hb}$ is more complex, since it is determined by the tertiary structure of individual subunits as well as by the tetramer quaternary structure. Crystallographic data have shown that the structure of the fully unliganded tense $(\mathrm{T})$ state of $\mathrm{Hb}$ and the fully ligated relaxed (R) states differ at both the tertiary and quaternary level (Park et al., 2006). Crystallographic and NMR studies suggest that the fully ligated relaxed state corresponds to the ensemble of conformations with distinct structures (Mueser et al., 2000; Silva et al., 1992). Moreover, Hb interactions with diatomic ligands is modulated by physiological effectors such as protons, chloride and phosphate ions, and non-physiological ligands including inositol hexakisphosphate (IHP) and bezafibrate (BZF) (Yonetani et al., 2002). Despite a structural homology between $\mathrm{Hb}$ and $\mathrm{Mb}$, the network of internal hydrophobic cavities identified in $\mathrm{Mb}$ is not conserved in $\mathrm{Hb}$ suggesting distinct ligand migration pathways in this protein (Mouawad et al., 2005; Savino et al., 2009).

Neuroglobin $(\mathrm{Ngb})$ is a recently discovered heme protein that belongs to the family of vertebrate hexa-coordinate hemoglobins (Burmester et al., 2000). Although the physiological function of $\mathrm{Ngb}$ is unclear, a neuroprotective role against oxidative stress induced damage has been proposed for Ngb (Greenberg et al., 2008; Nienhaus and Nienhaus, 2007). In spite of the low sequence homology between $\mathrm{Ngb}$ and $\mathrm{Mb}$, the three 
dimensional structure of $\mathrm{Ngb}$ is almost superimposable to that of $\mathrm{Mb}$ exhibiting the characteristic 3-over-3 $\alpha$ helix globin fold (Figure 7.1). However, Ngb exhibits structural features not previously observed in $\mathrm{Mb}$ and $\mathrm{Hb}$, including heme iron hexacoordination, the presence of an internal disulfide bond connecting the $\mathrm{CD}$ loop with the D-helix, large hydrophobic cavities, and heme sliding mechanism (Hamdane et al., 2003; Pesce et al., 2003; Vallone et al., 2004b). Previous spectroscopic and computational studies propose that the molecular mechanism of $\mathrm{CO}$ migration in $\mathrm{Ngb}$ includes fast diffusion of the photo-dissociated $\mathrm{CO}$ molecule into the internal network of hydrophobic cavities, which then returns into the distal pocket (Abbruzzetti et al., 2009; Astudillo et al., 2012). The subsequent rebinding to heme iron or escape from the protein through occurs through the wide hydrophic tunnel linking the heme binding site to the surrounding solvent, instead of the so-called "histidine gate" (Astudillo et al., 2012).

Here, I present a photoacoustic calorimetry study of $\mathrm{O}_{2}$ photo-dissociation from $\mathrm{Mb}$ and $\mathrm{Ngb}$ that has not been previously investigated using photothermal methods, despite the fact that oxygen is the physiological ligand for $\mathrm{Mb}$ and some studies indicate that $\mathrm{O}_{2}$ may be the physiological ligand in $\mathrm{Ngb}$. In addition, photoacoustic calorimetry was employed to determine the thermodynamic profiles of $\mathrm{CO}$ photo-dissociation from human $\mathrm{Hb}$ in the presence of heterotrophic allosteric effectors IHP and BZF. 


\section{A}

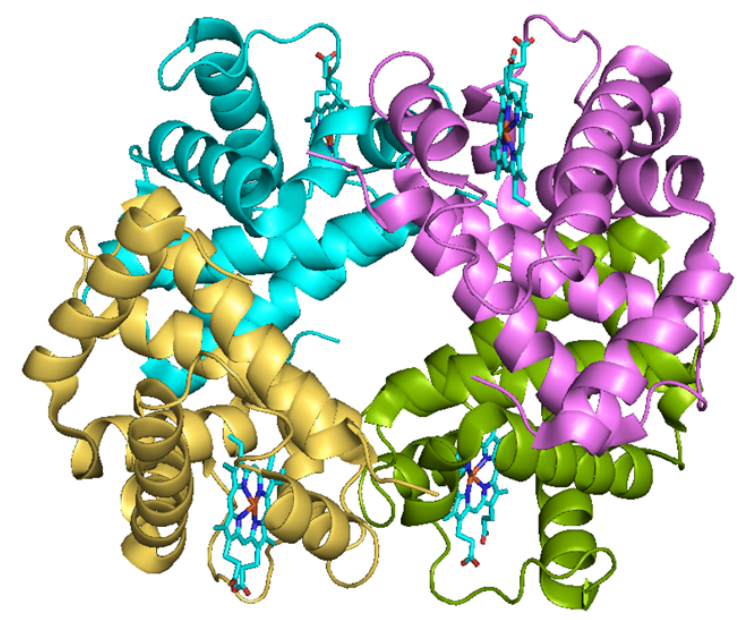

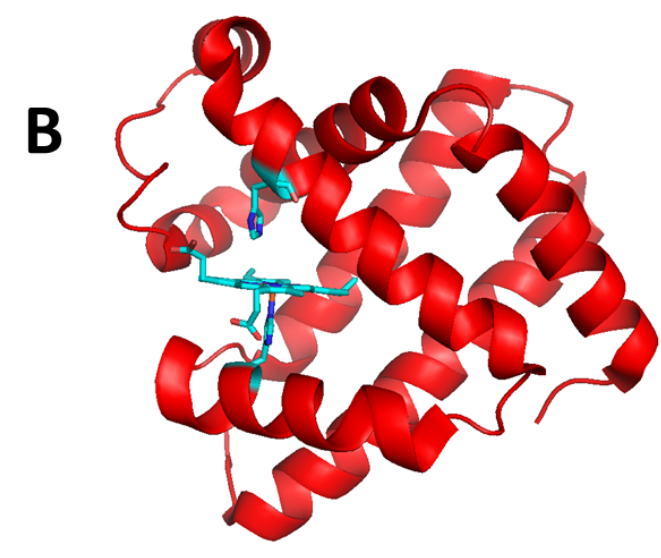

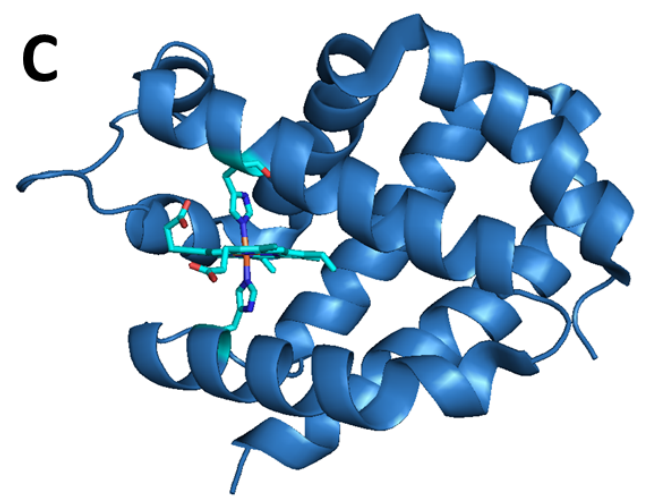

Figure 7.1 Ribbon representation of the tetrameric human $\mathrm{Hb}$ structure (A, PBD entry $1 \mathrm{FDH})$, horse heart $\mathrm{Mb}$ structure (B, PDB entry 1WLA) and human Ngb structure (C, PDB entry 1JO6). The heme prosthetic groups are shown as sticks. In the case of $\mathrm{Mb}$ and $\mathrm{Ngb}$, the distal and proximal histidines are visualized.<smiles>CC(C)(Oc1ccc(CCNC(=O)c2ccc(Cl)cc2)cc1)C(=O)O</smiles>

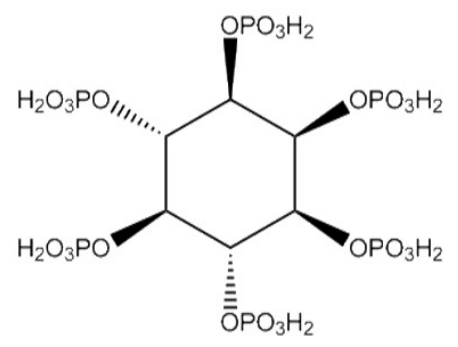

Figure 7.2 Structure of bezafibrate (left) and inositol hexakisphosphate (right). 


\subsection{Results}

\subsubsection{Steady-state absorption spectroscopy}

The steady-state absorption spectra of ferric, deoxy, and oxygen bound wild type $\mathrm{hNgb}$ at $\mathrm{pH} 7.0$ are displayed in Figure 7.3. The ferric form of $\mathrm{hNgb}$ exhibits a Soret band at $413 \mathrm{~nm}$ and two visible bands at $535 \mathrm{~nm}$ and $561 \mathrm{~nm}$. Reduction of the heme iron results in a bathochromic shift of the Soret band to $424 \mathrm{~nm}$ and the two Q bands in the visible part of the spectrum shift to shorter wavelengths (529 and $559 \mathrm{~nm})$. The absorption spectrum of oxygen bound $\mathrm{hNgb}$ exhibits a Soret band at the same position of the $\mathrm{CO}$ bound adduct $(413 \mathrm{~nm})$, but the two visible bands are shifted to longer wavelengths (539 $\mathrm{nm}$ and $571 \mathrm{~nm}$ ). The absorbance maxima observed for $\mathrm{hNgb}$ are identical to those previously reported by Dewilde et al. (2001) for the ferric, ferrous, and $\mathrm{O}_{2}$ adducts of $\mathrm{hNgb}$, and these spectral features are characteristic of hexacoordinate hemoglobins, including cytochromes, indicating that $\mathrm{Ngb}$ is a hexa-coordinated globin in which the distal His 64 is the endogenous ligand (Dewilde et al. 2001). 


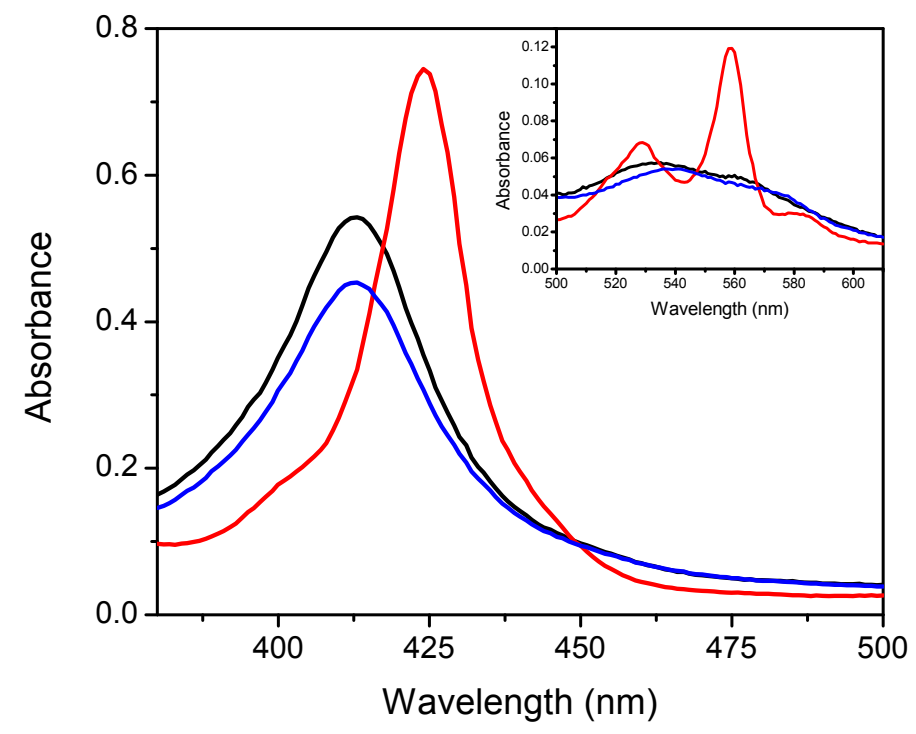

Figure 7.3 Absorption spectra of ferric (black line), ferrous (red line) and $\mathrm{O}_{2}$ bound (blue line) $\mathrm{hNgb}$ wild type in $50 \mathrm{mM}$ Tris buffer (pH 7.0).

\subsubsection{Thermodynamic parameters determined for $\mathrm{O}_{2}$ photo-release from vertebrate hemoglobins}

Ligand migration in heme proteins is often described using the sequential threestate model (Henry et al., 1983) shown in Figure 7.4. Upon cleavage of the coordination bond between the ligand and heme iron, the ligand is temporarily trapped within the protein matrix and then it either directly rebinds back to the heme iron in the so called "geminate rebinding" or diffuses from the protein matrix into the surrounding solvent. The subsequent bimolecular ligand binding to heme iron occurs on significantly longer time scales, hundreds of microseconds to milliseconds. The quantum yield for the geminate rebinding and for bimolecular association is strongly dependent on the character of the ligand and the protein. For example, $\mathrm{CO}$ rebinds to $\mathrm{Mb}$ predominantly 
through a bimolecular reaction with quantum yield close to unity $\left(\Phi_{\text {bim }}=0.96\right)($ Henry et al., 1983) and to Ngb with a lower quantum yield of 0.68 (Astudillo et al., 2010, 2012), whereas the quantum yield for bimolecular $\mathrm{O}_{2}$ rebinding to heme proteins is usually significantly lower (Carver et al., 1990; Walda et al., 1994), and NO rebinds predominantly through geminate rebinding ( $\mathrm{Ye}$ et al., 2002). To determine the thermodynamic parameters from acoustic data, the quantum yields for $\mathrm{CO}$ and $\mathrm{O}_{2}$ bimolecular rebinding to the proteins under investigation have to be known.

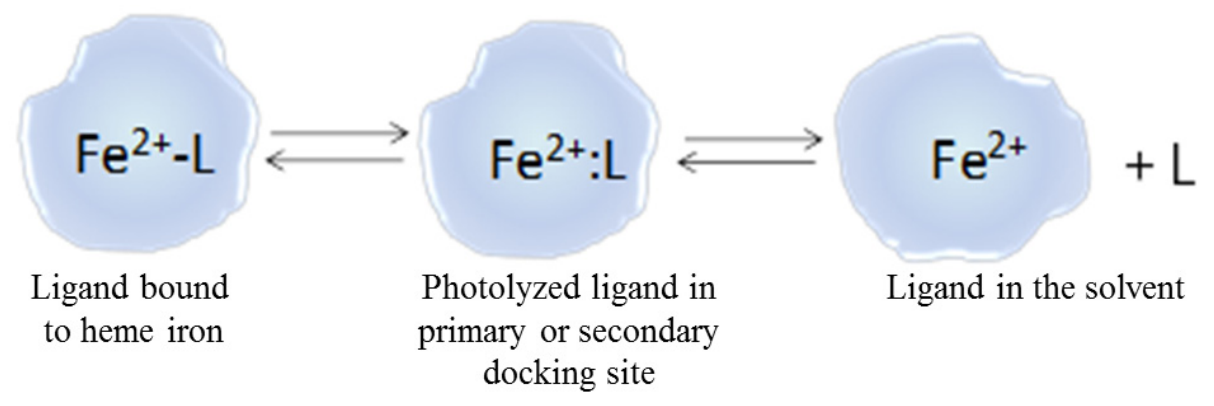

Figure 7.4 Schematic representation of ligand migration observed in heme proteins.

\subsubsection{Myoglobin}

The quantum yield for $\mathrm{O}_{2}$ bimolecular rebinding to $\mathrm{Mb}$ was measured in the temperature range from $5{ }^{\circ} \mathrm{C}$ to $20{ }^{\circ} \mathrm{C}$ using TA spectroscopy, as described in the Materials and Methods section. As shown in Figure 7.4, the values determined for the quantum yield show weak temperature dependence with the quantum yield decreasing with increasing temperature. At $20{ }^{\circ} \mathrm{C}$ the quantum yield is $0.09 \pm 0.01$ that is within the range of values reported previously $(\Phi=0.057$ (Walda et al., 1994) and $(\Phi=0.12$ (Carver et al., 1990)). 


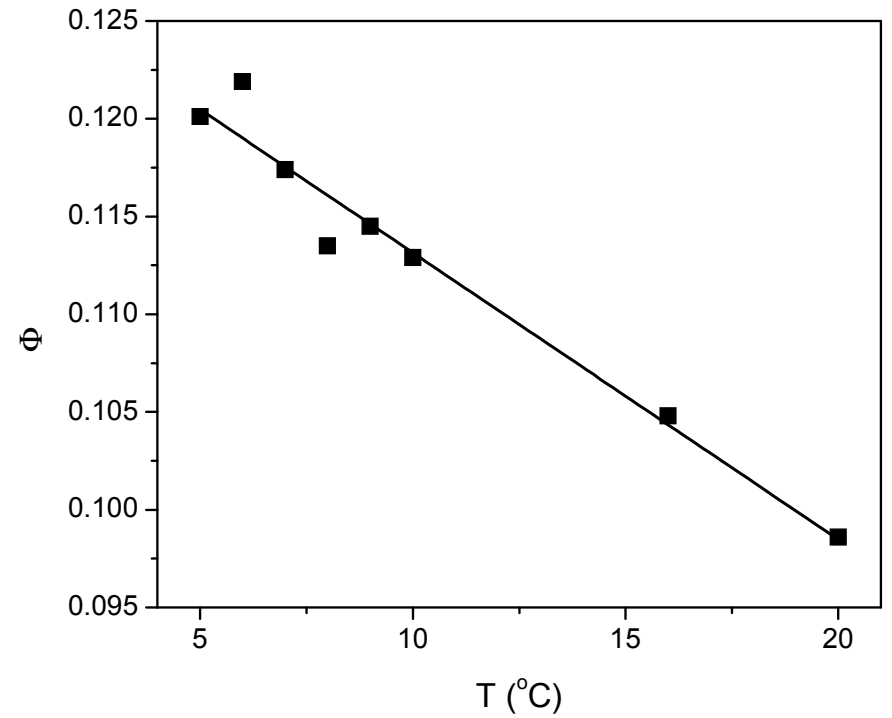

Figure 7.5 Quantum yield for bimolecular $\mathrm{O}_{2}$ rebinding to $\mathrm{Mb}$ as a function of temperature. The solid line demonstrates the trend.

Photo-acoustic traces for $\mathrm{O}_{2}$ dissociation from $\mathrm{Fe}^{2+} \mathrm{Mb}$ at $\mathrm{pH} 7.0$ are displayed in Figure 7.5. At low temperatures $\left(6{ }^{\circ} \mathrm{C}\right.$ to $\left.15^{\circ} \mathrm{C}\right)$, the sample photo-acoustic traces show a phase shift with respect to the reference trace indicating the presence of thermodynamic process(es) that occurs between $50 \mathrm{~ns}$ and $\sim 5 \mu$ s. The sample traces were deconvoluted as described in the Materials and Methods section and $\phi_{\mathrm{i}}$ values were plotted as a function of the temperature dependent factor $\left(C_{p} \rho / \beta\right)$ (Figure 7.6). The extrapolated volume and enthalpy changes are listed in Table 7.1. 


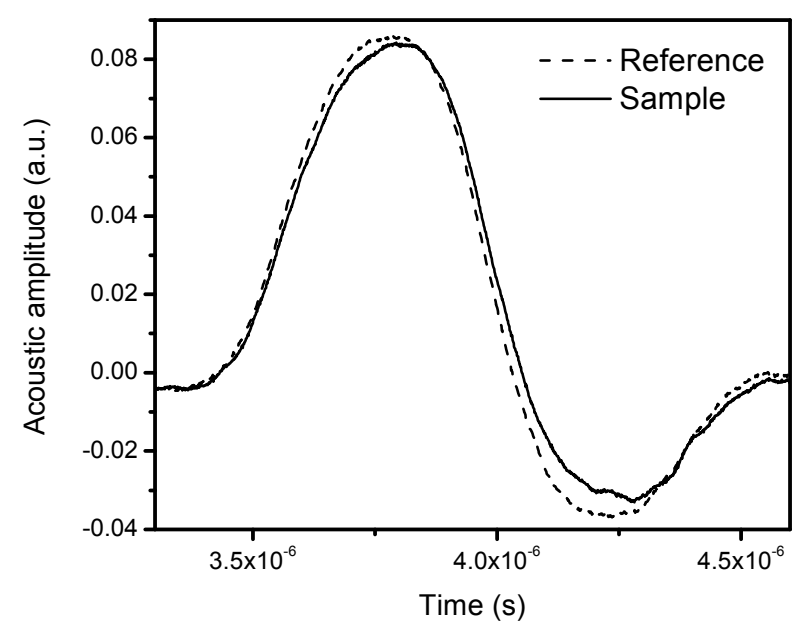

Figure 7.6 PAC traces for $\mathrm{O}_{2}$ photo-dissociation from $\mathrm{O}_{2}-\mathrm{Mb}$ at $9{ }^{\circ} \mathrm{C}$. Conditions: $40 \mu \mathrm{M}$ $\mathrm{Mb}$ dissolved in $50 \mathrm{mM}$ Hepes buffer $(\mathrm{pH}$ 7.0). The absorbance of the reference compound, $\mathrm{Fe}(\mathrm{III}) 4 \mathrm{SP}$, at the excitation wavelength of $532 \mathrm{~nm}$ was identical to that of $\mathrm{O}_{2}-\mathrm{Mb}$.

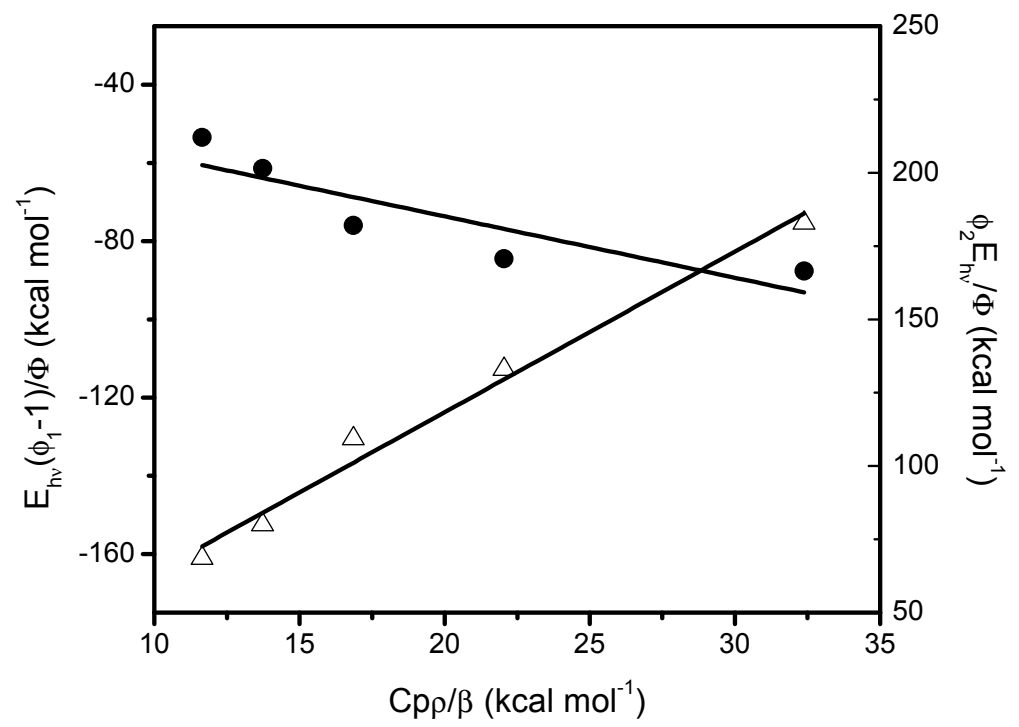

Figure 7.7 Plot of the ratio of the acoustic amplitude for $\mathrm{O}_{2}$ photo-release from $\mathrm{Mb}$ and the reference compound as a function of the $\mathrm{C}_{\mathrm{p}} \rho / \beta$ term. The values obtained for $\phi_{1}$ that correspond to the prompt phase are shown as solid circles and the $\phi_{2}$ values corresponding to the slow phase are shown as open triangles. 
Table 7.1 Volume and enthalpy changes associated with $\mathrm{O}_{2}$ dissociation from $\mathrm{Mb}$ in the temperature range of $6^{\circ} \mathrm{C}$ to $10^{\circ} \mathrm{C}$.

\begin{tabular}{ccc}
\hline & $\Delta \mathrm{V}\left(\mathrm{mL} \mathrm{mol}^{-1}\right)$ & $\Delta \mathrm{H}\left(\mathrm{kcal} \mathrm{mol}^{-1}\right)$ \\
\hline Fast phase $(\tau<20 \mathrm{~ns})$ & $-3.0 \pm 0.5$ & $20.5 \pm 8.5$ \\
Slow phase $(\tau>20 \mathrm{~ns})$ & $5.5 \pm 0.4$ & $-8.9 \pm 8.0$ \\
\hline
\end{tabular}

Photo-cleavage of the $\mathrm{Fe}-\mathrm{O}_{2}$ bond is associated with a fast structural relaxation ( $\tau<20 \mathrm{~ns}$ ) forming a transient "deoxy-Mb intermediate". This initial transition is endothermic $\left(\Delta \mathrm{H}=21 \pm 9 \mathrm{kcal} \mathrm{mol}^{-1}\right)$ and leads to a small volume contraction of $-3.0 \pm$ $0.5 \mathrm{~mL} \mathrm{~mol}^{-1}$. This initial relaxation is followed by a $\sim 250 \mathrm{~ns}$ kinetics that exhibit a volume increase of $5.5 \pm 0.4 \mathrm{~mL} \mathrm{~mol}^{-1}$ and a very small enthalpy change of $-8.9 \pm 8.0$ $\mathrm{kcal} \mathrm{mol}^{-1}$. The positive enthalpy change of the initial process is consistent with the photo-cleavage of $\mathrm{Fe}-\mathrm{O}_{2}$ bond and a similar volume decrease of approximately $-3 \mathrm{~mL}$ $\mathrm{mol}^{-1}$ has been observed previously for the photo-dissociation of $\mathrm{Fe}-\mathrm{CO}$ bond in $\mathrm{Mb}$ (Westrick and Peters, 1990; Westrick et al., 1990). The subsequent 250 ns kinetics may reflect either the nanosecond geminate rebinding of the $\mathrm{O}_{2}$ molecule or the ligand diffusion from the protein matrix into the surrounding solvent.

The reaction volume change observed for the slow phase includes several factors: i) volume change due to the $\mathrm{O}_{2}$ escape into the surrounding solvent, ii) volume change associated with heme hydration in deoxyMb, and iii) volume change resulting from structural changes. The reaction volume can be expressed as the difference between the partial molar volume of products and reactants according to:

$$
\Delta V_{\text {slow }}=V_{\mathrm{O}_{2}}^{o}+V_{\text {deoxyMb }}^{o}-V_{\mathrm{O}_{2} \mathrm{Mb}}^{o}-V_{\mathrm{H}_{2} \mathrm{O}}^{o}
$$


Where $\mathrm{V}_{\mathrm{O} 2}^{\circ}$ is the partial molar volume of oxygen, $\mathrm{V}^{\circ}{ }_{\mathrm{H} 2 \mathrm{O}}$ corresponds to the partial molar volume of water, $\mathrm{V}_{\text {deoxymb }}^{\circ}$ refers to the partial molar volume of transient "deoxyMb" intermediate, and $\mathrm{V}^{\circ}{ }_{\mathrm{O} 2 \mathrm{Mb}}$ is the partial molar volume of oxy-Mb. Using $\mathrm{V}_{\mathrm{O} 2}^{\circ}$ $=28 \mathrm{~mL} \mathrm{~mol}^{-1}$ (Projahn et al., 1990) and $\mathrm{V}_{\mathrm{H} 2 \mathrm{O}}^{\circ}=15 \mathrm{~mL} \mathrm{~mol}^{-1}$ (the partial molar volume of water scaled to the occupancy of water molecule hydrogen bound to distal histidine) (Belogortseva et al., 2007), the structural volume change associated with $\mathrm{O}_{2}$ photorelease from $\mathrm{Mb}\left(\mathrm{V}_{\text {deoxyMb }}^{\mathrm{o}}-\mathrm{V}_{\mathrm{O}}^{\mathrm{o}} \mathrm{Mb}\right)$ was estimated to be $-7.5 \mathrm{~mL} \mathrm{~mol}^{-1}$. This value is very similar to that reported previously for $\mathrm{CO}$ escape from $\mathrm{Mb}\left(\Delta \mathrm{V}_{\text {structural }}=\mathrm{V}_{\text {deoxyMb }}^{\mathrm{o}}-\right.$ $\mathrm{V}_{\mathrm{O} 2 \mathrm{Mb}}^{\mathrm{o}}=-6 \mathrm{~mL} \mathrm{~mol}^{-1}$ ) (Vetromile et al., 2011) demonstrating that the overall structural changes accompanying the ligand bound to ligand free transition in $\mathrm{Mb}$ are comparable for both ligands.

\subsubsection{Neuroglobin}

The quantum yield for $\mathrm{O}_{2}$ bimolecular rebinding to human $\mathrm{Ngb}$ was measured in the temperature range from $7{ }^{\circ} \mathrm{C}$ to $25{ }^{\circ} \mathrm{C}$ using TA spectroscopy, as described in the Materials and Methods section. The values determined for the quantum yield show weak temperature dependence with the quantum yield increasing with increasing temperature (Figure 7.7). The value determined for the quantum yield is $0.15 \pm 0.01$ at $20^{\circ} \mathrm{C}$, which is $\sim 4.5$ times lower than the quantum yield determined for $\mathrm{CO}$ bimolecular rebinding to human Ngb. 


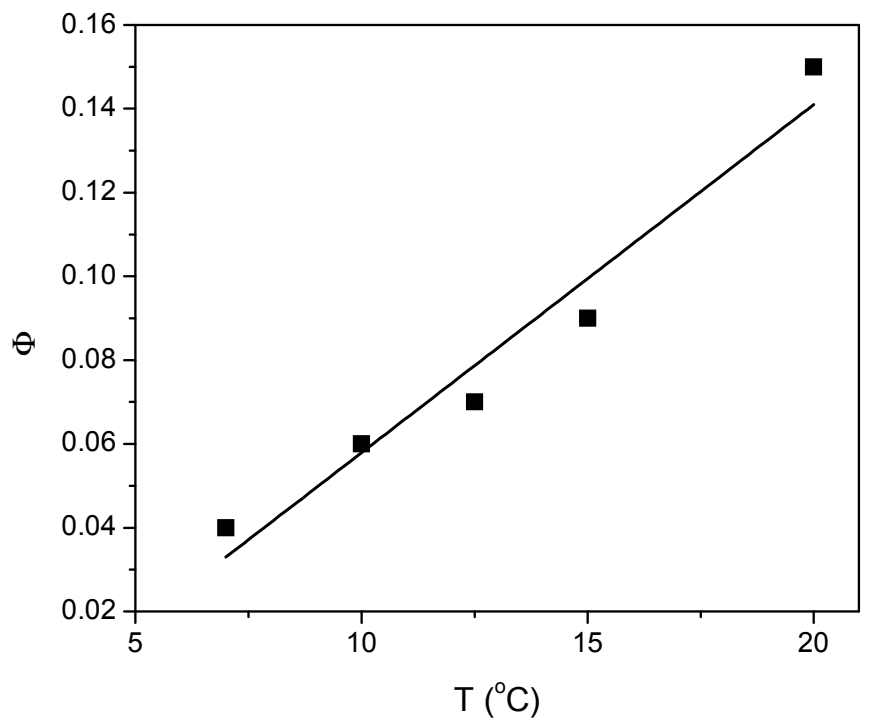

Figure 7.8 Quantum yield for bimolecular $\mathrm{O}_{2}$ rebinding to human $\mathrm{Ngb}$ as a function of temperature. The solid line demonstrates the trend.

Representative PAC traces for $\mathrm{O}_{2}$ photo-release from $\mathrm{hNgb}$ together with the reference compound, Fe(III)tetraphenylsulfonato porphyrin (Fe4SP), are shown in Figure 7.8. To probe the impact of electrostriction effects, $\mathrm{hNgb}$ sample was prepared in the presence of $500 \mathrm{mM} \mathrm{NaCl}$ and characterized using PAC. PAC traces determined for $\mathrm{O}_{2}$ photo-release from $\mathrm{hNgb}+500 \mathrm{mM} \mathrm{NaCl}$ together with the reference compound (Fe4SP) are displayed in Figure 7.9. 


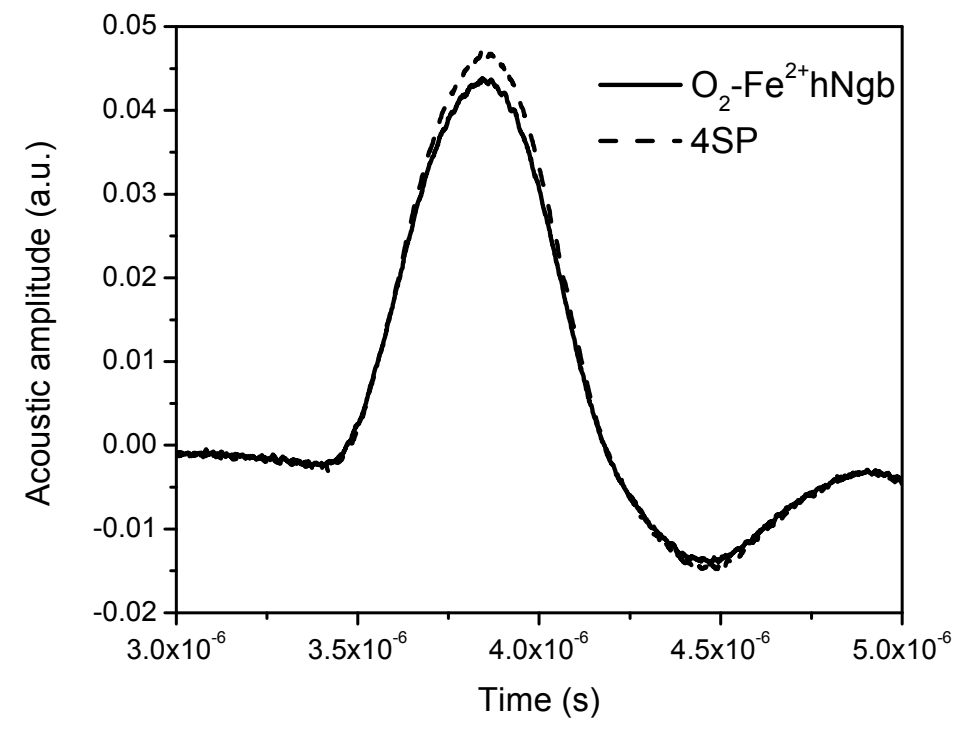

Figure 7.9 Photoacoustic traces for wild type $\mathrm{hNgb}$ and the reference compound 4SP. Conditions: $20 \mu \mathrm{M}$ protein in $50 \mathrm{mM}$ Tris buffer ( $\mathrm{pH} 7$ ). The absorbance of the sample matched that of the reference compound at $532 \mathrm{~nm}$.

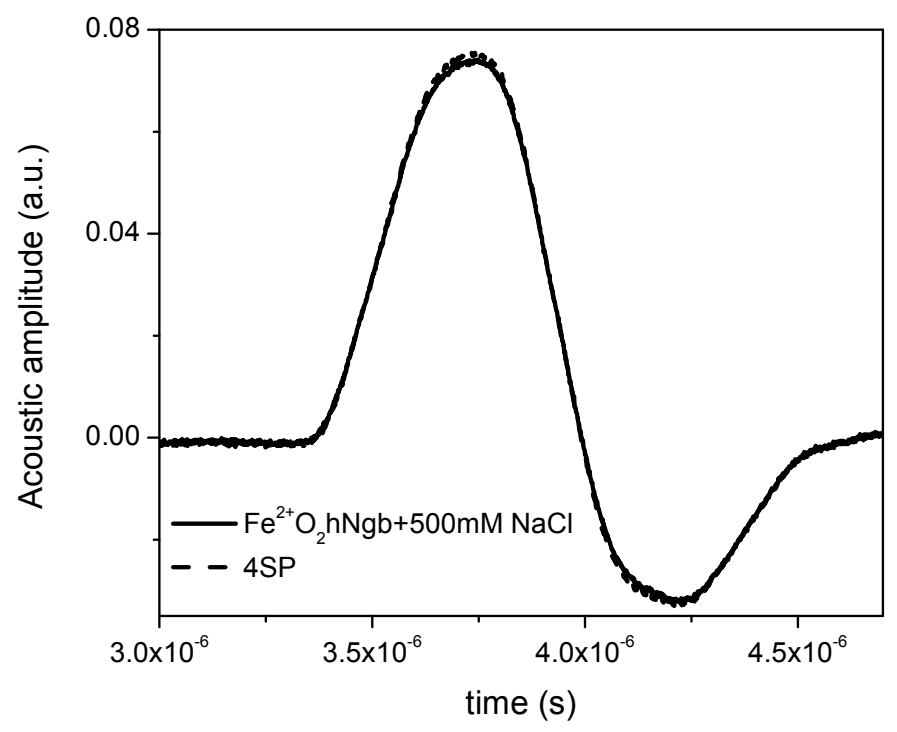

Figure 7.10 Photoacoustic traces for $\mathrm{hNgb}$ with $500 \mathrm{mM} \mathrm{NaCl}$ and the reference compound 4SP. Conditions: $20 \mu \mathrm{M}$ protein in $50 \mathrm{mM}$ Tris buffer, $500 \mathrm{mM} \mathrm{NaCl}$ (pH 7). The absorbance of the sample matched that of the reference compound at $532 \mathrm{~nm}$. 
As observed for CO photo-release from Ngbs, the sample and reference acoustic traces overlay in phase in both $\mathrm{hNgb}$ and $\mathrm{hNgb}+\mathrm{NaCl}$ indicating the absence of volume and enthalpy changes on a time scale between $\sim 50 \mathrm{~ns}$ and $\sim 5 \mathrm{~ms}$. The volume and enthalpy changes associated with $\mathrm{O}_{2}$ photo-release were determined from a plot of the $\left[\mathrm{E}_{\mathrm{hv}}(1-\phi)\right] / \Phi$ term as a function of the temperature dependent factor $(\mathrm{Cp} \rho / \beta)$ measured in the temperature range between $7{ }^{\circ} \mathrm{C}$ and $35^{\circ} \mathrm{C}$ (Figure 7.10), as described in the Materials and Methods section. The plot of $\left[\mathrm{E}_{\mathrm{hv}}(1-\phi)\right] / \Phi$ as a function of $(\mathrm{Cp} \rho / \beta)$ for hNgb shows a "V-shape" indicating that the thermodynamic parameters vary at low and high temperatures with a turning point at $\sim 16^{\circ} \mathrm{C}$.

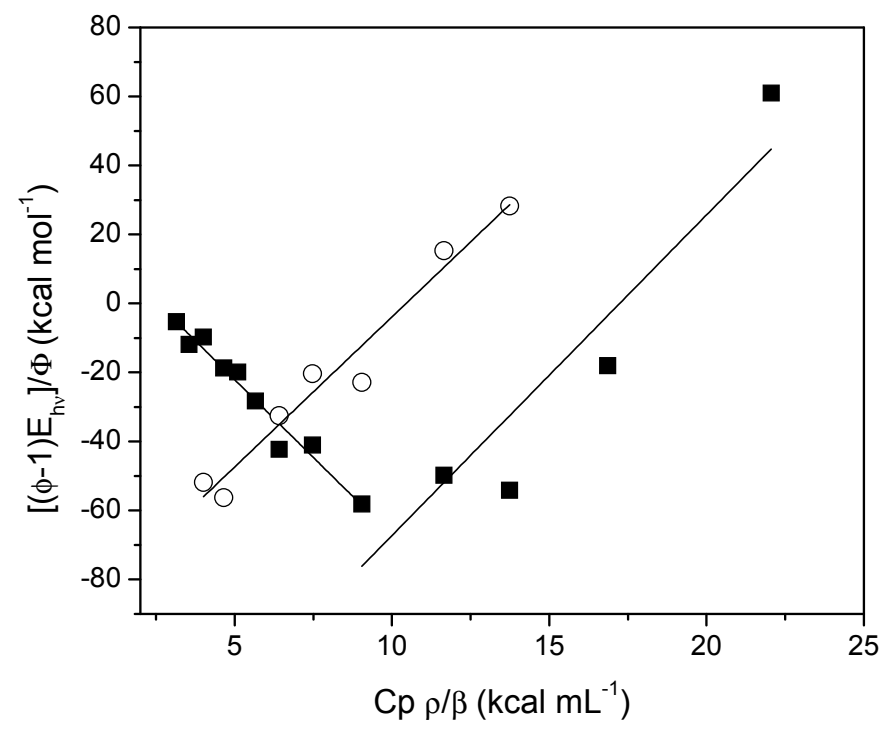

Figure 7.11 Plot of $\left[\mathrm{E}_{\mathrm{hv}}(\phi-1)\right] / \Phi$ as a function of $\mathrm{C}_{\mathrm{p}} \rho / \beta$ for $\mathrm{O}_{2}$ photo-release from $\mathrm{hNgb}$ (closed squares) and hNgb with $500 \mathrm{mM} \mathrm{NaCl}$ (open circles). 
Table 7.2 Thermodynamic parameters associated with $\mathrm{O}_{2}$ photo-release from $\mathrm{hNgb}$ and hNgb witn $500 \mathrm{mM} \mathrm{NaCl}$.

\begin{tabular}{lcc}
\hline Sample & $\Delta \mathrm{H}\left(\mathrm{kcal} \mathrm{mol}^{-1}\right)$ & $\Delta \mathrm{V}\left(\mathrm{mL} \mathrm{mol}^{-1}\right)$ \\
\hline $\mathrm{hNgb}\left(\mathrm{T}=16-35^{\circ} \mathrm{C}\right)$ & $-23 \pm 4$ & $-9.0 \pm 0.7$ \\
$\mathrm{hNgb}\left(\mathrm{T}=7-16^{\circ} \mathrm{C}\right)$ & $198 \pm 37$ & $11.3 \pm 2.4$ \\
$\mathrm{hNgb}+500 \mathrm{mM} \mathrm{NaCl}$ & $90 \pm 7$ & $8.7 \pm 0.8$ \\
\hline
\end{tabular}

At low temperatures $\left(7-16^{\circ} \mathrm{C}\right), \mathrm{O}_{2}$ photo-release from $\mathrm{hNgb}$ is associated with a large positive enthalpy change $\left(\Delta \mathrm{H}=198 \pm 37 \mathrm{kcal} \mathrm{mol}^{-1}\right)$ and leads to a volume expansion of $11.3 \pm 2.4 \mathrm{~mL} \mathrm{~mol}^{-1}$. At high temperatures $\left(16-35^{\circ} \mathrm{C}\right)$, the enthalpy change associated with $\mathrm{O}_{2}$ photo-dissociation is negative $\left(\Delta \mathrm{H}=-23 \pm 4 \mathrm{kcal} \mathrm{mol}^{-1}\right)$ and accompanied with a volume contraction of $-9.0 \pm 0.7 \mathrm{~mL} \mathrm{~mol}^{-1}$. In the presence of 500 $\mathrm{mM} \mathrm{NaCl}$, the plot of $\left[\mathrm{E}_{\mathrm{hv}}(1-\phi)\right] / \Phi$ as a function of $(\mathrm{Cp} \rho / \beta)$ is linear from $7{ }^{\circ} \mathrm{C}$ to $35^{\circ} \mathrm{C}$, indicating that temperature dependence observed for the thermodynamic parameters associated to $\mathrm{O}_{2}$ photo-release from $\mathrm{hNgb}$ results from electrostrictions effects upon ligand photo-dissociation. The observed reaction volume change at high ionic strength $(500 \mathrm{mM} \mathrm{NaCl})$ is comparable to the one observed at low temperatures, whereas the enthalpy change is $\sim$ two times lower.

The structural volume change $\left(\Delta \mathrm{V}_{\text {str }}\right)$, which describes the difference between the partial molar volume of the five-coordinate $\mathrm{Ngb}$ and $\mathrm{O}_{2}$-bound $\mathrm{Ngb}$, can be determined from the reaction volume change $(\Delta \mathrm{V})$, according to the equation 7.2.

$$
\Delta V=V^{o} O_{2}+V^{o}{ }_{N g b}-V^{o}{ }_{2} N g b
$$


where $\mathrm{V}_{\mathrm{O} 2}^{\circ}$ is the partial molar volume of $\mathrm{O}_{2}\left(28 \mathrm{~mL} \mathrm{~mol}^{-1}\right)$ (Projahn et al., 1990), $\mathrm{V}^{\circ}{ }_{\mathrm{O} 2 \mathrm{Ngb}}$ is the partial molar volume of oxy- $\mathrm{Ngb}$ and $\mathrm{V}^{\circ} \mathrm{Ngb}$ is the partial molar volume of penta-coordinate deoxy Ngb.

Similarly, the structural enthalpy change, $\Delta \mathrm{H}_{\text {str }}$, can be calculated from the reaction enthalpy change according to the following equation:

$$
\Delta H=\Delta H_{S t r}+\Delta H_{F e-O 2}
$$

Where $\Delta \mathrm{H}_{\mathrm{Fe}-\mathrm{O} 2}$ is the enthalpy of $\mathrm{Fe}-\mathrm{O}_{2}$ bond cleavage $\left(17.8 \mathrm{kcal} \mathrm{mol}^{-1}\right)$ (Radon and Pierloot, 2008).

At high temperatures $\left(\mathrm{T}=16-35{ }^{\circ} \mathrm{C}\right) \mathrm{O}_{2}$ photo-dissociation from $\mathrm{hNgb}$ is associated with a $\Delta \mathrm{V}_{\text {str }}$ of $-37.0 \pm 0.7 \mathrm{~mL} \mathrm{~mol}^{-1}$ and a $\Delta \mathrm{H}_{\text {str }}$ of $-41 \pm 4 \mathrm{kcal} \mathrm{mol}^{-1}$. On the other hand, at low temperatures $\left(\mathrm{T}=7-16{ }^{\circ} \mathrm{C}\right) \mathrm{O}_{2}$ photo-release from $\mathrm{hNgb}$ leads to a $\Delta \mathrm{V}_{\text {str }}$ of $-16.7 \pm 2.4 \mathrm{~mL} \mathrm{~mol}^{-1}$ and a $\Delta \mathrm{H}_{\text {str }}$ of $180 \pm 37 \mathrm{kcal} \mathrm{mol}^{-1}$. In the presence of 500 $\mathrm{mM} \mathrm{NaCl}$, the structural volume change $\left(\Delta \mathrm{V}_{\text {str }}=-19.3 \pm 0.8 \mathrm{~mL} \mathrm{~mol}^{-1}\right)$ comparable to the structural volume change observed at low temperatures and $0 \mathrm{mM} \mathrm{NaCl}$, whereas the structural enthalpy change $\left(\Delta \mathrm{H}_{\text {str }}=72 \pm 7 \mathrm{kcal} \mathrm{mol}^{-1}\right)$ is $\sim$ two times lower than the one observed in the absence of $\mathrm{NaCl}$. Interestingly, $\mathrm{CO}$ photo-dissociation from $\mathrm{hNgb}$ results in a structural volume change $\left(\Delta \mathrm{V}_{\text {str }}=-21.6 \pm 0.9 \mathrm{~mL} \mathrm{~mol}^{-1}\right)$ that is comparable to that observed upon $\mathrm{O}_{2}$ photo-dissociation at low temperatures and in the presence of $500 \mathrm{mM}$ $\mathrm{NaCl}$. The structural volume change determined for photo-dissociation of $\mathrm{CO}$ from $\mathrm{hNgb}$ was attributed to fast uptake of a water molecule, which is concomitant with $\mathrm{CO}$ escape from the protein matrix because of the absolute magnitude of the structural volume change that is similar to the partial molar volume of water $\left(\mathrm{V}_{\mathrm{m}}{ }^{\circ}=18 \mathrm{~mL} \mathrm{~mol}^{-1}\right)$ (Astudillo et al., 2012). 
The fact that the magnitude of the structural enthalpy change determined for $\mathrm{O}_{2}$ photo-release from $\mathrm{hNgb}$ at high ionic strength conditions is large $\left(\Delta \mathrm{H}_{\mathrm{str}}=72 \pm 7 \mathrm{kcal}\right.$ $\mathrm{mol}^{-1}$ ) suggests that proton uptake/release might take place upon $\mathrm{O}_{2}$ photo-dissociation.

\subsubsection{Hemoglobin and hemoglobin with effectors}

The quantum yield for $\mathrm{CO}$ bimolecular rebinding to $\mathrm{Hb}$, and to $\mathrm{Hb}$ in the presence of effector molecules were determined as a function of temperature (Fig. 7.10). The quantum yield increases linearly with temperature. At $20{ }^{\circ} \mathrm{C}, \mathrm{CO}$ binds to $\mathrm{Hb}$ with quantum yield of 0.68 and in the presence of IHP and BZF 0.62 and 0.46 , respectively. A similar quantum yield for $\mathrm{CO}$ bimolecular rebinding to $\mathrm{Hb}$ was reported previously by Unno et al. $\left(\Phi_{\text {bim }}=0.7\right.$ at $\left.20{ }^{\circ} \mathrm{C}\right)$ (Unno et al., 1990) and by Saffran and Gibson $(\Phi=0.7$ for $\mathrm{CO}$ binding to $\mathrm{Hb}$ and $\Phi=0.73$ for $\mathrm{CO}$ association to $\mathrm{Hb}$ in the presence of IHP at $\left.40{ }^{\circ} \mathrm{C}\right)$ (Saffran and Gibson, 1977).

The photo-acoustic traces for $\mathrm{CO}$ photo-dissociation from $\mathrm{Hb}$ are shown in Figure 7.11. The sample and the reference acoustic waves overlay in phase indicating that the observed thermodynamic processes take place within $50 \mathrm{~ns}$ upon $\mathrm{CO}$ photo-dissociation from $\mathrm{Hb}$, which is consistent with the fast $\mathrm{CO}$ diffusion from the heme matrix into the surrounding solvent. The fast ligand escape from the heme binding pocket was also observed in the presence of effectors. 


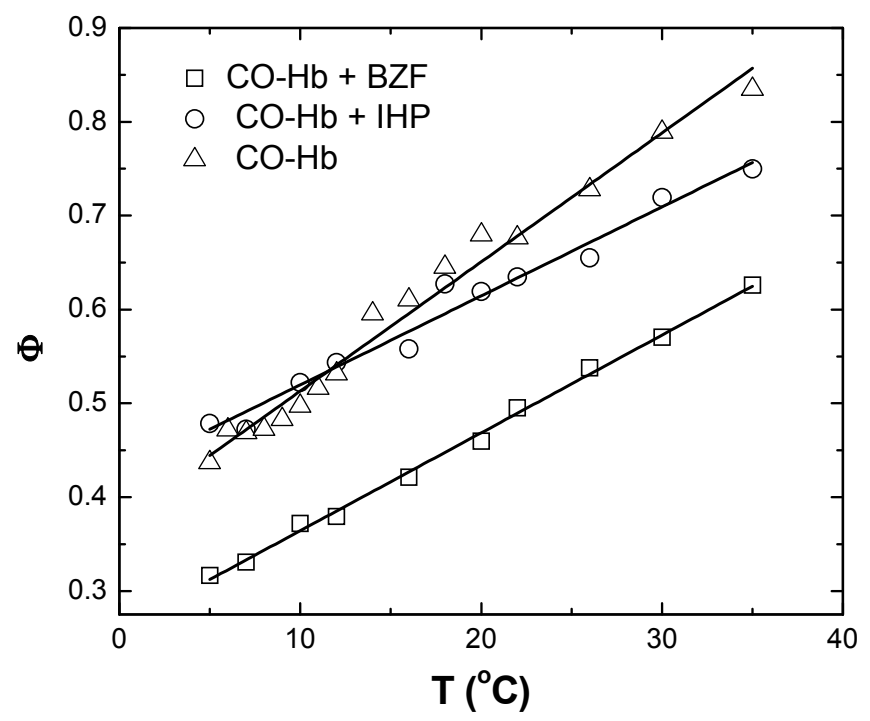

Figure 7.12 Quantum yield for bimolecular $\mathrm{CO}$ rebinding to $\mathrm{Hb}$ and $\mathrm{Hb}$ with effectors as a function of temperature. The error of quantum yield is \pm 0.05 . The solid line demonstrates the trend.

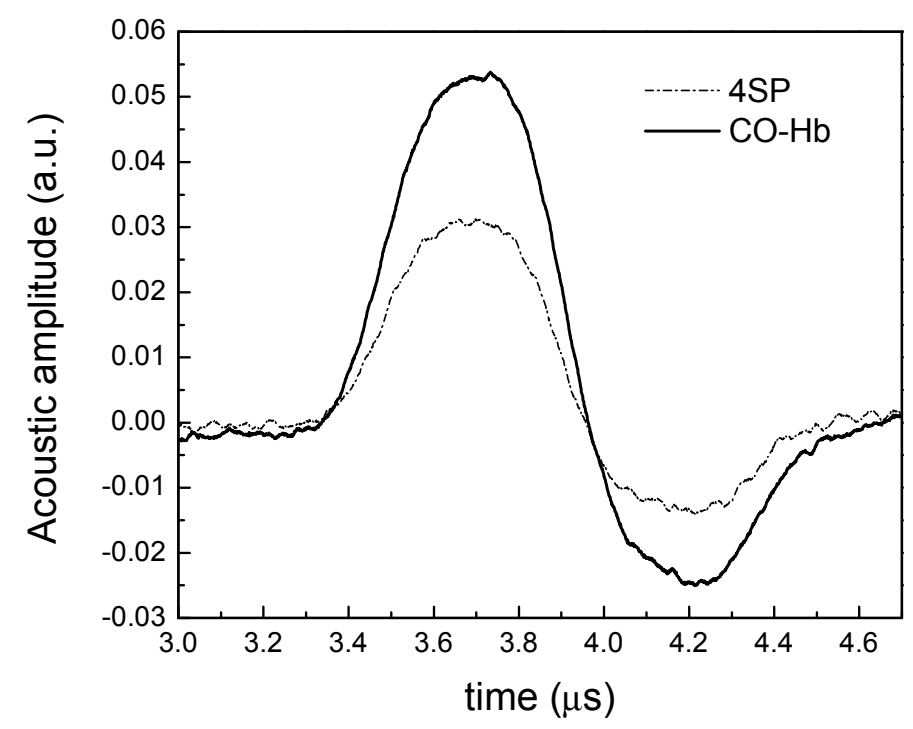

Figure 7.13 Photoacoustic traces for $\mathrm{CO}$ photo-dissociation from $\mathrm{CO}-\mathrm{Hb}$ complex and the reference compound Fe(III)4SP. Conditions: $40 \mu \mathrm{M}$ Hb in $100 \mathrm{mM}$ HEPES buffer $\mathrm{pH} 7.0$ and $20^{\circ} \mathrm{C}$. The absorbance of the reference compound matched the absorbance of the sample at $532 \mathrm{~nm}$. 
The volume and enthalpy changes associated with the diffusion of the photodissociated ligand to the surrounding solvent were determined from the plot of the ratio of the amplitude of the acoustic trace for $\mathrm{CO}$ photo-dissociation from $\mathrm{Hb}$ and the reference as a function of temperature, as described in the materials and methods section (Figure 7.12). The extrapolated thermodynamic values are shown in Table 7.3.

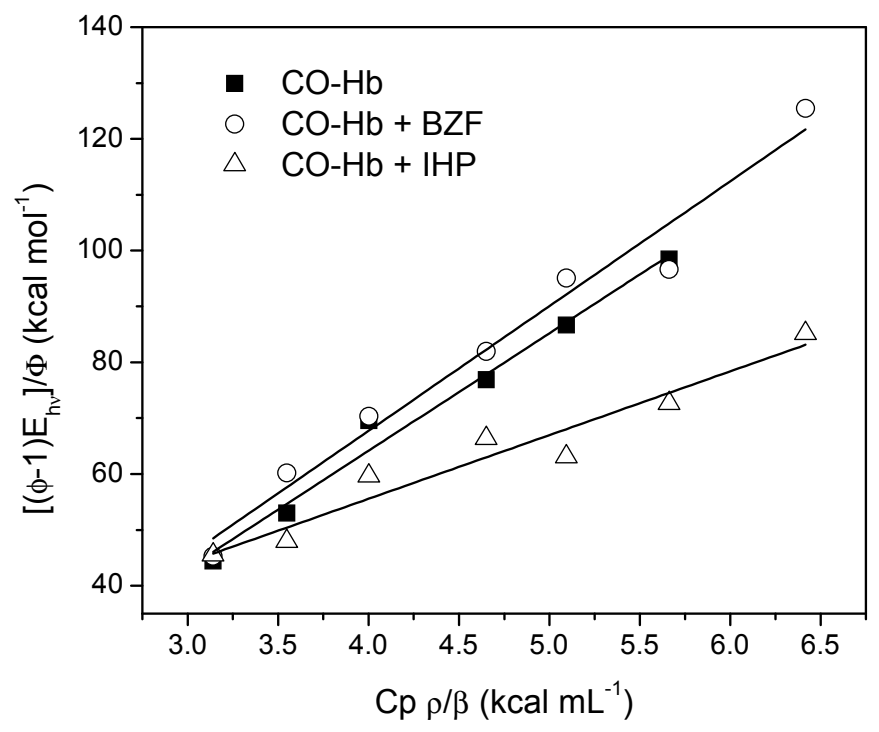

Figure 7.14 Plot of the ratio of the acoustic amplitude for the $\mathrm{CO}$ photo-dissociation from the $\mathrm{CO}-\mathrm{Hb}$ complex and the reference compound as a function of the temperature dependent factor $(\mathrm{Cp} \rho / \beta)$ term.

Table 7.3 Volume and enthalpy changes associated with $\mathrm{CO}$ photo-dissociation from $\mathrm{Hb}$.

$$
\Delta \mathrm{H}_{\text {prompt }}\left(\mathrm{kcal} \mathrm{mol}^{-1}\right) \quad \Delta \mathrm{V}_{\text {prompt }}\left(\mathrm{mL} \mathrm{mol}^{-1}\right)
$$

\begin{tabular}{lcc}
\hline $\mathrm{COHb}$ & $19.4 \pm 1.2$ & $21.5 \pm 0.9$ \\
\hline $\mathrm{COHb}+\mathrm{BZF}$ & $21.7 \pm 7.9$ & $22.3 \pm 1.7$ \\
\hline $\mathrm{COHb}+\mathrm{IHP}$ & $-9.9 \pm 6.1$ & $11.4 \pm 1.3$
\end{tabular}


The $\mathrm{CO}$ photo-release from $\mathrm{Hb}$ is associated with a positive volume change of $21.5 \pm 0.9 \mathrm{~mL} \mathrm{~mol}^{-1}$ and enthalpy change of $19.4 \pm 1.2 \mathrm{kcal} \mathrm{mol}^{-1}$. These results are in agreement with the thermodynamic parameters reported previously by Peters et al: $\Delta \mathrm{V}=$ $23.4 \pm 0.5 \mathrm{~mL} \mathrm{~mol}^{-1}$ and $\Delta \mathrm{H}=18.0 \pm 2.9 \mathrm{kcal} \mathrm{mol}^{-1}$ (Peters et al., 1992). Since the laser power used in this study was kept below $50 \mu \mathrm{J}$, the low level of photo-dissociation was achieved that corresponds to $1 \mathrm{CO}$ molecule per hemoglobin photo-released. Thus, the observed thermodynamic parameters reflect the transition between fully ligated $(\mathrm{CO})_{4} \mathrm{Hb}$ and triple ligated $(\mathrm{CO})_{3} \mathrm{Hb}$. Consequently, the observed reaction enthalpy corresponds to the enthalpy change due to the cleavage of the Fe-CO bond $\left(\Delta \mathrm{H}_{\mathrm{Fe}-\mathrm{CO}}=17.5 \mathrm{kcal} \mathrm{mol}{ }^{-1}\right.$ (Leung et al., 1987; Miksovska et al., 2005)), the enthalpy change due to the solvation of a CO molecule $\left(\Delta \mathrm{H}_{\text {solv }}=2.6 \mathrm{kcal} \mathrm{mol}^{-1}\right.$ (Leung et al., 1987)), the enthalpy change of structural relaxation associated with the ligand release from the protein matrix, and enthalpy of the distal pocket hydration. The occupancy of water molecules in the distal pocket of deoxyHb was determined to be significantly lower than that in $\mathrm{Mb}$ ( $\sim 0.64$ for the $\mathrm{Hb} \alpha$ - chain and $\sim 0.33$ for the $\mathrm{Hb} \beta$-chain (Esquerra et al., 2010)). Using an average occupancy of 0.48 , it can be estimated that the distal pocket hydration contributes to the overall enthalpy change by $\sim-3 \mathrm{kcal} \mathrm{mol}^{-1}$ (Vetromile et al., 2011). Therefore, the structural relaxation coupled to the $\mathrm{CO}$ dissociation and diffusion into the surrounding solvent is accompanied by a small enthalpy change of $2 \mathrm{kcal} \mathrm{mol}^{-1}$.

The observed reaction volume change for $\mathrm{CO}$ photo-release from $\mathrm{Hb}, \Delta \mathrm{V}=21.5$ $\mathrm{mL} \mathrm{mol}{ }^{-1}$, can be expressed as:

$$
\Delta V=V_{\mathrm{CO}}^{o}+V_{(\mathrm{CO})_{3} \mathrm{Hb}}^{o}-V_{(\mathrm{CO})_{4} \mathrm{Hb}}^{o}-V_{\mathrm{H}_{2} \mathrm{O}}^{o}
$$


where $\mathrm{V}^{\circ} \mathrm{CO}$ is the partial molar volume of $\mathrm{CO}$ and $\mathrm{V}^{\circ}{ }_{(\mathrm{CO}) 3 \mathrm{Hb}}$ and $\mathrm{V}^{\circ}{ }_{(\mathrm{CO}) 4 \mathrm{Hb}}$ are the partial molar volume of $(\mathrm{CO})_{3} \mathrm{Hb}$ and $(\mathrm{CO})_{4} \mathrm{Hb}$, respectively. Using $\mathrm{V}^{\circ} \mathrm{CO}=35 \mathrm{~mL} \mathrm{~mol}^{-1}$ (Projahn et al., 1990) and $\mathrm{V}_{\mathrm{H} 2 \mathrm{O}}^{\circ}=9 \mathrm{~mL} \mathrm{~mol}^{-1}$ (partial molar volume of water scaled by the average occupancy of the $\mathrm{Hb}$ chain), I estimate that upon release of one $\mathrm{CO}$ molecule per $\mathrm{Hb}$, the protein undergoes a small contraction of $-7 \mathrm{~mL} \mathrm{~mol}^{-1}$. The small volume change observed here is consistent with the minor structural changes due to deligation of $\mathrm{Hb}$ in the R-state as observed in the X-ray structure that are predominantly localized in the the $\alpha$-chain and include reposition of the F-helix and shift of the EF and CD corner (Wilson et al., 1996).

The volume and enthalpy changes associated with the $\mathrm{CO}$ photo-dissociation from $\mathrm{Hb}$ in the presence of heterogenous effectors BZT and IHP were also determined (Table 7.3). The photoacoustic data presented here show that binding of BZF to $\mathrm{CO}-\mathrm{Hb}$ complex does not impact the reaction volume and enthalpy changes associated with CO photorelease. On the other hand, binding of IHP has a significant impact on the observed volume and enthalpy changes. The reaction volume decreases by $10 \mathrm{~mL} \mathrm{~mol}^{-1}$ and the enthalpy change is more exothermic by nearly $30 \mathrm{kcal} \mathrm{mol}^{-1}$, compared to the thermodynamic parameters determined in the absence of effector molecules. Such negative reaction volume and exothermic enthalpy change indicates that electrostriction of solvent molecules caused by reorganization of salt bridges or redistribution of charges on protein surface contributes to the overall reaction volume and enthalpy change associated with $\mathrm{CO}$ photo-release. 


\subsection{Discussion}

On the basis of the PAC data, $\mathrm{O}_{2}$ photo-release from $\mathrm{Mb}$ is biphasic with the fast phase, occurring within $50 \mathrm{~ns}$, reflecting photo-cleavage of the $\mathrm{Fe}-\mathrm{O}_{2}$ bond and the slow phase $\left(\tau \sim 250 \mathrm{~ns}\right.$ at $\left.20{ }^{\circ} \mathrm{C}\right)$ resulting from either $\mathrm{O}_{2}$ nanosecond geminate rebinding or ligand migration from the protein matrix to the solvent. The observed volume contraction determined for the fast phase reflects a fast relaxation of the heme binding pocket that includes: i) cleavage of the hydrogen bond between the distal histidine and oxygen molecule (Phillips and Schoenborn, 1981) ii) reorientation of distal residues within the heme binding pocket (Olson et al., 2007), and iii) fast migration of the photo-released ligand into the primary docking site and then into the internal cavities $\left(\mathrm{Xe}_{4}\right.$ or $\left.\mathrm{Xe}_{1}\right)$ (Hummer et al., 2004).

The kinetics for the geminate $\mathrm{O}_{2}$ rebinding were studied on femtosecond timescale by Petrich et al. (1988), and on picosecond and nanosecond timescales (Carver et al., 1990; Miller et al., 1996). These studies identified two distinct sub-states of the "deoxyMb" intermediate: a "barrier-less" and a "photolyzable" sub-state. In the "barrierless" sub-state, oxygen rebinds to heme iron on sub-picosecond timescale, whereas oxygen association to the "photolyzable" sub-state occurs on nanosecond and microsecond timescales. Carver et al. (1990) have reported the time constant for $\mathrm{O}_{2}$ nanosecond geminate rebinding to be $52 \pm 14 \mathrm{~ns}$ at room temperature. This kinetic step has a lifetime that is comparable to the time resolution of the PAC instrument used in this study ( $\tau \sim 50 \mathrm{~ns}$ ), hence it was not resolved here. Therefore, the $250 \mathrm{~ns}$ step corresponds to $\mathrm{O}_{2}$ escape from the transient "deoxyMb" intermediate into the surrounding solvent and is approximately 3 times faster than the rate of CO escape (Westrick et al., 1990), which 
suggests that $\mathrm{O}_{2}$ diffuses from the protein matrix through a transient channel with a lower activation barrier than $\mathrm{CO}$. This result is consistent with the transient absorption studies that estimated the rate for $\mathrm{O}_{2}$ release to be approximately two times faster than that for CO (Carver et al., 1990). Interestingly, a similar time constant of $200 \mathrm{~ns}$ to $300 \mathrm{~ns}$ was determined for $\mathrm{CO}$ escape from $\mathrm{Mb}$ at $\mathrm{pH} 3.5$ (Angeloni and Feis, 2003). At acidic $\mathrm{pH}$, $\mathrm{Mb}$ adopts an open conformation with His 64 displaced toward the solvent giving a direct access to the distal cavity. These data suggest that reorientation of His 64 may not be a rate limiting step for $\mathrm{O}_{2}$ escape.

The small enthalpy change measured for the $250 \mathrm{~ns}$ relaxation $(\Delta \mathrm{H}=-8.9 \pm 8.0$ kcal mol $\left.{ }^{-1}\right)$ includes the enthalpy change for $\mathrm{O}_{2}$ solvation $\left(\Delta \mathrm{H}_{\text {solv }}=-2.9 \mathrm{kcal} \mathrm{mol}^{-1}(\mathrm{Mills}\right.$ et al., 1979)) and the enthalpy change associated with $\mathrm{H}_{2} \mathrm{O}$ binding to the heme binding pocket $\left(\Delta \mathrm{H}_{\text {solv }}=-7 \mathrm{kcal} \mathrm{mol}^{-1}\right.$ (Vetromile et al., 2011)) indicating that the structural relaxation coupled to the ligand escape from the protein is entropy driven. The similarity between the structural volume change determined for the slow phase of $\mathrm{O}_{2}$ photo-release from $\mathrm{Mb}\left(-7.5 \mathrm{~mL} \mathrm{~mol}^{-1}\right)$ and the volume change previously reported previously for $\mathrm{CO}$ escape from $\mathrm{Mb}\left(\Delta \mathrm{V}_{\text {structural }}=\mathrm{V}_{\text {doxyMb }}^{\circ} \mathrm{V}_{\mathrm{CO}-\mathrm{Mb}}^{\circ}=-6 \mathrm{~mL} \mathrm{~mol}^{-1}\right)$ (Vetromile et al., 2011) demonstrates that the overall structural changes accompanying the ligand bound to ligand free transition in $\mathrm{Mb}$ are similar for both ligands. These results are in agreement with the close resemblance of the X-ray structure of both the CO-bound and $\mathrm{O}_{2}$-bound $\mathrm{Mb}$ (Yang and Phillips, 1996).

The overall enthalpy change for $\mathrm{O}_{2}$ dissociation from $\mathrm{Mb}$ was determined to be $11.6 \pm 8.5 \mathrm{kcal} \mathrm{mol}^{-1}$ and this value is in agreement with the value of $10 \mathrm{kcal} \mathrm{mol}^{-1}$ reported previously (Projahn et al., 1990). The overall reaction volume change 
determined here $\left(\Delta \mathrm{V}_{\text {overall }}=+2.5 \mathrm{~mL} \mathrm{~mol}^{-1}\right)$ is somewhat larger than the reaction volume change determined from the measurement of the equilibrium constant as a function of pressure $\left(\Delta \mathrm{V}=-2.9 \mathrm{~mL} \mathrm{~mol}^{-1}\right)$ (Hasinoff, 1974) and significantly smaller than the reaction volume change determined as a difference between the activation volume for oxygen binding and dissociation from $\mathrm{Mb}$ that was reported to be $18 \mathrm{~mL} \mathrm{~mol}^{-1}$ (Projahn et al., 1990). Unlike photoacoustic studies that allow for reaction volume determination at ambient pressure, the high pressure measurements of equilibrium constant and/or rate constants (to determine activation volumes) may cause a pressure induced protein denaturation and/or structural changes, which may influence the magnitude of reaction volume changes in high pressure studies.

The photoacoustic data shows that $\mathrm{O}_{2}$ photo-release from $\mathrm{hNgb}$ is fast, occurring within $50 \mathrm{~ns}$, indicating that $\mathrm{O}_{2}$ escapes from the protein matrix through a direct pathway connecting the heme binding pocket with the surrounding solvent. Similarly, CO photorelease from $\mathrm{hNgb}$ is fast $(\tau<50 \mathrm{~ns})$ and it was proposed that an effective ligand migration pathway is provided through the wide hydrophobic tunnel that connects the distal pocket with the surrounding solvent in the deoxyNgb (Vallone et al., 2004b). The thermodynamic parameters associated with $\mathrm{O}_{2}$ photo-release from $\mathrm{hNgb}$ in $50 \mathrm{mM}$ Tris buffer ( $\mathrm{pH}$ 7.0) show temperature dependence in the range between $7{ }^{\circ} \mathrm{C}$ to $35^{\circ} \mathrm{C}$. At temperatures above $16{ }^{\circ} \mathrm{C}$, the reaction enthalpy and volume change are negative with values of $-23 \pm 4 \mathrm{kcal} \mathrm{mol}^{-1}$ and $-9.0 \pm 0.7 \mathrm{~mL} \mathrm{~mol}^{-1}$, respectively. Conversively, the reaction enthalpy and volume change become positive at temperatures below $16{ }^{\circ} \mathrm{C}$ with an enthalpy change of $198 \pm 34 \mathrm{kcal} \mathrm{mol}^{-1}$ and a volume change of $11.3 \pm 2.4 \mathrm{~mL} \mathrm{~mol}^{-1}$. These results indicate that the enthalpy change associated with oxygenation of penta- 
coordinate $\mathrm{hNgb}$ is exothermic at temperatures below $16{ }^{\circ} \mathrm{C}$ and endothermic at temperatures below $16{ }^{\circ} \mathrm{C}$. Temperature dependence of thermodynamic parameters associated with $\mathrm{O}_{2}$ binding to $\mathrm{hNgb}$ has been previously observed (Fago et al., 2004b), whereas $\mathrm{CO}$ association to $\mathrm{hNgb}$ does not show temperature dependence (Astudillo et al., 2010; Fago et al., 2004b). Fago et al. (2004b) reported that $\mathrm{O}_{2}$ association to hexacoordinate bishisdine $\mathrm{hNgb}$ at $\mathrm{pH} 7.4$ is exothermic at temperatures above $\sim 18{ }^{\circ} \mathrm{C}$ with $\Delta \mathrm{H}=-15.7 \mathrm{kcal} \mathrm{mol}^{-1}$, whereas endothermic enthalpy changes were observed at temperatures below $\sim 18{ }^{\circ} \mathrm{C}$ with $\Delta \mathrm{H}=12.7 \mathrm{kcal} \mathrm{mol}^{-1}$. These authors attributed the exothermic $\mathrm{O}_{2}$ binding to weakening of the electrostatic/hydrogen bonding interactions among His64, Lys67, and heme 7-propionate at high temperatures, whereas stronger polar interactions were proposed to contribute to the observed positive enthalpy for oxygen binding at low temperatures. The discrepancy between the data presented here and the studies of Fago et al. (2004b) may arise from the fact that Fago et al. (2004b) measured $\mathrm{O}_{2}$ association to hexa-coordinate bishisdine $\mathrm{hNgb}$, wheres the data shown here reflects $\mathrm{O}_{2}$ binding to penta-coordinate $\mathrm{hNgb}$. Therefore, the structural transitions occurring upon $\mathrm{O}_{2}$ photo-dissociation from hexacoordinate $\mathrm{O}_{2}$ bound $\mathrm{hNgb}$ and ferrous pentacoordinate $\mathrm{hNgb}$ are different than the ones occurring from hexacoordinate $\mathrm{O}_{2}$ bound $\mathrm{hNgb}$ to hexacoordinate bishistidine $\mathrm{hNgb}$.

To identify factors contributing to the temperature dependence observed for the enthalpy and volume changes associated with $\mathrm{O}_{2}$ photo-dissociation from $\mathrm{hNgb}$, thermodynamic parameters were determined at high ionic strength conditions $(500 \mathrm{mM}$ $\mathrm{NaCl}$ ). Interestingly, the thermodynamic parameters associated to $\mathrm{O}_{2}$ photo-release from $\mathrm{hNgb}$ do not show temperature dependence upon increase of the solution ionic strength in 
the range of $7{ }^{\circ} \mathrm{C}$ to $35^{\circ} \mathrm{C}$, indicating that the temperature depencence of the thermodynamic parameters is suppressed in high ionic strength conditions. Both reaction enthalpy and volume changes are positive in the presence of $500 \mathrm{mM} \mathrm{NaCl}$ with $\Delta \mathrm{H}=90$ $\pm 7 \mathrm{kcal} \mathrm{mol}^{-1}$ and $\Delta \mathrm{V}=8.7 \pm 0.8 \mathrm{~mL} \mathrm{~mol}^{-1}$. The reaction volume change determined for $\mathrm{hNgb}$ with $500 \mathrm{mM} \mathrm{NaCl}$ is comparable to the one determined for $\mathrm{hNgb}$ at temperatures below $16{ }^{\circ} \mathrm{C}$, whereas the enthalpy change in the presence of $500 \mathrm{mM} \mathrm{NaCl}$ is approximately 2 times lower than the one determined in the absence of $\mathrm{NaCl}$. The results shown here indicate that the magnitude of the volume and enthalpy change associated with $\mathrm{O}_{2}$ photo-release show dependence on the ionic strength of the solution, which suggests that electriction effects contribute to the observed photoacoustic signal. Generally, exposure of charges to the solvent result in negative reaction volume changes and the solvation of charges is an exothermic process (Lockney and Miksovska, 2006). Since $\mathrm{O}_{2}$ photo-dissociation from $\mathrm{hNgb}$ leads to a negative contribution to the overall volume and enthalpy changes at high temperatures $\left(\mathrm{T}=16-35^{\circ} \mathrm{C}\right)$ that are suppressed upon increase of the ionic strength of the solution, the $\mathrm{O}_{2}$ dissociation from the protein leads to conformational changes that involve solvation of charges. Examination of the crystal structure of $\mathrm{hNgb}$ indicates that Lys 67 forms a salt bridge with one of the heme propionate groups. Lys 67 residue and the heme group in Ngb are quite solvent exposed, compared with other globins such as Mb, and it was previously proposed that Lys 67 side chain acts as a barrier separating the distal pocket from the solvent through its electrostatic interaction with the heme propionate group (Nienhaus et al., 2004). Additionally, Lys 67 and the heme propionate groups have been proposed to be involved in larger hydrogen/electrostatic interactions network that include the distal His 64, Glu 60 
and Tyr 44 (Astudillo et al., 2012).The interaction between the distal His 64 and Tyr 44 is also involved in the displacement of the CD-D region upon $\mathrm{CO}$ association to the heme iron (Vallone et al., 2004b), hence structural changes may be relayed from the CD loop to the heme binding pocket. Therefore, at high temperatures $\mathrm{O}_{2}$ photo-dissociation from $\mathrm{hNgb}$ might result in disruption of the salt bridge between Lys 67 and the heme propionate group that is propagated to the protein surface through the larger electrostatic interaction discussed above. This alteration of electrostatic interactions may lead to exposure of charges on the protein surface that contributes to the observed electrostriction effects upon $\mathrm{O}_{2}$ photo-dissociation in $\mathrm{hNgb}$.

The magnitude of the structural enthalpy change upon $\mathrm{O}_{2}$ photo-release is large and positive in the presence of $500 \mathrm{mM} \mathrm{NaCl}$, indicating that other factors contribute to the structural enthalpy change in addition to electrostriction effects. A possible explanation for the large structural enthalpy change is protonation or deprotonation of ionizable groups in the protein upon $\mathrm{O}_{2}$ photo-dissociation. Fago et al. (2004b) reported that $\mathrm{hNgb}$ displays alkaline and Bohr acid effects that depend on both the temperature and the $\mathrm{pH}$ of the solution, since the $\mathrm{P}_{50}$ value increases and decreases upon a $\mathrm{pH}$ decrease. These authors suggest that the Bohr effect in Ngb arises from stabilization of bound $\mathrm{O}_{2}$ by the distal His 64 in its unprotonated form and protonation of the His 64 side chain favors $\mathrm{O}_{2}$ dissociation. Futhermore, replacement of the distal His 64 by Gln and Val residues has been shown to suppress the Bohr effect observed in $\mathrm{hNgb}$, whereas replacement of Lys 67 by Leu shows a reversed effect of the Bohr effect than the one observed for wild type $\mathrm{hNgb}$ in the $\mathrm{pH}$ range between 6.5 and 7.5 (i.e., $\mathrm{O}_{2}$ affinity 
increases with a $\mathrm{pH}$ increase), supporting a role of the distal His 64 and Lys 67 residues in the $\mathrm{pH}$ dependence observed for $\mathrm{O}_{2}$ affinity in $\mathrm{hNgb}$ (Fago et al., 2004b).

Protonation and deprotonation often accompany ligand binding reactions. In order to determine whether $\mathrm{O}_{2}$ photo-dissociation from $\mathrm{hNgb}$ leads to proton uptake or release, PAC studies could be perfomed in different buffers. Protonation of Tris buffer is associated with a small volume contraction of $-1 \mathrm{~mL} \mathrm{~mol}^{-1}$ and an exothermic enthalpy change of $-11.3 \mathrm{kcal} \mathrm{mol}^{-1}$, whereas protonation of phosphate buffer leads to a large volume expansion of $24 \mathrm{~mL} \mathrm{~mol}^{-1}$ and a small enthalpy change of $-1.8 \mathrm{kcal} \mathrm{mol}^{-1}$ . Therefore, if ligand photo-release is associated with a net change of protonation (proton release/uptake), a corresponding change in buffer protonation will contribute to the observed reaction enthalpy change thus different values for volume and enthalpy changes would be observed in different buffers (Lockney and Miksovska, 2006).

In case of $\mathrm{Hb}, \mathrm{PAC}$ data indicates that $\mathrm{CO}$ escapes from the protein matrix to the surrounding solvent within 50 ns. Previous transient absorption studies showed that the $\mathrm{CO}$ photo-release from the fully ligated $\mathrm{R}$-state $\mathrm{Hb}$ is followed by three relaxations with lifetimes of $50 \mathrm{~ns}, 1 \mu \mathrm{s}$, and $20 \mu \mathrm{s}$ that were assigned to the unimolecular geminate rebinding, the tertiary structural relaxation, and the $\mathrm{R} \rightarrow \mathrm{T}$ quaternary change, respectively (Goldbeck et al., 1996). The geminate rebinding occurs too fast to be resolved by the PAC detector used in this study, whereas the $20 \mu$ s $\mathrm{R} \rightarrow \mathrm{T}$ transition, which strongly depends on the extent of heme ligation, is too slow to be resolved in PAC measurements. The $1 \mu$ s relaxation is within the time-window accessible by the detection system, however I was unable to resolve this step. Since this relaxation was observed as a small perturbation of the deoxy-Soret band (Goldbeck et al., 1996), it may reflect the structural 
relaxation localized within the vicinity of the heme binding pocket, which does not lead to measurable volume and enthalpy changes.

The small volume change determined for $\mathrm{CO}$ photo-release from $\mathrm{Hb}$ was attributed to small structural changes resulting from deligation of $\mathrm{Hb}$ in the R-state. These results are in agreement with the reported crystal structure of $\mathrm{Hb}$ that shows that structural modifications occurring upon deligation are localized in the $\alpha$-chain, which include repositioning of the F-helix as well as movement of the EF and CD corner (Wilson et al., 1996).

The photoacoustic data presented here show that binding of BZF to $\mathrm{CO}-\mathrm{Hb}$ complex does not impact the reaction volume and enthalpy changes associated with $\mathrm{CO}$ photo-release. On the other hand, binding of IHP has a significant impact on the observed volume and enthalpy changes. The thermodynamic profiles for $\mathrm{CO}$ photo-dissociation from $\mathrm{CO}-\mathrm{Hb}$ complex in the presence and absence of effectors are presented in Fig.7.X. Both effectors bind to $\mathrm{Hb}$ in the $\mathrm{T}$-state and $\mathrm{R}$-state and modulate the interaction of $\mathrm{Hb}$ with diatomic ligands (Coletta et al., 1999b; Marden et al., 1990). For example, the binding of $\mathrm{BZF}$ or $\mathrm{IHP}$ to $\mathrm{CO}-\mathrm{Hb}$ complex decreases the $\mathrm{CO}$ association rate approximately four or eight times, respectively (Marden et al., 1990), and decreases the affinity of R state deoxy-Hb for oxygen (Tsuneshige et al., 2002). Coletta et al. (1999a) have reported that simultaneous binding or IHP and BZF effectors to $\mathrm{Hb}$ at ambient pressure leads to the $\mathrm{Hb}$ intermediate with tertiary T-like structure in the quaternary Rconformation. Recently, using NMR spectroscopy Song et al. have shown that binding of IHP to the fully ligated $\mathrm{Hb}$ increase the conformational fluctuation of the R-state in both the $\alpha$ - and $\beta$-chain (Song et al., 2008). 
The crystal structure of horse $\mathrm{CO}-\mathrm{Hb}$ in complex with BZF indicates that the structural changes due to BZF association to fully ligated $\mathrm{Hb}$ are localized in the $\alpha$ subunits (Shibayama et al., 2002). BZF binds to the surface of each $\alpha$-chain E-helix and decreases the distance between the heme iron and distal His and its binding site is surrounded by hydrophobic residues such as Ala 65, Leu 68, Leu 80 and Leu 83 (Shibayama et al., 2002). Such minor structural changes caused by BZF association are unlikely to alter the overall structural volume and enthalpy changes associated with the $\mathrm{CO}$ photo-release. However, due to the lower solubility of BZF, the effector concentration used is this study was $5 \mathrm{mM}$ that results in a $\mathrm{Hb}$ fractional saturation of 0.25 (using $\mathrm{K}_{\mathrm{D}}$ of $15 \mathrm{mM}$ (Ascenzi et al., 1993)). Such lower fractional saturation may prevent detection of BZF induced changes in $\mathrm{Hb}$ conformational dynamics.

The large decrease in the reaction volume and enthalpy changes observed for IHP in complex with $\mathrm{Hb}-\mathrm{CO}$ compared to the thermodynamic parameters determined in the absence of the effector, indicates that electrostriction of solvent molecules caused by reorganization of salt bridges or redistribution of charges on protein surface contributes to the overall reaction volume and enthalpy change associated with $\mathrm{CO}$ photo-release. Indeed, IHP interacts with charged residues along the $\mathrm{Hb}$ central cavity. At the $\mathrm{Hb} \mathrm{T}-$ state, the IHP binding site is located at the interface of the $\beta$-chains involving Val 1, His2, Lys 82 and His 141 from each chain (Riccio et al., 2001); whereas at the R-state Hb, the IHP molecule interacts with the charged residues Lys 99 and Arg 141 from each $\alpha$-chain (Laberge et al., 2005). In the absence of the X-ray structure of IHP bound fully ligated and partially photolyzed $\mathrm{CO}-\mathrm{Hb}$, it is difficult to point out the factors that contribute to the observed volume and enthalpy changes on the molecular level. Arg 141 forms a salt 
bridge with Asp 126 in the T-state deoxy Hb that is absent in the fully ligated R- state (Park et al., 2006). We speculate that the transition between the fully ligated (CO) ${ }_{4} \mathrm{Hb}$ and partially ligated $(\mathrm{CO})_{3} \mathrm{Hb}$ may be associated with a repositioning of the Arg 141 side chain leading to a partial exposure of either the IHP molecule and/or the Arg 141 side chain to the surrounding solvent molecules. Also, the ligand photo-release may be associated with the repositioning of the IHP molecule within the Hb central cavity. On the basis of a molecular dynamics simulation of IHP binding sides in south polar skua deoxyHb, an IHP migration pathway connecting the binding site at the interface between the $\alpha$-chains and the second binding site located between the $\beta$-chains was proposed suggesting that IHP interactions with $\mathrm{Hb}$ are dynamic and involve numerous positively charged residues situated along the central cavity (Riccio et al., 2001). Therefore, CO photo-release may trigger relocation of IHP within the central cavity resulting in larger exposure of IHP phosphate groups and/or charged amino acid residues and concomitant electrostriction of solvent molecules. 

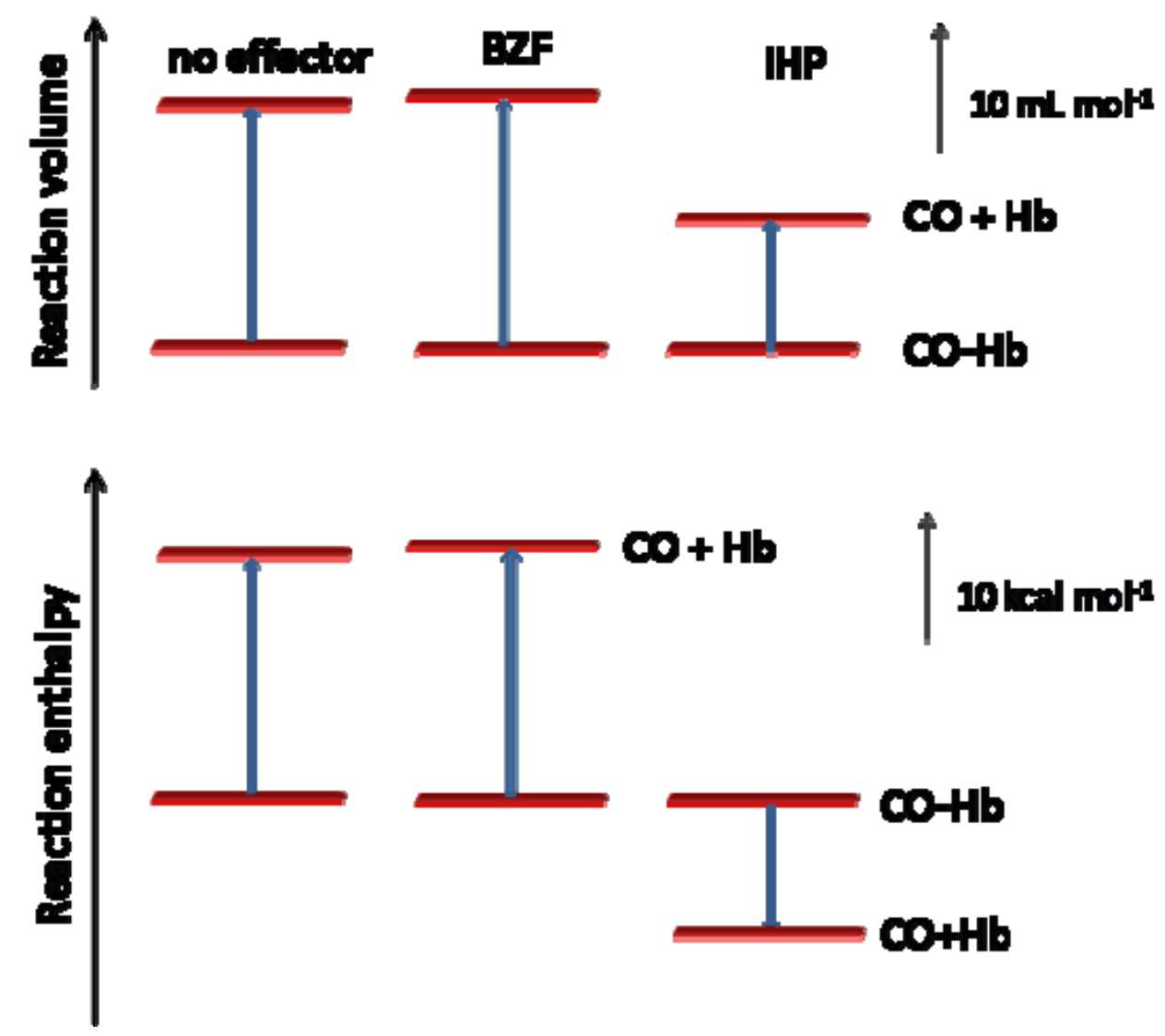

Figure 7.15 The thermodynamic profile for $\mathrm{CO}$ photo-dissociation from $\mathrm{Hb}$ in the absence of effector and in the presence of BZF and IHP. The arrows indicate the respective scale.

\subsection{Summary}

The photoacoustic data determined for the ligand photo-dissociation from $\mathrm{Mb}$ shows that the structural volume changes associated with $\mathrm{O}_{2}$ difussion from $\mathrm{Mb}$ active site are similar to those determined previously for $\mathrm{CO}$ in agreement with the crystallographic data. On the other hand, the time constant for $\mathrm{O}_{2}$ escape from the distal pocket to the surrounding solvent is two to three times faster than that for $\mathrm{CO}$, suggesting a distinct migration pathway for diatomic ligands in $\mathrm{Mb}$. In case of human $\mathrm{Ngb}$, the PAC data indicates that $\mathrm{O}_{2}$ photo-release from $\mathrm{Ngb}$ occurs within $50 \mathrm{~ns}$, as previously 
observed for $\mathrm{CO}$ photo-release. However, the thermodynamic parameters associated with $\mathrm{O}_{2}$ photo-dissociation from $\mathrm{Ngb}$ are temperature dependent exhibiting negative reaction and volume changes at temperatures above $16{ }^{\circ} \mathrm{C}$, whereas positive reaction volume and enthalpy changes are observed at temperatures below $16{ }^{\circ} \mathrm{C}$. This temperature dependence is attributed to electrostriction effects upon solvation of charges since increase of the solution ionic strength suppresses the temperature dependence. The large structural enthalpy change observed upon $\mathrm{O}_{2}$ photo-dissociation at high solution ionic strength conditions suggests that proton uptake/release occurs upon photo-dissociation of the ligand.

The PAC study shown here indicates that IHP binding to Hb-CO complex alters the volume and enthalpy changes associated with $\mathrm{CO}$ photo-dissociation from the heme iron indicating that the transition between the fully ligated $(\mathrm{CO})_{4} \mathrm{Hb}$ and partially ligated $(\mathrm{CO})_{3} \mathrm{Hb}$ complex is associated with the reorientation of IHP molecule within the central cavity and/ or charged amino acid residues interacting with IHP. 


\section{STUDY OF CYTOGLOBIN INTERACTIONS WITH FLUORESCENT PROBE 1-ANILINONAPHTHALENE-8-SULFONATE}

\subsection{Introduction}

Cytoglobin $(\mathrm{Cygb})$ is a heme protein expressed in humans and other vertebrates that belongs to the family of hexa-coordinate hemoglobins (Burmester et al., 2002). Cytoglobin is mainly localized in fibroblasts of connective tissue and cell lineages related to fibroblasts, such as hepatic stellate cells and myofibroblasts (Bosselut et al., 2010; Kawada et al., 2001). The localization of Cygb in connective tissue cells may explain its expression in several organs including brain, retina, lung, liver and heart at varying concentration levels, but the concentration of the protein is relatively low (usually in the micromolar range) (Oleksiewicz et al., 2011). At the subcellular level, Cygb can be found in both the cytoplasm and the nucleus upon over-expression of the protein, indicating that Cygb may translocate to the nucleus (Geuens et al., 2003; Kawada et al., 2001). The localization of Cygb within the nucleus suggests a possible role of the protein in regulation of gene transcription (Geuens et al., 2003).

Although the physiological function of Cygb remains unknown, increasing evidence suggests that Cygb has a cyto-protective role against cell damage induced by ischemic and hypoxic conditions, oxidative stress, and fibrosis induced changes (He et al., 2011; Hodges et al., 2008; Lv et al., 2008; McRonald et al., 2012). Under hypoxic and ischemic conditions, Cygb is up-regulated in vitro and in vivo in several organs, including brain, heart, liver, eyes and skeletal muscle (Fordel et al., 2004; Fordel et al., 2007). On the other hand, Cygb is down-regulated in some types of cancer (e.g., oesophageal, lung, head and neck cancer) and it was proposed to have a tumor suppressor 
role (McRonald et al., 2006; Shaw et al., 2009; Shivapurkar et al., 2008; Xinarianos et al., 2006).

The crystal structures of native ferric Cygb at $1.68 \AA$ (Makino et al., 2006) and $2.4 \AA ̊$ A (Sugimoto et al., 2004) resolution, CO bound ferrous Cygb (Makino et al., 2011), and ferric Cygb mutant in which Cys38 and Cys 83 have been replaced by Ser (de Sanctis et al., 2004a) have been elucidated. The X-ray structures show that Cygb displays the classical 3-over-3- $\alpha$-helical sandwich structure or "globin fold" (Figure 8.1). The presence of Fe-protoporphyrin IX as the heme prosthetic group enables Cygb to reversibly bind small gaseous ligands including $\mathrm{O}_{2}, \mathrm{CO}$ and $\mathrm{NO}$ (Smagghe et al., 2008; Trent and Hargrove, 2002). Interestingly, Cygb shares some peculiar structural features with other recently discovered hexa-coordinate hemoglobin, namely neuroglobin $(\mathrm{Ngb})$, that have not been previously observed in vertebrates, such as hexa-coordination in its ferric and ferrous state with a histidine (His 81) residue in the sixth position as an endogenous ligand, presence of an internal disulfide bond and large internal cavities (Ascenzi et al., 2004; Pesce et al., 2004a). The disulfide bond in Cygb forms between Cys 38 (B helix) and Cys 83 (E helix) and affects kinetics of ligand release and binding to the heme iron (Hamdane et al., 2003). Reduction of the disulfide bond in Cygb decreases the rate of His 81 dissociation from heme iron, but to a lesser extent than observed for $\mathrm{Ngb}$, by a factor of $\sim 2$, indicating a weaker coupling between the disulfide bond and ligand binding (Hamdane et al., 2003). Therefore, the mechanism of Cygb cyto-protection against oxidative stress may be linked to the redox chemistry of the protein within the cell. Moreover, formation of the disulfide bond alters the ligand migration in Cygb as well as the thermodynamic parameters associated with ligand escape from the protein 
matrix, suggesting a different mechanism of ligand migration that may vary in response to the redox conditions within the cell (Astudillo et al., 2013). Comparison of the thermodynamic profiles for photo-dissociation of $\mathrm{CO}$ as exogenous ligand suggests that in the presence of the disulfide bond the mechanism of ligand migration in Cygb is analogous to that of $\mathrm{Mb}$, through the His gate, whereas under reducing conditions the ligand migration mechanism in Cygb resembles that of human $\mathrm{Ngb}$ which was proposed to occur through the long internal hydrophobic tunnel (Astudillo et al., 2013).

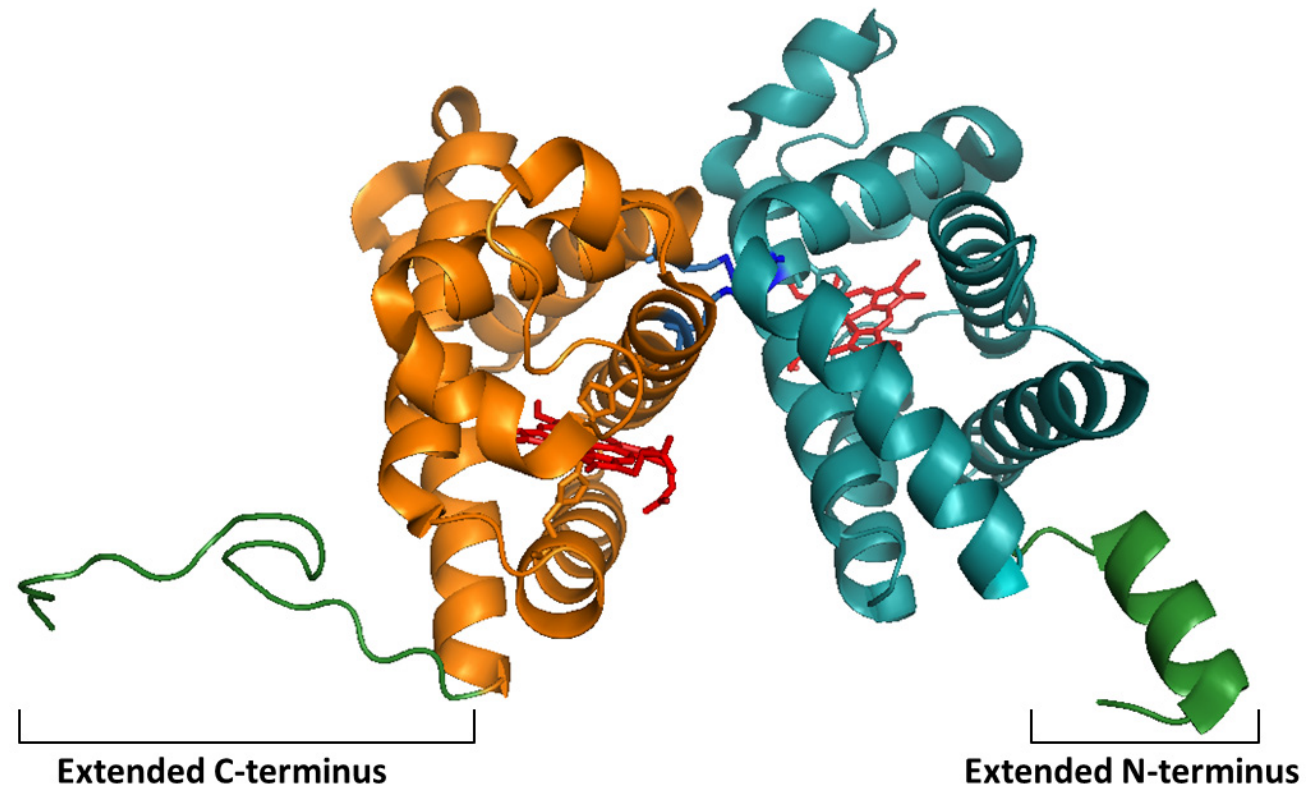

Figure 8.1 Three dimensional structure of Cygb (PDB entry: 2DC3, molecule A in orange and molecule B in light blue) homodimer. The heme prosthetic groups are shown in red and Cys residues in blue. Distal His 81and proximal His 113 residues are shown as orange sticks in molecule A and as blue sticks in molecule B. Extended C- and N-termini are highlighted in green.

One structural feature of Cygb that distinguishes it amongst other members of the globin family, including neuroglobin (Ngb), is that Cygb is composed of 190 amino acid residues making its sequence longer than other globins, which typically containing 140 to 160 residues (Trent and Hargrove, 2002). The crystal structure of Cygb at $1.68 \AA$ shows 
that Cygb forms a homodimer with N-terminal residues (4-20) forming a short $\alpha$-helix before the A helix and C-terminal residues (168-188) forming an ordered loop structure (Figure 8.1) (Makino et al., 2006). The crystal structure of Cygb mutant C38S/C83S (2.4 A) (Sugimoto et al., 2004) also shows the formation of a homodimer, however the dimerization modes in the two structures are different (Makino et al., 2006). The disruption of the disulfide bond by the mutation of Cys 38 and Cys 83 by Ser may result in different subunit orientations from the ones observed in the native protein (Makino et al., 2006). Noteworthy, it was proposed that only a small fraction of dimer might be formed in near-physiological conditions in vitro (Lechauve et al., 2010).

The function of the extended $\mathrm{N}$ - and $\mathrm{C}$ - termini regions in Cygb are not known, however they could be involved in lipid binding (Reeder et al., 2011) and/or proteinprotein interactions. Reeder et al. (2011) reported that ferric Cygb binds to lipids (sodium oleate and cardiolipin) and undergoes a conformational transition from hexacoordinate to penta-coordinate heme iron upon binding, suggesting a role of Cygb in lipid transport. Moreover, Cygb has been reported to have considerable peroxidase activity, consuming both hydrogen peroxide and lipid peroxides (Kawada et al., 2001). On the other hand, there is no evidence to date supporting Cygb interactions with other proteins. Previous studies using yeast two-hybrid assay and immune-precipitation combined with mass spectrometry did not find any interacting partners for Cygb (Hodges et al., 2008).

Although there is experimental evidence that supports Cygb association to lipids, the residues or regions involved in lipid binding have not yet been identified. Reeder et al. (2011) proposed that the extended N- and C-terminal may be responsible for the protein-lipid interactions, but this hypothesis has not been experimentally tested. Protein- 
ligand and protein-protein interactions are often facilitated by formation of hydrophobic patches or clefts on the protein's surface, leading to association of the ligand or protein through hydrophobic interactions with the target protein (Efremov et al., 2007). Therefore, characterization of the hydrophobic regions on the surface of proteins is essential to obtain insight into the molecular recognition process. In this context, extrinsic hydrophobic probes have been extensively utilized to characterize binding sites on the surface of proteins, including anilinonaphthalene dyes (Gasymov and Glasgow, 2007; Hawe et al., 2008). Amongst anilinonaphthalene probes, 1-anilino-8-naphthalene sulfonate $(1,8$-ANS) is one of the best known and most often used fluorescent probes for protein characterization and study of protein folding (Figure 8.2) (Hawe et al., 2008). 1,8ANS is a small molecule that is essentially non-fluorescent in water, but its fluorescence quantum yield increases upon association to hydrophobic regions of proteins and the emission maximum of 1,8-ANS shifts to shorter wavelengths upon binding (Daniel and Weber, 1966; Stryer, 1965). Although 1,8-ANS is mostly recognized as a hydrophobic probe, 1,8-ANS molecules can interact with both hydrophobic and hydrophilic groups (Matulis and Lovrien, 1998). The sulfonate group of 1,8-ANS molecule forms a salt bridge with positively charged amino acids (i.e., Lys, Arg and His), whereas the aromatic rings stabilize the binding with apolar groups (Matulis and Lovrien, 1998). As a result of this complementary interaction between aromatic groups and sulfonate groups of 1,8 ANS with the protein polar and apolar groups, 1,8-ANS has been shown to block binding sites in protein that associate with other substrates or partner proteins (Collini et al., 2003). Therefore, fatty acid binding properties can be investigated by using competitive fluorescence binding assays in which the fluorescence emission of 1,8-ANS in complex 
with the protein of interest is monitored upon addition of the lipid (Collini et al., 2003; Kane and Bernlohr, 1996; Li et al., 2012)
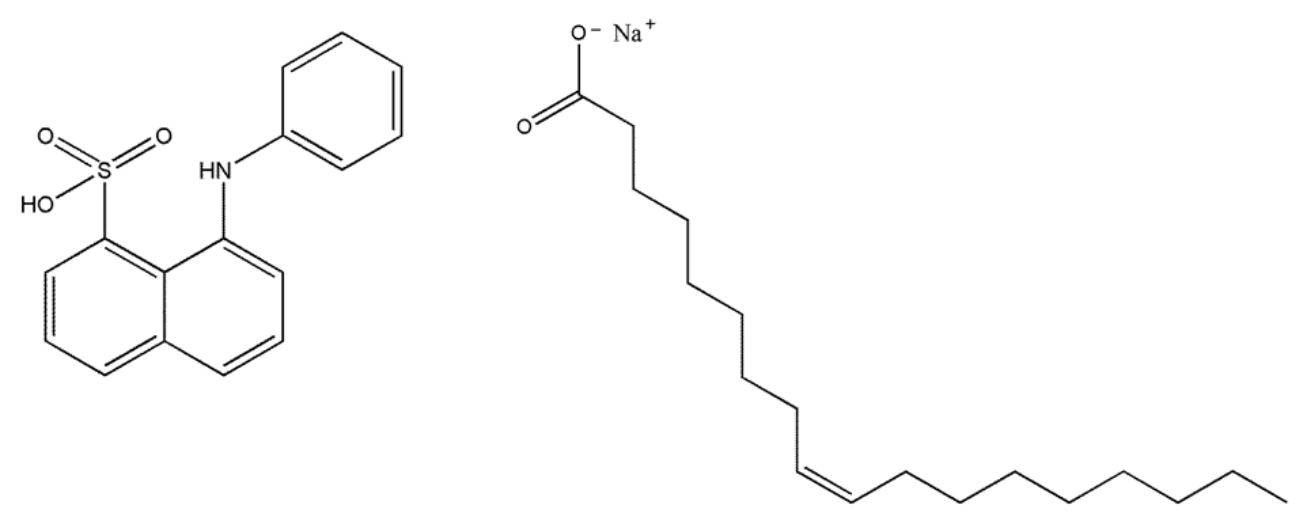

Figure 8.2 Structure of 1-anilino-8-naphthalene sulfonate (1,8-ANS) (left) and sodium oleate (right).

In this work, I characterize the interactions between 1,8-ANS and Cygb by employing fluorescence spectroscopy and isothermal titration calorimetry to obtain a better description of the surface of Cygb, which may be crucial for lipid and/or protein binding and obtain insight into the mechanism of Cygb interactions with lipids. Furthermore, the replacement of 1,8-ANS by oleate (Figure 8.2) in the 1,8-ANS:Cygb complex was monitored using steady-state fluorescence spectroscopy. The impact of the disulfide bond and exogenous ligand binding on 1,8-ANS-Cygb interactions was also probed by characterizing 1,8-ANS association to Cygb reduced with DTT (Cygb ${ }^{\text {red }}$ ) and cyanide bound Cygb (CNCygb). To identify the binding sites in Cygb, molecular docking was performed using Cygb as the receptor and 1,8-ANS and sodium oleate as ligands. Although 1,8-ANS probes have been usually employed in studies of globins in their apo forms, I believe that investigation of structural and dynamic properties of native Cygb is more physiologically relevant. I demonstrate that the extended termini in Cygb are 
crucial for association to 1,8-ANS and sodium oleate competes with 1,8-ANS for Cygb binding site.

\subsection{Results}

\subsubsection{Fluorescence properties of ANS binding to Cygb}

\subsubsection{Steady state fluorescence spectroscopy}

The 1,8-ANS dye was employed as an extrinsic fluorescent probe to monitor changes in the surface of Cygb structure. In order to investigate the impact of the oxidation state of the iron atom, presence of the disulfide bond, and binding of exogenous ligands on association of Cygb to 1,8-ANS, the following forms of the protein were characterized: ferric Cygb (Cygb), ferric Cygb reduced with DTT $\left(\mathrm{Cygb}^{\text {red }}\right.$ ), cyanide bound ferric Cygb in the presence (CNCygb) and absence of the disulfide bond $\left(\mathrm{CNCygb}^{\text {red }}\right)$, ferrous Cygb $\left(\mathrm{Fe}^{2+} \mathrm{Cygb}\right)$ and $\mathrm{CO}$ bound ferrous $\mathrm{Cygb}\left(\mathrm{CO}-\mathrm{Fe}^{2+} \mathrm{Cygb}\right)$. In addition, to probe the impact of the oligomerization state of Cygb on its interaction with 1,8-ANS, a high concentration of the protein $(100 \mu \mathrm{M})$ was tested. Solution studies indicate that Cygb is a monomer at low concentration of the protein $(\sim 20 \mu \mathrm{M})$, whereas at higher concentrations forms homodimers (Lechauve et al., 2010).

The emission intensity of free 1,8-ANS in solution is low, as a consequence of its small quantum yield of approximately 0.004 , and has an emission maximum located at $520 \mathrm{~nm}$ (Stryer, 1965). Addition of 1,8-ANS to Cygb $(20 \mu \mathrm{M})$ in $50 \mathrm{mM}$ Tris buffer, $\mathrm{pH}$ 7, leads to an increase in the emission intensity of ANS and a hypsochromic shift from $520 \mathrm{~nm}$ to $\sim 470 \mathrm{~nm}$ (Figure 8.3). Similar results were observed for all the other forms of the proteins studied and the emission maximum observed upon addition of 1,8-ANS to Cygb in its different forms is listed in Table 8.1. Such enhancement of the emission 
intensity of 1,8-ANS and the observed blue shift of the emission maximum in the presence of the protein are attributed to complexation of the fluorescent probe with Cygb in its different forms. These results are comparable to those reported for the association

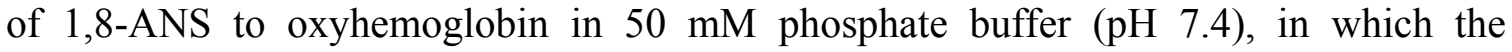
emission maximum of 1,8-ANS shifted to $\sim 486 \mathrm{~nm}$ upon addition of oxyhemoglobin (Syakhovich et al., 2004).

To further confirm that Cygb associates with the dye, steady-state polarization was also employed to characterize binding of 1,8-ANS to $\mathrm{Fe}^{3+} \mathrm{Cygb}, \mathrm{Fe}^{2+} \mathrm{Cygb}$ and $\mathrm{CO}-$ $\mathrm{Fe}^{2+} \mathrm{Cygb}$. The polarization value of free 1,8 -ANS in solution was 0.21 . Addition of $1,8-$ ANS to Cygb does not seem to impact the polarization values of 1,8-ANS as evident by the similarity of polarization between the free 1,8-ANS molecule and in complex with Cygb: $\mathrm{Fe}^{3+} \mathrm{Cygb}(0.25), \mathrm{Fe}^{2+} \mathrm{Cygb}(0.25)$ and $\mathrm{CO}-\mathrm{Fe}^{2+} \mathrm{Cygb}(0.34)$. The fact that the difference in polarization between free 1,8-ANS and 1,8-ANS in complex with Cygb is very small is likely a result light scattering of the free 1,8-ANS solution, which would increase the polarization value for free 1,8-ANS. Alternatively, the small difference in polarization may be a consequence of the short lifetime of the fluorophore. The shorter the lifetime of the fluorophore, the less the fluorophore will rotate between the absorption and emission of a photon and thus the higher the polarization of the emitted light will be (Jameson and Ross, 2010). 


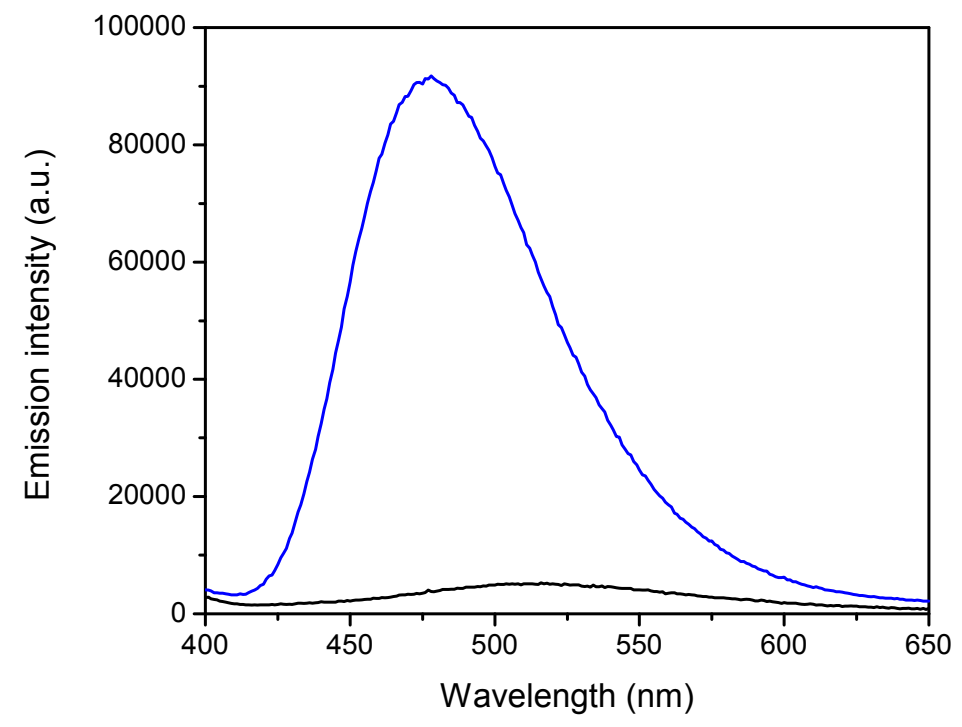

Figure 8.3 Fluorescence emission of $40 \mu \mathrm{M}$ of 1,8-ANS in buffer (black line) and in the presence of $20 \mu \mathrm{M}$ ferric Cygb (blue line). The buffer used was $50 \mathrm{mM}$ Tris, $\mathrm{pH}$ 7.0. The excitation wavelength was $350 \mathrm{~nm}$.

In order to determine the affinity of 1,8-ANS for Cygb in its different forms, a protein at constant concentration $(20 \mu \mathrm{M})$ was titrated with the fluorescent probe. Representative fluorescence emission spectra of 1,8-ANS in the presence of ferric Cygb, $\mathrm{Cygb}^{\text {red }}, \mathrm{CO}-\mathrm{Fe}^{2+} \mathrm{Cygb}$, and $\mathrm{CNCygb}^{\text {red }}$ are shown in Figure 8.4. The emission intensity of 1,8-ANS gradually increased upon addition of higher concentrations of the fluorescent probe. 

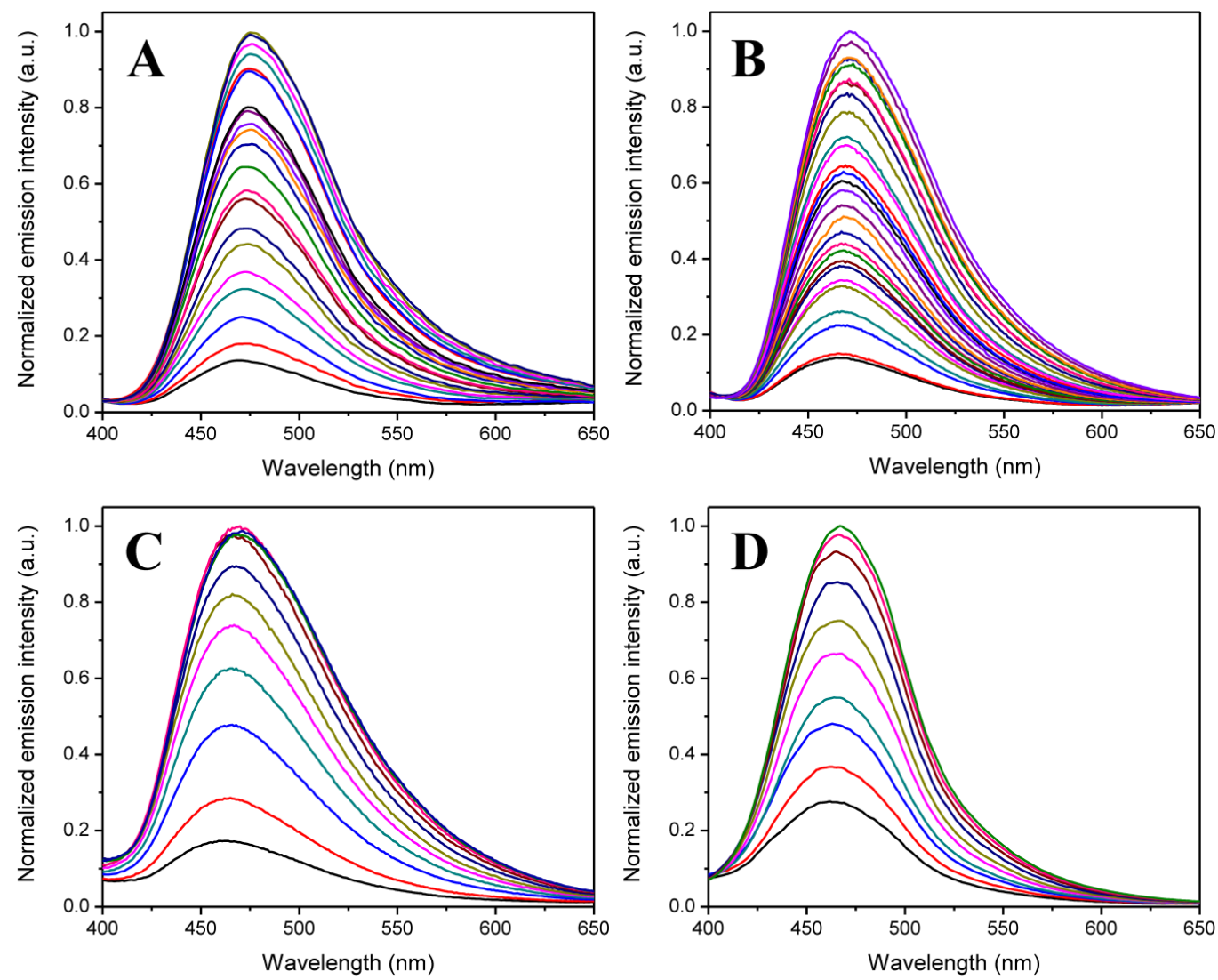

Figure 8.4 Fluorescence emission of different concentrations of 1,8-ANS in the presence of $\mathrm{Cygb}(\mathrm{A}), \mathrm{Cygb}^{\text {red }}$ (B), CO-Fe ${ }^{2+} \mathrm{Cygb}(\mathrm{C})$, and $\mathrm{CNCygb}^{\text {red }}$ (D). Conditions: $20 \mu \mathrm{M}$ protein in $50 \mathrm{mM}$ Tris ( $\mathrm{pH} 7.0$ ) for $\mathrm{Cygb}$ and $\mathrm{CO}-\mathrm{Fe}^{2+} \mathrm{Cygb}$, containing $10 \mathrm{mM}$ DTT for $\mathrm{Cygb}^{\text {red }}$, and containing $10 \mathrm{mM}$ DTT and $10 \mathrm{mM} \mathrm{KCN}$ for $\mathrm{CNCygb}^{\text {red }}$. The excitation wavelength was $350 \mathrm{~nm}$.

The maximum emission intensity of each concentration of 1,8-ANS in the presence of Cygb was plotted as a function of the concentration of 1,8-ANS (Figure 8.5) and the dissociation constant for each complex was determined from the fit of the curve to a single binding site according to equation 8.1. The apparent dissociation constants for 1,8-ANS in complex with $\mathrm{Cygb}, \mathrm{Fe}^{2+} \mathrm{Cygb}, \mathrm{CO}-\mathrm{Fe}^{2+} \mathrm{Cygb}, \mathrm{CNCygb}, \mathrm{Cygb}^{\text {red }}$ and $\mathrm{CN}-$ $\mathrm{Cygb}^{\text {red }}$ are listed in Table 8.1. 


$$
F=\frac{F_{\max }[1,8-A N S]}{K_{d}+[1,8-A N S]}
$$

where $\mathrm{F}$ represents the fluorescence intensity, $\mathrm{F}_{\max }$ is the maximum fluorescence intensity, [1,8-ANS] is the concentration of the fluorescent probe and $\mathrm{K}_{d}$ is the dissociation constant (Kane and Bernlohr, 1996).

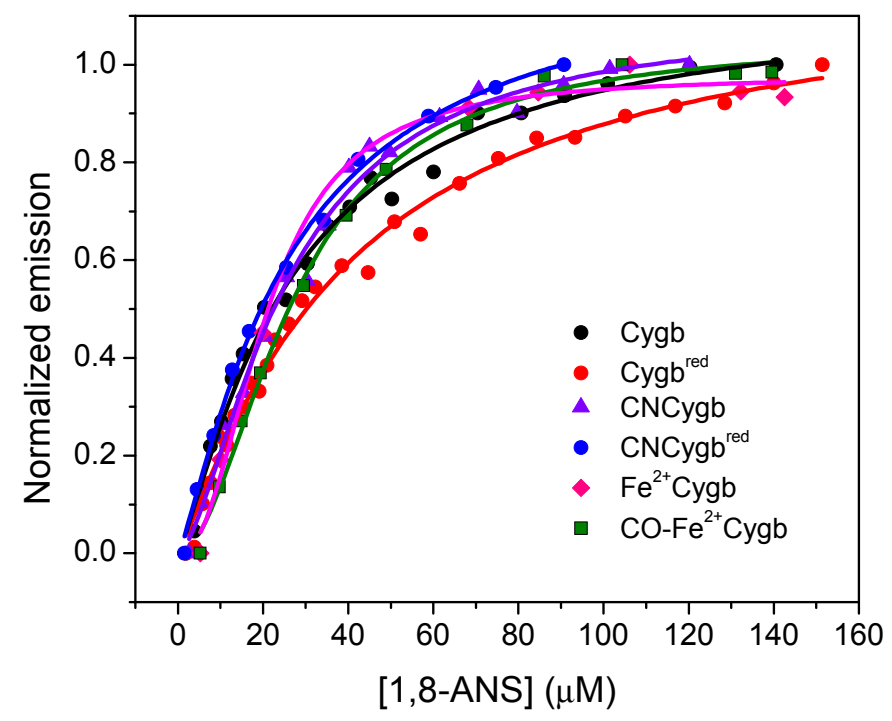

Figure 8.5 Titration curves of 1,8-ANS binding to $\mathrm{Cygb} \mathrm{Cygb}^{\text {red }}, \mathrm{CNCygb}^{\mathrm{CNCygb}}{ }^{\text {red }}$, $\mathrm{Fe}^{2+} \mathrm{Cygb}$ and $\mathrm{CO}-\mathrm{Fe}^{2+} \mathrm{Cygb}$. Conditions: $20 \mu \mathrm{M}$ protein in $50 \mathrm{mM}$ Tris, $\mathrm{pH}$ 7.0.

An apparent $\mathrm{K}_{\mathrm{d}}$ value of $20 \pm 2 \mu \mathrm{M}$ was determined for binding of 1,8-ANS to Cygb, indicating moderate affinity of the dye to the protein. A comparable $\mathrm{K}_{\mathrm{d}}$ value was obtained at higher Cygb concentration of the protein $\left(\mathrm{K}_{\mathrm{d}}=26 \pm 3\right)$ indicating that Cygb binding to 1,8 -ANS does not depend on Cygb concentration up to $100 \mu \mathrm{M}$. The fact that the $\mathrm{K}_{\mathrm{d}}$ for Cygb association to 1,8 -ANS does not depend on the concentration of the protein is somewhat surprising since it was proposed that Cygb forms homodimers at concentrations higher than $\sim 30 \mu \mathrm{M}$ (Lechauve et al., 2010). The $\mathrm{K}_{\mathrm{d}}$ value for 1,8-ANS 
binding to $\mathrm{Cygb}^{\text {red }}$, in which the disulfide bond was reduced by addition of DTT, was approximately two times higher than the $\mathrm{K}_{\mathrm{d}}$ observed in the presence of the disulfide bond. On the other hand, the $\mathrm{K}_{\mathrm{d}}$ value for 1,8-ANS binding to Cygb was not affected by the binding of exogenous ligands such as cyanide and $\mathrm{CO}$ or the oxidation state of the iron atom, as evident from the comparable affinity of the dye to the ferric (Cygb) and ferrous $\left(\mathrm{Fe}^{2+} \mathrm{Cygb}\right)$ forms of the protein in the absence and presence of exogenous ligand $\mathrm{CO}$ and $\mathrm{CN}^{-}$. These results suggest that disruption of the disulfide bond changes the properties of the protein surface in Cygb in a larger extent than binding of exogenous ligands.

Table 8.1 Dissociation constants and emission maximum for 1,8-ANS association to Cygb in its different forms determined using steady-state fluorescence spectroscopy.

\begin{tabular}{lcc}
\hline Sample & $\mathrm{K}_{\mathrm{d}}(\mu \mathrm{M})$ & $\lambda_{\max }(\mathrm{nm})$ \\
\hline Cygb $(100 \mu \mathrm{M})$ & $26 \pm 3$ & 473 \\
Cygb & $20 \pm 2$ & 470 \\
$\mathrm{Cygb}^{\text {red }}$ & $46 \pm 8$ & 468 \\
$\mathrm{CNCygb}^{\mathrm{N}}$ & $20 \pm 5$ & 470 \\
$\mathrm{CNCygb}^{\text {red }}$ & $19 \pm 1$ & 478 \\
$\mathrm{Fe}^{2+} \mathrm{Cygb}^{2+}$ & $21 \pm 2$ & 467 \\
$\mathrm{CO}^{-\mathrm{Fe}^{2+} \mathrm{Cygb}}$ & $26 \pm 2$ & 467 \\
\hline
\end{tabular}

Since human Ngb presents structural similarities with human Cygb (e.g., heme hexa-coordination and presence of disulfide bond), I tested the interaction of 1,8-ANS with human Ngb. Interestingly, no hypsochromic shift in the 1,8-ANS emission 
maximum occurred upon addition of the fluorescent probe 1,8-ANS to human Ngb even at high concentrations of the dye $(150 \mu \mathrm{M})$, as shown in Figure 8.6. A small increase in the emission intensity that was observed upon addition of the dye to hNgb can be attributed to a non-specific 1,8-ANS binding to the protein surface. The different spectral properties observed for 1,8-ANS in the presence of Cygb and Ngb indicates that 1,8-ANS does not associate to Ngb, suggesting specific interactions between 1,8-ANS and Cygb, which is also confirmed by the fact that other fluorescent hydrophobic probes such as 2,6-ANS and nile red do not bind to Cygb (data not shown).

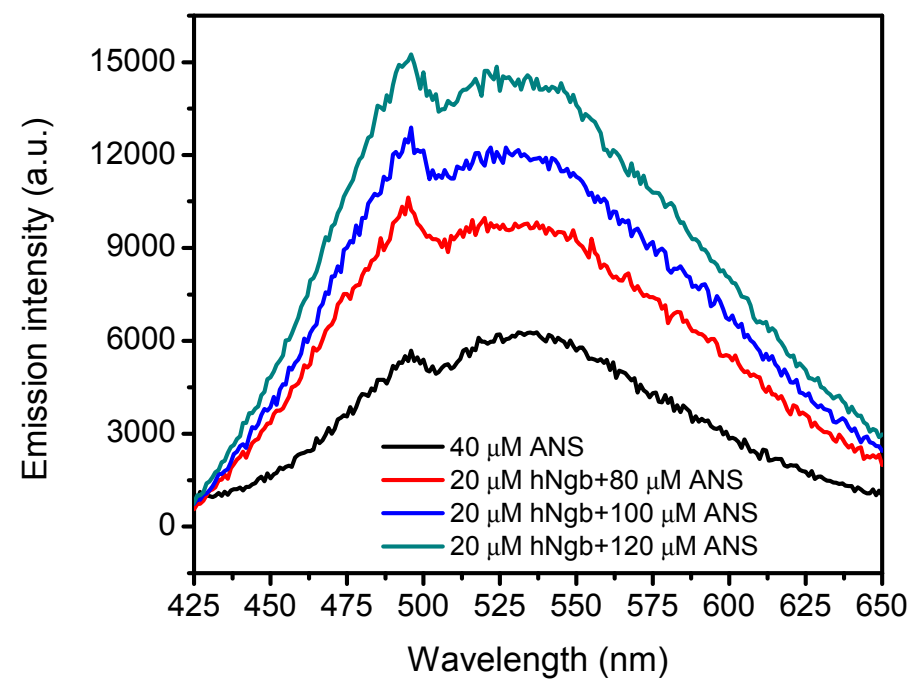

Figure 8.6 Fluorescence emission of $40 \mu \mathrm{M}$ of 1,8-ANS in buffer (black line) and in the presence of $20 \mu \mathrm{M} \mathrm{Ngb}$. The buffer used was $50 \mathrm{mM}$ Tris, $\mathrm{pH}$ 7.0. The excitation wavelength was $350 \mathrm{~nm}$. The sharp peak with a maximum at $485 \mathrm{~nm}$ corresponds to light scattering.

To obtain further insight into the structural features responsible for association of Cygb to 1,8-ANS, a truncated form of Cygb that does not contain the extended N- and Ctermini $(\Delta \mathrm{N} \Delta \mathrm{C}$-Cygb) was prepared in collaboration with Ms. Antonija Tangar 
(Chemistry and Biochemistry Department, FIU). Addition of 1,8-ANS to $\Delta \mathrm{N} \Delta \mathrm{C}$-Cygb results in a shift in the emission maximum of 1,8-ANS from $\sim 528 \mathrm{~nm}$ to $\sim 488 \mathrm{~nm}$ and an increase in emission intensity that is approximately 4.3 times lower than the emission intensity observed for Cygb (Figure 8.7), indicating that the formation of 1,8-ANS:Cygb complex is strongly enhanced in the presence of the extended $\mathrm{N}$ - and $\mathrm{C}$ - termini.

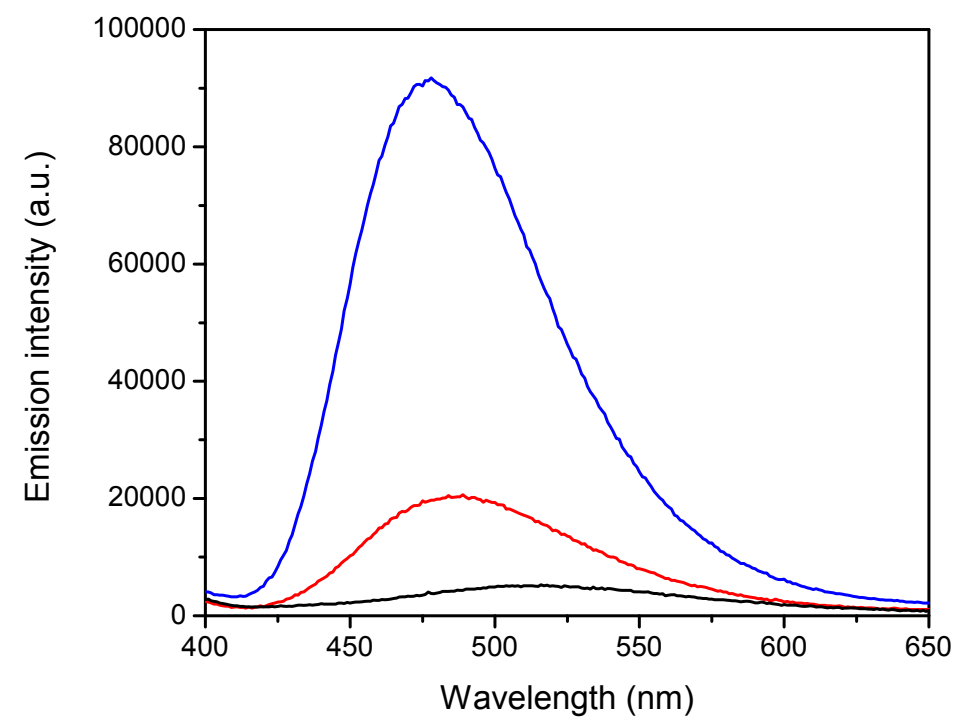

Figure 8.7 Fluorescence emission of $40 \mu \mathrm{M}$ of 1,8-ANS in buffer (black line), in the presence of $20 \mu \mathrm{M}$ ferric Cygb (blue line) and $20 \mu \mathrm{M}$ ferric $\Delta \mathrm{N} \Delta \mathrm{C}$-Cygb (red line). Conditions: $50 \mathrm{mM}$ Tris, $\mathrm{pH}$ 7.0. The excitation wavelength was $350 \mathrm{~nm}$.

\subsubsection{Time-resolved fluorescence spectroscopy}

The fluorescence lifetime of a dye provides valuable information regarding the polarity/hydrophobicity and conformational heterogeneity of a microenvironment surrounding (Lakowicz, 2006). Here, time-resolved fluorescence spectroscopy in the frequency domain was employed to characterize hydrophobic surfaces of Cygb at different oxidation and ligation states. The frequency modulation data for 1,8-ANS:Cygb, 
1,8-ANS:Cygb ${ }^{\text {red }}$, and 1,8-ANS:CNCygb complexes are displayed in Figure 8.8. The data were analyzed using a three discrete lifetime model that provided the best fits (as judged by residuals and $\chi^{2}$ values). The fluorescence decay parameters are summarized in Table 8.2 .

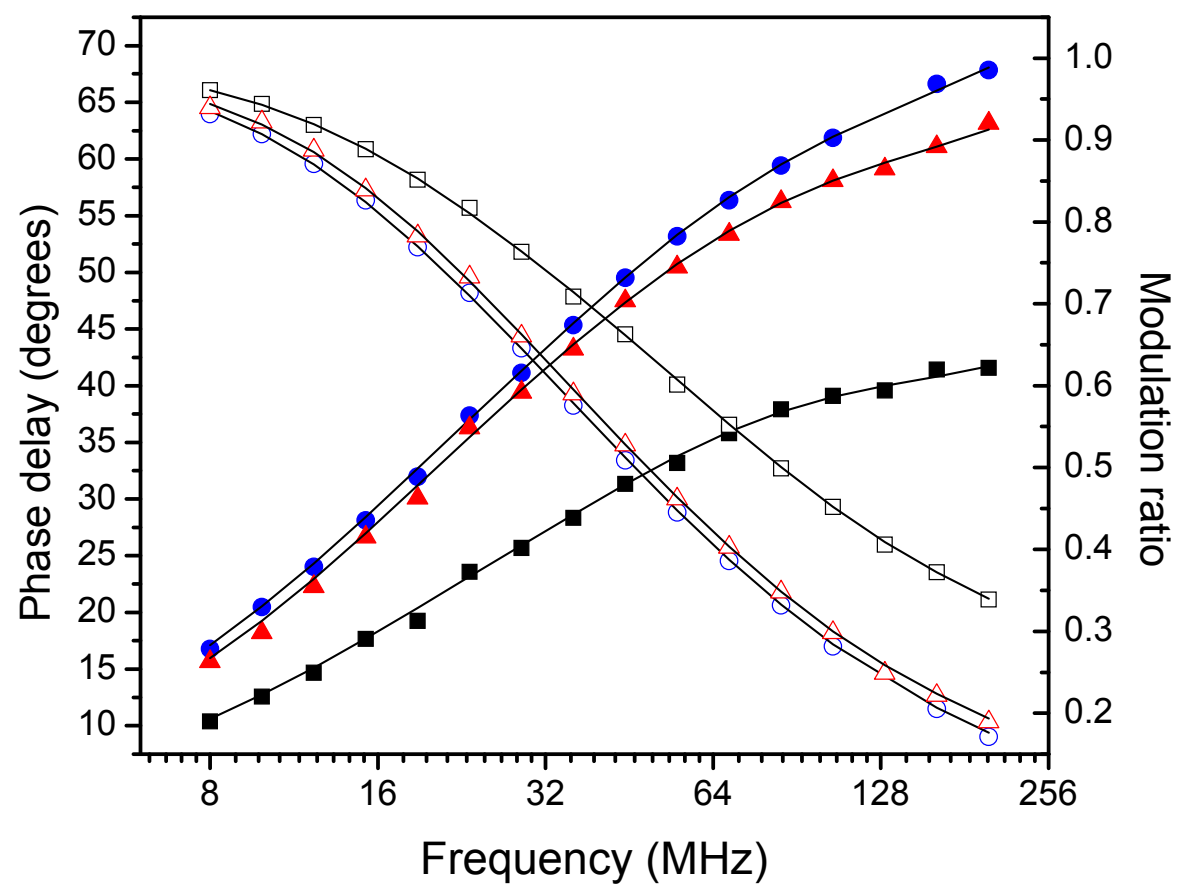

Figure 8.8 Time-resolved fluorescence in the frequency domain data determined for ANS in 1,8-ANS:Cygb (black squares), 1,8-ANS:Cygb ${ }^{\text {red }}$ (blue circles) and 1,8-ANS:CNCygb (red triangles) complexes. Phase delay data are shown as closed symbol and modulation ratio data are displayed as open symbols. Solid lines represent the fit to the data using a three exponential decay model. The data were analyzed using Globals software. Conditions: $20 \mu \mathrm{M}$ protein, $40 \mu \mathrm{M}$ 1,8-ANS in $50 \mathrm{mM}$ Tris (pH 7.0), containing $10 \mathrm{mM}$ DTT for $\mathrm{Cygb}^{\text {red }}$, and $10 \mathrm{mM} \mathrm{KCN}$ for CNCygb. The reference compound used was POPOP solubilized in ethanol ( $\tau=1.35 \mathrm{~ns}$ ) (Lakowicz, 2006). 
Table 8.2 Fluorescence decay parameters associated with 1,8-ANS in complex with Cygb, Cygb $^{\text {red }}$ and CNCygb.

\begin{tabular}{cccccccc}
\hline Sample & $\tau_{1}$ & $\alpha_{1}$ & $\tau_{2}$ & $\alpha_{2}$ & $\tau_{3}$ & $\alpha_{3}$ & $\chi^{2}$ \\
& $(\mathrm{~ns})$ & $(\%)$ & $(\mathrm{ns})$ & $(\%)$ & $(\mathrm{ns})$ & $(\%)$ & \\
\hline Cygb-ANS & $0.26 \pm 0.02$ & $24 \pm 1$ & $2.4 \pm 0.1$ & $38 \pm 1$ & $7.7 \pm 0.2$ & $38 \pm 2$ & 1.01 \\
Cygb $^{\text {red }}$-ANS & $0.25 \pm 0.02$ & $4 \pm 1$ & $2.6 \pm 0.1$ & $30 \pm 1$ & $8.2 \pm 0.1$ & $67 \pm 1$ & 0.98 \\
& & & & & & & \\
CNCygb-ANS & $0.29 \pm 0.09$ & $7 \pm 2$ & $2.6 \pm 0.5$ & $26 \pm 3$ & $7.6 \pm 0.3$ & $67 \pm 1$ & 1.50 \\
& & & & & & & \\
\hline
\end{tabular}

The lifetime of free 1,8-ANS in $50 \mathrm{mM}$ Tris buffer $(\mathrm{pH} \mathrm{7.0)}$ was $\sim 0.26 \mathrm{~ns}$ and matches well previously reported values (Kirk et al., 1996). In the presence of Cygb, two additional lifetimes were determined with the time constants of $2.4 \pm 0.1$ ns and $7.7 \pm 0.2$ ns and were attributed to the probe association to two distinct binding sites with different polarity. The shorter lifetime can be associated to an 1,8-ANS molecule bound to a polar binding site, whereas the longer lifetime can be attributed to 1,8-ANS bound to a hydrophobic site on the protein surface. The presence of numerous ANS binding sites on protein surface is not uncommon as several binding sites have been previously reported for 1,8-ANS association to other proteins (Syakhovich et al., 2004; Uversky et al., 1996). In particular, three lifetimes were also reported for 1,8-ANS association with oxyhemoglobin with values of $0.85 \mathrm{~ns}, 3.78 \mathrm{~ns}$, and $11.74 \mathrm{~ns}$ (Syakhovich et al., 2004). These values are much shorter than lifetimes of 1,8-ANS bound to non-heme proteins, for example, 1,8-ANS bound to apo-myoglobin exhibits a lifetime of 14 ns. Indeed, analysis of 1,8-ANS lifetimes in Cygb and in other heme proteins is more complex. The shorter 
1,8-ANS lifetimes determined for Cygb can be attributed to the increase in polarity of the 1,8-ANS binding site but may also reflect the efficient quenching of 1,8-ANS emission by the heme group. The quenching mechanism involves the resonance energy transfer between the 1,8-ANS donor and the heme acceptor since the emission spectrum of 1,8ANS overlays with the broad absorption spectrum of the heme group (data not shown). Since the energy transfer efficiency strongly depends on the distance between the donor and the acceptor as well as orientation of the transient dipole moments of the donor and acceptor (Lakowicz, 2006), the short lifetimes observed here may be associated to the close proximity of 1,8-ANS binding sites to the heme group.

Comparable values for $\tau_{2}$ and $\tau_{3}$ were observed in Cygb, Cygb ${ }^{\text {red }}$ and CNCygb suggesting that breakage of the disulfide bond and binding of an exogenous ligand does not largely impact the 1,8-ANS interactions with Cygb and the polarity of the two binding sites does not change upon disulfide bond reduction and cyanide association to Cygb. However, on the basis of the pre-exponential factors, the second binding site is more populated in $\mathrm{Cygb}^{\text {red }}$ and CNCygb, as evident by the ratio of $\alpha_{3}: \alpha_{2}$ of $\sim 2: 1$, than in Cygb where the ratio of $\alpha_{3}: \alpha_{2}$ is 1:1. In addition, the population of free 1,8-ANS in solution, represented by the value of $\alpha_{1}$, was $\sim 24 \%$ when the probe was in the presence of Cygb, whereas a smaller population of the free dye was observed in the presence of $\mathrm{Cygb}^{\text {red }}$ and $\mathrm{CNCygb}(\sim 3 \%$ and $\sim 7 \%$, respectively). These results suggest that a larger fraction of 1,8-ANS molecules are bound to Cygb upon disruption of the disulfide bond and binding of exogenous ligands. The fact that the second binding site is more populated in $\mathrm{Cygb}^{\text {red }}$ and CNCygb is likely a result of the conformational change of the protein in the reduced and ligand bound form leading to a higher accessibility of the probe to the 
binding site. Since the crystal structure of native Cygb containing the disulfide bond has not been resolved, it becomes difficult to assess the exact differences between native Cygb and Cygb ${ }^{\text {red }}$ or between Cygb and CNCygb.

\subsubsection{Thermodynamic parameters associated with ANS binding to Cygb}

Association of 1,8-ANS to Cygb, Cygb ${ }^{\text {red }}$, CNCygb and $\mathrm{CNCygb}^{\text {red }}$ were characterized employing isothermal titration calorimetry (ITC) to obtain information about the driving forces involved in the binding process between 1,8-ANS and the protein in these different forms. In ITC, the binding of the dye to the protein is determined by measuring the heat released upon ligand association to protein, which is independent of the fluorescence properties of the dye or the dye-protein complexes (Pierce et al., 1999). As a result, even association of ligands that bind weakly or either binding does not lead to a measurable change in fluorescence intensity can be detected using ITC (Hawe et al., 2011).

A solution of the protein at a constant concentration $(20 \mu \mathrm{M})$ was titrated with 1,8-ANS and the released heat was measured. Binding isotherms corresponding to ANS binding to $\mathrm{Cygb}, \mathrm{Cyg}^{\text {red }}, \mathrm{CN}-\mathrm{Cygb}$ and $\mathrm{CNCygb}^{\text {red }}$ in which the heat of dilution of $1,8-$ ANS was previously subtracted are shown in Figure 8.9. Each peak shown in the binding isotherm corresponds to a single injection of ANS solution and the negative heat signals with respect to the baseline indicate that heat was released during the reaction (Banerjee and Kishore, 2006). In all cases, the best fit of the ITC data was obtained using a twobinding sites model and the corresponding dissociation constants and thermodynamic parameters for the formation of 1,8-ANS:Cygb complexes are listed in Table 8.3. 
The $\mathrm{K}_{\mathrm{d}}$ values determined for the first binding site using ITC are in the range of 50 to $76 \mu \mathrm{M}$ and are somewhat larger than the ones determined by steady-state fluorescence spectroscopy, with the exception of $\mathrm{CNCygb}^{\text {red }}$ in which the $\mathrm{K}_{\mathrm{d}}$ value is approximately $103 \mu \mathrm{M}$ suggesting a weaker binding of 1,8-ANS to the $\mathrm{CN}$ bound form of the reduced protein. The Gibbs free energy change, $\Delta \mathrm{G}$, is in the range of -5.50 to -5.85 $\mathrm{kcal} \mathrm{mol}^{-1}$, indicating that there are no significant differences in the association of 1,8 ANS to Cygb in its different forms studied. At $25{ }^{\circ} \mathrm{C}$ the enthalpy change determined for the first binding site is small and negative with values ranging from -0.95 to $-2.6 \mathrm{kcal}$ $\mathrm{mol}^{-1}$, and the $\mathrm{T} \Delta \mathrm{S}$ term is positive and in the order of 2.9 to $4.9 \mathrm{kcal} \mathrm{mol}^{-1}$, indicating that 1,8-ANS binding to the first binding site is both enthalpically and entropically driven (Kinsley et al., 2008). In particular, the positive entropy change determined for association of 1,8-ANS to the first binding site in Cygb and its different forms can be attributed to release of water molecules hydrating nonpolar surfaces in Cygb upon 1,8ANS complexation to the protein (Kinsley et al., 2008).

The large $K_{d}$ value determined for the second binding site is in the millimolar range and likely reflects non-specific interactions between 1,8-ANS and Cygb in its different forms. The larger and more negative enthalpy changes determined for the second binding site in the range of -16 to $-77 \mathrm{kcal} \mathrm{mol}^{-1}$ as well as the negative and large values determined for $\mathrm{T} \Delta \mathrm{S}$ are indicative of a reaction that is mainly enthalpically driven (Kinsley et al., 2008). A non-specific binding of 1,8-ANS to the protein surface may be stabilized by electrostatic interactions between a negative charge of the sulfonyl 1,8-ANS group and positively charged amino acid residues on the protein surface (Matulis and Lovrien, 1998). Such interactions will result in negative enthalpy changes as well as 
negative entropy change because of the decrease in the number of molecules in the solution.

A

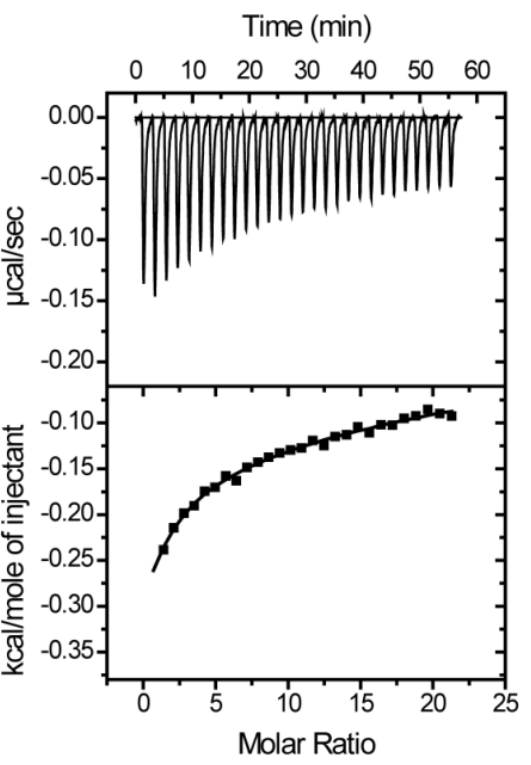

C

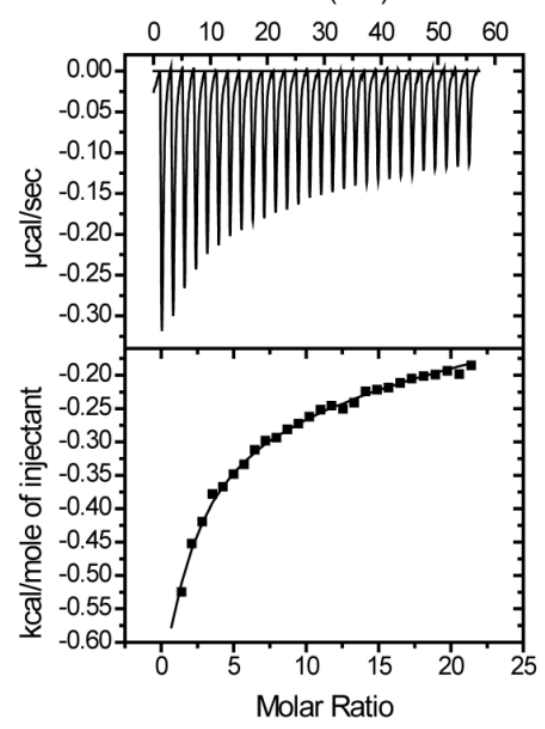

B

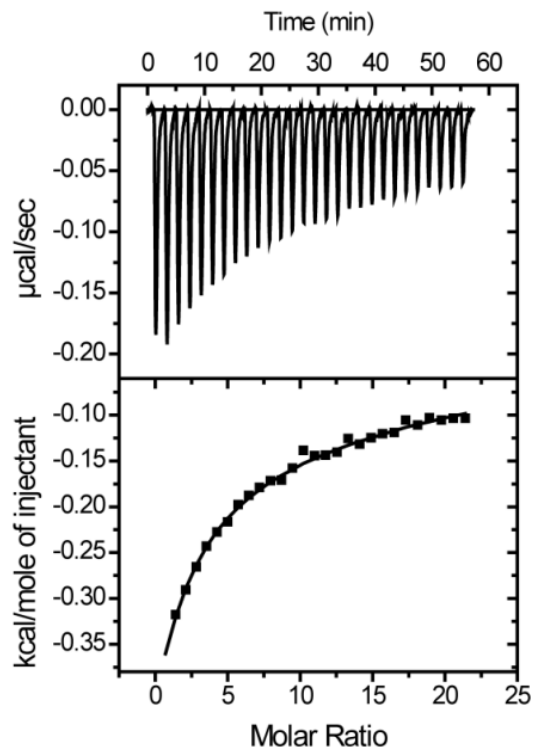

D

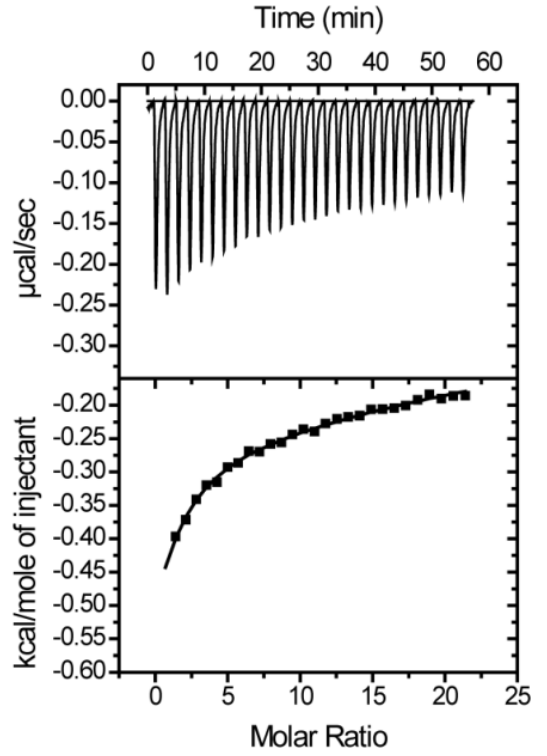

Figure 8.9 Isothermal titration calorimetry of ANS binding to Cygb (A), CNCygb (B), $\mathrm{Cygb}^{\text {red }}(\mathrm{C})$ and $\mathrm{CNCygb}^{\text {red }}(\mathrm{D})$. Top: Raw data for the titration of the protein $(20 \mu \mathrm{M})$ with $2 \mathrm{mM}$ ANS in $50 \mathrm{mM}$ Tris buffer $(\mathrm{pH} \mathrm{7})$ at $25^{\circ} \mathrm{C}$. Bottom: Integrated heats of binding obtained from fitting the raw data shown in the top panel after subtracting the heat of dilution. The solid line represents the best fit to the experimental data using a two sites binding model. The buffer used for $\mathrm{CN}^{-}$bound $\mathrm{Cygb}$ samples contained $10 \mathrm{mM}$ $\mathrm{KCN}$ and the buffer for $\mathrm{Cygb}^{\text {red }}$ samples contained a final concentration of DTT of 1 $\mathrm{mM}$. 
Table 8.3 Thermodynamic parameters determined for Cygb-ANS complexes using ITC.

\begin{tabular}{lcccccccc}
\hline Sample & $\begin{array}{c}\mathrm{K}_{\mathrm{d} 1} \\
(\mu \mathrm{M})\end{array}$ & $\begin{array}{c}\Delta \mathrm{H}_{1} \\
\left(\mathrm{kcal} \mathrm{mol}^{-1}\right)\end{array}$ & $\begin{array}{c}\mathrm{T} \Delta \mathrm{S}_{1} \\
\left(\mathrm{kcal} \mathrm{mol}^{-1}\right)\end{array}$ & $\begin{array}{c}\Delta \mathrm{G}_{1} \\
\left(\mathrm{kcal} \mathrm{mol}^{-1}\right)\end{array}$ & $\begin{array}{c}\mathrm{K}_{\mathrm{d} 2} \\
(\mathrm{mM})\end{array}$ & $\begin{array}{c}\Delta \mathrm{H}_{2} \\
\left(\mathrm{kcal} \mathrm{mol}^{-1}\right)\end{array}$ & $\begin{array}{c}\mathrm{T}_{2} \mathrm{~S}_{2} \\
\left(\mathrm{kcal} \mathrm{mol}^{-1}\right)\end{array}$ & $\begin{array}{c}\Delta \mathrm{G}_{2} \\
\left(\mathrm{kcal} \mathrm{mol}^{-1}\right)\end{array}$ \\
\hline Cygb & $50 \pm 8$ & $-1.0 \pm 0.1$ & $4.9 \pm 0.1$ & $-5.9 \pm 0.2$ & $1.9 \pm 0.2$ & $-16 \pm 1$ & $-12 \pm 1$ & $-3.7 \pm 0.9$ \\
Cygb $^{\text {red }}$ & $60 \pm 6$ & $-2.4 \pm 0.2$ & $3.4 \pm 0.2$ & $-5.8 \pm 0.4$ & $2.8 \pm 0.3$ & $-45 \pm 4$ & $-40 \pm 5$ & $-4.8 \pm 0.9$ \\
$\mathrm{CNCygb}$ & $76 \pm 7$ & $-1.8 \pm 0.1$ & $3.8 \pm 0.1$ & $-5.6 \pm 0.2$ & $2.0 \pm 0.2$ & $-17 \pm 2$ & $-13 \pm 2$ & $-3.5 \pm 0.8$ \\
$\mathrm{CNCygb}^{\text {red }}$ & $103 \pm 18$ & $-2.6 \pm 0.3$ & $2.9 \pm 0.3$ & $-5.5 \pm 0.6$ & $5.7 \pm 0.8$ & $-77 \pm 12$ & $-75 \pm 13$ & $-2.2 \pm 0.9$ \\
\hline
\end{tabular}

\subsubsection{Replacement of 1,8 -ANS with sodium oleate}

The affinity of other ligands for 1,8-ANS binding site have been probed using fluorescence spectroscopy by observing the decrease in emission of 1,8-ANS -protein complexes in the presence of increasing concentrations of competitor ligand (Kane and Bernlohr, 1996). Reeder et al. (2011) recently reported that sodium oleate binds to Cygb and induces a transition from hexa- to penta-coordinated, indicating a possible role of Cygb in lipid transport. However, the molecular mechanism of Cygb interactions with lipids as well as the lipid binding site has not been reported. To obtain further insight into the interaction of sodium oleate with Cygb, complexes of 1,8-ANS with Cygb were titrated with various concentration of sodium oleate. To prevent formation of micelles, the concentration of the stock solution of sodium oleate used was $0.7 \mathrm{mM}$, which is below the lowest critical micelle concentration $(\mathrm{cmc})$ and the buffer used was $1 \mathrm{mM}$ Hepes ( $\mathrm{pH} 7)$ to minimize the effect of ionic strength from the buffer.

Addition of sodium oleate to the Cygb-ANS, Cygr ${ }^{\text {red }}$-ANS and CNCygb-ANS complexes results in a decrease of the emission intensity of 1,8-ANS with increasing concentrations of oleate, as shown in Figures 8.10 to 8.12, which indicates that 1,8-ANS is replaced by oleate. These results suggest that oleate competes with the fluorescent 
probe 1,8-ANS for the same binding site or induces a conformational change that decreases 1,8-ANS binding to Cygb. Similar results have been observed for the interaction of 1,8-ANS with intestinal fatty acid binding protein (Kane and Bernlohr, 1996 and Kirk et al 1995).

Replacement studies with sodium oleate were also performed on 1,8-ANS :CNCygb ${ }^{\text {red }}$ complex, but the emission intensity of 1,8 -ANS in this complex increased upon addition of sodium oleate. The emission increase of 1,8-ANS suggests formation of micelles even at low concentration of buffer (1 mM Hepes), likely as a result of the increased ionic strength of the solution since it contains $1 \mathrm{mM}$ DTT and 10 $\mathrm{mM} \mathrm{KCN}$ to disrupt the disulfide bond and keep the protein in cyanide bound form, respectively.

The decay in corrected fluorescence intensity of 1,8-ANS:Cygb complexes (Cygb-ANS, Cygb ${ }^{\text {red }}$-ANS and CNCygb-ANS) were plotted as a function of the concentration of sodium oleate (competitor ligand), as shown in Figure 8.13. The apparent inhibitor contants $\left(\mathrm{K}_{\mathrm{i}}\right)$ were determined from the fit of the binding curves to equation 8.2 and are listed in Table 8.4.

$$
K_{i}=\frac{I_{50}}{1+\frac{[1,8-A N S]}{K_{d}}}
$$

where [1,8-ANS] is the concentration of free ANS, and $\mathrm{K}_{d}$ is the apparent dissociation constant of 1,8-ANS (from steady-state fluorescence measurements). $\mathrm{K}_{\mathrm{i}}$ refers to the apparent inhibitor constant, which corresponds to the concentration of sodium oleate that would bind to $50 \%$ of the protein molecules if no 1,8 -ANS were 
present. The midpoint of the competition curve corresponds to the $\mathrm{I}_{50}$ (Kane and Bernlohr, 1996).

The inhibition constant, $\mathrm{K}_{\mathrm{i}}$, determined for oleate displacement of 1,8-ANS from Cygb-ANS complexes is not significantly affected by the association of the exogenous ligand $\mathrm{CN}^{-}$, however a small effect was observed upon disruption of the disulfide bond in Cygb, suggesting a role of the disulfide bond in Cygb binding to lipids. The concentration of the protein does not affect the inhibition constant as evident by comparable values for $\mathrm{K}_{\mathrm{i}}$ for Cygb-ANS complexes with ratios 1:1 and 1:2.

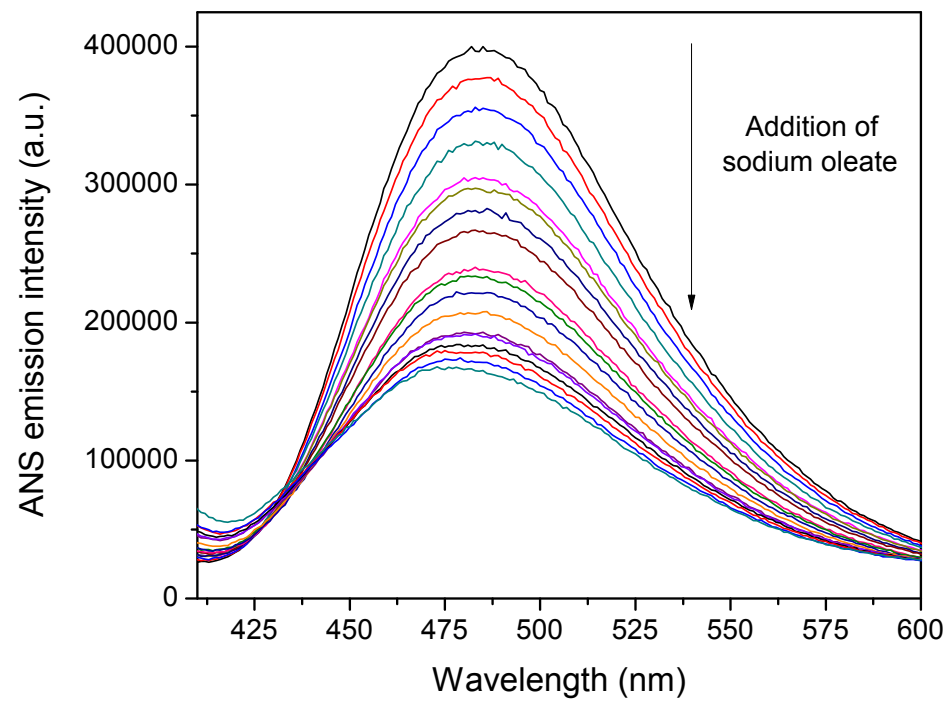

Figure 8.10 Fluorescence emission of 1,8-ANS in complex with Cygb (1:1) in the absence and presence of increasing concentration of sodium oleate. Conditions: $20 \mu \mathrm{M}$ Cygb, $20 \mu \mathrm{M}$ 1,8-ANS in $1 \mathrm{mM}$ Hepes, $\mathrm{pH}$ 7.0. 


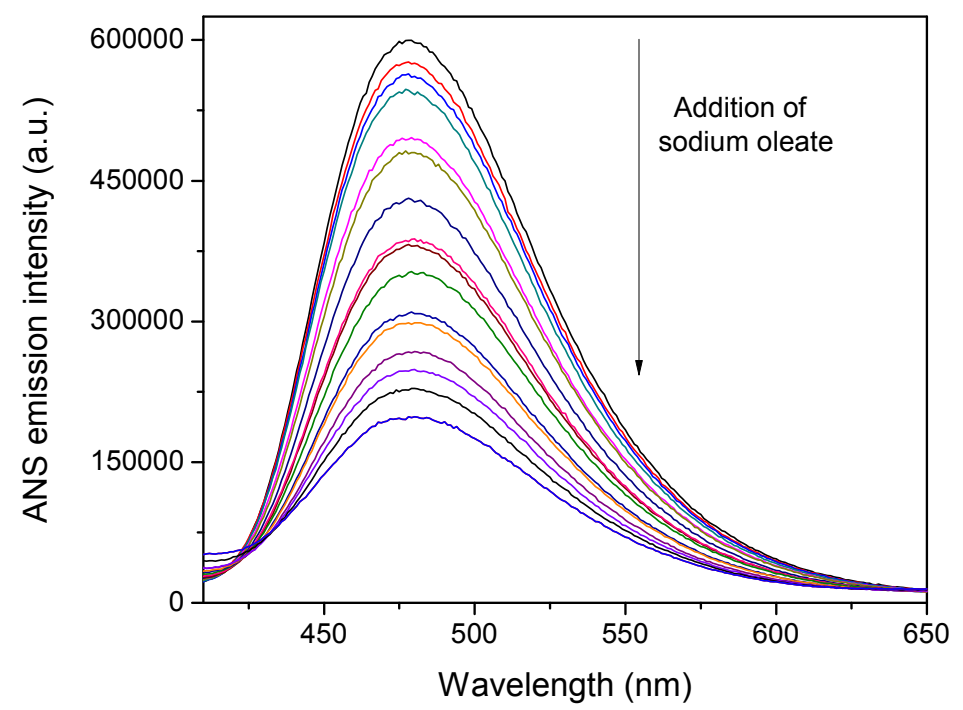

Figure 8.11 Fluorescence emission of 1,8-ANS in complex with $\mathrm{Cygb}^{\text {red }}(1: 1)$ in the absence and presence of increasing concentration of sodium oleate. Conditions: $20 \mu \mathrm{M}$ $\mathrm{Cygb}^{\text {red }}, 20 \mu \mathrm{M}$ 1,8-ANS in $1 \mathrm{mM}$ Hepes, $\mathrm{pH}$ 7.0, containing $1 \mathrm{mM}$ DTT.

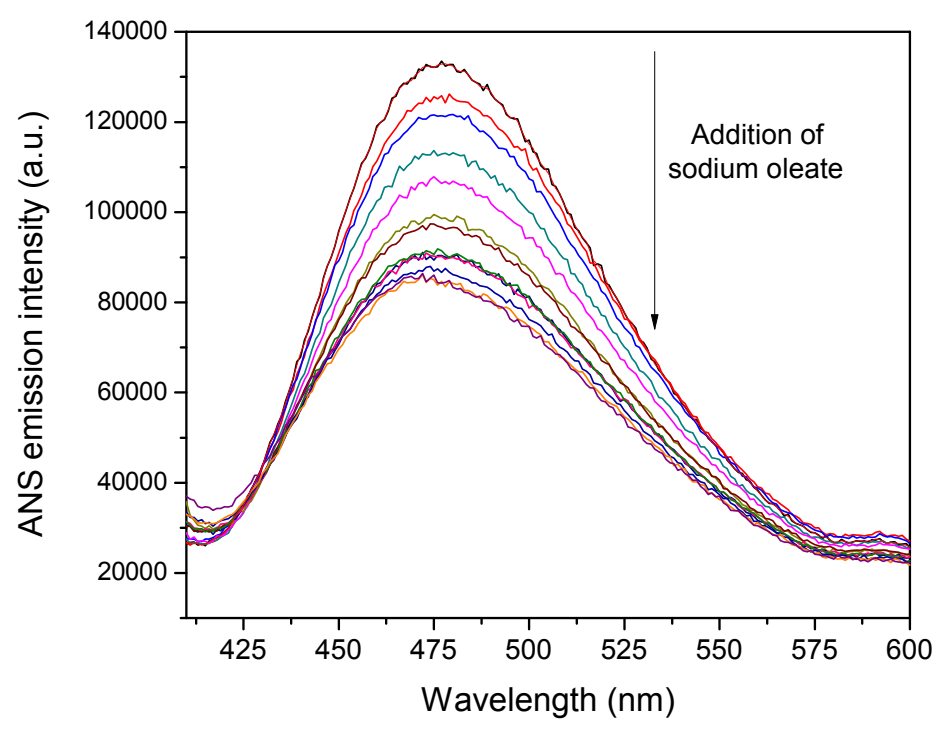

Figure 8.12 Fluorescence emission of 1,8-ANS in complex with CNCygb (1:1) in the absence and presence of increasing concentration of sodium oleate. Conditions: $20 \mu \mathrm{M}$ CNCygb, $20 \mu \mathrm{M}$ 1,8-ANS in $1 \mathrm{mM}$ Hepes, $\mathrm{pH}$ 7.0, containing $10 \mathrm{mM} \mathrm{KCN}$. 


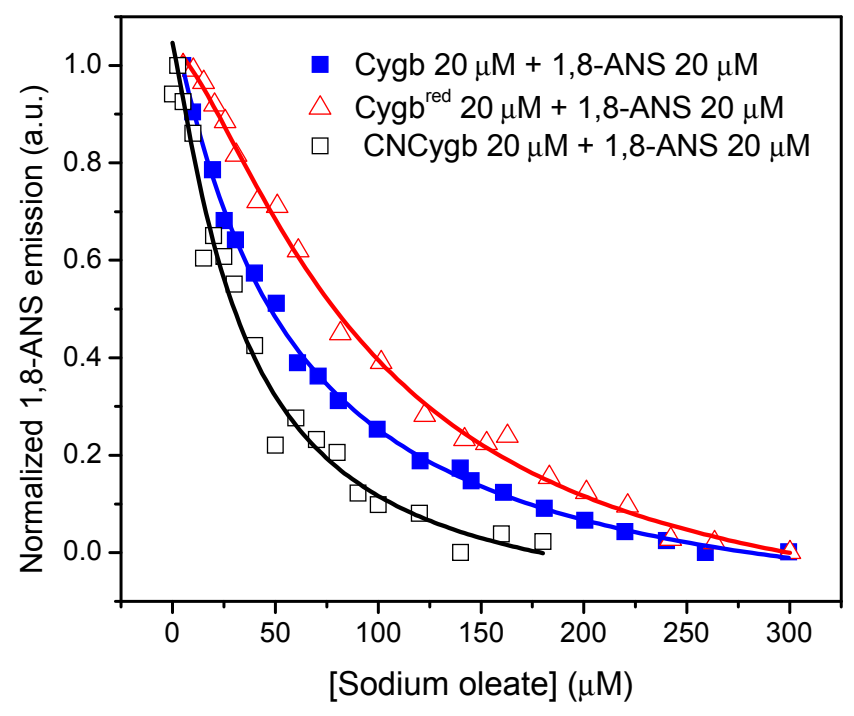

Figure 8.13 Titration curves for addition of sodium oleate to Cygb-ANS, Cygb ${ }^{\text {red }}$-ANS, and CNCybg-ANS complexes. The concentration of the protein and 1,8-ANS were 20 $\mu \mathrm{M}$. The buffer used was $1 \mathrm{mM}$ Hepes for Cygb-ANS, $1 \mathrm{mM}$ Hepes containing $10 \mathrm{mM}$ DTT for $\mathrm{Cygb}^{\text {red }}$-ANS, and $1 \mathrm{mM}$ Hepes containing $10 \mathrm{mM} \mathrm{KCN}$ for CNCygb-ANS.

Table 8.4 Inhibitor constants determined for displacement of 1,8-ANS with sodium oleate in Cygb-ANS complexes.

\begin{tabular}{lcc}
\hline Sample & $\mathrm{I}_{50}(\mu \mathrm{M})$ & $\mathrm{K}_{\mathrm{i}}(\mu \mathrm{M})$ \\
\hline Cygb-ANS (1:1) & $56 \pm 3$ & $26 \pm 4$ \\
Cygb-ANS (1:2) & $69 \pm 4$ & $21 \pm 4$ \\
CygbDTT-ANS (1:1) & $95 \pm 7$ & $66 \pm 8$ \\
CNCygb-ANS (1:1) & $35 \pm 6$ & $19 \pm 7$ \\
\hline
\end{tabular}

\subsubsection{Molecular docking studies}

Fluorescence and ITC data demonstrate that 1,8-ANS associates to Cygb, and the displacement studies with sodium oleate suggests that oleate binds to the same binding 
site, or a region similar to that in which 1,8-ANS binds, thereby competing with 1,8 ANS. To complement the experimental data and obtain structural information on the binding site of 1,8-ANS and oleate in Cygb, the molecular docking program AutoDock was employed using YASARA molecular modeling software for computational docking of 1,8-ANS and oleate on Cygb structure. The crystal structure of wild type Cygb (PDB: 2DC3, molecule B) was employed as the rigid "receptor", whereas 1,8-ANS and sodium oleate were used as the flexible ligands. The structures of 1,8-ANS and sodium oleate were minimized employing Hyperchem software prior docking simulations. The structure of the protein was minimized using Amber03 force field in YASARA molecular modeling software.

The molecular model of the complex corresponding to the highest affinity binding site, predicted from the docking simulation of 1,8-ANS binding to Cygb, is shown in Figure 8.14, and is located at the interface of $\alpha$-helices $G$ and $H$. The 1,8-ANS anilino and naphthyl rings are stabilized by the side chains of Val 122, Ile 126 and Ala 152 as well as the backbone of Lys 125 through hydrophobic interactions, whereas the negatively charged sulfonate group seems to be stabilized by the side chain of Arg 155 through electrostatic interactions. Comparison of the sequence of human Cygb with that of human $\mathrm{Ngb}$ and $\mathrm{Mb}$ shows that the residues occupying the positions mentioned above are different for the three proteins. In Ngb, these residues are Ser 122, Ser 125, Thr 126, Gly 128, Gly 129 and Ser 130 . In case of Mb, the residues are Lys 122, Glu 125, Phe 126, Ser 128, Glu 129 and Cys 130. Although the homology in this region is higher between $\mathrm{Cygb}$ and $\mathrm{Mb}$ than between $\mathrm{Cygb}$ and $\mathrm{Ngb}$, these differences may explain the higher affinity of 1,8-ANS for Cygb than for Mb and Ngb. 


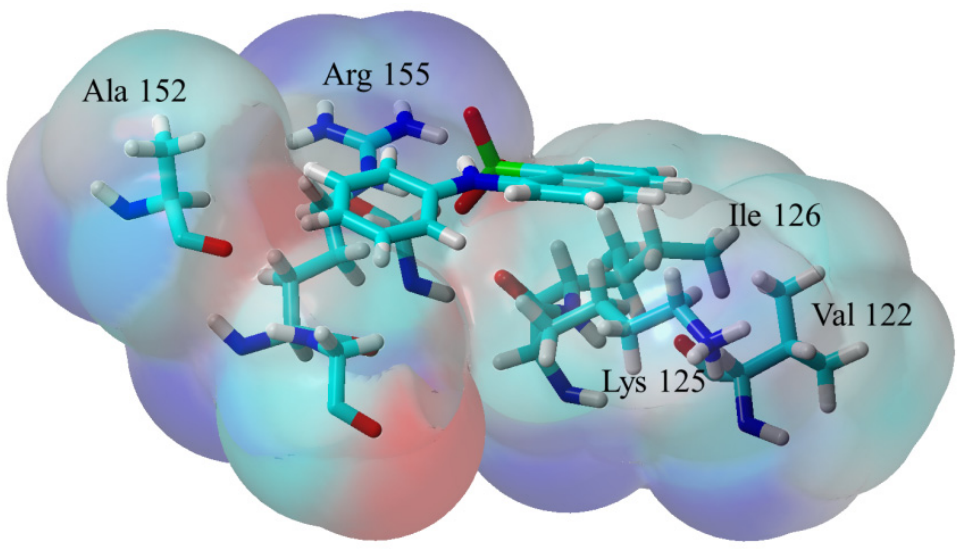

Figure 8.14 Representation of binding site with highest affinity determined from the docking of 1,8-ANS to Cygb (PDB file: 2DC3, molecule B).

The molecular docking model showing the highest affinity of oleate binding to Cygb is shown in Figure 8.15. Oleate binding site is located at the interface between the A-helix and the small $\alpha$-helical structure formed by the extended N-terminus of Cygb. The long hydrophobic alkyl chain of oleate is highly flexible and covers the cavity on the surface on Cygb formed by the side chains of residues Pro 5 and Leu 19, as well as the backbone of Glu 9, Arg 12, Arg 13, Ser 16, and Glu 18. The negatively charged carboxyl group of oleate points toward the side chain of Arg 24 and Glu 21, indicating that association of oleate to Cygb is also stabilized through electrostatic and/or hydrogen bonding with the side chain of Arg 24 and Glu 21.

My results indicate that sodium oleate displaces 1,8-ANS from Cygb-ANS complex, as evidenced by the decrease in the emission intensity of 1,8-ANS in the presence of the protein upon addition of sodium oleate. Therefore, the molecular models obtained from the docking simulation of 1,8-ANS binding to Cygb were further inspected 
to determine a binding site for 1,8-ANS occupying a position where oleate also binds to the protein. One of the clusters formed by 1,8-ANS molecules binding to Cygb is located close to the extended N-terminus of Cygb (Figure 8.16). The anilino and naphthalene rings are partially buried within the cavity formed by the side chains and backbone of Val 4, Pro 5, Ile 10, Glu 9, Arg 13, Ser 16, Glu 18, Ser 20 and Glu 21 through hydrophobic interactions. On the other hand, the sulfonate group of 1,8-ANS is mostly solvent exposed and stabilized by interactions with Arg 24 side chain. It has been previously reported that 1,8 -ANS binding to proteins is stabilized by both hydrophobic and hydrophilic interactions, in which the side chains of Arg, Lys and His residues stabilize the sulfonate group of 1,8-ANS (Matulis and Lovrien, 1998). Although this binding site for 1,8-ANS does not correspond to the highest affinity binding site, it would explain the decreased intensity emission of 1,8-ANS association to the truncated form of Cygb $\Delta \mathrm{N} \Delta \mathrm{C}$-Cygb. Interestingly, residues Arg 24, Glu 21 and Ser 20 are also observed as residues involved in the association of oleate to Cygb (Figure 8.18), suggesting a competitive binding between 1,8-ANS and oleate. 


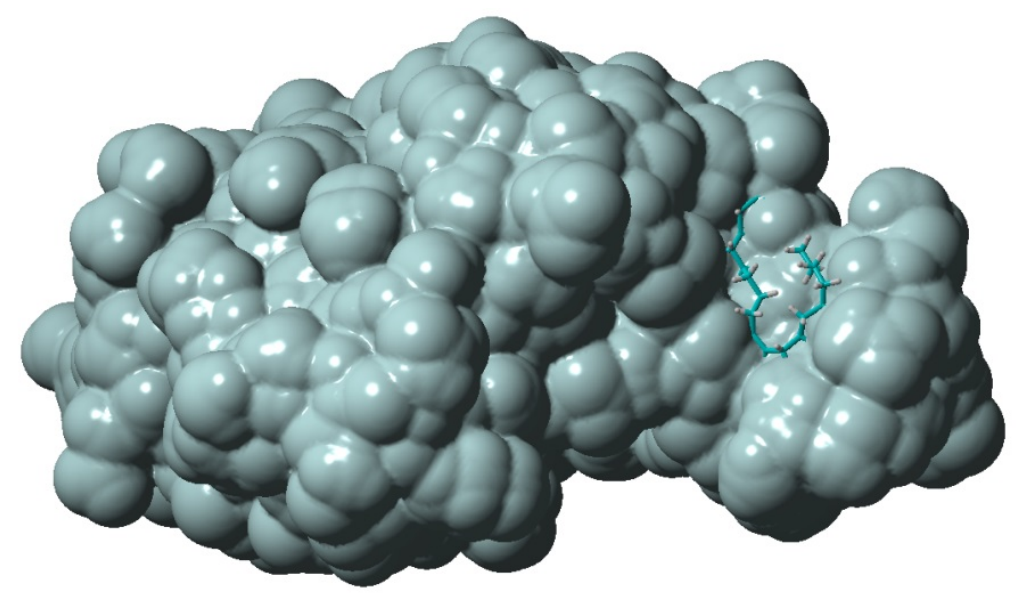

Figure 8.15 Docking of oleate to Cygb (PDB file: 2DC3, molecule B). The solvent accessible surface of Cygb structure is shown in light blue color, whereas oleate structure is shown as sticks.

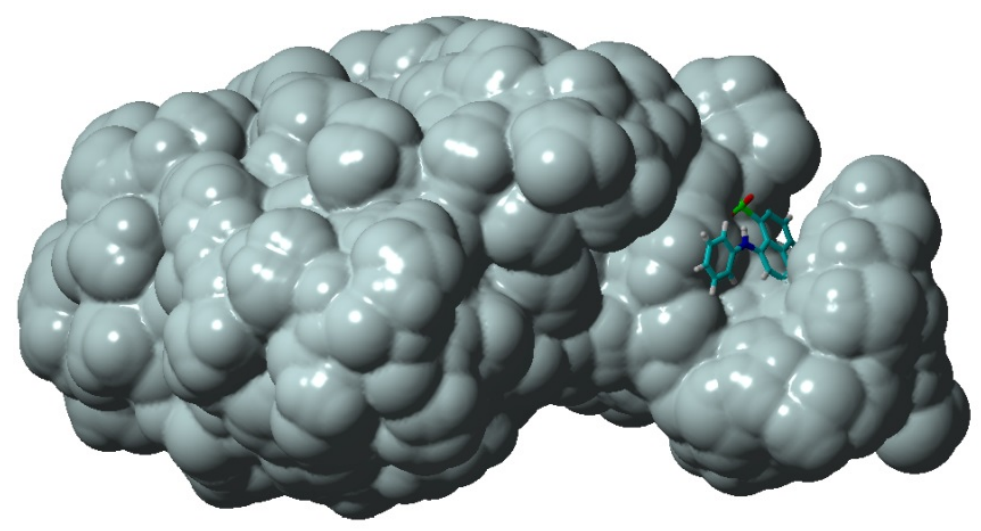

Figure 8.16 Docking of 1,8-ANS to Cygb (PDB file: 2DC3, molecule B). The solvent accessible surface of Cygb structure is shown in light blue color, whereas 1,8-ANS structure is shown as sticks. 


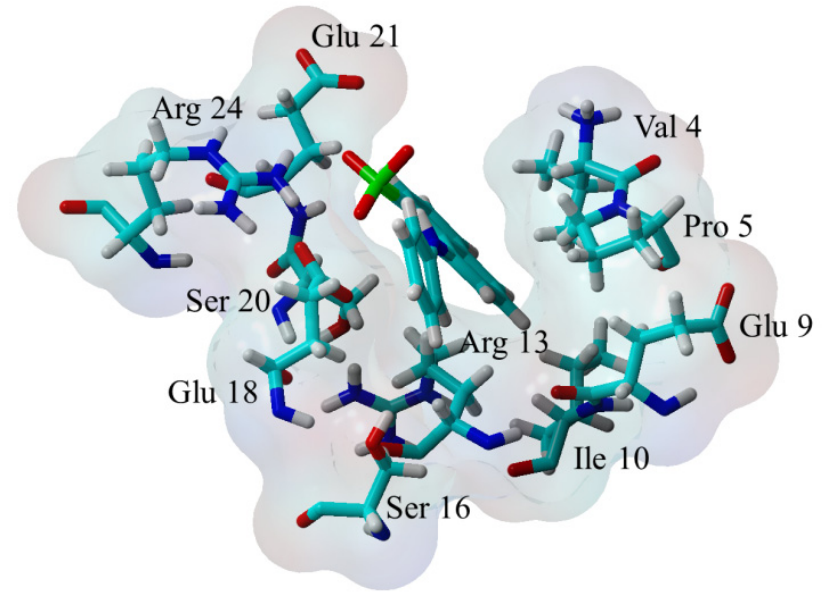

Figure 8.17 Representation of solvent accessible surface area of residues involved in the interaction of Cygb (PDB file: 2DC3, molecule B) with 1,8-ANS.

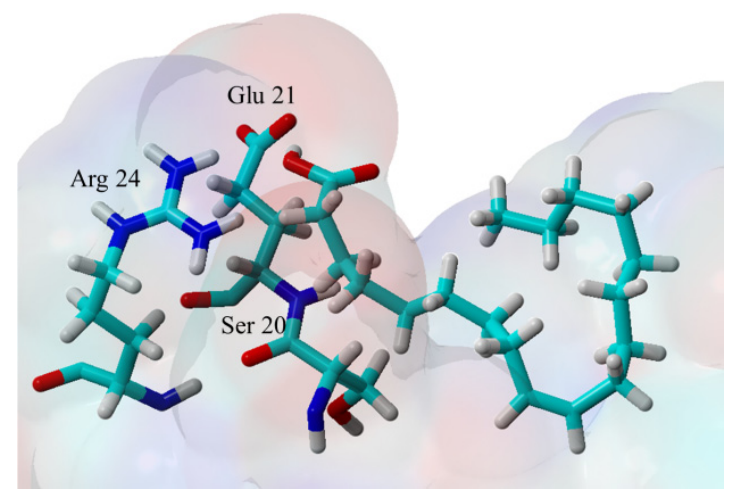

Figure 8.18 Representation of solvent accessible surface area of residues involved in the interaction of Cygb (PDB file: 2DC3, molecule B) with oleate that are also involved in the interaction of Cygb with 1,8-ANS.

\subsection{Discussion}

The compound 1,8-ANS is a widely employed fluorescent probe that noncovalently attaches to the hydrophobic cavities and/or clefts on protein surfaces and are commonly used to monitor solvent exposed hydrophobic patches as well as protein unfolding mechanism (Cardamone and Puri, 1992; Hawe et al., 2008; Pastukhov and Ropson, 2003; Uversky et al., 1996). Steady-state and time-resolved fluorescence 
spectroscopy, as well as ITC were employed to characterize the interactions of 1,8-ANS with Cygb. Molecular docking simulations were performed to complement the experimental data and obtain structural information about ANS and oleate binding sites.

The substantial enhancement of the fluorescence intensity of 1,8-ANS and the shift to shorter wavelengths of the emission maximum of the probe in the presence of Cygb indicates association of 1,8-ANS to the protein. Similar results were observed for the different forms of Cygb tested: $\mathrm{Fe}^{2+} \mathrm{Cygb}, \mathrm{CO}-\mathrm{Fe}^{2+} \mathrm{Cygb}, \mathrm{CNCygb}, \mathrm{Cygb}^{\text {red }}$ and $\mathrm{CN}-$ $\mathrm{Cygb}^{\text {red }}$. A single binding site was determined for 1,8-ANS association to Cygb in its different forms employing steady-state fluorescence and a $K_{d}$ value of $20 \pm 2$ was determined for 1,8-ANS association to ferric Cygb, indicating that 1,8-ANS binds to the protein with moderate affinity. The oxidation state of the heme iron and binding of exogenous ligand does not impact the affinity of 1,8-ANS for the protein as evident by comparable $\mathrm{K}_{\mathrm{d}}$ values determined for 1,8 -ANS association to $\mathrm{Fe}^{2+} \mathrm{Cygb}$, $\mathrm{CO}-\mathrm{Fe}^{2+} \mathrm{Cygb}$ and CNCygb. Conversely, reduction of the disulfide bond in Cygb decreases the affinity of Cygb for 1,8-ANS as evident from the approximately two-fold increase of the $\mathrm{K}_{\mathrm{d}}$ value of $\mathrm{Cyg}^{\text {red }}$.

Since the extended $\mathrm{N}$ - and $\mathrm{C}$ - terminal were proposed to have a role in association of Cygb to lipids (Reeder et al., 2011), the impact of the extended N- and C-termini in Cygb was probed by characterizing 1,8-ANS association to a truncated form of Cygb in which the extended termini are missing ( $\Delta \mathrm{N} \Delta \mathrm{C}$-Cygb) by using steady-state fluorescence spectroscopy. Although addition of 1,8-ANS to $\Delta \mathrm{N} \Delta \mathrm{C}$-Cygb results in a blue shift in the emission maximum of ANS and enhanced fluorescence intensity, the increment in emission intensity is approximately 4.3 times lower for $\Delta \mathrm{N} \Delta \mathrm{C}$-Cygb than for native $\mathrm{Cygb}$ 
under the same experimental conditions. In addition, the emission maximum of 1,8-ANS in the presence of $\Delta \mathrm{N} \Delta \mathrm{C}$-Cygb is less blue shifted (488 nm) than observed for Cygb (470 $\mathrm{nm})$. These results suggest a role of the extended $\mathrm{N}$ - or C-terminus of Cygb in association with ANS, likely providing a binding pocket to accommodate an 1,8-ANS molecule. In the absence of these extended termini, the three dimensional structure of Cygb is almost superimposable with that of $\mathrm{Mb}$ and $\mathrm{Ngb}$. Interestingly, 1,8-ANS does not associate to $\mathrm{Ngb}$ as evident by no blue shift of the emission maximum nor enhanced fluorescence intensity of 1,8-ANS in the presence of Ngb. Furthermore, the $\mathrm{K}_{\mathrm{d}}$ value reported for 1,8ANS binding to native $\mathrm{Mb}$ is $\sim 600 \mu \mathrm{M}$ (Mukherjee et al., 2006), which indicates that 1,8-ANS associates to Cygb with $~ 30$ times higher affinity than $\mathrm{Mb}$.

The fact that different binding sites may not contribute equally to the fluorescence signal arising from the 1,8-ANS probe in complex with the protein, it may be difficult to evaluate the number of binding sites by only employing steady-state fluorescence. In particular, populations of weakly interacting dye molecules may result in a minor increase in the overall emission intensity (Hawe et al., 2011; Lakowicz, 2006). Therefore, time-resolved fluorescence spectroscopy was employed to evaluate those sites that may have a minor contribution to the overall fluorescence intensity. The time-resolved fluorescence data in the frequency domain was better fitted employing a three discrete decay model, indicating that 1,8 -ANS is present in three microenvironments, one corresponding to the free molecule in the bulk solution and the other two correspond to two binding sites with different polarity. 
The two additional lifetimes correspond to two distinct binding sites of the 1,8ANS to Cygb, reflecting heterogeneity of 1,8 -ANS association to the protein. This heterogeneity has been previously observed in other systems, including oxyhemoglobin, bovine carbonic anhydrase, and human $\alpha$-lactoglobulin (Syakhovich et al., 2004; Uversky et al., 1996).

Two binding sites were identified with ITC for association of 1,8-ANS to Cygb. The $\mathrm{K}_{\mathrm{d}}$ values determined for the first binding site are somewhat larger than the ones obtained employing fluorescence spectroscopy, with the exception of $\mathrm{CNCygb}^{\text {red }}$ in which the $K_{d}$ value is close to $100 \mu \mathrm{M}$ suggesting a weaker binding of 1,8 -ANS to the cyanide bound form of the reduced protein. The $\mathrm{K}_{\mathrm{d}}$ values for the second binding site determined using ITC are in the order of 2 and $5.7 \mathrm{mM}$ indicating a weaker non-specific binding of the probe to the protein. In ITC, dye binding to protein is measured by the heat released upon binding, independent of their fluorescence properties. Therefore, weakly binding fractions of 1,8 -ANS that may contribute to a lower degree to the overall fluorescence intensity can also be detected (Hawe et al., 2011). However, ITC might be less sensitive to pick up small fractions of strongly binding and highly fluorescence dye molecules, which can be detected in steady-state, and more specifically in time-resolved fluorescence (Hawe et al., 2011).

The thermodynamic parameters determined for the first binding site indicate that 1,8-ANS binds to the protein through both enthalpic and entropic contributions, since the enthalpy change is small and negative and $\mathrm{T} \Delta \mathrm{S}$ is small and positive. On the other hand, the second binding site is attributed to non-specific binding of ANS to the protein and is mainly entropy driven. 
Since several studies employing fatty acid binding proteins indicate that fatty acids displace 1,8-ANS from these proteins in complex with 1,8-ANS (Kane and Bernlohr, 1996), a displacement fluorescence study was employed to characterize dissociation of 1,8-ANS from 1,8-ANS:Cygb complexes by sodium oleate. It was previously reported that Cygb associates with sodium oleate in a 1:1 ratio with a dissociation constant that ranges from 0.7 to $110 \mu \mathrm{M}$ (Reeder et al., 2011). Fatty acids interactions with Cygb may be relevant for its physiological function since Cygb may have a role in fatty acid transport which could be related to its involvement in cancer (Oleksiewicz et al., 2011). In addition, the interaction of Cygb with fatty acids may be involved in a pathway that aids in translocation of the protein from the cytoplasm to the nucleus. Steady-state fluorescence results demonstrate that sodium oleate displaces 1,8ANS from the 1,8-ANS:Cygb complexes. The inhibition constants determined for Cygb, $\mathrm{Cygb}^{\text {red }}$ and CNCygb are in the range of $\sim 20$ to $\sim 61 \mu \mathrm{M}$, thereby reduction of the disulfide bind and association of exogenous ligands do not largely impact the association of the protein to oleate. Although there is no structural data available for 1,8-ANS:Cygb complexes, since oleate competes with 1,8-ANS binding, one can reasonably speculate that 1,8-ANS resides somewhere in the fatty acid binding site (Kane and Bernlohr, 1996). Docking of 1,8-ANS to Cygb indicates that the binding site with highest affinity is located on the interface between helices $\mathrm{G}$ and $\mathrm{H}$, however, the second binding site identified in docking studies is located at the same position retrieved from the highest affinity binding site determined for oleate binding to Cygb using the same docking simulation method. Therefore, oleate may compete with 1,8-ANS for this binding site. Docking of oleate to Cygb indicates that the fatty acid interacts with the side chains of 
residues located at the extended $\mathrm{N}$-termini and some residues involved in Cygb-oleate interactions are also involved in 1,8-ANS-Cygb interactions, such as Arg 24, Glu 21 and Ser 20 .

Since the time-resolved fluorescence data indicates that 1,8-ANS binds to two sites of different polarity, the 1,8-ANS binding site with highest affinity retrieved from 1,8-ANS docking to Cygb might correspond to the more solvent exposed binding site with shorter lifetime. Comparison of the sequence homology between Cygb and other globins, such as $\mathrm{Mb}$ and $\mathrm{Ngb}$, suggests that this binding site is specific for Cygb since few residues involved in 1,8-ANS-Cygb interactions are also present in $\mathrm{Mb}$ and $\mathrm{Ngb}$ sequence. On the other hand, the ANS binding site located at the same position where oleate associates to Cygb might correspond to the more apolar binding site with longer lifetime because 1,8-ANS molecule seems to be more buried at the interface between the extended $\mathrm{N}$-termini and the $\mathrm{A} \alpha$-helix in Cygb.

Noteworthy, since the C-terminus of Cygb has not been resolved in the crystal structure of Cygb showing the extended termini; it is possible that the binding site in which oleate and ANS compete to associate with the protein could be located in the proximity of the extended C-terminus. Further investigation is needed using the truncated form of each extended terminus (i.e., $\Delta \mathrm{N}$-Cygb and $\Delta \mathrm{C}$-Cygb) to determine the effect of each mutation on association of the protein with ANS.

\subsection{Summary}

Steady-state and time-resolved fluorescence spectroscopy, as well as ITC were employed to characterize the interactions of 1,8-ANS with Cygb. A fluorescence displacement assay was used to characterize the association of oleate to Cygb by taking 
advantage of the enhanced fluorescence emission of 1,8-ANS in the presence of Cygb. In addition, molecular docking simulations were performed to complement the experimental data and obtain structural information about 1,8-ANS and oleate binding sites.

Spectroscopic and thermodynamic data demonstrates that 1,8-ANS associates to Cygb with a moderate affinity. A single binding site was retrieved from the steady-state fluorescence data, but the time-resolved fluorescence data indicates that 1,8-ANS in complex with Cygb is present in two environments with different polarity likely as a result of the presence of two binding sites. Similarly, the ITC data were best fitted with a set of two binding sites model with one site of moderate affinity, whereas the other set of binding sites was attributed to non-specific binding of the probe to the protein. The first binding site determined with ITC has both enthalpic and entropic contributions indicating hydrophobic and hydrophilic interactions between 1,8-ANS and Cygb for this binding site. Visual inspection of the molecular models retrieved from docking of 1,8-ANS and oleate to Cygb in combination with the experimental results suggest that 1,8-ANS might bind to two sites, one located at the interface between helices $\mathrm{G}$ and $\mathrm{H}$ and the other one located between the extended N-terminus and A-helix of Cygb. The binding site located close to the extended $\mathrm{N}$-terminus of Cygb shares amino acid residues involved in the interaction between oleate and Cygb, indicating that this is the site where 1,8-ANS and oleate compete for Cygb binding site. The extended termini in Cygb may form a binding pocket for ANS and oleate interactions; therefore this structural feature not found in other globins has a crucial role in the interaction of Cygb with lipids that might be important for the physiological function of the protein. 


\section{GUANIDINIUM HYDROCHLORIDE INDUCED UNFOLDING STUDY OF HUMAN CYTOGLOBIN AND NEUROGLOBIN}

\subsection{Introduction}

Protein folding and unfolding is one of the most studied areas of biochemistry and biophysics. For many proteins, polypeptide chains must typically fold into their threedimensional structure, once released from the ribosome in vivo or denatured in vitro, in order to function (Gregersen et al., 2006). Some pathological conditions have been linked to protein unfolding and accumulation of mis-folded proteins that lead to formation of aggregates, such as the so-called mis-folding diseases (e.g., Alzheimer's, Parkinson's, Huntington's and Creutzfeldt-Jakob disease) (Gregersen et al., 2006), ), and cancer (Hinton et al., 2013). Therefore, investigation of the mechanism by which proteins fold from a disordered denatured state to a more ordered native state is important to understand the factors that promote folding (Nolting, 2006).

Unfolding transitions can be described using a two-state, three-state or more complex models. The two-state model assumes that only the native $(\mathrm{N})$ and unfolded (U) states are present at equilibrium ( $N \leftrightarrow U)$, whereas the three-state model assumes a multistep process that includes the formation of an intermediate species (I) described by the following reaction: $N \leftrightarrow I \leftrightarrow U$ (Nolting, 2006).

The unfolding process in proteins can be induced by adding chemical denaturants, or by changing the $\mathrm{pH}$ or temperature of the solution (Kelly and Price, 1997; Mu et al., 2010; Santoro and Bolen, 1988). Hence, a method used to determine the stability of a protein and investigate unfolding transitions consists in titration of the protein with denaturants and the spectroscopic signal is measured as a function of denaturant 
concentration. The most commonly used chemical denaturants to study protein unfolding are guanidinium hydrochloride (GuHCl) and urea (Monera et al., 1994). Urea and $\mathrm{GuHCl}$ denaturation curves are generally used to obtain an estimate of the conformational stability of proteins by measuring the differences in conformational stabilities between the folded (native) and the unfolded (denatured) states. The free energy change in the absence of denaturant $\left(\Delta \mathrm{G}_{2}^{\mathrm{H}}{ }^{\mathrm{O}}\right)$ is calculated from analysis of denaturation curves to determine the stability of proteins (Pace, 1975). Urea and $\mathrm{GuHCl}$ molecules are presumed to bind to peptide bonds and, as a protein unfolds, more peptide groups are exposed to denaturant molecules (Monera et al., 1994; Pace, 1975). However, the mechanism though which these denaturants destabilize protein structure is still controversial. Lim et al. (2009) have proposed that only urea destabilizes protein structure through hydrogen bonding interactions with the peptide groups, whereas guanidinium stacks with other planar groups found in aromatic residues and with itself.

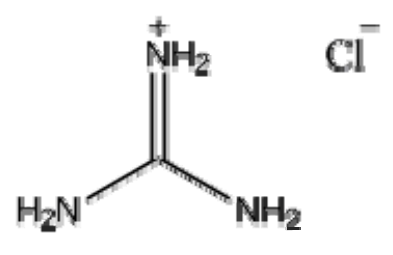

Figure 9.1 Structure of guanidinium hydrochloride $(\mathrm{GuHCl})$.

Several studies have focused on understanding the mechanism of protein folding and unfolding in globins, especially myoglobin $(\mathrm{Mb})$ and hemoglobin $(\mathrm{Hb})$, since the synthesis of these proteins involves expression of the apo-form of the protein (without the heme group) and incorporation of the heme cofactor. A better understanding of how these proteins fold and incorporate the heme group into their structure is of interest in the field 
of biotechnology to express stable proteins that could be used as blood substitutes (Culbertson and Olson, 2010). Despite the fact that most studies focused and folding of heme proteins has been performed on their apo-forms (without the heme group), it has been demonstrated that heme affinity is important for stability of $\mathrm{Mb}$ (Hargrove and Olson, 1996; Hargrove et al., 1996). Unfolding of Mb has been described using a twostate model and it was proposed that the heme group maintains its interaction with the unfolded polypeptide chain through the proximal His (Moczygemba et al., 2000).

Although there are several studies focusing on unfolding of vertebrate globins, there are only few studies that focus on folding/unfolding of the two new members of the vertebrate globin family, namely neuroglobin (Ngb) and cytoglobin (Cygb). Thermally induced denaturation of $\mathrm{Ngb}$ and Cygb was investigated by Hamdane et al. (2005) and their results show that these proteins present enhanced thermal stability with a melting temperature $\left(\mathrm{T}_{\mathrm{m}}\right)$ of $100{ }^{\circ} \mathrm{C}$ for human $\mathrm{Ngb}(\mathrm{hNgb})$ and $95{ }^{\circ} \mathrm{C}$ for Cygb. In addition, this group observed a single transition between the folded state to the unfolded state in Ngb and Cygb. A recent study by Guca et al. (2013) describes the GuHCl-induced denaturation of hNgb with a two-state model. However, the acid-induced and GuHClinduced denaturation of apoNgb was proposed to occur through a three-state mechanism in which the transition from the folded state to the unfolding state occurs through the formation of a molten globule intermediate, whereas urea-induced denaturation occurs through a two-state transition (Mu et al., 2010; Zhang et al., 2013a).

In this work, I studied the GuHCl-induced unfolding of hNgb and human Cygb employing UV-visible absorption spectroscopy and circular dichroism spectroscopy. To determine the impact of the disulfide bond and association of exogenous ligands on the 
stability of Cygb, the protein in the presence and absence of the disulfide bond (Cygb ${ }^{\text {red }}$ ) as well as the cyanide adduct of Cygb (CNCygb) were also characterized. Far-UV circular dichroism (CD) spectroscopy provides information about the secondary structure of a protein and is extensively used as a tool to monitor protein folding/unfolding (Kelly and Price, 1997). In case of heme proteins, the sensitivity of the Soret band to changes in the environment (e.g., chemical changes, $\mathrm{pH}$, temperature) allows the use of absorption spectroscopy to characterize unfolding transitions (Guca et al., 2013; Hamdane et al., 2005; Hargrove and Olson, 1996).

To obtain insight about ligand-induced global transitions in $\mathrm{hNgb}$, time-resolved fluorescence in the frequency domain was employed to characterize bishistidyl $\mathrm{Fe}^{3+} \mathrm{hNgb}$ and cyanide bound $\mathrm{Fe}^{3+} \mathrm{hNgb}$. Since the sequence of $\mathrm{hNgb}$ contains three Trp residues (W13, W133 and W148), three hNgb mutants were constructed to obtain hNgb constructs carrying a single Trp residue (W13Y/ W133Y, W13Y/W148Y, and W133Y/W148Y) and also characterized in the absence and presence of cyanide using time-resolved fluorescence spectroscopy. As shown in Figure 9.2, residues Trp 13 is located in the A helix at $\sim 7 \AA$ from the heme group, $\operatorname{Trp} 133$ is located in the $\mathrm{H}$ helix at $\sim 20 \AA$ from the heme iron, whereas $\operatorname{Trp} 148$ is located at the end of $\alpha$-helix $\mathrm{H}$ in the C-terminus at $\sim 20 \AA$ from the heme iron. The impact of the substitution of two Trp residues with Tyr on the stability of $\mathrm{hNgb}$ was determined by monitoring GuHCl-induced unfolding of the protein. 

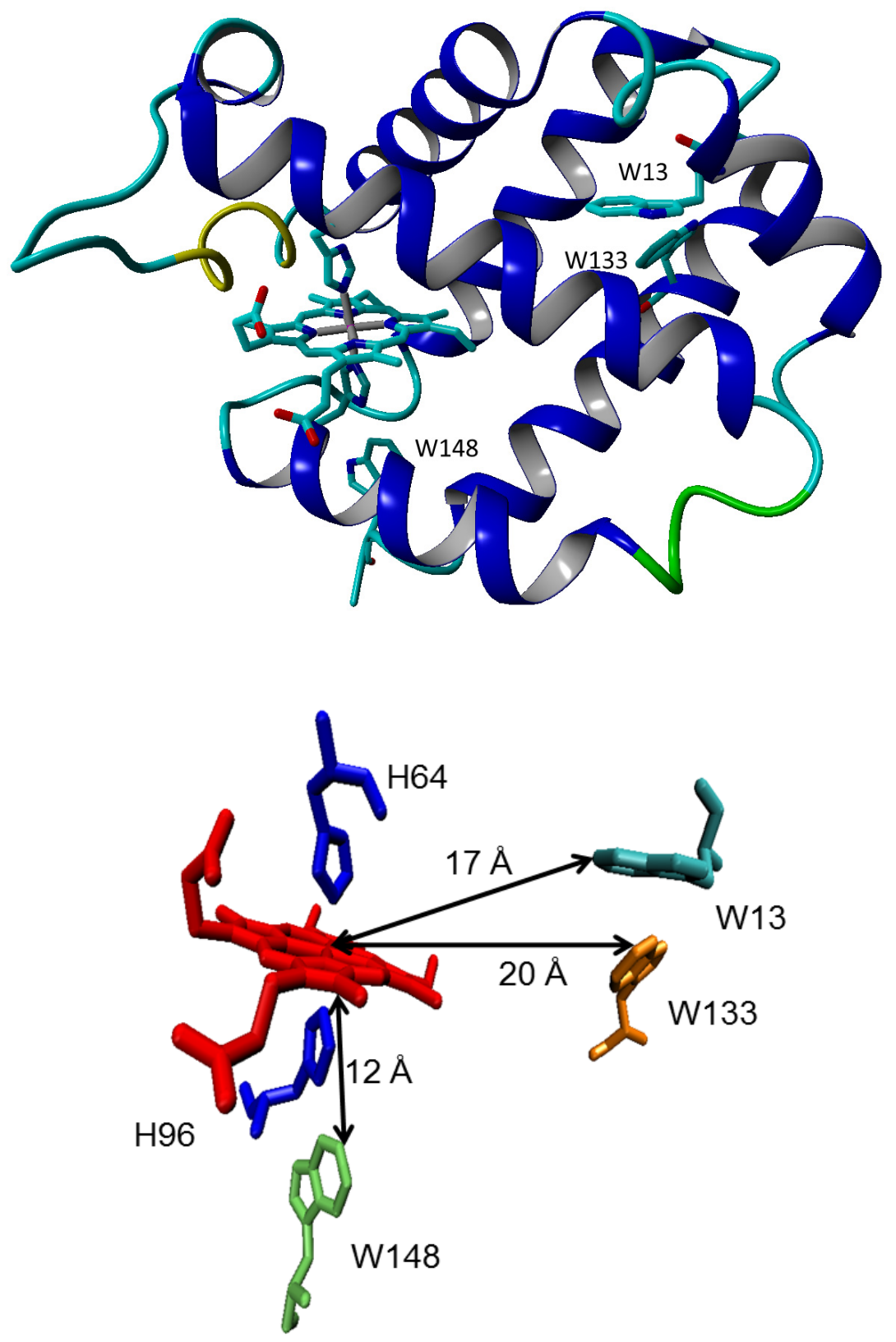

Figure 9.2 Top: Three dimensional structure of hNgb (PDB entry 1OJ6, molecule A) depicting the heme prosthetic group, distal His 64, proximal His 96, and residues Trp 13, Trp 133 and Trp 148 as sticks. Botton: Distance between the heme iron and Trp residues in $\mathrm{hNgb}$. 


\subsection{Results}

\subsubsection{Cytoglobin}

The GuHCl-induced structural changes of Cygb were investigated by using UV-visible absorption spectroscopy and CD spectroscopy. To assess the impact of the disulfide bond and binding of exogenous ligand on the stability of Cygb, the protein was studied in the presence (Cygb) and absence of the disulfide bond by reducing the disulfide bond with 10 mM DTT $\left(\mathrm{Cygb}^{\text {red }}\right)$, as well as the cyanide bound adduct (CNCygb).

\subsubsection{UV-Visible absorption spectroscopy}

The Soret band in heme proteins is characterized by a large extinction coefficient and is sensitive to changes in the environment such as chemical changes, hence the changes in absorbance of the Soret band upon addition of chaotropic agents (e.g., urea and guanidinium hydrochloride) or changes in $\mathrm{pH}$ has been extensively used in unfolding studies of heme proteins (Guca et al., 2013; Hargrove and Olson, 1996). Therefore, the GuHCl-induced unfolding of Cygb was monitored by the change in intensity of the Soret band upon addition of the caotropic agent $\mathrm{GuHCl}$ in $50 \mathrm{mM}$ Tris buffer ( $\mathrm{pH}$ 7.0).

Addition of $\mathrm{GuHCl}$ to $\mathrm{Cygb}$ results in a decreased intensity and a hypsochromic shift in the maximum wavelength of the Soret band (Figure 9.3), which is consistent with exposure of the heme group to the solvent as previously observed in $\mathrm{Mb}$ (Hargrove and

Olson, 1996). Similar results were observed for $\mathrm{Cygb}^{\text {red }}$ and CNCygb, as shown in Figures 9.4 and 9.5, respectively. 


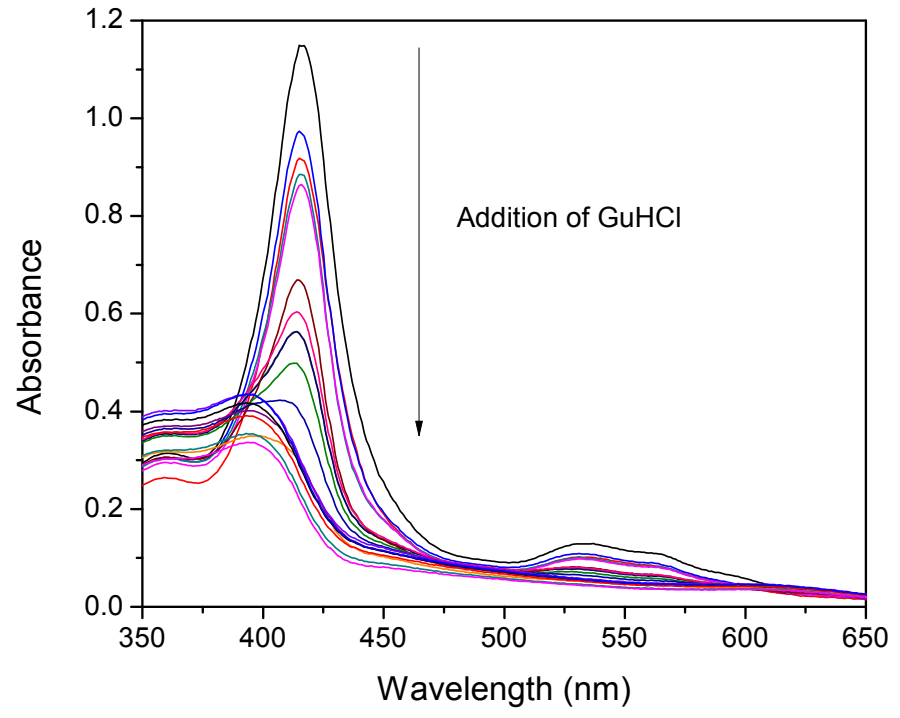

Figure 9.3 UV-visible absorption spectra of Cygb in the presence of increasing concentrations of $\mathrm{GuHCl}$ from 0 to $6 \mathrm{M}$. The concentration of the proteins was approximately $10 \mu \mathrm{M}$ in $10 \mathrm{mM}$ Tris buffer (pH 7.0).

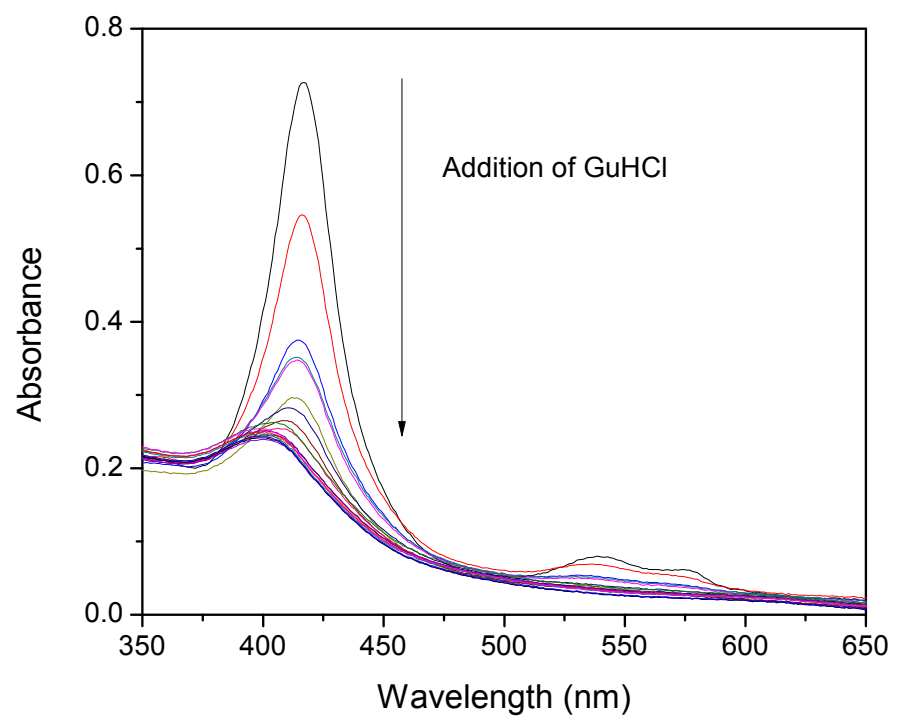

Figure 9.4 UV-visible absorption spectra of $\mathrm{Cygb}^{\text {red }}$ in the presence of increasing concentrations of $\mathrm{GuHCl}$ from 0 to $6 \mathrm{M}$. The concentration of the proteins was approximately $10 \mu \mathrm{M}$. The buffer used was $10 \mathrm{mM}$ Tris buffer (pH 7.0) containing 10 mM DTT. 


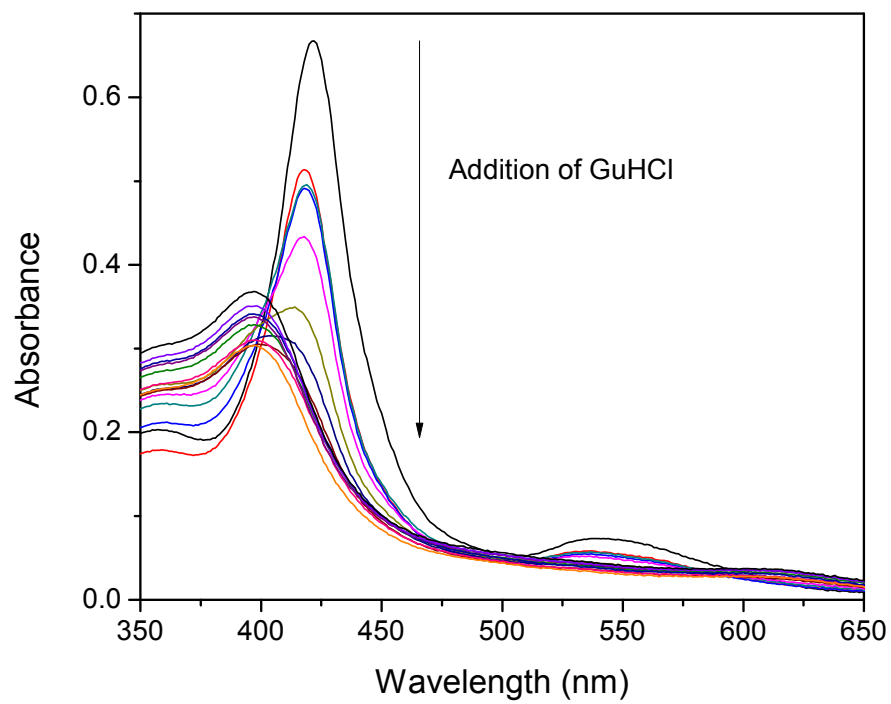

Figure 9.5 UV-visible absorption spectra of CNCygb in the presence of increasing concentrations of $\mathrm{GuHCl}$ from 0 to $6 \mathrm{M}$. The concentration of the proteins was approximately $10 \mu \mathrm{M}$. The buffer used for Cygb was $10 \mathrm{mM}$ Tris buffer $(\mathrm{pH} 7.0$ ) containing $10 \mathrm{mM} \mathrm{KCN}$.

The fraction of unfolded protein was plotted as a function of the concentration of denaturant and the resulting denaturation curves are shown in Figure 9.6. A single transition, from folded to unfolded, is observed from the denaturation curves. Therefore, the equilibrium unfolding data determined for wild type Cygb, $\mathrm{Cygb}^{\text {red }}$ and $\mathrm{CNCygb}$ were analyzed using a two-state model, according to the method reported by Santoro \& Bolen (1988) as described below.

The variation of the signal, $\mathrm{Y}$, with respect to the concentration of $\mathrm{GuHCl}$ is described by:

$$
Y=\frac{\left(Y_{N, H_{2} O}+\beta_{N}[G u H C l]\right)+\left(Y_{D, H_{2} O}+\beta_{D}[G u H C l]\right) \exp \left(-\left(\Delta G_{H_{2} O}^{o}+m[G u H C l]\right) / R T\right)}{1+\exp \left(-\left(\Delta G_{H_{2} O}^{o}+m[G u H C l]\right) / R T\right)}
$$


where $Y_{N, H_{2} O}$ and $\beta_{N}$ are the intercept and slope of the pre-transition baseline, $Y_{D, H_{2} O}$ and $\beta_{D}$ are the intercept and slope of the post-transition baseline, $[\mathrm{GuHCl}]$ is the concentration of $\mathrm{GuHCl}, \Delta G_{\mathrm{H}_{2} \mathrm{O}}^{o}$ corresponds to the the free energy of unfolding in the absence of denaturant, and $m$ represents the dependence on the denaturant concentration (Santoro and Bolen, 1988). Origin software was employed to fit the denaturation curves.

Noteworthy, the absence of a single isosbestic point in the denaturation curves in Cygb, Cygbred and CNCygb suggests that an intermediate may be formed during the unfolding process; however it could not be resolved using UV-visible spectroscopy likely as a result of a low fraction of the intermediate.

The determined values for free energy of unfolding $\left(\Delta G^{o}{ }_{H_{2}} \mathrm{O}\right)$ and the degree of hydrophobic exposure upon unfolding $(\mathrm{m})$ are listed in Table 9.1.

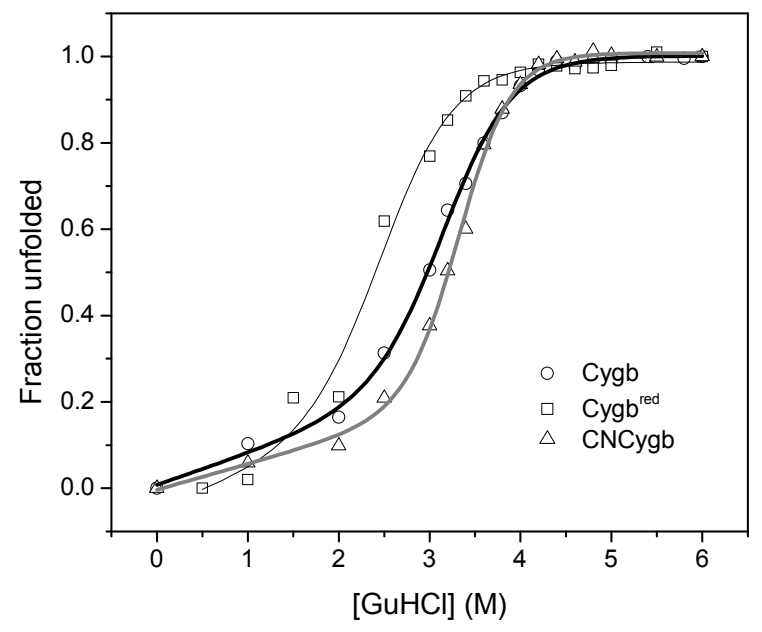

Figure 9.6 Unfolding curves of Cygb, Cygb ${ }^{\text {red }}$ and CNCygb monitored using UV-visible absorption spectroscopy as a function of $\mathrm{GuHCl}$ concentration. The concentration of the protein was $\sim 10 \mu \mathrm{M}$ in $10 \mathrm{mM}$ Tris buffer, $\mathrm{pH}$ 7.0. The buffer used for Cygb ${ }^{\text {red }}$ samples contained $10 \mathrm{mM}$ DTT and for CNCygb samples contained $10 \mathrm{mM} \mathrm{KCN}$. 
Table 9.1 Free energy of unfolding $\left(\Delta G^{o}{ }_{H_{2} O}\right.$ ) and degree of hydrophobic exposure upon GuHCl-induced unfolding $(\mathrm{m})$ determined for $\mathrm{Cygb}, \mathrm{Cygb}^{\text {red }}$ and $\mathrm{CNCygb}$ employing UV-visible absorption spectroscopy.

\begin{tabular}{lcc}
\hline & $\Delta G^{o}{ }_{H_{2} O}\left(\mathrm{kcal} \mathrm{mol}^{-1}\right)$ & $\mathrm{m}\left(\mathrm{kcal} \mathrm{mol}^{-1} \mathrm{M}^{-1}\right)$ \\
\hline Cygb & $4.8 \pm 0.4$ & $1.5 \pm 0.1$ \\
$\mathrm{Cygb}^{\text {red }}$ & $3.6 \pm 0.9$ & $1.4 \pm 0.3$ \\
$\mathrm{CNCygb}$ & $6.7 \pm 0.7$ & $2.0 \pm 0.2$ \\
\hline
\end{tabular}

The change in free energy $\left(\Delta G^{o} \mathrm{H}_{2} \mathrm{O}\right)$ determined for the transition of Cygb is $4.8 \pm$ $0.4 \mathrm{kcal} \mathrm{mol}{ }^{-1}$ with an $\mathrm{m}$ value of $1.5 \pm 0.1 \mathrm{kcal} \mathrm{mol}^{-1} \mathrm{M}^{-1}$. The value of $\Delta G_{\mathrm{H}_{2} \mathrm{O}}^{o}$ determined for Cygb is similar to the one reported for horse heart $\mathrm{Mb}$ at $\mathrm{pH} 8$ of $4.95 \pm$ $0.13 \mathrm{kcal} \mathrm{mol}^{-1}$ (Roncone et al., 2005). However, the m value determined by Roncone et al. (2005) for $\mathrm{Mb}$ of $3.45 \pm 0.09 \mathrm{kcal} \mathrm{mol}^{-1} \mathrm{M}^{-1}$ is $\sim 2$ times higher than the value determined for Cygb, suggesting a higher solvent-exposed surface area of $\mathrm{Mb}$ than in Cygb. Alternatively, larger $\mathrm{m}$ values have been attributed to the existence of some intermediate state during the unfolding process (Roncone et al., 2005).

Reduction of the disulfide bond in Cygb does not impact the stability of the protein as evidenced by the comparable values of $\Delta G^{o}{ }_{2} \mathrm{O}$, within the error, determined for Cygb and $\mathrm{Cygb}^{\text {red }}$. This result is somewhat surprising and points out that the disulfide bond in Cygb does not have a role in protein stabilization. Protein folding studies have pointed out that disruption of disulfide bonds usually destabilize the native structure of proteins (Li et al., 2013; Vinther et al., 2013; Zhang et al., 2011b). The value of the $\mathrm{m}$ parameter, which is related to the exposure of the protein surface area to the solvent, was 
comparable for $\mathrm{Cygb}$ and $\mathrm{Cyg}^{\text {red }}$, whereas a slightly larger value was determined for CNCygb, suggesting an increase in the solvent-exposed surface area for the $\mathrm{CN}^{-}$bound form of Cygb.

On the other hand, binding of $\mathrm{CN}^{-}$to Cygb leads to a small increase in stability by $\sim 1.9 \mathrm{kcal} \mathrm{mol}^{-1}$. The cyanide adduct of $\mathrm{Mb}$ also showed a higher stability than $\mathrm{Mb}$, which was attributed to a greater ability of cyanide to accept $\pi$ electrons from the heme iron, which results in stabilization through the so-called "trans effect" of the bond between the heme iron and the proximal His residue $\left(\mathrm{Fe}^{3+}-\mathrm{His} 93\right)$ (Hargrove and Olson, 1996).

\subsubsection{Far-UV CD spectroscopy}

The structural changes induced by addition of $\mathrm{GuHCl}$ to $\mathrm{Cygb}$, $\mathrm{Cygb}^{\text {red }}$ and CNCygb were also monitored using far-UV CD spectroscopy. Far-UV CD spectroscopy has been extensively used in protein unfolding/folding studies because this technique provides information about changes in the secondary structure upon denaturation (Kelly and Price, 1997).

The far-UV CD spectrum of human Cygb exhibits two negative bands at $209 \mathrm{~nm}$ and $222 \mathrm{~nm}$ that are characteristic of $\alpha$-helical proteins (Figure 9.13). The $\alpha$-helical content of Cygb was calculated to be $\sim 70 \%$ from the mean ellipticity at $222 \mathrm{~nm}$, as described in chapter 4 , and this value matches well the $\alpha$-helical content estimated by Sawai et al. (2003) of $72 \%$ as expected for globins. However, the CD spectrum for Cygb showed slightly less $\alpha$-helical content than Ngb, which was attributed to a less $\alpha$-helical nature of the extra residues at the $-\mathrm{N}$ and $-\mathrm{C}$ termini $(\sim 20$ amino acid residues at each termini) (Hamdane et al., 2005). As described in chapter 4, the $\alpha$-helical content 
calculated for $\mathrm{hNgb}$ is $76 \%$, which is comparable to the reported value of $78 \%$ (Hamdane et al., 2005).

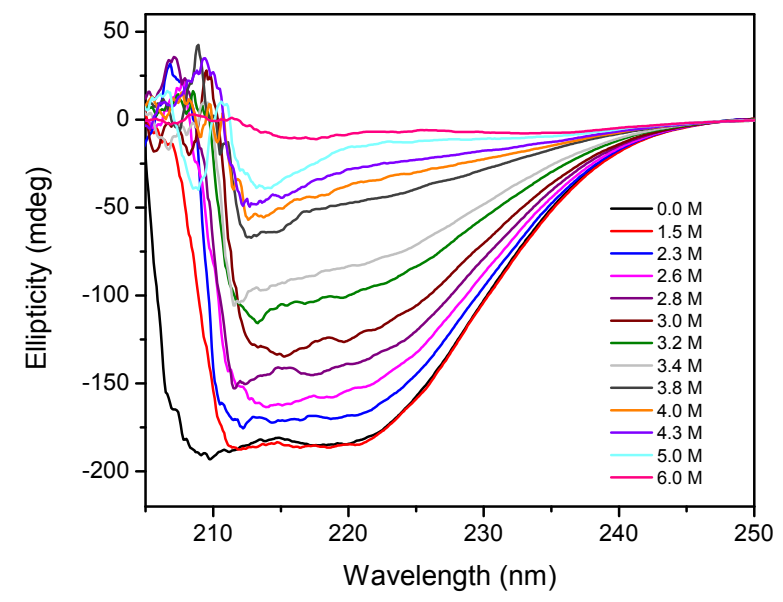

Figure 9.7 Far-UV Circular dichroism spectra of Cygb in the presence of increasing concentrations of $\mathrm{GuHCl}$. The concentration of the proteins was $40 \mu \mathrm{M}$ in $10 \mathrm{mM}$ Tris buffer ( $\mathrm{pH} 7.0)$.

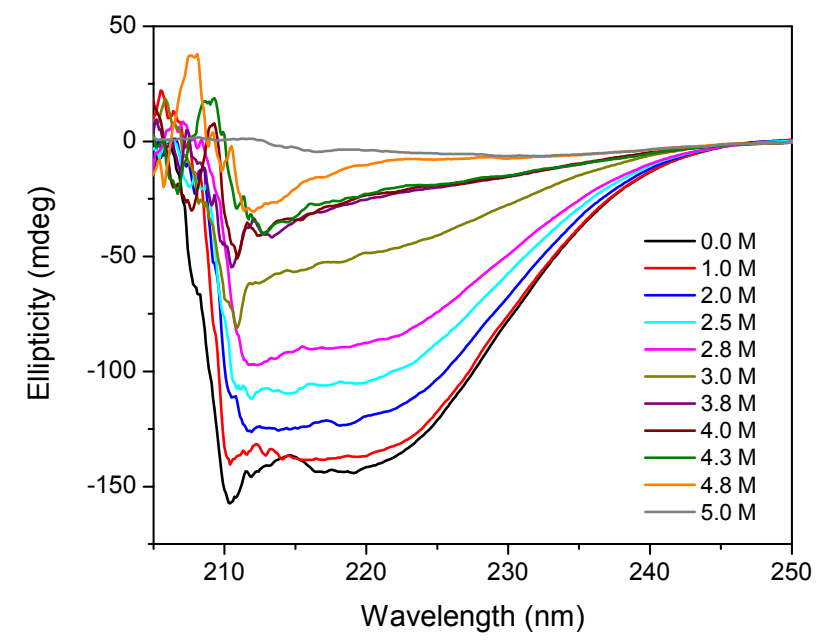

Figure 9.8 Far-UV Circular dichroism spectra of $\mathrm{Cygb}^{\text {red }}$ in the presence of increasing concentrations of $\mathrm{GuHCl}$. The concentration of the proteins was $40 \mu \mathrm{M}$. The buffer used was $10 \mathrm{mM}$ Tris buffer (pH 7.0) containing $10 \mathrm{mM}$ DTT. 


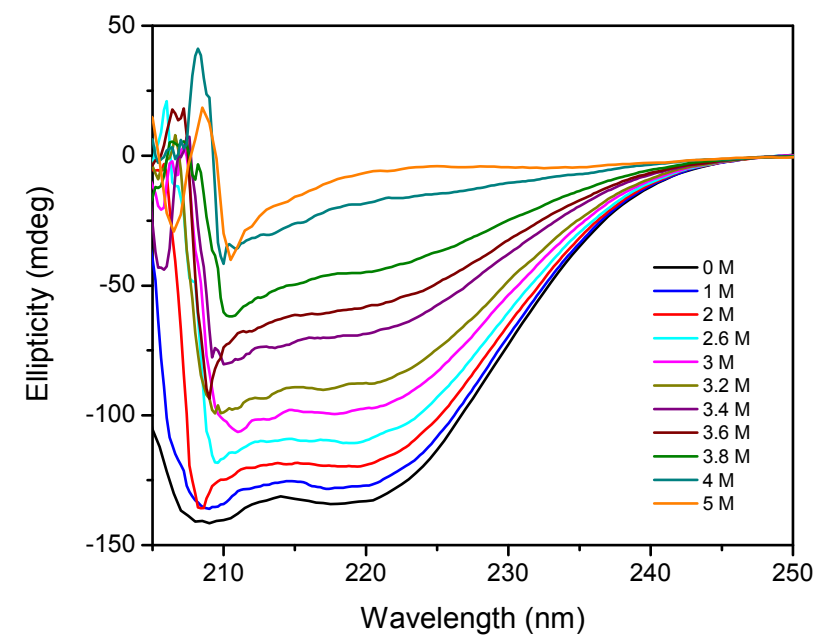

Figure 9.9 Far-UV Circular dichroism spectra of CNCygb in the presence of increasing concentrations of $\mathrm{GuHCl}$. The concentration of the proteins was $40 \mu \mathrm{M}$. The buffer used for Cygb was $10 \mathrm{mM}$ Tris buffer ( $\mathrm{pH} 7.0)$ containing $10 \mathrm{mM} \mathrm{KCN}$.

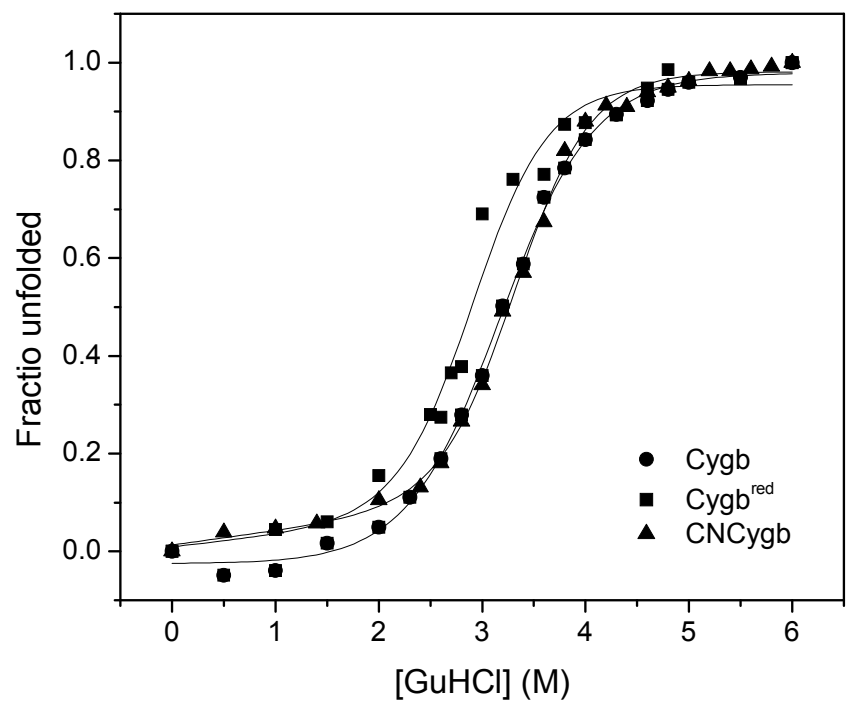

Figure 9.10 Unfolding curves of $\mathrm{Cygb}, \mathrm{Cygb}^{\text {red }}$ and CNCygb monitored using CD spectroscopy as a function of $\mathrm{GuHCl}$ concentration. The concentration of the protein was $40 \mu \mathrm{M}$ in $10 \mathrm{mM}$ Tris buffer, $\mathrm{pH}$ 7.0. The buffer used for $\mathrm{Cygb}^{\text {red }}$ samples contained 10 $\mathrm{mM}$ DTT and for CNCygb samples contained $10 \mathrm{mM} \mathrm{KCN}$. 
The GuHCl-induced denaturation curves determined for $\mathrm{Cygb}, \mathrm{Cygb}^{\text {red }}$ and CNCygb determined employing Far-UV CD spectroscopy indicate a single transition from the folded state to unfolded state. The free energy of unfolding and $m$ parameter determined using a two-state model are listed in table 9.2.

Table 9.2 Free energy of unfolding $\left(\Delta G^{o}{ }_{H_{2}} \mathrm{O}\right)$ and degree of hydrophobic exposure upon $\mathrm{GuHCl}$-induced unfolding (m) determined for $\mathrm{Cygb}, \mathrm{Cygb}^{\text {red }}$ and $\mathrm{CNCygb}$ employing $\mathrm{CD}$ spectroscopy.

\begin{tabular}{lcc}
\hline & $\Delta G^{o} \mathrm{H}_{2} \mathrm{O}\left(\mathrm{kcal} \mathrm{mol}^{-1}\right)$ & $\mathrm{m}\left(\mathrm{kcal} \mathrm{mol}^{-1} \mathrm{M}^{-1}\right)$ \\
\hline Cygb & $5.0 \pm 0.4$ & $1.7 \pm 0.3$ \\
$\mathrm{Cygb}^{\text {red }}$ & $4.6 \pm 0.9$ & $1.7 \pm 0.4$ \\
$\mathrm{CNCygb}$ & $5.3 \pm 0.4$ & $1.6 \pm 0.1$ \\
\hline
\end{tabular}

The change in free energy $\left(\Delta G_{H_{2} O}^{o}\right)$ determined using CD spectroscopy for Cygb is $5.0 \pm 0.4 \mathrm{kcal} \mathrm{mol}^{-1}$, is comparable to the value determined employing absorption spectroscopy of $4.8 \pm 0.4 \mathrm{kcal} \mathrm{mol}^{-1}$. The $\mathrm{CD}$ data indicates that reduction of the disulfide bond in Cygb and binding of cyanide does not seem to impact the stability of the proteins as evident by the comparable values determined for $\Delta G_{H_{2} O}^{o}$ for Cygb, $\mathrm{Cygb}^{\text {red }}$ and CNCygb. The difference observed in the $\Delta G^{o}{ }_{H_{2} O}$ values determined using $\mathrm{CD}$ spectroscopy and UV-visible absorption spectroscopy, suggest that an intermediate may be formed during Cygb unfolding exhibiting a low fraction and thus were not resolved in these measurements. Indeed, overlay of the denaturation curves determined using UVvisible absorption spectroscopy and $\mathrm{CD}$ spectroscopy show a large shift for Cygb and $\mathrm{Cygb}^{\text {red }}$, whereas a small difference was observed in case of CNCygb unfolding curves (Figure 9.11), suggesting the presence of an intermediate in the transition from folded to 
unfolded in $\mathrm{Cygb}, \mathrm{Cygb}^{\text {red }}$ and $\mathrm{CNCygb}$ that could not be resolved here, likely because there is a low fraction of the intermediate. Each technique reflects different conformational changes in the protein structure. For instance, the changes in absorption monitored in the Soret band upon denaturation reflect the release of the heme from Cygb, whereas changes in the $\mathrm{CD}$ signal at $222 \mathrm{~nm}$ measure modifications in the secondary structure of Cygb (Hargrove and Olson, 1996; Hargrove et al., 1996).

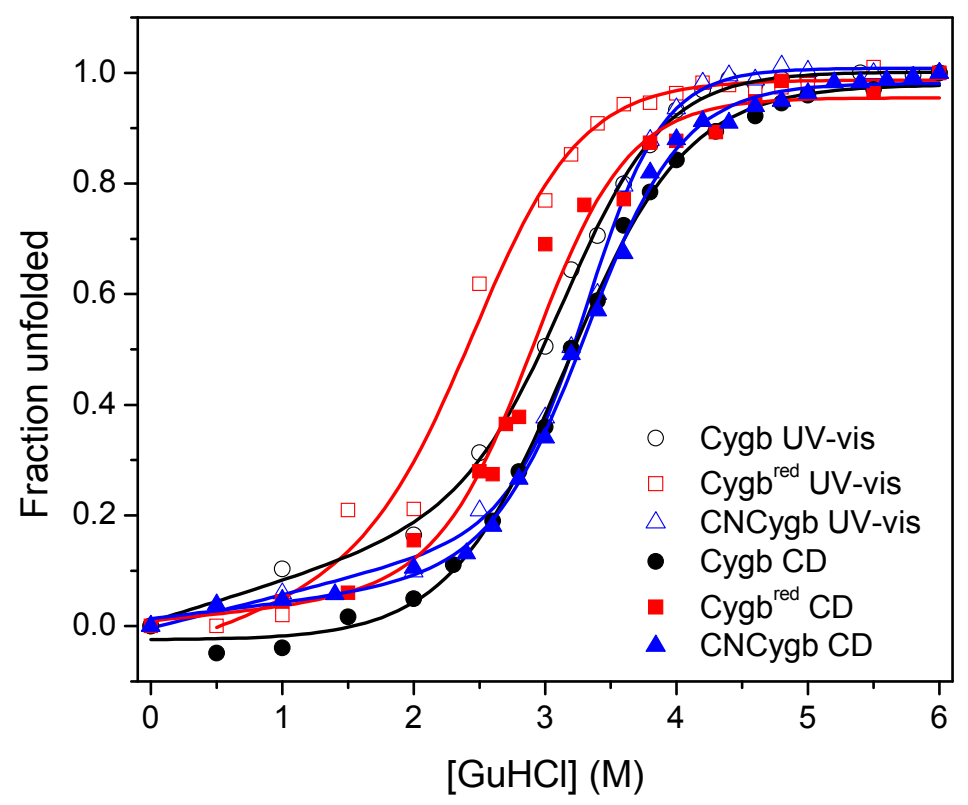

Figure 9.11 Overlay of unfolding curves of Cygb, Cygb ${ }^{\text {red }}$ and CNCygb determined using UV-visible spectroscopy (open symbols) and CD spectroscopy (closed symbols) as a function of $\mathrm{GuHCl}$ concentration.

\subsubsection{Neuroglobin}

\subsubsection{UV-visible absorption spectroscopy}

The GuHCl-induced structural changes of $\mathrm{Ngb}$ were determined recording the change in the intensity of the Soret band as a function of the denaturant concentration. To 
determine the impact of substitution of two Trp residues with Tyr on hNgb stability, the following hNgb double Trp mutants were also characterized: W13Y/W133Y, W13Y/W148Y and W133Y/W148Y. The overlay of absorption spectra of wild type $\mathrm{hNgb}$ and double Trp mutants, shown in Figure 9.7, indicates that there are no significant differences between the absorption spectrum of wild type $\mathrm{hNgb}$ and all Trp mutants studied. Therefore, the replacement of W13, W133 and W148 by Tyr residue does not modify the heme pocket in $\mathrm{hNgb}$.

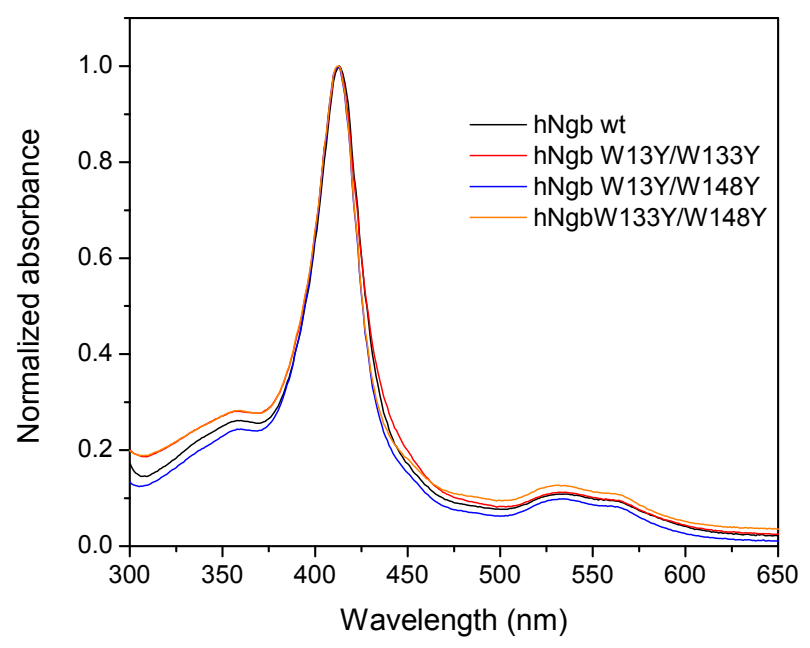

Figure 9.12 Overlay of UV-visible absorption spectra of wild type hNgb and Trp mutants $\mathrm{W} 13 \mathrm{Y} / \mathrm{W} 133 \mathrm{Y}, \mathrm{W} 13 \mathrm{Y} / \mathrm{W} 148 \mathrm{Y}$, and W133Y/W148Y. The concentration of the protein was $10 \mu \mathrm{M}$ in $50 \mathrm{mM}$ Tris buffer (pH 7.0).

As observed for Cygb, addition of GuHCl to hNgb and the double Trp mutants investigated results in a decreased intensity as well as a hypsochromic shift in the maximum wavelength of the Soret band (Figures 9.8 to 9.11), indicating exposure of the heme group to the solvent upon denaturation of the protein (Hargrove and Olson, 1996) 


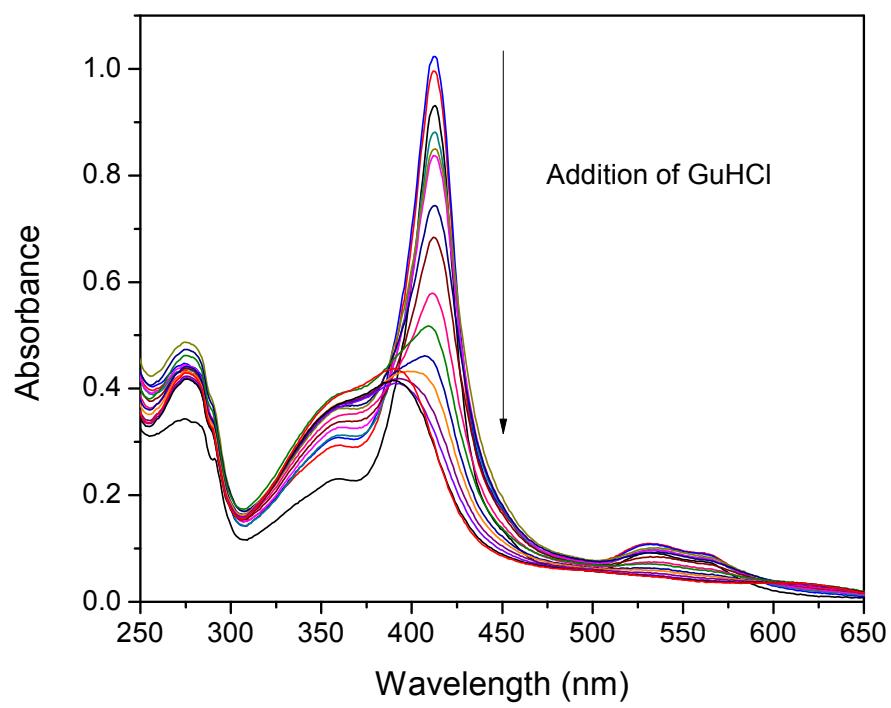

Figure 9.13 UV-visible absorption spectra of wild type $\mathrm{hNgb}$ in the presence of increasing concentrations of $\mathrm{GuHCl}$ from 0 to $6 \mathrm{M}$. The concentration of the proteins was approximately $10 \mu \mathrm{M}$ in $10 \mathrm{mM}$ Tris buffer ( $\mathrm{pH} 7.0$ ).

The fraction of unfolded protein was plotted as a function of the concentration of denaturant and the resulting unfolding curves are shown in Figure 9.12. The equilibrium unfolding data obtained using UV-visible absorption spectroscopy for wild type hNgb and all Trp mutants studied indicate a single transition from the folded to unfolded state upon addition of $\mathrm{GuHCl}$. Therefore, the unfolding data was analyzed according to a twostate model as previously described for Cygb and the free energy of unfolding and $\mathrm{m}$ values are listed in table 9.3.

The free energy of unfolding and $\mathrm{m}$ value determined for $\mathrm{hNgb}$ are comparable to the values determined for all Trp mutants studied: W13Y/W133Y, W13Y/W148Y and W133Y/W148Y. These results indicate that the replacement of W13, W133 and W148 with Tyr does not have a large impact on the stability of the protein. 
The GuHCl-induced unfolding of $\mathrm{hNgb}$ was recently reported by Guca et al. (2013). The denaturing curves reported by this group also show a single transition between the folded and unfolded state and the determined free energy of unfolding was $9.8 \pm 0.4 \mathrm{kcal} \mathrm{mol}^{-1}$ with an $\mathrm{m}$ value of $3.0 \pm 0.1 \mathrm{kcal} \mathrm{mol}^{-1} \mathrm{M}^{-1}$. These values are higher than the ones determined in this work, which could be attributed to the fact that the method used by Guca et al. (2013) to fit their denaturation curves does not take into consideration the dependence of unfolding on the denaturant concentration (especially at low and high concentration of denaturant), thereby they might obtain a higher value for $\Delta G^{o}{ }_{H_{2}} \mathrm{O}$.

Comparison of the free energy of unfolding between $\mathrm{hNgb}\left(\Delta G^{o}{ }_{\mathrm{H}_{2} \mathrm{O}}=7.3 \pm 1.1\right.$ $\left.\mathrm{kcal} \mathrm{mol}{ }^{-1}\right)$ and $\mathrm{Cygb}\left(\Delta G_{\mathrm{H}_{2} \mathrm{O}}^{o}=4.8 \pm 0.4 \mathrm{kcal} \mathrm{mol}^{-1}\right)$ provides evidence that $\mathrm{hNgb}$ is more stable towards GuHCl-induced denaturation than Cygb by $\sim 2.5 \mathrm{kcal} \mathrm{mol}^{-1}$. Although the unfolding study of Hamdane et al. (2005) used thermal denaturation of Ngb and Cygb, this group demonstrated that $\mathrm{hNgb}$ has a higher thermal stability $\left(\mathrm{T}_{\mathrm{m}}=100\right.$ $\left.{ }^{\circ} \mathrm{C}\right)$ than Cygb $\left(\mathrm{T}_{\mathrm{m}}=95^{\circ} \mathrm{C}\right)$. Hamdane et al. (2005) did not report the free energy of unfolding for Cygb and Ngb. 


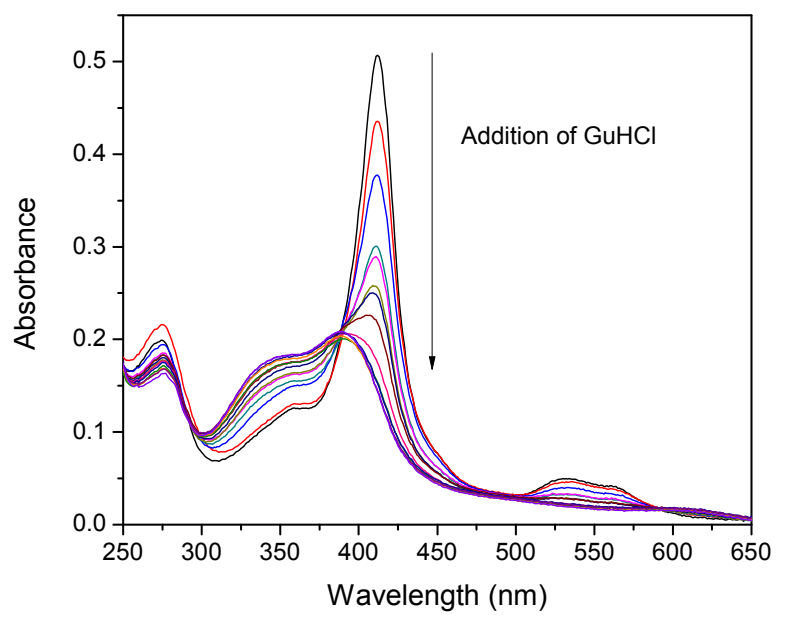

Figure 9.14 UV-visible absorption spectra of $\mathrm{hNgb} \mathrm{W} 13 \mathrm{Y} / \mathrm{W} 133 \mathrm{Y}$ in the presence of increasing concentrations of $\mathrm{GuHCl}$ from 0 to $6 \mathrm{M}$. The concentration of the proteins was approximately $10 \mu \mathrm{M}$ in $10 \mathrm{mM}$ Tris buffer ( $\mathrm{pH} 7.0)$.

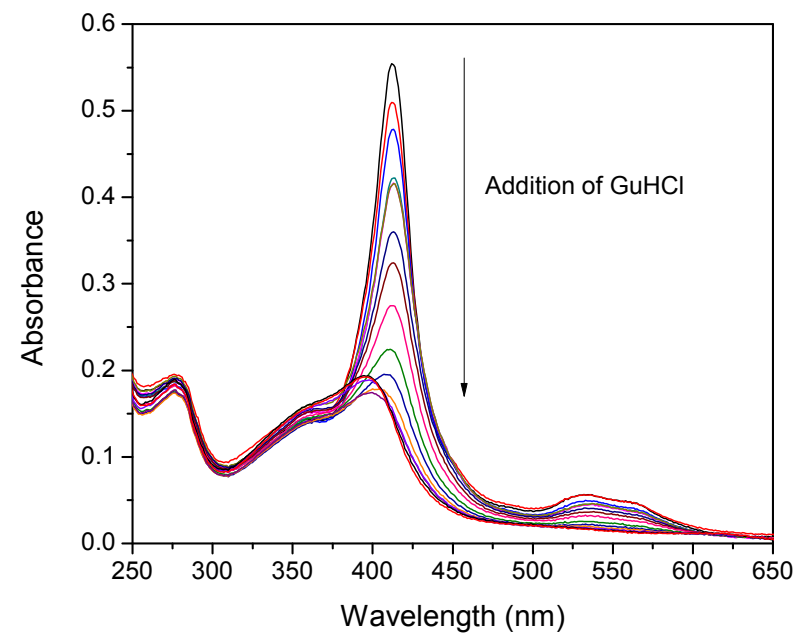

Figure 9.15 UV-visible absorption spectra of $\mathrm{hNgb} \mathrm{W} 13 \mathrm{Y} / \mathrm{W} 148 \mathrm{Y}$ in the presence of increasing concentrations of $\mathrm{GuHCl}$ from 0 to $6 \mathrm{M}$. The concentration of the proteins was approximately $10 \mu \mathrm{M}$ in $10 \mathrm{mM}$ Tris buffer $(\mathrm{pH}$ 7.0). 


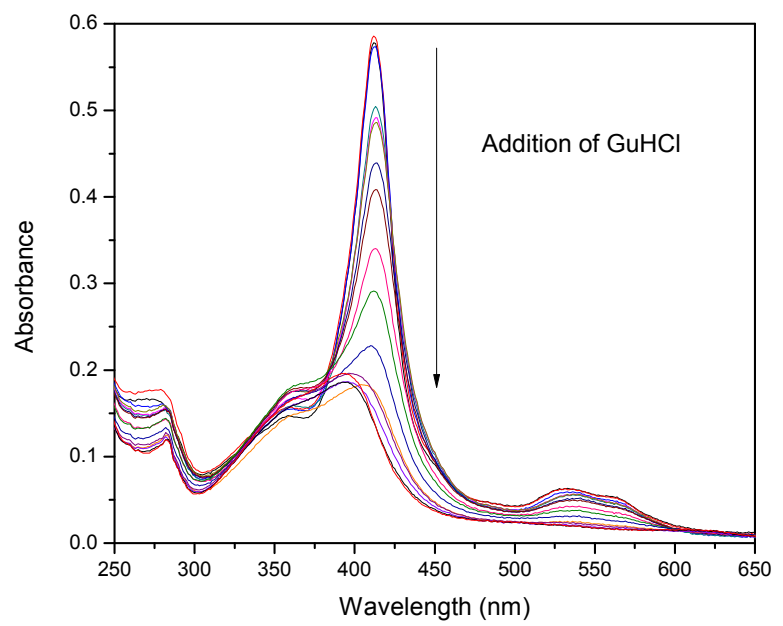

Figure 9.16 UV-visible absorption spectra of hNgb W133Y/W148Y in the presence of increasing concentrations of $\mathrm{GuHCl}$ from 0 to $6 \mathrm{M}$. The concentration of the proteins was approximately $10 \mu \mathrm{M}$ in $10 \mathrm{mM}$ Tris buffer $(\mathrm{pH} 7.0)$.

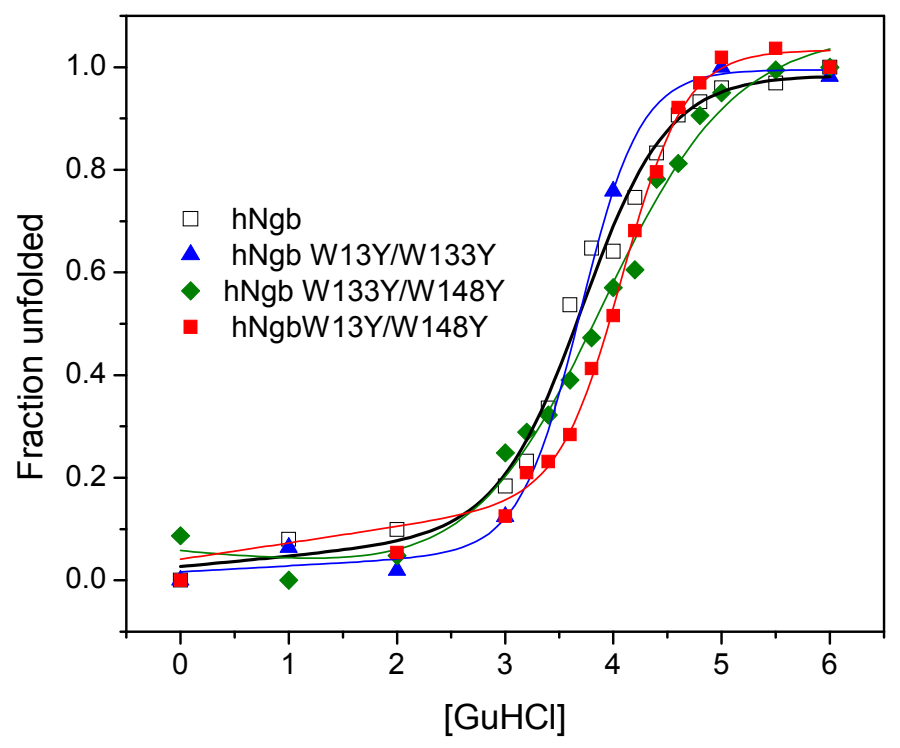

Figure 9.17 Unfolding curves of wild type hNgb and Trp mutants monitored using UVvisible absorption spectroscopy as a function of $\mathrm{GuHCl}$ concentration. The concentration of the protein was $\sim 10 \mu \mathrm{M}$ in $10 \mathrm{mM}$ Tris buffer, $\mathrm{pH} 7.0$. 
Table 9.3 Free energy of unfolding $\left(\Delta G^{o}{ }_{H_{2} O}\right)$ and degree of hydrophobic exposure upon GuHCl-induced unfolding (m) determined for wild type hNgb and Trp mutants employing UV-visible absorption spectroscopy.

\begin{tabular}{lcc}
\hline & $\Delta G^{o}{ }_{H_{2} O}\left(\mathrm{kcal} \mathrm{mol}^{-1}\right)$ & $\mathrm{m}\left(\mathrm{kcal} \mathrm{mol} \mathrm{M}^{-1}\right)$ \\
\hline $\mathrm{hNgb}$ & $7.3 \pm 1.1$ & $1.9 \pm 0.2$ \\
$\mathrm{hNgb} \mathrm{W13Y/W133Y}$ & $7.2 \pm 1.6$ & $1.8 \pm 0.4$ \\
$\mathrm{hNgb} \mathrm{W13Y/W148Y}$ & $7.7 \pm 3.1$ & $2.1 \pm 0.8$ \\
$\mathrm{hNgb} \mathrm{W133Y/W148Y}$ & $7.0 \pm 1.2$ & $1.7 \pm 0.3$ \\
\hline
\end{tabular}

\subsubsection{Far-UV CD spectroscopy}

The far-UV CD spectra of wild type hNgb and mutants W13Y/W133Y, $\mathrm{W} 13 \mathrm{Y} / \mathrm{W} 148 \mathrm{Y}$ and $\mathrm{W} 133 \mathrm{Y} / \mathrm{W} 148 \mathrm{Y}$ in $10 \mathrm{mM}$ Tris buffer $(\mathrm{pH} 7.0)$ are shown in Figure 9.17. The CD spectrum of wild type hNgb exhibits two negative peaks at $208 \mathrm{~nm}$ and 222 $\mathrm{nm}$ that are characteristic of $\alpha$-helical structure, as previously reported for $\mathrm{hNgb}$ (Hamdane et al., 2005; Zhao et al., 2006).

The $\alpha$-helical content was calculated from the mean ellipticity value at $222 \mathrm{~nm}$ for wild type hNgb and double Trp mutants as previously described in Chapter 4. The calculate $\alpha$-helical content of wild type $\mathrm{hNgb}$ was $\sim 76 \%$ that matches well previously reported value of $\sim 78 \%$ (Hamdane et al., 2005). The $\alpha$-helical content of the double Trp mutants is lower than the one determined for the wild type protein with values of $\sim 69 \%$ for $\mathrm{W} 13 \mathrm{Y} / \mathrm{W} 133 \mathrm{Y}, \sim 74 \%$ for $\mathrm{W} 13 \mathrm{Y} / \mathrm{W} 148 \mathrm{Y}$ and $~ 73 \%$ for W133Y/W148Y. Therefore, the largest effect on the secondary structure of $\mathrm{hNgb}$ was observed when W13 and W133 were replaced by Tyr. Since W13 and W133 are part of $\alpha$-helices A and H, respectively, whereas W148 does not form part of one the $\alpha$-helices in the structure of hNgb (Figure 
9.2) is not surprising that substation of both W13 and W133 would affect the protein's $\alpha$ helical content.

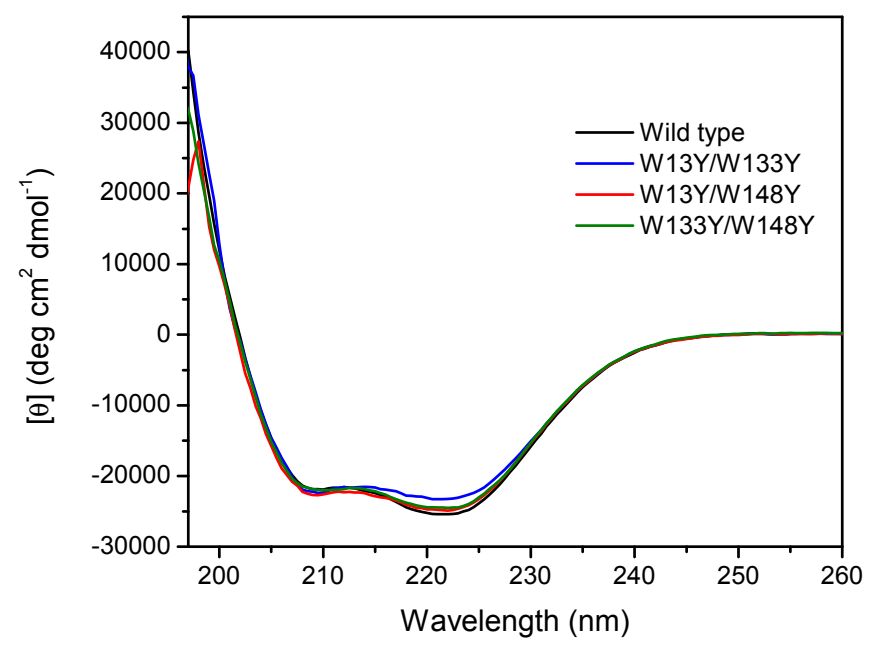

Figure 9.18 Far-UV CD spectra of wild type hNgb and mutants W13Y/W133Y, $\mathrm{W} 13 \mathrm{Y} / \mathrm{W} 148 \mathrm{Y}$ and $\mathrm{W} 133 \mathrm{Y} / \mathrm{W} 148 \mathrm{Y}$. The concentration of the protein was $40 \mu \mathrm{M}$ in 10 $\mathrm{mM}$ Tris buffer ( $\mathrm{pH}$ 7.0). Spectra were corrected for the baseline of the buffer.

\subsubsection{Fluorescence spectroscopy}

There are three Trp residues in the amino acid sequence of $\mathrm{hNgb}$ (W13, W133 and W148). Since Trp fluorescence properties are strongly dependent on the distance between Trp and the heme group, monitoring of Trp lifetimes allows characterization occurring close to the heme group.

Data analysis of fluorescence data for Ngb may be complex because of the presence of the three Trp residues. Therefore, single Trp hNgb constructs were prepared by collaboration with Dr. Pierre Sebban's group from University of Paris IX (Orsay, France). Characterization of the single Trp hNgb constructs allows monitoring of global structural changes in $\mathrm{hNgb}$ upon binding of exogenous ligands. 
The time-resolved data in the frequency domain were measured for bishistidine $\mathrm{Fe}^{3+} \mathrm{hNgb}$ and double Trp mutants W13Y/W133Y, W13Y/W148Y and W133Y/W148Y are displayed in Figure 9.18, whereas the data measured for the cyanide adducts of the proteins are shown in Figure 9.19. The data was analyzed employing a sum of discrete exponential decays and the quality of the fit was inspected by the chi square $\left(\chi^{2}\right)$ value and the randomness of the resulting residuals. The lifetimes and pre-exponential factors retrieved from the fit are listed in table 9.4.

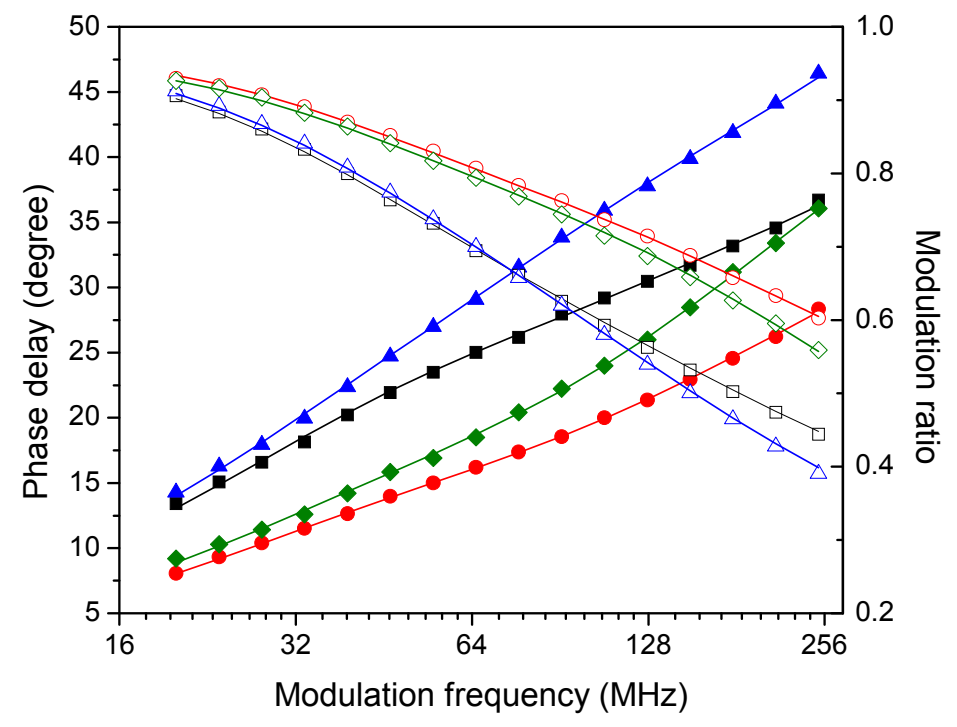

Figure 9.19 Time-resolved fluorescence in the frequency domain data determined for wild type hNgb (black squares) and double Trp mutants W13Y/W133Y (blue triangles), W13Y/W148Y (red circles) and W133Y/W148Y (green diamonds). Phase delay data are shown as open symbol and modulation ratio data are displayed as closed symbols. Solid lines represent the fit to the data using a sum of three exponential decay model retrieved using Vinci software. The concentration of the protein was $7 \mu \mathrm{M}$ in $50 \mathrm{mM}$ Tris (pH 7). 


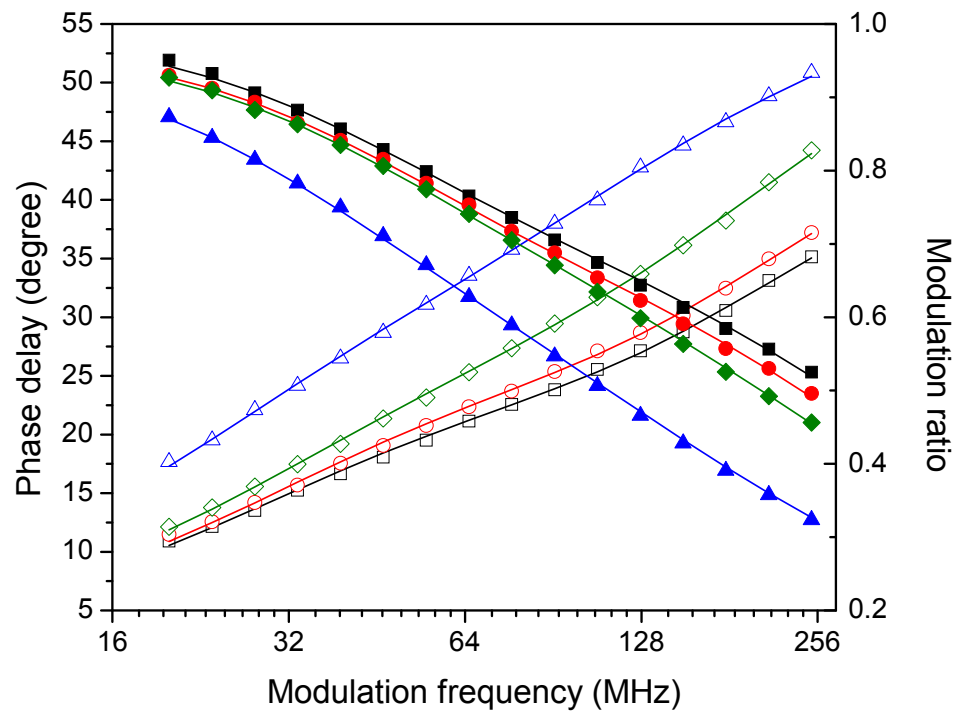

Figure 9.20 Time-resolved fluorescence in the frequency domain data determined for $\mathrm{CN}$ - adducts of wild type hNgb (black squares) and double Trp mutants W13Y/W133Y (blue triangles), W13Y/W148Y (red circles) and W133Y/W148Y (green diamonds). Phase delay data are shown as open symbol and modulation ratio data are displayed as closed symbols. Solid lines represent the fit to the data using a sum of three exponential decay model retrieved using Globals software. The concentration of the protein was $7 \mu \mathrm{M}$ in $50 \mathrm{mM}$ Tris $(\mathrm{pH} 7)$ containing $10 \mathrm{mM} \mathrm{KCN}$.

Table 9.4 Fluorescence lifetime of $\mathrm{hNgb}$ and Trp mutants as a function of $\mathrm{CN}$ - binding.

\begin{tabular}{lccccccc}
\hline \multicolumn{1}{c}{ Sample } & $\tau_{1}(\mathrm{~ns})$ & $\alpha_{1}(\%)$ & $\tau_{2}(\mathrm{~ns})$ & $\alpha_{2}(\%)$ & $\tau_{3}(\mathrm{~ns})$ & $\alpha_{3}(\%)$ & $\chi^{2}$ \\
\hline Wild type & $0.21 \pm 0.02$ & 82.0 & $1.1 \pm 0.1$ & 12.7 & $4.0 \pm 0.1$ & 5.3 & 1.02 \\
W13Y/W148Y & $0.16 \pm 0.02$ & 87.0 & $0.8 \pm 0.1$ & 11.1 & $3.9 \pm 0.2$ & 1.9 & 0.50 \\
W13Y/W133Y & $0.31 \pm 0.03$ & 71.5 & $1.4 \pm 0.1$ & 21.3 & $4.2 \pm 0.3$ & 7.2 & 0.98 \\
W133Y/W148Y & $0.18 \pm 0.04$ & 71.8 & $0.8 \pm 0.1$ & 25.4 & $3.8 \pm 0.2$ & 2.9 & 0.80 \\
Wild type + KCN & $0.06 \pm 0.03$ & 79.3 & $0.61 \pm 0.04$ & 18.1 & $3.6 \pm 0.1$ & 2.6 & 1.28 \\
W13Y/W148Y + KCN & $0.03 \pm 0.01$ & 92.4 & $0.60 \pm 0.03$ & 6.6 & $3.5 \pm 0.1$ & 1.0 & 1.17 \\
W13Y/W133Y + KCN & $0.24 \pm 0.04$ & 61.7 & $1.3 \pm 0.1$ & 28.7 & $4.8 \pm 0.2$ & 9.6 & 1.08 \\
W133Y/W148Y + KCN & $0.19 \pm 0.06$ & 58.6 & $0.80 \pm 0.07$ & 34.1 & $3.6 \pm 0.1$ & 7.2 & 1.05 \\
\hline
\end{tabular}


The time-resolved fluorescence data indicates multiphasic Trp lifetime in wild type $\mathrm{hNgb}$ as well as in individual mutants, which suggests conformational heterogeneity of the protein. The shorter lifetime $\tau_{1}$ is in the range of 0.16 to $0.31 \mathrm{~ns}$ for the bishistidyl ferric form of $\mathrm{hNgb}$ and Trp mutants. Cyanide binding to the wild type hNgb and Trp mutant W13Y/W148Y results in decrease of $\tau_{1}$, however there was no effect on $\tau_{2}$ and $\tau_{3}$. The longer lifetime $\tau_{3}$ was approximately $4 \mathrm{~ns}$ and is comparable to the value determined for horse heart $\mathrm{Mb}$ at $\mathrm{pH} 7.2$ of $4.89 \mathrm{~ns}$, which was attributed to the presence of a small fraction of the apo-protein (Gryczynski et al., 1995). The fact that $\tau_{1}$ and $\tau_{2}$ are in the order of sub-nanosecond to nanosecond range are a consequence of quenching of Trp fluorescence by the heme group. For instance, a mean lifetime as short as $\sim 0.1 \mathrm{~ns}$ has been reported for cases where there is a strong intramolecular quenching reaction, such as the case of energy transfer to a heme (Hirsch, 2000).

The heterogeneity observed for each single Trp construct studied here has been observed in other proteins. It is well known that a single Trp residue in proteins can display bi-exponential decay. Similarly, the fluorescence decay of the free Trp in water is bi-exponential, which has been attributed to the presence of two rotamers of the side chain of Trp molecule (Hirsch, 2000). However, the heterogeneity in Trp emission in proteins is associated with the heterogeneity of the protein structure and not to the two rotamers of the Trp residue (Hirsch, 2000).

\subsection{Discussion}

The GuHCl-induced unfolding curves determined for Cygb, $\mathrm{Cygb}^{\mathrm{re}} \mathrm{d}$ and CNCygb using UV-visible absorption and CD spectroscopy exhibit a single transition between the folded state and unfolded state, suggesting that $\mathrm{GuHCl}$-induced denaturation of Cygb can 
be described using a two-state model. Comparison of the free energy of unfolding for Cygb, Cygb ${ }^{\text {red }}$ and CNCygb indicate that reduction of the disulfide does not impact the stability of the protein, whereas cyanide binding slightly increases the Cygb. These results suggest that in Cygb the role of the disulfide bond is not to provide structural stability to the protein. In general, disruption of disulfide bonds in proteins decrease the resistance of the protein towards denaturation, likely as a result of destabilization of the unfolded state by reducing its entropy. Furthermore, hydrophobic residues are generally buried inside the folded proteins containing disulfide bonds and are less accessible to denaturants (Wedemeyer et al., 2000). It was previously reported that the disruption of the disulfide bond in Ngb upon reduction with DTT or replacement of Cys residues by Ser resulted in higher melting temperature than the melting temperature determined for wild type Ngb containing the internal disulfide bond (Hamdane et al., 2005). The authors proposed that the protein stability in Ngb may be directly related to hexa-coordination of the heme iron rather than the presence of the disulfide bond. Therefore, these results shown here do not support a role of the disulfide bond in Cygb stability.

On the other hand, ligand binding has been previously shown to enhance the stability of heme proteins towards denaturation (Hargrove and Olson, 1996). Although the denaturation curves shown in this study for Cygb, $\mathrm{Cygb}^{\text {red }}$ and CNCygb exhibit a single transition and were analyzed according to a two-state model, the lack of a clear isosbestic point in the titration curves monitored using UV-visible absorption spectroscopy suggest the presence of a low fraction of an intermediate between the folded and unfolded state. Further evidence of the presence of an intermediate during Cygb unfolding process is provided by the fact that the denaturation curves determined using 
UV-visible absorption spectroscopy are not superimposable with those determined employing CD spectroscopy. Since absorption spectroscopy and CD spectroscopy reflect different conformational changes in the protein structure, each technique may detect to a larger extent the presence of an intermediate during the unfolding process than the other. The changes in absorption monitored in the Soret band as a result of denaturation reflect the release of the heme from the protein, whereas changes in the CD signal at $222 \mathrm{~nm}$ measure modifications in the secondary structure of the protein (Hargrove and Olson, 1996; Hargrove et al., 1996).

The CD changes determined by Hargrove \& Olson (1996) for denaturation of $\mathrm{Mb}$ indicate the presence of an intermediate, as previously observed for denaturation of the apo form of $\mathrm{Mb}(\mathrm{apoMb})$, suggesting that unfolding of $\mathrm{Mb}$ occurs through a threestep process. Hargrove \& Olson (1996) proposed a mechanism for the unfolding of $\mathrm{Mb}$ that involves heme loss as the first transition, followed by formation of a partially folded intermediate state of apoMb and a subsequent transition to the unfolded state at high concentration of $\mathrm{GuHCl}$.

The free energy of unfolding determined for Cygb is comparable to the value previously reported for $\mathrm{Mb}$ by Roncone et al. (2005), but it is lower than the one determined for $\mathrm{Ngb}$ by approximately $2.3 \mathrm{kcal} \mathrm{mol}^{-1}$ indicating that $\mathrm{Ngb}$ structure is more stable towards denaturation than $\mathrm{Cygb}$ and $\mathrm{Mb}$. These results are consistent with the results obtained using thermal denaturation of Cygb and $\mathrm{Ngb}$, in which a $\mathrm{T}_{\mathrm{m}}$ of $100{ }^{\circ} \mathrm{C}$ was reported to $\mathrm{Ngb}$ compared to a $\mathrm{T}_{\mathrm{m}}$ of $95{ }^{\circ} \mathrm{C}$ for Cygb (Hamdane et al., 2005). Previous spectroscopic studies on the unfolding of the apoform of $\mathrm{Mb}$ have proposed that $\mathrm{Mb}$ unfolds through a three-state mechanism in which a molten globule intermediate is 
formed. The molten globule intermediate has been characterized by CD and NMR spectroscopy in combination with site-directed mutagenesis studies, indicating that helices $\mathrm{A}, \mathrm{G}$ and $\mathrm{H}$ remain folded forming the molten globule hydrophobic core, while the other helices of the protein exhibit high flexibility and are mostly disordered (Eliezer and Wright, 1996; Eliezer et al., 1998; Hughson and Baldwin, 1989; Hughson et al., 1991; Hughson et al., 1990). In order to understand the factors leading to a higher stability in Ngb than Cygb and $\mathrm{Mb}$ towards GuHCl-induced unfolding, the position of hydrophobic residues from helices $\mathrm{A}, \mathrm{G}$ and $\mathrm{H}$ in the three dimensional structure of $\mathrm{Ngb}$, Cygb and $\mathrm{Mb}$ were visualized using Pymol software (Figure 9.21). Inspection of Ngb structure suggests that that the heme group in this protein is stabilized by hydrophobic interactions Phe 106 (G-helix) and Trp 148 (H-helix) on the proximal site because of their proximity to the heme group, whereas in Cygb and $\mathrm{Mb}$ structures a single residue hydrophobic residue is found in the vicinity of the proximal site (Val 161 in Cygb and Phe 137 in Mb). However, replacement of Trp 148 by Tyr in hNgb does not affect the stability of the protein. In addition, the packing of helices $\mathrm{A}, \mathrm{G}$ and $\mathrm{H}$ in $\mathrm{Ngb}$ seems to be more compact than in Cygb.

The denaturation curves determined for $\mathrm{hNgb}$ and double Trp mutants indicate that GuHCl-induced unfolding of Ngb and the Trp mutants can be described using a twostate model. The UV-visible spectra of $\mathrm{Ngb}$ at various concentrations of $\mathrm{GuHCl}$ show one isosbestic point. The change in free energy upon unfolding with $\mathrm{GuHCl}$ determined for hNgb and Trp mutants using UV-visible absorption spectroscopy are similar, indicating that replacement of the Trp W13, W133 and W148 residues by Tyr do not destabilize the protein structure. Although the denaturation curves recorded in this study show a single 
transition from folded state to unfolded state in $\mathrm{Ngb}$, these results do not guarantee that denaturation follows a two-state mechanism. It is important to complement the stability studies with kinetics studies to determine the steps involved in the unfolding mechanism.

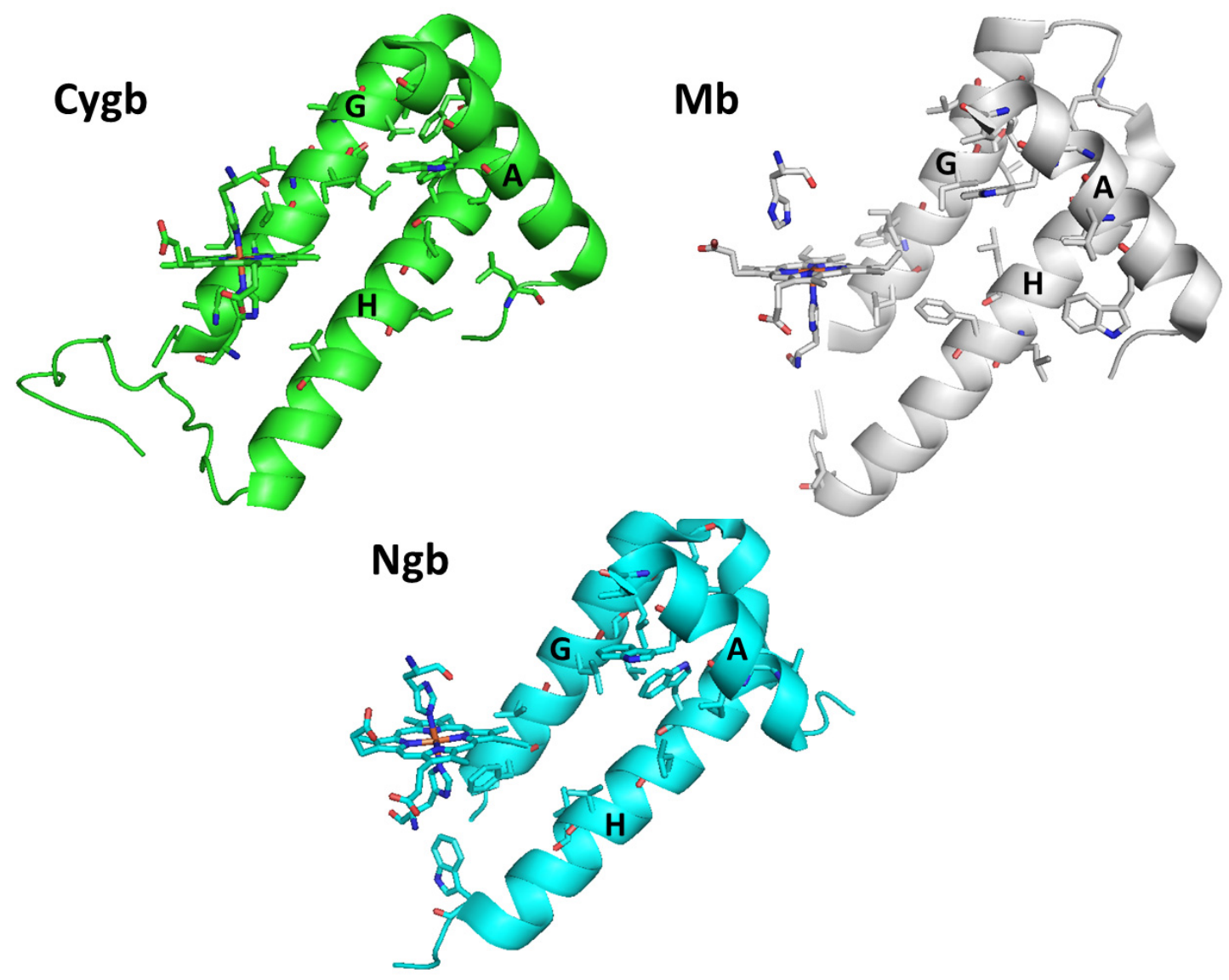

Figure 9.21 Ribbon representation of the dimensional structure of human Ngb (PDB entry 1OJ6), Cygb (PDB entry 2DC3) and $\mathrm{Mb}$ (PDB entry 1WLA) depicting hydrophobic residues in helices $\mathrm{A}, \mathrm{G}$ and $\mathrm{H}$ as sticks.

The time-resolved data in the frequency domain measured for bishistidine $\mathrm{Fe}^{3+} \mathrm{hNgb}$ and double Trp mutants W13Y/W133Y, W13Y/W148Y and W133Y/W148Y indicate multiphasic Trp lifetime in the wild type protein as well as in double Trp 
mutants. The fact that three lifetimes were retrieved even for the single Trp constructs of $\mathrm{hNgb}$ suggests that Trp samples two conformations, one that is in a more polar environment and exhibits a shorter lifetime and one that is located in more hydrophobic environment and results in a longer lifetime. The fact that cyanide binding to single Trp construct W13Y/W148Y results in a decrease in the shorter Trp lifetime suggests that Trp 133 is more efficiently quenched by the heme group upon formation of the cyanide adduct, likely as a result of a conformational change upon ligand binding.

\subsection{Summary}

$\mathrm{GuHCl}$-induced denaturation of $\mathrm{hNgb}$ and Cygb can be described with a two-state model and $\mathrm{hNgb}$ is more stable towards $\mathrm{GuHCl}$-induced denaturation than Cygb by approximately $2.3 \mathrm{kcal} \mathrm{mol}^{-1}$. In addition, the presence of the disulfide bond do not affect stability of Cygb, and whereas a small stabilization was observed upon cyanide binding.

The stability of $\mathrm{hNgb}$ is not largely affected by the replacement of Trp 13, Trp 133 and Trp 148 by Tyr and the single Trp constructs. The time-resolved fluorescence data indicates multiphasic Trp lifetime in the wild type $\mathrm{hNgb}$ protein as well as in double Trp mutants, suggesting conformational heterogeneity of $\mathrm{hNgb}$. 


\section{CONCLUSIONS}

Ligand escape from the protein matrix in human Ngb is significantly faster than in $\mathrm{Mb}$, suggesting that in human $\mathrm{Ngb}$ the so-called "histidine gate" mechanism does not regulate the ligand escape from the distal pocket to the surrounding solvent. Investigation of the role of conserved amino acid residues His 64 and Val 68 on ligand migration in $\mathrm{Ngb}$ indicates that Val 68 residue appears to have an analogous role in human Ngb as previously observed in other penta-coordinate hemoglobins by regulating the internal movement of the photo-released ligand between the heme distal pocket and the surrounding hydrophobic cavities. On the other hand, the distal His 64 has a moderate effect on the kinetics of ligand rebinding to human $\mathrm{Ngb}$ and no effect on the $\mathrm{CO}$ geminate quantum yield, suggesting that the distal His does not modulate ligand association to penta-coordinate Ngb. Replacement of Cys 120 by Ser residue may modify the ligand migration pathway in $\mathrm{hNgb}$ through the interface between helix $\mathrm{G}$ and the $\mathrm{AB}$ loop. In addition, disruption of the electrostatic network formed by the heme propionate groups, Lys 67, His 64 and Tyr 44 modifies the thermodynamic profiles for $\mathrm{CO}$ association to the protein.

The internal disulfide bond in Ngb modulates the magnitude of the overall structural changes upon ligand binding/release and the presence of the disulfide bond increases the rate constant for distal histidine dissociation in $\mathrm{hNgb}$ relative to $\mathrm{rNgb}$. On the other hand, the disulfide bond in Cygb modulates the rate for diatomic ligand escape from the protein matrix and the quantum yield for $\mathrm{CO}$ geminate rebinding. The volume and enthalpy changes associated with $\mathrm{CO}$ photo-release from $\mathrm{Cygb}^{\text {red }}$ are comparable to those measured for $\mathrm{Ngb}^{\text {red }}$, suggesting a similar mechanism of ligand migration that 
occurs through an intraprotein hydrophobic channel. The thermodynamic parameters determined for $\mathrm{CO}$ escape from $\mathrm{Cygb}^{\mathrm{ox}}$ resemble those observed for ligand photodissociation from $\mathrm{Mb}$, suggesting that the photo-dissociated ligand may escape through the so-called "histidine gate". Therefore, the presence of the disulfide bond in Cygb finetunes cytoglobin interactions with diatomic ligands through modulation of the structural dynamics and accessibility of the internal hydrophobic cavities which may define the Cygb intracellular function under physiological and pathological conditions.

Photodissociation of $\mathrm{O}_{2}$ in $\mathrm{Mb}$ leads to a volume change associated with $\mathrm{O}_{2}$ difussion from $\mathrm{Mb}$ active site that is comparable to that previously determined for $\mathrm{CO}$, however $\mathrm{O}_{2}$ escape from the distal pocket to the surrounding solvent is $\sim 2-3$ times faster than that observed for $\mathrm{CO}$, suggesting a distinct migration pathway for both diatomic ligands in $\mathrm{Mb}$. As previously observed for $\mathrm{CO}$ photo-release from Ngb, photodissociation of $\mathrm{O}_{2}$ occurs within $50 \mathrm{~ns}$, suggesting that migration of $\mathrm{O}_{2}$ and $\mathrm{CO}$ occurs through the wide hydrophobic tunnel linking the heme pocket with the surrounding solvent. The thermodynamic parameters associated with ligand photo-dissociation from human Ngb exhibit temperature-dependence in the temperature range between 7 and 35 ${ }^{\circ} \mathrm{C}$ with a turning point at $\sim 16^{\circ} \mathrm{C}$. This temperature dependence is attributed to electrostriction effects upon solvation of charges since increase of the solution ionic strength suppresses the temperature dependence. The large structural enthalpy change observed upon $\mathrm{O}_{2}$ photo-dissociation at high solution ionic strength conditions suggests that proton uptake/release occurs upon photo-dissociation of the ligand.

The thermodynamic profiles determined for $\mathrm{Hb}-\mathrm{CO}$ in the presence and absence of allosteric effectors indicates that BZF does not impact the thermodynamic parametes 
associated with ligand photo-release, whereas IHP association to $\mathrm{Hb}-\mathrm{CO}$ complex alters the volume and enthalpy changes associated with CO photo-dissociation from the heme iron indicating that the transition between the fully ligated $(\mathrm{CO})_{4} \mathrm{Hb}$ and partially ligated $(\mathrm{CO})_{3} \mathrm{Hb}$ complex leads to reorientation of IHP molecule within the central cavity and/ or charged amino acid residues interacting with IHP.

The hydrophobic probe 1,8-ANS binds to Cygb with moderate affinity, but it does not associate to $\mathrm{Ngb}$, suggesting different properties of the protein surface between $\mathrm{Ngb}$ and Cygb. Interestingly, truncation of the $\mathrm{N}$ - and C-termini in Cygb leads to decreased binding to 1,8-ANS and competitive binding was observed between sodium oleate and 1,8-ANS. Two binding sites were identified using ITC and time-resolved fluorescence spectroscopy and to obtain further insight into the binding sites docking simulation of 1,8-ANS to Cygb were performed. Docking simulation of 1,8-ANS with Cygb indicate that one of 1,8-ANS binding sites is located close to the extended N-termini in Cygb and a similar binding site was identified for oleate by docking oleate to the protein. These results suggest that the extended $\mathrm{N}$-terminus in Cygb facilitates a binding pocket for 1,8ANS and oleate.

Guanidine hydrochloride induced unfolding of $\mathrm{Ngb}$ and Cygb indicates that Ngb is more stable towards GuHCl-induced unfolding than Cygb, likely as a result of a larger degree of buried amino acid residues within the Ngb core than in Cygb. Interestingly, the presence of the disulfide bond in Cygb does not have an impact on the stability of the protein, whereas association of cyanide increases the stability of Cygb towards GuHClinduced unfolding. 


\section{LIST OF REFERENCES}

Abbruzzetti, S., Bruno, S., Faggiano, S., Ronda, L., Grandi, E., Mozzarelli, A., and Viappiani, C. (2008). Characterization of Ligand Migration Mechanisms Inside Hemoglobins from the Analysis of Geminate Rebinding Kinetics. Globins and Other Nitric Oxide-Reactive Proteins, Part B, 437: 329-345.

Abbruzzetti, S., Faggiano, S., Bruno, S., Spyrakis, F., Mozzarelli, A., Dewilde, S., Moens, L., and Viappiani, C. (2009). Ligand Migration through the Internal Hydrophobic Cavities in Human Neuroglobin. Proceedings of the National Academy of Sciences of the United States of America, 106 (45): 18984-18989.

Abbruzzetti, S., Viappiani, C., Murgida, D. H., Erra-Balsells, R., and Bilmes, G. M. (1999). Non-Toxic, Water-Soluble Photocalorimetric Reference Compounds for UV and Visible Excitation. Chemical Physics Letters, 304 (3-4): 167-172.

Al-Attar, S., and de Vries, S. (2013). Energy Transduction by Respiratory MetalloEnzymes: From Molecular Mechanism to Cell Physiology. Coordination Chemistry Reviews, 257 (1): 64-80.

Anderson, J. L., and Chapman, S. K. (2005). Ligand Probes for Heme Proteins. Dalton Transactions, 13-24.

Angeloni, L., and Feis, A. (2003). Protein Relaxation in the Photodissociation of Myoglobin-CO Complexes. Photochemical \& Photobiological Sciences, 2 (7): 730-740.

Anselmi, M., Brunori, M., Vallone, B., and Di Nola, A. (2007). Molecular Dynamics Simulation of Deoxy and Carboxy Murine Neuroglobin in Water. Biophysical Journal, 93 (2): 434-441.

Anselmi, M., Di Nola, A and Amadei, A. (2011). Kinetics of Carbon Monoxide Migration and Binding in Solvated Neuroglobin as Revealed by Molecular Dynamics Simulations and Quantum Mechanical Calculations. Journal of Physical Chemistry B, 115 (10): 2436-2446.

Antonini, E., and Brunori, M. (1971). Hemoglobin and Myoglobin in their Reactions with Ligands. Frontiers of Biology. Amsterdam, North-Holland Publishing Company.

Arcovito, A., Moschetti, T., D'Angelo, P., Mancini, G., Vallone, B., Brunori, M. and Della Longa, S. (2008). An X-ray Diffraction and X-ray Absorption Spectroscopy Joint Study of Neuroglobin. Archives of Biochemistry and Biophysics, 475 (1): 7-13. 
Ascenzi, P., Bertollini, A., Santucci, R., Amiconi, G., Coletta, M., Desideri, A., Giardina, B., Polizio, F., and Scatena, R. (1993). Cooperative Effect of Inositol Hexakisphosphate, Bezafibrate, and Clofibric Acid on the Spectroscopic Properties of the Nitric Oxide Derivative of Ferrous Human Hemoglobin. Journal of Inorganic Biochemistry, 50 (4): 263-272.

Ascenzi, P., Bocedi, A., de Sanctis, D., Pesce, A., Bolognesi, M., Marden, M. C., Dewilde, S., Moens, L., Hankeln, T., and Burmester, T. (2004). Neuroglobin and Cytoglobin - Two New Entries in the Hemoglobin Superfamily. Biochemistry and Molecular Biology Education, 32 (5): 305-313.

Astudillo, L., Bernad, S., Derrien, V., Pierre, S., and Miksovska, J. (2013). Reduction of the Internal Disulfide Bond between Cys 38 And 83 Switches the Ligand Migration Pathway in Cytoglobin. Journal of Inorganic Biochemistry, 129: 23-29.

Astudillo, L., Bernad, S., Derrien, V., Sebban, P., and Miksovska, J. (2010). Probing the Role of the Internal Disulfide Bond in Regulating Conformational Dynamics in Neuroglobin. Biophysical Journal, 99 (2): L16-L18.

Astudillo, L., Bernad, S., Derrien, V., Sebban, P., and Miksovska, J. (2012). Conformational Dynamics in Human Neuroglobin: Effect of His64, Val68, and Cys120 on Ligand Migration. Biochemistry, 51 (50): 9984-9994.

Banerjee, T., and Kishore, N. (2006). Binding of 8-Anilinonaphthalene Sulfonate to Dimeric and Tetrameric Concanavalin A: Energetics and its Implications on Saccharide Binding Studied by Isothermal Titration Calorimetry and Spectroscopy. Journal of Physical Chemistry B, 110 (13): 7022-7028.

Belogortseva, N., Rubio, M., Terrell, W., and Miksovska, J. (2007). The Contribution of Heme Propionate Groups to the Conformational Dynamics Associated with CO Photodissociation from Horse Heart Myoglobin. Journal of Inorganic Biochemistry, 101 (7): 977-986.

Bocahut, A., Bernad, S., Sebban, P., and Sacquin-Mora, S. (2009). Relating the Diffusion of Small Ligands in Human Neuroglobin to its Structural and Mechanical Properties. Journal of Physical Chemistry B, 113 (50): 16257-16267.

Bocahut, A., Derrien, V., Bernad, S., Pierre, S., Sacquin-Mora, S., Guittet, E., and Lescop, E. (2013). Heme Orientation Modulates Histidine Dissociation and Ligand Binding Kinetics in the Hexacoordinated Human Neuroglobin. Journal of Biological Inorganic Chemistry, 18 (1): 111-122. 
Boens, N., Qin, W. W., Basaric, N., Hofkens, J., Ameloot, M., Pouget, J., Lefevre, J. P., Valeur, B., Gratton, E., Vandeven, M., Silva, N. D., Engelborghs, Y., Willaert, K., Sillen, A., Rumbles, G., Phillips, D., Visser, A., van Hoek, A., Lakowicz, J. R., J. R., Malak, J. R., Gryczynski, I., Szabo, A. G., Krajcarski, D. T., Tamai, N., and Miura, A. (2007). Fluorescence Lifetime Standards for Time and Frequency Domain Fluorescence Spectroscopy. Analytical Chemistry, 79 (5): 2137-2149.

Bondarenko, V., Dewilde, S., Moens, L., and La Mar, G. N. (2006). Solution 1H NMR Characterization of the Axial Bonding of the Two His in Oxidized Human Cytoglobin. Journal of the American Chemical Society, 128 (39): 12988-12999.

Bonding, S. H., Henty, K., Dingley, A. J., and Brittain, T. (2008). The Binding of Cytochrome C to Neuroglobin: A Docking and Surface Plasmon Resonance Study. International Journal of Biological Macromolecules, 43 (3): 295-299.

Bossa, C., Anselmi, M., Roccatano, D., Amadei, A., Vallone, B., Brunori, M., and Di Nola, A. (2004). Extended Molecular Dynamics Simulation of the Carbon Monoxide Migration in Sperm Whale Myoglobin. Biophysical Journal, 86 (6): 3855-3862.

Bosselut, N., Housset, C., Marcelo, P., Rey, C., Burmester, T., Vinh, J., Vaubourdolle, M., Cadoret, A., and Baudin, B. (2010). Distinct Proteomic Features of Two Fibrogenic Liver Cell Populations: Hepatic Stellate Cells and Portal Myofibroblasts. Proteomics, 10 (5): 1017-1028.

Braslavsky, S. E. (1986). Photoacoustic and Photothermal Methods Applied to the Study of Radiationless Deactivation Processes in Biological-Systems and in Substances of Biological Interest. Photochemistry and Photobiology, 43 (6): 667-675.

Braslavsky, S. E., and Heibel, G. E. (1992). Time-Resolved Photothermal and Photoacoustic Methods Applied to Photoinduced Processes in Solution. Chemical Reviews, 92 (6): 1381-1410.

Brittain, T., Skommer, J., Henty, K., Birch, N., and Raychaudhuri, S. (2010a). A Role for Human Neuroglobin in Apoptosis. IUBMB Life, 62 (12): 878-885.

Brittain, T., Skommer, J., Raychaudhuri, S., and Birch, N. (2010b). An Antiapoptotic Neuroprotective Role for Neuroglobin. International Journal of Molecular Sciences, 11 (6): 2306-2321.

Brunori, M. (2000). Structural Dynamics of Myoglobin. Biophysical Chemistry, 86 (2-3): 221-230. 
Brunori, M., and Gibson, Q. H. (2001). Cavities and Packing Defects in the Structural Dynamics of Myoglobin. EMBO Reports, 2 (8): 674-679.

Brunori, M., and Vallone, B. (2007). Neuroglobin, Seven Years After. Cellular and Molecular Life Sciences, 64 (10): 1259-1268.

Burmester, T., Ebner, B., Weich, B., and Hankeln, T. (2002). Cytoglobin: A Novel Globin Type Ubiquitously Expressed in Vertebrate Tissues. Molecular Biology and Evolution, 19 (4): 416-421.

Burmester, T., and Hankeln, T. (2009). What is the Function of Neuroglobin?. Journal of Experimental Biology, 212 (10): 1423-1428.

Burmester, T., Weich, B., Reinhardt, S., and Hankeln, T. (2000). A Vertebrate Globin Expressed in the Brain. Nature, 407 (6803): 520-523.

Bykova, N. V., Igamberdiev, A. U., Ens, W., and Hill, R. D. (2006). Identification of an Intermolecular Disulfide Bond in Barley Hemoglobin. Biochemical and Biophysical Research Communications, 347 (1): 301-309.

Cao, W. X., Ye, X., Sjodin, T., Christian, J. F., Demidov, A. A., Berezhna, S., Wang, W., Barrick, D., Sage, J. T., and Champion, P. M. (2004). Investigations of Photolysis and Rebinding Kinetics in Myoglobin using Proximal Ligand Replacements. Biochemistry, 43 (34): 11109-11117.

Cardamone, M., and Puri, N. K. (1992). Spectrofluorometric Assessment of the Surface Hydrophobicity of Proteins. Biochemical Journal, 282: 589-593.

Carver, T. E., Rohlfs, R. J., Olson, J. S., Gibson, Q. H., Blackmore, R. S., Springer, B. A., and Sligar, S. G. (1990). Analysis of the Kinetic Barriers for Ligand Binding to Sperm Whale Myoglobin using Site-Directed Mutagenesis and Laser Photolysis Techniques. Journal of Biological Chemistry, 265 (32): 20007-20020.

Chekmarev, S. F., Krivov, S. V., and Karplus, M. (2005). Folding Time Distributions as an Approach to Protein Folding Kinetics. Journal of Physical Chemistry B, 109 (11): 5312-5330.

Chen, E. F., Goldbeck, R. A., and Kliger, D. S. (1997). Nanosecond Time-Resolved Spectroscopy of Biomolecular Processes. Annual Review of Biophysics and Biomolecular Structure, 26: 327-355. 
Chen, Y. H., Yang, J., and Martinez, H. (1972). Determination of the Secondary Structures of Proteins by Circular Dichroism and Optical Rotatory Dispersion. Biochemistry, 11 (22): 4120-4131.

Chen, Y. P., Woodin, S. A., Lincoln, D. E., and Lovell, C. R. (1996). An Unusual Dehalogenating Peroxidase from the Marine Terebellid Polychaete Amphitrite Ornata. Journal of Biological Chemistry, 271 (9): 4609-4612.

Chodera, J. D., and Mobley, D. L. (2013). Entropy-Enthalpy Compensation: Role and Ramifications in Biomolecular Ligand Recognition and Design. Annual Review of Biophysics, 42: 121-142.

Ciaccio, C., De Sanctis, G., Marini, S., Sinibaldi, F., Santucci, R., Arcovito, A., Bellelli, A., Ghibaudi, E., Rosa, P. F., and Coletta, M. (2004). Proton Linkage for CO Binding and Redox Properties of Bovine Lactoperoxidase. Biophysical Journal, 86 (1): 448-454.

Coletta, M., Angeletti, M., Ascenzi, P., Bertollini, A., Della Longa, S., De Sanctis, G., Priori, A. M., Santucci, R., and Amiconi, G. (1999a). Coupling of the Oxygen-Linked Interaction Energy for Inositol Hexakisphosphate and Bezafibrate Binding to Human HbA0. Journal of Biological Chemistry, 274 (11): 6865-6874.

Coletta, M., Angeletti, M., Ascone, I., Boumis, G., Castellano, A. C., Dell Ariccia, M., Della Longa, S., De Sanctis, G., Priori, A. M., Santucci, R., Feis, A., and Amiconi, G. (1999b). Heterotropic Effectors Exert More Significant Strain on Monoligated than on Unligated Hemoglobin. Biophysical Journal, 76 (3): 1532-1536.

Collini, M., D'Alfonso, L., Molinari, H., Ragona, L., Catalano, M., and Baldini, G. (2003). Competitive Binding of Fatty Acids and the Fluorescent Probe 1-8Anilinonaphthalene Sulfonate to Bovine Beta-Lactoglobulin. Protein Science, 12 (8): 1596-1603.

Cornwell, T. J., and Evans, K. F. (1985). A Simple Maximum-Entropy Deconvolution Algorithm. Astronomy \& Astrophysics, 143 (1): 77-83.

Culbertson, D. S., and Olson, J. S. (2010). Role of Heme in the Unfolding and Assembly of Myoglobin. Biochemistry, 49 (29): 6052-6063.

Daniel, E., and Weber, G. (1966). Cooperative Effects in Binding by Bovine Serum Albumin. I. The Binding of 1-Aniline-8-Naphthalenesulfonate. Fluorimetric titrations. Biochemistry, 5 (6): 1893-1900. 
Dantsker, D., Roche, C., Samuni, U., Blouin, G., Olson, J. S., and Friedman, J. M. (2005). The Position 68(E11) Side Chain in Myoglobin Regulates Ligand Capture, Bond Formation with Heme Iron, and Internal Movement into the Xenon Cavities. Journal of Biological Chemistry, 280 (46): 38740-38755.

de Sanctis, D., Dewilde, S., Pesce, A., Moens, L., Ascenzi, P., Hankeln, T., Burmester, T., and Bolognesi, M. (2004a). Crystal Structure of Cytoglobin: The Fourth Globin Type Discovered in Man Displays Heme Hexa-Coordination. Journal of Molecular Biology, 336 (4): 917-927.

de Sanctis, D., Dewilde, S., Pesce, A., Moens, L., Ascenzi, P., Hankeln, T., Burmester, T., and Bolognesi, M. (2004b). Mapping Protein Matrix Cavities in Human Cytoglobin Through Xe Atom Binding. Biochemical and Biophysical Research Communications, 316 (4): 1217-1221.

de Sanctis, D., Pesce, A., Nardini, M., Bolognesi, M., Bocedi, A., and Ascenzi, P. (2004c). Structure-Function Relationships in the Growing Hexa-Coordinate Hemoglobin Sub-Family. IUBMB Life, 56 (11-12): 643-651.

Dewilde, S., Kiger, L., Burmester, T., Hankeln, T., Baudin-Creuza, V., Aerts, T., Marden, M. C., Caubergs, R., and Moens, L. (2001) Biochemical Characterization and Ligand Binding Properties of Neuroglobin, A Novel Member of the Globin Family. Journal of Biological Chemistry, 276 (42): 38949-38955.

Du, W. H., Syvitski, R., Dewilde, S., Moens, L., and La Mar, G. N. (2003). Solution 1H NMR Characterization of Equilibrium Heme Orientational Disorder with Functional Consequences in Mouse Neuroglobin. Journal of the American Chemical Society, 125 (27): 8080-8081.

Dunford, B. (1999). Heme Peroxidases. USA, Wiley-VCH.

Eaton, W. A., Henry, E. R., Hofrichter, J., Bettati, S., Viappiani, C., and Mozzarelli, A. (2007). Evolution of Allosteric Models for Hemoglobin. IUBMB Life, 59 (8-9): 586-599.

Efremov, R. G., Chugunov, A. O., Pyrkov, T. V., Priestle, J. P., Arseniev, A. S., and Jacoby, E. (2007). Molecular Lipophilicity in Protein Modeling and Drug Design. Current Medicinal Chemistry, 14 (4): 393-415.

Eliezer, D., and Wright, P. E. (1996). Is Apomyoglobin a Molten Globule? Structural Characterization by NMR. Journal of Molecular Biology, 263 (4): 531-538. 
Eliezer, D., Yao, J., Dyson, H. J., and Wright, P. E. (1998). Structural and Dynamic Characterization of Partially Folded States of Apomyoglobin and Implications for Protein Folding. Nature Structural Biology, 5 (2): 148-155.

Emara, M., Salloum, N., and Allalunis-Turner, J. (2009). Expression and Hypoxic UpRegulation of Neuroglobin in Human Glioblastoma Cells. Molecular Oncology, 3 (1): 45-53.

Emara, M., Turner, A. R., and Allalunis-Turner, J. (2010). Hypoxic Regulation of Cytoglobin and Neuroglobin Expression in Human Normal and Tumor Tissues. Cancer Cell International, 10: 33-48.

Esquerra, R. M., Lopez-Pena, I., Tipgunlakant, P., Birukou, I., Nguyen, R. L., Soman, J., Olson, J. S., Kliger, D. S., and Goldbeck, R. A. (2010). Kinetic Spectroscopy of Heme Hydration and Ligand Binding in Myoglobin and Isolated Hemoglobin Chains: An Optical Window into Heme Pocket Water Dynamics. Physical Chemistry Chemical Physics, 12 (35): 10270-10277.

Everse, J. (2004). Heme Proteins. Encyclopedia of Biological Chemistry. New York, USA. Elsevier.

Ezhevskaya, M., Trandafir, F., Moens, L., Dewilde, S., and Van Doorslaer, S. (2011). EPR Investigation of the Role of B10 Phenylalanine in Neuroglobin - Evidence that B10Phe Mediates Structural Changes in the Heme Region upon Disulfide-Bridge Formation. Journal of Inorganic Biochemistry, 105 (9): 1131-1137.

Fago, A., Herold, S., Weber, R. E., Dewilde, S., and Moens, L. (2004a). Reactions between Neuroglobin and Nitric Oxide: A Protective Role Against Brain Hypoxia. Comparative Biochemistry and Physiology B-Biochemistry \& Molecular Biology, 139 (1): $140-140$.

Fago, A., Hundahl, C., Dewilde, S., Gilany, K., Moens, L., and Weber, R. E. (2004b). Allosteric Regulation and Temperature Dependence of Oxygen Binding in Human Neuroglobin and Cytoglobin - Molecular Mechanisms and Physiological Significance. Journal of Biological Chemistry, 279 (43): 44417-44426.

Fago, A., Hundahl, C., Malte, H., and Weber, R. E. (2004c). Functional Properties of Neuroglobin and Cytoglobin. Insights into the Ancestral Physiological Roles of Globins. IUBMB Life, 56 (11-12): 689-696. 
Fago, A., Mathews, A. J., and Brittain, T. (2008). A Role for Neuroglobin: Resetting the Trigger Level for Apoptosis in Neuronal and Retinal Cells. IUBMB Life, 60 (6): 398401.

Fago, A., Mathews, A. J., Moens, L., Dewilde, S., and Brittain, T. (2006). The Reaction of Neuroglobin with Potential Redox Protein Partners Cytochrome $\mathrm{B}_{5}$ and Cytochrome C. FEBS Letters, 580 (20): 4884-4888.

Fordel, E., Geuens, E., Dewilde, S., Rottiers, P., Carmeliet, P., Grooten, J., and Moens, L. (2004). Cytoglobin Expression is Upregulated in All Tissues Upon Hypoxia: An in Vitro and in vivo Study by Quantitative Real-Time PCR. Biochemical and Biophysical Research Communications, 319 (2): 342-348.

Fordel, E., Thijs, L., Martinet, W., Lenjou, M., Laufs, T., Van Bockstaele, D., Moens, L., and Dewilde, S. (2006). Neuroglobin and Cytoglobin Overexpression Protects Human SH-SY5Y Neuroblastoma Cells Against Oxidative Stress-Induced Cell Death. Neuroscience Letters, 410 (2): 146-151.

Fordel, E., Thijs, L., Moens, L., and Dewilde, S. (2007). Neuroglobin and Cytoglobin Expression in Mice - Evidence for a Correlation with Reactive Oxygen Species Scavenging. FEBS Journal, 274 (5): 1312-1317.

Franzen, S., Belyea, J., Gilvey, L. B., Davis, M. F., Chaudhary, C. E., Sit, T. L., and Lommel, S. A. (2006). Proximal Cavity, Distal Histidine, and Substrate HydrogenBonding Mutations Modulate the Activity of Amphitrite Ornata Dehaloperoxidase. Biochemistry, 45 (30): 9085-9094.

Gabba, M., Abbruzzetti, S., Spyrakis, F., Forti, F., Bruno, S., Mozzarelli, A., Luque, F. J., Viappiani, C., Cozzini, P., Nardini, M., Germani, F., Bolognesi, M., Moens, L., and Dewilde, S. (2013). CO Rebinding Kinetics and Molecular Dynamics Simulations Highlight Dynamic Regulation of Internal Cavities in Human Cytoglobin. PLOS One, 8 (1): e49770.

Gallagher, S. R. (2001). One-Dimensional SDS Gel Electrophoresis of Proteins. Current Protocols in Protein Science. John Wiley \& Sons, Inc.

Gasymov, O. K., and Glasgow, B. J. (2007). ANS Fluorescence: Potential to Augment the Identification of the External Binding Sites of Proteins. Biochimica et Biophysica Acta-Proteins and Proteomics, 1774 (3): 403-411. 
Gensch, T., and Viappiani, C. (2003). Time-Resolved Photothermal Methods: Accessing Time-Resolved Thermodynamics of Photoinduced Processes in Chemistry And Biology. Photochemical \& Photobiological Sciences, 2 (7): 699-721.

Geuens, E., Brouns, I., Flamez, D., Dewilde, S., Timmermans, J. P., and Moens, L. (2003). A Globin in the Nucleus!. Journal of Biological Chemistry, 278 (33): 3041730420 .

Gibson, Q. H., Olson, J. S., McKinnie, R. E., and Rohlfs, R. J. (1986). A Kinetic Description of Ligand Binding to Sperm Whale Myoglobin. Journal of Biological Chemistry, 261 (22): 10228-10239.

Giuffre, A., Moschetti, T., Vallone, B., and Brunori, M. (2008). Neuroglobin: Enzymatic Reduction and Oxygen Affinity. Biochemical and Biophysical Research Communications, 367 (4): 893-898.

Goldbeck, R. A., Bhaskaran, S., Ortega, C., Mendoza, J. L., Olson, J. S., Soman, J., Kliger, D. S., and Esquerra, R. M. (2006). Water and Ligand Entry in Myoglobin: Assessing the Speed and Extent of Heme Pocket Hydration after CO Photodissociation. Proceedings of the National Academy of Sciences of the United States of America, 103 (5): 1254-1259.

Goldbeck, R. A., Paquette, S. J., Björling, S. C., and Kliger, D. S. (1996). Allosteric Intermediates in Hemoglobin. 2. Kinetic Modeling of $\mathrm{HbCO}$ Photolysis. Biochemistry, 35 (26): 8628-8639.

Greenberg, D. A., Jin, K., and Khan, A. A. (2008). Neuroglobin: An Endogenous Neuroprotectant. Current Opinion in Pharmacology, 8 (1): 20-24.

Gregersen, N., Bross, P., Vang, S., and Christensen, J. (2006). Protein Misfolding and Human Disease. Annual Review of Genomics and Human Genetics, 17:103-124.

Gryczynski, Z., Lubkowski, J., and Bucci, E. (1995). Heme-Protein Interactions in Horse Heart Myoglobin at Neutral $\mathrm{pH}$ and Exposed to Acid Investigated by Time-Resolved Fluorescence in the Picosecond to Nanosecond Time Range. Journal of Biological Chemistry, 270 (33): 19232-19237.

Guca, E., Roumestand, C., Vallone, B., Royer, C. A., and Dellarole, M. (2013). LowCost Equilibrium Unfolding of Heme Proteins Using $2 \mu \mathrm{L}$ Samples. Analytical Biochemistry, 443 (1): 13-15. 
Gull, S. F., and Daniell, G. J. (1978). Image-Reconstruction from Incomplete and Noisy Data. Nature, 272 (5655): 686-690.

Halcrow, M. A. (2008). Trapping and Manipulating Excited Spin States of Transition Metal Compounds. Chemical Society Reviews, 37 (2): 278-289.

Halligan, K., Jourd'heuil, F., Vincent, C., and Jourd'heuil, D. (2007). Nitric Oxide Bioavailability in the Vascular Wall is Regulated by Cytoglobin. Free Radical Biology and Medicine, 43: S166-S166.

Hamdane, D., Kiger, L., Dewilde, S., Green, B. N., Pesce, A., Uzan, J., Burmester, T., Hankeln, T., Bolognesi, M., Moens, L., and Marden, M. C. (2003). The Redox State of the Cell Regulates the Ligand Binding Affinity of Human Neuroglobin and Cytoglobin. Journal of Biological Chemistry, 278 (51): 51713-51721.

Hamdane, D., Kiger, L., Dewilde, S., Uzan, J., Burmester, T., Hankeln, T., Moens, L., and Marden, M. C. (2005). Hyperthermal Stability of Neuroglobin and Cytoglobin. FEBS Journal, 272 (8): 2076-2084.

Han, S., Rousseau, D. L., Giacometti, G., and Brunori, M. (1990). Metastable Intermediates in Myoglobin at Low $\mathrm{pH}$. Proceedings of the National Academy of Sciences of the United States of America, 87 (1): 205-209.

Hankeln, T., Ebner, B., Fuchs, C., Gerlach, F., Haberkamp, M., Laufs, T. L., Roesner, A., Schmidt, M., Weich, B., Wystub, S., Saaler-Reinhardt, S., Reuss, S., Bolognesi, M., De Sanctis, D., Marden, M. C., Kiger, L., Moens, L., Dewilde, S., Nevo, E., Avivi, A., Weber, R. E., Fago, A., and Burmester, T. (2005). Neuroglobin and Cytoglobin in Search of their Role in the Vertebrate Globin Family. Journal of Inorganic Biochemistry, 99 (1): $110-119$.

Hara, T., Hirota, N., and Terazima, M. (1996). New Application of the Transient Grating Method to a Photochemical Reaction: The Enthalpy, Reaction Volume Change, and Partial Molar Volume Measurements. Journal of Physical Chemistry, 100 (24): 1019410200.

Hardison, R. (1998). Hemoglobins from Bacteria to Man: Evolution of Different Patterns of Gene Expression. Journal of Experimental Biology, 201 (8): 1099-1117.

Hargrove, M. S. (2000). A Flash Photolysis Method to Characterize Hexacoordinate Hemoglobin Kinetics. Biophysical Journal, 79 (5): 2733-2738. 
Hargrove, M. S., Brucker, E. A., Stec, B., Sarath, G., Arredondo-Peter, R., Klucas, R. V., Olson, J. S., and Phillips, G. N. (2000). Crystal Structure of a Nonsymbiotic Plant Hemoglobin. Structure with Folding \& Design, 8 (9): 1005-1014.

Hargrove, M. S., and Olson, J. S. (1996). The Stability of Holomyoglobin is Determined by Heme Affinity. Biochemistry, 35 (35): 11310-11318.

Hargrove, M. S., Wilkinson, A. J., and Olson, J. S. (1996). Structural Factors Governing Hemin Dissociation from Metmyoglobin. Biochemistry, 35 (35): 11300-11309.

Hasinoff, B. B. (1974). Kinetic Activation Volumes of the Binding of Oxygen and Carbon Monoxide to Hemoglobin and Myoglobin Studied on a High-Pressure Laser Flash Photolysis Apparatus. Biochemistry, 13 (15): 3111-3117.

Hawe, A., Rispens, T., Herron, J. N., and Jiskoot, W. (2011). Probing Bis-ANS Binding Sites of Different Affinity on Aggregated IgG by Steady-State Fluorescence, TimeResolved Fluorescence and Isothermal Titration Calorimetry. Journal of Pharmaceutical Sciences, 100 (4): 1294-1305.

Hawe, A., Sutter, M., and Jiskoot, W. (2008). Extrinsic Fluorescent Dyes as Tools for Protein Characterization. Pharmaceutical Research, 25 (7): 1487-1499.

He, X., Lv, R. Y., Wang, K., Huang, X. F., Wu, W. T., Yin, L. F., and Liu, Y. (2011). Cytoglobin Exhibits Anti-Fibrosis Activity on Liver in Vivo and in Vitro. Protein Journal, 30 (7): 437-446.

Henry, E. R., Sommer, J. H., Hofrichter, J., Eaton, W. A., and Gellert, M. (1983). Geminate Recombination of Carbon Monoxide to Myoglobin. Journal of Molecular Biology, 166 (3): 443-451.

Henzler-Wildman, K. A., Lei, M., Thai, V., Kerns, S. J., Karplus, M., and Kern, D. (2007). A Hierarchy of Timescales in Protein Dynamics is Linked to Enzyme Catalysis. Nature, 450 (7171): 913-6.

Hepler, J. R., and Gilman, A. G. (1992), G-Proteins. Trends in Biochemical Science, 17 (10): 383-387.

Herold, S., Fago, A., Weber, R. E., Dewilde, S., and Moens, L. (2004). Reactivity Studies of the Fe(III) and Fe(II)NO Forms of Human Neuroglobin Reveal a Potential Role Against Oxidative Stress. Journal of Biological Chemistry, 279 (22): 22841-22847. 
Hinton, C., Antony, H., Hashimi, S. M., Munn, A., and Wei, M. Q. (2013). Significance of Prion and Prion-Like Proteins in Cancer Development, Progression and Multi-Drug Resistance. Current Cancer Drug Targets, 13 (8): 895-904.

Hirsch, R. E. (2000). Heme-Protein Fluorescence. Topics in fluorescence spectroscopy, vol. 6. New York, Kluwer Academic/Plenum Publishers.

Hodges, N. J., Innocent, N., Dhanda, S., and Graham, M. (2008). Cellular Protection from Oxidative DNA Damage by Over-Expression of the Novel Globin Cytoglobin in vitro. Mutagenesis, 23 (4): 293-298.

Hoogewijs, D., Ebner, B., Germani, F., Hoffmann, F. G., Fabrizius, A., Moens, L., Burmester, T., Dewilde, S., Storz, J. F., Vinogradov, S. N., and Hankeln, T. (2012). Androglobin: A Chimeric Globin in Metazoans that is Preferentially Expressed in Mammalian Testes. Molecular Biology and Evolution, 29 (4): 1105-1114.

Horovitz, A., Serrano, L., Avron, B., Bycroft, M., and Fersht, A. R. (1990). Strength and Cooperativity of Contributions of Surface Salt Bridges to Protein Stability. Journal of Molecular Biology, 216 (4): 1031-1044.

Hou, S. B., Freitas, T., Larsen, R. W., Piatibratov, M., Sivozhelezov, V., Yamamoto, A., Meleshkevitch, E. A., Zimmer, M. G., Ordal, W., and Alam, M. (2001). Globin-coupled sensors: A Class of Heme-containing Sensors in Archaea and Bacteria. Proceedings of the National Academy of Sciences of the United States of America, 98 (16): 9353-9358.

Hou, S. B., Larsen, R. W., Boudko, D., Riley, C. W., Karatan, E., Zimmer, M., Ordal, G. W., and Alam, M. (2000). Myoglobin-like Aerotaxis Transducers in Archaea and Bacteria. Nature, 403 (6769): 540-544.

Hughson, F. M., and Baldwin, R. L. (1989). Use of Site-Directed Mutagenesis to Destabilize Native Apomyoglobin Relative to Folding Intermediates. Biochemistry, 28 (10): 4415-4422.

Hughson, F. M., Barrick, D., and Baldwin, R. L. (1991). Probing the Stability of a Partly Folded Apomyoglobin Intermediate by Site-Directed Mutagenesis. Biochemistry, 30 (17): 4113-4118.

Hughson, F. M., Wright, P. E., and Baldwin, R. L. (1990). Structural Characterization of a Partly Folded Apomyoglobin Intermediate. Science, 249 (4976): 1544-1548.

Hummer, G., Schotte, F., and Anfinrud, P. A. (2004). Unveiling Functional Protein Motions with Picosecond X-Ray Crystallography and Molecular Dynamics Simulations. 
Proceedings of the National Academy of Sciences of the United States of America, 101 (43): 15330-15334.

Igamberdiev, A. U., Bykova, N. V., and Hill, R. D. (2006). Nitric Oxide Scavenging by Barley Hemoglobin is Facilitated by a Monodehydroascorbate Reductase-Mediated Ascorbate Reduction of Methemoglobin. Planta, 223 (5): 1033-1040.

Ishikawa, H., Kim, S., Kwak, K., Wakasugi, K., and Fayer, M. D. (2007). Disulfide Bond Influence on Protein Structural Dynamics Probed with 2D-IR Vibrational Echo Spectroscopy. Proceedings of the National Academy of Sciences of the United States of America, 104 (49): 19309-19314.

Jameson, D. M., and Ross, V. (2010). Fluorescence Polarization/Anisotropy in Diagnostics and Imaging. Chemical Reviews, 110 (5): 2685-2708.

Jongeward, K. A., Magde, D., Taube, D. J., Marsters, J. C., Traylor, T. G., and Sharma, V. S. (1988). Picosecond and Nanosecond Geminate Recombination of Myoglobin with $\mathrm{CO}, \mathrm{O}_{2}, \mathrm{NO}$, and Isocyanides. Journal of the American Chemical Society, 110 (2): 380387.

Kakar, S., Hoffman, F. G., Storz, J. F., Fabian, M., and Hargrove, M. S. (2010). Structure and Reactivity of Hexacoordinate Hemoglobins. Biophysical Chemistry, 152 (1-3): 1-14.

Kane, C. D., and Bernlohr, D. A. (1996). A Simple Assay for Intracellular Lipid-Binding Proteins using Displacement of 1-Anilinonaphthalene 8-Sulfonic Acid. Analytical Biochemistry, 233 (2): 197-204.

Katz, A., Wu, D. Q., and Simon, M. I. (1992). Subunits Beta Gamma of Heterotrimeric G-Protein Activate Beta-2 Isoform of Phospholipase C. Nature, 360 (6405): 686-689.

Kawada, N., Kristensen, D. B., Asahina, K., Nakatani, K., Minamiyama, Y., Seki, S., and Yoshizato, K. (2001). Characterization of a Stellate Cell Activation-Associated Protein (STAP) with Peroxidase Activity Found in Rat Hepatic Stellate Cells. Journal of Biological Chemistry, 276 (27): 25318-25323.

Kawada, N., and Le, T. T. (2011). Cytoglobin as a Novel Tumor Suppressor in the Liver. Hepatology, 54: 757A-757A.

Kelly, S. M., and Price, N. C. (1997). The Application of Circular Dichroism to Studies of Protein Folding and Unfolding. Biochimica et Biophysica Acta-Protein Structure and Molecular Enzymology, 1338 (2): 161-185. 
Khan, A. A., Sun, Y. J., Jin, K. L., Mao, X. O., Chen, S. V., Ellerby, L. M., and Greenberg, D. A. (2007). A Neuroglobin-Overexpressing Transgenic Mouse. Gene, 398 (1-2): 172-176.

Kiger, L., Uzan, J., Dewilde, S., Burmester, T., Hankeln, T., Moens, L., Hamdane, D., Baudin-Creuza, V., and Marden, M. C. (2004). Neuroglobin Ligand Binding Kinetics. IUBMB Life, 56 (11-12): 709-719.

Kinsley, N., Sayed, Y., Mosebi, S., Armstrong, R. N., and Dirr, H. W. (2008). Characterization of the Binding of 8-Anilinonaphthalene Sulfonate to Rat Class Mu GST M1-1. Biophysical Chemistry, 137 (2-3): 100-104.

Kirk, W. R., Kurian, E., and Prendergast, F. G. (1996). Characterization of the Sources of Protein-Ligand Affinity: 1-Sulfonato-8-(1')Anilinonaphthalene Binding to Intestinal Fatty Acid Binding Protein. Biophysical Journal, 70 (1): 69-83.

Kitatsuji, C., Kurogochi, M., Nishimura, S. I., Ishimori, K., and Wakasugi, K. (2007). Molecular Basis of Guanine Nucleotide Dissociation Inhibitor Activity of Human Neuroglobin by Chemical Cross-Linking and Mass Spectrometry. Journal of Molecular Biology, 368 (1): 150-160.

Kriegl, J. M., Bhattacharyya, A. J., Nienhaus, K., Burmester, T., Hankeln, T., and Nienhaus, G. U. (2002). Ligand Binding and Protein Dynamics in Neuroglobin. Biophysical Journal, 82 (1): 449A-449A.

Laberge, M., Kövesi, I., Yonetani, T., and Fidy, J. (2005). R-State Hemoglobin Bound to Heterotropic Effectors: Models of the DPG, IHP and RSR13 Binding Sites. FEBS Letters, 579 (3): 627-632.

LaCount, M. W., Zhang, E. L., Chen, Y. P., Han, K. P., Whitton, M. M., Lincoln, D. E., Woodin, S. A., and Lebioda, L. (2000). The Crystal Structure and Amino Acid Sequence of Dehaloperoxidase from Amphitrite Ornata Indicate Common Ancestry with Globins. Journal of Biological Chemistry, 275 (25): 18712-18716.

Lakowicz, J. (2006). Principles of Fluorescence Spectroscopy. Baltimore, U.S.A. Springer.

Lakowicz, J. R., Laczko, G., Cherek, H., Gratton, E., and Limkeman, M. (1984). Analysis of Fluorescence Decay Kinetics from Variable-Frequency Phase-Shift and Modulation Data. Biophysical Journal, 46 (4): 463-477. 
Laman, D. M., and Falvey, D. E. (1996). Photothermal Beam Deflection Apparatus for the Time-Resolved Kinetic Study of Fast Photophysical and Photochemical Processes. Review of Scientific Instruments, 67 (9): 3260-3269.

Lamb, D. C., Ostermann, A., Prusakov, V. E., and Parak, F. G. (1998). From Metmyoglobin to Deoxymyoglobin: Relaxations of an Intermediate State. European Biophysics Journal with Biophysics Letters, 27 (2): 113-125.

Lambright, D. G., Balasubramanian, S., Decatur, S. M., and Boxer, S. G. (1994). Anatomy and Dynamics of a Ligand-Binding Pathway in Myoglobin - The Roles of Residue-45, Residue-60, Residue-64, and Residue-68. Biochemistry, 33 (18): 5518-5525.

Lardinois, O. M., Tomer, K. B., Mason, R. P., and Deterding, L. J. (2008). Identification of Protein Radicals Formed in the Human Neuroglobin - $\mathrm{H}_{2} \mathrm{O}_{2}$ Reaction using ImmunoSpin Trapping and Mass Spectrometry. Biochemistry, 47 (39): 10440-10448.

Larsen, R. W., and Miksovska, J. (2007). Time Resolved Thermodynamics of Ligand Binding to Heme Proteins. Coordination Chemistry Reviews, 251 (9-10): 1101-1127.

Lavalette, D., Tetreau, C., Brochon, J. C., and Livesey, A. (1991). Conformational Fluctuations and Protein Reactivity - Determination of the Rate-Constant Spectrum and Consequences in Elementary Biochemical Processes. European Journal of Biochemistry, 196 (3): 591-598.

Lechauve, C., Chauvierre, C., Dewilde, S., Moens, L., Green, B. N., Marden, M. C., Celier, C., and Kiger, L. (2010). Cytoglobin Conformations and Disulfide Bond Formation. FEBS Journal, 277 (12): 2696-2704.

Lecomte, J. T., Vuletich, D. A., and Lesk, A. M. (2005). Structural Divergence and Distant Relationships in Proteins: Evolution of the Globins. Current Opinion in Structural Biology, 15 (3): 290-301.

Leung, W. P., Cho, K. C., Chau, S. K., and Choy, C. L. (1987). Measurement of the Protein-Ligand Bond Energy of Carboxymyoglobin by Pulsed Photoacoustic Calorimetry. Chemical Physics Letters, 141 (3): 220-224.

Li, J. Y., Henry, E., Wang, L. M., Delelis, O., Wang, H., Simon, F., Tauc, P., Brochon, J. C., Zhao, Y. L., and Deprez, E. (2012). Comparative Study of the Fatty Acid Binding Process of a New FABP from Cherax Quadricarinatus by Fluorescence Intensity, Lifetime and Anisotropy. PLOS One, 7 (12): e51079. 
Li, Y., Yan, J., Zhang, X., and Huang, K. (2013). Disulfide Bonds in Amyloidogenesis Diseases Related Proteins. Proteins-Structure Function and Bioinformatics, 81 (11): 1862-1873.

Lim, W. K., Rösgen, J., and Englander, S. W. (2009). Urea, but not Guanidinium, Destabilizes Proteins by Forming Hydrogen Bonds to the Peptide Group. Proceedings of the National Academy of Sciences, 106 (8): 2595-2600.

Lippard, S., and Berg, J. (1994). Principles of Bioinorganic Chemistry. California, USA. University Science Books.

Livesey, A. K., and Brochon, J. C. (1987). Analyzing the Distribution of Decay Constants in Pulse-Fluorometry using the Maximum-Entropy Method. Biophysical Journal, 52 (5): 693-706.

Lockney, D., and Miksovska, J. (2006). Characterization of Carbon Monoxide Photodissociation from Fe(II)LPO with Photoacoustic Calorimetry. Journal of Physical Chemistry B, 110 (47): 24165-24170.

Louis-Jeune, C., Andrade-Navarro, M. A., and Perez-Iratxeta, C. (2012). Prediction of Protein Secondary Structure from Circular Dichroism using Theoretically Derived Spectra. Proteins-Structure Function and Bioinformatics, 80 (12): 2818-2818.

Lv, Y. H., Wang, Q. Z., Diao, Y., and Xu, R. A. (2008). Cytoglobin: A Novel Potential Gene Medicine for Fibrosis and Cancer Therapy. Current Gene Therapy, 8 (4): 287-294.

Makino, M., Sawai, H., Shiro, Y., and Sugimoto, H. (2011). Crystal Structure of the Carbon Monoxide Complex of Human Cytoglobin. Proteins-Structure Function and Bioinformatics, 79 (4): 1143-1153.

Makino, M., Sugimoto, H., Sawai, H., Kawada, N., Yoshizato, K., and Shiro, Y. (2006). High-Resolution Structure of Human Cytoglobin: Identification of Extra N- and CTermini and a New Dimerization Mode. Acta Crystallographica Section D-Biological Crystallography, 62: 671-677.

Mammen, P. P., Graham, S., Kanatous, S. B., McGrath, A. J., Shelton, J. M., Richardson, J. A. , and Garry, D. J. (2004). Cytoglobin: A Novel Cardioprotective Factor in the Stressed Heart. Circulation, 110 (17): 169-169.

Marcelli, A., Abbruzzetti, S., Bustamante, J. P., Feis, A., Bonamore, A., Boffi, A., Gellini, C., Salvi, P. R., Estrin, D. A., Bruno, S., Viappiani, C., and Foggi, P. (2012). 
Following Ligand Migration Pathways from Picoseconds to Milliseconds in Type II Truncated Hemoglobin from Thermobifida fusca. PLOS One, 7 (7): e39884.

Marden, M. C., Bohn, B., Kister, J., and Poyart, C. (1990). Effectors of Hemoglobin. Separation of Allosteric and Affinity Factors. Biophysical Journal, 57 (3): 397-403.

Matulis, D., and Lovrien, R. (1998). 1-Anilino-8-Naphthalene Sulfonate Anion-Protein Binding Depends Primarily on Ion Pair Formation. Biophysical Journal, 74 (1): 422-429.

McRonald, F. E., Liloglou, T., Xinarianos, G., Hill, L., Rowbottom, L., Langan, J. E., Ellis, A., Shaw, J. M., Field, J. K., and Risk, J. M. (2006). Down-Regulation of the Cytoglobin Gene, Located on 17q25, in Tylosis with Oesophageal Cancer (TOC): Evidence for Trans-Allele Repression. Human Molecular Genetics, 15 (8): 1271-1277.

McRonald, F. E., Risk, J. M., and Hodges, N. J. (2012). Protection from Intracellular Oxidative Stress by Cytoglobin in Normal and Cancerous Oesophageal Cells. PLOS One, 7 (2): e30587.

Meloun, M., and Ferencikova, Z. (2012). Enthalpy-Entropy Compensation for Some Drugs Dissociation in Aqueous Solutions. Fluid Phase Equilibria, 328: 31-41.

Miksovska, J., and Astudillo, L. (2011). Time Resolved Thermodynamics Associated with Diatomic Ligand Dissociation from Globins. Thermodynamics - Interaction Studies - Solids, Liquids and Gases. Juan Carlos Moreno-Pirajan, Ed. InTech.

Miksovska, J., Horsa, S., Davis, M. F., and Franzen, S. (2008). Conformational Dynamics Associated with Photodissociation of CO from Dehaloperoxidase Studied using Photoacoustic Calorimetry. Biochemistry, 47 (44): 11510-11517.

Miksovska, J., and Larsen, R. W. (2003). Structure-Function Relationships in Metalloproteins. Methods Enzymology, 360: 302-29.

Miksovska, J., Norstrom, J., and Larsen, R. W. (2005). Thermodynamic Profiles for CO Photodissociation from Heme Model Compounds: Effect of Proximal Ligands. Inorganic Chemistry, 44 (4): 1006-1014.

Milani, M., Nardini, M., Pesce, A., Mastrangelo, E., and Bolognesi, M. (2008). Hemoprotein Time-Resolved X-Ray Crystallography. IUBMB Life, 60 (3): 154-158.

Miller, L. M., Patel, M., and Chance, M. R. (1996). Identification of Conformational Substates in Oxymyoglobin Through The pH-Dependence of the Low-Temperature Photoproduct Yield. Journal of the American Chemical Society, 118 (19): 4511-4517. 
Mills, F. C., Ackers, G. K., Gaud, H. T., and Gill, S. J. (1979). Thermodynamic Studies on Ligand Binding and Subunit Association of Human Hemoglobins. Enthalpies of Binding $\mathrm{O}_{2}$ and $\mathrm{CO}$ to Subunit Chains of Hemoglobin A. Journal of Biological Chemistry, 254 (8): 2875-2880.

Moczygemba, C., Guidry, J., and Wittung-Stafshede, P. (2000). Heme Orientation Affects Holo-Myoglobin Folding and Unfolding Kinetics. FEBS Letters, 470 (2): 203206.

Mokdad, A., Nissen, M., Satterlee, J. D., and Larsen, R. W. (2007). Evidence for Fast Conformational Change Upon Ligand Dissociation in the HemAT Class of Bacterial Oxygen Sensors. FEBS Letters, 581 (23): 4512-4518.

Monera, O. D., Kay, C. M., and Hodges, R. S. (1994). Protein Denaturation with Guanidine-Hydrochloride or Urea Provides a Different Estimate of Stability Depending on the Contributions of Electrostatic Interactions. Protein Science, 3 (11): 1984-1991.

Morikis, D., Champion, P. M., Springer, B. A., and Sligar, S. G. (1989). Resonance Raman Investigations of Site-Directed Mutants of Myoglobin - Effects of Distal Histidine Replacement. Biochemistry, 28 (11): 4791-4800.

Moschetti, T., Mueller, U., Schulze, J., Brunori, M., and Vallone, B. (2009). The Structure of Neuroglobin at High Xe and Kr Pressure Reveals Partial Conservation of Globin Internal Cavities. Biophysical Journal, 97 (6): 1700-1708.

Mouawad, L., Maréchal, J. D., and Perahia, D. (2005). Internal Cavities and Ligand Passageways in Human Hemoglobin Characterized by Molecular Dynamics Simulations. Biochimica et Biophysica Acta (BBA), 1724 (3): 385-393.

Mu, J., Li, L., Guo, Y., Qiu, Z., and Tan, X. (2010). Spectroscopic Study on AcidInduced Unfolding and Refolding of Apo-Neuroglobin. Spectrochimica Acta Part AMolecular and Biomolecular Spectroscopy, 75 (5): 1600-1604.

Mueser, T. C., Rogers, P. H., and Arnone, A. (2000). Interface Sliding as Illustrated by the Multiple Quaternary Structures of Liganded Hemoglobin. Biochemistry, 39 (50): 15353-64.

Mukherjee, P., Haider, M., Hargrove, M. S., and Petrich, J. W. (2006). Characterization of the Interactions of Fluorescent Probes with Proteins: Coumarin 153 and 1,8-ANS in Complex with Holo- and Apomyoglobin. Photochemistry and Photobiology, 82 (6): 1586-1590. 
Nadra, A. D., Marti, M. A., Pesce, A., Bolognesi, M., and Estrin, D. A. (2008). Exploring the Molecular Basis of Heme Coordination in Human Neuroglobin. Proteins-Structure Function and Bioinformatics, 71 (2): 695-705.

Nagahara, N. (2011). Intermolecular Disulfide Bond to Modulate Protein Function as a Redox-Sensing Switch. Amino Acids, 41 (1): 59-72.

Nakatani, K., Okuyama, H., Shimahara, Y., Saeki, S., Kim, D. H., Nakajima, Y., Seki, S., Kawada, N., and Yoshizato, K. (2004). Cytoglobin/STAP, Its Unique Localization in Splanchnic Fibroblast-Like Cells and Function in Organ Fibrogenesis. Laboratory Investigation, 84 (1): 91-101.

Nguyen, B. D., Zhao, X. F., Vyas, K., La Mar, G. N., Lile, R. A., Brucker, E. A., Phillips, G. N., Olson, J. S., and Wittenberg, J. B. (1998). Solution and Crystal Structures of a Sperm Whale Myoglobin Triple Mutant that Mimics the Sulfide-Binding Hemoglobin from Lucina pectinata. Journal of Biological Chemistry, 273 (16): 9517-9526.

Nienhaus, K., Deng, P. C., Olson, J. S., Warren, J. J., and Nienhaus, G. U. (2003). Structural Dynamics of Myoglobin - Ligand Migration and Binding in Valine 68 Mutants. Journal of Biological Chemistry, 278 (43): 42532-42544.

Nienhaus, K., Kriegl, J. M., and Nienhaus, G. U. (2004). Structural Dynamics in the Active Site of Murine Neuroglobin and its Effects on Ligand Binding. Journal of Biological Chemistry, 279 (22): 22944-22952.

Nienhaus, K., Lutz, S., Meuwly, M., and Nienhaus, G. U. (2010). Structural Identification of Spectroscopic Substates in Neuroglobin. ChemPhysChem, 11 (1): 119129.

Nienhaus, K., and Nienhaus, G. U. (2005a). A Spectroscopic Study of Structural Heterogeneity and Carbon Monoxide Binding in Neuroglobin. Journal of Biological Physics, 31 (3-4): 417-432.

Nienhaus, K., and Nienhaus, G. U. (2005b). Probing Heme Protein-Ligand Interactions by UV-Visible Absorption Spectroscopy. G. U. Nienhaus, Ed. Methods in Molecular Biology, 305, Humana Press.

Nienhaus, K., and Nienhaus, G. U. (2007). Searching for Neuroglobin's Role in the Brain. IUBMB Life, 59 (8-9): 490-497.

Nolting, B. (2006). Protein Folding Kinetics: Biophysical Methods. Germany. Springer Berlin Heidelberd. 
Oleksiewicz, U., Liloglou, T., Field, J. K., and Xinarianos, G. (2011). Cytoglobin: Biochemical, Functional and Clinical Perspective of the Newest Member of the Globin Family. Cellular and Molecular Life Sciences, 68 (23): 3869-3883.

Oleksiewicz, U., Liloglou, T., Tasopoulou, K. M., Daskoulidou, N., Bryan, J., Gosney, J. R., Field, J. K., and Xinarianos, G. (2013). Cytoglobin Has Bimodal: Tumour Suppressor and Oncogene Functions in Lung Cancer Cell Lines. Human Molecular Genetics, 22 (16): 3207-3217.

Olson, J. S., Soman, J., and Phillips, G. N. (2007). Ligand Pathways in Myoglobin: A Review of Trp Cavity Mutations. IUBMB Life, 59 (8-9): 552-562.

Osborne, R. L., Taylor, L. O., Han, K. P., Ely, B., and Dawson, J. H. (2004). Amphitrite Ornata Dehaloperoxidase: Enhanced Activity for the Catalytically Active Globin Using MCPBA. Biochemical and Biophysical Research Communications, 324 (4): 1194-1198.

Ostermann, A., Waschipky, R., Parak, F. G., and Nienhaus, G. U. (2000). Ligand Binding and Conformational Motions in Myoglobin. Nature, 404 (6774): 205-208.

Pace, C. N. (1975). The Stability of Globular Proteins. CRC Critical Reviews in Biochemistry, 3 (1): 1-43.

Park, S. Y., Yokoyama, T., Shibayama, N., Shiro, Y., and Tame, J. R. (2006). $1.25 \AA$ Resolution Crystal Structures of Human Haemoglobin in the Oxy, Deoxy and Carbonmonoxy Forms. Journal of Molecular Biology, 360 (3): 690-701.

Pastukhov, A. V., and Ropson, I. J. (2003). Fluorescent Dyes as Probes to Study LipidBinding Proteins. Proteins-Structure Function and Genetics, 53 (3): 607-615.

Perry, C. B., Chick, T., Ntlokwana, A., Davies, G., and Marques, H. M. (2002). The Coordination of Ligands by Iron Porphyrins: A Comparison of Ligand Binding by Myoglobin from Sperm Whale and the Haem Undecapeptide from Cytochrome C. Journal of the Chemical Society- Dalton Transactions, 449-457.

Perutz, M. F.. (1989). Myoglobin and Hemoglobin - Role of Distal Residues in Reactions with Heme Ligands. Trends in Biochemical Sciences, 14 (2): 42-44.

Perutz, M. F., Wilkinson, A. J., Paoli, M., and Dodson, G. G. (1998). The Stereochemical Mechanism of the Cooperative Effects in Hemoglobin Revisited. Annual Review of Biophysics and Biomolecular Structure, 27: 1-34. 
Pesce, A., Bolognesi, M., Bocedi, A., Ascenzi, P., Dewilde, S., Moens, L., Hankeln, T., and Burmester, T. (2002). Neuroglobin and Cytoglobin - Fresh Blood for the Vertebrate Globin Family. EMBO Reports, 3 (12): 1146-1151.

Pesce, A., De Sanctis, D., Nardini, M., Dewilde, S., Moens, L., Hankeln, T., Burmester, T., Ascenzi, P., and Bolognesi, M. (2004a). Reversible Hexa- to Penta-Coordination of the Heme Fe Atom Modulates Ligand Binding Properties of Neuroglobin and Cytoglobin. IUBMB Life, 56 (11-12): 657-664.

Pesce, A., Dewilde, S., Nardini, M., Moens, L., Ascenzi, P., Hankeln, T., Burmester, T., and Bolognesi, M. (2003). Human Brain Neuroglobin Structure Reveals a Distinct Mode of Controlling Oxygen Affinity. Structure, 11 (9): 1087-1095.

Pesce, A., Dewilde, S., Nardini, M., Moens, L., Ascenzi, P., Hankeln, T., Burmester, T., and Bolognesi, M. (2004b). The Human Brain Hexacoordinated Neuroglobin ThreeDimensional Structure. Micron, 35 (1-2): 63-65.

Peters, K. S., Watson, T., and Logan, T. (1992). Photoacoustic Calorimetry Study of Human Carboxyhemoglobin. Journal of the American Chemical Society, 114 (11): 42764278 .

Peters, K. S., Watson, T., and Marr, K. (1991). Time-Resolved Photoacoustic Calorimetry - A Study of Myoglobin and Rhodopsin. Annual Review of Biophysics and Biophysical Chemistry, 20: 343-362.

Petrich, J. W., Poyart, C., and Martin, J. L. (1988). Photophysics and Reactivity of HemeProteins-A Femtosecond Absorption Study of Hemoglobin, Myoglobin, and Protoheme. Biochemistry, 27 (11): 4049-4060.

Phillips, S. E., and Schoenborn, B. P. (1981). Neutron Diffraction Reveals OxygenHistidine Hydrogen Bond in Oxymyoglobin. Nature, 292 (5818): 81-82.

Pierce, M. M., Raman, C. S., and Nall, B. T. (1999). Isothermal Titration Calorimetry of Protein-Protein Interactions. Methods, 19 (2): 213-221.

Projahn, H. D., Dreher, C., and Van Eldik, R. (1990). Effect of Pressure on the Formation and Deoxygenation Kinetics of Oxymyoglobin. Mechanistic Information from a Volume Profile Analysis. Journal of the American Chemical Society, 112 (1): 17-22.

Quillin, M. L., Arduini, R. M., Olson, J. S., and Phillips, G. N. (1993). High Resolution Crystal Structures of Distal Histidine Mutants of Sperm Whale Myoglobin. Journal of Molecular Biology, 234 (1): 140-155. 
Quillin, M. L., Li, T. S., Olson, J. S., Phillips, G. N., Dou, Y., Ikedasaito, M., Regan, R., Carlson, M., Gibson, Q. H., Li, H. Y., and Elber, R. (1995). Structural and FunctionalEffects of Apolar Mutations of the Distal Valine in Myoglobin. Journal of Molecular Biology, 245 (4): 416-436.

Radon, M., and Pierloot, K. (2008). Binding of CO, NO, And O2 to Heme by Density Functional and Multireference Ab Initio Calculations. Journal of Physical Chemistry A, 112 (46): 11824-11832.

Ranjbar, B., and Gill, P. (2009). Circular Dichroism Techniques: Biomolecular and Nanostructural Analyses - A Review. Chemical Biology \& Drug Design, 74 (2): 101-120.

Raychaudhuri, S., Skommer, J., Henty, K., Birch, N., and Brittain, T. (2010). Neuroglobin Protects Nerve Cells from Apoptosis by Inhibiting the Intrinsic Pathway of Cell Death. Apoptosis, 15 (4): 401-411.

Reeder, B. J., Svistunenko, D. A., and Wilson, M. T. (2011). Lipid Binding to Cytoglobin Leads to a Change in Haem Coordination: A Role for Cytoglobin in Lipid Signalling of Oxidative Stress. Biochemical Journal, 434: 483-492.

Riccio, A., Tamburrini, M., Giardina, B., and di Prisco, G. (2001). Molecular Dynamics Analysis of a Second Phosphate Site in the Hemoglobins of the Seabird, South Polar Skua. Is There a Site-Site Migratory Mechanism along the Central Cavity?. Biophysical Journal, 81 (4): 1938-1946.

Rodgers, K. R. (1999). Heme-Based Sensors in Biological Systems. Current Opinion in Chemical Biology, 3 (2): 158-167.

Roesner, A., Fuchs, C., Hankeln, T., and Burmester, T. (2005). A Globin Gene of Ancient Evolutionary Origin in Lower Vertebrates: Evidence for Two Distinct Globin Families in Animals. Molecular Biology and Evolution, 22 (1): 12-20.

Rohlfs, R. J., Mathews, A. J., Carver, T. E., Olson, J. S., Springer, B. A., Egeberg, K. D., and Sligar, S. G. (1990). The Effects of Amino-Acid Substitution at Position-E7 (Residue-64) on the Kinetics of Ligand-Binding to Sperm Whale Myoglobin. Journal of Biological Chemistry, 265 (6): 3168-3176.

Roncone, R., Monzani, E., Labo, S., Sanangelantoni, A. M., and Casella, L. (2005). Catalytic Activity, Stability, Unfolding, and Degradation Pathways of Engineered and Reconstituted Myoglobins. Journal of Biological Inorganic Chemistry, 10 (1): 11-24. 
Rosell, F. I., and Mauk, A. G. (2011). Photochemical Reagents for the Study of Metalloproteins by Flash Photolysis. Coordination Chemistry Reviews, 255 (7-8): 737756.

Ross, J. A., and Jameson, D. M. (2008). Time-Resolved Methods in Biophysics. 8. Frequency Domain Fluorometry: Applications to Intrinsic Protein Fluorescence. Photochemical \& Photobiological Sciences, 7 (11): 1301-1312.

Saffran, W. A., and Gibson, Q. H. (1977). Photodissociation of Ligands from Heme and Heme Proteins. Effect of Temperature and Organic Phosphate. Journal of Biological Chemistry, 252 (22): 7955-7958.

Safo, M. K., Ahmed, M. H., Ghatge, M. S., and Boyiri, T. (2011). Hemoglobin-Ligand Binding: Understanding $\mathrm{Hb}$ Function and Allostery on Atomic Level. Biochimica et Biophysica Acta-Proteins and Proteomics, 1814 (6): 797-809.

Sage, J. T., Morikis, D., and Champion, P. M. (1991). Spectroscopic Studies of Myoglobin at Low pH - Heme Structure and Ligation. Biochemistry, 30 (5): 1227-1237.

Sakakura, M., Morishima, I., and Terazima, M. (2002). Structural Dynamics of Distal Histidine Replaced Mutants of Myoglobin Accompanied with the Photodissociation Reaction of the Ligand. Biochemistry, 41 (15): 4837-4846.

Salter, M. D., Blouin, G. C., Soman, J., Singleton, E. W., Dewilde, S., Moens, L., Pesce, A., Nardini, M., Bolognesi, M., and Olson, J. S. (2012). Determination of Ligand Pathways in Globins Apolar Tunnels Versus Polar Gates. Journal of Biological Chemistry, 287 (40): 33163-33178.

Santoro, M. M., and Bolen, D. W. (1988). Unfolding Free Energy Changes Determined by the Linear Extrapolation Model. 1. Unfolding of Phenylmethanesulfonyl AlphaChymotrypsin Using Different Denaturants. Biochemistry, 27 (21): 8063-8068.

Savino, C., Miele, A. E., Draghi, F., Johnson, K. A., Sciara, G., Brunori, M., and Vallone, B. (2009). Pattern of Cavities in Globins: The Case of Human Hemoglobin. Biopolymers, 91 (12): 1097-1107.

Sawai, H., Kawada, N., Yoshizato, K., Nakajima, H., Aono, S., Mizutani, Y., and Shiro, Y. (2003). Characterization of Heme Environmental Structure of Cytoglobin, a Fourth Globin in Human. Biochemistry, 42(17): 5133-5142.

Schaad, O., Zhou, H. X., Szabo, A., Eaton, W. A., and Henry, E. R. (1993). Simulation of the Kinetics of Ligand Binding to a Protein by Molecular Dynamics: Geminate 
Rebinding of Nitric Oxide to Myoglobin. Proceedings of the National Academy of Sciences of the United States of America, 90 (20): 9547-9551.

Schaberle, F. A., Nunes, R. M., Barroso, M., Serpa, C., and Arnaut, L. G. (2010). Analytical Solution for Time-Resolved Photoacoustic Calorimetry Data and Applications to Two Typical Photoreactions. Photochemical \& Photobiological Sciences, 9 (6): 812822.

Schay, G., Smeller, L., Tsuneshige, A., Yonetani, T., and Fidy, J. (2006). Allosteric Effectors Influence the Tetramer Stability of Both R- and T-States of Hemoglobin A. Journal of Biological Chemistry, 281 (36): 25972-25983.

Schmidt, M., Gerlach, F., Avivi, A., Laufs, T., Wystub, S., Simpson, J. C., Nevo, E., Saaler-Reinhardt, S., Reuss, S., Hankeln, T., and Burmester, T. (2004). Cytoglobin is a Respiratory Protein in Connective Tissue and Neurons, which is Up-Regulated by Hypoxia. Journal of Biological Chemistry, 279 (9): 8063-8069.

Schmidt, M., Nienhaus, K., Pahl, R., Krasselt, A., Anderson, S., Parak, F., Nienhaus, G. U., and Srajer, V. (2005). Ligand Migration Pathway and Protein Dynamics in Myoglobin: A Time-Resolved Crystallographic Study on L29W MbCO. Proceedings of the National Academy of Sciences of the United States of America, 102 (33): 1170411709 .

Schotte, F., Soman, J., Olson, J. S., Wulff, M., and Anfinrud, P. A. (2004). Picosecond Time-Resolved X-Ray Crystallography: Probing Protein Function in Real Time. Journal of Structural Biology, 147 (3): 235-246.

Scott, E. E., Gibson, Q. H., and Olson, J. S. (2001). Mapping the Pathways for $\mathrm{O}_{2}$ Entry Into and Exit from Myoglobin. Journal of Biological Chemistry, 276 (7): 5177-5188.

Shaw, R. J., Omar, M. M., Rokadiya, S., Kogera, F. A., Lowe, D., Hall, G. L., Woolgar, J. A., Homer, J., Liloglou, T., Field, J. K., and Risk, J. M. (2009). Cytoglobin is Upregulated by Tumour Hypoxia and Silenced by Promoter Hypermethylation in Head and Neck Cancer. British Journal of Cancer, 101 (1): 139-144.

Shibayama, N., Miura, S., Tame, J. R., Yonetani, T., and Park, S. Y. (2002). Crystal Structure of Horse Carbonmonoxyhemoglobin-Bezafibrate Complex at $1.55-\AA$ Resolution. Journal of Biological Chemistry, 277 (41): 38791-38796.

Shivapurkar, N., Stastny, V., Okumura, N., Girard, L., Xie, Y., Prinsen, C., Thunnissen, F. B., Wistuba, I. I., Czerniak, B., Frenkel, E., Roth, J. A., Liloglou, T., Xinarianos, G., Field, J. K., Minna, J. D., and Gazdar, A. F. (2008). Cytoglobin, the Newest Member of 
the Globin Family, Functions as a Tumor Suppressor Gene. Cancer Research, 68 (18): 7448-7456.

Silva, M. M., Rogers, P. H., and Arnone, A. (1992). A Third Quaternary Structure of Human Hemoglobin A at $1.7 \AA$ Resolution. Journal of Biological Chemistry, 267 (24): $17248-17256$.

Smagghe, B. J., Hoy, J. A., Percifield, R., Kundu, S., Hargrove, M. S., Sarath, G., Hilbert, J. L., Watts, R. A., Dennis, E. S., Peacock, W. J., Dewilde, S., Moens, L., Blouin, G. C., Olson, J. S., and Appleby, C. A. (2009). Correlations between Oxygen Affinity and Sequence Classifications of Plant Hemoglobins. Biopolymers, 91 (12): 1083-1096.

Smagghe, B. J., Trent III, J. T., and Hargrove, M. S. (2008). NO Dioxygenase Activity in Hemoglobins Is Ubiquitous In Vitro, but Limited by Reduction In Vivo. PLOS One, 3 (4): e2039.

Song, X. J., Simplaceanu, V., Ho, N. T., and Ho, C. (2008). Effector-Induced Structural Fluctuation Regulates the Ligand Affinity of an Allosteric Protein: Binding of Inositol Hexaphosphate Has Distinct Dynamic Consequences for the $\mathrm{T}$ and $\mathrm{R}$ States of Hemoglobin. Biochemistry, 47 (17): 4907-4915.

Sottini, S., Viappiani, C., Ronda, L., Bettati, S., and Mozzarelli, A. (2004). CO Rebinding Kinetics to Myoglobin and R-State Hemoglobin Doped Silica Gels in the Presence of Glycerol. Journal of Physical Chemistry B, 108 (24): 8475-8484.

Sprang, S. R. (1997). G Protein Mechanisms: Insights from Structural Analysis. Annual Review of Biochemistry, 66: 639-678.

Srajer, V., Ren, Z., Teng, T. Y., Schmidt, M., Ursby, T., Bourgeois, D., Pradervand, C., Schildkamp, W., Wulff, M., and Moffat, K. (2001). Protein Conformational Relaxation and Ligand Migration in Myoglobin: A Nanosecond to Millisecond Molecular Movie from Time-Resolved Laue X-Ray Diffraction. Biochemistry, 40 (46): 13802-13815.

Srajer, V., Teng, T. Y., Ursby, T., Pradervand, C., Ren, Z., Adachi, S., Schildkamp, W., Bourgeois, D., Wulff, M., and Moffat, K. (1996). Photolysis of the Carbon Monoxide Complex of Myoglobin: Nanosecond Time-Resolved Crystallography. Science, 274 (5293): 1726-1729.

Steinbach, P. J. (1996). Two-Dimensional Distributions of Activation Enthalpy and Entropy from Kinetics by the Maximum Entropy Method. Biophysical Journal, 70 (3): 1521-1528. 
Steinbach, P. J. (2012). Filtering Artifacts from Lifetime Distributions when Maximizing Entropy Using a Bootstrapped Model. Analytical Biochemistry, 427 (1): 102-105.

Steinbach, P. J., Chu, K., Frauenfelder, H., Johnson, J. B., Lamb, D. C., Nienhaus, G. U., Sauke, T. B., and Young, R. D. (1992). Determination of Rate Distributions from Kinetic Experiments. Biophysical Journal, 61 (1): 235-245.

Steinbach, P. J., Ionescu, R., and Matthews, C. R. (2002). Analysis of Kinetics Using a Hybrid Maximum-Entropy/Nonlinear-Least-Squares Method: Application to Protein Folding. Biophysical Journal, 82 (4): 2244-2255.

Strickland, N., and Harvey, J. N. (2007). Spin-Forbidden Ligand Binding to the FerrousHeme Group: Ab Initio and DFT Studies. Journal of Physical Chemistry B, 111 (4): 841852.

Stryer, L. (1965). The Interaction of a Naphthalene Dye with Apomyoglobin and Apohemoglobin. A Fluorescent Probe of Non-Polar Binding Sites. Journal of Molecular Biology, 13 (2): 482-495.

Sugimoto, H., Makino, M., Sawai, H., Kawada, N., Yoshizato, K., and Shiro, Y. (2004). Structural Basis of Human Cytoglobin for Ligand Binding. Journal of Molecular Biology, 339 (4): 873-885.

Sugimoto, T., Unno, M., Shiro, Y., Dou, Y., and Ikeda-Saito, M. (1998). Myoglobin Mutants Giving the Largest Geminate Yield in CO Rebinding in the Nanosecond Time Domain. Biophysical Journal, 75 (5): 2188-2194.

Sun, Y. J., Jin, K. L., Mao, X. O., Zhu, Y. H., and Greenberg, D. A. (2001). Neuroglobin is Up-Regulated by and Protects Neurons from Hypoxic-Ischemic Injury. Proceedings of the National Academy of Sciences of the United States of America, 98 (26): 1530615311.

Sun, Y. J., Jin, K. L., Peel, A., Mao, X. O., Xie, L., and Greenberg, D. A. (2003). Neuroglobin Protects the Brain from Experimental Stroke In Vivo. Proceedings of the National Academy of Sciences of the United States of America, 100 (6): 3497-3500.

Syakhovich, V. E., Parul, D. A., Ruta, E. Y., Bushuk, B. A., and Bokut, S. B. (2004). 1,8Anilinonaphthalene Sulfonate Binds to Central Cavity of Human Hemoglobin. Biochemical and Biophysical Research Communications, 317 (3): 761-767.

Tada, T., Watanabe, Y., Matsuoka, A., Ikeda- Saito, M., Imai, K., Yukio, N., and Shikama, K. (1998). African Elephant Myoglobin with an Unusual Autoxidation 
Behavior: Comparison with the H64Q Mutant of Sperm Whale Myoglobin. Biochimica et Biophysica Acta -Protein Structure and Molecular Enzymology, 1387 (1-2): 165-176.

Takahashi, N., Watanabe, S., and Wakasugi, K. (2013). Crucial Roles of Glu60 in Human Neuroglobin as a Guanine Nucleotide Dissociation Inhibitor and Neuroprotective Agent. PLOS One, 8 (12): e83698.

Takano, K., Tsuchimori, K., Yamagata, Y., and Yutani, K. (2000). Contribution of Salt Bridges Near the Surface of a Protein to the Conformational Stability. Biochemistry, 39 (40): 12375-12381.

Tian, W. D., Sage, J. T., and Champion, P. M. (1993). Investigations of Ligand Association and Dissociation Rates in the Open and Closed States of Myoglobin. Journal of Molecular Biology, 233 (1): 155-166.

Tiso, M., Tejero, J., Basu, S., Azarov, I., Wang, X. D., Simplaceanu, V., Frizzell, S., Jayaraman, T., Geary, L., Shapiro, C., Ho, C., Shiva, S., Kim-Shapiro, D. B., and Gladwin, M. T. (2011). Human Neuroglobin Functions as a Redox-regulated Nitrite Reductase. Journal of Biological Chemistry, 286 (20): 18277-18289.

Trent, J. T., and Hargrove, M. S. (2002). A Ubiquitously Expressed Human Hexacoordinate Hemoglobin. Journal of Biological Chemistry, 277 (22): 19538-19545.

Trent, J. T., Watts, R. A., and Hargrove, M. S. (2001). Human Neuroglobin, a Hexacoordinate Hemoglobin that Reversibly Binds Oxygen. Journal of Biological Chemistry, 276 (32): 30106-30110.

Tsuneshige, A., Park, S., and Yonetani, T. (2002). Heterotropic Effectors Control the Hemoglobin Function by Interacting with Its $\mathrm{T}$ And R States- A New View on the Principle Of Allostery. Biophysical Chemistry, 98 (1-2): 49-63.

Unno, M., Ishimori, K., and Morishima, I. (1990). High-Pressure Laser Photolysis Study of Hemoproteins. Effects of Pressure on Carbon Monoxide Binding Dynamics for R- and T-State Hemoglobins. Biochemistry, 29 (44): 10199-10205.

Uno, T., Ryu, D., Tsutsumi, H., Tomisugi, Y., Ishikawa, Y., Wilkinson, A. J., Sato, H., and Hayashi, T. (2004). Residues in the Distal Heme Pocket of Neuroglobin Implications for the Multiple Ligand Binding Steps. Journal of Biological Chemistry, 279 (7): 5886-5893. 
Uversky, V. N., Winter, S., and Lober, G. (1996). Use of Fluorescence Decay Times of 8-ANS Protein Complexes to Study the Conformational Transitions in Proteins Which Unfold Through The Molten Globule State. Biophysical Chemistry, 60 (3): 79-88.

Uzan, J., Dewilde, S., Burmester, T., Hankeln, T., Moens, L., Hamdane, D., Marden, M. C., and Kiger, L. (2004). Neuroglobin and Other Hexacoordinated Hemoglobins Show a Weak Temperature Dependence of Oxygen Binding. Biophysical Journal, 87 (2): 11961204.

Valeur, B. (2001). Principles of Steady-State and Time-Resolved Fluorometric Techniques, in Molecular Fluorescence: Principles and Applications. Wiley-VCH Verlag $\mathrm{GmbH}$, Weinheim, FRG.

Vallone, B., Nienhaus, K., Brunori, M., and Nienhaus, G. U. (2004a). The Structure of Murine Neuroglobin: Novel Pathways for Ligand Migration and Binding. ProteinsStructure Function and Bioinformatics, 56 (1): 85-92.

Vallone, B., Nienhaus, K., Matthes, A., Brunori, M., and Nienhaus, G. U. (2004b). The Structure of Carbonmonoxy Neuroglobin Reveals a Heme-Sliding Mechanism for Control of Ligand Affinity. Proceedings of the National Academy of Sciences of the United States of America, 101 (50): 17351-17356.

Van Doorslaer, S., Dewilde, S., Kiger, L., Nistor, S. V., Goovaerts, E., Marden, M. C., and Moens, L. (2003). Nitric Oxide Binding Properties of Neuroglobin - A Characterization by EPR and Flash Photolysis. Journal of Biological Chemistry, 278 (7): 4919-4925.

Vazquez-Limon, C., Hoogewijs, D., Vinogradov, S. N., and Arredondo-Peter, R. (2012). The Evolution of Land Plant Hemoglobins. Plant Science, 191: 71-81.

Vetromile, C. M., Miksovska, J., and Larsen, R. W. (2011). Time Resolved Thermodynamics Associated with Ligand Photorelease in Heme Peroxidases and Globins: Open Access Channels Versus Gated Ligand Release. Biochimica et Biophysica Acta-Proteins and Proteomics, 1814 (8): 1065-1076.

Vinogradov, S. N., Fernandez, I., Hoogewijs, D., and Arredondo-Peter, R. (2011). Phylogenetic Relationships of 3/3 and 2/2 Hemoglobins in Archaeplastida Genomes to Bacterial and Other Eukaryote Hemoglobins. Molecular Plant, 4 (1): 42-58.

Vinogradov, S. N., Tinajero-Trejo, M., Poole, R. K., and Hoogewijs, D. (2013). Bacterial and Archaeal Globins - A Revised Perspective. Biochimica et Biophysica Acta-Proteins and Proteomics, 1834 (9): 1789-1800. 
Vinther, T. N., Norrman, M., Ribel, U., Huus, K., Schlein, M., Steensgaard, D. B., Pedersen, T. A., Pettersson, I., Ludvigsen, S., Kjeldsen, T., Jensen, K. J., and Hubalek, F. (2013). Insulin Analog with Additional Disulfide Bond has Increased Stability and Preserved Activity. Protein Science, 22 (3): 296-305.

Wakasugi, K., Kitatsuji, C., and Morishima, I. (2005). Possible Neuroprotective Mechanism of Human Neuroglobin. Annals of the New York Academy of Sciences, 1053: 220-230.

Wakasugi, K., and Morishima, I. (2005). Identification of Residues in Human Neuroglobin Crucial for Guanine Nucleotide Dissociation Inhibitor Activity. Biochemistry, 44 (8): 2943-2948.

Wakasugi, K., Nakano, T., Kitatsuji, C., and Morishima, I. (2004). Human Neuroglobin Interacts with Flotillin-1, a Lipid Raft Microdomain-Associated Protein. Biochemical and Biophysical Research Communications, 318 (2): 453-460.

Wakasugi, K., Nakano, T., and Morishima, I. (2003). Oxidized Human Neuroglobin Acts as a Heterotrimeric G Alpha Protein Guanine Nucleotide Dissociation Inhibitor. Journal of Biological Chemistry, 278 (38): 36505-36512.

Wakasugi, K., Takahashi, N., Uchida, H., and Watanabe, S. (2011). Species-Specific Functional Evolution of Neuroglobin. Marine Genomics, 4 (3): 137-142.

Walda, K. N., Liu, X. Y., Sharma, V. S., and Magde, D. (1994). Geminate Recombination of Diatomic Ligands CO, $\mathrm{O}_{2}$, NO with Myoglobin. Biochemistry, 33 (8): 2198-2209.

Watanabe, S., Takahashi, N., Uchida, H., and Wakasugi, K. (2012). Human Neuroglobin Functions as an Oxidative Stress-responsive Sensor for Neuroprotection. Journal of Biological Chemistry, 287 (36): 30128-30138.

Weber, G., and Joung, L. (1964). Fragmentation Of Bovine Serum Albumin By Pepsin. Journal of Biological Chemistry, 239 (5): 1424-1431.

Wedemeyer, W. J., Welker, E., Narayan, M., and Scheraga, H. A. (2000). Disulfide Bonds and Protein Folding. Biochemistry, 39 (15): 4207-4216.

Westrick, J. A., and Peters, K. S. (1990). A Photoacoustic Calorimetric Study of Horse Myoglobin. Biophysical Chemistry, 37 (1-3): 73-79. 
Westrick, J. A., Peters, K. S., Ropp, J. D., and Sligar, S. G. (1990). Role of the Arginine45 Salt Bridge in Ligand Dissociation from Sperm Whale Carboxymyoglobin as Probed by Photoacoustic Calorimetry. Biochemistry, 29 (28): 6741-6746.

Wilson, J., Phillips, K., and Luisi, B. (1996). The Crystal Structure of Horse Deoxyhaemoglobin Trapped in the High-affinity (R) State. Journal of Molecular Biology, 264 (4): 743-756.

Wouters, M. A., Fan, S. W., and Haworth, N. L. (2010). Disulfides as Redox Switches: From Molecular Mechanisms to Functional Significance. Antioxidants \& Redox Signaling, 12 (1): 53-91.

Xinarianos, G., McRonald, F. E., Risk, J. M., Bowers, N. L., Nikolaidis, G., Field, J. K., and Liloglou, T. (2006). Frequent Genetic and Epigenetic Abnormalities Contribute to the Deregulation of Cytoglobin in Non-Small Cell Lung Cancer. Human Molecular Genetics, 15 (13): 2038-2044.

Xu, J., Li, L. Z., Yin, G. W., Li, H. L., and Du, W. H. (2009). Ligand Orientation of Human Neuroglobin Obtained from Solution NMR and Molecular Dynamics Simulation as Compared with X-Ray Crystallography. Journal of Inorganic Biochemistry, 103 (12): 1693-1701.

Xu, J., Yin, G. W., and Du, W. H. (2011). Distal Mutation Modulates the Heme Sliding in Mouse Neuroglobin Investigated by Molecular Dynamics Simulation. ProteinsStructure Function and Bioinformatics, 79 (1): 191-202.

Xu, R., Harrison, P. M., Chen, M., Li, L. Y., Tsui, T. Y., Fung, P. C., Cheung, P. T., Wang, G. J., Li, H., Diao, Y., Krissansen, G. W., Xu, S., and Farzaneh, F. (2006). Cytoglobin Overexpression Protects Against Damage-Induced Fibrosis. Molecular Therapy, 13 (6): 1093-1100.

Yang, F., and Phillips, G. N. (1996). Crystal Structures Of CO-, Deoxy- and MetMyoglobins at Various pH Values. Journal of Molecular Biology, 256 (4): 762-774.

Ye, X., Demidov, A., and Champion, P. M. (2002). Measurements of the Photodissociation Quantum Yields of $\mathrm{MbNO}$ and $\mathrm{MbO}_{2}$ and the Vibrational Relaxation of the Six-Coordinate Heme Species. Journal of the American Chemical Society, 124 (20): 5914-5924.

Yin, G. W., Li, Y. J., Li, J., Du, W. H., Wei, Q., and Fang, W. H. (2008). Solution ${ }^{1}$ H NMR Study of the Active Site Structure for the Double Mutant H64Q/V68F Cyanide Complex from Mouse Neuroglobin. Biophysical Chemistry, 136 (2-3): 115-123. 
Yonetani, T., and Laberge, M. (2008). Protein Dynamics Explain the Allosteric Behaviors of Hemoglobin. Biochimica et Biophysica Acta-Proteins and Proteomics, 1784 (9): 1146-1158.

Yonetani, T., Park, S. I., Tsuneshige, A., Imai, K., and Kanaori, K. (2002). Global Allostery Model of Hemoglobin. Modulation of $\mathrm{O}_{2}$ Affinity, Cooperativity, and Bohr Effect by Heterotropic Allosteric Effectors. Journal of Biological Chemistry, 277 (37): $34508-34520$.

Yu, H. S., Saw, J. H., Hou, S. B., Larsen, R. W., Watts, K. J., Johnson, M. S., Zimmer, M. A., Ordal, G. W., Taylor, B. L., and Alam, M. (2002). Aerotactic Responses in Bacteria to Photoreleased Oxygen. FEMS Microbiology Letters, 217 (2): 237-242.

Zhang, B. B., Xu, J., Li, Y. M., Du, W. H., and Fang, W. H. (2011a). Molecular Dynamics Simulation of Carboxy and Deoxy Human Cytoglobin in Solution. Journal of Inorganic Biochemistry, 105 (7): 949-956.

Zhang, C., Gao, C. H., Mu, J. S., Qiu, Z. L., and Li, L. Z. (2013a). Spectroscopic Studies on Unfolding Processes of Apo-Neuroglobin Induced by Guanidine Hydrochloride and Urea. Biomedical Research International. 349542.

Zhang, J., Lan, S. J., Liu, Q. R., Liu, J. M., and Chen, X. Q. (2013b). Neuroglobin, a Novel Intracellular Hexa-Coordinated Globin, Functions as a Tumor Suppressor in Hepatocellular Carcinoma via Raf/MAPK/Erk. Molecular Pharmacology, 83 (5): 11091119.

Zhang, L., Chou, C. P., and Moo-Young, M. (2011b). Disulfide Bond Formation and its Impact on the Biological Activity and Stability of Recombinant Therapeutic Proteins Produced by Escherichia coli Expression System. Biotechnology Advances, 29 (6): 923929.

Zhang, W., and Phillips, G. N. (2003a). Crystallization and X-Ray Diffraction Analysis of the Sensor Domain of the HemAT Aerotactic Receptor. Acta Crystallographica Section D, 59 (4): 749-751.

Zhang, W., and Phillips, G. N. (2003b). Structure of the Oxygen Sensor in Bacillus subtilis: Signal Transduction of Chemotaxis by Control of Symmetry. Structure, 11 (9): 1097-1110.

Zhao, C., Li, L. Z., Wang, L., and Ji, H. W. (2006). Circular Dichroism Spectral Studies on the Recomhinant Human Neuroglobin. Chinese Science Bulletin, 51 (21): 2581-2585. 
Šrajer, V., Ren, Z., Teng, T. Y., Schmidt, M., Ursby, T., Bourgeois, D., Pradervand, C., Schildkamp, W., Wulff, M., and Moffat, K. (2001). Protein Conformational Relaxation and Ligand Migration in Myoglobin: A Nanosecond to Millisecond Molecular Movie from Time-Resolved Laue X-ray Diffraction. Biochemistry, 40 (46): 13802-13815. 
VITA

\section{LUISANA ASTUDILLO}

2006 B.Sc. in Chemistry.

Universidad Simón Bolívar.

Caracas, Venezuela.

2006-2007 Quality Control Analyst.

Pepsi-Cola Venezuela.

Caracas, Venezuela.

2008-2010 Teaching Assistant.

Florida International University.

Miami, Florida.

2009-2010 Outstanding General Chemistry Teaching Assistant (TA)

2009 Third Prize for Poster Presentation. FAME.

Orlando, FL,

2010-2014 Research Assistant and Teaching Assistant.

Florida International University.

Miami, Florida.

$2012 \quad$ SoFLACS Graduate Student Travel Award

$2012 \quad$ BRI Student Summer Research Award

2012 Outstanding Graduate Poster Presentation.

University of South Florida, FL.

\section{PUBLICATIONS}

REDUCTION OF THE INTERNAL DISULFIDE BOND BETWEEN CYS 38 AND 83 SWITCHES THE LIGAND MIGRATION PATHWAYS IN CYTOGLOBIN. Luisana Astudillo, Sophie Sebban, Valerie Derrien, Pierre Sebban, Jaroslava Miksovska. Journal of Inorganic Biochemistry, 2013, 129:23-29.

CONFORMATIONAL DYNAMICS IN NEUROGLOBIN: THE ROLE OF VAL 68, HIS 64 AND CYS 120 ON LIGAND MIGRATION. Luisana Astudillo, Sophie Sebban, Valerie Derrien, Pierre Sebban, Jaroslava Miksovska. Biochemistry, 2012, 51:9984-9994. 
TIME RESOLVED THERMODYNAMICS ASSOCIATED WITH DIATOMIC LIGAND DISSOCIATION FROM GLOBINS. Jaroslava Miksovska and Luisana Astudillo. In: Themodynamic - Interactions Studies - Solids, Liquids and Gases, Juan Carlos Moreno-Pirajan (Ed.), 2011, InTech.

PROBING THE ROLE OF THE INTERNAL DISULFIDE BOND IN REGULATING CONFORMATIONAL DYNAMICS IN NEUROGLOBIN. Luisana Astudillo, Sophie Sebban, Valerie Derrien, Pierre Sebban, Jaroslava Miksovska. Biophysical Journal, 2010, 99:L16-L18.

QUANTITATIVE STUDY OF CYTOCHROME C AND NEUROGLOBIN INTERACTIONS BY QUARTZ NANOPIPETTES. Purushutam Tiwari, Luisana Astudillo, Jaroslava Miksovska, Xuegen Wang, Yesim Darici and Jin He. Submitted.

A SURFACE PLASMON RESONANCE STUDY FOR THE CHARACTERIZATION OF MOLECULAR MECHANISM OF NEUROGLOBIN BINDING TO CYTOCHROME C. Purushutam Tiwari, Luisana Astudillo, Jaroslava Miksovska, Sophie Sebban, Valerie Derrien, Pierre Sebban, Xuegen Wang, Yesim Darici and Jin He. Submitted.

\section{SELECTED CONFERENCE PRESENTATIONS}

CYTOGLOBIN INTERACTIONS WITH HYDROPHOBIC PROBE 1.8-ANS AND LIPIDS. Luisana Astudillo, Khoa Pham, Sophie Bernad, Valerie Derrien, Pierre Sebban and Jaroslava Miksovska. 245th ACS National Meeting. New Orleans, LA. April 2013.

CYTOGLOBIN INTERACTIONS WITH HYDROPHOBIC PROBE 1.8-ANS. Luisana Astudillo, Khoa Pham, Sophie Bernad, Valerie Derrien, Pierre Sebban and Jaroslava Miksovska. Biophysical Society 57th Annual Meeting. Philadelphia, PA. February 2013.

IMPACT OF THE INTERNAL DISULFIDE BOND ON STRUCTURAL DYNAMICS IN VERTEBRATE HEXA-COORDINATE HEMOGLOBINS. Luisana Astudillo, Sophie Bernad, Valerie Derrien, Pierre Sebban and Jaroslava Miksovska. Biophysical Society 56th Annual Meeting. San Francisco, CA. February 2012.

TIME-RESOLVED THERMODYNAMICS OF CO BINDING TO NEUROGLOBIN. Luisana Astudillo, Sophie Bernad, Valerie Derrien, Pierre Sebban and Jaroslava Miksovska. FAME. Tarpon Springs, FL. May 2011.

CONFORMATIONAL DYNAMICS IN NEUROGLOBIN. Luisana Astudillo, Pierre Sebban and Jaroslava Miksovska. Biophysical Society 55th Annual Meeting. Baltimore, MD. February 2011.

ROLE OF THE INTERNAL DISULFIDE BOND IN THE CONFORMATIONAL DYNAMICS OF NEUROGLOBIN. Luisana Astudillo, Pierre Sebban and Jaroslava 
Miksovska. Biophysical Society 54th Annual Meeting. San Francisco, CA. February 2010. 